\title{
Biomimetische Oxidationsreaktionen mit zweikernigen
}

\section{Kupferpyrazolatkomplexen}

\author{
Dissertation \\ zur Erlangung des Doktorgrades \\ der Mathematisch-Naturwissenschaftlichen Fakultäten \\ der Georg-August-Universität zu Göttingen \\ vorgelegt von
}

Diplom-Chemiker

Jens Ackermann

aus Weinheim

Göttingen, den 23.09.03 
D7

Referent: Prof. Dr. Franc Meyer

Korreferent: Prof. Dr. Ulf Diederichsen

Tag der mündlichen Prüfung: 04.11.03 
Ich erkläre hiermit an Eides statt, daß ich diese Arbeit selbständig und ohne unerlaubte Hilfsmittel angefertigt habe. 
Die vorliegende Arbeit entstand im Zeitraum von Oktober 1999 bis November 2003 unter Anleitung von Prof. Dr. Franc Meyer am Anorganisch-Chemischen Institut der Ruprecht-Karls-Universität Heidelberg und dem Anorganisch-Chemischen Institut der Universität Göttingen. 


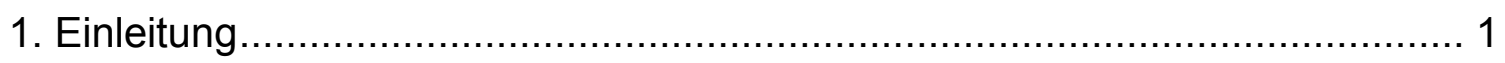

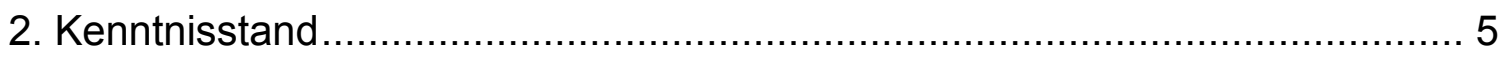

2.1 Sauerstofftransport in vivo durch Metalloproteine ................................... 5

$2.2 \mathrm{O}_{2}$-Anbindung an Kupferkomplexe - Modellsysteme für Hämocyanin......... 7

2.3 Enzyme mit aktiven Kupferzentren ............................................... 13

2.4 Biomimetische Modellsysteme von Enzymen mit Typ 3 Kupferzentrum .. 21

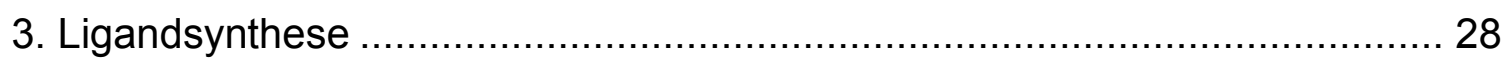

3.1 Eigenschaften der Pyrazolat-Kompartimentliganden ............................ 28

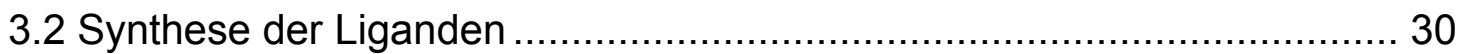

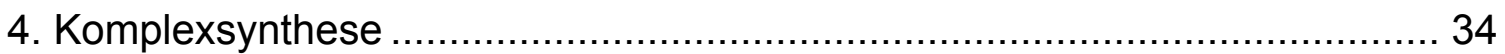

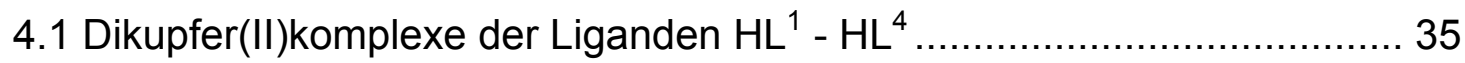

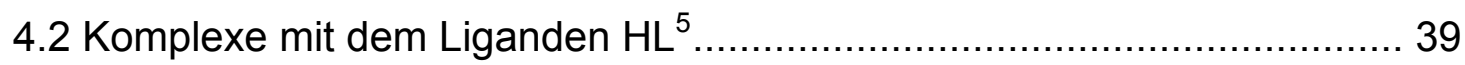

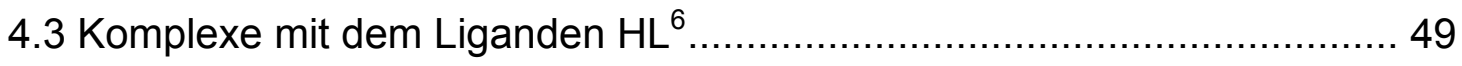

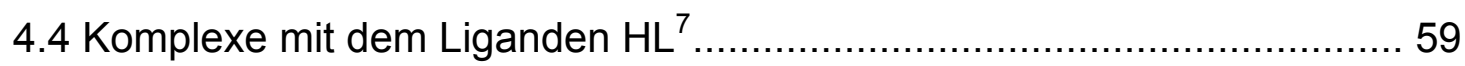

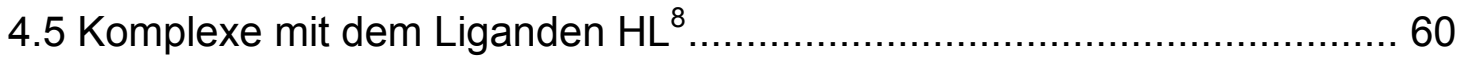

4.6 Vergleich spektroskopischer und struktureller Eigenschaften der

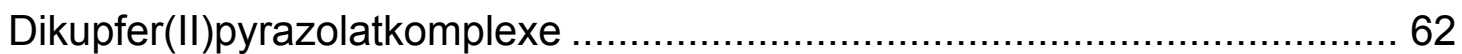

5. Kinetische Untersuchungen zur Catecholase-Aktivität ................................ 64

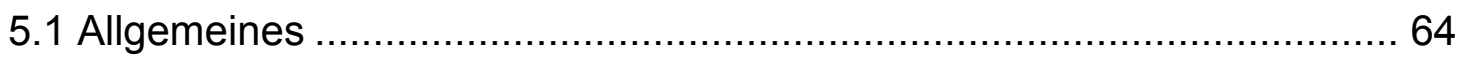

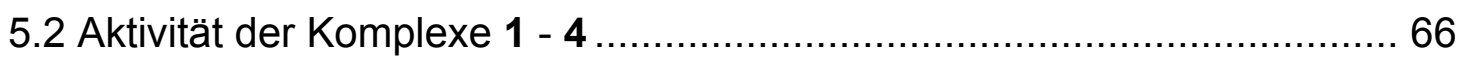

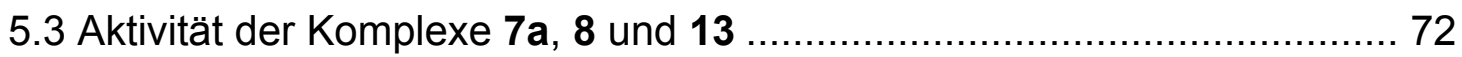

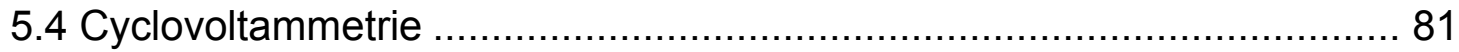

5.5 Aktivität eines Dikupferkomplexes mit einem Triazacyclononan/Pyrazolat-

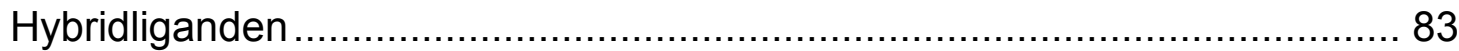

5.6 Aktivität eines einkernigen Kupferkomplexes...................................... 90

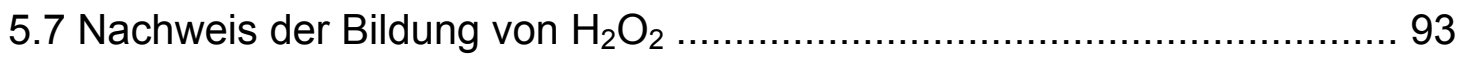

5.8 Diskussion der katalytischen Aktivität der Dikupferpyrazolatkomplexe .... 96

6. Charakterisierung von Disauerstoffaddukten.......................................102

6.1 Spektroskopische Eigenschaften von Dikupfersauerstoffaddukten.........102

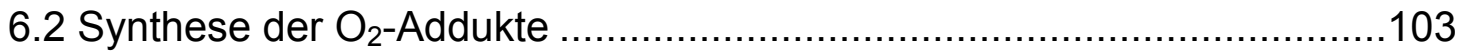

6.3 UV/Vis-Spektroskopie und Stopped-Flow-Messungen ........................105

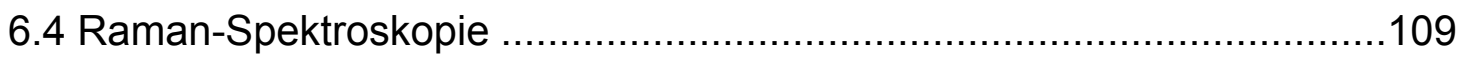

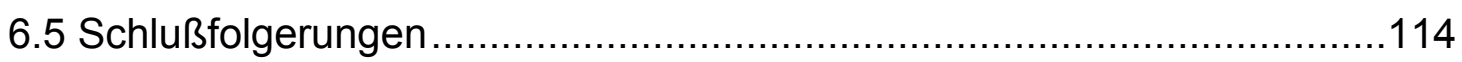

7. Addukte mit substratanalogen Molekülen.............................................117 
7.2 Charakterisierung von $\mathrm{tbc}^{2-}$ - und $\mathrm{tcc}^{2-}$-Addukten an Dikupferpyrazolatkomplexe

8. Überlegungen zum Mechanismus der Catecholase-Reaktion der

Dikupferpyrazolatkomplexe 137

9. Reaktionen der Dikupferpyrazolatkomplexe mit Phenolen 141

9.1 Versuche zur o-Hydroxylierung von Phenolen

9.2 Dinukleare Kupferkomplexe als Katalysatoren bei der oxidativen Kupplung von 2,6-Dimethylphenol 145

10. Zusammenfassung 149

10.1 Zielsetzung. 149

10.2 Synthese von Pyrazolliganden und ihrer Dikupfer(II)komplexe. 149

10.3 Katalytische Aktivität von Dikupfer(II)pyrazolatkomplexen.... 155

10.4 Charakterisierung und Aktivität von Dikupfersauerstoffaddukten. 159

10.5 Synthese von Addukten substratanaloger Moleküle 161 10.6 Versuche zur Umsetzung von Phenolen 163

11. Experimenteller Teil 166

11.1 Allgemeines 166

11.2 Die Pyrazolliganden $\mathrm{HL}^{5}-\mathrm{HL}^{8}$ 170

11.3 Synthese der Komplexe 175

11.4 Kinetische Messungen 210

11.5 Synthese und Charakterisierung von Dikupferperoxoverbindungen .....212 11.6 Reaktivität der Dikupfersauerstoffverbindungen 213

11.7 Röntgenstrukturanalyse .213

11.8 Kristalldaten und Angaben zur Struktur im Festkörper. 215

12. Literaturverzeichnis .223 
Verzeichnis der verwendeten Abkürzungen

\begin{tabular}{|ll|ll|}
\hline Ar & Aromat & $k$ & Geschwindigkeitskonstante \\
ber. & berechnet & {$[$ Kat] } & Konzentration des Katalysators \\
BPEA & Bis[2-(2-pyridyl)ethyl]amin & $K_{\mathrm{M}}$ & Michaelis-Konstante \\
BPEMA & (2-Pyridylmethyl)[2-(2- & KO'Bu & Kalium-tert-butylat \\
& pyridyl)ethyl]amin & & \\
BPMA & Bis(2-pyridylmethyl)amin & Konz. & Konzentration \\
tBu & tert-Butyl & L & Ligand \\
CT & Charge Transfer & LMCT & Ligand-Metall-Charge-Transfer \\
CV & Cyclovoltammetrie & $m$ & meta \\
d & Abstand & M & molar \\
DMP & Dimethylphenol & max & Maximum \\
DPQ & 3,3',5,5'-Tetra-methyl-4,4'- & Me & Methyl \\
& diphenochinon & & \\
dtboc & Di-tert-butyl-o-chinon & MeCN & Acetonitril \\
$\varepsilon$ & Extinktionskoeffizient & MePEA & Methyl-[2-(2-pyridyl)ethyl]amin \\
E & elektrochemisches Potential & MES & 2-Morpholinoethansulfonsäure \\
EA & Elementaranalyse & MOPS & 3-Morpholinopropansulfonsäure \\
El & Electron Impact & MS & Massenspektrometrie \\
ESI & Electron Spray lonisation & Nibeol & 4-Nitrobenzylalkohol \\
ESR & Elektronen-Spin-Resonanz & NMR & Nuclear Magnetic Resonance \\
Et & Ethyl & $\tilde{v}$ & Wellenzahl \\
exp. & experimentell & o & ortho \\
FAB & Fast Atom Bombardment & OAc & Acetat \\
GC & Gaschromatographie & PPE & Poly(1,4)phenylether \\
$\mathrm{H}_{2}$ dtbc & 3,5-Di-tert-butylcatechol & Pr & iso-Propyl \\
$\mathrm{H}_{2}$ tbc & Tetrabromcatechol & py & Pyridyl \\
$\mathrm{H}_{2}$ tcc & Tetrachlorcatechol & $\mathrm{pz}$ & Pyrazolyl \\
$\mathrm{HOAc}$ & Essigsäure & $\mathrm{R}$ & Rest \\
'Pr & Isopropyl & Red & Reduktion \\
IR & Infrarot & sh & Schulter \\
$J$ & Kopplungskonstante & $\mathrm{T}$ & Temperatur \\
\hline
\end{tabular}




\begin{tabular}{|ll|}
\hline THF & Tetrahydrofuran \\
THP & Tetrahydropyran \\
TMPA & Tripyridylmethylamin \\
Triflat & Trifluormethylsulfonat \\
TRIS & 2-Amino-2-(hydroxy-methyl)propan-1,3- \\
& diol \\
UV/Vis & Ultraviolett/Visible \\
$v$ & Geschwindigkeit \\
\hline
\end{tabular}




\section{Einleitung}

Kupfer ist schon seit langem ein wichtiger Rohstoff für den Menschen, obwohl es zu den seltenen Elementen auf unserem Planeten gehört - es macht nur ca. 0.007 \% der Erdkruste aus. Von Bedeutung sind vor allem seine Legierungen mit anderen Metallen, insbesondere Kupfer-Zinn-Legierungen, sog. Bronzen, welche schon seit der nach innen benannten „Bronzezeit“ (ca. 2200 - 800 v.Chr.) ein wichtiger Werkstoff zur Herstellung von Arbeitsgeräten, Schmuck und Waffen sind. In der modernen Gesellschaft findet das Kupfer in seiner elementaren Form z.B. aufgrund seiner hervorragenden elekrischen Leitfähigkeit Verwendung zur Herstellung elektrischer Leitungen, sowie als guter Wärmeleiter u.a. in Heiz- und Kühlschlangen und Destillationsapparaturen. ${ }^{[1,2]}$

In der chemischen Forschung und Industrie spielen Kupferverbindungen seit vielen Jahren eine Rolle in der organischen Synthese. So katalysieren z.B. Kupfer(I)salze die Zersetzung von Diazoniumsalzen, ein wichtiger Teil der Sandmeyer-Reaktion, bei welcher die Diazogruppe in aromatischen Verbindungen durch andere Nucleophile wie $\mathrm{Cl}^{-}, \mathrm{Br}^{-}$oder $\mathrm{CN}^{-}$substituiert wird. Bei der Ullmann-Kupplung werden mit Hilfe von Kupferbronze Aryle über Arylkupferzwischenstufen miteinander gekuppelt. Von besonderer Bedeutung sind Organokupfer(I)-Verbindungen, die Cuprate, welche aus Organolithiumverbindungen durch Reaktion mit Kupfer(I)salzen gewonnen werden:

$2 \mathrm{RLi}+\mathrm{Cu}^{+} \rightarrow\left[\mathrm{R}_{2} \mathrm{CuLi}\right]+\mathrm{Li}^{+}$

Die Cuprate finden in einer Vielzahl von Reaktionen Verwendung, von besonderer Bedeutung sind die nucleophile Substitution von Halogeniden und Sulfonaten, die Öffnung von Epoxiden, die 1,4-Addition an $\alpha, \beta$-ungesättigte Carbonylverbindungen, sowie die Addition an Alkine. ${ }^{[3,4]}$

In Koordinationsverbindungen bevorzugt Kupfer $(\mathrm{I})$ eine tetraedrische Koordinationsgeometrie, wie z.B. in $\left[\mathrm{Cu}(\mathrm{CN})_{4}\right]^{3-}$ oder $\left[\mathrm{Cu}(\mathrm{Py})_{4}\right]^{3-}$, aber auch kleinere Koordinationszahlen (KZ) sind möglich ( $\mathrm{KZ}$ 2: $\left[\mathrm{CuCl}_{2}\right]^{-}, \mathrm{KZ}$ 3: $\left.\left[\mathrm{Cu}(\mathrm{CN})_{3}\right]^{2-}\right)$. Für Kupfer(II) werden vor allem die Koordinationszahlen 4, 5 und 6 gefunden. Die 
Koordinationsgeometrie wird durch den Jahn-Teller-Effekt beeinflußt, welcher aufgrund der ungleichen Besetzung der $e_{g}$-Orbitale im oktaedrischen Kristallfeld zu einer Verzerrung in Form einer Dehnung des Oktaeders führt. Somit weisen die apicalen Liganden bei fünf- oder sechsfacher Koordination des Kupfers meist einen größeren Abstand als die basalen Liganden auf, eine quadratisch planare Koordination kann als Extremfall eines tetragonal verzerrten Oktaeders betrachtet werden. ${ }^{[2]}$

Ist Kupfer im Alltag und der chemischen Praxis ein gewohntes Element, so ist die Tatsache, daß es für viele Lebensarten - darunter auch den Menschen - ein essentielles Spurenelement ist, zunächst überraschend - zumal Kupfer(II)salze schon in geringen Mengen für Algen, Pilze und Bakterien äußerst giftig wirken. ${ }^{[1]}$ Kupfer ist nach Eisen und Zink das dritthäufigste Übergangsmetall in Organismen. Im menschlichen Körper findet man es z.B. in der Cytochrom c-Oxidase, einem Enyzm der Mitochondrienmembran, das drei koordinativ gebundene Kupfer- und zwei Eisenzentren enthält und das als Endstufe der Atmungskette $\mathrm{O}_{2}$ und $\mathrm{H}^{+}$zu $\mathrm{H}_{2} \mathrm{O}$ umwandelt. Im erwachsenen menschlichen Körper befinden sich ca. 150 mg Kupfer. Weitere bekannte kupferhaltige Proteine sind Z.B. Cu,Zn-Superoxid-Dismutase (Antioxidationsfunktion), Hämocyanin $\left(\mathrm{O}_{2}\right.$-Transport), Tyrosinase (Oxygenierung und Oxidase-Aktivität) und Catechol-Oxidase (Oxidase-Aktivität). Kupferproteine spielen auch bei der Elektronenübertragung eine wichtige Rolle. ${ }^{[5]}$

Da die Funktionen von Kupfer und anderen „anorganischen“ Elementen in biologischen Organismen immer größere Beachtung finden, versucht die bioanorganische Chemie, das Wirken dieser Elemente in biologischen Systemen besser zu verstehen. Außerdem sollen Funktionsprinzipien der Metalloproteine auf andere, einfachere synthetische Modellsysteme übertragen werden. Zum einen werden dazu Struktur und Funktion des jeweiligen Proteins selbst untersucht. Zum anderen werden einfache, sog. biomimetische, Modellverbindungen konstruiert, die entweder die Reaktionszentren der Metalloproteine strukturell und elektronisch möglichst genau nachahmen, oder die sich zwar vom aktiven Zentrum des Proteins unterscheiden, aber eine ähnliche Funktion erfüllen. Da Enzyme über einen langen Zeitraum durch evolutionäre Prozesse optimiert wurden, sollte die Entwicklung 
analoger, biomimetischer Modellverbindungen $\mathrm{zu}$ effizienten und wirtschaftlich interessanten Katalysatoren führen.

Ziel dieser Arbeit ist es, neue Modellsysteme vorzustellen, die auf den Erkenntnissen über Kupferproteine beruhen, bei denen sich zwei Kupferzentren in enger Nachbarschaft zueinander befinden (Typ 3 Kupferzentrum) und so bei der Vermittlung von Redoxreaktionen miteinander kooperieren können. ${ }^{[5]}$ Vorbilder sind hier das Sauerstoff transportierende Hämocyanin, sowie die Tyrosinase und die Catechol-Oxidase, welche mit Hilfe molekularen Sauerstoffes die Oxygenierung von Phenolen zu 1,2-Diphenolen bewirken (nur Tyrosinase) und die Oxidation von 1,2Diphenolen („Catecholen“) zu o-Chinonen katalysieren (Tyrosinase und CatecholOxidase).

Verwendet wird in dieser Arbeit eine Reihe von Kompartimentliganden, deren gemeinsames Merkmal ein zentraler Pyrazolkern ist, an dem in 3- und 5-Position verschiedene Seitenarme angebracht sind, die ähnlich zu den entsprechenden Kupferproteinen weitere N-Donoratome enthalten. Durch diesen Aufbau weist der Chelatligand zwei „Taschen“ auf, in welchen zwei Kupferionen koordinativ gebunden werden können (Abbildung 1).

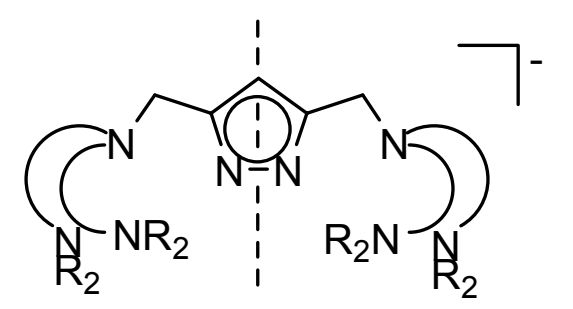

I<smiles>[R20]CCN(C)Cc1n[nH]c(CN(C)CCC)c1I</smiles>

II

Abbildung 1: Schematische Darstellung der verwendeten Kompartimentligandsysteme.

Jeder der beiden Koordinationshalbräume entspricht dabei formal einem einfachen Aminchelatliganden wie z.B. dem Tris(aminoethyl)amin (TREN) in Typ I oder dem Diethylentriamin (DIEN) in Typ II. Die Diazingruppe des Pyrazolats gibt den Bereich vor, in welchem sich der Kupfer-Kupfer-Abstand bewegt. Dieser soll dann durch Wahl unterschiedlich langer Seitenarme variiert werden. Ebenso werden Seitenarme mit unterschiedlichen koordinativen und elektronischen Eigenschaften an den Pyrazolkern angebracht. 
Schwerpunkt dieser Arbeit ist die Untersuchung der Auswirkungen all dieser Parameter auf die katalytischen Eigenschaften der entsprechenden dinuklearen Kupferzentren. Zu diesem Zweck wurde eine Reihe von Dikupfer(II)pyrazolatkomplexen synthetisiert, charakterisiert und auf ihre katalytische Aktivität getestet.

Zur weiteren Aufklärung der Abläufe am Reaktionszentrum der Katalysatoren sollten aus Dikupfer(I)komplexen und $\mathrm{O}_{2}$ Sauerstoffaddukte synthetisiert, spektroskopisch charakterisiert und auf ihre Aktivität überprüft werden. Ebenso sollten Addukte mit substratanalogen Molekülen, welche selbst nicht umgesetzt werden, Aufschlüsse über Intermediate des Katalysezyklus geben.

Ziel ist es, durch Kombination der erhaltenen Ergebnisse ein detaillierteres Bild über die Voraussetzungen für katalytische Oxidations- bzw. Oxygenierungsaktivität sowie über den Mechanismus von Sauerstoffaktivierung und Substratumsatz bei zweikernigen Kupfersystemen zu erhalten. 


\section{Kenntnisstand}

\subsection{Sauerstofftransport in vivo durch Metalloproteine}

Sauerstoff ist für fast alle biologischen Systeme ein lebensnotwendiger Energielieferant. Während in der Uratmosphäre unseres Planeten Sauerstoff keine besondere Rolle spielte, macht dieser mittlerweile etwa $21 \%$ der Atmosphäre unseres Planeten aus. Dieser Konzentrationsanstieg resultiert hauptsächlich aus der Photosynthese der Pflanzen, welche unter Ausnutzung von Sonnenlicht als Energiequelle aus $\mathrm{CO}_{2}$ und $\mathrm{H}_{2} \mathrm{O}$ Kohlenhydrate und $\mathrm{O}_{2}$ gewinnen. In Folge dieses Anstiegs entwickelten sich Organismen, welche in der Lage waren, nicht nur zunächst oxidative Schäden durch dieses Diradikal zu verhindern, sondern dieses sogar als Oxidationsmittel im eigenen Stoffwechsel als wesentlich effizientere Alternative zum anaeroben Stoffwechsel zu verwenden. ${ }^{[6]}$ Aerobe Organismen „verbrennen“ Kohlenhydrate mit Hilfe molekularen Sauerstoffes vollständig zu $\mathrm{CO}_{2}$ und $\mathrm{H}_{2} \mathrm{O}$, während aus Fettsäuren durch fortschreitende Oxidation an deren $\beta$-CAtom Acetyl-CoA gewonnen wird.

Hierzu muß im Organismus der Sauerstoff von außen dorthin transportiert werden, wo er für oxidative Reaktionen benötigt wird. Während bei kleinen Organismen der Sauerstoff durch Diffusion transportiert werden kann, müssen bei höherentwickelten Organismen $\mathrm{O}_{2}$-Transportproteine in einem Kreislaufsystem die Aufgabe übernehmen, das $\mathrm{O}_{2}$ vom Atmungsorgan zu den Zellen zu transportieren. Oft werden solche Transportfunktionen von Metalloproteinen übernommen, welche in ihrem aktiven Zentrum Kupfer oder Eisen aufweisen. ${ }^{[5,7]}$ So weist das Hämoglobin - ein tetrameres Protein aus vier Proteinuntereinheiten - in jeder Untereinheit eine sog. Hämgruppe auf. In der oxygenierten Form des Proteins ist in dieser Gruppe ein Eisenion in Form einer quadratischen Bipyramide basal durch vier N-Atome eines umgebenden Porphyrinringes und in den apicalen Positionen durch einen Histidinrest, sowie den gebundenen molekularen Sauerstoff koordiniert (Abbildung 2). Der Sauerstoff bindet end on an das Eisenzentrum mit einem Winkel von $115^{\circ}{ }^{[8]}$ Resonanz-Ramanspektren ergaben hierbei eine O-O-Streckschwingung bei 1105 $\mathrm{cm}^{-1}$, charakteristisch für ein koordiniertes Superoxidion $\mathrm{O}_{2}{ }^{-}$, wonach dem Eisen eine Oxidationsstufe von +3 zukommt. Das $\mathrm{O}_{2}^{-}$-lon wird wiederum durch eine $\mathrm{H}$-Brücke zu einem benachbarten Histidinrest stabilisiert. Weitere eisenhaltige Transportproteine 
sind das dem Hämoglobin eng verwandte Myoglobin, welches für den Sauerstofftransport im Gewebe zuständig ist, sowie das nur intrazellulär vorkommende Hämerythrin, welches zwei benachbarte Nicht-Häm-Eisenzentren aufweist.

Die dritte Variante der $\mathrm{O}_{2}$-transportierenden Metalloproteine ist die der Hämocyanine. Kupfer(I) und Kupfer(II) sind leicht ineinander umzuwandeln $\left(E^{0}\left(\mathrm{Cu}^{\prime \prime} / \mathrm{Cu}^{\prime}\right)=+0.153 \mathrm{~V}\right)$ und deshalb für die Vermittlung von Redoxvorgängen besonders geeignet, in diesem Falle die reversible Bindung molekularen Sauerstoffs. Die Festkörperstrukturen sowohl des Deoxy- als auch des Oxyhämocyanins konnten röntgenographisch ermittelt werden. ${ }^{[9,10,11,12]}$ Aus der Struktur der Oxyform geht hervor, daß der Sauerstoff peroxidisch in Form einer $\mu-\eta^{2}: \eta^{2}$-Koordination zwei Kupfer(II)ionen im Abstand von $3.6 \AA$ (Typ 3 Kupferzentrum) verbrückt. Jedes Kupferion ist weiterhin von drei Histidinresten koordiniert (Abbildung 2).

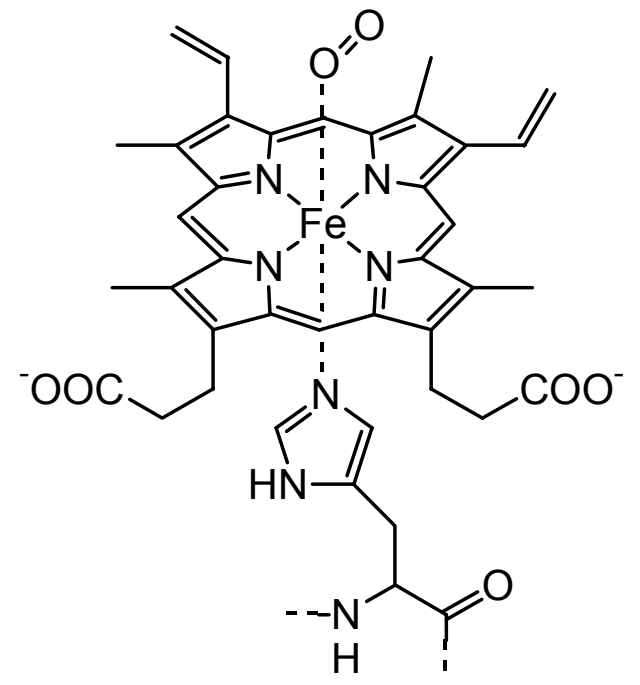

III

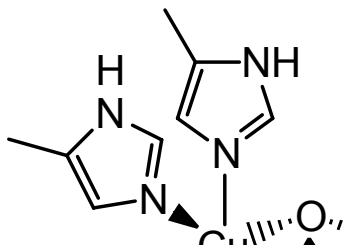<smiles>Cc1cnc[nH]1</smiles>

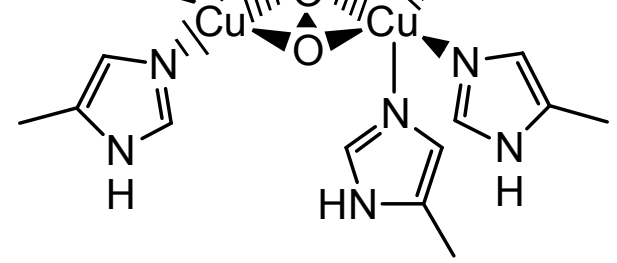

Abbildung 2: Struktur der Hämgruppe mit gebundenem $\mathrm{O}_{2}$ im Hämoglobin (III), $\mathrm{O}_{2}$-Bindungsstelle im Oxyhämocyanin (IV). ${ }^{[8]}$

In einer Zweielektronenredoxreaktion wird somit das vereinte Redoxvermögen zweier in enger Nachbarschaft befindlicher Kupferzentren unter Oxidation beider $\mathrm{Cu}(\mathrm{l})$ des Deoxyhämocyanin zu $\mathrm{Cu}(\mathrm{II})$ genutzt, um den kinetisch inerten Triplett-Sauerstoff zu aktivieren und in Form einer Peroxidbrücke reversibel zu binden. 


\title{
$2.2 \mathrm{O}_{2}$-Anbindung an Kupferkomplexe - Modellsysteme für Hämocyanin
}

Um die Prinzipien bei der Sauerstoffanbindung- und -aktivierung an solche Metalloproteine besser zu verstehen und die gewonnen Erkenntnisse bei neuen synthetischen Verbindungen anzuwenden, wurden in den letzten Jahrzehnten zahlreiche einfache, biomimetische Verbindungen synthetisiert, welche die natürlichen Vorbilder in ihrer Struktur nachahmen oder zumindest vergleichbare strukturelle und elektronische Parameter aufweisen und damit ähnliche Funktionen wie diese haben können.

Molekularer Sauerstoff kann auf viele verschiedene Arten an Kupferzentren solcher Modellkomplexe anbinden, von denen mittlerweile eine Vielzahl röntgenographisch und spektroskopisch charakterisiert sind (Abbildung 3). ${ }^{[13-36]}$<smiles>OO[AlH2]O[GaH]</smiles>

$\eta^{1}$-Superoxo*<smiles>[AlH2]O[SiH2]1O[AlH2]O1</smiles>

$\mu-\eta^{2}: \eta^{2}-$ Peroxo

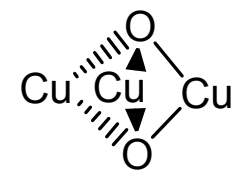

$\operatorname{Bis}\left(\mu_{3}-0 \times 0\right)$<smiles>[SiH2]O[SiH2]O[GeH2]</smiles>

$\operatorname{Bis}(\mu-0 \times 0)$<smiles>[AlH2]O[GeH3]</smiles>

$\mu_{4}$-Peroxo<smiles></smiles>
$\eta^{2}$-Superoxo $\mu-1,1-$ Hydroperoxo<smiles>[AlH2]OO[AlH2]</smiles><smiles>[AlH2]O[AlH2]</smiles>
$\mu-\eta^{1}: \eta^{1}-$ trans-Peroxo $\mu-\eta^{1}: \eta^{1}-$ cis-Peroxo*

\begin{abstract}
Abbildung 3: Mögliche Addukte von $\mathrm{O}_{2}$ an Kupferzentren, ${ }^{*}$ noch nicht röntgenographisch charakterisiert).
\end{abstract}

Einer der großen Erfolge der bioanorganischen Chemie war die korrekte Vorhersage des Bindungsmodus von Sauerstoff im Oxyhämocyanin. Kitajima et al. synthetisierten im Jahre 1988 durch Reaktion des Kupfer(II)komplexes des Liganden $\left[\mathrm{HB}\left(3,5-\mathrm{Me}_{2} \mathrm{pz}\right)_{3}\right]^{-}$mit $\mathrm{H}_{2} \mathrm{O}_{2}$, aber auch durch direkte Reaktion des entsprechenden Kupfer(I)komplexes mit $\mathrm{O}_{2}$, einen Komplex V, dessen UV/Vis-Spektrum erstaunliche Ähnlichkeit mit jenem von Oxyhämocyanin aufwies, mit Banden bei $551 \mathrm{~nm}$ 
$\left(\varepsilon=800 \mathrm{~mol} \mathrm{l}^{-1} \mathrm{~cm}^{-1}\right)$ und $349 \mathrm{~nm}\left(\varepsilon=21000 \mathrm{~mol} \mathrm{l}^{-1} \mathrm{~cm}^{-1}\right)$. Ebenso ergaben sich aus dem Resonanz-Ramanspektrum und den magnetischen Eigenschaften des Komplexes so große Ähnlichkeiten, daß der Komplex als Modellverbindung für Oxyhämocyanin gelten konnte ${ }^{[13]}$ - trotz der Irreversibilität der Sauerstoffanbindung. Die strukturelle Aufklärung dieses Komplexes im Jahre $1989^{[14]}$ ergab eine neuartige $\mu-\eta^{2}: \eta^{2}$-peroxidische Anbindung des Sauerstoffes zwischen zwei Kupferzentren der monomeren Einheiten $\mathrm{Cu}\left(\mathrm{HB}\left(3,5-{ }^{i} \mathrm{Pr}_{2} \mathrm{pz}\right)_{3}\right)$ (Abbildung 4, Komplex V).

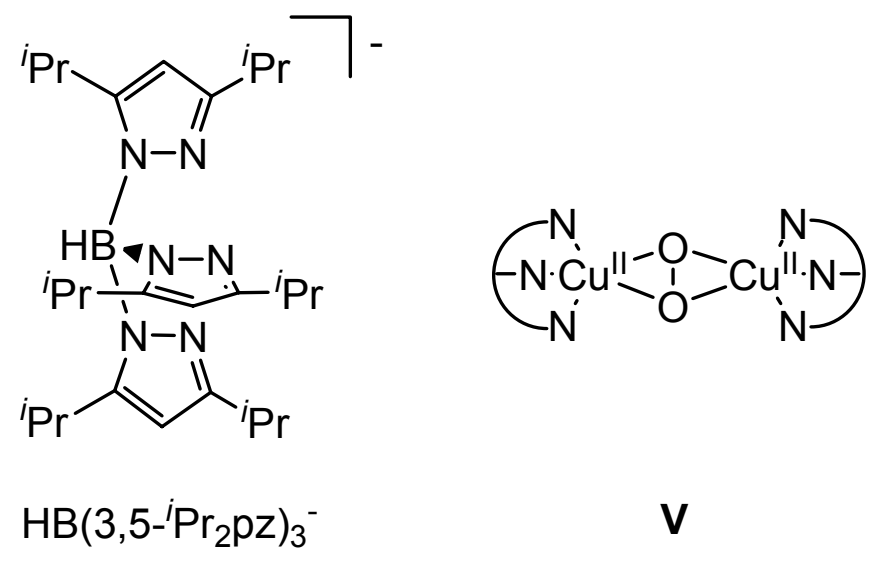

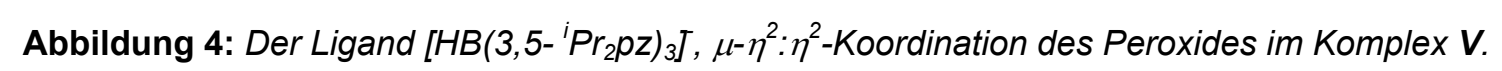

In der Tat wurde dieser postulierte Bindungsmodus für Oxyhämocyanin im Jahre 1994 anhand einer Festkörperstruktur des Metalloproteins bestätigt (Abbildung 2). ${ }^{[0]}$ Kodera et al. entwickelten einen Liganden VI mit zwei Kavitäten, welche beide in der Lage sind, Kupferionen aufzunehmen (Abbildung 5). ${ }^{[15]}$ Hier gelang die Synthese eines Sauerstoffadduktes durch Reaktion des Kupfer(II)komplexes mit $\mathrm{H}_{2} \mathrm{O}_{2}$ oder der Kupfer(I)verbindung mit $\mathrm{O}_{2}$. Aus der Festkörperstruktur ergab sich auch hier eine $\mu-\eta^{2}: \eta^{2}$-Koordination des Peroxids zwischen die Kupferzentren. Das Besondere an diesem Komplex ist, daß er zum einen - im Gegensatz zum weitaus größten Teil der bislang bekannten Kupfer-Peroxo-Verbindungen - noch bei Raumtemperatur stabil ist $\left(t_{1 / 2\left(25^{\circ} \mathrm{C}\right)}=25.5 \mathrm{~h}\right)$ und zum anderen in der Lage ist, den molekularen Sauerstoff reversibel zu binden. Die Reversibilität wurde zurückgeführt auf eine starke Verzerrung der $\mathrm{Cu}_{2} \mathrm{O}_{2}$-Einheit, durch welche die $\mathrm{O}_{2}$-Affinität des Komplexes verringert wird. 


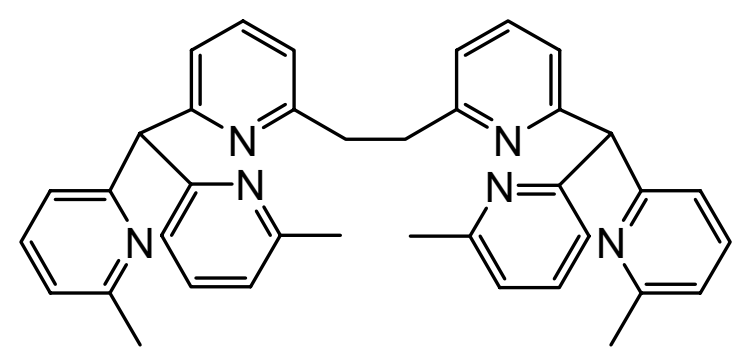

Abbildung 5: Der Ligand VI von Kodera et al.

Unerwartet war die Entdeckung von Tolman et al., daß bei manchen Dikupfersauerstoffaddukten beide Kupfer(I)zentren durch den molekularen Sauerstoff zu Kupfer(III) unter Bildung einer Bis( $\mu$-oxo)-verbrückten Spezies mit deutlich anderen spektroskopischen Eigenschaften oxidiert werden. Die röntgenographisch charakterisierten $\mathrm{Bis}(\mu$-oxo)dikupferkomplexe weisen einen verringerten $\mathrm{Cu}-\mathrm{O}-$ Abstand von durchschnittlich $1.81 \AA$ auf - ein Indiz für eine Kupfer(III)spezies. Außerdem findet man einen außerordentlich langen O-O-Abstand von durchschnittlich $2.327 \AA$, so daß eine O-O-Bindung unwahrscheinlich ist. ${ }^{[16]}$ So wurden für Kupfer(I)komplexe von verschiedenen 1,4,7-trisubstituierten Triazacyclononanen durch Umsetzen mit $\mathrm{O}_{2}$ nicht nur die zuvor bereits bekannten $\mu-\eta^{2}: \eta^{2}$-Anbindungen gefunden. Es wurden vielmehr auch Liganden entwickelt, mit denen entweder ausschließlich Bis( $\mu$-oxo)-Anbindungen gebildet wurden, oder auch einige Liganden wie das 1,4,7-Triisopropyl-1,4,7-triazacyclononan $\left.\left(\mathrm{L}^{i} \operatorname{Pr}_{3}\right)\right)$, bei welchen je nach verwendetem Lösungsmittel, Gegenion und Temperatur eines der beiden Isomeren in das andere umgewandelt werden kann. ${ }^{[17,18,19,20]}$ Mit dem Liganden $m-X Y L^{i P r 4}$, einem Liganden mit zwei Tetraazacyclononantaschen, welche durch einen $m$-Xylyl-Spacer verbunden sind, wurde in Dichlormethan oder Aceton bei niedrigen Konzentrationen anhand seiner typischen UV/Vis- und Ramanspektroskopischen Eigenschaften eine intramolekulare $\mu-\eta^{2}: \eta^{2}$-Brücke gefunden, bei höheren Konzentrationen bildeten sich intermolekulare Bis( $\mu$-oxo)-Brücken (Schema 1). ${ }^{[21]}$ Kurze Brücken in zweikernigen Kupferkomplexen verhindern die Ausbildung eines $\mu-\eta^{2}: \eta^{2}$-Peroxo-dikupferkomplexes und begünstigen die Bis( $\mu$-oxo)-Struktur. ${ }^{[22]}$ 


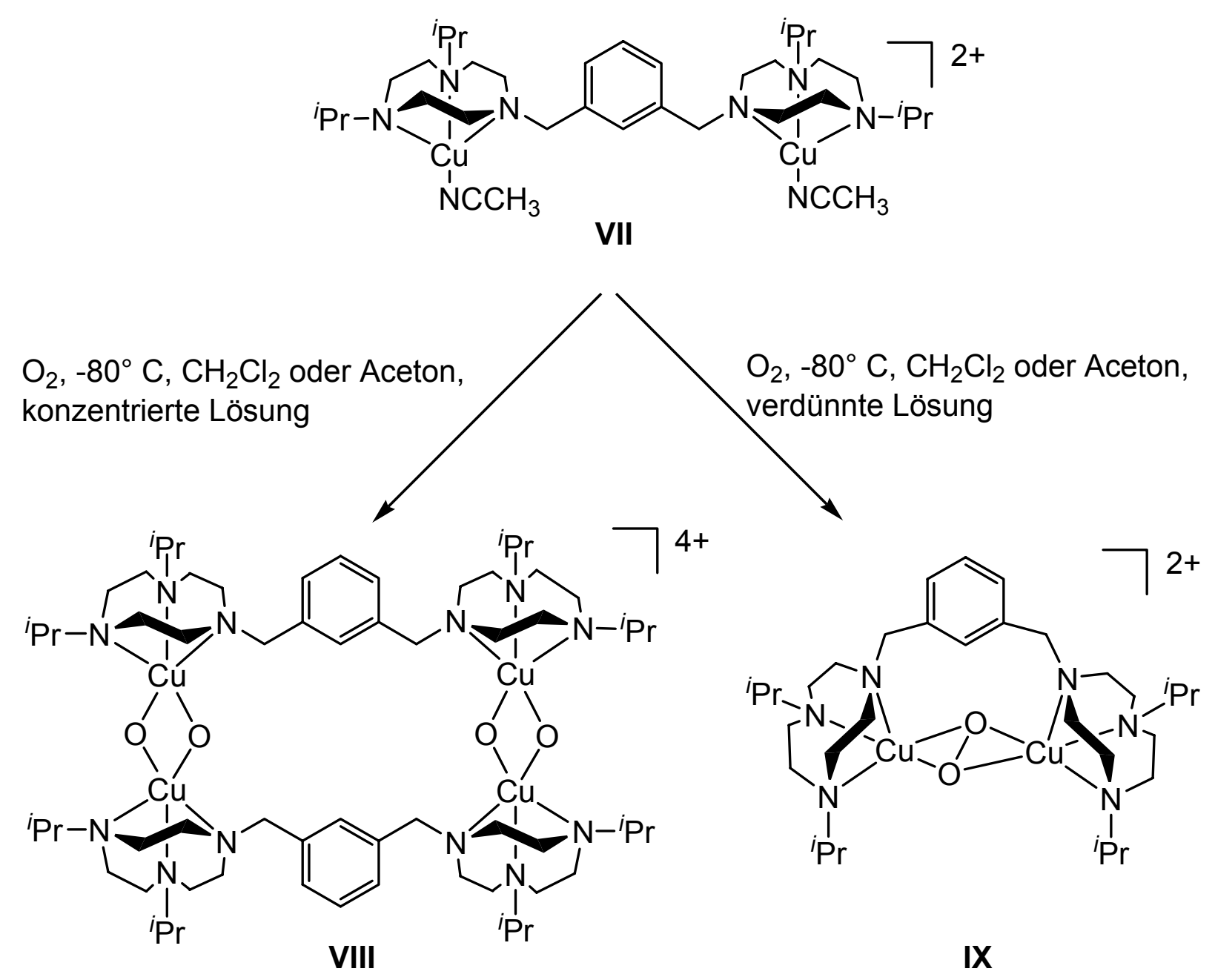

Schema 1: Kontrolle der Art der Sauerstoffanbindung durch Variation der Konzentration.

Ausgehend von temperaturabhängigen kinetischen Experimenten unter Verwendung der Stopped-Flow-Technik postuliert Tolman als Mechanismus der Oxygenierung bei einigen der untersuchten mononuklearen Kupfer(I)komplexe, daß zunächst eine (Superoxo)-Kupfer(II)spezies gebildet wird, wonach sich dann zunächst ein $\mu-\eta^{2}: \eta^{2}$ Intermediat bildet, welches im Gleichgewicht mit der unter Bindungsbruch gebildeten isomeren $\mathrm{Bis}\left(\mu\right.$-oxo)-Spezies liegt (Schema 2). ${ }^{[21,22,23,24,25]}$

$\left[\mathrm{LCu}(\text { Donor })^{\mathrm{n}+}\right] \underset{\substack{\text { niedrige } \\ \text { Temp. }}}{\stackrel{\mathrm{O}_{2}}{\longrightarrow}}\left[\mathrm{LCu}\left(\mathrm{O}_{2}\right)\right]^{\mathrm{n}+} \stackrel{\left[\mathrm{LCu}(\text { Donor })^{\mathrm{n}+}\right]}{\longrightarrow}[\mathrm{LCu}>\mathrm{O}=\mathrm{CuL}]^{2 \mathrm{n}+} \rightleftharpoons\left[\mathrm{LCu}>\mathrm{O}^{2 \mathrm{O}+}\right.$

Schema 2: Bildung von Bis( $\mu$-oxo)-Dikupfer(III)komplexen aus Kupfer(I)komplexen und $\mathrm{O}_{2}$.

Die Entdeckung der Bis( $\mu$-oxo)-Spezies wirft die Frage auf, ob ein solches Zwischenprodukt auch bei Metalloproteinen wie Hämocyanin und verwandten 
Enzymen wie Tyrosinase und Catechol-Oxidase eine Rolle spielen könnte. Diese Frage ist aber noch nicht abschließend geklärt. ${ }^{[25]}$

Mittlerweile sind eine Reihe weiterer Bis( $\mu$-oxo)-Dikupferkomplexe mit unterschiedlichen Liganden gefunden worden. Bei einem Kompex von Karlin et al. mit einem Bis(2-pyridin-2-yl-ethyl)amin-Liganden wurde im Kristall ein Gemisch beider Isomere aus $80 \% \operatorname{Bis(\mu -oxo)-~und~} 20 \% \mu-\eta^{2}: \eta^{2}$-Form gefunden. ${ }^{[26,27]}$ Die Verwendung von $\beta$-Diketiminat-Anionen $\mathbf{X}$ (Abbildung 6 ) erlaubt je nach Art der Substituenten am $\beta$-Diketiminatzentrum die gezielte Synthese von entweder $\eta^{2}$ Superoxo-Addukten $\left(1 \mathrm{R}_{1}=\mathrm{Me}, \quad \mathrm{R}_{2}=\mathrm{H}\right)$, oder neutralen $\operatorname{Bis}(\mu$-oxo)-Dikupferkomplexen $\left(2 \mathrm{R}_{1}=\mathrm{H}, \mathrm{R}_{2}=\mathrm{Ar}\right)$. Die Art der Produkte wurde mittels UV/VisResonanz-Raman und EPR-Spektroskopie sowie mittels $\mathrm{O}_{2}$-Titration bestimmt. ${ }^{[28,29]}$

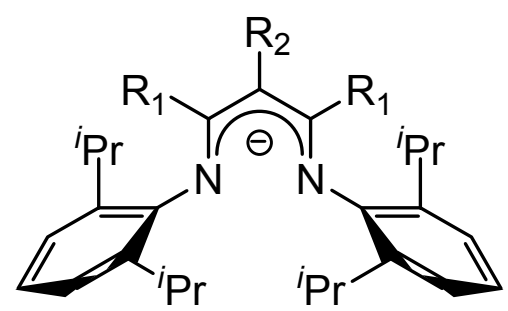

Abbildung 6: $\beta$-Diketiminatligand X, 1: $R_{1}=M e, R_{2}=H, 2: R_{1}=H, R_{2}=A r$.

Die erste röntgenographische Charakterisierung einer Kupferperoxoverbindung gelang Karlin et al. 1988, indem sie den Kupfer(I)komplex [Cu(TMPA) $(R C N)] \mathrm{PF}_{6}(\mathrm{R}=$ $\mathrm{Me}$, Et) mit $\mathrm{O}_{2}$ bei tiefer Temperatur umsetzten. In der Festkörperstruktur des erhaltenen Produktes XI verbindet der Sauerstoff in Form einer peroxidischen trans$\mu-\eta^{1}: \eta^{1}$-Brücke zwei Cu"TMPA-Einheiten (Abbildung 7). ${ }^{[30]}$<smiles>c1ccc(CN(Cc2ccccn2)Cc2ccccn2)nc1</smiles>

TMPA

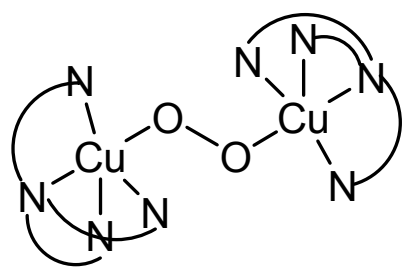

XI

Abbildung 7: Der Ligand TMPA, $\mu-\eta^{1}: \eta^{1}$-Koordination des Peroxides im Komplex XI. 
Ein Modellkomplex XII für die oxidierte Form der Kupferenzyme Ascorbat Oxidase, Ceruloplasmin und Laccase mit einem dreikernigen Kupferzentrum, ${ }^{[31]}$ deren Aufgabe die Vierelektronenreduktion von $\mathrm{O}_{2}$ zu $\mathrm{H}_{2} \mathrm{O}$ ist, wurde 1996 von Stack et al. vorgestellt. ${ }^{[32]}$ Hier finden sich drei Kupferionen in einer annähernd quadratisch planaren Umgebung, koordiniert von zwei O-Donoren des als $\mu_{3}$-Oxobrücke vorliegenden Sauerstoffs und zwei N-Donoren. Der O-O-Abstand von $2.37 \AA$ zeigt deutlich, daß die O-O-Bindung gebrochen ist. ${ }^{[16]}$<smiles>C[C@@H]1CCCC[C@@H]1N(C)C</smiles>

$\mathrm{L}_{\text {TM }}$

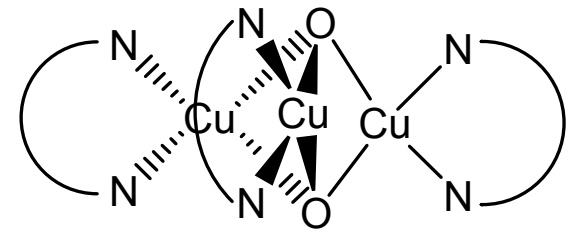

XII

Abbildung 8: Der Ligand $L_{T M}, \mu_{3}$-Oxo-Koordination des Sauerstoffes im Komplex XII.

Die Oxidationsstufen der Kupferzentren werden aufgrund von magnetischen Messungen als $2 \times \mathrm{Cu}^{\prime \prime}$ und $1 \times \mathrm{Cu}^{\text {III }}$ beschrieben.

Für eine $\mu_{4}$-peroxidische Verbrückung des aufgenommenen $\mathrm{O}_{2}$ sind die Festkörperstrukturen zweier ungewöhnlich stabiler Modellkomplexe bekannt. Der eine stammt von Krebs et al. und weist eine cis- $\mu_{4}$-Peroxobrücke auf, welche über einer aus den vier Kupferzentren aufgespannten Ebene liegt. ${ }^{[33]}$ Des weiteren ist die Struktur eines $\mathrm{Cu}_{4}$-Komplexes mit trans- $\mu_{4}$-verbrückendem Peroxid und dem auch in der vorliegenden Arbeit verwendeten Liganden $\mathrm{HL}^{3}$ bekannt (Abbildung 9). ${ }^{[34]}$ 


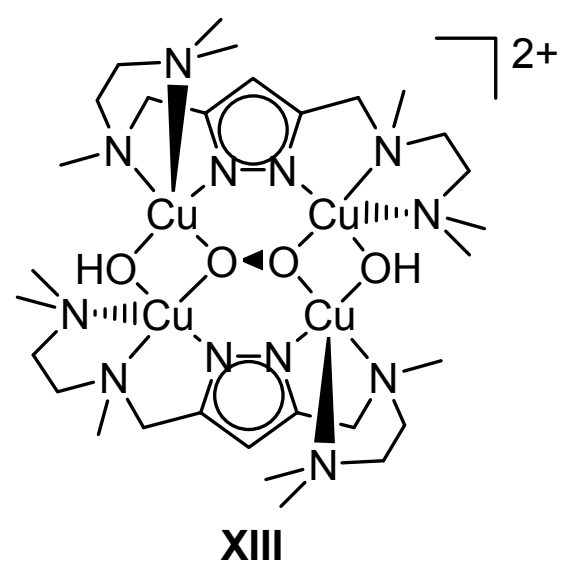

Abbildung 9: Komplex XIII mit dem Liganden $\mathrm{HL}^{3}$ mit trans- $\mu_{4}$-peroxidischer Koordination des Sauerstoffes.

Diese Verbindung XIII ist bei Raumtemperatur unter Luftatmosphäre mehrere Tage stabil und wandelt sich nur langsam in einen Komplex um, in welchem anstelle der Peroxoeinheit zwei $\mathrm{OH}^{-}$-Brücken jeweils zwei Kupferzentren verbinden.

Auch Komplexe, in denen der Sauerstoff end on als $\eta^{1}$-Hydroperoxid und in side-onKoordination als $\eta^{2}$-Superoxid an mononukleare Kupferzentren bindet, sind bekannt. Der dreizähnige, sterisch anspruchsvollere Ligand $\left[\mathrm{HB}\left(3-{ }^{t} \mathrm{Bu}-5-^{i} \mathrm{Prpz}\right)_{3}\right]^{-}$von Kitajima et al. führt zur side-on-Koordination, der vierzähnige Ligand TMPA zur end-onKoordination. ${ }^{[35,36]}$

Zusammenfassend kann man sagen, daß die Wahrung einer fünffachen Koordination an den Kupferzentren ein mitbestimmender Faktor bei der Frage ist, welche KupferSauerstoffspezies gebildet wird. Dreizähnige Chelatliganden ergeben bevorzugt eine $\eta^{2}$-Koordination und können $\mu-\eta^{2}: \eta^{2}$-Peroxo oder durch Bindungsbruch auch Bis $(\mu-$ oxo)-Brücken ausbilden, während vierzähnige Liganden zu einer $\eta^{1}$-Koordination und somit in der Regel zu $\mu-\eta^{1}: \eta^{1}-$ Komplexen führen. Ein weiterer Faktor bei dinuklearen Komplexen ist der Abstand der Kupferzentren zueinander. Große Abstände sollten die end on verbrückende Koordination favorisieren.

\subsection{Enzyme mit aktiven Kupferzentren}

Enzyme, die in der Lage sind, den gebundenen Sauerstoff bei der Katalyse von Redoxreaktionen zu nutzen, können in drei Klassen aufgeteilt werden. ${ }^{[8]}$ 
Zu den sogenannten Typ 1 Kupferproteinen gehören zum einen Proteine wie das Azurin und das Plastocyanin, welche an Elektronentransferreaktionen beteiligt sind. So werden bei der Photosynthese die im Photosystem II durch Oxidation von $\mathrm{H}_{2} \mathrm{O}$ freigewordenen Elektronen u. a. durch das mobile Elektronenträgerprotein Plastocyanin zum Photosystem I transportiert, wo diese $\mathrm{NAD}^{+}$zu NADH reduzieren. ${ }^{[6]}$ Während in der oxidierten Form des Plastocyanins das Kupferzentrum durch zwei Histidin-, einen Cystein- und einen Methioninrest verzerrt tetraedrisch koordiniert ist, so kommt beim Azurin als fünfter Ligand noch das O-Atom einer Amidgruppe hinzu. Gemeinsames Merkmal aller dieser sogenannten „blauen Kupferproteine" ist eine Bande bei ca. $600 \mathrm{~nm}$ von besonders großer Intensität $(\varepsilon>$ $2000 \mathrm{~mol} \mathrm{I}^{-1} \mathrm{~cm}^{-1}$ ), welche auf einen LMCT-Übergang von Cys $S$ auf das Kupfer(II) zurückzuführen ist. ${ }^{[37]}$

Typ II Kupferzentren werden in verschiedenen Oxidasen wie der Galactose-Oxidase und Oxygenasen wie der Dopamin- $\beta$-hydroxylase gefunden. Galactose-Oxidase ist ein Enzym mit einem einkernigen Kupferzentrum, in welchem im inaktiven Zustand bei $\mathrm{pH} 7$ das Kupferion quadratisch pyramidal koordiniert ist. Apical koordiniert ein Tyrosinrest, in den basalen Positionen sitzen zwei Histidinreste, sowie ein modifizierter Tyrosinrest mit kovalenter Bindung zum S-Atom eines nahen Cysteinrestes. An einer weiteren basalen Koordinationsstelle koordiniert ein $\mathrm{H}_{2} \mathrm{O}$ Molekül. Im aktiven Zustand des Enzyms wird diese Stelle durch das O-Atom des Galactosesubstrates besetzt. Während dem Kupferzentrum in der oxidierten Form des Enzyms zunächst die Oxidationsstufe + III zugeordnet wurde, wurde aufgrund spektroskopischer Messungen entdeckt, daß das Kupfer die Oxdiationsstufe +II aufweist, während der Tyrosinrest als Radikal vorliegt, welches mit dem Kupfer eine antiferromagnetische Wechselwirkung eingeht (XIV, Abbildung 10). ${ }^{[38]}$ 


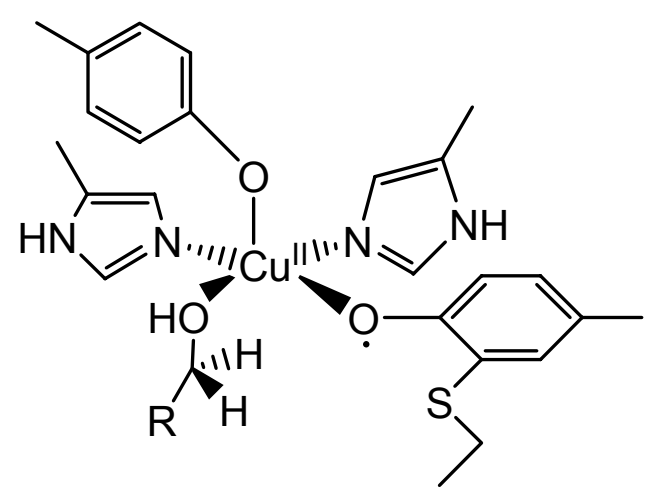

XIV

Abbildung 10: Aktives Zentrum (XIV) der Galactose-Oxidase in der oxidierten Form.

Galactose-Oxidase katalysiert die Oxidation von Galactose $(\mathbf{X V})$ und anderen primären Alkoholen zu Aldehyden unter Reduktion von $\mathrm{O}_{2} \mathrm{zu} \mathrm{H}_{2} \mathrm{O}_{2}$ (Schema 3). $\mathrm{Da}$ für diese Zweielektronenredoxreaktion das $\operatorname{Kupfer}(\mathrm{I})$ der reduzierten Form des Enzyms nur ein Elektron zur Verfügung stellen kann, wird der Tyrosinrest als weiterer Elektronenlieferant unter Bildung des Radikals benötigt.

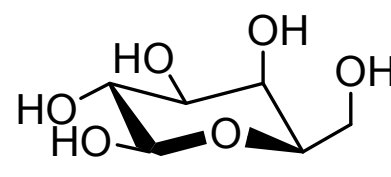

XV
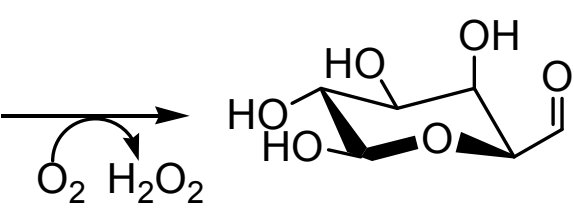

$\mathbf{X V I}$

Schema 3: : Oxidation von Galactose (XV) durch Galactose-Oxidase

Die Dopamin- $\beta$-hydroxylase katalysiert die Hydroxylierung von aus L-Dopa durch Decarboxylierung gebildetem Dopamin (XVII) zum Noradrenalin (XVIII). Beide Verbindungen sind wichtige Neurotransmitter. Noradrenalin kann durch Methylierung der Amingruppe zum Hormon Adrenalin umgesetzt werden (Schema 4). ${ }^{[6]}$<smiles>[NH3+]CCc1ccc(O)c(O)c1</smiles>

Dopamin (XVII)<smiles>[NH3+]C[C@H](O)c1ccc(O)c(O)c1</smiles>

Noradrenalin (XVIII)

Schema 4: Hydroxylierung von Dopamin zu Noradrenalin durch Dopamin- $\beta$-hydroxylase.

Proteine mit Typ 2 Kupferzentrum weisen ähnliche spektroskopische Eigenschaften wie der einfache Kupfer(II)pentaquakomplex auf. 
Mit dem aus einigen Arthropoden und Mollusken isolierten Hämocyanin wurde bereits ein Protein mit einem Typ 3 Kupferzentrum vorgestellt (siehe Abbildung 2). Hier befinden sich zwei Kupferzentren in so enger Nachbarschaft, daß zwar eine direkte Metall-Metall-Bindung nicht möglich ist, wohl aber das Zusammenwirken beider Zentren bei der Vermittlung von Zweielektronenredoxreaktionen. Zwei andere Kupferproteine - die Tyrosinase und die Catechol-Oxidase - sind strukturell dem Hämocyanin eng verwandt, haben aber enzymatische Eigenschaften.

Die Tyrosinase ist zwar strukturell noch nicht charaktersiert, weist aber die typischen spektroskopischen Eigenschaften der Typ 3 Kupferproteine auf: eine starke antiferromagnetische Kopplung zwischen den Kupferzentren, in der Oxyform eine O-O-Streckschwingung bei $\sim 750 \mathrm{~cm}^{-1}$ und Absorptionsbanden bei $\sim 350 \mathrm{~nm}$ $\left(\varepsilon \sim 20000 \mathrm{~mol} \mathrm{l}^{-1} \mathrm{~cm}^{-1}\right)$ und bei $600 \mathrm{~nm}\left(\varepsilon \sim 1000 \mathrm{~mol} \mathrm{l}^{-1} \mathrm{~cm}^{-1}\right) \cdot{ }^{[39]}$ Die Tyrosinase katalysiert die ortho-Hydroxylierung von Tyrosin (XIX) zu 3,4-Dihydroxyphenylalanin (L-DOPA (XX), Cresolase-Aktivität) und ist in der Lage, dieses anschließend zum o-Chinon (XXI) zu oxidieren (Catecholase-Aktivität). Diese Chinone polymerisieren letztlich zu Melaninen, welche wiederum z.B. bei der Wundheilung von Pflanzen eine wichtige Rolle spielen, indem sie die Wunde vor weiteren Angriffen durch Insekten schützen (Schema 5). So ist u.a. die Braunfärbung angeschnittener Äpfel auf die Bildung von Melaninen zurückzuführen. Die Catechol-Oxidase katalysiert ausschließlich den zweiten Schritt, die Oxidation von entstandenem 1,2-Diphenol ("Catechol“) zum o-Chinon. Während die Catechol-Oxidase nur in Pflanzengewebe, einigen Insekten und Crustaceen gefunden wurde, weisen eine große Zahl von Pflanzen, Pilzen, Bakterien, Säugetieren, Crustaceen und Insekten das Enzym Tyrosinase auf. ${ }^{[37]}$ 


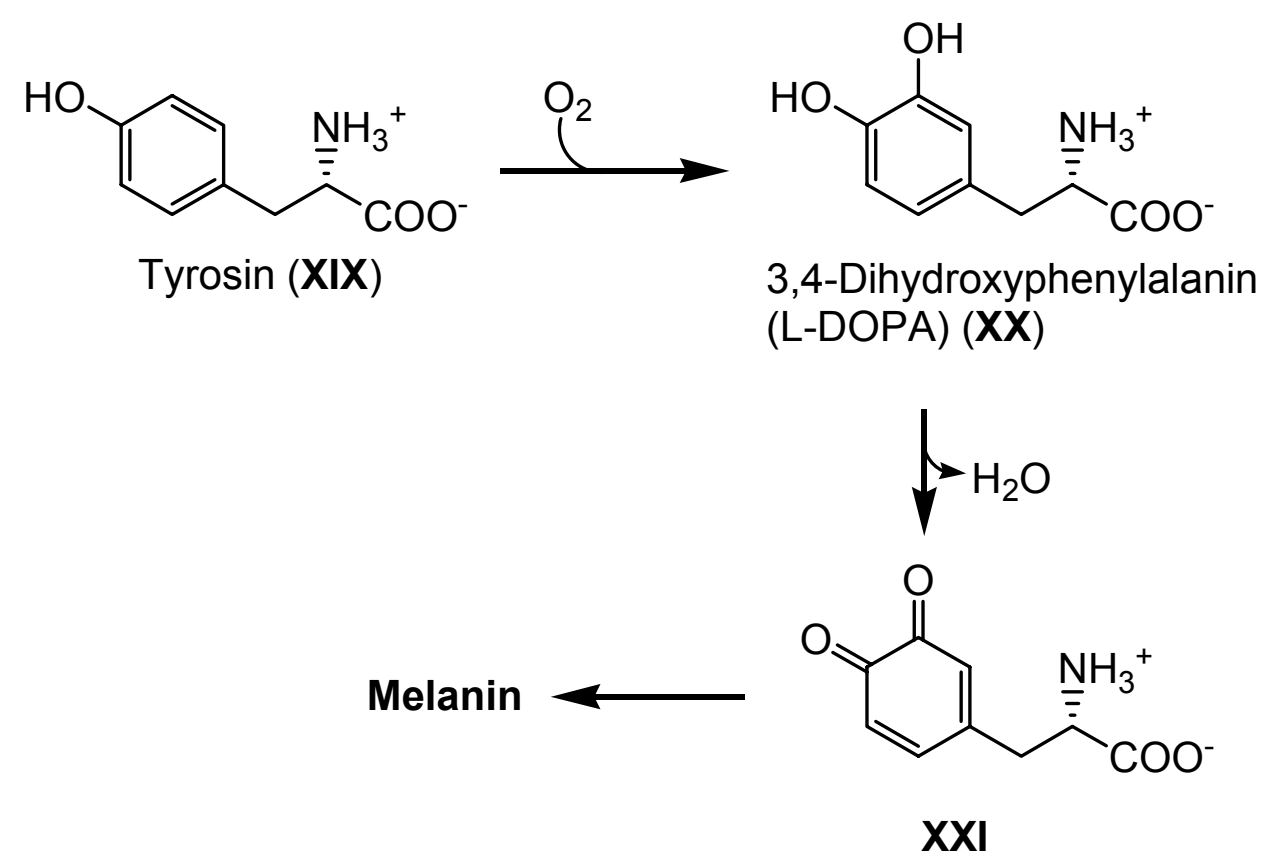

Schema 5: Synthese von Melanin aus Tyrosin durch Tyrosinase.

Die Struktur von Catechol-Oxidase der Süßkartoffel Ipomoea batatas konnte sowohl in der met- als auch in der deoxy-Form von Krebs et al. röntgenographisch aufgeklärt werden, und in der Tat weist das aktive Zentrum große Ähnlichkeit zu jenem des Hämocyanins auf. ${ }^{[39]}$ In der oxidierten met-Form findet man zwei Kupfer(II)zentren in einem Abstand von $2.9 \AA$, wobei beide durch je drei Histidinliganden sowie einen Brückenliganden trigonal pyramidal koordiniert sind. Bei dem Brückenliganden handelt es sich wahrscheinlich um ein $\mathrm{OH}^{-}$-lon (Abbildung 11). In der deoxy-Form haben beide Kupferzentren die Oxidationsstufe +1 und einen Metall-Metall-Abstand von $4.4 \AA$. Ein Kupferion ist verzerrt trigonal pyramidal von drei Histidinresten und einem $\mathrm{H}_{2} \mathrm{O}$ koordiniert, das andere quadratisch planar von drei Histidinliganden, wobei eine Koordinationsstelle unbesetzt bleibt.

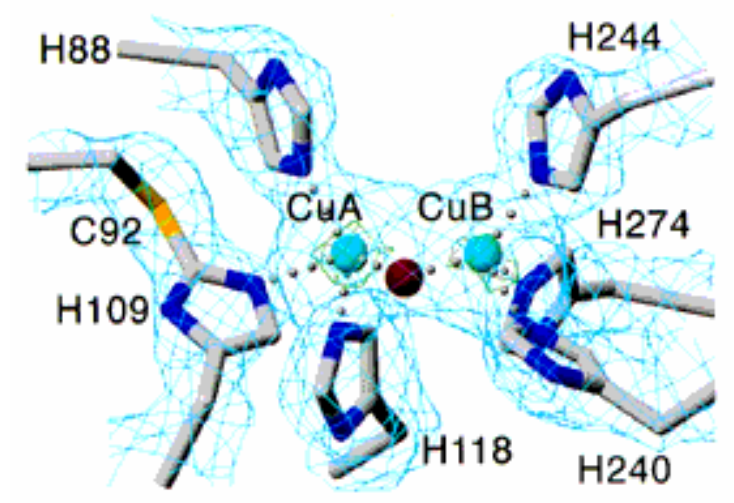

Abbildung 11: Struktur des aktiven Zentrums in der met-Form der Catechol-Oxidase. 
Vergleicht man die Strukturen von Hämocyanin und Catechol-Oxidase miteinander, so wird deutlich, warum sich beide Proteine in ihren Eigenschaften so stark unterscheiden. Während bei der Catechol-Oxidase das aktive Zentrum offen für die Anbindung größerer Substratmoleküle ist, so weißt das Hämocyanin von Limulus polyphemus ein $\mathrm{N}$-terminales Peptid auf, welches einen Phenylalaninrest beinhaltet, der die Kupferionen abschirmt, so daß zwar genügend Platz für $\mathrm{O}_{2}$, nicht aber für ein großes Phenol- oder Catecholsubstrat ist. ${ }^{[40]}$ Unterstützt wird diese Hypothese durch die Tatsache, daß Spinnen-Hämocyanin nach begrenzter Proteolyse sowohl Cresolase- als auch Catecholase-Aktivität aufweist.

Ebenso ist bei der Catechol-Oxidase eines der beiden Kupferzentren von einem Phenylalaninrest abgeschirmt, somit ist nur eines der beiden Kupferionen zugänglich für den Angriff durch ein Substrat. Da für eine erfolgreiche ortho-Hydroxylierung von Phenolen postuliert wird, daß eine große Nähe der ortho-Position des Substrats zum bereits koordinierten $\mu-\eta^{2}: \eta^{2}$-verbrückten Peroxid erforderlich ist, könnte dies einen Grund für die fehlende Cresolase-Aktivität der Catechol-Oxidase darstellen.

Für den Mechanismus der durch Tyrosinase- bzw. Catechol-Oxidase vermittelten Phenol- und Catecholoxidation durch molekularen Sauerstoff existieren unterschiedliche Vorschläge. Der zuerst von Solomon et al. vorgeschlagene Mechanismus für die katalytische Aktivität von Tyrosinase ist in Schema 6 vorgestellt.

Dargestellt sind sowohl Cresolase- als auch Catecholase-Aktivität, die oxy-Form des Enzyms kann sowohl ein Phenol als auch ein Catechol umsetzen. Im CresolaseZyklus (innerer Kreis) bindet zunächst ein Phenol an die oxy-Form und wird unter Bildung der $\mathrm{OH}^{-}$-verbrückten met-Form des Enzyms ortho-hydroxyliert. Das entstandene Catechol kann die Kupferzentren bidentat verbrücken und wird unter Reduktion der met-Form zur deoxy-Form zum o-Chinon oxidiert. Die deoxy-Form kann wiederum durch Aufnahme von $\mathrm{O}_{2}$ zur oxy-Form oxidiert werden. Der Catecholase-Zyklus kann sowohl von der oxy- als auch der met-Form starten. Nachdem das erste Catechol an die met-Form angreift und oxidiert wird, kann nach Bildung der oxy-Form ein zweites Catechol anbinden und wird unter Rückbildung der met-Form ebenfalls zum o-Chinon oxidiert. Ein Überschuß von Phenolsubstrat 
inhibiert das Enzym, dagegen kann gebildetes Catechol das Phenol vom met-Tyrosin wieder verdrängen, wodurch das Enzym wieder in den Katalysezyklus eintreten kann. Dieser Mechanismus geht von einem bidentat verbrückenden Angriff des Catechols an beide Kupferzentren aus. Krebs et al. postulieren dagegen aufgrund der Anbindung des Inhibitors Phenylthioharnstoff (PTU) einen nur monodentaten Angriff des Catechols an eines der beiden Kupferionen bei der Catechol-Oxidase (XXII, Abbildung 12).

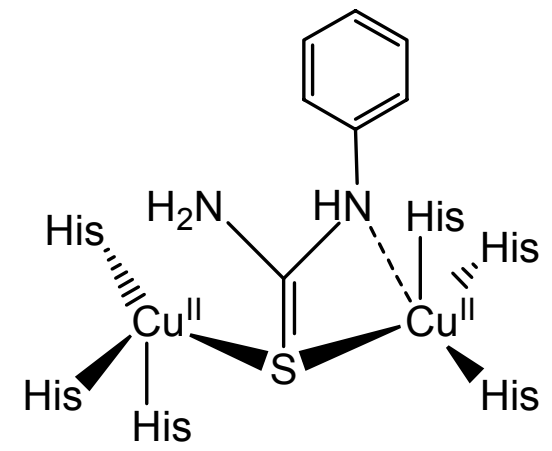

XXII

Abbildung 12: PTU-Inhibitorkomplex XXII.

Jedoch sind der Mechanismus und damit auch die Gründe für die unterschiedlichen Eigenschaften von Tyrosinase und Catechol-Oxidase noch nicht endgültig geklärt und bedürfen weiterer Experimente. So existieren z.B. von Kitajima et al. und Tolman et al. auf Basis ihrer jeweiligen biomimetischen Modellsysteme alternative Vorschläge, die einen Radikalmechanismus bzw. ein Kupfer(III)intermediat beinhalten. ${ }^{[162,41]}$ 


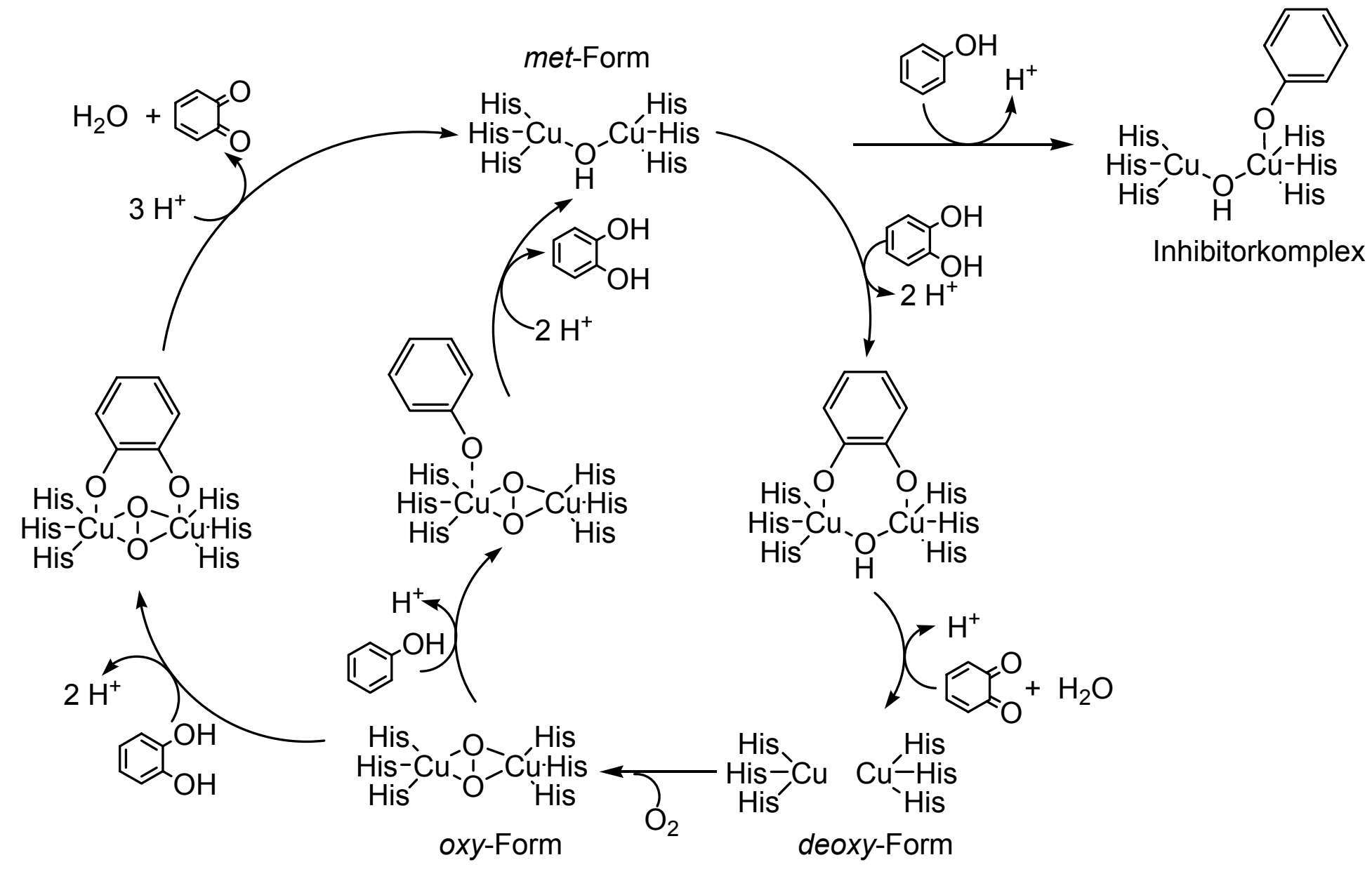

Schema 6: vorgeschlagener Mechanismus der Cresolase- und Catecholase-Aktivität von Tyrosinase. ${ }^{[31,37]}$ 
Einige Kupferproteine lassen sich keinem der drei Typen zuordnen. So weisen die bereits erwähnten Enzyme Laccase, Ascorbat-Oxidase und Ceruloplasmin ein dreikerniges Kupferzentrum auf, welches als Kombination aus Typ 2 und Typ 3 beschrieben werden kann. Ebenso läßt sich das in Cytochrom $c$ Oxidase und $\mathrm{N}_{2} \mathrm{O}$ Reduktase gefundene CuA Zentrum keinem der drei Typen zuordnen. Beide Proteine sind Teil von Atmungsketten unterschiedlicher Organismen. Es handelt sich bei CuA um eine gemischt-valentes Zentrum aus zwei zweifach $S$ (Cys) verbrückten Kupferionen, in welchem den Kupferzentren formal die Oxidationsstufe +1.5 zukommt (Abbildung 13). ${ }^{[42]}$

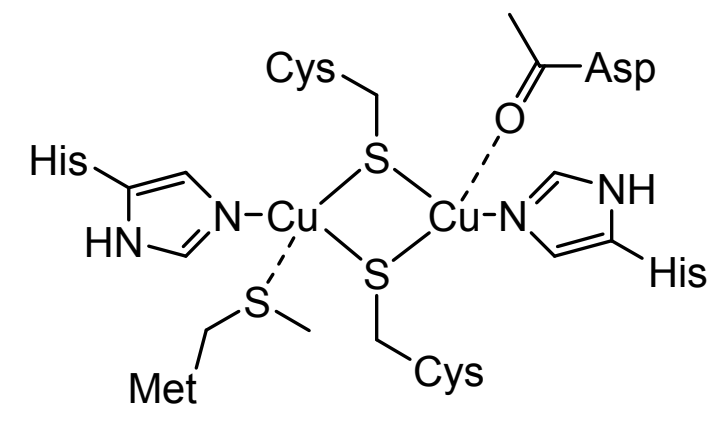

Abbildung 13: Strukturmodell des CuA-Zentrums der $\mathrm{N}_{2} \mathrm{O}$-Reduktase.

Aufgabe des CuA-Zentrums ist der Elektronentransfer zum katalytisch aktiven Zentrum, an welchem die Reduktion von $\mathrm{O}_{2} \mathrm{zu} \mathrm{H}_{2} \mathrm{O}$ (bei der Cytochrom $c$ Oxidase) bzw. von $\mathrm{N}_{2} \mathrm{O}$ zu $\mathrm{N}_{2}$ (bei der $\mathrm{N}_{2} \mathrm{O}$-Reduktase) stattfindet. Im letzteren Fall weist das katalytisch aktive Zentrum einen einzigartigen $\mathrm{Cu}_{4} \mathrm{~S}$-Cluster auf (CuZZentrum). ${ }^{[43,44,45,46]}$

\subsection{Biomimetische Modellsysteme von Enzymen mit Typ 3 Kupferzentrum}

Im folgenden soll näher auf die vielfältigen biomimetischen Ansätze eingegangen werden, mit denen die Funktion von Tyrosinase und Catechol-Oxidase bereits erfolgreich nachgeahmt wurden. $\mathrm{C}$-H-Bindungsaktivierung durch KupferDisauerstoffkomplexe wurde in einer Vielzahl von Fällen nachge-wiesen. ${ }^{[17,21,26 \text {, }}$ 47,48,49,50,51,52,53,54,55,56,57,58,59] Meist wird hierbei der Ligand selbst angegriffen, es sind aber auch C-H-Aktivierungen bei exogenen Substraten bekannt. ${ }^{[17,51,52,57]}$

Ein frühes Beispiel für eine intramolekulare Hydroxylierung an der 2-Position eines $m$-Xylylspacers zwischen zwei koordinierenden Ligandseitenarmen stammt von 
Karlin et al. im Komplex XXIII $\left(\left[\mathrm{Cu}_{2}^{\prime}(\mathrm{R}-\mathrm{XYL}-\mathrm{H})\right]^{2+}\right)($ Schema 7$) .{ }^{[47,60]}$

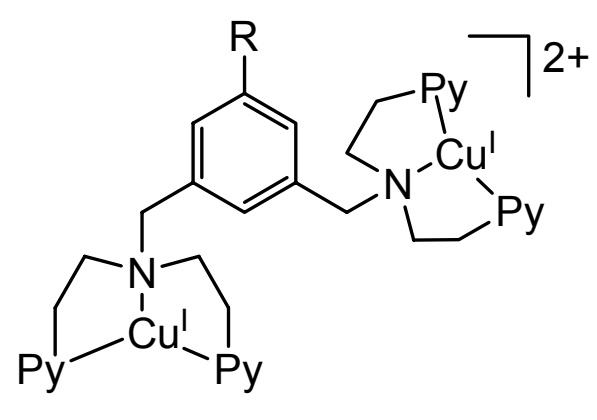

XXIII, Py = 2-PyridyI

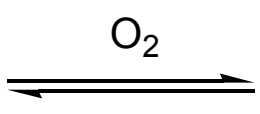

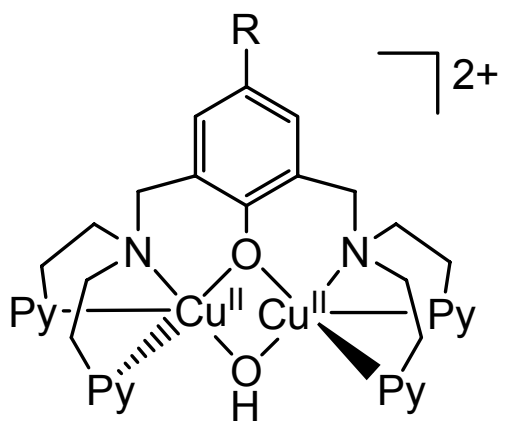

XXV

Schema 7: Hydroxylierung des Liganden $R-X Y L-H\left(R=H, \mathrm{NO}_{2}, \mathrm{C}\left(\mathrm{CH}_{3}\right)_{3}, \mathrm{~F}\right)$.

Wird dieser Komplex mit Sauerstoff umgesetzt, so entsteht Komplex XXIV, welcher aufgrund seiner spektroskopischen Eigenschaften als $\mu-\eta^{2}: \eta^{2}$-Peroxoverbindung charakterisiert und von Karlin als Modell für die Tyrosinase vorgeschlagen wurde. Die Hydroxylierung unter Entstehung des Komplexes XXV wird als elektrophiler Angriff des side on gebundenen Peroxids an den aromatischen Ring beschrieben.

Besonders vielfältig ist die Chemie der $\operatorname{Bis}(\mu-0 x 0)-V e r b i n d u n g e n$, von denen bekannt ist, daß sie Arene hydroxylieren, ${ }^{[58]}$ Substituenten des Liganden oxidativ unter Bildung von Aldehyden oder Ketonen $\mathrm{N}$-dealkylieren, ${ }^{[20,22]}$ unter Luftausschluß intermolekular H-Atome von 1,4-Cyclohexadien unter Bildung der Bis( $\mu$-hydroxo)Spezies und von Benzol abstrahieren, Phenole dimerisieren ${ }^{[26]}$ und mit Catecholen zu Monokupfersemichinonkomplexen reagieren können. ${ }^{[57]}$ Mittlerweile gelang mit einem Bis( $\mu$-oxo)-Dikupfer(III)komplex auch die intramolekulare aliphatische Hydroxylierung des Liganden XXVI (Schema 8). ${ }^{[59]}$ 
<smiles>[X]c1ccc(CCN(CC)CCc2ccccn2)cc1</smiles>

XXVI, X = OMe, Me, $\mathrm{H}, \mathrm{Cl}, \mathrm{NO}_{2}$<smiles>N[Al]1O[Ge]2(N)NCC(c3ccccc3)C1(N)O2</smiles>

$\mathrm{Ph}$

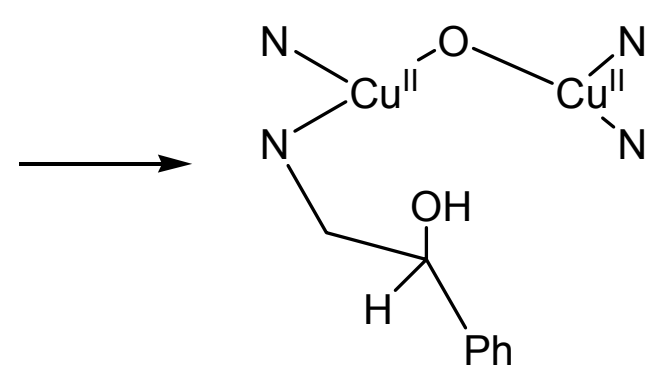

$\downarrow$

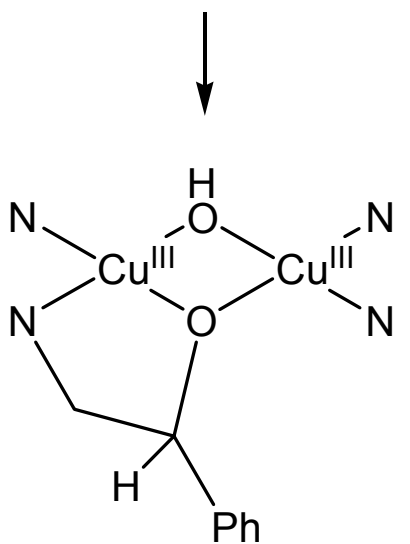

Schema 8: Aliphatische Hydroxylierung des Liganden XXVI.

Von besonderem Interesse als biomimetische Modellverbindungen sind jedoch Kupfer-Disauerstoffkomplexe, welche in der Lage sind, exogene Substrate analog zur Tyrosinase in ortho-Stellung zu hydroxylieren, bzw. Catechole zu o-Chinonen zu oxidieren. Die erste Erwähnung eines Modellsystems, das sowohl Cresolase- als auch Catecholase-Aktivität bei dem exogenen Substrat 2,4-Di-tert-butylphenol aufweist, stammt von Réglier et al. aus dem Jahre 1990. ${ }^{[61]}$ Mit einem röntgenographisch allerdings nicht charakterisierten - zweikernigen Kupfer(I)komplex wurden Turnover-Raten von $11-16 \mathrm{~h}^{-1}$ berichtet.

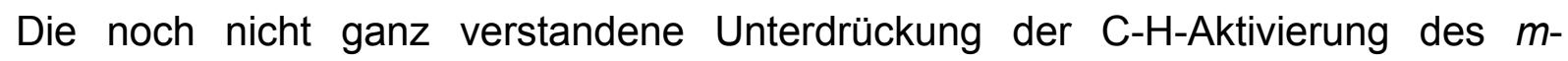
Xylylspacers in Karlin's Modellkomplex bei Verwendung leicht modifizierter NHeterocyclen $^{[50]}$ führte zur Entwicklung eines Liganden mit 1-MethylbenzimidazolSeitenarmen. ${ }^{[52,62,63,64]}$ Der zweikernige Kupfer(I)komplex XXVII dieses Liganden ist in der Lage, molekularen Sauerstoff reversibel aufzunehmen. Das bei $-80^{\circ} \mathrm{C}$ stabile Produkt XXVIII wurde aufgrund seines UVIVis- und Ramanspektrums als $\mu-\eta^{2}: \eta^{2}-$ side on verbrückte Peroxoverbindung identifiziert. Bei $-60^{\circ} \mathrm{C}$ zersetzt sich der Komplex, ist aber bei dieser Temperatur in der Lage, das Substrat 4Carbomethoxyphenolat zum entsprechenden Catechol umzusetzen. 3,5-Di-tertbutylcatechol wird bei $-60^{\circ} \mathrm{C}$ zum o-Chinon oxidiert (Schema 9). 


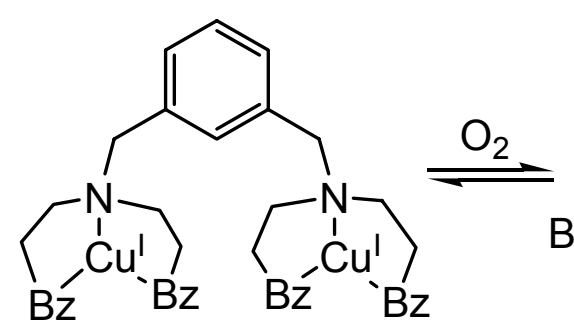

XXVII

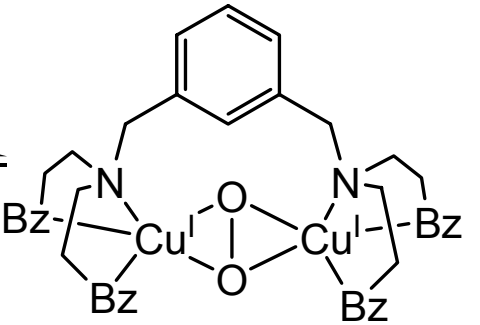

XXVIII

$\mathrm{Bz}=1-$ Methyl-benzimidazol<smiles>COC(=O)c1ccc(O)cc1</smiles><smiles>[CH]1CC[Co]1</smiles>

$\mathrm{MeOOC}$<smiles>Cc1ccc(O)c(O)c1</smiles><smiles>CC(C)(C)c1cc(O)c(O)c(Br)c1</smiles><smiles>O=C1C=C(Br)C=C(Br)C1=O</smiles>

Schema 9: Modellkomplex von Casella et al. und eingesetzte Substrate.

Damit ist dies das erste Modellsystem, welches sowohl ein stabiles Sauerstoffaddukt bildet, als auch Tyrosinase-Aktivität gegenüber exogenen Substraten aufweist. Jedoch wurde das Produkt der Cresolase-Reaktion nur in schlechter Ausbeute von ca. $20 \%$ in Bezug auf den eingesetzten Kupferkomplex erhalten.

Etwas später wurde mit einem spektroskopisch charakterisierten $\mu-\eta^{2}: \eta^{2}$ Peroxodikupferkomplex mit dem tridentaten Liganden $\mathrm{N}, \mathrm{N}$-Bis[2-(2-pyridyl)-ethyl]$\alpha, \alpha$-dideuteriobenzylamin ebenfalls - hier allerdings in fast quantitativer Ausbeute die ortho-Hydroxylierung exogener Phenolate zu den entsprechenden Catecholen beschrieben. ${ }^{[65]}$

Dagegen ist eine wesentlich größere Anzahl von zweikernigen Kupferkomplexen bekannt, die ausschließlich eine katalytische Catecholase-Aktivität, nicht jedoch eine Cresolase-Aktivität besitzen. Eine exemplarische Auswahl dafür verwendeter Liganden befindet sich in Abbildung 14. Einige der entsprechenden Liganden koordinieren nur jeweils ein Kupferzentrum (XXXII, XXXIII und XXXX) ${ }^{[66,67]}$ dennoch erhält man mit ihnen mehrkernige Kupfer(II)komplexe durch Dimerisierung einzelner 
Ligand-Metall-Fragmente. Andere (XIX - XXXX, XXXIV, XXXXI und XXXVII $)^{[62,63,67,68,69]}$ bieten bereits zwei Kompartimente zur Ausbildung eines Dikupferkomplexes. Der hierbei verwendete Spacer zwischen den Kompartimenten kann entweder ein nichtfunktionalisierter Aromat (XXX) oder eine aliphatische Kette (XXXI) sein, der Spacer kann aber Z.B. auch schon die bei der Catechol-Oxidase vorgefundene Hydroxidbrücke zwischen den Kupferzentren bereits in ähnlicher Weise durch eine Alkoxo- oder Phenoxogruppe zur Verfügung stellen (XXXIV,

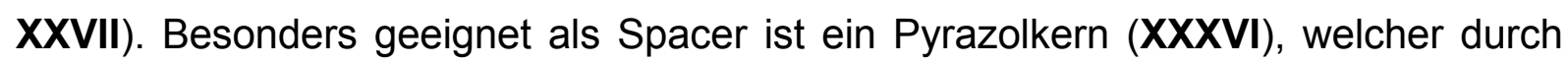
seine Diazinfunktion bereits einen Kupfer-Kupfer-Abstand zwischen ca. 3.4 - $4.5 \AA$ vorgibt und damit gute Voraussetzungen für eine kooperative Wechselwirkung zwischen beiden Metallzentren schafft. ${ }^{[70]}$ Bei einer Reihe weiterer Ligandsysteme wurde ebenfalls Catecholase-Aktivität nachgewiesen und untersucht. ${ }^{[71,72,73,74,75,76,77,78,79,80,81,82,83,84,85]}$ Es wird auch von Catecholase-Aktivität bei einem zweikernigen Zinkkomplex berichtet, bei welchem das Zink selbst nicht an Redoxreaktionen beteiligt ist. Das zinkgebundene Catecholat reagiert direkt mit $\mathrm{O}_{2}$ unter Bildung eines freien Superoxid-Anions. ${ }^{[86]}$

Trotz der Vielzahl untersuchter Systeme sind die Zusammenhänge zwischen strukturellen und elektronischen Parametern der Kupferkatalysatoren noch nicht vollständig geklärt. Beobachtet wird eine größere Aktivität zweikerniger Kupferkomplexe im Vergleich zu einkernigen bei einem Kupfer-Kupfer-Abstand $<5 \AA .{ }^{[68,77]}$ Zudem wird im Falle zweikerniger Komplexe im allgemeinen eine Abhängigkeit 1. Ordnung von der Katalysatorkonzentration gefunden, während bei mononuklearen Kupfer(II)komplexen von einer Abhängigkeit 2. Ordnung berichtet wird. ${ }^{[62,87,88]}$ Beides weist auf die Notwendigkeit der Kooperation zweier eng benachbarter Kupferzentren bei der Oxidation von Catecholen hin und könnte ein Hinweis auf eine verbrückende Anbindung des Catecholsubstrates im katalytischen Zyklus sein. 


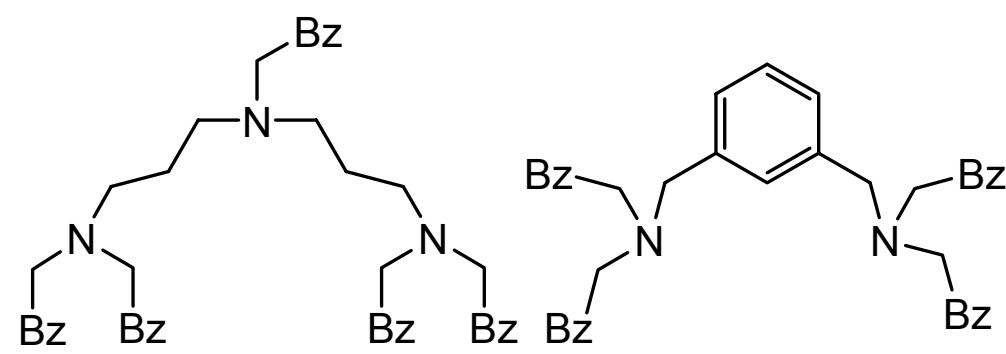

XIX<smiles>COC1OC(CNC=CC(=O)C(C)=O)C(OC)C(OC)C1OC</smiles>

$\mathrm{H}_{2}$ XXXII<smiles>CC(=O)C(=CNCC1OC2OC3C(O)OC(C3O)C1O2)C(C)=O</smiles>

$\mathrm{H}_{2}$ XXXIII

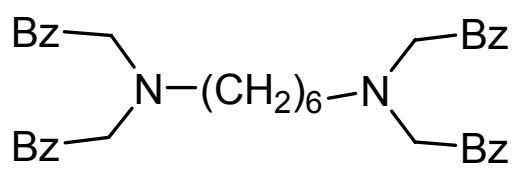

XXXI<smiles>c1ccc(CCSCc2cc[nH]n2)nc1</smiles>

HXXXV

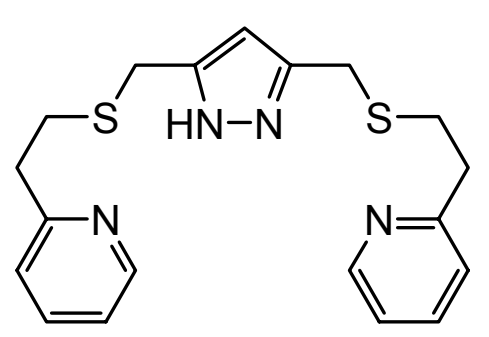

HXXXVI<smiles>Oc1ccccc1CN(Cc1ccccn1)CC(O)CN(Cc1ccccn1)Cc1ccccc1O</smiles>

HXXXVII

Abbildung 14: Ausgewählte Ligandsysteme, deren Kupferkomplexe Catecholase-Aktivität aufweisen.

Die katalytische Aktivität des Dikupfer(II)komplexes von XXXIV wurde auf den entatischen Zustand des Liganden im Komplex zurückgeführt, was zu einer starken Verzerrung der quadratisch pyramidalen Koordinationsumgebung der Kupferzentren führt und eine leichte Abdissoziation der $\mu$-Hydroxid-Zweitbrücke zwischen den Kupferzentren und den erleichterten Angriff eines größeren Catechols unter Ausbildung einer entspannteren Ligandkonformation ermöglicht. ${ }^{[68]}$ Untersuchungen der Redoxeigenschaften von Katalysatoren ergaben zwar keine direkte Korrelation zwischen $E^{0}\left(\mathrm{Cu}^{\prime \prime} / \mathrm{Cu}^{\prime}\right)$ der Katalysatoren mit ihrer katalytischen Aktivität, jedoch muß das Redoxpotential in einem geeigneten Fenster für die leichte Reduktion des 
Catechols und anschließende Oxidation des Komplexes durch $\mathrm{O}_{2}$ liegen. ${ }^{[6]}$ Sicher ist, daß für die katalytische Aktivität nicht nur ein Parameter bestimmend ist, sondern daß das Zusammenwirken verschiedener - teils voneinander abhängiger - Faktoren, wie der Abstand der Kupferzentren zueinander, geometrische Effekte (Spannung im Ligandgerüst, Verzerrung der idealen Koordinationsgeometrie der Kupferzentren), Basizität der Liganden, sterische Behinderungen durch große Ligandreste und elektrochemische Eigenschaften des Katalysators, die Aktivität des jeweiligen Katalysators bestimmt. Aufgrund der Komplexität dieser Zusammenhänge sollte ein Ligandsystem hergestellt werden, welches gezielt die Variation nur eines Parameters erlaubt, während die anderen Parameter weitgehend konstant gehalten werden. 


\section{Ligandsynthese}

\subsection{Eigenschaften der Pyrazolat-Kompartimentliganden}

Die im Rahmen dieser Arbeit verwendeten Liganden $\mathrm{HL}^{1}-\mathrm{HL}^{10}$ (Abbildung 15) erlauben ein gezieltes Studium der Auswirkung des Kupfer-Kupfer-Abstandes sowie unterschiedlicher Koordinationsgeometrien und elektronischer Umgebungen an den Kupferzentren auf die Strukturen und Reaktivitäten der Komplexe. Innerhalb der Reihen $\mathrm{HL}^{1}-\mathrm{HL}^{4}$ und $\mathrm{HL}^{5}-\mathrm{HL}^{8}$ erhält man Dikupferkomplexe mit unterschiedlichen Kupfer-Kupfer-Abständen, bedingt durch die unterschiedliche Länge der Seitenarme der einzelnen Liganden. ${ }^{[70]}$ Zugleich können je nach Länge des Seitenarmes Chelatringe mit 5 oder 6 beteiligten Atomen gebildet werden, was die Stärke der Koordination der N-Donoren beeinflussen sollte. Zudem bieten die Liganden $\mathrm{HL}^{5}$ $\mathrm{HL}^{8}$ Pyridin-N-Donoren mit der Möglichkeit zur $\mathrm{M} \rightarrow \mathrm{L}$ Rückbindung an, während dies bei $\mathrm{HL}^{1}-\mathrm{HL}^{4}, \mathrm{HL}^{9}$ und $\mathrm{HL}^{10}$ mit rein aliphatischen Seitenketten nicht möglich ist. Die aus der Fähigkeit des Pyridins zur $\pi$-Wechselwirkung mit dem Metall resultierende leichte Polarisierbarkeit des Pyridins sollte das weichere Kupfer $(I)$ gegenüber dem harten Kupfer(II) stabilisieren und somit die Redoxpotentiale zu positiveren Werten verschieben. ${ }^{[89]}$ Die Liganden $\mathrm{HL}^{9}$ und $\mathrm{HL}^{10}$ beinhalten Triazacyclononan-Seitenarme und können als makrozyklische Analoga zum offenkettigen Liganden $\mathrm{HL}^{4}$ betrachtet werden. ${ }^{\left[{ }^{90]}\right.}$ Durch den makrozyklischen Effekt und die damit einhergehenden großen Stabilitätskonstanten der entsprechenden Komplexe kann die Auswirkung der Stärke der Ligand-Metall-Bindung auf die katalytische Aktivität untersucht werden.

Die Liganden $\mathrm{HL}^{1}-\mathrm{HL}^{8}$ wurden im Rahmen dieser Arbeit über mehrstufige Syntheserouten selbst hergestellt. 


\section{LIGANDSYNTHESE}

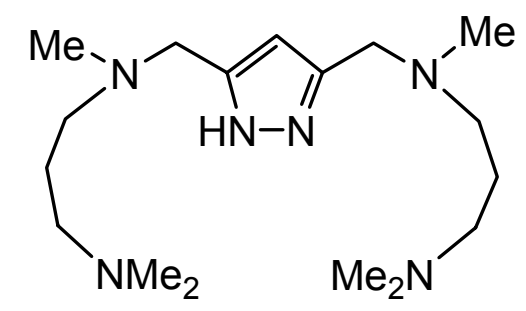

$H L^{1}$<smiles>CN(C)CCN(C)Cc1cc(CN(C)C)[nH]n1</smiles>

$\mathrm{HL}^{3}$<smiles></smiles>

$\mathrm{HL}^{5}$

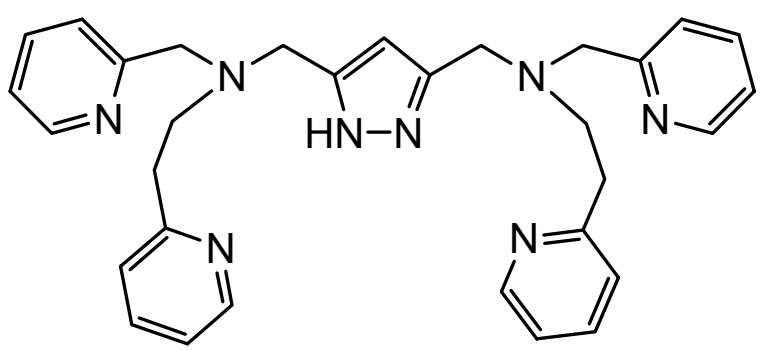

$\mathrm{HL}^{7}$

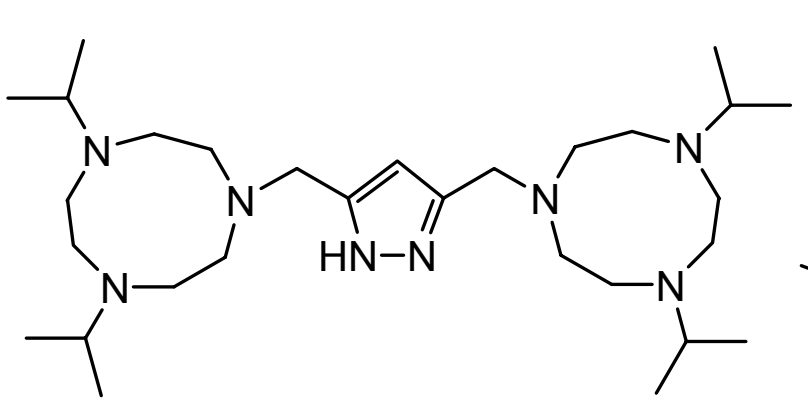

$\mathrm{HL}^{9}$

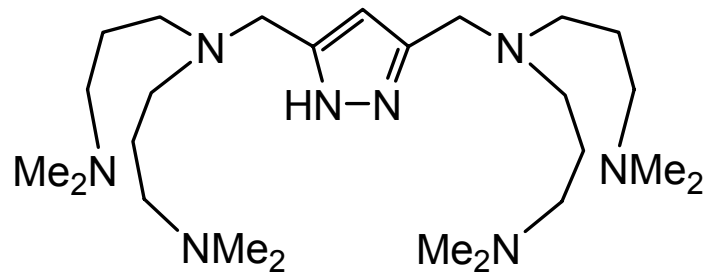

$\mathrm{HL}^{2}$

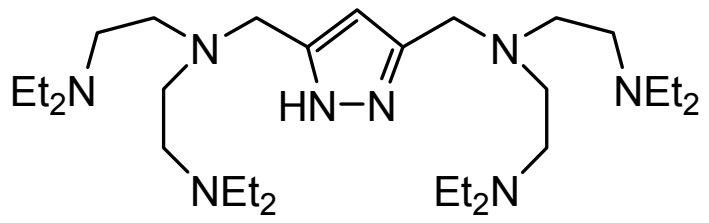

$\mathrm{HL}^{4}$

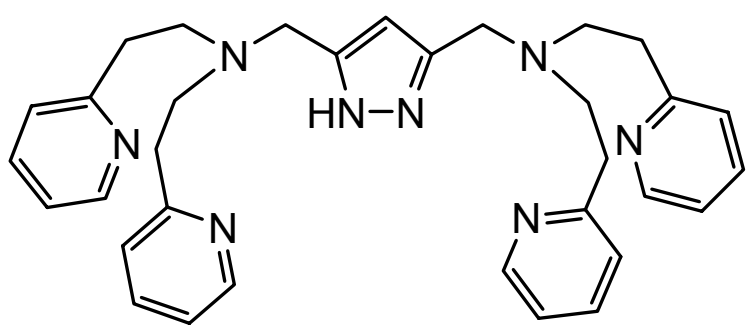

$\mathrm{HL}^{6}$

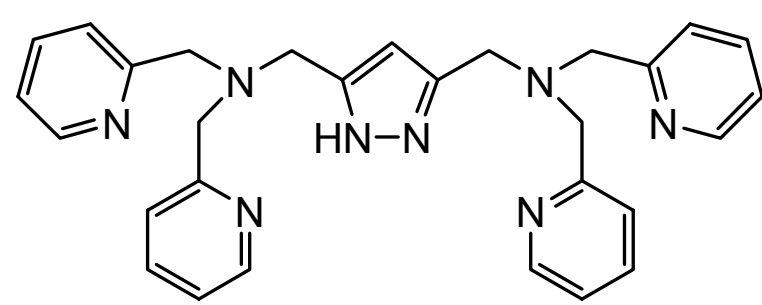

$\mathrm{HL}^{8}$

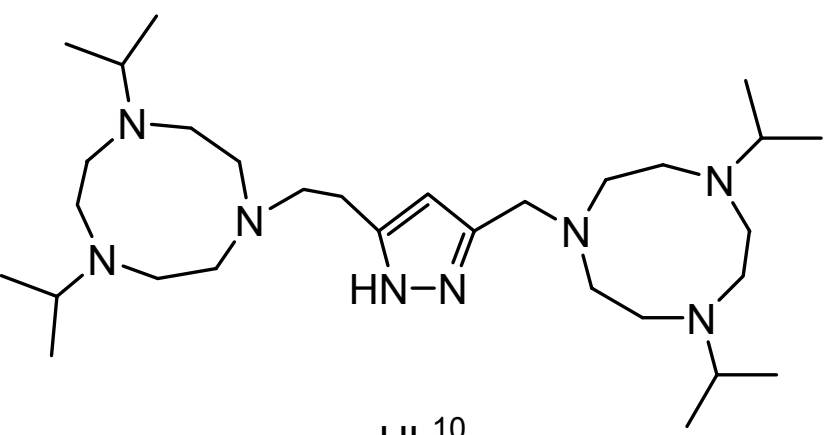

$\mathrm{HL}^{10}$

Abbildung 15: Die Kompartimentliganden $H L^{1}-H L^{10}$. 


\subsection{Synthese der Liganden}

Zur Herstellung der Liganden wurden zwei Verfahren verwendet. Die Synthese der Pyrazolliganden mit aliphatischen Seitenarmen erfolgte nach Literaturvorschriften. ${ }^{[91,92,93]}$ Zunächst wurde die Pyrazol-3,5-dicarbonsäure XXXVIII mit Thionylchlorid in das Pyrazol-3,5-dicarbonyl-dichlorid XXXIX umgewandelt. Anschließend wurde in Gegenwart eines Überschusses der Hilfsbase Triethylamin mit dem aliphatischen Amin $H N R^{1} R^{2}$ umgesetzt und dann das entstandene Amid XL mit $\mathrm{LiAlH}_{4}$ zu XLI reduziert (Schema 10). Die Amine $H N R^{1} R^{2}$ können käuflich erworben werden. Das $\mathrm{N}, \mathrm{N}, \mathrm{N}^{\prime}, \mathrm{N}^{\prime}$-Tetraethyldiethylentriamin wurde z.T. aus Bis(chlorethyl)amin-Hydrochlorid mit Diethylamin und $\mathrm{KOH}$ hergestellt.

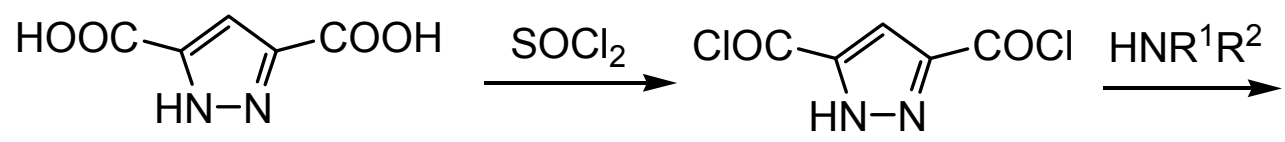
XXXVIII XXXIX<smiles>[R12]NCc1cc(CN=[R12])[nH]n1</smiles>

$\mathbf{X L}$

$$
\begin{aligned}
& \mathrm{N}_{\mathrm{R}^{2}}^{-\mathrm{R}^{1}}=\mathrm{N}^{-\mathrm{Me}} \mathrm{NMe}_{2} \quad\left(\mathrm{HL}^{1}\right) \quad \mathrm{N}^{\mathrm{Me}} \sim_{\mathrm{NMe}_{2}} \\
& \mathrm{~N}\left(\mathrm{NMe}_{2}\right)_{2}\left(\mathrm{HL}^{2}\right) \quad \mathrm{N}\left(\mathrm{NEt}_{2}\right)_{2}
\end{aligned}
$$

XLI

Schema 10: Synthese der Pyrazolliganden mit aliphatischen Seitenarmen.

Diese Syntheseroute erwies sich für Liganden mit aromatischen Pyridin-haltigen Seitenarmen als ungünstig, da es hier aufgrund des ungeschützten aziden H-Atoms am Heterozyklus verstärkt zur Spaltung der Amidbindung bei der Reduktion mit $\mathrm{LiAlH}_{4} \mathrm{kam}$. Daher wurde eine andere Syntheseroute gewählt, bei welcher das $\mathrm{H}$ Atom durch eine Tetrahydropyran-Gruppe (THP-Gruppe) geschützt wurde.

Zunächst wird hier nach Literaturvorschrift das 3,5-Bis(chlormethyl)-1(tetrahydropyran-2-yl)pyrazol hergestellt. ${ }^{[94,95,96]}$ Hierbei wird zuerst das 3,5- 
Dimethylpyrazol XLII durch $\mathrm{KMnO}_{4}$ zum Pyrazol-3,5-dicarbonsäuremonokaliumsalz XLIII oxidiert, welches anschließend säurekatalysiert mit Methanol verestert wird. Der Ester XLIV kann nun durch $\mathrm{LiAlH}_{4}$ zum Alkohol XLV reduziert werden, welcher dann durch $\mathrm{SOCl}_{2}$, das zugleich als Solvens dient, chloriert wird (Schema 11).
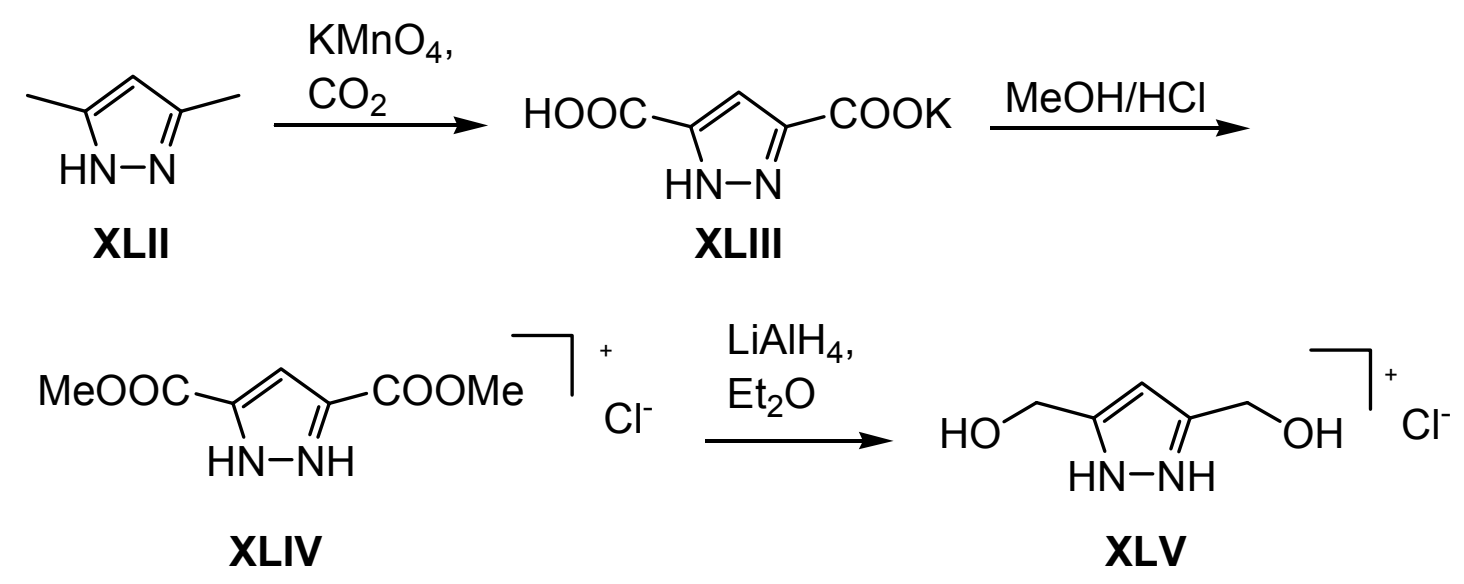

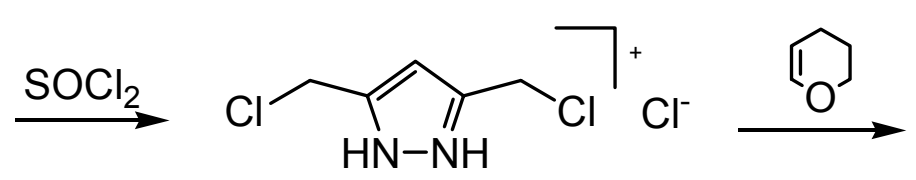

XLVI

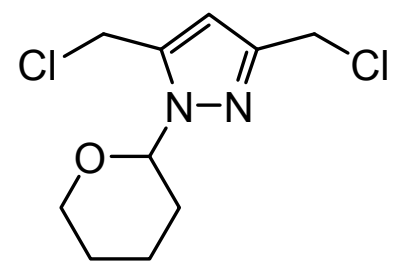

XLVII

Schema 11: Synthese von 3,5-Bis(chlormethyl)-1-(tetrahydropyran-2-yl)pyrazol.

Das entstandene 3,5-Bis(chlormethyl)pyrazol Hydrochlorid XLVI wird mit 3,4Dihydropyran in Dichlormethan zum THP-geschützten 3,5-Bis(chlormethyl)-1(tetrahydropyran-2-yl)pyrazol XLVII umgesetzt. Die anschließend als Seitenarme einzuführenden Amine wurden ebenfalls nach Literaturvorschrift hergestellt. ${ }^{[97,98]}$ Bis[2-(2-pyridyl)ethyl]amin (BPEA) kann aus 2-Vinylpyridin (XLVIII) und Ammoniumchlorid in Methanol gewonnen werden. Bis(2-pyridylmethyl)amin (BPMA) und (2-Pyridylmethyl)[2-(2-pyridyl)ethyl]amin (BPEMA) werden aus Pyridin-2carboxaldehyd (L) und dem entsprechenden (2-Pyridylalkyl)amin in absolutem Ethanol gewonnen (Schema 12). Ein weiteres Amin, das Methyl-[2-(2pyridyl)ethyl]amin (MePEA), kann käuflich erworben werden. 
<smiles>COCCOCCO</smiles>

XLVIII<smiles>c1ccc(CCNCCc2ccccn2)nc1</smiles>

BPEA (IL)

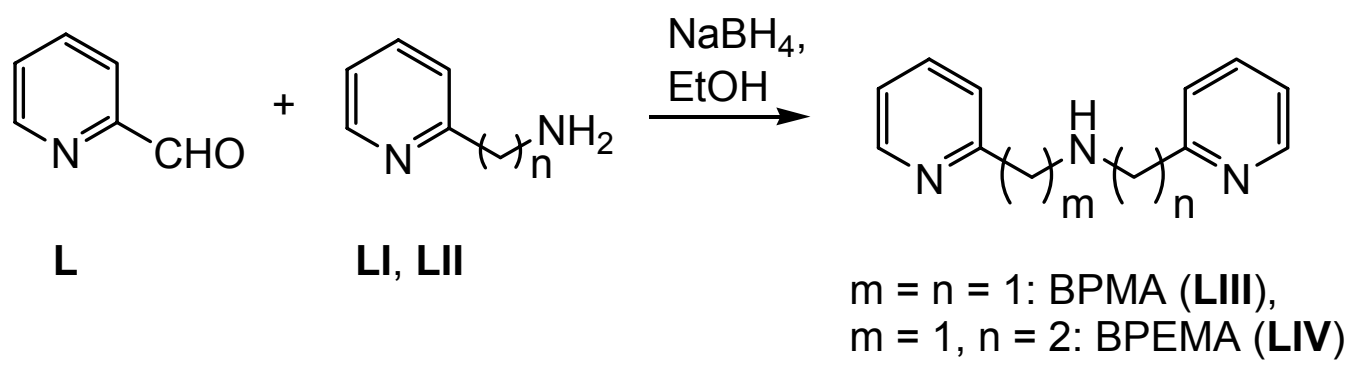

Schema 12: Synthese der Amine mit Pyridylresten.

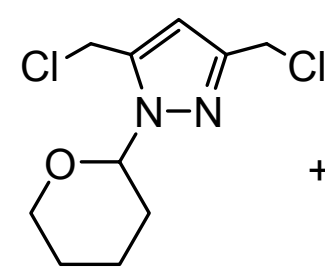
1) $\mathrm{Na}_{2} \mathrm{CO}_{3}, \mathrm{MeCN}$
2) $\mathrm{EtOH} / \mathrm{HCl}$
3) $\mathrm{NaOH}$<smiles>[R][R10]NCc1cc(CN[R12])[nH]n1</smiles>

\section{XLVII}

N- ${ }_{\mathrm{R}^{2}}^{\mathrm{R}^{1}}=$<smiles>CNCCc1ccccn1</smiles>

$\left(\mathrm{HL}^{5}\right)$<smiles>c1ccc(CCNCc2ccccn2)nc1</smiles><smiles>CCCc1ccccn1</smiles>
$\left(\mathrm{HL}^{6}\right)$<smiles>CN(C)c1ccccn1</smiles>

Schema 13: Synthese der Pyrazolliganden mit Pyridinseitenarmen.

Zur Synthese der neuen Liganden wurden zunächst das jeweilige Amin und die geschützte Pyrazolverbindung über trockenem $\mathrm{Na}_{2} \mathrm{CO}_{3}$ in Acetonitril unter Rückfluß erhitzt. Nach Abkühlen, Filtration und Entfernen des Solvens liegt der THP- 
geschützte Ligand als gelbes Öl vor. Die Schutzgruppe wird durch $\mathrm{EtOH} / \mathrm{HCl}$ abgespalten und anschließend das entstandene Hydrochlorid mit wäßriger $\mathrm{NaOH}$ bei pH 12 deprotoniert. Den Liganden erhält man durch Extraktion mit Dichlormethan und Entfernen des Solvens im Vakuum (Schema 13). Die Liganden $\mathrm{HL}^{1}-\mathrm{HL}^{4}$ können - alternativ zur in Schema 10 skizzierten Syntheseroute - ebenfalls auf diese Weise erhalten werden. 


\section{Komplexsynthese}

Zur Darstellung der Dikupfer(II)komplexe aus den synthetisierten Liganden $\mathrm{HL}^{1}-\mathrm{HL}^{8}$ versetzt man eine Lösung der Pyrazolverbindung zunächst mit zwei Äquivalenten einer Base. Dies geschieht zum einen, um das azide Proton des Pyrazolkernes zu entfernen und zum anderen, um durch Abstraktion eines Protons aus $\mathrm{H}_{2} \mathrm{O}$ aus dem Kristallwasser des eingesetzten Kupfersalzes oder auch aus einem protischen Lösungsmittel wie z.B. Methanol, den späteren Einbau eines zweiten verbrückenden Liganden zwischen den Metallzentren zu ermöglichen (Schema 14). Der deprotonierte Ligand kann mit verschiedenen Kupfersalzen zum gewünschten Produkt umgesetzt werden.<smiles>[R12]NCc1cc(CN[R12])[nH]n1</smiles>

Schema 14: Synthese der Dikupfer(II)komplexe

Die erhaltenen Komplexe sind in der Regel nur dann ausreichend rein für nachfolgende Reaktivitätsstudien, wenn sie in einkristalliner Form erhalten werden können. Hierbei bieten sich Salze mit großen, schlecht koordinierenden Anionen wie z. B. $\mathrm{ClO}_{4}^{-}$oder $\mathrm{BF}_{4}^{-}$an. Wird als Base Kalium-tert-butylat verwendet, fällt als Nebenprodukt der Reaktion das Kaliumsalz des eingesetzten Anions aus und kann durch Filtration großenteils abgetrennt werden. Um das Produkt kristallin zu gewinnen, wird es durch Überschichten der Lösung mit einem langsam 
eindiffundierenden Solvens mit schlechten Lösungseigenschaften über einen längeren Zeitraum ausgefällt.

\subsection{Dikupfer(II)komplexe der Liganden $\mathrm{HL}^{1}-\mathrm{HL}^{4}$}

Im Rahmen der Diplomarbeit wurden bereits vier zweikernige Kupferkomplexe mit den Liganden $\mathrm{HL}^{1}-\mathrm{HL}^{4}$ synthetisiert (Abbildung 16). ${ }^{[99,100]}$

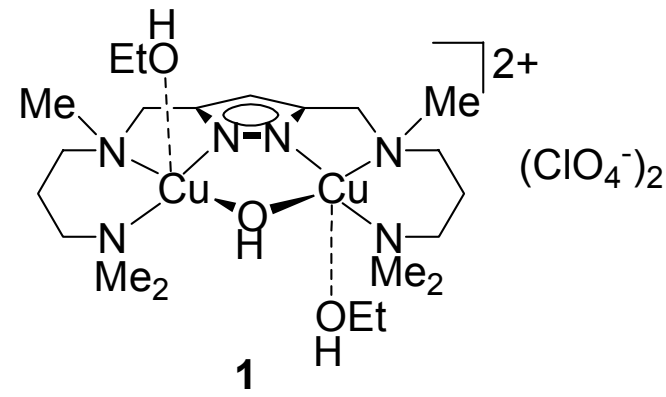

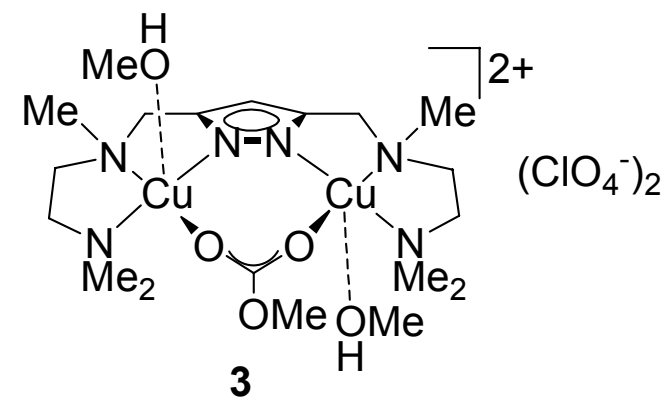
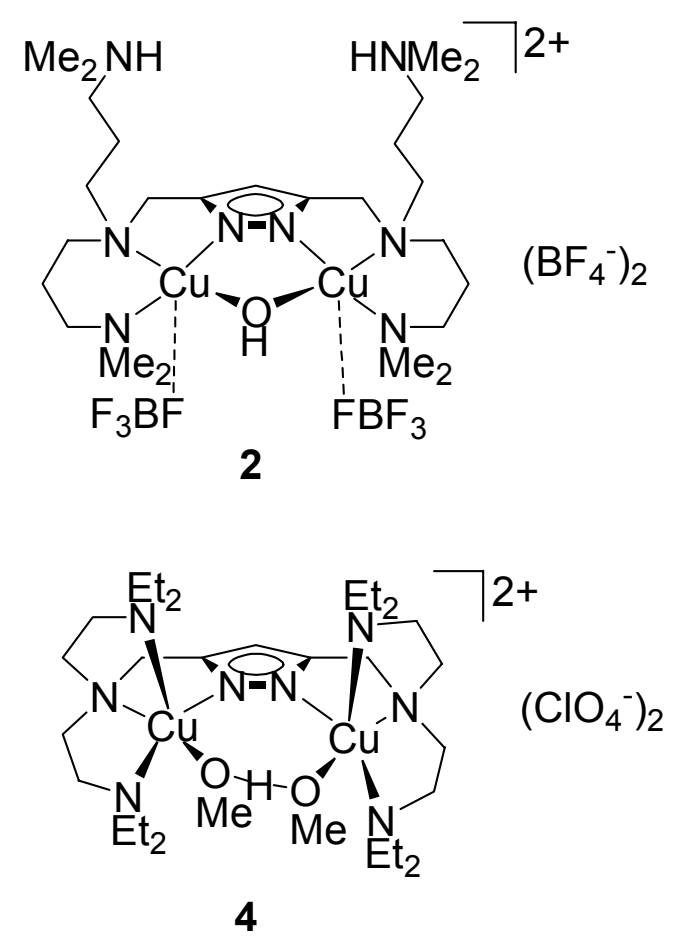

Abbildung 16: Die in früheren Arbeiten hergestellten Komplexe 1- 4.

Aus den röntgenographisch ermittelten Strukturen im Festkörper war ersichtlich, daß die Pyrazolatliganden $\mathrm{HL}^{1}$ und $\mathrm{HL}^{2}$ mit langen Seitenarmen zu einem kürzeren $\mathrm{Cu}$ Cu-Abstand von 3.540(1) $\AA$ in Komplex 1 und 3.447(2) $\AA$ in Komplex 2 führen. Die Kupferzentren beider Komplexe sind $\mathrm{OH}^{-}$-verbrückt. Im Gegensatz zur Synthese von 1 war bei der Synthese von 2 der Zusatz einer externen Base nicht notwendig, da die zusätzlichen Amin-Seitenarme als intramolekulare Base wirken. $\mathrm{HL}^{3}$ und $\mathrm{HL}^{4}$ ergaben aufgrund ihrer kurzen Ligandseitenarme Kupferkomplexe mit längerem $\mathrm{Cu}$ Cu-Abstand (4.088(1) $\AA$ bei Komplex 3 und 4.533(1) $\AA$ bei Komplex 4), da hier die Kupferzentren auseinandergezogen werden. Dies führt dazu, daß in diesen Komplexen als Zweitbrücken größere Liganden eingebaut werden müssen. Wurde $\mathrm{HL}^{3}$ mit $\mathrm{Cu}\left(\mathrm{ClO}_{4}\right)_{2} \cdot 6 \mathrm{H}_{2} \mathrm{O}$ und einer Hilfsbase umgesetzt und anschließend $\mathrm{CO}_{2}$ in die Lösung eingeleitet, so wurde beobachtet, daß der entstandene Komplex 3 eine 
Methylcarbonatbrücke zwischen den Kupferzentren aufwies, welche durch Veresterung des $\mathrm{CO}_{2}$ mit Methanol entstanden war. In 4 werden die Kupferzentren durch einen zusätzlichen Ligandseitenarm koordiniert, wodurch diese noch weiter auseinandergezogen werden. Komplex 4 weist dementsprechend eine große MeO$\mathrm{H}-\mathrm{OMe}$-Brücke zwischen den Kupferzentren auf. Die Koordinationsgeometrie der Kupferzentren von 1 - 3 kann als verzerrt quadratisch pyramidal beschrieben werden, während jene von 4 eher einer verzerrt trigonal bipyramidalen Geometrie entspricht. Dies kann durch den Parameter $\tau$ quantifiziert werden. ${ }^{[101]}$ Er ergibt sich aus: $\tau=(\beta-\alpha) / 60^{\circ}$, wobei $\alpha$ und $\beta$ die beiden größten Winkel an den fünffach koordinierten Metallzentren sind, die von den Bindungen zu den Donoren eingeschlossen werden, wobei gilt: $\beta>\alpha$. Für eine ideale trigonale Bipyramide ergibt sich damit der Wert $\tau=\left(180^{\circ}-120^{\circ}\right) / 60^{\circ}=1$ und für eine ideale quadratische Pyramide $\tau=\left(180^{\circ}-180^{\circ}\right) / 60^{\circ}=0$. Die erhaltenen $\tau$-Werte für $1-4$ sind in Tabelle 1 zusammengefaßt.

\begin{tabular}{|c|c|c|}
\hline Komplex & $d_{\mathrm{Cu} 1 \cdots \mathrm{Cu} 2}[\AA]$ & $\tau($ Cu1/Cu2) \\
\hline $\mathbf{1}$ & $3.540(1)$ & $0.23 / 0.34$ \\
$\mathbf{2}$ & $3.447(2)$ & $0.38 / 0.35$ \\
$\mathbf{3}$ & $4.088(1)$ & $0.21 / 0.09$ \\
$\mathbf{4}$ & $4.533(1)$ & $0.64 / 0.64$ \\
\hline
\end{tabular}

Tabelle 1: $d_{C u \cdots C u}[\AA]$ und $\tau$-Werte für die Koordinationsgeometrien der Komplexe 1 - 4.

Leitet man in eine Lösung von $\mathrm{HL}^{3}, \mathrm{KO}^{t} \mathrm{Bu}$ und $\mathrm{Cu}\left(\mathrm{ClO}_{4}\right)_{2} \cdot 6 \mathrm{H}_{2} \mathrm{O}$ in Methanol eine halbe Stunde $\mathrm{CO}_{2}$ ein, entfernt dann das Methanol im Vakuum und läßt in Aceton durch Pentandiffusion auskristallisieren, kann eine Veresterung des entstehenden Carbonats verhindert werden. Das entstandene $\mathrm{CO}_{3}{ }^{2-}$ verbrückt die in einer Ligandeinheit eingebetteten Kupferzentren Cu1 und Cu2 mit dem Cu4-Zentrum einer weiteren Dikupferpyrazolateinheit in Komplex $\left[\left(\mathrm{L}^{3} \mathrm{Cu}_{2}\right)\left(\mathrm{CO}_{3}\right)\left(\mathrm{H}_{2} \mathrm{O}\right)_{2}\left(\mathrm{ClO}_{4}\right)\right]\left(\mathrm{ClO}_{4}\right)_{3}$ (5) (Abbildung 17) in Form einer $\mu_{3}$-Brücke. Dabei liegen die Abstände $\mathrm{d}_{\mathrm{Cu1-017}}=$ 1.911(2) $\AA, d_{\text {Cu2-018 }}=1.940(2) \AA$, und $d_{\text {Cu3-019 }}=1.923(2) \AA$ im Bereich der für $\mu_{3^{-}}$ Carbonat-verbrückte Kupferkomplexe literaturbekannten Werte. ${ }^{[102,103,104,105,106]}$ 


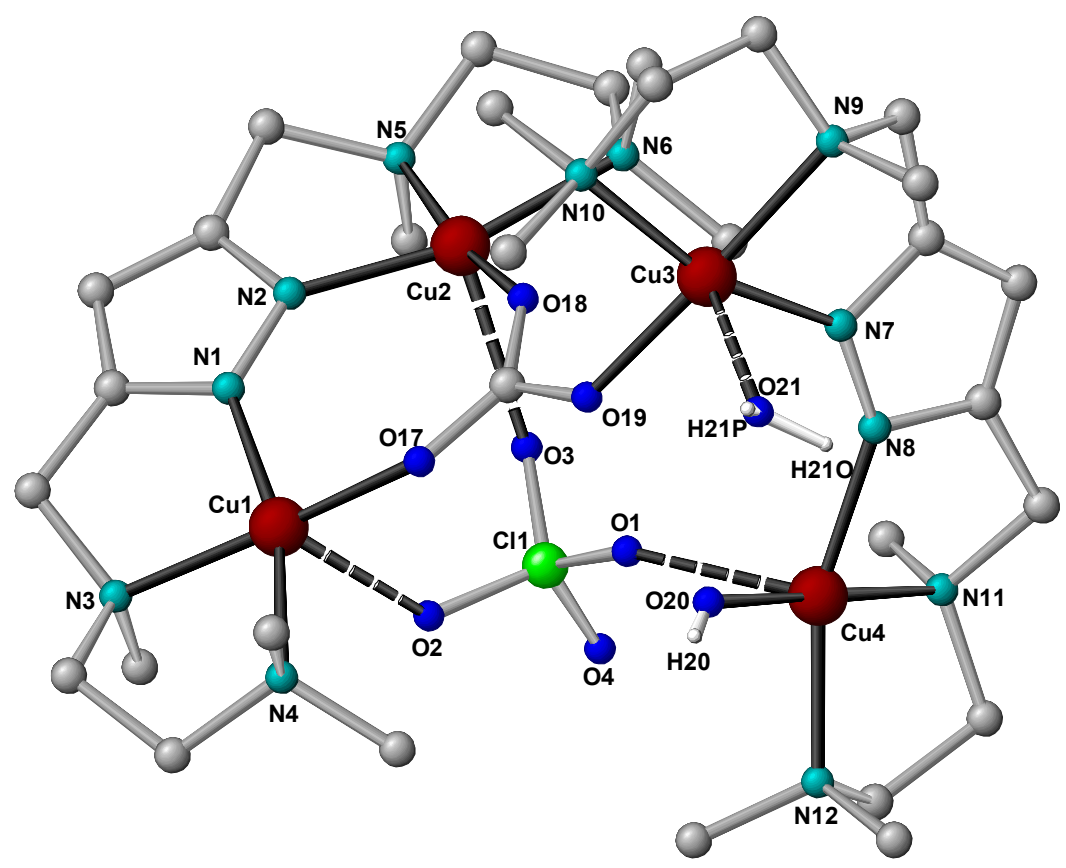

Abbildung 17: Festkörperstruktur des Kations von 5.

Die Veresterung mit dem Lösungsmittel Methanol findet also langsam genug statt, um durch frühzeitiges Entfernen des alkoholischen Solvens verhindert werden zu können. Vergleichbare $\mathrm{CO}_{2}$-Aktivierung mit Dinickelkomplexen wurde auch in der eigenen Arbeitsgruppe festgestellt ${ }^{[107]}$ und ist zudem bereits für einige weitere Übergangsmetalle ${ }^{[108,109,110,111,112]}$ sowie das Zinkenzym Carboanhydrase ${ }^{[5]}$ bekannt, das die hydrolytische Umwandlung von $\mathrm{CO}_{2}$ in Hydrogencarbonat katalysiert. Ein dreikerniger Kupferkomplex mit einem 2,2'-Bipyridinliganden, in dem nach Aufnahme von $\mathrm{CO}_{2}$ ebenfalls eine $\mu_{3}-\mathrm{CO}_{3}$-Brücke festgestellt wurde, wurde von Reedijk et al. synthetisiert. ${ }^{[104]}$ Komplex 5 kristallisiert in der Raumgruppe C2/c mit acht Molekülen in einer monoklinen Zelle. An jedes Kupferzentrum koordiniert ein N-Atom eines Pyrazolatkernes, sowie zwei N-Donoren eines Seitenarmes. Zusätzlich sind die Kupferzentren Cu1, Cu2 und Cu4 in Form einer $\mu_{3}$-Brücke durch ein Perchloratanion verknüpft $\left(d_{\mathrm{Cu} 1-\mathrm{O} 2}=2.504(2) \AA, d_{\mathrm{Cu2-O3}}=2.528(3) \AA, d_{\mathrm{Cu} 4-01}=2.431(2) \AA\right)$. Ein röntgenographisch charakterisierter, ebenfalls $\mu_{3}$-Perchlorato-verbrückter, dreikerniger Kupferkomplex weist sehr ähnliche Abstände zwischen den Kupferzentren und den Perchlorat-O-Atomen auf. ${ }^{[113]}$ An Cu3 und auch Cu4 bindet noch jeweils ein $\mathrm{H}_{2} \mathrm{O}$-Molekül an, so daß sich in allen Fällen eine verzerrte quadratisch pyramidale Koordination aller Kupferzentren ergibt $\left(\tau_{\mathrm{Cu} 1}=0.12, \tau_{\mathrm{Cu} 2}=\right.$ $0.09, \tau_{\mathrm{Cu} 3}=0.23, \tau_{\mathrm{Cu} 4}=0.24$ ). Desweiteren findet man pro $\mathrm{Cu}_{4}$-Einheit noch zwei 
Acetonmoleküle, von denen eines einen Abstand von $2.765 \AA$ zum O-Atom des an Cu3 koordinierten $\mathrm{H}_{2} \mathrm{O}$-Moleküls hat, so daß das Vorliegen einer $\mathrm{H}$-Brücke wahrscheinlich ist. Im Vergleich mit 3 ( $d_{\mathrm{Cu} 1 \ldots \mathrm{Cu} 2}=4.088(1) \AA$ ) ist der Abstand der durch ein Pyrazolat und das Carbonat verbrückten Kupferzentren Cu1 und Cu2 mit 4.001(1) A sehr ähnlich, während derjenige von Cu3 und Cu4, welche nicht durch eine zusätzliche Zweitbrücke verbunden sind, mit $4.279 \AA$ deutlich höher liegt. Im FAB-Massenspektrum ist bei $\mathrm{m} / \mathrm{z}=1199$ mit geringer Intensität von $12 \%$ das Fragment $\left[\left(\mathrm{L}^{3} \mathrm{Cu}_{2}\right)_{2}\left(\mathrm{CO}_{3}\right)\left(\mathrm{ClO}_{4}\right)_{3}\right]^{+} \mathrm{zu}$ sehen, die größte Intensität weist das Fragment $\left[\mathrm{L}^{3} \mathrm{Cu}_{2}\right]^{+}$bei $m / z=421$ auf.

\begin{tabular}{|c|c|c|c|}
\hline \multicolumn{4}{|l|}{ Abstände } \\
\hline Cu1-017 & $1.911(2)$ & Cu3-019 & $1.923(2)$ \\
\hline Cu1-N1 & $1.914(3)$ & Cu3-N7 & $1.968(3)$ \\
\hline Cu1-N3 & $2.039(2)$ & Cu3-N10 & $2.050(3)$ \\
\hline Cu1-N4 & $2.050(3)$ & Cu3-N9 & $2.064(3)$ \\
\hline Cu1-O2 & $2.5036(24)$ & Cu3-O21 & $2.318(4)$ \\
\hline Cu2-N2 & $1.926(3)$ & Cu4-N8 & $1.955(3)$ \\
\hline Cu2-018 & $1.940(2)$ & Cu4-O20 & $1.961(3)$ \\
\hline Cu2-N6 & $2.049(3)$ & Cu4-N12 & $2.036(3)$ \\
\hline Cu2-N5 & $2.065(3)$ & Cu4-N11 & $2.045(3)$ \\
\hline Cu2-O3 & $2.5279(25)$ & Cu4-O1 & $2.431(2)$ \\
\hline Cu1‥Cu2 & $4.0014(5)$ & Cu3 ‥Cu4 & 4.279 \\
\hline \multicolumn{4}{|l|}{ Winkel } \\
\hline O17-Cu1-N1 & 101.08(11) & O19-Cu3-N7 & $98.21(10)$ \\
\hline O17-Cu1-N3 & $171.28(10)$ & O19-Cu3-N10 & $93.63(11)$ \\
\hline N1-Cu1-N3 & 81.94(11) & N7-Cu3-N10 & 162.82(13) \\
\hline O17-Cu1-N4 & $89.84(10)$ & O19-Cu3-N9 & $176.75(13)$ \\
\hline N1-Cu1-N4 & $164.27(11)$ & N7-Cu3-N9 & $81.57(12)$ \\
\hline N3-Cu1-N4 & $85.69(11)$ & N10-Cu3-N9 & $85.90(13)$ \\
\hline O2-Cu1-O17 & $86.35(9)$ & O19-Cu3-O21 & $86.34(11)$ \\
\hline O2-Cu1-N1 & $97.57(10)$ & N7-Cu3-O21 & $96.21(13)$ \\
\hline O2-Cu1-N3 & 101.44(9) & N10-Cu3-O21 & $96.93(13)$ \\
\hline O2-Cu1-N4 & $94.37(11)$ & N9-Cu3-O21 & $96.90(14)$ \\
\hline N2-Cu2-O18 & $102.93(10)$ & N8-Cu4-O20 & $96.14(11)$ \\
\hline N2-Cu2-N6 & $164.07(12)$ & N8-Cu4-N12 & $161.75(12)$ \\
\hline O18-Cu2-N6 & $90.03(10)$ & O20-Cu4-N12 & $94.30(12)$ \\
\hline N2-Cu2-N5 & $80.95(11)$ & N8-Cu4-N11 & $82.52(11)$ \\
\hline O18-Cu2-N5 & $169.67(10)$ & O20-Cu4-N11 & 176.24(11) \\
\hline N6-Cu2-N5 & $84.76(11)$ & N12-Cu4-N11 & $86.11(13)$ \\
\hline O3-Cu2-N2 & $93.26(0.10)$ & N8-Cu4-O1 & $98.57(10)$ \\
\hline O3-Cu2-O18 & $90.58(0.09)$ & O20-Cu4-O1 & $82.87(10)$ \\
\hline O3-Cu2-N6 & $95.86(0.10)$ & N12-Cu4-O1 & $97.55(11)$ \\
\hline O3-Cu2-N5 & $98.81(0.09)$ & N11-Cu4-O1 & $100.79(10)$ \\
\hline
\end{tabular}

Tabelle 2: Ausgewählte Bindungslängen $[\AA]$ und -winkel $\left[^{\circ}\right]$ von 5. 
Im UV/Vis-Spektrum in Aceton ist für alle vier Kupferzentren nur eine Bande für einen d-d-Übergang bei $614 \mathrm{~nm}\left(\varepsilon=450 \mathrm{~mol} \mathrm{I}^{-1} \mathrm{~cm}^{-1}\right)$ zu sehen, sie liegt damit im typischen Bereich für quadratisch pyramidal koordinierte Kupfer(II)zentren. Die Bande bei 226 $\mathrm{nm}\left(\varepsilon=18090 \mathrm{~mol} \mathrm{I}^{-1} \mathrm{~cm}^{-1}\right)$ liegt im Bereich der internen Ligandenübergänge und ist einem $\pi-\pi^{*}$-Übergang des Pyrazolatkernes zuzuordnen. Die Schulter bei $286 \mathrm{~nm}$ liegt ebenfalls noch in diesem Bereich, könnte aber auch von einem Charge-TransferÜbergang herrühren. ${ }^{[14]}$

Für den literaturbekannten Komplex $\mathrm{Cu}_{3}(\mathrm{dpt})_{3}\left(\mu_{3}-\mathrm{CO}_{3}\right)\left(\mathrm{ClO}_{4}\right)_{4} \cdot 2 \mathrm{H}_{2} \mathrm{O}$ (dpt = Dipropylentriamin) wird die Lage der IR-Banden der $\mu_{3}-\mathrm{CO}_{3}-$ Brücke bei 1475,1425 , 837, 782 und $672 \mathrm{~cm}^{-1}$ angegeben. ${ }^{[115]} \mathrm{Im}$ hier synthetisierten Komplex liegen entsprechende Banden bei 1471, 1422, 847 und $776 \mathrm{~cm}^{-1}$.

\subsection{Komplexe mit dem Liganden $\mathrm{HL}^{5}$}

Der Pyrazolligand $\mathrm{HL}^{5}$ weist zwei $\left\{\mathrm{N}_{3}\right\}$-Koordinationstaschen auf. Im Falle eines zweikernigen Metallkomplexes ist somit zu erwarten, daß jedes Metallzentrum durch drei Ligand-N-Donoren koordiniert wird. Aufgrund der von Kupfer(II) favorisierten Fünffachkoordination bleiben zwei Koordinationsstellen für die Koordination von weiteren verbrückenden oder endständigen Liganden, z.B. Solvensmolekülen oder Gegenionen, frei. Ausgehend von den bisher synthetisierten dinuklearen Komplexen mit aliphatischen Seitenarmen ist für Kupfer(II) eine verzerrt quadratisch pyramidale oder trigonal bipyramidale Koordination zu erwarten. ${ }^{\text {[99] }}$

Setzt man den Liganden $\mathrm{HL}^{5}$ in der zuvor beschriebenen Weise mit $\mathrm{Cu}\left(\mathrm{ClO}_{4}\right)_{2} \cdot 6 \mathrm{H}_{2} \mathrm{O}$ in Methanol um, so entsteht ein in diesem Solvens sowie in Aceton äußerst schlecht lösliches Produkt, so daß andere Lösungsmittel gewählt werden müssen. Ein gutes Lösungsverhalten zeigt das Reaktionsprodukt in Acetonitril. Nach Ablauf der Reaktion und Entfernen des Solvens im Vakuum kann man das Produkt durch Aufnehmen in Dichlormethan und Überschichten mit Diethylether isolieren. Der erhaltene Komplex $\left[{ }^{5} \mathrm{Cu}_{2}(\mathrm{OAc})\left(\mathrm{ClO}_{4}\right)\right] \mathrm{ClO}_{4}(6)$ kristallisiert in der Raumgruppe $P_{1}^{-}$ mit zwei Molekülen in der triklinen Zelle. Auch hier befinden sich Kupferzentren in beiden Koordinationstaschen, die durch jeweils ein Pyrazol-N-Atom sowie zwei NAtome eines Seitenarmes koordiniert sind. Zusätzlich wirkt in größerem Abstand ein 
Perchloratanion als $\mu_{2}$-verbrückender Ligand zwischen den Kupferzentren ( $d_{\mathrm{Cu} 1-03}=$ 2.372(2) $\left.\AA, d_{\mathrm{Cu2-06}}=2.568(1) \AA\right)$. Unerwartet ist der zweite Brückenligand: Durch vollständige oder teilweise Hydrolyse des Acetonitrils im basischen Milieu während der Komplexsynthese ist offensichtlich entweder ein Acetamid oder ein Acetat entstanden, welches ebenfalls $\mu_{2}$-verbrückend zwischen beide Kupferzentren tritt (Abbildung 18). Pro Komplexeinheit findet sich noch jeweils ein Dichlormethanmolekül in der Festkörperstruktur.

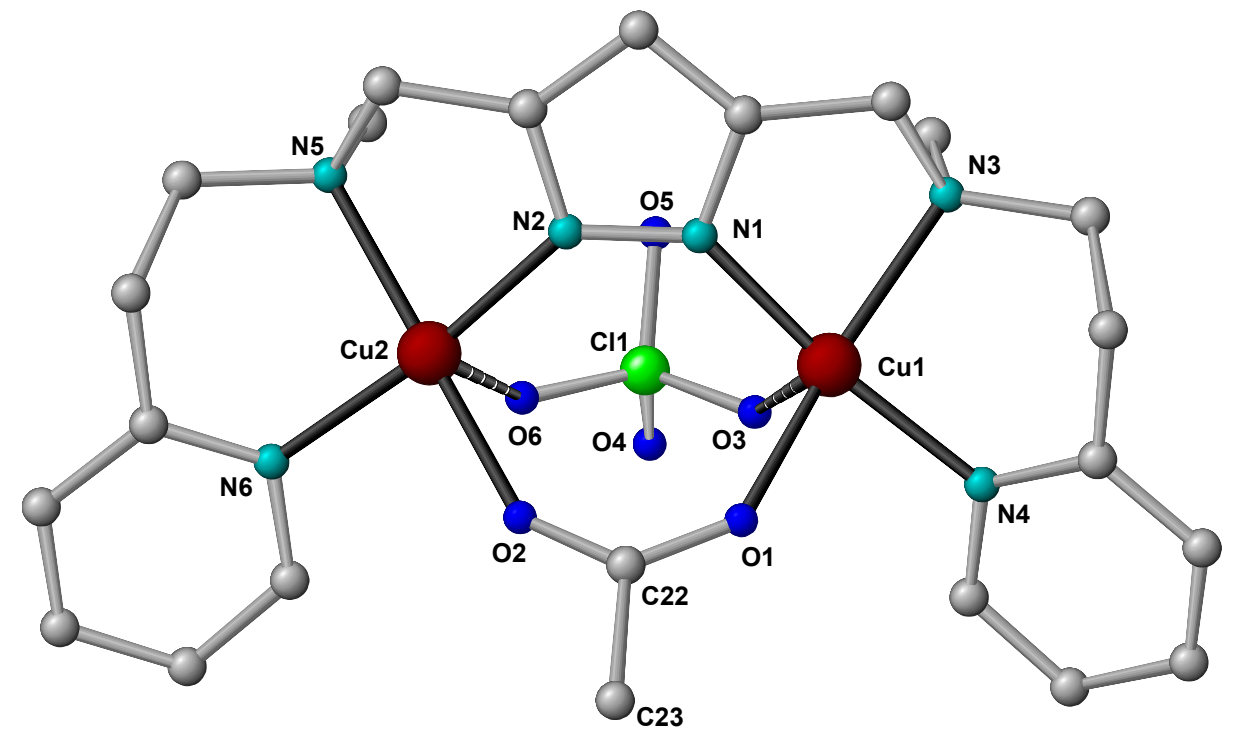

Abbildung 18: Festkörperstruktur des Kations von 6.

Eine eindeutige Aussage über die Art des Hydrolyseprodukts läßt sich anhand der Festkörperstruktur jedoch nicht treffen, da sich die Elektronendichte von NH-Gruppen von der des O-Atoms kaum unterscheidet. Auch das FAB-Massenspektrum kann hier keinen Aufschluß geben. Es sind nur Peaks für $\mathrm{L}^{5} \mathrm{Cu}_{2}{ }^{+}$bei $\mathrm{m} / \mathrm{z} 489$, sowie zwei Peaks für $\mathrm{L}^{5} \mathrm{Cu}_{2} \mathrm{ClO}_{4}{ }^{+}$und $\mathrm{L}^{5} \mathrm{Cu}_{2}\left(\mathrm{ClO}_{4}\right)_{2}{ }^{+}$bei $\mathrm{m} / \mathrm{z} 588$ und $687 \mathrm{im} \mathrm{FAB-Spektrum} \mathrm{zu}$ sehen. Im IR-Spektrum liegen zwei starke Banden bei 1571 bzw. $1449 \mathrm{~cm}^{-1}$. Dies entspricht fast genau den entsprechenden Banden für $\tilde{v}_{a}\left(\mathrm{COO}^{-}\right)$und $\tilde{v}_{\mathrm{s}}\left(\mathrm{COO}^{-}\right)$eines $\mu$-Acetat-verbrückten dinuklearen Kupferkomplexes von Neves et al. ${ }^{[16]}$

Beide Kupferzentren sind auch in diesem Komplex in Form einer nur leicht verzerrten quadratischen Pyramide fünffach koordiniert ( $\tau=0.05$ bzw. 0.16). Der Abstand zwischen den Kupferzentren ist mit 4.045(1) $\AA$ angesichts der Länge der Seitenarme überraschend groß. Möglicherweise werden die Metallzentren durch die Brückenliganden auseinandergedrückt. Zudem schränkt die sp²-Hybridisierung der 
Pyridin-C- und -N-Atome die Beweglichkeit der Ligandseitenarme im Vergleich zum entsprechenden Dikupferkomplex mit flexibleren aliphatischen Seitenarmen ein. Beim Komplex 1 liegt der Chelatsechsring in der entspannten Sesselkonformation vor und erlaubt einen CuCu-Abstand von 3.540(1) $\AA$, während der Pyrazolatring mit den Kupferzentren und der OH-Zweitbrücke fast in einer Ebene zu liegen kommt. ${ }^{[100]}$ Dagegen liegen bei 6 auf Grund der $\mathrm{sp}^{2}$-Hybridisierung der Aromatenatome die Atome des Pyridinringes, sowie das C6- bzw. C16-Atom in der meta-Position und das jeweilige koordinierte Kupferion in einer Ebene, die im Vergleich mit der durch den Pyrazolkern, die in 3- und 5-Stellung befindlichen Methylengruppen und die Kupferzentren aufgespannten Ebene leicht gekippt sind. Die Bindungswinkel an den beteiligten $\mathrm{sp}^{2}$-Zentren bewegen sich zwischen $116.6^{\circ}$ und $122.2^{\circ}$ (Abbildung 19).
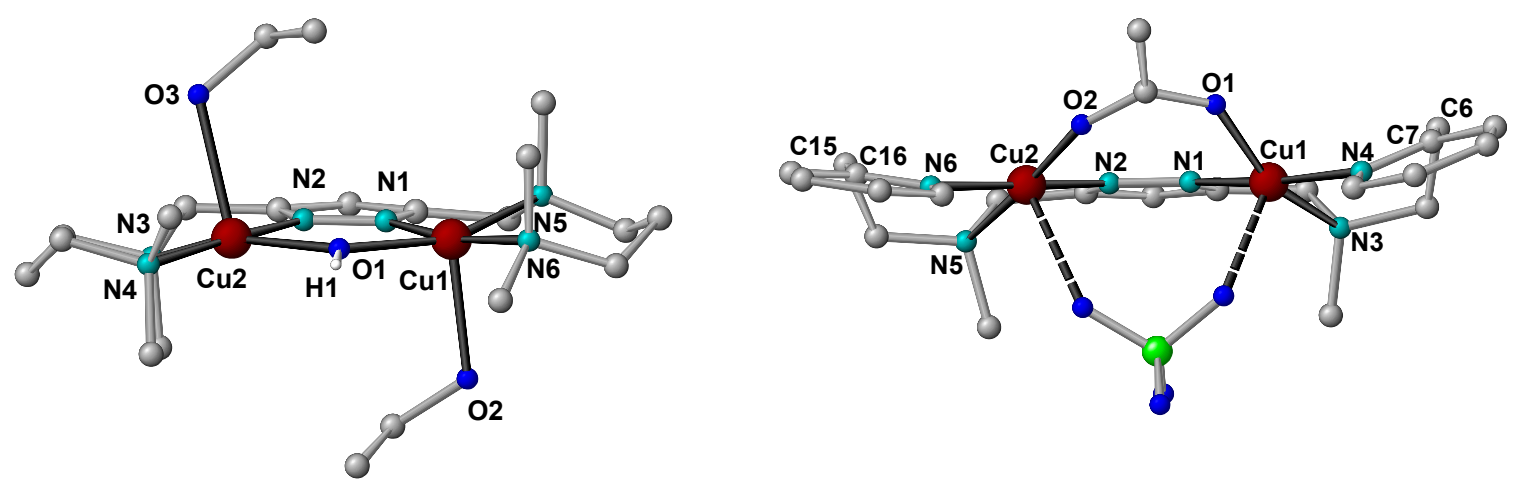

Abbildung 19: Vergleich von 1 und 6.

Ob allerdings die unterschiedliche Hybridisierung der Seitenarme ursächlich zum großen Cu‥Cu-Abstand führt oder der Einbau einer größeren Zweitbrücke, läßt sich anhand dieser Struktur noch nicht beantworten.

\begin{tabular}{|l|l|l|l|}
\hline Abstände \\
\hline Cu1-N1 & $1.917(2)$ & Cu2-N2 & $1.915(2)$ \\
\hline Cu1-N4 & $1.978(2)$ & Cu2-O2 & $1.960(2)$ \\
\hline Cu1-O1 & $1.981(2)$ & Cu2-N6 & $1.982(2)$ \\
\hline Cu1-N3 & $2.105(2)$ & Cu2-N5 & $2.098(2)$ \\
\hline Cu1-O3 & $2.372(2)$ & Cu2-O6 & $2.5676(23)$ \\
\hline Cu1‥Cu2 & $4.0449(5)$ & & \\
\hline Winkel \\
\hline N1-Cu1-N4 & $170.09(9)$ & N2-Cu2-O2 & $98.62(9)$ \\
\hline N1-Cu1-O1 & $97.73(9)$ & N2-Cu2-N6 & $172.75(10)$ \\
\hline N4-Cu1-O1 & $85.21(9)$ & O2-Cu2-N6 & $87.54(9)$ \\
\hline N1-Cu1-N3 & $81.12(9)$ & N2-Cu2-N5 & $80.44(9)$ \\
\hline N4-Cu1-N3 & $92.92(9)$ & O2-Cu2-N5 & $175.66(10)$ \\
\hline O1-Cu1-N3 & $160.37(9)$ & N6-Cu2-N5 & $93.14(10)$ \\
\hline N1-Cu1-O3 & $98.90(9)$ & O6-Cu2-N2 & $94.48(9)$ \\
\hline \multicolumn{5}{|c}{41} \\
\hline
\end{tabular}




\begin{tabular}{|l|l|l|l|}
\hline N4-Cu1-O3 & $90.28(8)$ & O6-Cu2-O2 & $84.14(9)$ \\
\hline O1-Cu1-O3 & $94.31(8)$ & O6-Cu2-N6 & $89.95(9)$ \\
\hline N3-Cu1-O3 & $105.26(8)$ & O6-Cu2-N5 & $100.14(9)$ \\
\hline
\end{tabular}

Tabelle 3: Ausgewählte Bindungslängen $[\AA]]$ und -winkel $\left[^{\circ}\right]$ von 6.

Um Aufschluß über die Art der Zweitbrücke bei Komplex 6 zu gewinnen, wurde dieser auf andere Weise ohne Vewendung von Acetonitril direkt hergestellt. Dazu wurde der Ligand $\mathrm{HL}^{5}$ in Aceton mit 1.5 Äquivalenten $\mathrm{Cu}\left(\mathrm{ClO}_{4}\right)_{2} \cdot 6 \mathrm{H}_{2} \mathrm{O}$, sowie mit einem Äquivalent $\mathrm{Cu}(\mathrm{OAc})_{2} \cdot \mathrm{H}_{2} \mathrm{O}$ umgesetzt und aus Dichlormethan mit Diethylether auskristallisiert. Im ESI-Massenspektrum aus der Methanollösung des Produkts findet man mit einer Intensität von $37 \%$ den Peak einer Spezies $\left[\mathrm{L}^{5} \mathrm{Cu}_{2}\left(\mathrm{O}_{2} \mathrm{CCH}_{3}\right)\left(\mathrm{ClO}_{4}\right)\right]^{+}$, sowie mit einer Intensität von $10 \%$ die Spezies $\left[\mathrm{L}^{5} \mathrm{Cu}_{2}\left(\mathrm{O}_{2} \mathrm{CCH}_{3}\right)\right]^{+}$, allerdings auch mit einer Intensität von $100 \%$ die Spezies $\left[\mathrm{L}^{5} \mathrm{Cu}_{2}\left(\mathrm{O}_{2} \mathrm{CCH}_{3}\right)_{2}\right]^{+}$, so daß hier entweder ein Produkt mit zwei Acetat- und einem Perchlorat-Gegenion entstanden ist, oder - was ausgehend von der eingesetzten Stöchiometrie der Edukte wahrscheinlicher ist - ein Gemisch von Komplexen mit einer unterschiedlichen Kombination von Gegenionen entstanden ist. Bei der röntgenographischen Charakterisierung des entstandenen Produktes wurde dagegen nur ein Komplex nachgewiesen - dieser Dikupferkomplex entspricht exakt dem schon auf andere Weise hergestellten Komplex 6. Dagegen bestätigt das IRSpektrum die Annahme, daß bei der Reaktion mehrere Produkte entstanden sind. Zusätzlich zu den bekannten $\tilde{v}_{\mathrm{a}}\left(\mathrm{COO}^{-}\right)$- und $\tilde{v}_{\mathrm{s}}\left(\mathrm{COO}^{-}\right)$- Banden bei 1566 und $1447 \mathrm{~cm}^{-1}$ findet man zwei weitere Peaks bei 1488 und $1415 \mathrm{~cm}^{-1}$. Die Elementaranalyse stimmt gut mit der Festkörperstruktur überein. Hier könnte z.B. neben der kristallographisch charakterisierten Verbindung eine weitere Spezies vorliegen, in der das Acetat einen anderen Bindungsmodus aufweist, deren Konstitution sich aber nicht von 6 unterscheidet. Die Lagen der Banden in den UVSpektren in Acetonitril stimmen ebenfalls gut überein. Man findet bei ca. 235 und 260 $\mathrm{nm}$ die Banden von $\pi-\pi^{*}$-Übergängen des Pyrazolat- und Pyridinkernes, einen CTÜbergang bei ca. $290 \mathrm{~nm}$ und den d-d-Übergang bei ca. $630 \mathrm{~nm}$. Trotz der Abweichungen in Massen- und IR-Spektrum konnte aufgrund der fast vollständigen Übereinstimmung beider Festkörperstrukturen belegt werden, daß es sich bei der Zweitbrücke von 6 um ein Acetat handelt.

Durch Reaktion von $\mathrm{HL}^{5}$ mit $\mathrm{Cu}\left(\mathrm{BF}_{4}\right)_{2} \cdot 6 \mathrm{H}_{2} \mathrm{O}$ in Methanol und anschließende 
Etherdiffusion erhält man blaue Kristalle. Bei der massenspektrometrischen Analyse mit der FAB-Methode erhält man einen Peak bei $m / z=489$ mit der Intensität $100 \%$, was einem $\left[\mathrm{L}^{5} \mathrm{Cu}_{2}\right]^{+}$-Fragment entspricht. Zwei weitere Peaks entsprechen einem $\left[\mathrm{L}^{5} \mathrm{Cu}_{2} \mathrm{~F}\right]^{+}$-Fragment bei $\mathrm{m} / \mathrm{z}=508$ mit einer Intensität von $79 \%$ und einem $\left[\mathrm{L}^{5} \mathrm{Cu}_{2} \mathrm{~F}(\mathrm{BF})_{4}\right]^{+}$-Fragment bei $\mathrm{m} / \mathrm{z}=595$ mit einer Intensität von $32 \%$. Hier hat offenbar die Abstraktion eines Fluorid-lons vom $\mathrm{BF}_{4}^{-}$-Anion stattgefunden. Eine solche Reaktion ist seit langem literaturbekannt, ebenso wie für das $\mathrm{PF}_{6}-$ Anion. ${ }^{[117,118,119,120,121]}$ In der Festkörperstruktur von $\left[\mathrm{L}^{5} \mathrm{Cu}_{2} \mathrm{~F}(\mathrm{MeOH})_{2}\left(\mathrm{BF}_{4}\right)\right] \mathrm{BF}_{4}(\mathbf{7 a})$ findet man das Fluorid koordiniert an eines der beiden Kupferzentren. Gleichzeitig bildet es eine Wasserstoffbrücke zum H-Atom einer an das benachbarte Kupferzentrum koordinierten methanolischen Hydroxygruppe (Abbildung 20). Das Fluorid wirkt nicht verbrückend, obwohl durchaus eine große Zahl zweikerniger Kupfer(II)komplexe röntgenographisch charakterisiert sind, in welchen das Fluor verbrückend zwischen beide Kupferzentren tritt. ${ }^{[117,118,119,120]}$ In der Tat wird oft sogar die Fluoridabspaltung von $\mathrm{BF}_{4}{ }^{-}$zur Bildung verbrückender Fluoridgruppen zwischen zwei Metallzentren ausgenutzt. ${ }^{[18]}$ Die erste Struktur eines $\mu_{3}$-fluoridverbrückten trinuklearen Kupfer(I)komplexes stammt von Hofmann at al. aus dem Jahre 2000. ${ }^{[122]}$

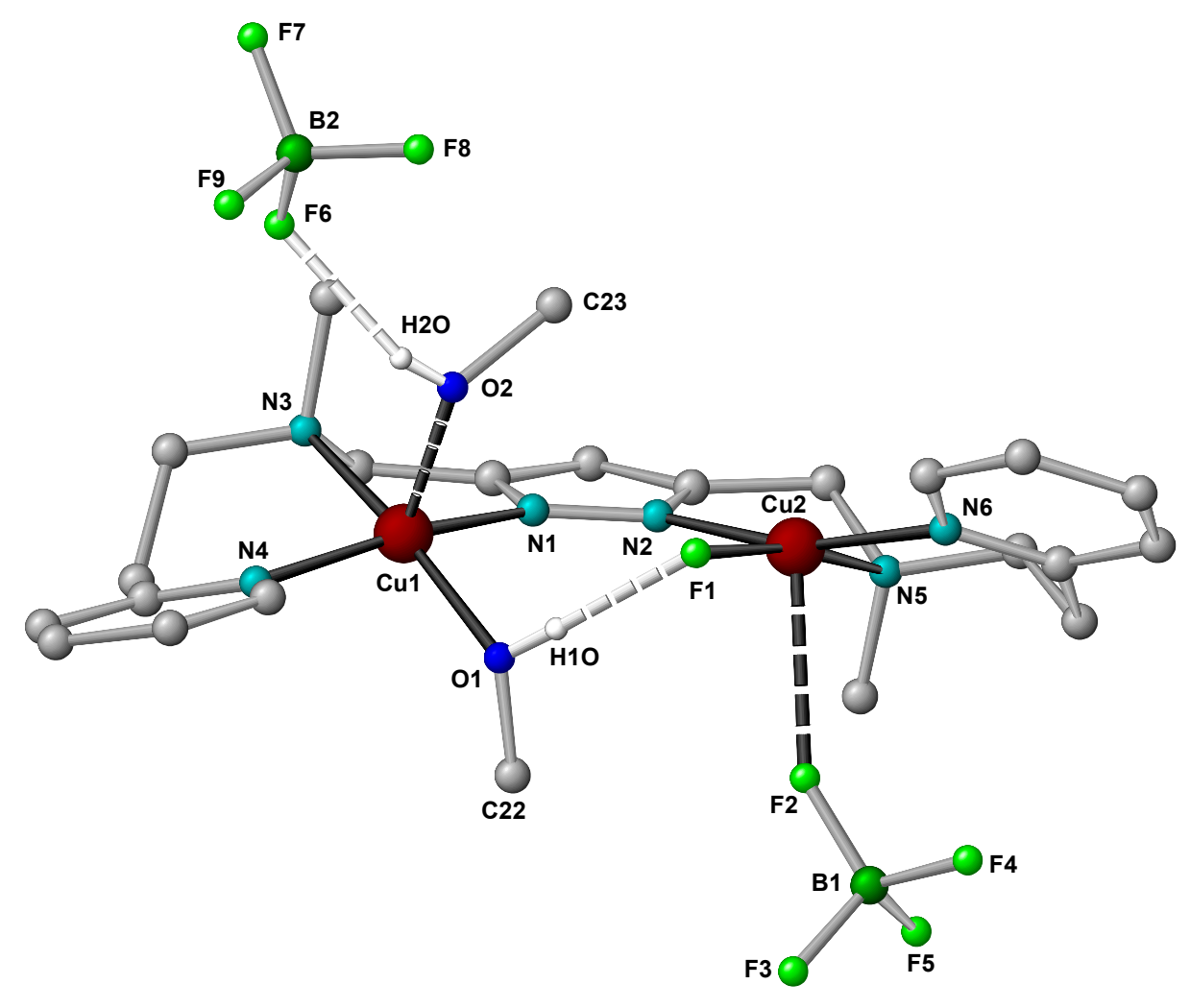

Abbildung 20: Festkörperstruktur von 7a. 
Der Komplex 7a kristallisiert in der Raumgruppe Pbca mit 8 Molekülen in der orthorhombischen Zelle. Obwohl hier kein großer zweiter Brückenligand zur Verfügung steht, besteht auch in diesem Komplex ein großer Abstand von 4.290(1) $\AA$ zwischen den Metallzentren, so daß auch hier nicht - wie im vergleichbaren Komplex

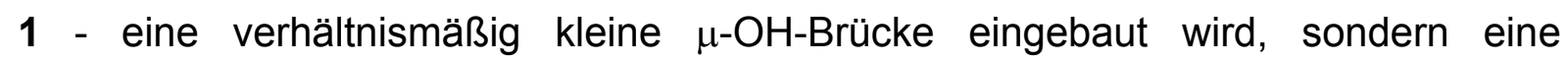
verbrückende MeO-H $\cdots$ F-Einheit zwischen die Kupferzentren tritt. Beide Metallionen befinden sich in einer nur leicht verzerrt quadratisch pyramidalen Koordinationsumgebung $\left(\tau_{\mathrm{Cu} 1}=0.01, \tau_{\mathrm{Cu} 2}=0.09\right.$ ). Cu1 wird hierbei basal durch ein Pyrazol-N-Atom, zwei Seitenarm-N-Atome und das O-Atom der MeO-H $\cdots$ F-Brücke koordiniert. In apicaler Position sitzt ein locker koordiniertes Methanol-Molekül $\left(d_{\mathrm{Cu} 1-\mathrm{O} 2}=2.271(2) \AA\right)$. Cu2 wird in der basalen Ebene durch ein Pyrazol-N-Atom, zwei Seitenarm-N-Atome und das Fluorid koordiniert. Apical bindet ein F-Atom eines $\mathrm{BF}_{4}{ }^{-}$-Gegenions an $\left(d_{\mathrm{Cu} 2-\mathrm{F} 2}=2.602(2) \AA\right)$. Ein weiteres $\mathrm{BF}_{4}{ }^{-}$-Anion bildet über das F6Atom eine $\mathrm{H}$-Brücke zum $\mathrm{H}$-Atom $\mathrm{H} 2 \mathrm{O}$ des apical koordinierten Methanol $\left(d_{\mathrm{F} 6-\mathrm{O} 2}=\right.$ $2.739 \AA)$.

\begin{tabular}{|c|c|c|c|}
\hline \multicolumn{4}{|l|}{ Abstände } \\
\hline Cu1-N1 & $1.948(2)$ & Cu2-F1 & $1.8967(15)$ \\
\hline Cu1-O1 & $2.000(2)$ & Cu2-N2 & \begin{tabular}{|l|}
$1.976(2)$ \\
\end{tabular} \\
\hline Cu1-N4 & $2.003(2)$ & Cu2-N6 & \begin{tabular}{|l|}
$2.000(3)$ \\
\end{tabular} \\
\hline Cu1-N3 & $2.091(2)$ & Cu2-N5 & \begin{tabular}{|l|}
$2.047(2)$ \\
\end{tabular} \\
\hline Cu1-O2 & $2.271(2)$ & Cu2-F2 & $2.6024(19)$ \\
\hline 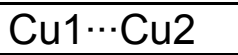 & $4.2903(8)$ & 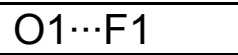 & $2.4678(27)$ \\
\hline \multicolumn{4}{|l|}{ Winkel } \\
\hline N1-Cu1-O1 & $93.09(9)$ & F1-Cu2-N2 & \begin{tabular}{|l|}
$94.28(8)$ \\
\end{tabular} \\
\hline N1-Cu1-N4 & $167.66(10)$ & F1-Cu2-N6 & \begin{tabular}{|l|}
$90.73(8)$ \\
\end{tabular} \\
\hline O1-Cu1-N4 & $88.37(9)$ & N2-Cu2-N6 & \begin{tabular}{|l}
$163.60(10)$ \\
\end{tabular} \\
\hline N1-Cu1-N3 & $81.73(9)$ & F1-Cu2-N5 & $168.97(9)$ \\
\hline O1-Cu1-N3 & $168.17(9)$ & N2-Cu2-N5 & \begin{tabular}{|l|}
$81.63(10)$ \\
\end{tabular} \\
\hline N4-Cu1-N3 & $94.46(9)$ & N6-Cu2-N5 & $96.03(10)$ \\
\hline N1-Cu1-O2 & $100.74(9)$ & F2-Cu2-F1 & $78.46(7)$ \\
\hline O1-Cu1-O2 & $90.87(9)$ & F2-Cu2N2 & $110.21(9)$ \\
\hline N4-Cu1-O2 & $91.47(9)$ & F2-Cu2-N6 & $86.09(9)$ \\
\hline N3-Cu1-O2 & $100.52(9)$ & F2-Cu2-N5 & $93.28(8)$ \\
\hline
\end{tabular}

Tabelle 4: Ausgewählte Bindungslängen $[\AA]$ und -winkel $\left[^{\circ}\right]$ von $7 a$.

Das UV/Vis-Spektrum in Methanol zeigt zwei Banden bei $206 \mathrm{~nm}\left(\varepsilon=14800 \mathrm{~mol} \mathrm{I}^{-1}\right.$ $\left.\mathrm{cm}^{-1}\right)$ und $224 \mathrm{~nm}\left(\varepsilon=14540 \mathrm{~mol} \mathrm{I}^{-1} \mathrm{~cm}^{-1}\right)$ für $\pi-\pi^{*}$-Übergänge der Pyridin- und 
Pyrazolheterozyklen, zwei Banden bei $259 \mathrm{~nm}\left(\varepsilon=11820 \mathrm{~mol} \mathrm{I}^{-1} \mathrm{~cm}^{-1}\right)$ und $284 \mathrm{~nm}$ (Schulter, $\varepsilon=4710 \mathrm{~mol} \mathrm{I}^{-1} \mathrm{~cm}^{-1}$ ), welche bereits Charge-Transfer-Übergängen entsprechen könnten, sowie eine im Bereich von quadratisch pyramidalen Koordinationsgeometrien des Kupfers liegende Bande bei $644 \mathrm{~nm}$ $\left(\varepsilon=200 \mathrm{~mol} \mathrm{l}^{-1} \mathrm{~cm}^{-1}\right)$.

Nachdem die Lösung von 7a einige Tage unter Luftatmosphäre stehengelassen wurde, kristallisierte in geringer Menge noch eine weitere Spezies $\left[\mathrm{L}^{5} \mathrm{Cu}_{2} \mathrm{~F}\left(\mathrm{H}_{2} \mathrm{O}\right)_{2}(\mathrm{MeOH})\left(\mathrm{BF}_{4}\right)_{2}\right] \mathbf{7 b}$ aus, deren Struktur im Festkörper ebenfalls röntgenographisch charakterisiert werden konnte (Abbildung 21). Die Verbindung kristallisiert in der Raumgruppe $P 2_{1} / c$ mit vier Molekülen in der monoklinen Zelle. Beide Kupferzentren sind nahezu quadratisch pyramidal koordiniert ( $\tau_{\mathrm{Cu} 1}=0.02, \tau_{\mathrm{Cu} 2}$ $=0.14) . \mathrm{Im}$ Gegensatz zu 7a finden sich in $7 \mathrm{~b}$ zwei an $\mathrm{Cu} 2$ koordinierte $\mathrm{H}_{2} \mathrm{O}$ Moleküle. Ein $\mathrm{H}_{2} \mathrm{O}$ koordiniert in apicaler Position, während das andere zusammen mit einem Pyrazol-N-Atom und den zwei N-Atomen eines Seitenarmes die basale Koordinationsebene bildet. An Cu2 koordinieren auch hier basal ein Fluorid sowie die restlichen N-Donoren des Liganden. Apical koordiniert ein Solvensmolekül über das O1-Atom. Der Cu ‥Cu-Abstand bleibt mit 4.287(1) A fast identisch zu jenem von 7a.

$\mathrm{H}_{2} \mathrm{O}$ wird zum einen durch das wasserhaltige $\mathrm{Cu}\left(\mathrm{BF}_{4}\right)_{2} \cdot 6 \mathrm{H}_{2} \mathrm{O}$ schon während der Herstellung des Komplexes eingeführt und sollte den wohl zunächst koordinierenden Liganden Methanol schnell verdrängen können (Kupfer(II) tauscht den Liganden $\mathrm{H}_{2} \mathrm{O}$ mit $k>10^{8} \mathrm{~s}^{-1}$ sehr schnell aus). ${ }^{[123]}$ Eine vergleichbare Beobachtung wurde bereits während der Diplomarbeit bei einem anderen Dikupferkomplex gemacht. ${ }^{[99]}$ Zum anderen konnte im nicht geschlossenen System Luftfeuchtigkeit langsam in die Reaktionslösung eindiffundieren, während das Solvens verdunstete. 


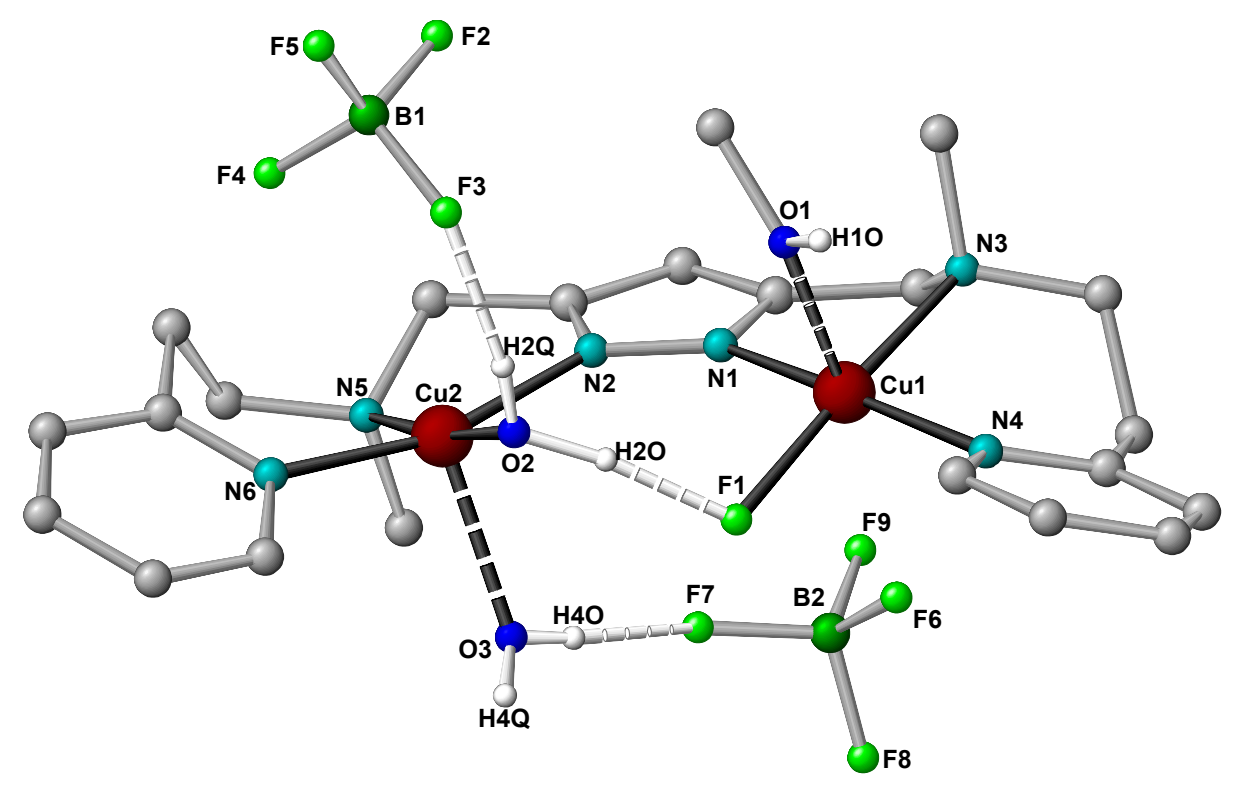

Abbildung 21: Festkörperstruktur von $\mathbf{7 b .}$

\begin{tabular}{|c|c|c|c|}
\hline \multicolumn{4}{|l|}{ Abstände } \\
\hline Cu1-F1 & $1.9157(17)$ & Cu2-N2 & $1.979(2)$ \\
\hline Cu1-N1 & $1.964(2)$ & Cu2-O2 & $1.998(2)$ \\
\hline Cu1-N4 & $1.992(2)$ & Cu2-N6 & $2.001(2)$ \\
\hline Cu1-N3 & $2.081(2)$ & Cu2-N5 & $2.086(2)$ \\
\hline Cu1-O1 & $2.364(3)$ & Cu2-O3 & $2.222(2)$ \\
\hline 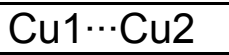 & $4.2865(11)$ & $\mathrm{F} 1 \cdots \mathrm{O} 2$ & $2.5066(29)$ \\
\hline \multicolumn{4}{|l|}{ Winkel } \\
\hline F1-Cu1-N1 & $94.93(9)$ & N2-Cu2-O2 & $94.93(9)$ \\
\hline F1-Cu1-N4 & $88.37(9)$ & N2-Cu2-N6 & $164.34(10)$ \\
\hline N1-Cu1-N4 & $171.46(10)$ & O2-Cu2-N6 & $88.01(10)$ \\
\hline F1-Cu1-N3 & 172.91(9) & N2-Cu2-N5 & $82.21(9)$ \\
\hline N1-Cu1-N3 & $82.30(10)$ & O2-Cu2-N5 & $172.77(10)$ \\
\hline N4-Cu1-N3 & $93.50(10)$ & N6-Cu2-N5 & $93.01(10)$ \\
\hline F1-Cu1-O1 & $90.49(10)$ & N2-Cu2-O3 & $97.83(9)$ \\
\hline N1-Cu1-O1 & $99.10(10)$ & O2-Cu2-O3 & $88.53(10)$ \\
\hline N4-Cu1-O1 & $88.73(10)$ & N6-Cu2-O3 & 97.62(9) \\
\hline N3-Cu1-O1 & $96.39(11)$ & N5-Cu2-O3 & $98.41(9)$ \\
\hline
\end{tabular}

Tabelle 5: Ausgewählte Bindungslängen $[\AA]$ und -winkel $\left[{ }^{\circ}\right]$ von $\mathbf{7 b}$.

Dieses Beispiel zeigt, wie leicht der Ligandenaustausch an den Kupfer(II)zentren offensichtlich vonstatten geht. Die einfache Abstraktion von Solvensmolekülen sollte die Ausbildung freier Koordinationsstellen und damit die katalytische Aktivität der Komplexe begünstigen. Abschließend bleibt festzuhalten, daß - obwohl durchaus mehrkernige Kupferkomplexe mit verbrückendem Fluorid bei einem $\mathrm{Cu} \cdots \mathrm{Cu}$-Abstand bis zu $4 \AA$ literaturbekannt sind ${ }^{[117,118,119,120]}$ - bei den hier vorgestellten Komplexen 
der Einbau einer größeren Zweitbrücke bevorzugt wird, während das Fluorid nur an einem Kupferzentrum sitzt.

Bei der Synthese des Dikupferkomplexes von $\mathrm{HL}^{5}$ mit zwei Äquivalenten Kalium-tertbutylat und zwei Äquivalenten $\mathrm{Cu}\left(\mathrm{NO}_{3}\right)_{2} \cdot 3 \mathrm{H}_{2} \mathrm{O}$ wird - trotz des Einsatzes zweier Äquivalente der Base - die Spezies $\left[\mathrm{L}^{5} \mathrm{Cu}_{2}(\mathrm{MeOH})_{2}\left(\mathrm{NO}_{3}\right)_{2}\right] \mathrm{NO}_{3}$ (8) gebildet, bei der beide Kupferzentren jeweils zusätzlich zu einem Pyrazol-N-Atom und zwei Seitenarm-N-Atomen des Liganden $\left[\mathrm{L}^{5}\right]^{-}$durch ein O-Atom eines Nitrat-Anions in der basalen Ebene, sowie durch das O-Atom eines Methanolmoleküls in der apicalen Position einer verzerrten quadratisch pyramidalen Koordinationssphäre koordiniert sind $\left(\tau_{\mathrm{Cu} 4}=0.38, \tau_{\mathrm{Cu} 2}=0.02\right)$. Nitrat-O-Atom und Methanol-O-Atom verbrücken auf beiden Seiten der durch den Pyrazolkern aufgespannten Ebene durch H-Brücken die Kupferzentren $\left(d_{\mathrm{O} 12 \cdots 015}=2.745(6), d_{\mathrm{O} 13 \cdots 014}=2.761(6)\right)$. Der Dikupferkomplex kristallisiert in der Raumgruppe $P_{1}^{-}$mit zwei Molekülen in der triklinen Zelle (Abbildung 22).

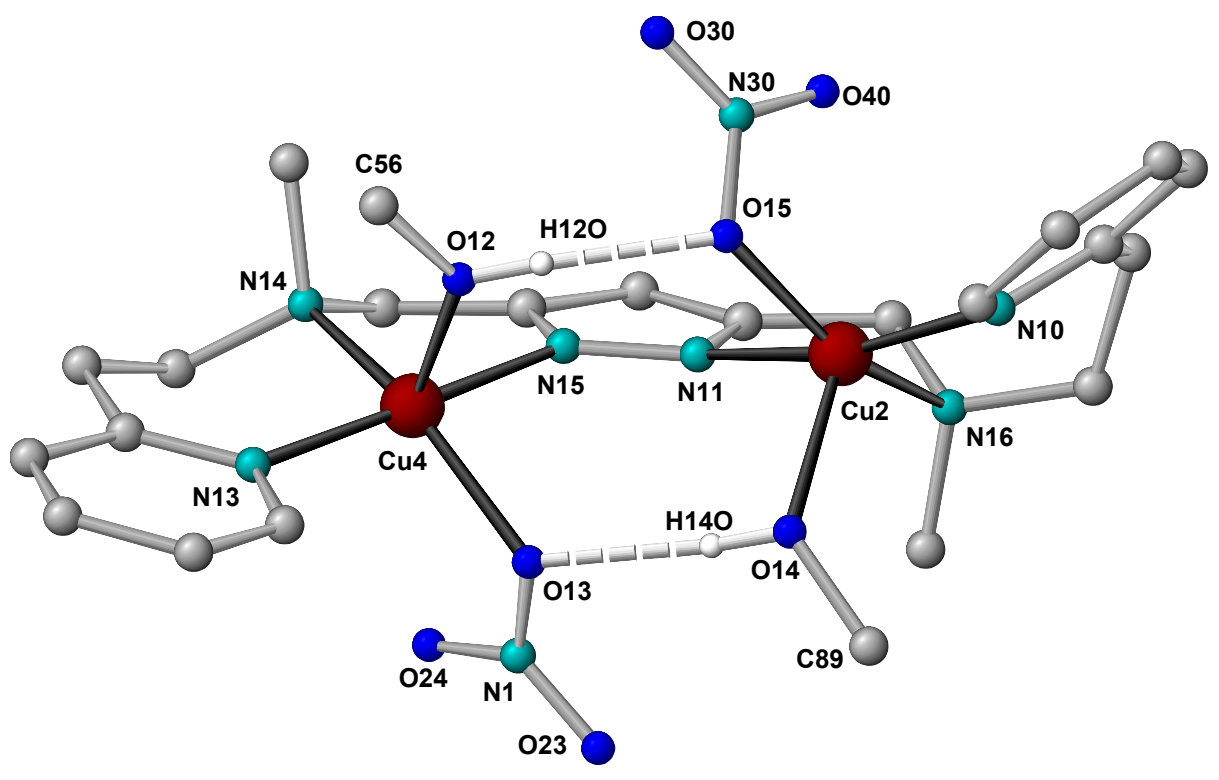

Abbildung 22: Festkörperstruktur des Kations von 8.

Trotz Verwendung zweier Äquivalente der Base findet man in der Festkörperstruktur nur den deprotonierten Liganden $\left[\mathrm{L}^{5}\right]^{-}$, jedoch keine deprotonierte Zweitbrücke. Anstattdessen treten zwei MeO-H $\cdots \mathrm{O}\left(\mathrm{NO}_{2}\right)$-Brücken auf. Dies ist ein weiterer Beleg dafür, daß die in diese Ligandmatrize eingebetteten Kupferzentren große Abstände 
$\left(d_{\mathrm{Cu} \cdots \mathrm{Cu}}=4.357(1) \AA\right)$ und damit große Zweitbrücken bevorzugen. Die Ausbildung der $\mathrm{MeO}-\mathrm{H} \cdots \mathrm{O}\left(\mathrm{NO}_{2}\right)$-Brücken unter gleichzeitiger Wahrung der Fünffachkoordination beider Kupferzentren bewirkt eine starke Verzerrung der Struktur des Komplexes, ersichtlich am Torsionswinkel Cu4-N15-N11-Cu2 $=35.1^{\circ}$ (zum Vergleich: der entsprechende Winkel bei $\mathbf{5}, \mathbf{6}, 7 \mathrm{a}$ und $\mathbf{7 b}$ beträgt $1.2^{\circ}, 1.6^{\circ}, 2.0^{\circ}$ und $12.7^{\circ}$ ) Dies könnte bedeuten, daß die Zweitbrücken bei der Umsetzung mit einem Substrat leicht zu entfernen sind und somit der Angriff des Substrates erleichtert wird.

Setzt man $\mathrm{HL}^{5}$ mit nur einem Äquivalent Kalium-tert-butylat und Kupfernitrat-Trihydrat um, so so entsteht ebenfalls das Produkt 8. Bei weiteren Synthesen konnte somit auf das zweite Basenäquivalent verzichtet werden.

\begin{tabular}{|c|c|c|c|}
\hline \multicolumn{4}{|l|}{ Abstände } \\
\hline Cu2-N11 & $1.971(4)$ & Cu4-N15 & $1.987(5)$ \\
\hline Cu2-N10 & $2.007(4)$ & Cu4-N13 & $2.027(5)$ \\
\hline Cu2-N16 & $2.046(4)$ & \begin{tabular}{|l|} 
Cu4-N14 \\
\end{tabular} & $2.029(5)$ \\
\hline Cu2-O15 & $2.057(4)$ & \begin{tabular}{|l} 
Cu4-O13 \\
\end{tabular} & $2.077(4)$ \\
\hline Cu2-O14 & $2.201(4)$ & Cu4-O12 & $2.241(4)$ \\
\hline \multirow[t]{2}{*}{ Cu2 $\cdots$ Cu4 } & $4.357(1)$ & $012 \cdots 015$ & $2.745(6)$ \\
\hline & & $013 \cdots 014$ & $2.761(6)$ \\
\hline \multicolumn{4}{|l|}{ Winkel } \\
\hline N11-Cu2-N10 & $165.00(17)$ & N15-Cu4-N13 & $177.55(18)$ \\
\hline N11-Cu2-N16 & $81.10(18)$ & N15-Cu4-N14 & $82.58(19)$ \\
\hline N10-Cu2-N16 & $95.19(18)$ & N13-Cu4-N14 & $95.1(2)$ \\
\hline N11-Cu2-O15 & $89.87(16)$ & N15-Cu4-O13 & $88.79(17)$ \\
\hline N10-Cu2-O15 & $88.43(16)$ & N13-Cu4-O13 & $93.08(17)$ \\
\hline N16-Cu2-O15 & 158.22(16) & N14-Cu4-O13 & 154.51(16) \\
\hline N11-Cu2-O14 & $100.92(17)$ & N15-Cu4-O12 & $96.57(16)$ \\
\hline N10-Cu2-O14 & $94.02(17)$ & N13-Cu4-O12 & $84.89(17)$ \\
\hline N16-Cu2-O14 & $109.50(16)$ & N14-Cu4-O12 & $111.32(17)$ \\
\hline O15-Cu2-O14 & $91.60(15)$ & O13-Cu4-O12 & $93.42(15)$ \\
\hline N11-Cu2-Cu4 & $39.95(13)$ & N15-Cu4-Cu2 & $40.39(13)$ \\
\hline N10-Cu2-Cu4 & 147.77(13) & N13-Cu4-Cu2 & $142.06(13)$ \\
\hline N16-Cu2-Cu4 & $116.83(13)$ & N14-Cu4-Cu2 & $120.56(14)$ \\
\hline O15-Cu2-Cu4 & $63.36(11)$ & O13-Cu4-Cu2 & $60.79(11)$ \\
\hline O14-Cu2-Cu4 & $72.83(11)$ & O12-Cu4-Cu2 & $70.97(12)$ \\
\hline
\end{tabular}

Tabelle 6: Ausgewählte Bindungslängen $[\AA]$ ] und -winkel [] von 8.

Im FAB-Massenspektrum von 8 findet man als stärksten Peak bei $\mathrm{m} / \mathrm{z}=613$ das Fragment $\left[\mathrm{L}^{5} \mathrm{Cu}_{2}\left(\mathrm{NO}_{3}\right)_{2}\right]^{+}$. Die nur schwach koordinierenden Methanolmoleküle können hier nicht nachgewiesen werden. Im UV/Vis-Spektrum in Methanollösung 
findet man die Lage des $d$-d-Überganges im Bereich quadratisch pyramidal koordinierter Kupfer(II)zentren bei $635 \mathrm{~nm}\left(\varepsilon=290 \mathrm{~mol} \mathrm{I}^{-1} \mathrm{~cm}^{-1}\right)$.

\subsection{Komplexe mit dem Liganden $\mathrm{HL}^{6}$}

Der Pyrazolligand $\mathrm{HL}^{6}$ bietet pro Metallzentrum einen zusätzlichen Pyridin-N-Donor an und sättigt dementsprechend jeweils eine zusätzliche Koordinationsstelle ab. Die Länge der Seitenarme des Liganden ist identisch mit denen von $\mathrm{HL}^{5}$.

Setzt man $\mathrm{HL}^{6}$ mit zwei Äquivalenten der Base Kalium-tert-butylat sowie zwei Äquivalenten $\mathrm{Cu}\left(\mathrm{ClO}_{4}\right)_{2} \cdot 6 \mathrm{H}_{2} \mathrm{O}$ in Acetonitril um, so erhält man nach Kristallisation in Acetonitril durch Etherdiffusion die zu 6 analoge Verbindung $\left[\mathrm{L}^{6} \mathrm{Cu}_{2}(\mathrm{OAc})\right]\left(\mathrm{ClO}_{4}\right)_{2}(\mathbf{9})$, die ebenso in Methanol schwerlöslich ist. Beide Kupferzentren sind durch jeweils einen Pyrazol-N-Donor, ein N-Atom aus dem aliphatischen Teil des Seitenarmes, sowie zwei Pyridin-N-Atome koordiniert. Zusätzlich findet man wie schon bei 6 einen zweiten die Kupferionen verbrückenden Acetat- oder Acetamid-Liganden, welcher durch Hydrolyse von Acetonitril entstanden sein muß (Abbildung 23).

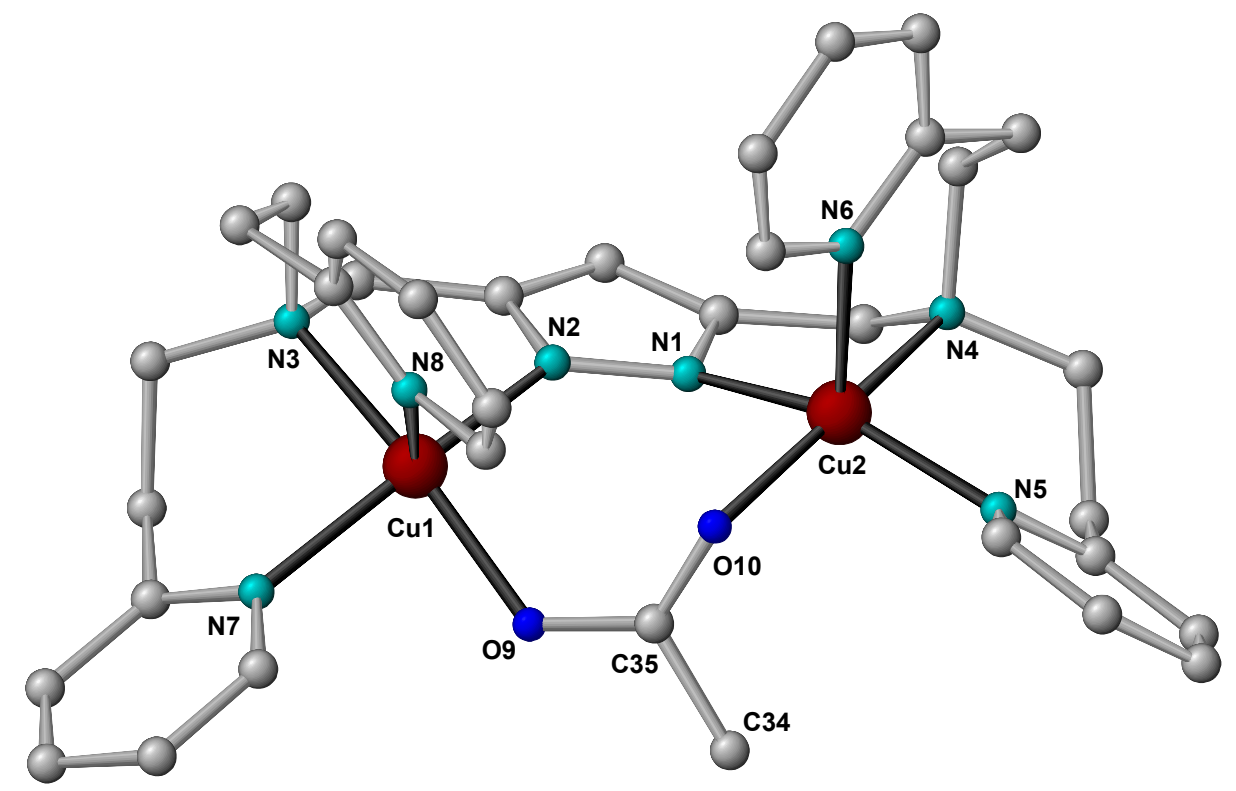

Abbildung 23: Festkörperstruktur des Kations von 9.

Der Komplex kristallisiert in der Raumgruppe C2/c mit acht Molekülen in der monoklinen Zelle. Beide Kupferzentren sind leicht verzerrt quadratisch pyramidal von vier N-Donoren des Pyrazolatliganden sowie einem Heteroatom eines verbrückenden Acetats bzw. Amids umgeben $\left(\tau_{\mathrm{Cu} 1}=0.07, \tau_{\mathrm{Cu} 2}=0.15\right)$. Hierbei besetzen die Pyridin- 
N-Atome N8 und N6 die apicalen Positionen. Bei dem Heteroatom dürfte es sich analog zu 6 auch hier um ein O-Atom eines durch vollständige Hydrolyse des Solvens Acetonitril im basischen Milieu entstandenen Acetats handeln. Der Abstand der Metallzentren liegt bei 4.268(1) A. Der Komplex ist stark verzerrt, der Torsionswinkel Cu1-N2-N1-Cu2 beträgt $44.6^{\circ}$. Dies dürfte in erster Linie auf die sterischen Ansprüche der Zweitbrücke zurückzuführen sein. Die Zweitbrücke selbst ist in einem großen Winkel im Vergleich mit der Pyrazolebene eingebaut, möglicherweise, weil die Kupferzentren in dieser Ligandmatrize nicht mehr weiter auseinandergedrückt werden können. Die Komplexe dieses Liganden mit sterisch weniger anspruchsvollen Zweitbrücken zeigen eine weit kleinere Verzerrung, wie die folgenden Beispiele zeigen werden.

\begin{tabular}{|l|l|l|l|}
\hline Abstände \\
\hline Cu1-O9 & $1.972(3)$ & Cu2-N1 & $1.951(3)$ \\
\hline Cu1-N2 & $1.987(4)$ & Cu2-N5 & $1.997(4)$ \\
\hline Cu1-N7 & $2.049(4)$ & Cu2-O10 & $2.001(3)$ \\
\hline Cu1-N3 & $2.092(4)$ & Cu2-N4 & $2.094(4)$ \\
\hline Cu1-N8 & $2.219(4)$ & Cu2-N6 & $2.227(4)$ \\
\hline Cu11..Cu2 & $4.2675(10)$ & & \\
\hline Winkel & \multicolumn{3}{|l|}{} \\
\hline O9-Cu1-N2 & $95.08(14)$ & N1-Cu2-N5 & $158.13(17)$ \\
\hline O9-Cu1-N7 & $86.18(14)$ & N1-Cu2-O10 & $87.43(14)$ \\
\hline N2-Cu1-N7 & $158.60(17)$ & N5-Cu2-O10 & $91.37(14)$ \\
\hline O9-Cu1-N3 & $162.82(15)$ & N1-Cu2-N4 & $82.79(14)$ \\
\hline N2-Cu1-N3 & $80.61(14)$ & N5-Cu2-N4 & $94.58(14)$ \\
\hline N7-Cu1-N3 & $91.90(15)$ & O10-Cu2-N4 & $167.09(13)$ \\
\hline O9-Cu1-N8 & $99.26(14)$ & N1-Cu2-N6 & $105.98(15)$ \\
\hline N2-Cu1-N8 & $108.29(15)$ & N5-Cu2-N6 & $95.87(15)$ \\
\hline N7-Cu1-N8 & $92.53(16)$ & O10-Cu2-N6 & $95.16(13)$ \\
\hline N3-Cu1-N8 & $97.88(15)$ & N4-Cu2-N6 & $95.61(15)$ \\
\hline
\end{tabular}

Tabelle 7: Ausgewählte Bindungslängen $[\AA]$ und -winkel $\left[{ }^{\circ}\right]$ von 9.

Im ESI-Massenspektrum der Lösung in Methanol wird hier eindeutig bei $m / z=789$ das Fragment $\left[\mathrm{L}^{6} \mathrm{Cu}_{2}(\mathrm{OAc})_{2}\right]^{+}$mit einer Intensität von $24 \%$, sowie bei $\mathrm{m} / \mathrm{z}=829$ mit einer Intensität von $27 \%$ die Spezies $\left[\mathrm{L}^{6} \mathrm{Cu}_{2}(\mathrm{OAc})\left(\mathrm{ClO}_{4}\right)\right]^{+}$gefunden, womit die Zweitbrücke als Acetat identifiziert werden kann. Im IR-Spektrum liegen die Banden für $\tilde{v}_{\mathrm{a}}\left(\mathrm{COO}^{-}\right)$und $\tilde{v}_{\mathrm{s}}\left(\mathrm{COO}^{-}\right)$bei $1570 \mathrm{bzw} .1444 \mathrm{~cm}^{-1}$. Das UV-Spektrum in Acetonitril zeigt $\pi-\pi^{*}$-Übergänge von Pyrazolat- und Pyridinkern bei $225 \mathrm{~nm}(\varepsilon=$ $\left.18430 \mathrm{~mol} \mathrm{I}^{-1} \mathrm{~cm}^{-1}\right)$ und $257 \mathrm{~nm}\left(\varepsilon=20650 \mathrm{~mol} \mathrm{I}^{-1} \mathrm{~cm}^{-1}\right)$, einen CT-Übergang bei 267 
$\mathrm{nm}$ (Schulter, $\varepsilon=16080 \mathrm{~mol} \mathrm{I}^{-1} \mathrm{~cm}^{-1}$ ) und die Bande eines $\mathrm{d}$-d-Überganges bei $646 \mathrm{~nm}\left(\varepsilon=280 \mathrm{~mol} \mathrm{I}^{-1} \mathrm{~cm}^{-1}\right)$.

Zum weiteren Beleg für die Identität der Zweitbrücke als Acetat wurde ein acetatverbrückter Dikupferkomplex von $\mathrm{HL}^{6}$ gezielt hergestellt. $\mathrm{HL}^{6}$ wurde mit zwei Äquivalenten Kalium-tert-butylat, sowie 1.5 Äquivalenten $\mathrm{Cu}\left(\mathrm{ClO}_{4}\right)_{2} \cdot 6 \mathrm{H}_{2} \mathrm{O}$ und einem halben Äquivalent $\mathrm{Cu}(\mathrm{OAc})_{2} \cdot \mathrm{H}_{2} \mathrm{O}$ in Aceton umgesetzt und das Produkt nach Entfernen des Solvens im Vakuum durch Etherdiffusion aus Dichlormethan ausgefällt. Obwohl es nicht gelang, diese Verbindung kristallin zu erhalten, so entspricht doch ihr IR- und UV-Spektrum demjenigen des Komplexes 9. Im ESIMassenspektrum dieser Verbindung in Methanollösung lassen sich die Fragmente $\left[\mathrm{L}^{6} \mathrm{Cu}_{2}(\mathrm{OAc})\right]^{+}$und $\left[\mathrm{L}^{6} \mathrm{Cu}_{2}(\mathrm{OAc})\left(\mathrm{ClO}_{4}\right)\right]^{+}$bei $\mathrm{m} / \mathrm{z}=730$ mit einer Intensität von $71 \%$ bzw. bei 829 mit einer Intensität von ebenfalls $71 \%$ nachweisen. Der stärkste Peak mit $100 \%$ bei $m / z=787$ dürfte allerdings einer Spezies $\left[\mathrm{L}^{6} \mathrm{Cu}_{2}(\mathrm{OH})\left(\mathrm{ClO}_{4}\right)\right]^{+}$gehören, so daß offensichtlich unter den hier gewählten Reaktionsbedingungen bzw. unter den Meßbedingungen bei der ESI-Messung neben dem acetatverbrückten Dikupferkomplex noch ein Komplex mit einem Hydroxidliganden entstanden ist.

Geht man bei der Herstellung des Dikupferkomplexes zunächst in der gleichen Weise vor wie bei der Synthese von 9 beschrieben, entfernt aber nach sechs Stunden das Acetonitril im Vakuum, um das Produkt dann aus Dichlormethan durch Etherdiffusion auskristallisieren zu lassen, so läßt sich die Hydrolyse von Acetonitril und damit der Einbau von Acetat in den Komplex verhindern. Der so erhaltene Komplex $\left[\mathrm{L}^{6} \mathrm{Cu}_{2}(\mathrm{OH})\right]\left(\mathrm{ClO}_{4}\right)_{2}$ (10) kristallisiert in der Raumgruppe $P 2_{1} / n$ mit vier Molekülen in der monoklinen Zelle. Die Koordinationsgeometrie der Kupferzentren durch den Pyrazolatliganden ist mit derjenigen des Komplexes 9 weitgehend identisch: in einer leicht verzerrt quadratisch pyramidalen Koordinationsumgebung $\left(\tau_{\mathrm{Cu} 1}=0.09, \tau_{\mathrm{Cu} 2}=0.18\right)$ sitzen die Atome N5 bzw. N7 eines Pyridinrestes in der apicalen Position, während die Atome N1, N3 und N4 bzw. N2, N6 und N8 in der basalen Ebene liegen. Den vierten basalen Donor teilen sich beide Kupferzentren in Form einer verbrückend koordinierenden $\mathrm{OH}^{-}$-Gruppe, die wiederum eine schwache $\mathrm{H}$-Brücke zu einem Perchloration bildet $\left(d_{\mathrm{O} 1-\mathrm{O} 2}=3.141 \AA\right)$. Der Kupfer-KupferAbstand beträgt 3.441(1) $\AA$ (Abbildung 24), der Torsionswinkel Cu1-N1-N2-Cu2 nur $3.1^{\circ}$. 


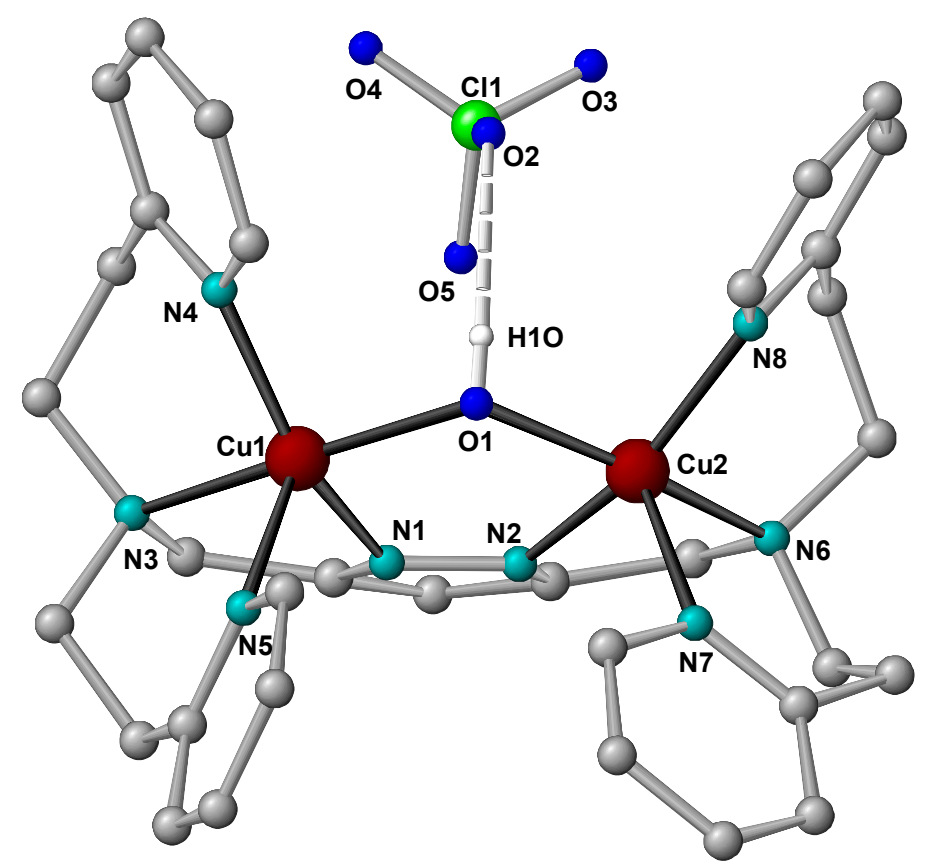

Abbildung 24: Festkörperstruktur des Kations von 10.

Der gefundene Metall-Metall-Abstand und damit der Einbau der kleinen $\mathrm{OH}^{-}$-Brücke zwischen den Metallzentren entspricht bei diesem Komplex den Erwartungen: lange Pyrazolatligand-Seitenarme erlauben kürzere Kupfer-Kupfer-Abstände. Während bei den Komplexen von $\mathrm{HL}^{5}$ auch bei Vorhandensein kleiner Liganden wie $\mathrm{F}^{-}$und $\mathrm{OH}^{-}$ immer der Einbau einer großen Brücke gefunden wurde, so wird bei 10 - obwohl auch z.B. die Koordination der Kupferzentren mit einem $\mathrm{OH}^{-}$- und einem AcetonitrilLiganden denkbar wäre - der kleine Brückenligand bevorzugt. Im Falle von 9 rührt somit der große Abstand der Metallzentren nicht etwa da her, daß diese durch die Seitenarme „auseinandergezogen“ werden - sie werden im Gegenteil durch die Acetatzweitbrücke auseinandergedrückt. Er hat also eine andere Ursache als im Fall von 6.

\begin{tabular}{|l|l|l|l|}
\hline Abstände \\
\hline Cu1-N1 & $1.909(3)$ & Cu2-N2 & $1.902(3)$ \\
\hline Cu1-N4 & $1.983(3)$ & Cu2-N8 & $1.975(3)$ \\
\hline Cu1-O1 & $1.991(2)$ & Cu2-O1 & $1.990(2)$ \\
\hline Cu1-N3 & $2.160(3)$ & Cu2-N6 & $2.135(3)$ \\
\hline Cu1-N5 & $2.215(3)$ & Cu2-N7 & $2.210(3)$ \\
\hline Cu1‥Cu2 & $3.4406(6)$ & & \\
\hline Winkel & & \\
\hline N1-Cu1-N4 & $152.23(11)$ & N2-Cu2-N8 & $157.56(12)$ \\
\hline N1-Cu1-O1 & $86.94(10)$ & N2-Cu2-O1 & $86.58(10)$ \\
\hline
\end{tabular}




\begin{tabular}{|l|l|l|l|}
\hline N4-Cu1-O1 & $90.97(11)$ & N8-Cu2-O1 & $93.66(11)$ \\
\hline N1-Cu1-N3 & $79.80(11)$ & N2-Cu2-N6 & $79.32(11)$ \\
\hline N4-Cu1-N3 & $95.87(11)$ & N8-Cu2-N6 & $95.68(11)$ \\
\hline O1-Cu1-N3 & $163.08(10)$ & O1-Cu2-N6 & $162.86(10)$ \\
\hline N1-Cu1-N5 & $102.99(11)$ & N2-Cu2-N7 & $103.42(11)$ \\
\hline N4-Cu1-N5 & $104.60(10)$ & N8-Cu2-N7 & $98.79(11)$ \\
\hline O1-Cu1-N5 & $100.83(10)$ & O1-Cu2-N7 & $97.66(10)$ \\
\hline N3-Cu1-N5 & $92.42(10)$ & N6-Cu2-N7 & $95.07(11)$ \\
\hline
\end{tabular}

Tabelle 8: Ausgewählte Bindungslängen $[\AA]]$ und -winkel $\left[{ }^{\circ}\right]$ von 10.

Im FAB-Massenspektrum von $\mathbf{1 0}$ kann die $\mathrm{OH}^{-}$-Brücke nicht detektiert werden, stärkster Peak bei $m / z=671$ gehört zur Spezies $\left[\mathrm{L}^{6} \mathrm{Cu}_{2}\right]^{+}$. Das UV/Vis-Spektrum in Dichlormethan zeigt die d-d-Bande bei $644 \mathrm{~nm}\left(\varepsilon=270 \mathrm{~mol} \mathrm{I}^{-1} \mathrm{~cm}^{-1}\right)$.

Aufgrund der nicht völlig auszuschließenden Hydrolyse von Acetonitril während der Komplexsynthese wurden weitere Dikupferkomplexe mit diesem Liganden in anderen Lösungsmitteln und mit anderen Gegenionen hergestellt. Zunächst wurde $\mathrm{HL}^{6}$ mit $\mathrm{Cu}\left(\mathrm{BF}_{4}\right)_{2} \cdot 6 \mathrm{H}_{2} \mathrm{O}$ und einer Hilfsbase umgesetzt. Der Komplex wurde in Methanol synthetisiert und durch Überschichten mit Diethylether kristallin gewonnen. Die Verbindung $\left[{ }^{6} \mathrm{Cu}_{2} \mathrm{~F}(\mathrm{MeOH})\right]\left(\mathrm{BF}_{4}\right)_{2}$ (11) kristallisiert in der Raumgruppe $P_{1}^{1}$ mit zwei Molekülen in der triklinen Zelle. Beide Kupferzentren sind durch verzerrt quadratisch pyramidal angeordnete Donoren umgeben $\left(\tau_{\mathrm{Cu} 1}=0.25, \tau_{\mathrm{Cu} 2}=0.23\right)$. Cu2 ist in apicaler Position durch ein Pyridin-N-Atom koordiniert, in basaler Position durch das andere Pyridin-N-Atom desselben Seitenarmes, das Atom N6, sowie das O1-Atom eines koordinierenden Methanolmoleküls. An der entsprechenden Stelle bindet im Falle von Cu1 ein Fluorid an, ansonsten ist die koordinative Umgebung dieses Metallzentrums identisch. Das Fluorid und Methanol-O1-Atom sind durch eine $\mathrm{H}$ Brücke miteinander verbunden $\left(d_{F 1 \ldots 01}=2.434(2) \AA\right)$. Der Abstand der Kupferzentren beträgt 4.430(1) $\AA$, auch hier werden die Kupferzentren durch die Zweitbrücke auseinandergedrückt, was auch hier wieder zu einer großen Verzerrung des Moleküls führt, der Torsionswinkel Cu1-N2-N1-Cu2 beträgt 43.6 . Die Anwesenheit von Fluorid als Ligand ist auch hier auf seine Abspaltung vom $\mathrm{BF}_{4}^{-}$-Ion zurückzuführen. Da wie schon in den bereits vorgestellten Beispielen in diesem Komplex das Fluorid nicht verbrückend wirkt, muß zum Erreichen einer ausreichenden Koordination beider Kupferzentren Methanol als weiterer Ligand 
eingebaut werden, wodurch die Kupferzentren auseinandergedrückt werden (Abbildung 25).

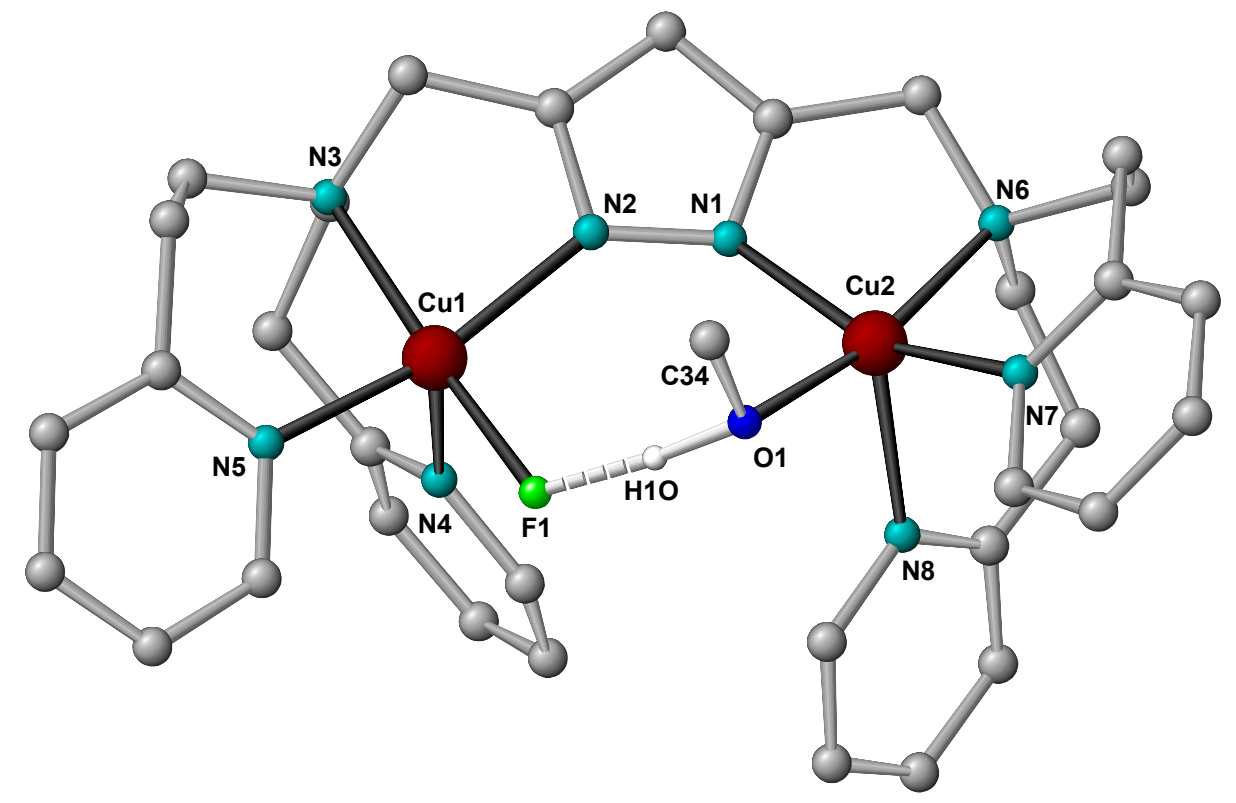

Abbildung 25: Festkörperstruktur des Kations von 11.

Im FAB-Massenspektrum von 11 läßt sich die Spezies $\left[\mathrm{L}^{6} \mathrm{Cu}_{2} \mathrm{~F}\right]^{+}$bei $\mathrm{m} / \mathrm{z}=690$ mit einer Intensität von $100 \%$, sowie das Fragment $\left[{ }^{6} \mathrm{Cu}_{2} \mathrm{~F}\left(\mathrm{BF}_{4}\right)\right]^{+}$bei $\mathrm{m} / \mathrm{z}=777$ mit $46 \%$ nachweisen. Das Fragment $\left[\mathrm{L}^{6} \mathrm{Cu}_{2}\right]^{+}$taucht bei $\mathrm{m} / \mathrm{z}=671$ mit einer Intensität von 52 \% auf. Die d-d-Bande des Komplexes in Methanollösung ist im UV/VisSpektrum bei $669 \mathrm{~nm}\left(\varepsilon=210 \mathrm{~mol} \mathrm{I}^{-1} \mathrm{~cm}^{-1}\right) \mathrm{zu}$ finden, im erwarteten Bereich der quadratisch pyramidal koordinierten Kupferzentren.

$\mid$\begin{tabular}{l|l|l|l|}
\hline Abstände \\
\hline Cu1-F1 & $1.9191(15)$ & Cu2-O1 & $1.9956(16)$ \\
\hline Cu1-N2 & $1.9969(18)$ & Cu2-N1 & $2.001(2)$ \\
\hline Cu1-N5 & $2.0265(19)$ & Cu2-N7 & $2.043(2)$ \\
\hline Cu1-N3 & $2.085(2)$ & Cu2-N6 & $2.0601(19)$ \\
\hline Cu1-N4 & $2.2470(19)$ & Cu2-N8 & $2.221(2)$ \\
\hline Cu1‥Cu2 & $4.4302(10)$ & O1‥F1 & $2.4343(21)$ \\
\hline Winkel \\
\hline F1-Cu1-N2 & $93.63(7)$ & O1-Cu2-N1 & $91.71(7)$ \\
\hline F1-Cu1-N5 & $87.28(7)$ & O1-Cu2-N7 & $86.98(7)$ \\
\hline N2-Cu1-N5 & $157.99(8)$ & N1-Cu2-N7 & $154.23(8)$ \\
\hline F1-Cu1-N3 & $173.03(7)$ & O1-Cu2-N6 & $167.70(7)$ \\
\hline N2-Cu1-N3 & $83.45(7)$ & N1-Cu2-N6 & $82.41(8)$ \\
\hline N5-Cu1-N3 & $93.06(8)$ & N7-Cu2-N6 & $93.62(8)$ \\
\hline
\end{tabular}




\begin{tabular}{|l|l|l|l|}
\hline F1-Cu1-N4 & $91.82(7)$ & O1-Cu2-N8 & $93.97(7)$ \\
\hline N2-Cu1-N4 & $105.51(7)$ & N1-Cu2-N8 & $107.75(8)$ \\
\hline N5-Cu1-N4 & $96.43(7)$ & N7-Cu2-N8 & $98.01(8)$ \\
\hline N3-Cu1-N4 & $95.06(7)$ & N6-Cu2-N8 & $98.10(8)$ \\
\hline
\end{tabular}

Tabelle 9: Ausgewählte Bindungslängen $[\AA]$ und -winkel $\left[^{\circ}\right]$ von 11.

Um - wie im Falle von 11 - unerwünschte Nebenreaktionen des gewählten Gegenions zu verhindern, wurde im Folgenden $\mathrm{Cu}\left(\mathrm{CF}_{3} \mathrm{SO}_{3}\right)_{2}$ („Kupfer(II)triflat“) als Edukt gewählt. Das Triflatanion gewährleistet eine gute Löslichkeit der hergestellten Dikupferkomplexe in Methanol und auch weniger protischen Lösungsmitteln. Zunächst wurde der Ligand $\mathrm{HL}^{6}$ mit zwei Äquivalenten Kalium-tert-butylat und zwei Äquivalenten Kupfer(II)triflat in Dichlormethan umgesetzt. Durch Etherdiffusion erhält man das Produkt $\left[\mathrm{L}^{6} \mathrm{Cu}_{2} \mathrm{Cl}\right]\left(\mathrm{CF}_{3} \mathrm{SO}_{3}\right)_{2}$ (12) in kristalliner Form in der Raumgruppe $P 2_{1} / c$ mit vier Molekülen in der monoklinen Zelle. Überraschend ist zunächst der Fund eines Dikupfer(II)komplexes mit einem verbrückenden Chlorid zwischen den Kupferzentren. Allerdings entsteht aus Dichlormethan durch Lichteinwirkung in Spuren $\mathrm{HCl}$, so daß nach Protonenabstraktion das entstehende Chlorid in den Komplex eingebaut werden kann. Zum anderen sind Dikupfer(II)komplexe von $\mathrm{HL}^{6}$ offensichtlich selbst in der Lage, eine Halogenidabstraktion von halogenhaltigen Substratmolekülen zu bewirken (siehe Kapitel 7). Die Koordinationsgeometrie beider Kupferzentren im Dikupferkomplex ist trotz der Symmetrie des eingesetzten Liganden sehr unterschiedlich: Cu2 befindet sich in einer fast ideal quadratisch pyramidalen Koordinationsumgebung mit dem apicalen Pyridin-N5-Atom und den basalen Atomen Pyridin-N6, N7, dem Pyrazol-N3 und dem verbrückenden Chlorid $\left(\tau_{\text {Cu2 }}=0.01\right.$ ). Dagegen weist Cu1 mit $\tau=0.47$ eine Koordination auf, welche zwischen einer quadratisch pyramidalen und einer trigonal bipyramidalen Koordination liegt (Abbildung 26). 


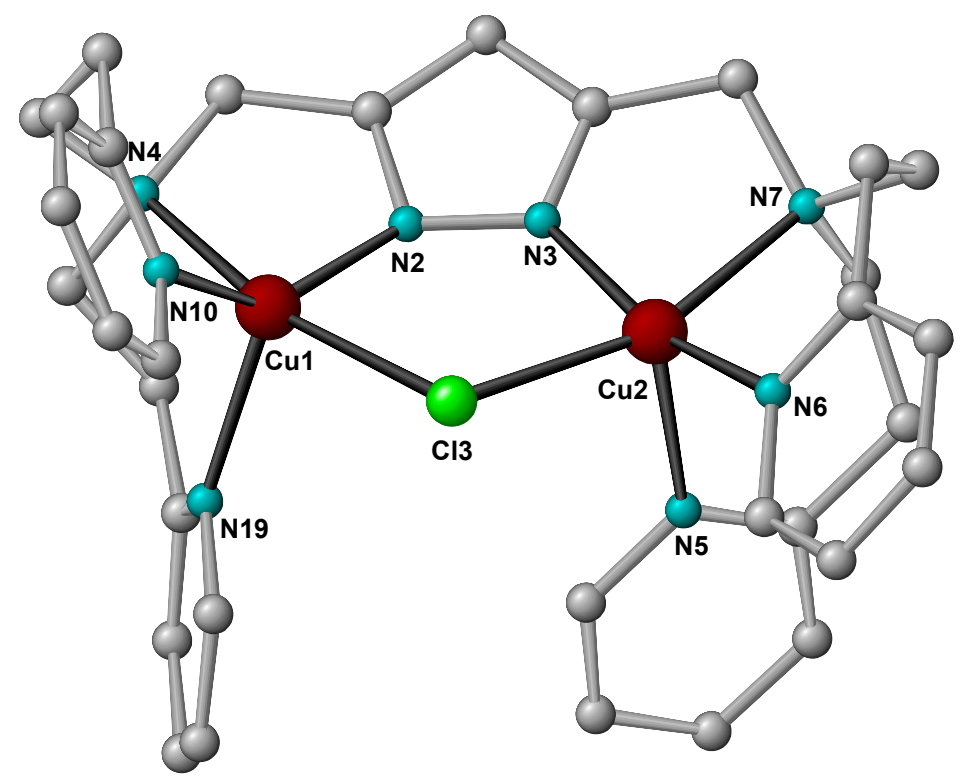

Abbildung 26: Festkörperstruktur des Kations von 12.

Zu dieser Anordnung könnte es zum einen durch Packungseffekte kommen. Zum anderen können auch die sterischen Ansprüche des Liganden einerseits und des großen Chloridions andererseits eine Rolle spielen. Abbildung 27 zeigt, daß sich die Kovalenzradien des Chloridions und der H-Atome der vier Pyridinreste berühren, so daß diese aus energetisch ansonsten günstigeren Positionen herausgedrückt werden. Bei der kleineren Zweitbrücke $\mathrm{OH}^{-}$hat der Ligand mehr Spielraum. Im Vergleich zum $\mathrm{OH}^{-}$-verbrückten Dikupferkomplex ist der Kupfer-Kupferabstand mit 3.829(2) $\AA$ auch deutlich größer, während die Abstände der Kupferzentren zu den Liganddonoren im üblichen Rahmen liegen. Dieses Beispiel belegt die große koordinative Flexibilität des Pyrazolatligandgerüstes.
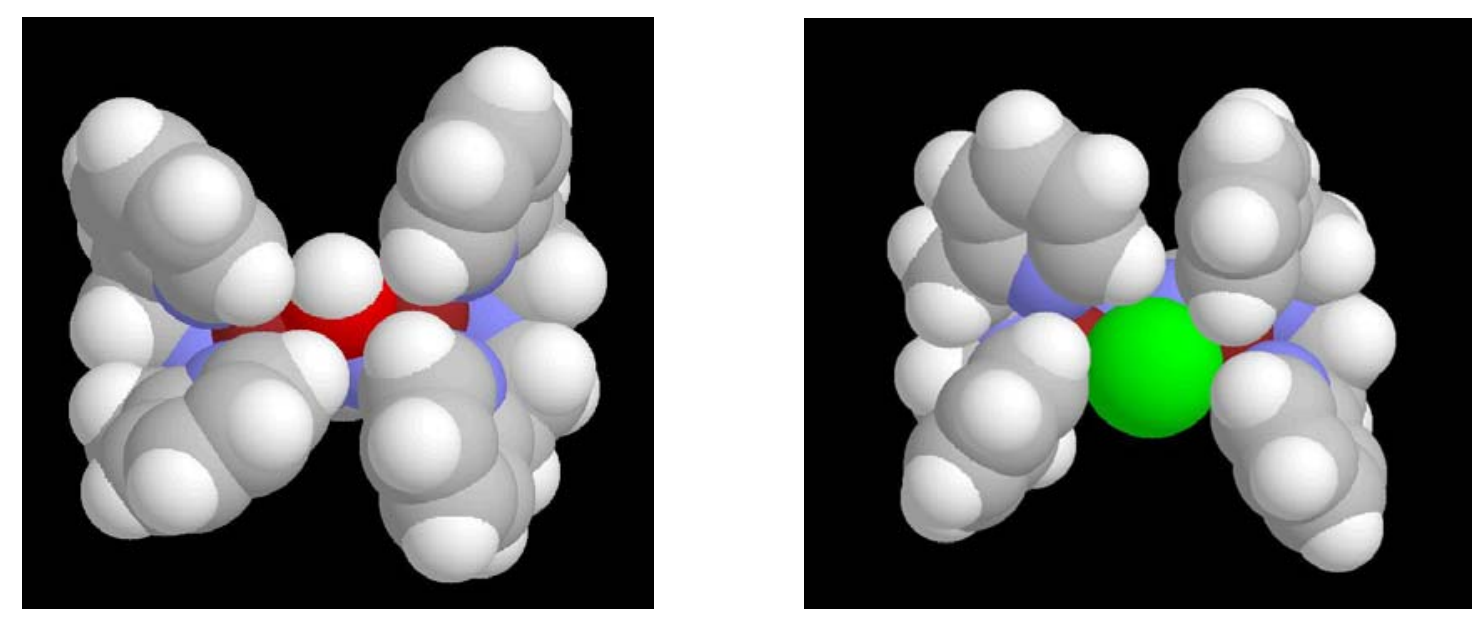

Abbildung 27: Raumerfüllende Darstellung der Kationen von 10 (links) und 12 (rechts). 
Im ESI-Massenspektrum der Methanollösung von 12 ist bei $\mathrm{m} / \mathrm{z}=855$ mit einer Intensität von $35 \%$ das Fragment $\left[\mathrm{L}^{6} \mathrm{Cu}_{2} \mathrm{Cl}\left(\mathrm{CF}_{3} \mathrm{SO}_{3}\right)\right]^{+} \mathrm{zu}$ sehen, wesentlich stärker als die Peaks der chloridfreien Fragmente bei $\mathrm{m} / \mathrm{z}=671$ mit einer Intensität von $9 \%$ und 767 mit $15 \%$, die sich den Spezies $\left.\left[\mathrm{L}^{6} \mathrm{Cu}_{2}\right]^{+}\right]$bzw. $\left[\mathrm{L}^{6} \mathrm{Cu}_{2}(\mathrm{MeOH})_{3}\right]^{+}$zuordnen lassen. Im UV/Vis-Spektrum in Methanol findet man eine d-d-Bande bei $652 \mathrm{~nm}$ $\left(\varepsilon=270 \mathrm{~mol} \mathrm{I}^{-1} \mathrm{~cm}^{-1}\right)$. Durch die starke Anbindung von Chlorid wird das zweikernige Kupferzentrum hinsichtlich der Anbindung von Substraten im allgemeinen blockiert und die katalytische Aktivität der entsprechenden Komplexe enorm eingeschränkt. So weiß man z.B., daß das Enyzm Catechol-Oxidase von Chlorid inhibiert wird. ${ }^{[124,125]}$

\begin{tabular}{|l|l|l|l|}
\hline \multicolumn{4}{|l|}{ Abstände } \\
\hline Cu1-N2 & $1.932(3)$ & Cu2-N3 & $1.900(3)$ \\
\hline Cu1-N10 & $2.044(3)$ & Cu2-N6 & $1.980(3)$ \\
\hline Cu1-N4 & $2.115(3)$ & Cu2-N7 & $2.158(4)$ \\
\hline Cu1-N19 & $2.179(4)$ & Cu2-N5 & $2.207(4)$ \\
\hline Cu1-Cl3 & $2.4398(12)$ & Cu2-Cl3 & $2.4120(14)$ \\
\hline Cu1-Cu2 & $3.8294(15)$ & & \\
\hline Winkel & \multicolumn{3}{|l|}{} \\
\hline N2-Cu1-N10 & $139.38(13)$ & N3-Cu2-N6 & $160.56(14)$ \\
\hline N2-Cu1-N4 & $81.48(13)$ & N3-Cu2-N7 & $79.99(15)$ \\
\hline N10-Cu1-N4 & $95.97(13)$ & N6-Cu2-N7 & $95.03(15)$ \\
\hline N2-Cu1-N19 & $111.58(14)$ & N3-Cu2-N5 & $102.01(14)$ \\
\hline N10-Cu1-N19 & $109.04(14)$ & N6-Cu2-N5 & $97.22(14)$ \\
\hline N4-Cu1-N19 & $94.10(14)$ & N7-Cu2-N5 & $96.82(15)$ \\
\hline N2-Cu1-Cl3 & $86.93(10)$ & N3-Cu2-Cl3 & $87.18(11)$ \\
\hline N10-Cu1-Cl3 & $89.45(10)$ & N6-Cu2-Cl3 & $92.63(11)$ \\
\hline N4-Cu1-Cl3 & $167.31(10)$ & N7-Cu2-Cl3 & $161.37(12)$ \\
\hline N19-Cu1-Cl3 & $94.96(10)$ & N5-Cu2-Cl3 & $99.04(10)$ \\
\hline
\end{tabular}

Tabelle 10: Ausgewählte Bindungslängen $[\AA]$ und -winkel $\left[^{\circ}\right]$ von 12.

Bei Umsetzung von Kupfer(I)triflat mit $\left[\mathrm{L}^{6}\right]^{-}$in Dichlormethan bei tiefer Temperatur und anschließender Luftzugabe konnte Komplex $\left[\mathrm{L}^{6} \mathrm{Cu}_{2}(\mathrm{OH})\right]\left(\mathrm{CF}_{3} \mathrm{SO}_{3}\right)_{2}$ (13) durch anschließende Etherdiffusion in kristalliner, allerdings unreiner, Form erhalten werden. Glücklicherweise läßt sich dieser Komplex in wesenlich größerer Reinheit aber auch direkt über das Kupfer(II)triflat in Methanol herstellen und anschließend durch Pentandiffusion aus Aceton in kristalliner Form erhalten. Die Struktur von 13 entspricht - abgesehen vom verwendeten Anion - im wesentlichen der des 
Komplexes 10. Er kristallisiert in der $P n a 2_{1}$ Raumgruppe im orthorhombischen Kristallsystem. Pro Elementarzelle finden sich acht Moleküle, die aus vier Paaren zweier fast identischer Komplexeinheiten bestehen. Man findet eine verzerrt quadratisch pyramidale Koordination an den zwei Kupferzentren: $\tau_{\text {Cu21 }}=0.13$ [0.15] und $\tau_{\text {Cu22 }}=0.22[0.19]$. Die Werte in Klammern entsprechen hierbei jenen der zweiten unabhängigen Moleküleinheit. Koordiniert sind die Kupferzentren dabei in der basalen Ebene von einem Pyrazol-N-Atom, dem O-Atom der Hydroxidbrücke, einem Pyridin-N-Atom und dem N-Atom aus dem aliphatischen Teil der Seitenkette. In apicaler Position bindet in etwas größerer Entfernung von ca. $2.20-2.25 \AA$ das zweite Pyridin-N-Atom an. Über eine $\mathrm{H}$-Brücke ist ein O-Atom des Triflat-Anions an das H1O- und das H2-Atom gebunden $\left(d_{0201 \ldots 041}=2.955(5)\right.$ [3.127(5)] $\AA$ ) (Abbildung 28). Wichtig ist hier, daß der Abstand der Kupferzentren mit $d_{\mathrm{Cu} \cdots \mathrm{Cu}}=3.515(5)$ [3.518(5)] $\AA$ in einem Bereich liegt, der nicht sehr weit entfernt liegt von dem bei der oxidierten Form der Catechol-Oxidase gefundenen (2.9 A). Der Komplex könnte sich somit als besonders interessant hinsichtlich seiner katalytischen Aktivität erweisen.

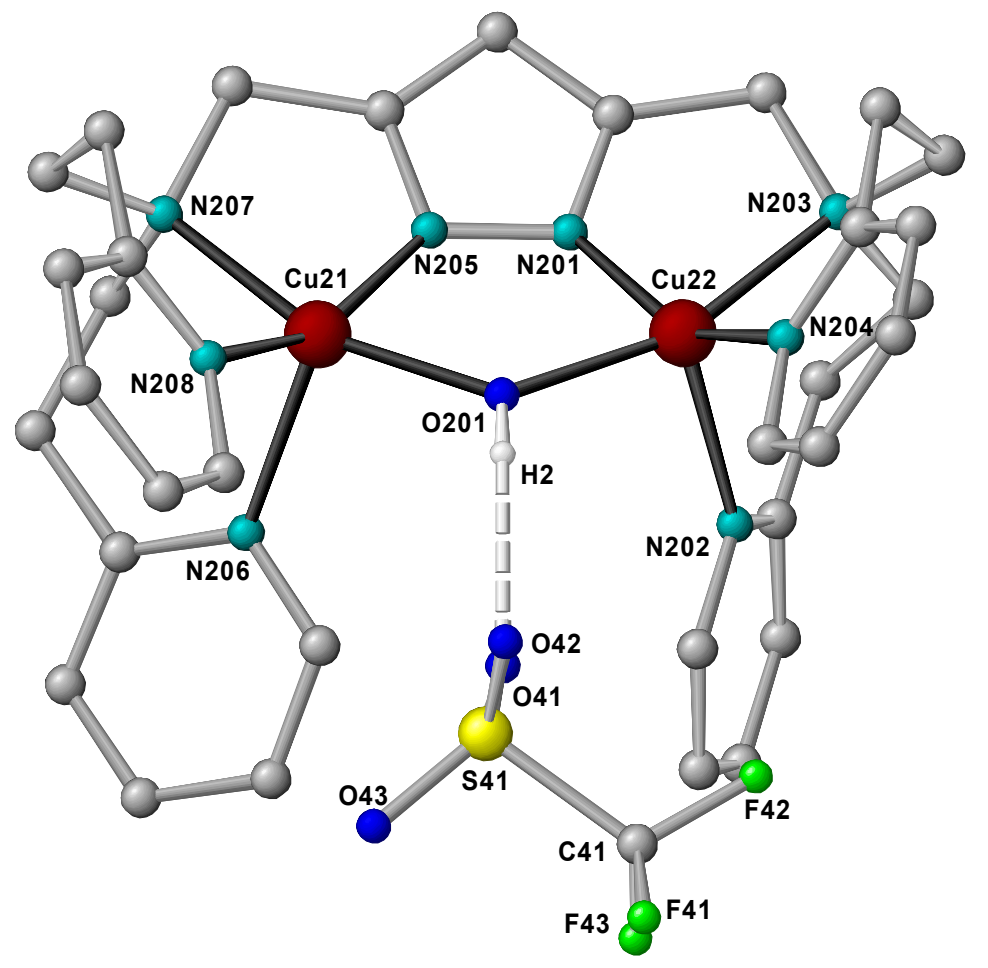

Abbildung 28: Festkörperstruktur eines Kations von 13. 


\begin{tabular}{|c|c|c|c|c|c|}
\hline \multicolumn{6}{|l|}{ Abstände } \\
\hline Cu21-N205 & $1.912(4)$ & {$[1.905(3)]$} & Cu22-N201 & $1.913(4)$ & {$[1.921(4)]$} \\
\hline Cu21-N208 & $2.016(3)$ & {$[2.007(3)]$} & Cu22-N204 & $2.010(4)$ & {$[1.999(4)]$} \\
\hline Cu21-O201 & $2.003(4)$ & {$[2.002(4)]$} & Cu22-O201 & $2.000(3)$ & {$[2.002(3)]$} \\
\hline Cu21-N207 & $2.120(4)$ & {$[2.141(4)]$} & Cu22-N203 & $2.161(4)$ & {$[2.132(3)]$} \\
\hline Cu21-N206 & $2.222(4)$ & {$[2.248(4)]$} & Cu22-N202 & $2.224(4)$ & {$[2.201(4)]$} \\
\hline $\mathrm{O} 41 \cdots \mathrm{O} 201$ & $2.955(5)$ & {$[3.127(5)]$} & Cu21… & $3.515(5)$ & {$[3.518(5)]$} \\
\hline \multicolumn{6}{|l|}{ Winkel } \\
\hline N205-Cu21-N208 & $153.45(14)$ & [152.15(13)] & N201-Cu22-N204 & $148.36(13)$ & {$[150.20(13)]$} \\
\hline N205-Cu21-O201 & $83.74(13)$ & {$[84.04(13)]$} & N201-Cu22-O201 & $84.45(16)$ & {$[83.74(15)]$} \\
\hline N208-Cu21-O201 & $96.96(15)$ & {$[95.85(15)]$} & N204-Cu22-O201 & $94.79(14)$ & {$[96.25(14)]$} \\
\hline N205-Cu21-N207 & $79.03(15)$ & {$[78.23(14)]$} & N201-Cu22-N203 & $79.03(15)$ & {$[79.36(15)]$} \\
\hline N208-Cu21-N207 & $94.96(15)$ & {$[96.59(15)]$} & N204-Cu22-N203 & $95.15(14)$ & {$[94.93(14)]$} \\
\hline O201-Cu21-N207 & $161.03(13)$ & {$[160.94(12)]$} & O201-Cu22-N203 & $161.69(12)$ & [161.68(12)] \\
\hline N205-Cu21-N206 & $111.80(15)$ & {$[113.91(14)]$} & N201-Cu22-N202 & $102.89(16)$ & [101.82(16)] \\
\hline N208-Cu21-N206 & $94.30(16)$ & {$[93.51(15)]$} & N204-Cu22-N202 & $108.48(17)$ & [107.77(17)] \\
\hline O201-Cu21-N206 & $99.79(13)$ & {$[101.09(13)]$} & O201-Cu22-N202 & $98.51(13)$ & {$[96.48(13)]$} \\
\hline N207-Cu21-N206 & $93.95(14)$ & {$[92.50(13)]$} & N203-Cu22-N202 & $92.86(13)$ & {$[93.86(13)]$} \\
\hline
\end{tabular}

Tabelle 11: Ausgewählte Bindungslängen $[\AA]$ und -winkel $\left[{ }^{\circ}\right]$ von 13, Werte für das zweite unabhängige Molekül sind in Klammern angegeben.

Der stärkste Peak im FAB-Massenspektrum von 13 gehört zum Fragment $\left[\mathrm{L}^{6} \mathrm{Cu}_{2}\right]^{+}$ bei $m / z=671$, aber mit geringer Intensität von nur $2 \%$ taucht auch bei $m / z=986$ die Spezies $\left[\mathrm{L}^{6} \mathrm{Cu}_{2}(\mathrm{OH})\left(\mathrm{CF}_{3} \mathrm{SO}_{3}\right)_{2}\right]^{+}$auf. Das UV/Vis-Spektrum in Methanol entspricht den Spektren quadratisch pyramidal koordinierter Kupferverbindungen mit einem d-dÜbergang bei $648 \mathrm{~nm}\left(\varepsilon=250 \mathrm{~mol} \mathrm{I}^{-1} \mathrm{~cm}^{-1}\right)$.

\subsection{Komplexe mit dem Liganden $\mathrm{HL}^{7}$}

Der Ligand $\mathrm{HL}^{7}$ lieferte im Rahmen dieser Arbeit trotz vielfältiger Versuche keine reinen Produkte, es wurden ausschließlich Komplexe in Form von Ölen oder Pulvern erhalten. Nachweisen ließen sich erhaltene Dikupferkomplexe dieses Liganden nur massenspektrometrisch. Setzt man z.B. $\mathrm{HL}^{7}$ in Methanol mit zwei Äquivalenten $\mathrm{Cu}\left(\mathrm{NO}_{3}\right)_{2} \cdot 3 \mathrm{H}_{2} \mathrm{O}$ sowie zwei Äquivalenten Kalium-tert-butylat um, so erhält man nach Etherdiffusion ein Pulver, in dessen FAB-Massenspektrum die Spezies $\left[\mathrm{L}^{7} \mathrm{Cu}_{2}\right]^{+}$bei $\mathrm{m} / \mathrm{z}=643$ mit einer Intensität von $22 \%$ enthalten ist, ebenso wie die Spezies $\left[\mathrm{L}^{7} \mathrm{Cu}_{2}\left(\mathrm{NO}_{3}\right)\right]^{+}$bei $\mathrm{m} / \mathrm{z}=705$ (19\%) und $\left[\mathrm{L}^{7} \mathrm{Cu}_{2}\left(\mathrm{NO}_{3}\right)_{2}\right]^{+}$bei $\mathrm{m} / \mathrm{z}=767(8 \%)$. Jedoch lassen sich aufgrund dieser Befunde keine verläßlichen Aussagen über die Konstitution der erhaltenen Koordinationsverbindung machen. 


\subsection{Komplexe mit dem Liganden $\mathrm{HL}^{8}$}

Der Ligand $\mathrm{HL}^{8}$ mit kurzen Ligandseitenarmen wurde ebenfalls mit verschiedenen Kupfer(II)salzen umgesetzt. Durch Deprotonierung des Pyrazols mittels der Base Kalium-tert-butylat und anschließendes Umsetzen mit $\mathrm{Cu}\left(\mathrm{BF}_{4}\right)_{2} \cdot 6 \mathrm{H}_{2} \mathrm{O}$ in Methanol entstand der Dikupferkomplex $\left[\mathrm{L}^{8} \mathrm{Cu}_{2} \mathrm{~F}(\mathrm{MeOH})\right]\left(\mathrm{BF}_{4}\right)_{2} \quad$ (14), welcher durch Etherdiffusion in kristalliner Form ausgefällt wurde und röntgenographisch charakterisiert werden konnte. Der Komplex kristallisiert in der Raumgruppe $P 2_{1} / c$ mit vier Molekülen in der monoklinen Zelle. Man findet hier eine trigonal bipyramidale Koordinationsumgebung der Kupferzentren mit $\tau_{\mathrm{Cu} 1}=0.92$ und $\tau_{\mathrm{Cu} 2}=0.91$. Cu1 hat in den apicalen Positionen auf einer Seite ein Fluorid, welches offensichtlich wieder durch Abspaltung vom $\mathrm{BF}_{4}$-lon entstanden ist, und das aliphatische $\mathrm{N}$-Atom eines der beiden Chelatseitenarme des Liganden. Basal koordinieren die aromatischen $\mathrm{N}$ Atome (Pyrazol-N, zweimal Pyridin-N). Im Falle von Cu2 wird der Platz des Fluorids vom O1-Atom eines koordinierenden Methanolmoleküles eingenommen. Zwischen $\mathrm{F} 1$ und $\mathrm{O} 1$ ist aufgrund des geringen Abstandes von $d_{\mathrm{O} 1 \ldots \mathrm{F} 1}=2.384(6) \AA$ eine $\mathrm{H}$ Brücke wahrscheinlich (Abbildung 29).

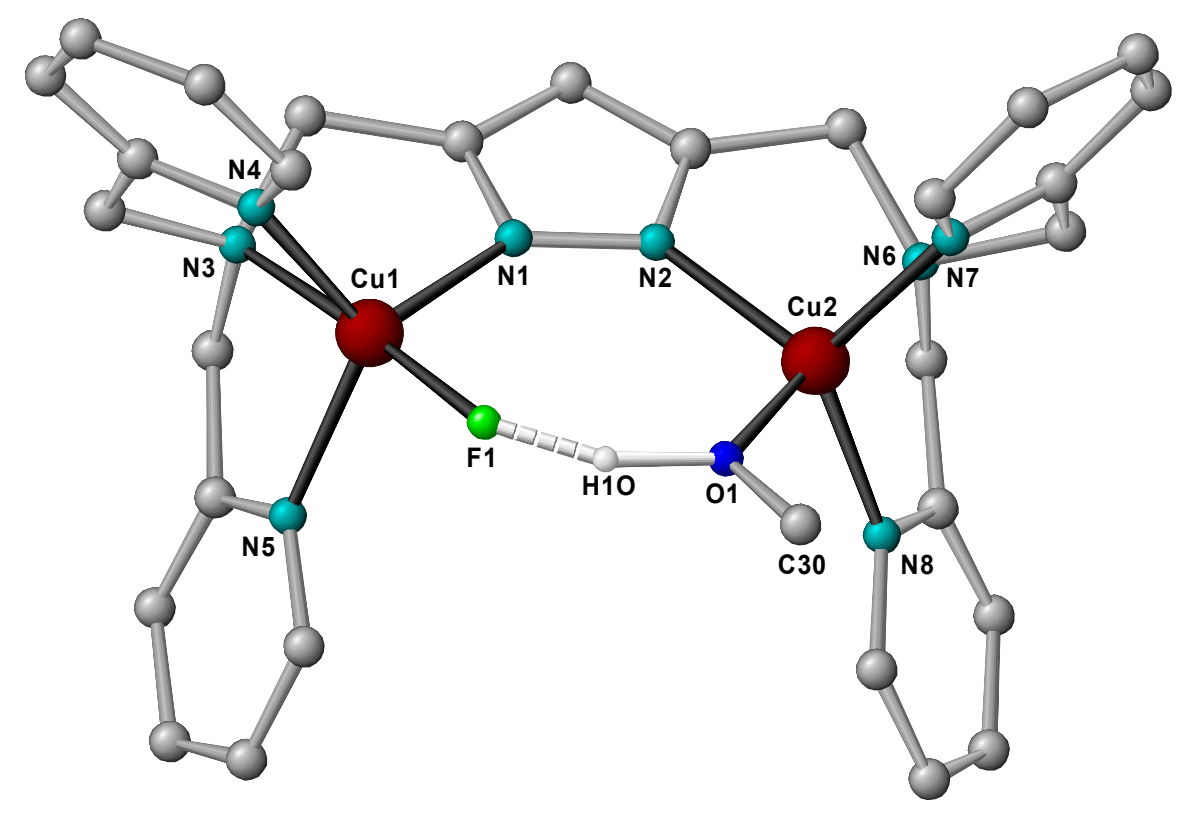

Abbildung 29: Festkörperstruktur des Kations von 14.

Die trigonal bipyramidale Koordination ist durch die sterischen Zwänge des Liganden gegeben. Bei dem bereits zuvor hergestellten Dikupferkomplex 4 mit aliphatischen Seitenarmen gleicher Länge und identischer Anzahl von Donoratomen wurde die 
gleiche Art der Koordination ebenfalls gefunden. ${ }^{[99]}$ Auch einfache Kupfer(II)komplexe mit dem Tris(methylpyridylamin)-Liganden (TMPA) weisen bevorzugt trigonal bipyramidale Koordinationsgeometrien auf. ${ }^{[30,126,127,128,129,130]}$

Der Abstand der Kupferzentren in 14 ist mit 4.291(1) A recht groß, vergleichbar mit $d_{\mathrm{Cu} \cdots \mathrm{Cu}}=4.533 \AA$ bei Komplex 4. Die Abstände der Ligand-N-Donoren von den Kupferzentren sind mit ca. $2.00-2.08 \AA$ allesamt fast gleich lang und relativ klein. Dies und der Torsionswinkel Cu1-N1-N2-Cu2 von nur $8.5^{\circ}$ sprechen für eine entspannte Konformation des Liganden.

\begin{tabular}{|c|c|c|c|}
\hline \multicolumn{4}{|l|}{ Abstände } \\
\hline Cu1-F1 & $1.911(4)$ & Cu2-O1 & $1.900(4)$ \\
\hline Cu1-N1 & $2.005(5)$ & Cu2-N2 & $2.004(5)$ \\
\hline Cu1-N3 & $2.040(5)$ & Cu2-N8 & $2.027(5)$ \\
\hline Cu1-N4 & $2.042(5)$ & Cu2-N6 & $2.042(5)$ \\
\hline Cu1-N5 & $2.050(5)$ & Cu2-N7 & $2.079(5)$ \\
\hline Cu1‥Cu2 & 4.291(1) & F1…01 & $2.384(6)$ \\
\hline \multicolumn{4}{|l|}{ Winkel } \\
\hline F1-Cu1-N1 & 102.18(19) & O1-Cu2-N2 & $100.89(19)$ \\
\hline F1-Cu1-N3 & $176.5(2)$ & O1-Cu2-N8 & $96.2(2)$ \\
\hline N1-Cu1-N3 & $81.26(19)$ & N2-Cu2-N8 & 122.00(18) \\
\hline F1-Cu1-N4 & $97.6(2)$ & O1-Cu2-N6 & 177.08(19) \\
\hline N1-Cu1-N4 & 117.0(2) & N2-Cu2-N6 & 82.04(19) \\
\hline N3-Cu1-N4 & $81.3(2)$ & N8-Cu2-N6 & 82.16(19) \\
\hline F1-Cu1-N5 & $95.6(2)$ & O1-Cu2-N7 & $98.2(2)$ \\
\hline N1-Cu1-N5 & 115.08(19) & N2-Cu2-N7 & 109.38(18) \\
\hline N3-Cu1-N5 & $82.2(2)$ & N8-Cu2-N7 & $122.40(19)$ \\
\hline N4-Cu1-N5 & 121.6(2) & N6-Cu2-N7 & $80.8(2)$ \\
\hline
\end{tabular}

Tabelle 12: Ausgewählte Bindungslängen $[\AA]$ und -winkel $\left[{ }^{\circ}\right]$ von 14.

Im FAB-Massenspektrum von 14 zeigt sich ein mit einer Intensität von $24 \%$ recht starkes Signal für $\left[\mathrm{L}^{8} \mathrm{Cu} \mathrm{H}_{2} \mathrm{~F}\right]^{+}$, sowie ein Peak für $\left[\mathrm{L}^{8} \mathrm{Cu}_{2} \mathrm{~F}\left(\mathrm{BF}_{4}\right)\right]^{+}$mit $7 \%$ bei $\mathrm{m} / \mathrm{z}=634$ bzw. 721. Das UVNVis-Spektrum des Komplexes in Methanol ist deutlich verschieden von jenem der quadratisch pyramidal koordinierten Kupferkomplexe. Bei $900 \mathrm{~nm}$ $\left(\varepsilon=280 \mathrm{~mol} \mathrm{I}^{-1} \mathrm{~cm}^{-1}\right.$ ) liegt ein $\mathrm{d}$-d-Übergang vor, der einem trigonal bipyramidal koordinierten Kupferzentrum entspricht. Zugleich findet man aber bei wesentlich kleineren Wellenlängen eine Bande bei $691 \mathrm{~nm}$ (Schulter, $\varepsilon=190 \mathrm{~mol} \mathrm{I}^{-1} \mathrm{~cm}^{-1}$ ), die auf eine quadratisch planare Koordination hindeutet. In Lösung könnten daher möglicherweise zwei Spezies mit unterschiedlicher Koordinationsgeometrie im 
Gleichgewicht vorliegen.

\subsection{Vergleich spektroskopischer und struktureller Eigenschaften der Dikupfer(II)pyrazolatkomplexe}

Die wichtigsten strukturellen und UV/Vis-spektroskopischen Ergebnisse der vorgestellten Komplexe sind in Tabelle 13 zusammengefaßt. Wie aus dem Vergleich zwischen $\lambda_{\max }$ der jeweiligen d-d-Übergänge der Komplexe in Lösung einerseits und der $\tau$-Werte der Kupferzentren im Festkörper andererseits ersichtlich ist, bleibt die Koordinationsgeometrie der Kupferzentren in Lösung im Wesentlichen erhalten.

\begin{tabular}{|llll|}
\hline Komplex & $d_{\mathrm{Cu} \cdots \mathrm{Cu}}[\AA]$ & $\tau$ & $\lambda\left(\mathrm{max}[\mathrm{nm}]\left(\varepsilon\left[\mathrm{mol} \mathrm{I}^{-1} \mathrm{~cm}^{-1}\right]\right)\right.$ \\
\hline $\mathbf{1}$ & $3.540(1)$ & $0.23 / 0.34$ & $621(210)$ \\
$\mathbf{3}$ & $3.447(2)$ & $0.38 / 0.35$ & $589(270)$ \\
4 & $4.088(1)$ & $0.21 / 0.09$ & $638(280)$ \\
$\mathbf{5}$ & $4.533(1)$ & $0.64 / 0.64$ & $855(530)$ \\
6 & $4.001(1), 4.279$ & $0.12 / 0.09 / 0.23 / 0.24$ & $614(450)$ \\
$7 \mathbf{a}$ & $4.045(1)$ & $0.05 / 0.16$ & $631(440)$ \\
8 & $4.290(1)$ & $0.01 / 0.09$ & $644(200)$ \\
9 & $4.357(1)$ & $0.38 / 0.02$ & $635(290)$ \\
10 & $4.268(1)$ & $0.07 / 0.15$ & $646(280)$ \\
11 & $3.441(1)$ & $0.09 / 0.18$ & $644(270)$ \\
12 & $4.430(1)$ & $0.25 / 0.23$ & $669(210)$ \\
13 & $3.829(2)$ & $0.47 / 0.01$ & $652(270)$ \\
14 & $3.515(5)[3.518(5)]$ & $0.13[0.15] / 0.22[0.19]$ & $638(290)$ \\
\hline
\end{tabular}

Tabelle 13: Strukturelle und UVNVis Daten (d-d-Übergänge) der Komplexe 1 - 14; die Daten für 1 - 4 stammen aus früheren Arbeiten. ${ }^{[100]}$

Die Komplexe 4 und 14, bei welchen im Festkörper eine trigonal bipyramidale Geometrie beobachtet wird, zeigen in Lösung ein $\lambda_{\max }$ im für ebendiese Koordinationsgeometrie typischen Bereich. Die anderen - im festen Zustand quadratisch pyramidal koordinierten - Dikupfer(II)komplexe zeigen in Lösung Banden, die auf eine quadratisch pyramidale Koordinationsgeometrie hinweisen. ${ }^{[14]}$ 
Diese Beobachtung legt nahe, daß eine ausreichende Ähnlichkeit der Strukturen der Komplexe in Lösung und im festen Zustand gegeben ist, so daß eine spätere Korrelation kinetischer Daten der gelösten Komplexe mit ihren - aus der jeweiligen Festkörperstruktur gewonnenen - strukturellen Parametern möglich wird. 


\section{Kinetische Untersuchungen zur Catecholase-Aktivität}

In diesem Kapitel werden die pH-Abhängigkeit, Katalysatorabhängigkeit und Substratabhängigkeit der Catecholase-Aktivität von acht verschiedenen Dikupfer(II)pyrazolatkomplexen bestimmt und Zusammenhänge mit ihren strukturellen und elektronischen Eigenschaften untersucht. Zudem wird zum Vergleich die katalytische Aktivität eines mononuklearen Kupferkomplexes bestimmt. Desweiteren wird die Detektion des Nebenproduktes der Catecholase-Reaktion beschrieben.

\subsection{Allgemeines}

Es wurden acht verschiedene Dikupfer(II)komplexe auf ihre Fähigkeit hin untersucht, die Oxidation eines Catechols durch molekularen Sauerstoff zu vermitteln. Obwohl eine Vielzahl verschiedener Catechole zur Verfügung steht und auch einige davon für diesen Zweck Verwendung gefunden haben, ${ }^{[75,77,79,80,82]}$ so bietet sich doch insbesondere das 3,5-Di-tert-butylcatechol $\left(\mathrm{H}_{2} \mathrm{dtbc}\right)$ als Testsubstrat der Catecholasereaktion an, ${ }^{[66,73,74,78,81,131,132,133]}$ da es durch sein niedriges Redoxpotential von -1.19 $\mathrm{V}^{[134]}$ leicht zum Di-tert-butyl-o-chinon (dtboc) zu oxidieren ist (Schema 15).<smiles>CC(C)(C)C1=CC(=O)C(=O)C(Br)=C1</smiles>

Schema 15: Oxidation von $\mathrm{H}_{2} d t b c$ zu dtboc.

Seine sterisch anspruchsvollen tert-Butylgruppen verhindern unerwünschte Nebenreaktionen wie z.B. die Ringöffnung, wie sie in der Natur von den eisenhaltigen Catechol-Dioxygenasen bzw. deren Modellverbindungen bekannt ist. ${ }^{[135]}$ 
Zur Analyse der katalytischen Aktivität wird die Methode der Anfangsgeschwindigkeiten verwendet (initial rate method). ${ }^{[136,137]}$

Ist eine Reaktion gegeben durch:

(1) $x_{1} \mathrm{~A}+x_{2} \mathrm{~B} \rightarrow x_{3} \mathrm{C}$

so gilt für die Reaktionsgeschwindigkeit in Bezug auf das Produkt C:

(2) $v=\frac{\mathrm{d}(\mathrm{C})}{\mathrm{d}(\mathrm{t})}=k \cdot[\mathrm{A}]^{a} \cdot[\mathrm{B}]^{b}$.

Bevor man den Betrag des Geschwindigkeitskoeffizienten $k$ der Reaktion ermitteln kann, muß man die Exponenten $a$ und $b$ der Gleichung (2) kennen. Handelt es sich bei der Reaktion nicht um einen Elementarprozeß - d.h. läuft sie in mehreren Schritten ab - so entsprechen $a$ und $b$ nicht den stöchiometrischen Koeffizienten $x_{1}$ und $x_{2}$. Zur experimentellen Ermittlung von a geht man folgendermaßen vor:

In einer Meßreihe hält man die Anfangskonzentration $[B]_{0}$ konstant und variiert $[A]_{0}$, so daß gilt:

(3) $v_{0}=k[\mathrm{~A}]_{0}{ }^{a} \cdot$ const. $=k_{\exp } \cdot[\mathrm{A}]_{0}{ }^{a}$.

und

(4) $\lg \left(v_{0}\right)=\lg \left(k_{\exp }\right)+a \cdot \lg \left([\mathrm{A}]_{0}\right)$.

Trägt man $\lg \left(v_{0}\right)$ gegen $\lg \left([\mathrm{A}]_{0}\right)$ auf, so erhält man als Steigung der erhaltenen Geraden die Reaktionsordnung a bezüglich der Substanz A und aus dem yAchsenabschnitt die Geschwindigkeitskonstante $k_{\text {exp }}$. Entsprechend geht man mit B vor.

Bei Verwendung von $\mathrm{H}_{2} \mathrm{dtbc}$ als Substrat wird die Entstehung des gelben dtboc UV/Vis-spektroskopisch anhand der starken Absorptionsbande bei $\lambda_{\max }=400 \mathrm{~nm}$ $\left(\varepsilon=1810 \mathrm{M}^{-1} \mathrm{~cm}^{-1}\right)$ verfolgt.

Laut Lambert-Beer'schen Gesetz gilt hierbei:

(5) $A=\varepsilon \cdot[\mathrm{dtboc}] \cdot d$

wobei $A$ die erhaltene Absorption des Produktes ist, $\varepsilon$ der molare Extinktionskoeffizient (in $\mathrm{I} \mathrm{mol}^{-1} \mathrm{~cm}^{-1}$ ) und $d$ die Schichtdicke der Probe (in $\mathrm{cm}$ ), d.h. der Küvettendurchmesser.

Die Konzentration des o-Chinons zu einem beliebigen Zeitpunkt ergibt sich dann durch: 
(6) $[\mathrm{dtboc}]=A \cdot \varepsilon^{-1} \cdot d^{-1}$.

Das vereinfachte Geschwindigkeitsgesetz der Catecholasereaktion lautet dann:

(7) $v=\frac{\mathrm{d}\left(A \cdot \varepsilon^{-1} \cdot d^{-1}\right)}{\mathrm{d} t}=\frac{\mathrm{d}([\mathrm{dtboc}])}{\mathrm{d} t}=k^{\prime} \cdot[\mathrm{Kat}]^{\mathrm{x}} \cdot\left[\mathrm{H}_{2} \mathrm{dtbc}\right]^{\mathrm{y}}$,

wobei die Konzentration des zur Oxidation benötigten molekularen Sauerstoffs durch Rühren der Probe an Luftatmosphäre während der Reaktion auf konstantem Niveau gehalten wird und in $k^{\prime}$ beinhaltet ist.

In zwei Meßreihen wird zum einen die Anfangskonzentration des Substrats $\left[\mathrm{H}_{2} \mathrm{dtbc}\right]_{0}$ konstant gehalten und jene des Katalysators [Kat $]_{0}$ variiert und zum anderen bei konstantem $[K a t]_{0}$ die $\left[\mathrm{H}_{2} \mathrm{dtbc}\right]_{0}-$ Konzentration verändert, woraus sich die Gleichungen

(8) $v_{0}=k^{\prime} \cdot$ const $_{1} \cdot[\text { Kat }]_{0}=k_{\text {exp }} \cdot[\text { Kat }]_{0}$ und

(9) $v_{0}=k^{\prime} \cdot$ const $_{2} \cdot\left[\mathrm{H}_{2} \mathrm{dtbc}\right]_{0}=k_{\text {cat }} \cdot\left[\mathrm{H}_{2} \mathrm{dtbc}\right]_{0}$ ergeben.

Die Anfangssteigung $v_{0}$ kann durch das Anlegen einer Fitkurve an die erhaltene Meßkurve ermittelt werden. Jede beliebige unbekannte Funktion kann durch eine Potenzreihe ausgedrückt werden. In einem Konzentrations-Zeit-Diagramm erhält man zunächst:

(10) $[\mathrm{dtboc}]=\mathrm{c}_{1}+\mathrm{c}_{2} t+\mathrm{c}_{3} t^{2}+\mathrm{c}_{4} t^{3}+\ldots$

Die Steigung der Kurve bei einem beliebigen Zeitpunkt t ergibt sich dann zu :

(11) $v=\frac{\mathrm{d}([\mathrm{dtboc}])}{\mathrm{d} t}=\mathrm{c}_{2}+2 \mathrm{c}_{3} t+3 \mathrm{c}_{4} t^{2}+\ldots$

Zum Zeitpunkt $\mathrm{t}=0$ erhält man:

(12) $v_{0}=\left(\frac{\mathrm{d}([\mathrm{dtboc}])}{\mathrm{d} t}\right)_{0}=\mathrm{c}_{2}$

Die Meßkurven wurden auf diese Weise mit den Programmen Sigma Plot und Origin ausgewertet. ${ }^{[138,139]}$

\subsection{Aktivität der Komplexe 1 - 4}

Die Komplexe 1, 2, 3 und 4 (Abbildung 30) besitzen Pyrazolatliganden mit unterschiedlichen aliphatischen Seitenarmen, welche Stickstoff als Donoratome 
enthalten. Erste Untersuchungen der katalytischen Aktivität der Komplexe wurden bereits im Rahmen der eigenen Diplomarbeit durchgeführt und mit strukturellen und elektrochemischen Parametern der Komplexe verglichen. ${ }^{[99]}$ Hierbei stellte sich ein Zusammenhang zwischen dem Abstand der Kupferzentren und der CatecholaseAktivität der Katalysatoren heraus. So weisen 1 und 2 mit langen aliphatischen Seitenarmen und einem Kupfer-Kupfer-Abstand von 3.540(1) bzw. 3.447(2) $\AA$ einen deutlich schnelleren Substratumsatz auf als $\mathbf{3}$ und $\mathbf{4}$ mit kurzen Seitenarmen und einem daraus folgenden großen intermetallischen Abstand von 4.088(1) bzw. 4.533(1) A. Am deutlich inaktivsten ist 4, welcher nicht nur den ungünstigsten MetallMetall-Abstand aufweist, sondern auch eine zusätzliche Koordination beider Kupferzentren durch jeweils einen chelatisierenden Seitenarm, was die erfolgreiche Anbindung eines Substratmoleküls weiter erschweren dürfte.
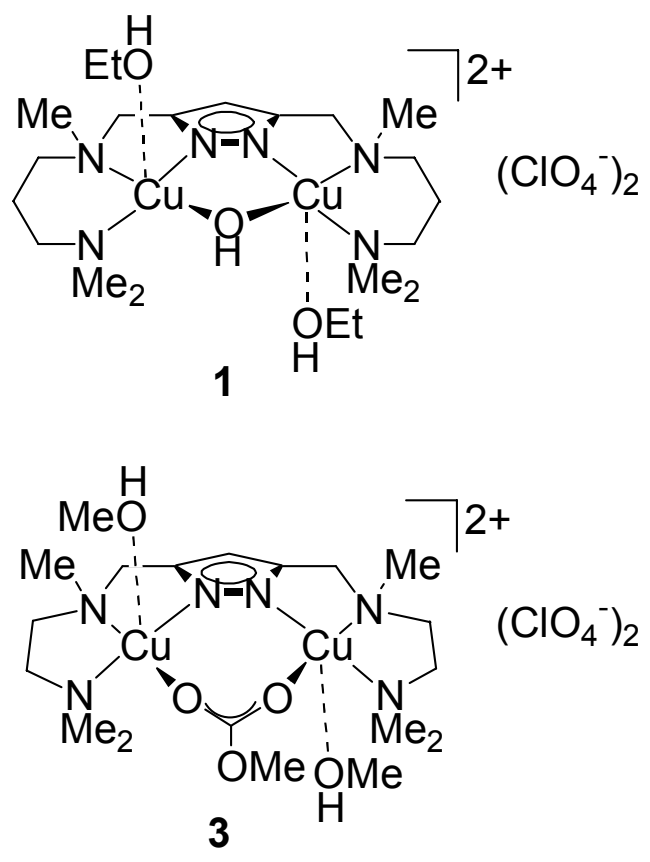
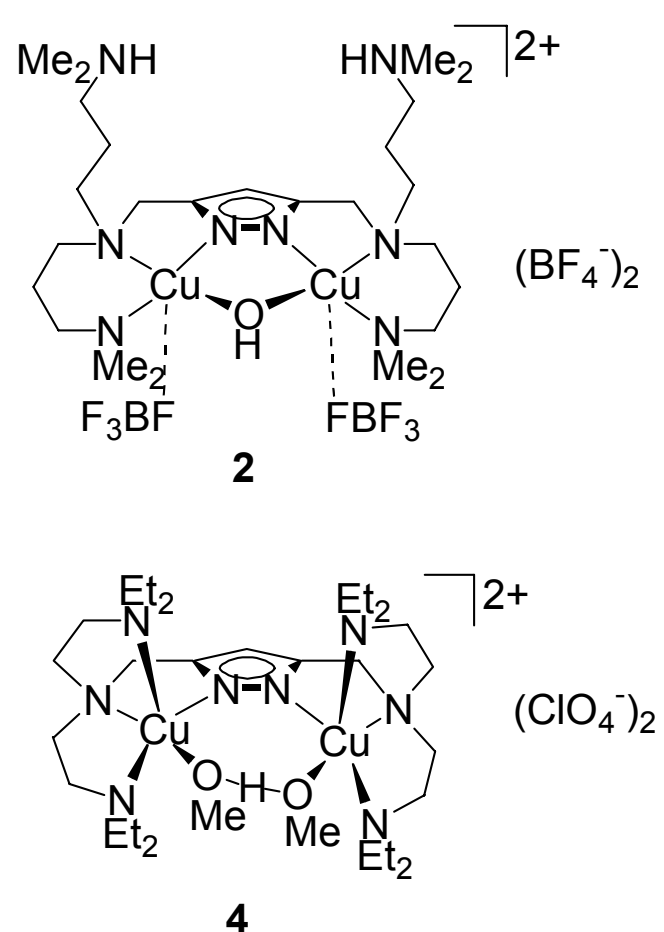

Abbildung 30: Strukturen der Katalysatoren 1 - 4, für die Katalyse wurde das $\mathrm{ClO}_{4}^{-}$-Salz von 2 verwendet.

Im Folgenden sollten die zunächst erhaltenen - aufgrund der Schnelligkeit der Umsetzung des Substrates jedoch noch recht ungenauen - Daten für die katalytische Aktivität der Komplexe quantifiziert werden, indem bei deutlich niedrigeren Katalysatorkonzentrationen und einem größeren Substratüberschuß gearbeitet wurde. 
Alle kinetischen Messungen erfolgten in luftgesättigtem Methanol bei $20^{\circ} \mathrm{C}$ in einer offenen Küvette, was die Eindiffusion von $\mathrm{O}_{2}$ aus der Luft in das Lösungsmittel erleichtern sollte. In der ersten Meßreihe wurde die Startkonzentration des Katalysators [Kat $]_{0}$ zwischen $2 \cdot 10^{-6}$ und $2 \cdot 10^{-5} \mathrm{M}$ variiert, während die Startkonzentration des Substrats $\left[\mathrm{H}_{2} \mathrm{dtbc}\right]_{0}$ konstant bei $10^{-3} \mathrm{M}$ gehalten wurde. In allen Fällen wurde eine lineare Abhängigkeit von $v_{0}$ von der Katalysatorkonzentration gefunden, welche durch lineare Regression ausgewertet wurde (Abbildung 31). Für die Zweielektronenoxidation des Substrats im ersten Schritt der Katalyse sollte ein bimetallisches Katalysatormolekül benötigt werden, was durch die gefundene Abhängigkeit 1. Ordnung in Bezug auf den Katalysator bestätigt wird.

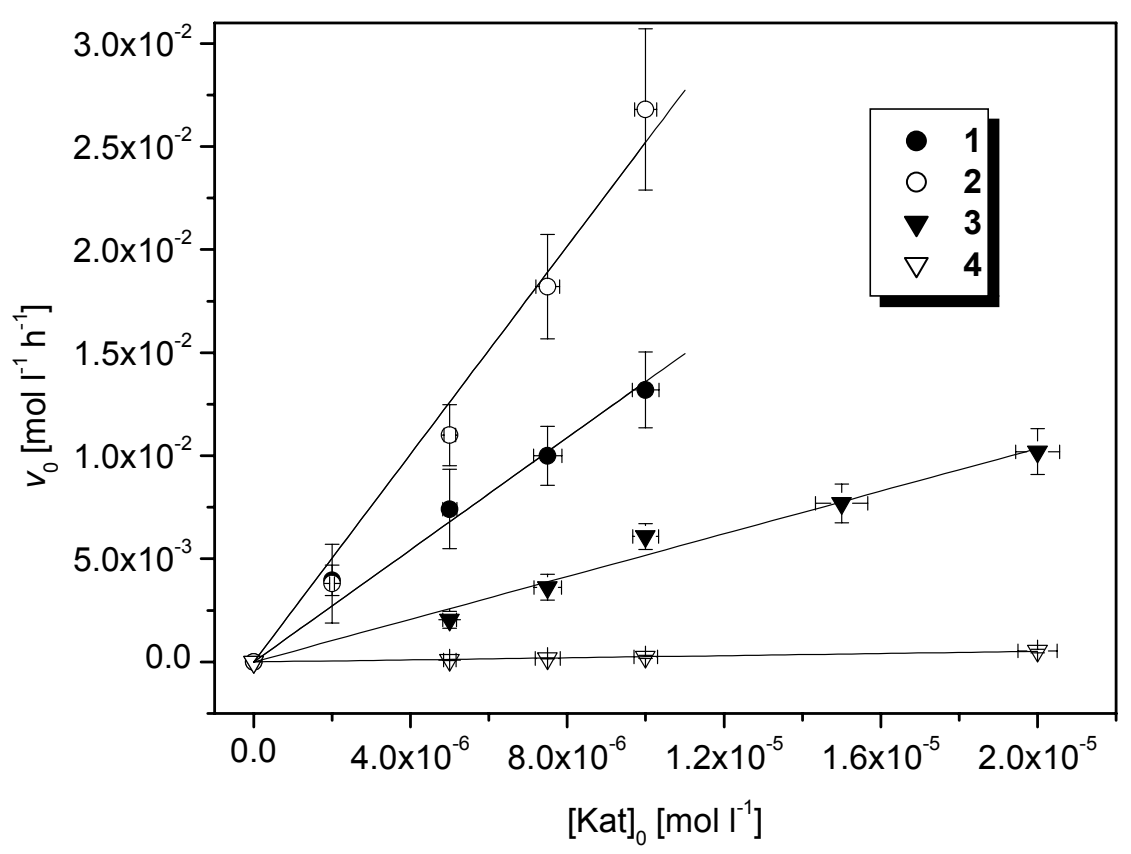

Abbildung 31: Abhängigkeit von vo von der gewählten Katalysator-Anfangskonzentration [Kat] $]_{0}$ bei konstanter Substrat-Anfangskonzentration von $10^{-3} \mathrm{M}$ für die Komplexe 1 - 4 in Methanol; $T=20^{\circ} \mathrm{C}$.

Komplex 2 erweist sich bei dieser Meßreihe als besonders reaktiv mit einer Geschwindigkeitskonstanten $k_{\exp }$ von $2430 \mathrm{~h}^{-1}$ (Tabelle 14). Die langen Seitenarme des Liganden erlauben ebenso im Falle von 1 einen kleinen Abstand der Kupferzentren zueinander. Daher verwundert es nicht, daß auch 1 eine große katalytische Aktivität aufweist $\left(k_{\exp }=1400 \mathrm{~h}^{-1}\right)$. Prominentester Unterschied zwischen 1 und $\mathbf{2}$ ist die Anwesenheit zweier zusätzlicher protonierter Seitenarme bei 2. Dies führt zum einen zu einer höheren Gesamtladung des Komplexes und zum anderen kann die große Nähe der aziden H-Atome zum reaktiven Zentrum des Katalysators 
die Reaktion beeinflussen. So könnte die Protonenübertragung auf die während der Reaktion gebildete Sauerstoffspezies unter Bildung von $\mathrm{H}_{2} \mathrm{O}$ oder $\mathrm{H}_{2} \mathrm{O}_{2}$ erleichtert werden.

Im Vergleich dazu ist 3 mit $k_{\text {exp }}=514 \mathrm{~h}^{-1}$ schon deutlich weniger aktiv. 3 kann aufgrund des großen Cu...Cu-Abstandes von 4.088(1) $\AA$ keine $\mathrm{OH}^{-}$-Brücke mehr zwischen die Metallzentren einbauen. Dagegen nimmt der Komplex unter Raumatmosphäre langsam $\mathrm{CO}_{2}$ auf, welches durch Veresterung mit dem Solvens Methanol in Form einer Methylcarbonatbrücke zwischen die Kupferzentren tritt. Für die kinetischen Untersuchungen wurde dieser Komplex gezielt hergestellt. Auffallend ist bei 3, daß die Abnahme an Aktivität mit der Vergrößerung des Cu...Cu-Abstandes aufgrund der kurzen Ligandseitenarme einhergeht. Da sich $\mathbf{1}$ und $\mathbf{3}$ in ihrem Aufbau ansonsten nicht wesentlich unterscheiden - in beiden Fällen sind die Kupferzentren durch jeweils zwei aliphatische und ein Pyrazol-N-Atom, sowie ein O-Atom basal koordiniert, und in der apicalen Position der quadratisch pyramidalen Koordinationsumgebung durch das O-Atom eines locker gebundenen Solvensmoleküls - dürfte beim Vergleich der Aktivität beider Katalysatoren der unterschiedliche Metall-Metall-Abstand die herausragende Rolle spielen, zumal die Methylcarbonatbrücke aufgrund der angespannten sterischen Situation in $\mathbf{3}$ relativ leicht zu entfernen sein sollte. ${ }^{[100]}$

Die Länge der Ligandseitenarme in $\mathbf{4}$ ist identisch zu jener in 3, jedoch erzwingt die Koordination der Kupferzentren durch einen weiteren koordinierenden Seitenarm einen noch weit größeren Metall-Metall-Abstand, damit einhergehend den Einbau einer größeren Zweitbrücke, hier in Form einer MeO ...H...OMe-Brücke. Als Grund für die weitaus geringere Aktivität dieses Komplexes im Vergleich zu den anderen eingesetzten Katalysatoren bieten sich mehrere Ursachen an: Der Abstand der Metallzentren zueinander ist mit 4.533(1) A weit entfernt vom „idealen“ Abstand 2.9 $\AA$, wie man inn bei der Catechol-Oxidase findet. Weiterhin ist bei beiden Kupferzentren jeweils eine Koordinationsstelle durch zusätzliche Chelatdonoren blockiert, während bei den anderen drei Katalysatoren immer eine durch leicht zu verdrängende Solvens- oder Perchlorationen besetzte Koordinationsstelle für den Angriff durch ein Substrat zur Verfügung steht. Zu beachten ist auch die veränderte Koordinationsgeometrie von $\mathbf{4}$, bei welchem als einzigem der vier Katalysatoren eine 
verzerrte trigonal bipyramidale Koordinationsumgebung an den Kupferzentren vorliegt, sowie sein niedriges Redoxpotential (siehe Kapitel 5.4).

Die zweite Meßreihe wurde bei konstantem $[K a t]_{0}=10^{-5} \mathrm{M}$ durchgeführt, $\left[\mathrm{H}_{2} \mathrm{dtbc}\right]_{0}$ lag zwischen $4 \cdot 10^{-5} \mathrm{M}$ und $2 \cdot 10^{-3} \mathrm{M}$. Für 2 und 4 wurden Sättigungskinetiken gefunden, während bei 1 und 3 im untersuchten Konzentrationsbereich keine Abhängigkeit von $v_{0}$ von der Substratkonzentration festgestellt werden konnte (Abbildung 32).

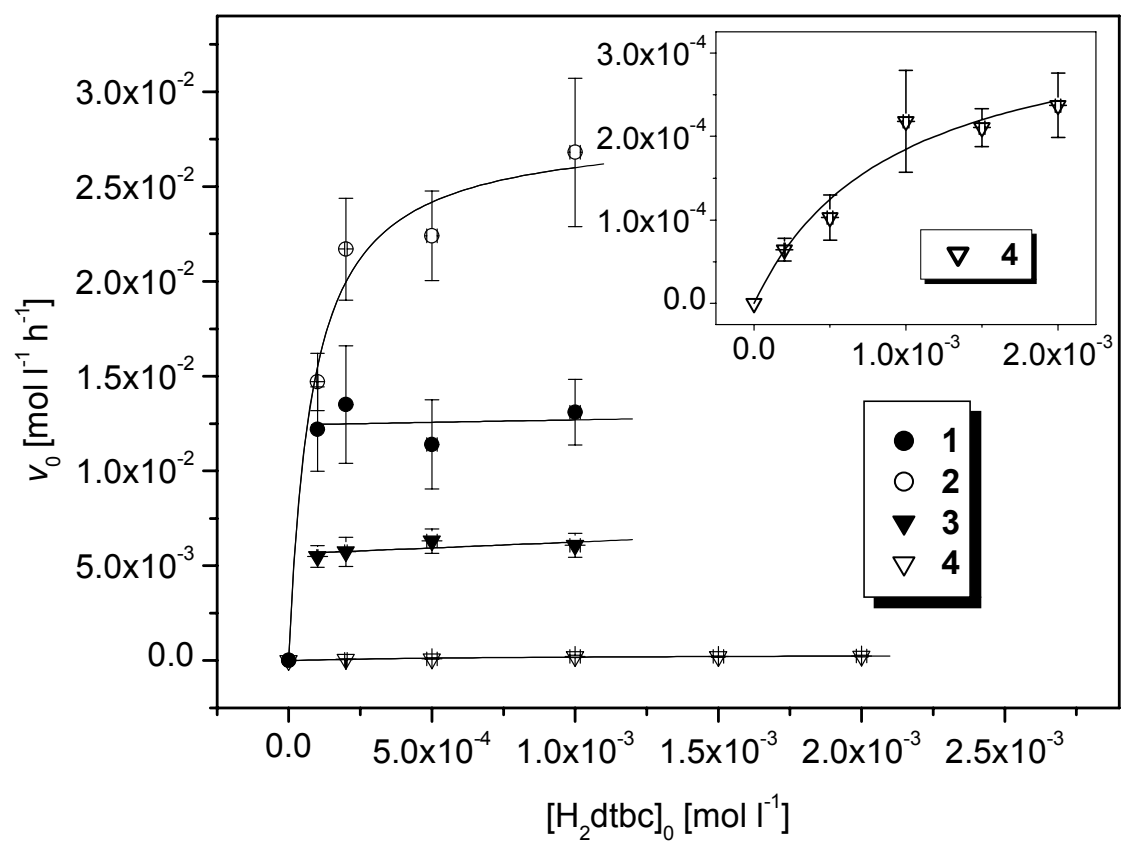

Abbildung 32: Abhängigkeit von $v_{0}$ von der gewählten Substrat-Anfangskonzentration $\left[\mathrm{H}_{2} \mathrm{dtbc}\right]_{0}$ bei konstanter [Kat]o von $10^{-5} \mathrm{M}$ für die Komplexe 1 - 4 in Methanol; $T=20^{\circ} \mathrm{C}$.

Die für 2 und $\mathbf{4}$ gefundenen Kinetiken ähneln denen enzymkatalysierter Reaktionen, ${ }^{[140,141]}$ so daß zur ihrer Auswertung von einer Michaelis-Menten-Kinetik ausgegangen wurde. Hierbei handelt es sich um Reaktionen mit einem vorgelagerten Gleichgewicht zwischen Katalysator (Kat) und Substrat (S) auf der einen und einem Katalysator-Substrat-Addukt auf der anderen Seite, welches dann irreversibel im geschwindigkeitsbestimmenden Schritt zum Produkt weiterreagiert:

(13) Kat $+S \frac{k_{1}}{k_{-1}}$ Kat $\cdot S \stackrel{k_{\text {cat }}}{\longrightarrow}$ Kat $+\mathrm{P}$ 
Mit der Näherung, daß die Konzentration [Kat-S] sehr gering ist und sich während der Reaktion nicht mehr ändert, d.h. d([Kat·S]/dt $=0$ („Quasistationarität“) ergibt sich:

(14) $v=k_{\text {cat }} \cdot[\text { Kat }]_{0} \cdot \frac{[\mathrm{S}]}{K_{\mathrm{M}}+[\mathrm{S}]}=v_{\max } \cdot \frac{[\mathrm{S}]}{K_{\mathrm{M}}+[\mathrm{S}]}$,

(„Michaelis-Menten-Gleichung“, $K_{\mathrm{M}}$ : Michaelis-Konstante; $\left.K_{\mathrm{M}}=\left(k_{-1}+k_{\text {cat }}\right) / k_{1}\right)$.

Bei geringen Substratkonzentrationen, d.h. [S] $\ll K_{\mathrm{M}}$, ist $v$ proportional zu [S], bei hoher Substratkonzentration, d.h. [S] >> $K_{M}$ wird $v$ unabhängig von [S], woraus sich die beobachtete Sättigungskurve der Substratabhängigkeit ergibt. Zugleich ergibt sich aus der Michaelis-Menten-Gleichung eine lineare Abhängigkeit von der Katalysatorkonzentration, was ebenfalls den Beobachtungen entspricht. Zur Auswertung trägt man den Reziprokwert der Reaktionsgeschwindigkeit gegen den Reziprokwert der Substratkonzentration auf („Lineweaver-Burke-Plot“):

(15) $\frac{1}{v}=\frac{1}{v_{\max }}+\frac{K_{\mathrm{M}}}{v_{\max }} \frac{1}{[\mathrm{~S}]}$

So erhält man eine Gerade, aus deren Ordinatenabschnitt $v_{\max }$ und aus deren Steigung die Michaelis-Konstante ermittelt werden können.

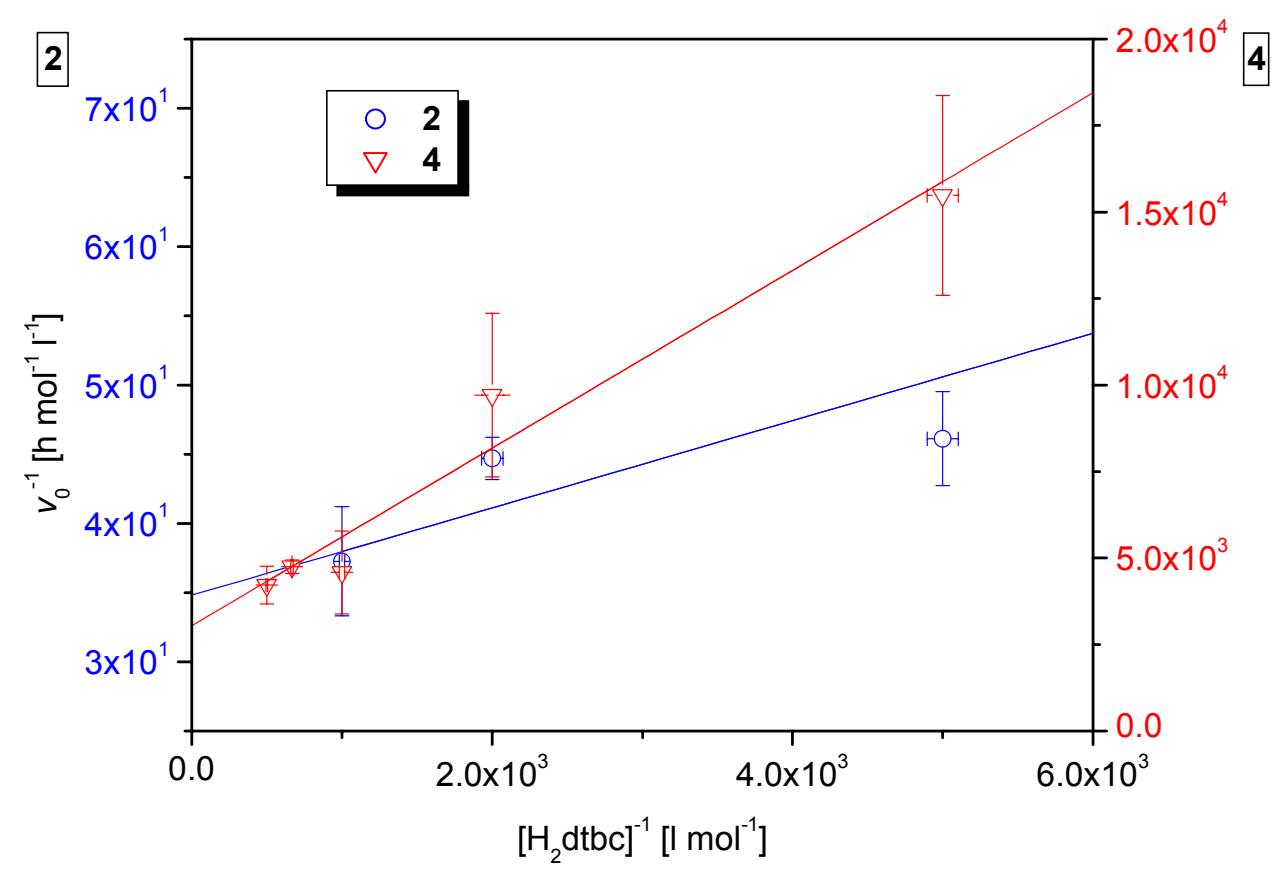

Abbildung 33: Lineweaver-Burke-Plots für die Katalysatoren 2 und 4. 
Die auf diese Weise erhaltenen Werte von $K_{M}$ und $v_{\max }$ sind in Tabelle 14 aufgeführt. Die fehlende Abhängigkeit von der Substratkonzentration bei 1 und 3 könnte durch eine besonders niedrige Michaelis-Konstante zu erklären sein. Da diese in etwa der reziproken Bindungskonstante von Substrat und Katalysator entspricht, bedeutet das eine starke thermodynamische Begünstigung der Anbindung des Substrats an den Katalysator in diesen Fällen. Da die Michaelis-Menten-Kinetik nur eine Vereinfachung der tatsächlich vorliegenden Kinetik darstellt, ist es aber auch möglich, daß ein anderer Schritt des katalytischen Zyklus geschwindigkeitsbestimmend ist, wie z.B. die Reoxidation der Kupfer(I)zentren mit molekularem Sauerstoff.

\begin{tabular}{cccccc}
\hline & $k_{\exp } / \mathrm{h}^{-1}$ & $d_{\mathrm{Cu} \ldots \mathrm{Cu}} / \AA$ & $k_{\mathrm{cat}} / \mathrm{h}^{-1}$ & $K_{\mathrm{M}} / \mathrm{mol} \mathrm{I}^{-1}$ & $v_{\max } / \mathrm{mol} \mathrm{I}^{-1} \mathrm{~min}^{-1}$ \\
\hline $\mathbf{1}$ & $1400 \pm 200$ & 3.54 & - & - & - \\
$\mathbf{2}$ & $2430 \pm 202$ & 3.45 & $2804 \pm 388$ & $(8.9 \pm 3.5) \cdot 10^{-5}$ & $(4.7 \pm 0.6) \cdot 10^{-4}$ \\
3 & $514 \pm 70$ & 4.09 & - & - & - \\
4 & $22.8 \pm 1.2$ & 4.53 & $31.6 \pm 11.3$ & $(7.9 \pm 4.0) \cdot 10^{-4}$ & $(5.3 \pm 1.9) \cdot 10^{-6}$ \\
\hline
\end{tabular}

Tabelle 14: Kinetische Parameter der Oxidation von $\mathrm{H}_{2} \mathrm{dtbc}$ bei $20^{\circ} \mathrm{C}$ in Methanol und $\mathrm{Cu}$... CuAbstand der Katalysatoren 1 - 4, $v_{\max }$ wurde durch einen Lineweaver-Burke Plot erhalten.

\subsection{Aktivität der Komplexe 7a, 8 und 13}

Die Komplexe 7a und 8 weisen lange Ligandseitenarme mit endständigen Pyridylresten auf und haben große Kupfer-Kupfer-Abstände von 4.290(1) A bei 7a bzw. 4.357(1) $\AA$ bei 8. Durch Abspaltung eines Fluorids vom $\mathrm{BF}_{4}^{-}$erhält man im Falle von 8 ein Fluorid-Addukt des Katalysators (Abbildung 34). 7a und 8 bieten sich an, um die Auswirkungen unterschiedlicher Zweitbrücken - insbesondere den Einfluß des koordinierten Fluorids - auf die Aktivität der Katalysatoren zu untersuchen. 


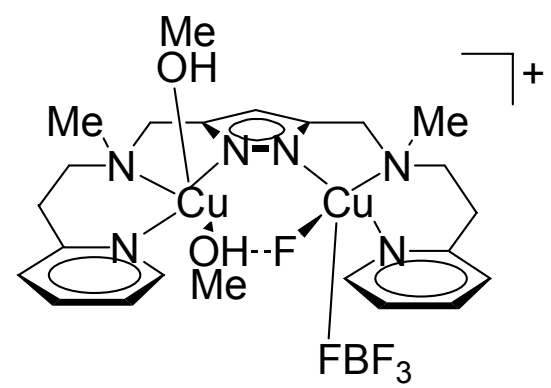

$7 a$

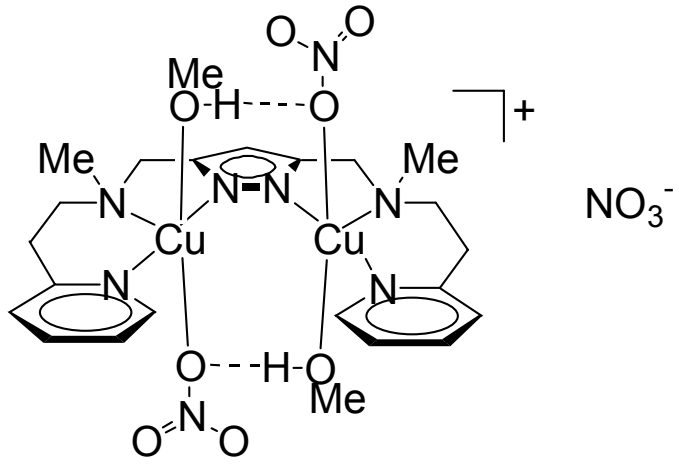

8

Abbildung 34: Die Katalysatoren 7a und 8, beide gebildet mit dem Liganden $\left[L^{5} T\right.$.

Komplex 13 weist einen für kooperative Wechselwirkungen der Metallzentren günstigen Kupfer-Kupfer-Abstand von 3.515(5) $\AA$ auf, allerdings ist jeweils eine Koordinationsstelle beider Kupferzentren durch einen N-Donor des Chelatseitenarmes blockiert, was wie im Falle des Komplexes 4 das Anbinden des Substrates erschweren kann, wenn hierfür die Verdrängung eines N-Donors erforderlich ist (Abbildung 35).

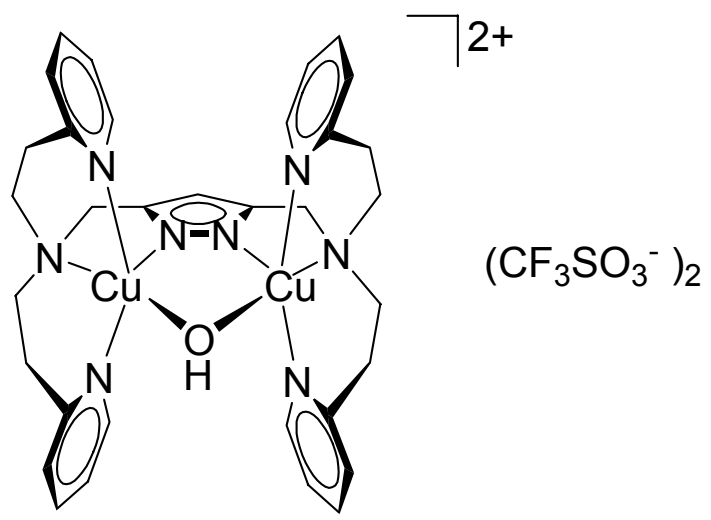

Abbildung 35: Katalysator 13 mit dem Liganden $\left[L^{6} J\right.$.

Untersucht man zunächst die katalytische Aktivität der Komplexe 7a, 8 und 13 in reinem, luftgesättigtem Methanol, so stellen sich die Komplexe als wenig aktiv heraus. Auch bei hoher Katalysatorkonzentration von $10^{-4} \mathrm{M}$ ist innerhalb eines Zeitintervalls von $15 \mathrm{~min}$ kaum Substratumsatz festzustellen. Zudem ist keine Entwicklung eines klaren Maximums bei $400 \mathrm{~nm} \mathrm{zu}$ beobachten, welches dem gebildeten 0 -Chinon zugeordnet werden könnte. Vielmehr bildet sich bei $\lambda=400 \mathrm{~nm}$ eine schlecht definierte Schulter in einer Charge-Transfer Bande des Komplexes (Abbildung 36). Bei 13 konnte unter vergleichbaren Bedingungen keine wesentliche zeitliche Änderung des UV/Vis-Spektrums beobachtet werden. 


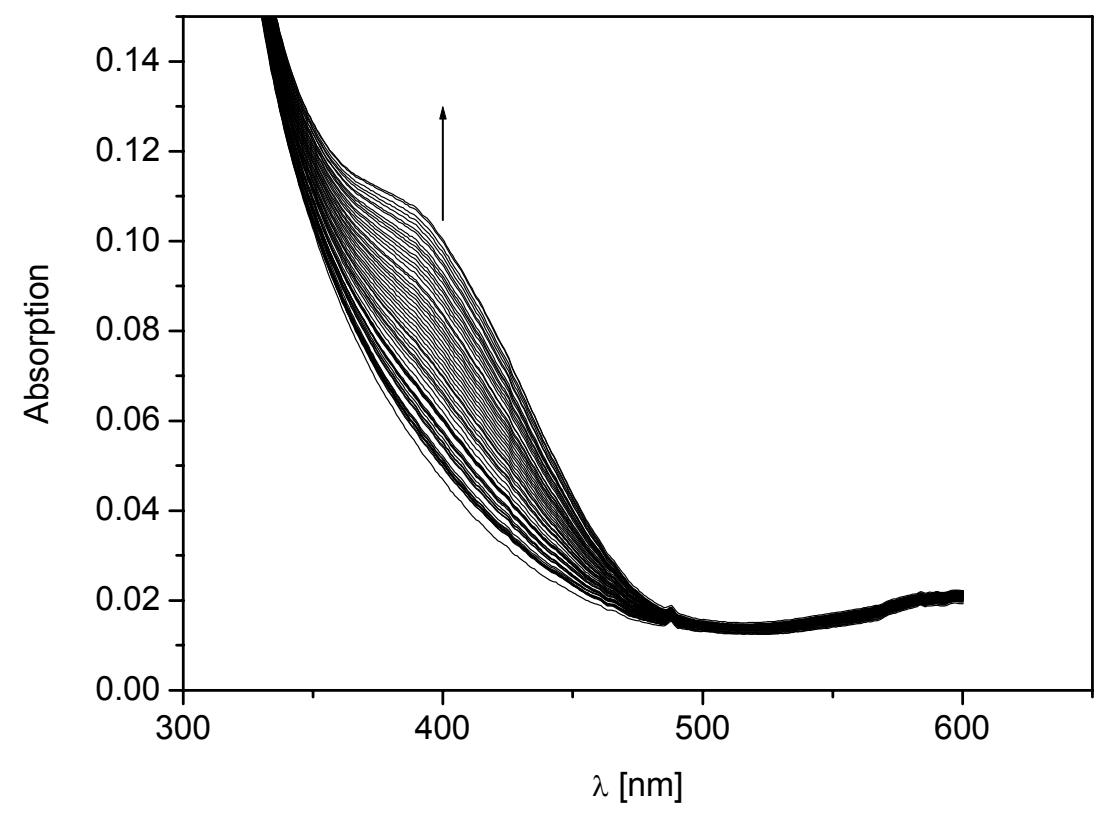

Abbildung 36: Verlauf der Catecholase-Reaktion von 7a in reinem Methanol, ([7a $]_{0}=10^{-4} \mathrm{M}\left[\mathrm{H}_{2} \mathrm{dtbc}\right]_{0}$ $\left.=10^{-3} \mathrm{M}\right)$ - Der Kurvenverlauf für 8 ist ähnlich und daher aus Gründen der Übersichtlichkeit nicht aufgeführt.

Eine deutliche Steigerung der Aktivität der Komplexe wird im gepufferten Milieu erhalten, wobei eine 29:1 Mischung von Methanol und wäßrigem Puffer mit der Konzentration $0.047 \mathrm{M}$ verwandt wurde. Die Aktivität wurde in einem Bereich von $\mathrm{pH}=4$ bis 9 mit verschiedenen Puffersystemen untersucht, wobei im sauren Bereich der Essigsäure-Acetat-Puffer, im neutralen Bereich verschiedene Puffer mit Sulfonsäurefunktionen, wie MES- und MOPS-Puffer (MES $=2$-Morpholinoethansulfonsäure, MOPS = 3-Morpholinopropansulfonsäure), sowie im basischen Bereich der TRIS-Puffer (TRIS = 2-Amino-2-(hydroxymethyl)propan-1,3-diol) zum Einsatz kamen.
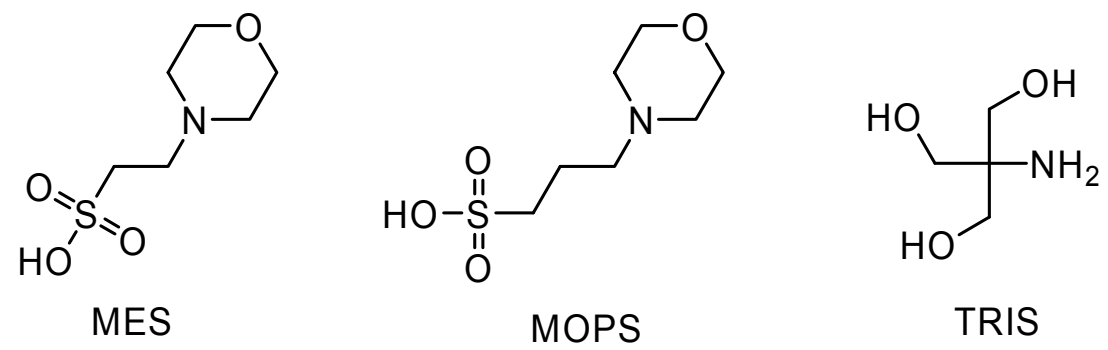

Abbildung 37: Strukturen der eingesetzten Puffer. 
Zusätzlich wurde im Falle des TRIS-Puffers die Auswirkung unterschiedlicher PufferGegenionen auf die katalytische Aktivität der Dikupferkomplexe untersucht. Bei Verwendung von $\mathrm{Cl}^{-}$als Gegenion sollte eine geringere Aktivität als beim $\mathrm{NO}_{3}{ }^{-}-$ Gegenion zu erwarten sein, da $\mathrm{Cl}^{-}$durch starke Koordination an die Metallzentren diese deaktiviert. ${ }^{[66]}$

Im Prinzip steigt die Aktivität der Komplexe 7a und 8 innerhalb der einzelnen Pufferbereiche mit steigendem $\mathrm{pH}$ bis ca. $\mathrm{pH} 7.5$ und fällt danach wieder $\mathrm{ab}$ (Abbildung 38).

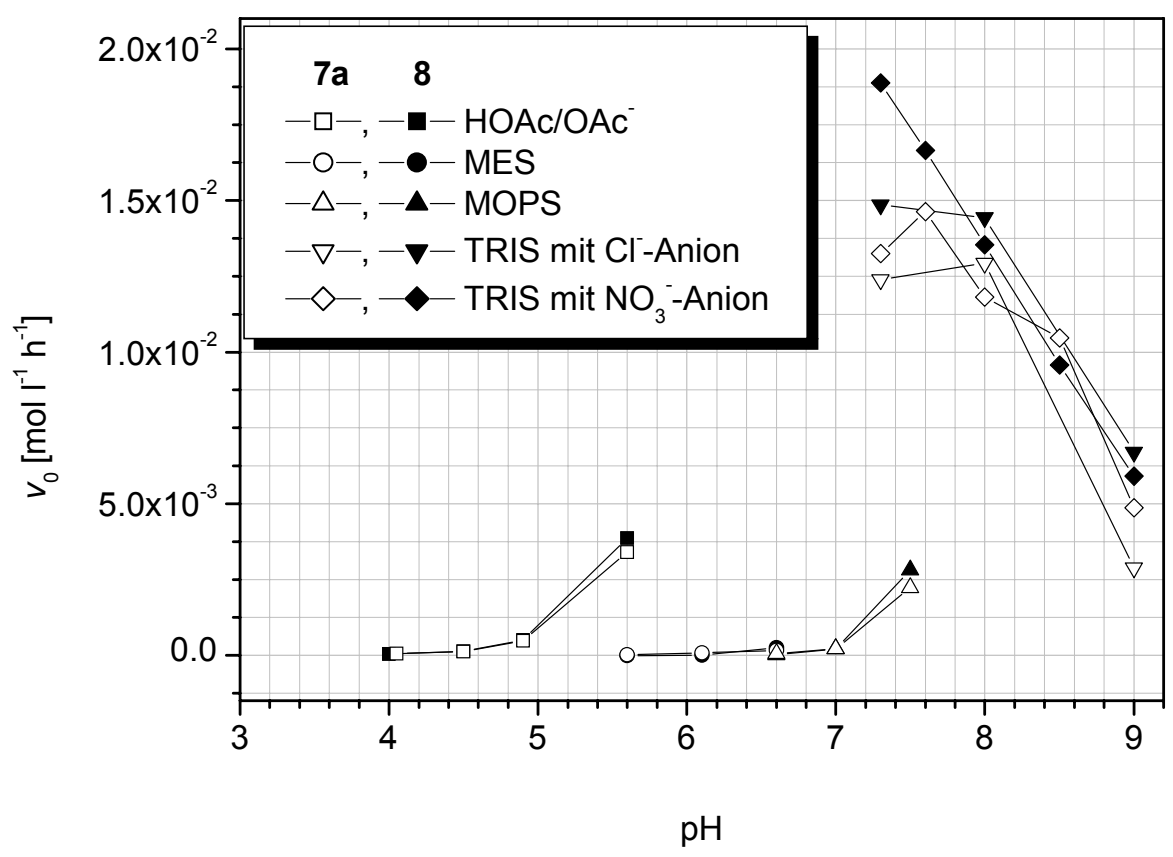

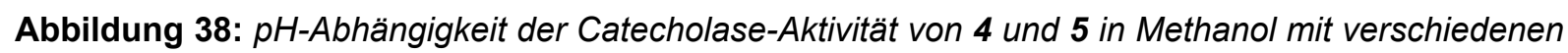
Puffersystemen, $[\mathrm{Kat}]_{0}=2 \cdot 10^{-5} \mathrm{M},\left[\mathrm{H}_{2} \mathrm{dtbc}\right]_{0}=2 \cdot 10^{-3} \mathrm{M} ; \mathrm{T}=20^{\circ} \mathrm{C}$.

Im Sauren sollte eine Substratanbindung erschwert sein, da unter diesen Bedingungen keine ausreichende Konzentration an teilweise deprotoniertem Substrat vorliegt, während $\mathrm{H}_{2} \mathrm{dtbc}$ nur schlecht an die Kupferzentren koordiniert. In Anbetracht der pKs-Werte von 10.4 bzw. 14.7 für die aziden H-Atome des Substrats könnte der Anstieg der Aktivität darauf zurückzuführen sein, daß erst bei höheren $\mathrm{pH}-$ Werten eine der OH-Funktionen des Substrates zumindest in geringem Anteil deprotoniert ist und eine Anbindung von hdtbc stattfinden kann. ${ }^{[142]}$ Offensichtlich hat jedoch der eingesetzte Puffer einen wesentlichen Einfluß auf das untersuchte System. So kann im Falle des gut koordinierenden Essigsäure-Acetat-Puffers z.B. 
die Zweitbrücke des Dikupferkomplexes gegen ein Acetation ausgetauscht werden. Die geringe Aktivität bei pH 6 - 7 liegt also eher am verwendeten MES- bzw. MOPSPuffer. Leider wurde für den entsprechenden $\mathrm{pH}$-Bereich kein geeigneteres Puffersystem gefunden, bei Verwendung des BIS-TRIS-Puffers (2,2Bis(hydroxyethyl)-iminotris(hydroxy-methyl)methan), welcher dem TRIS-Puffer ähnlicher ist, lagen die Aktivitäten in der gleichen Größenordnung, bei Verwendung des $\mathrm{H}_{2} \mathrm{PO}_{3}{ }^{-} / \mathrm{HPO}_{3}{ }^{2-}$-Puffers wurde eine starke Präzipitatbildung beobachtet. Die Auswirkung von Puffern auf katalytische Reaktionen wird in der Literatur oft vernachlässigt, was aber - wie der hier vorliegende Fall verdeutlicht - in vielen Fällen eigentlich nicht zulässig ist.

Ein analoges Bild ergibt sich für den Katalysator 13, nur wird hier noch eine wesentlich stärkere Aktivitätssteigerung unter Verwendung des TRIS-Puffers bei pHWerten $>7.3$ beobachtet. Das Aktivitätsmaximum liegt hier im basischen Bereich bei $\mathrm{pH}=8$ (Abbildung 39).

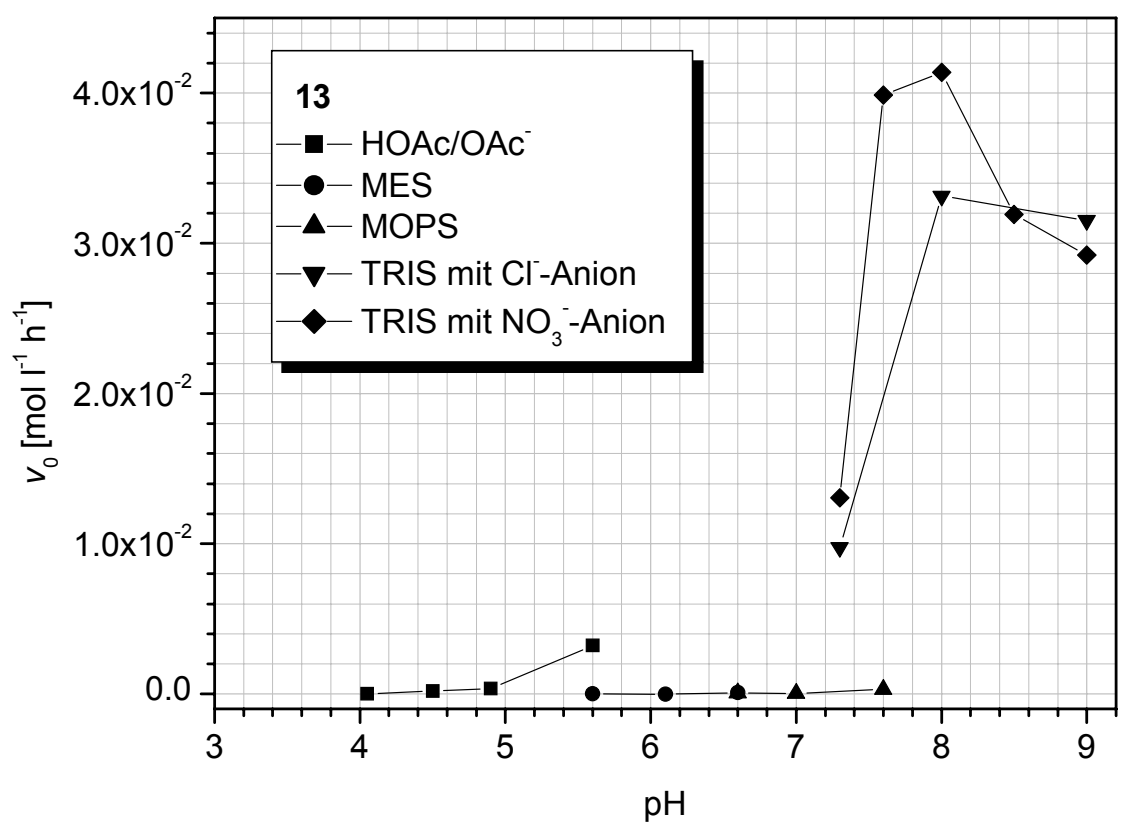

Abbildung 39: pH-Abhängigkeit der Catecholase-Aktivität von 13 in Methanol mit verschiedenen Puffersystemen, $[K a t]_{0}=10^{-5} \mathrm{M},\left[\mathrm{H}_{2} \mathrm{dtbc}\right]_{0}=2 \cdot 10^{-3} \mathrm{M}, \mathrm{T}=20^{\circ} \mathrm{C}$.

Auch mit Komplex 13 wurde bei Verwendung von MES- und MOPS-Puffern kaum katalytische Aktivität erhalten. Auch in diesem Falle ist somit ein großer Einfluß des Puffers auf das System vorhanden. Dies verdeutlicht, daß - sofern möglich - bei 
Untersuchung der pH-Abhängigkeit von Reaktionen immer die gleiche Klasse von Puffern für den gesamten $\mathrm{pH}-$ Bereich verwendet werden sollte. Im Prinzip eignen sich hierfür eine Reihe von Puffern mit Sulfonsäurefunktionen wie MES und MOPS, die einerseits einen großen $\mathrm{pH}$-Bereich abdecken und andererseits kaum komplexieren. Allerdings ergaben diese eine äußerst schwache katalytische Aktivität für die hier untersuchten Komplexe. Dagegen aktivieren der Essigsäure/Acetat- und insbesondere der TRIS-Puffer die Katalysatoren deutlich.

Im Extremfall könnte ein gut komplexierender Puffer die Metallionen sogar aus Chelatligandgerüst herauslösen. Daher wurden Blindproben mit Kupfernitrat, -tetrafluoroborat und -triflat im gepufferten Milieu durchgeführt. Zwar wurde auch hier katalytische Aktivität festgestellt, jedoch war diese deutlich geringer als unter Verwendung der Dikupfer(II)komplexe. Somit kann ein gravierender Einfluß des für die katalytischen Untersuchungen verwendeten Puffers auf die Zusammensetzung des Katalysators ausgeschlossen werden, d.h. die Kupferzentren verbleiben in den Kavitäten des Pyrazolatliganden. Jedoch bleibt festzuhalten, daß bei Verwendung von Puffern in der Katalyse immer deren mögliche Interaktion mit dem Katalysator in Betracht gezogen werden muß.

Über den gesamten untersuchten $\mathrm{pH}$-Bereich verhalten sich die Katalysatoren $\mathbf{7 a}$ und 8 sehr ähnlich. Insbesondere unter Verwendung des TRIS-Puffers im leicht bis stark basischen Milieu ist eine große Aktivität beider Komplexe festzustellen, wobei das $\mathrm{Cl}^{-}$-Anion nicht zu wesentlich anderen Aktivitäten führt als das $\mathrm{NO}_{3}{ }^{-}$-Gegenion. Insbesondere im optimalen pH-Bereich von 7.3 - 7.6 ergibt das $\mathrm{NO}_{3}{ }^{-}$-lon aber schnelleren Substratumsatz. Sowohl bei $\mathbf{7 a}$ als auch bei 8 liegt hier auch die stärkste Aktivität vor, im Falle von $7 \mathrm{a}$ bei $\mathrm{pH} 7.6$ und bei 8 bei 7.3. Um beide Komplexe besser vergleichen zu können, wurden die folgenden katalytischen Untersuchungen in beiden Fällen bei pH 7.3 mit dem TRIS-Puffer mit $\mathrm{NO}_{3}{ }^{-}$-Gegenion durchgeführt. Auch bei 13 erhält man unter Verwendung des $\mathrm{Cl}^{-}$-Anions im allgemeinen schlechtere Aktivitäten. Nur bei pH-Werten ab 9 scheint sich die Aktivität umzukehren. Da aber in diesem Bereich schon verstärkte Autoxidation des Substrates beobachtet wurde, weisen die Ergebnisse hier einen größeren Fehler auf, so daß keine sichere Aussage möglich ist. Die folgenden kinetischen Untersuchungen mit 13 wurden bei pH 8 durchgeführt. 
In einer ersten Meßreihe wurde die Konzentration von $7 \mathrm{a}$ und 8 zwischen $10^{-5}$ und $10^{-4} \mathrm{M}$ und die Konzentration von 13 zwischen $10^{-6}$ und $10^{-5} \mathrm{M}$ variiert, während $\left[\mathrm{H}_{2} \mathrm{dtbc}\right]_{0}$ konstant bei $2 \cdot 10^{-3} \mathrm{M}$ gehalten wurde. Die Reaktionstemperatur betrug $20^{\circ} \mathrm{C}$. Die Komplexe $7 \mathrm{a}$ und 8 zeigen dabei ein sehr ähnliches Verhalten (Abbildung 40). Die gefundene Abhängigkeit von $v_{0}$ von der Katalysatorkonzentration ergibt keine ideale lineare Abhängigkeit bei größeren Katalysatorkonzentrationen ab ca. $7.5 \mathrm{~mol} \mathrm{l}^{-1}$, d.h. nur geringem Substratüberschuß. Möglicherweise kommt es hier zur Bildung oligomerer Komplexspezies. Die lineare Regression liefert aber noch akzeptable Werte mit $R=0.990$ für $7 a$ bzw. $R=0.996$ für 8 . Als Geschwindigkeitskonstanten erhält man für $7 \mathrm{a}$ den Wert $k_{\exp }=629 \mathrm{~h}^{-1}$ und für 8 den Wert $k_{\exp }=593 \mathrm{~h}^{-1}$ (Tabelle 15). Damit liegen sie immerhin noch bezüglich ihrer Aktivität im Bereich des Komplexes $\mathbf{3}$ und das trotz des deutlich größeren Abstands zwischen ihren beiden Kupferzentren. Jedoch sollte bei 7a und 8 das Ligandgerüst flexibel genug sein, um während der Katalyse auch kleinere Kupfer-Kupfer-Abstände zu realisieren.

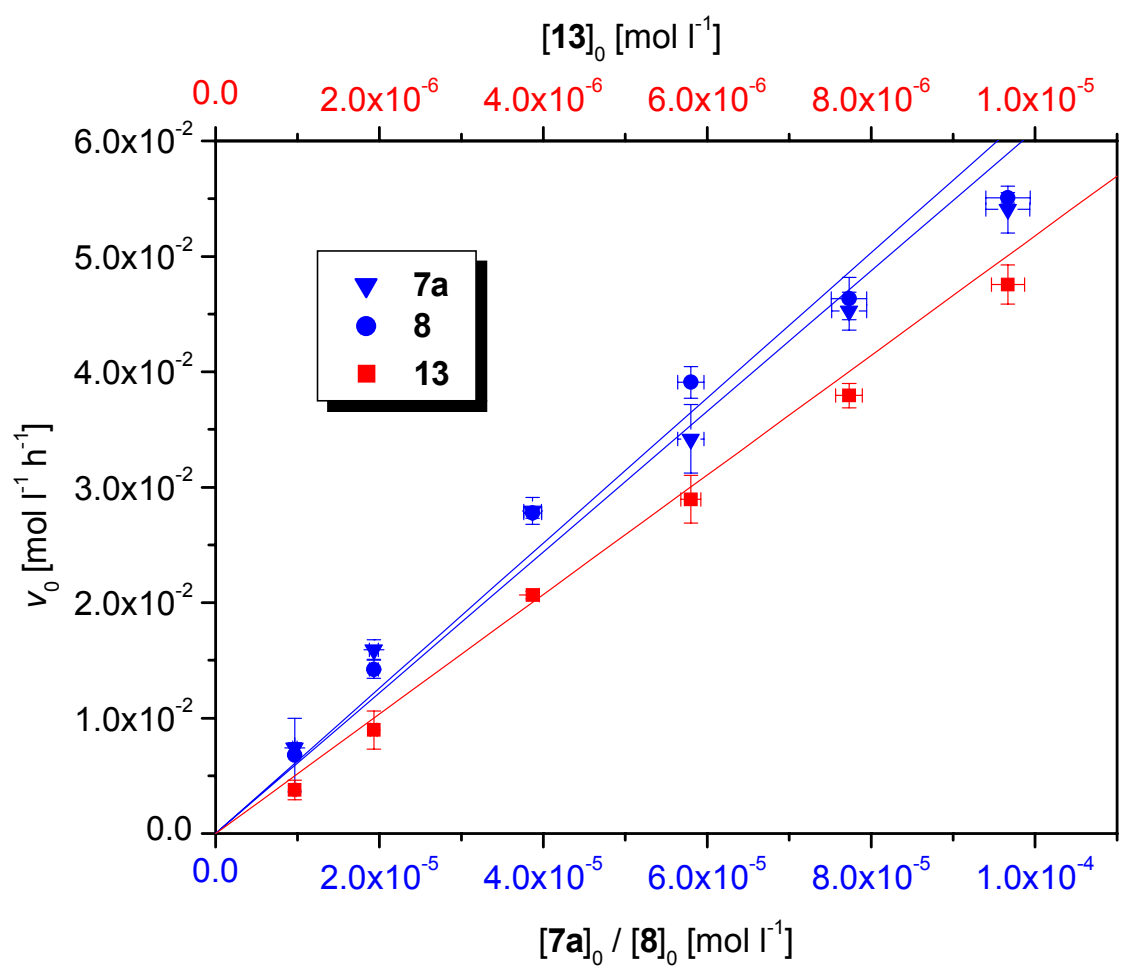

Abbildung 40: Abhängigkeit von $v_{0}$ von der gewählten Katalysator-Anfangskonzentration [Kat] $]_{0}$ von $7 \mathrm{a}$ und 8 bei $\mathrm{pH} 7.3$ und 11 bei $\mathrm{pH} 8.0$ bei konstanter Substrat-Anfangskonzentration von $2 \cdot 10^{-3} \mathrm{M}$ in Methanol mit TRIS-Puffer (0.047 M); $T=20^{\circ} \mathrm{C}$. 
Eine lineare Abhängigkeit der Catecholase-Aktivität von der Komplexkonzentration ist bei 13 über das gesamte Meßintervall gegeben, die katalytische Aktivität ist zudem erstaunlich hoch mit $k_{\exp }=5178 \mathrm{~h}^{-1}$. Damit ist 13 der aktivste von allen der im Rahmen dieser Arbeit untersuchten Komplexe und fast doppelt so aktiv wie Komplex 2.

Die zweite Meßreihe wurde mit einer Katalysatoranfangskonzentration von [Kat $]_{0}=$ $2 \cdot 10^{-5} \mathrm{M}$ bei $7 \mathrm{a}$ und 8 , sowie $10^{-5} \mathrm{M}$ bei 13 durchgeführt, wobei $\left[\mathrm{H}_{2} \mathrm{dtbc}\right]_{0}$ zwischen $4 \cdot 10^{-4}$ und $2.8 \cdot 10^{-3}$ lag. In allen Fällen wurden Sättigungskinetiken gefunden, auf welche wie in den zuvor beschriebenen Versuchen eine Michaelis-MentenAuswertung angewandt wurde (Abbildung 41).

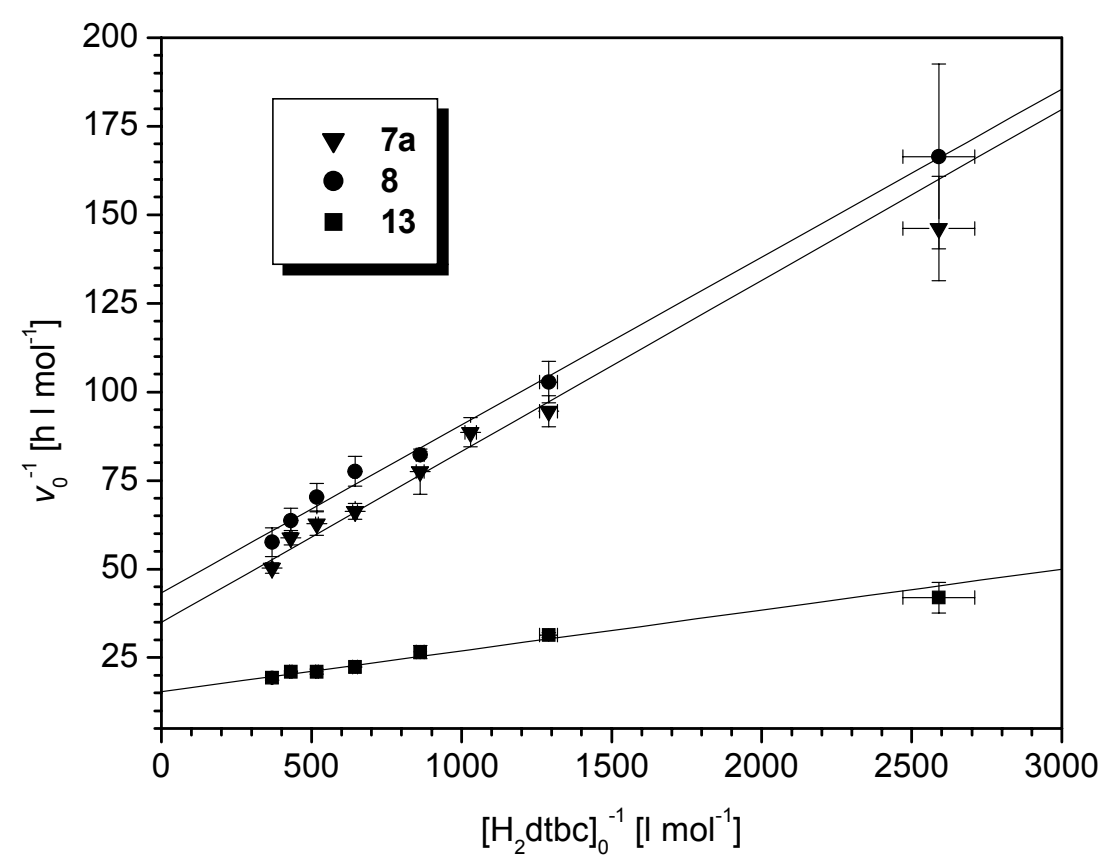

Abbildung 41: Lineweaver-Burke-Plots für die Katalysatoren 7a, $8(\mathrm{pH} 7.3)$ und $13(\mathrm{pH} \text { 8), [7a }]_{0}=$ $[8]_{0}=2 \cdot 10^{-5} \mathrm{M},[13]_{0}=10^{-5} \mathrm{M} ; \mathrm{T}=20^{\circ} \mathrm{C}$.

Auch hier zeigen beide Komplexe 7a und 8 ein sehr ähnliches Verhalten. Die erhaltenen $k_{\text {cat }}$ Werte liegen alle höher als die $k_{\text {exp }}$-Werte, welche in Meßreihe 1 erhalten wurden. Dies erklärt sich dadurch, daß gilt:

$v=k_{\text {exp }} \cdot[\text { Kat }]_{0}$ bzw. $v_{\max }=k_{\text {cat }} \cdot[\text { Kat }]_{0}$,

$v=v_{\max }$ gilt nur für große Substratüberschüsse, d.h. [S] $>K_{\mathrm{M}}$ (siehe Gleichung 14), ansonsten muß $k_{\exp }$ kleiner als $k_{\text {cat }}$ sein. Die erhaltenen Werte sind in Tabelle 15 
zusammengefaßt:

\begin{tabular}{cccccc}
\hline & $k_{\exp } / \mathrm{h}^{-1}$ & $d_{\mathrm{Cu} \ldots \mathrm{Cu}} / \AA$ & $k_{\mathrm{cat}} / \mathrm{h}^{-1}$ & $K_{\mathrm{M}} / \mathrm{mol} \mathrm{I}^{-1}$ & $v_{\max } / \mathrm{mol} \mathrm{I}^{-1} \mathrm{~min}^{-1}$ \\
\hline $\mathbf{7 a}$ & $629 \pm 12$ & 4.29 & $1432 \pm 93$ & $(1.4 \pm 0.1) \cdot 10^{-3}$ & $(4.8 \pm 0.3) \cdot 10^{-4}$ \\
$\mathbf{8}$ & $593 \pm 4$ & 4.36 & $1125 \pm 102$ & $(1.0 \pm 0.1) \cdot 10^{-3}$ & $(3.8 \pm 0.3) \cdot 10^{-4}$ \\
$\mathbf{1 3}$ & $5178 \pm 62$ & $3.51 / 3.52$ & $6502 \pm 239$ & $(7.5 \pm 0.5) \cdot 10^{-4}$ & $(10.8 \pm 0.3) \cdot 10^{-4}$ \\
\hline
\end{tabular}

Tabelle 15: Kinetische Parameter der Oxidation von $\mathrm{H}_{2} \mathrm{dtbc}$ bei $20^{\circ} \mathrm{C}$ in Methanol mit $0.047 \mathrm{M}$ TRISPuffer und Cu...Cu-Abstand der Katalysatoren 7a, 8 und 13, $v_{\max }$ wurde durch einen Lineweaver-Burke Plot erhalten.

Die nahezu identischen Aktivitäten der Komplexe 7a und $\mathbf{8}$ lassen darauf schließen, daß bei Anwesenheit von $\mathrm{H}_{2} \mathrm{O}$ oder des Puffers die schwach koordinierenden $\mathrm{NO}_{3}{ }^{-}-$ und $\mathrm{F}^{-}$-lonen rasch gegen andere Liganden ausgetauscht werden und folglich identische katalytisch aktive Spezies entstehen. Aufschluß über eine mögliche Veränderung der Komplexstruktur im gepufferten Milieu kann durch UV/VisSpektroskopie erhalten werden. In reinem Methanol unterscheiden sich Intensität und Lage der d-d-Absorptionsbanden von 7a und 8, während man bei Einsatz eines Puffers nahezu identische Spektren erhält (Abbildung 42).

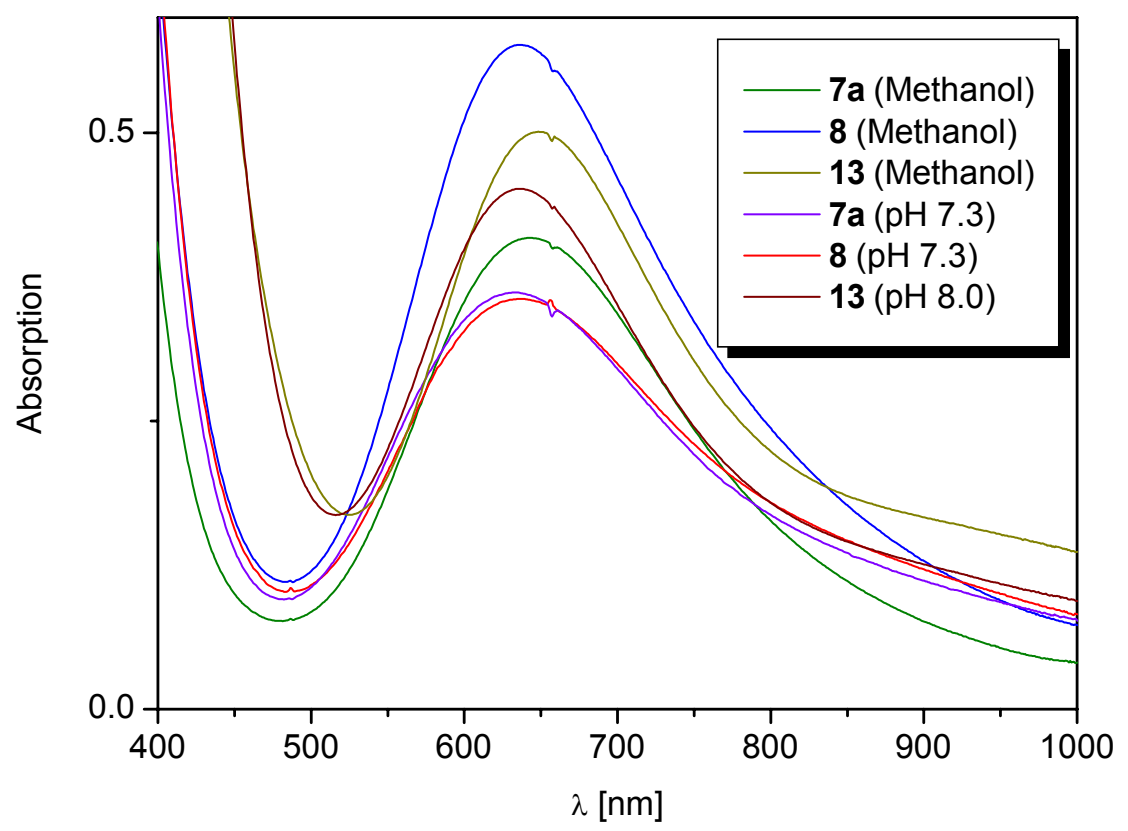

Abbildung 42: UV/Vis-Spektrum von 7a, 8 und 13 in reinem Methanol und mit TRIS-Puffer.

Wahrscheinlich liegt also unter den gewählten Katalysebedingungen die gleiche 
reaktive Spezies vor. Die Lage der d-d-Banden von $7 \mathrm{a}$ und 8 bei $\mathrm{pH} 7.3$ ist identisch mit der d-d-Bande von 8 in Methanol, da sich jedoch die Intensität deutlich ändert, muß eine Veränderung in der direkten koordinativen Umgebung der Kupferzentren stattgefunden haben. Dasselbe gilt für 13, wo ebenfalls eine leichte Verschiebung der d-d-Banden zu kleineren Wellenlängen und eine geringe Intensitätsänderung zu beobachten sind, so daß auch hier ein Ligandenaustausch an den Kupferzentren wahrscheinlich ist.

Einen weiteren Hinweis auf die in Lösung vorliegenden Spezies kann die ESIMassenspektrometrie liefern. 7a und $\mathbf{8}$ ergeben im TRIS-gepufferten Medium mit $\mathrm{NO}_{3}{ }^{-}$-Gegenion sehr ähnliche Spektren. Man sieht in beiden Fällen das Fragment $\left[\mathrm{L}^{5} \mathrm{Cu}_{2}\right]^{+}$bei $m / z=489$, sowie ein Fragment $\left[\mathrm{L}^{5} \mathrm{Cu}_{2}\left(\mathrm{NO}_{3}\right)_{2}\right]^{+}$bei $\mathrm{m} / \mathrm{z}=613$. Während man im Spektrum von 5 in reinem Methanol das koordinierte $\mathrm{F}^{-}$noch deutlich im Massenspektrum sehen kann, fehlt der entsprechende Peak hier völlig.

\subsection{Cyclovoltammetrie}

Die cyclovoltammetrischen Untersuchungen der Dikupfer(II)komplexe zeigten durchweg einen irreversiblen Reduktionsprozeß von Kupfer(II) zu Kupfer(I). Dies ist wahrscheinlich zurückzuführen auf eine schnelle Dissoziation der O-haltigen Zweitbrücken aus der Koordinationssphäre der Kupferzentren bei der Reduktion von Kupfer(II) zu Kupfer(I), da harte O-Liganden nur eine geringe Affinität zum weichen Kupfer(I) aufweisen. ${ }^{[68,143,144]}$ Weiterhin hat auch die Bevorzugung unterschiedlicher Koordinationsgeometrien durch Kupfer(I) und Kupfer(II) Auswirkungen auf die Wechselwirkung von Zweitbrücke und Kupferzentren. Die Irreversibilität der elektrochemischen Redoxprozesse bedeutet jedoch nicht grundsätzlich eine mangelnde Eignung der Komplexe als Oxidationskatalysatoren, entscheidend ist hier nur deren prinzipielle Reoxidierbarkeit. Nach Malachowski et al. muß das Redoxpotential in einem geeigneten Fenster für die leichte Reduktion des Catechols und anschließende Oxidation des Komplexes durch den molekularen Sauerstoff liegen. ${ }^{[76]}$ Im Falle von Malachowskis einkernigen Kupfer(II)komplexen mit tripodalen Pyrazol- und Pyridinliganden liegt dieses Fenster zwischen $E_{1 / 2}=-0.71$ und $-0.08 \mathrm{~V}$ mit einem Maximum der Aktivität bei ca. $-0.40 \mathrm{~V}$ (gegen Ferrocen), da außerhalb dieses Bereichs die Kupfer(II)- bzw. die Kupfer(I)-Spezies zu sehr stabilisiert werden. 
Es sollte jedoch erwähnt werden, daß andere Untersuchungen keinen klaren Zusammenhang zwischen Redoxpotential des Katalysators und katalytischer Aktivität herstellen konnten. ${ }^{[62,68,76,78]}$ Die für $\mathbf{1}-\mathbf{4}, \mathbf{7 a}, 8$ und 13 beobachteten $E_{\mathrm{p}}{ }^{\text {Red }}$-Werte sind in Tabelle 14 zusammengefaßt.

\begin{tabular}{lll}
\hline Komplex & $E_{\mathrm{p}}^{\text {Red,1 }}[\mathrm{V}]$ & $E_{\mathrm{p}}^{\text {Red,2 }}[\mathrm{V}]$ \\
\hline $\mathbf{1}$ & -0.75 & - \\
$\mathbf{2}$ & -0.68 & -1.08 \\
$\mathbf{3}$ & -0.76 & -1.03 \\
$\mathbf{4}$ & -0.90 & - \\
$\mathbf{7 a}$ & $*$ & $*$ \\
$\mathbf{8}$ & -0.67 & -1.14 \\
$\mathbf{1 3}$ & -0.53 & -1.11
\end{tabular}

Tabelle 16: Elektrochemische Eigenschaften der Komplexe in Acetonitril $\left(0.1 \mathrm{M} \mathrm{N}^{n} \mathrm{Bu}_{4} P F_{6}, 200 \mathrm{mV}^{1}\right.$ bei 1 - 4, $100 \mathrm{mV}^{1}$ bei 7a, 8, 13); Peak Potentiale in V vs. Ferrocen; * 7a lieferte keine verwertbaren Daten.

Trotz der elektrochemischen Irreversibilität der Redoxprozesse kann man das Redoxverhalten der einzelnen Komplexe doch zumindest qualitativ mit Hilfe ihres $E_{\mathrm{p}}{ }^{\text {Red }}$-Wertes miteinander vergleichen. Die Werte der Komplexe 1 - $\mathbf{4}$ wurden bereits im Rahmen der Diplomarbeit gemessen. ${ }^{[99]}$ Während man für 1, 2 und 3 ähnliche Werte von $-0.75,-0.68$ und $-0.76 \vee$ (gegen Ferrocen) findet, weist 4 mit $E_{\mathrm{p}}^{\text {Red }}=-0.90 \mathrm{~V}$ eine größere Stabilisierung der Kupfer(II)spezies auf, was mit dessen deutlich geringerer Aktivität gut konform geht. Der Komplex 13 mit dem höchsten $E_{\mathrm{p}}{ }^{\text {Red }}$ von $-0.53 \vee$ hat die größte Aktivität. 8 hat einhergehend mit seiner kleineren Catecholase-Aktivität auch ein niedrigeres $E_{p}{ }^{\text {Red }}$ von $-0.67 \vee$ (Abbildung 43). Bei 7a wurden nur schlecht definierte Reduktion- und Reoxidationspeaks gefunden. In fast allen Spektren findet man zwei Reduktionspeaks, von denen der erste bereits die Zweielektronenreduktion von $\mathrm{Cu}^{\prime \prime} \mathrm{Cu}^{\prime \prime} \mathrm{zu} \mathrm{Cu}^{\prime} \mathrm{Cu}^{\prime}$ darstellen könnte, während im nächsten Schritt eine Reduktion am Liganden stattfindet. Eine andere Möglichkeit ist die Reduktion zu Cu'Cu", einhergehend mit einer strukurellen Veränderung des Liganden, welche das Redoxpotential des zweiten Kupferions verschiebt. Der zweite Peak ist dann der Einelektronenreduktion von $\mathrm{Cu}^{\prime} \mathrm{Cu}^{\prime \prime} \mathrm{zu} \mathrm{Cu}^{\prime} \mathrm{Cu}^{\prime}$ zuzuordnen. Dies wurde im Rahmen der vorliegenden Arbeit nicht weiter untersucht. 


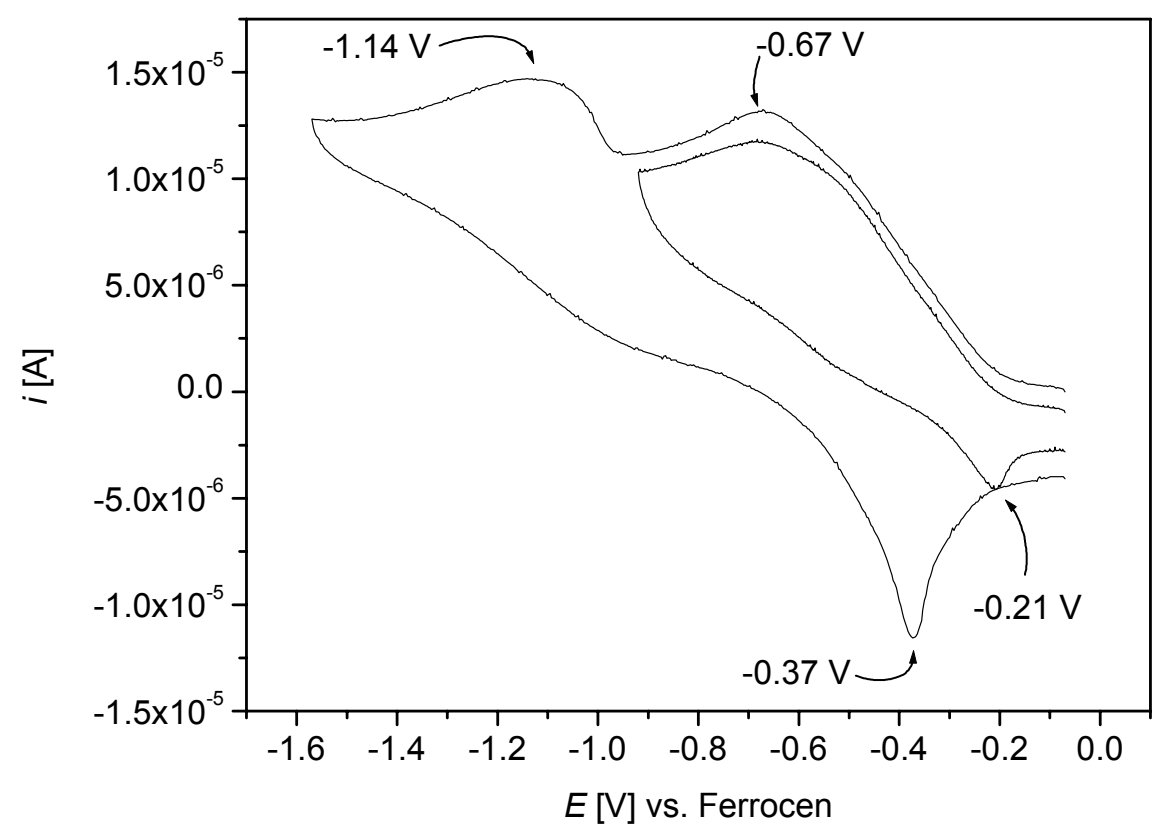

Abbildung 43: Cyclovoltammogramm von 8 in Acetonitril (0.1 $\left.\mathrm{M} \mathrm{N}^{n} \mathrm{Bu}_{4} P F_{6}, 100 \mathrm{mV}^{1}\right)$; Peak Potentiale in V vs. Ferrocen.

\subsection{Aktivität eines Dikupferkomplexes mit einem Triazacyclononan/Pyrazolat- Hybridliganden}

Die bisher untersuchten Dikupfer(II)komplexe haben offenkettige Liganden. Erste Untersuchungen des Komplexes LV mit dem Pyrazolatliganden $\mathrm{HL}^{9}$, der makrozyklische Tetraazacyclononan-Seitenarme aufweist (Abbildung 44), wurden bereits durchgeführt. ${ }^{[00,107]}$

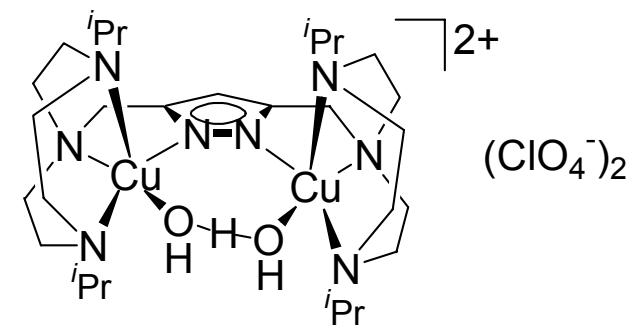

Abbildung 44: Struktur des Dikupferkomplexes $L V$ von $\left[L^{9} T\right.$.

Für die Catecholase-Testreaktion wurde auch hier eine lineare Abhängigkeit der Reaktionsgeschwindigkeit von der Katalysatorkonzentration gefunden, doch für die Abhängigkeit von $\left[\mathrm{H}_{2} \mathrm{dtbc}\right]_{0}$ konnte aufgrund des ungewöhnlichen Kurvenverlaufs mit Hilfe der limitierten Methode der Anfangsgeschwindigkeiten keine klare Aussage getroffen werden. Möglicherweise liegen hier mehrere konkurrierende 
Reaktionskanäle vor. Die äußerst geringe Aktivität dieses Komplexes mit $k_{\exp }=2.2 \pm$ $0.2 \mathrm{~h}^{-1}$ wurde zurückgeführt auf den großen Kupfer-Kupfer-Abstand von $4.411 \AA$, das sehr negative Reduktionspotential von $E_{p}{ }^{R e d}=-1.05 \mathrm{~V}$ (gegen Ferrocen), was einer ausgeprägten Stabilisierung der Kupfer(II)spezies entspricht, sowie auf die Rigidität des Komplexes, die durch den konformativ unbeweglichen Makrozyklus bedingt ist. Nicht zuletzt könnte die hohe Stabilitätskonstante des Kupferkomplexes verhindern, daß ausreichend freie Koordinationsstellen für die Anbindung des Substrats vorliegen.

Will man die Auswirkung der Länge des Kupfer-Kupfer-Abstandes solcher makrozyklischen Systeme auf deren katalytische Aktivität untersuchen, bietet es sich an, den Abstand nicht durch Wahl eines anderen - weniger stabilen - Makrozyklus, sondern durch Verwendung unsymmetrischer Liganden wie $\mathrm{HL}^{10} \mathrm{zu}$ variieren (Abbildung 45). ${ }^{[90,107]}$

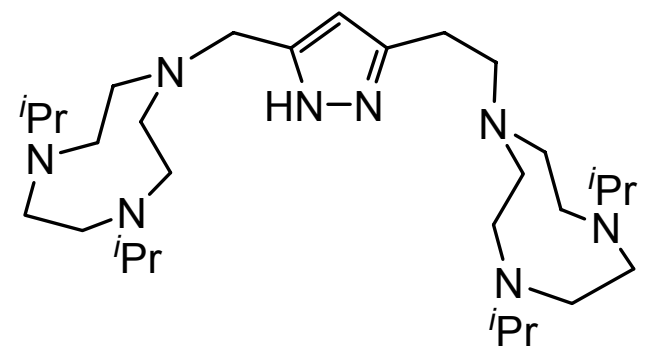

Abbildung 45: Der unsymmetrische Ligand $H L^{10}$.

Setzt man diesen Liganden in Ethanol mit zwei Äquivalenten Kalium-tert-butylat und anschließend mit zwei Äquivalenten Kupfer(II)perchlorat-Hexahydrat um und läßt nach Entfernen des Solvens im Vakuum aus Aceton durch Etherdiffustion auskristallisieren, so erhält man eine grüne Verbindung, die jedoch nicht in kristalliner Form erhalten werden konnte. Hinweise auf die Zusammensetzung dieses Komplexes ergeben sich aus dem Massenspektrum, der Elementaranalyse und dem Vergleich mit der Struktur eines röntgenographisch bereits charakterisierten Komplexes LVI von $\mathrm{HL}^{10}$ mit Nickel(II) (Abbildung 46). 


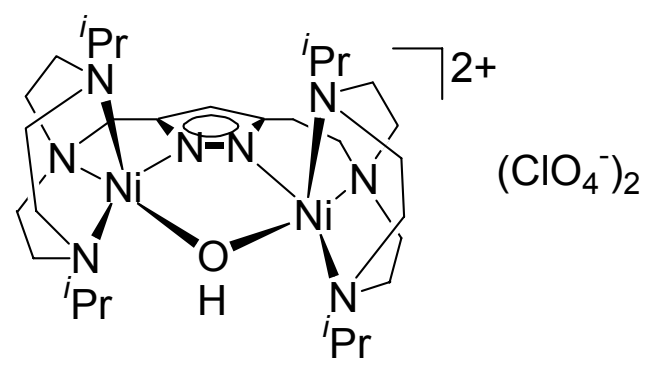

Abbildung 46: Der Dinickelkomplex LVI.

Aufgrund der größeren Länge eines Ligandseitenarms, sowie auch durch die größere Flexibilität, welche die zusätzliche Methylengruppe bedingt, ist eine Annäherung der Metallzentren möglich $\left(d_{\mathrm{N} i \cdots \mathrm{Ni}}=3.500 \AA\right)$. Daher wird eine kleinere $\mathrm{OH}^{-}$-Zweitbrücke zwischen die Nickelzentren eingebaut. Die Nickelzentren sind beide verzerrt quadratisch pyramidal koordiniert $\left(\tau_{\mathrm{Ni} 1}=0.40, \tau_{\mathrm{Ni} 2}=0.24\right) .{ }^{[90]}$ Eine entsprechende Struktur ist für den Dikupfer(II)komplex wahrscheinlich, da sich die Radien von $\mathrm{Ni}(I I)$ mit $0.69 \AA$ und $\mathrm{Cu}(\mathrm{II})$ mit $0.73 \AA$ kaum unterscheiden und Komplexe mit $\mathrm{HL}^{10}$ aufgrund der Rigidität des Makrozyklus auch eine analoge Koordinationsgeometrie aufweisen sollten. Im FAB-Massenspektrum findet man bei $m / z=773$ den Peak für die Spezies $\left[\mathrm{L}^{10} \mathrm{Cu}_{2}(\mathrm{OH})\left(\mathrm{ClO}_{4}\right)\right]^{+}$mit der Intensität $100 \%$ und bei $\mathrm{m} / \mathrm{z}=674$ mit $44 \%$ das Fragment $\left[\mathrm{L}^{10} \mathrm{Cu}_{2}(\mathrm{OH})\right]^{+}$. Zusammen mit der Elementaranalyse (ber.: $\mathrm{C} 41.19 \mathrm{H}$ $6.91 \mathrm{~N} 12.81$, exp.: $\mathrm{C} 41.26 \mathrm{H} 6.97 \mathrm{~N}$ 12.62) unterstützt dies die Annahme, daß der $\mathrm{OH}$-verbrückte Dikupfer(II)komplex $\left[\mathrm{L}^{10} \mathrm{Cu}_{2}(\mathrm{OH})\right]\left(\mathrm{ClO}_{4}\right)_{2}$ (15) vorliegt (Abbildung 47).

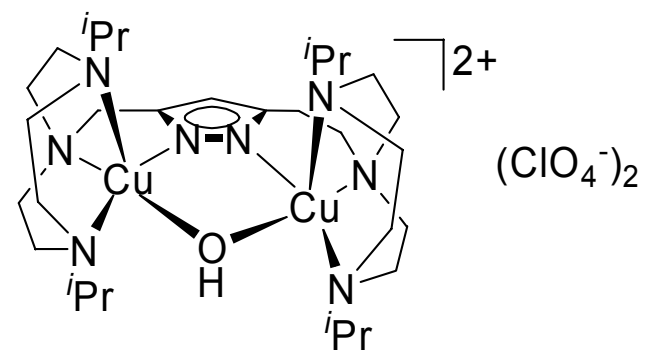

Abbildung 47: Postulierte Struktur des Komplexes 15.

Bei der cyclovoltammetrischen Analyse erwies sich die Reduktion der Kupfer(II)spezies als irreversibel, vermutlich aufgrund der Abdissoziation der $\mathrm{OH}$ Brücke bei der Reduktion. Ein $E_{\mathrm{p}}^{\text {Red }}$ wurde bei $-1.38 \mathrm{~V}$ gefunden. Aufgrund dieses stark negativen Potentials dürfte durch die starke Stabilisierung von Kupfer(II) nur eine sehr geringe Catecholase-Aktivität zu erwarten sein.

Bei Raumtemperatur in reinem Methanol konnte erwartungsgemäß keine 
nennenswerte Aktivität beim Umsetzen mit dem $\mathrm{H}_{2} \mathrm{dtbc}$-Testsubstrat festgestellt werden, zudem weist der Komplex eine Schulter bei $328 \mathrm{~nm}$ und eine starke Bande bei $375 \AA$ auf, so daß für die Absorptionsbande des erwarteten Reaktionsproduktes dtboc das Absorptionsmaximum bei $400 \mathrm{~nm}$ nicht deutlich erkennbar ist. Bei $210 \mathrm{~nm}$ und $267 \mathrm{~nm}$ sieht man weiterhin Banden, welche $\pi-\pi^{*}$-Übergängen des Liganden zugeordnet werden können. Die Lösung des freien Liganden in Methanol weist eine Bande bei $224 \mathrm{~nm}\left(\varepsilon=1170 \mathrm{~mol} \mathrm{l}^{-1} \mathrm{~cm}^{-1}\right)$ und eine Bande bei $280 \mathrm{~nm}$ (Schulter, $\varepsilon=190 \mathrm{~mol} \mathrm{I}^{-1} \mathrm{~cm}^{-1}$ ) auf. Die Banden bei $328 \mathrm{~nm}$ und $375 \mathrm{~nm}$ könnten von ChargeTransfer-Übergängen zwischen Ligand und den beiden Kupferzentren herrühren. Kupfer(II)komplexe von anderen Liganden mit der Triazacyclononangruppe weisen Übergänge im gleichen Bereich auf. ${ }^{[19,145,146]}$ Die Bande des d-d-Überganges der Kupferzentren liegt mit $629 \mathrm{~nm}$ in dem für Kupfer(II)verbindungen üblichen Bereich (Abbildung 48).

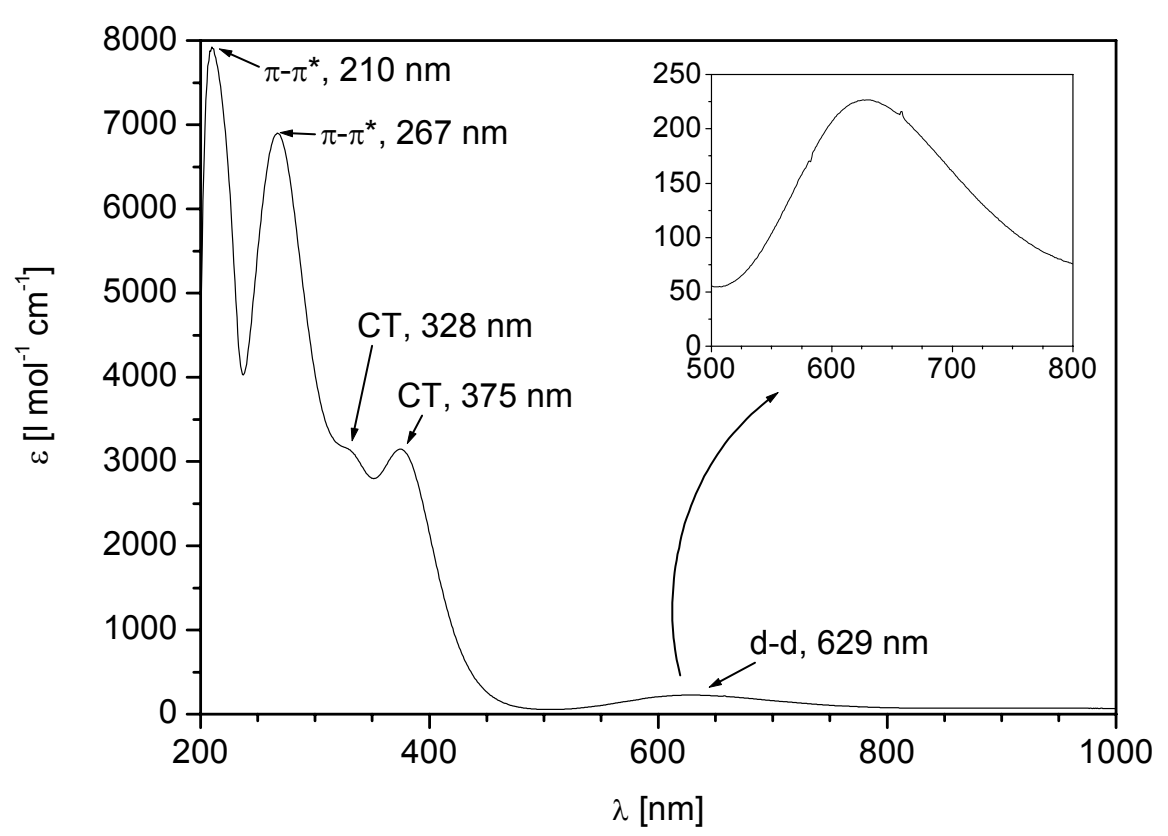

Abbildung 48: UVNVis-Spektrum von 15 in Methanol und vorgeschlagene Zuordnung der Banden.

Auch dieser Komplex zeigt eine gewisse Steigerung der Aktivität bei Durchführung der Reaktion im gepufferten Milieu. Alle folgenden Untersuchungen wurden bei erhöhter Temperatur von $40^{\circ} \mathrm{C}$ in luftgesättigtem Methanol in geschlossener Küvette gemacht, um eine Beschleunigung der Reaktion zu erreichen.

Die Untersuchung der pH-Abhängigkeit zeigt ein Maximum der Aktivität im Sauren 
bei $\mathrm{pH}=5.6 \mathrm{im}$ Essigsäure-Acetat-Puffer (Abbildung 49). Verwendung eines $\mathrm{Cl}^{-}-$ Gegenions bei Einsatz des TRIS-Puffers im neutralen und basischen Bereich führt hier zu einer deutlichen Verringerung der Aktivität, jedoch wird der Katalysator nicht vollständig durch Koordination des $\mathrm{Cl}^{-}$inhibiert.

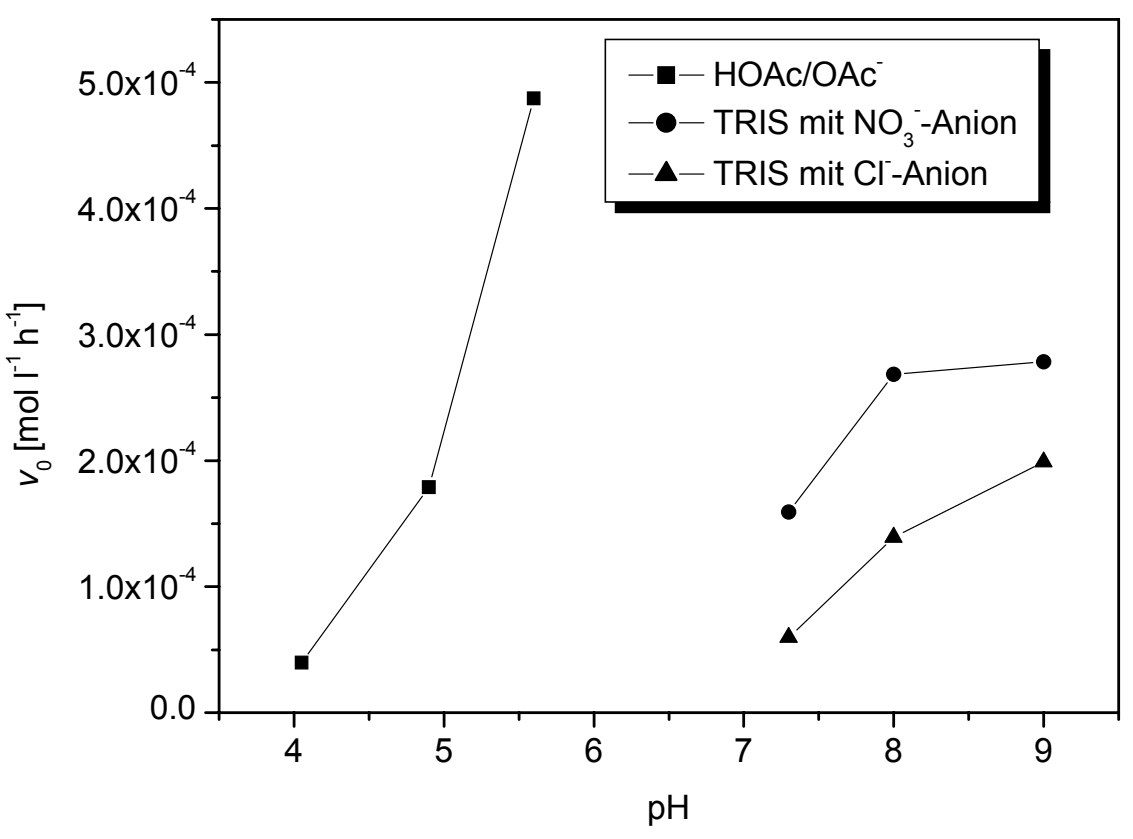

Abbildung 49: $\mathrm{pH}$-Abhängigkeit der Catecholase-Aktivität von 15 in Methanol mit verschiedenen Puffersystemen, $[\text { Kat }]_{0}=10^{-4} \mathrm{M},\left[\mathrm{H}_{2} \mathrm{dtbc}\right]_{0}=2 \cdot 10^{-3} \mathrm{M}$.

Eine erste Meßreihe mit variabler Komplexkonzentration von $4.8 \cdot 10^{-5} \mathrm{M}$ bis $2.4 \cdot 10^{-4} \mathrm{M}$ wurde bei $\mathrm{pH} 5.6$ durchgeführt. Die erhaltenen Kurvenverläufe waren jedoch nicht eindeutig. Man erhält zu Beginn einen sehr steilen Anstieg der Produktkonzentration. Danach nimmt die Aktivität des Komplexes stark ab, der Katalysator wird deaktiviert. Nur bei niedriger Katalysatorkonzentration von 4.8·10-5 $\mathrm{M}$ kann man das erhaltene Ergebnis so erklären, daß zunächst im ersten Schritt ein Äquivalent des Substrats umgesetzt wird, während der nächste Schritt die Reoxidierung der entstandenen Kupfer(I)-Spezies zu Kupfer(II) - nur sehr langsam abläuft (Abbildung 50), aber diese Interpretation widerspricht dem stark negativen $E_{\mathrm{p}}{ }^{\text {Red }}$ von 15, wonach die Kupfer(II)-Spezies eigentlich gegenüber Kupfer(I) stabilisiert sein sollte. Zudem scheint bei höheren Katalysatorkonzentrationen zunächst ein vielfaches der Substratmenge oxidiert zu werden, bevor der Katalysator seine Aktivität verliert. Eine weitere Möglichkeit ist, 
daß sich während der Reaktion oligomere Komplexspezies mit unterschiedlicher Aktivität bilden. Eine verläßliche Auswertung der erhaltenen Kurven mit Hilfe von polynomischen Fitkurven war aufgrund des ungewöhnlichen Kurvenverlaufes nicht möglich.

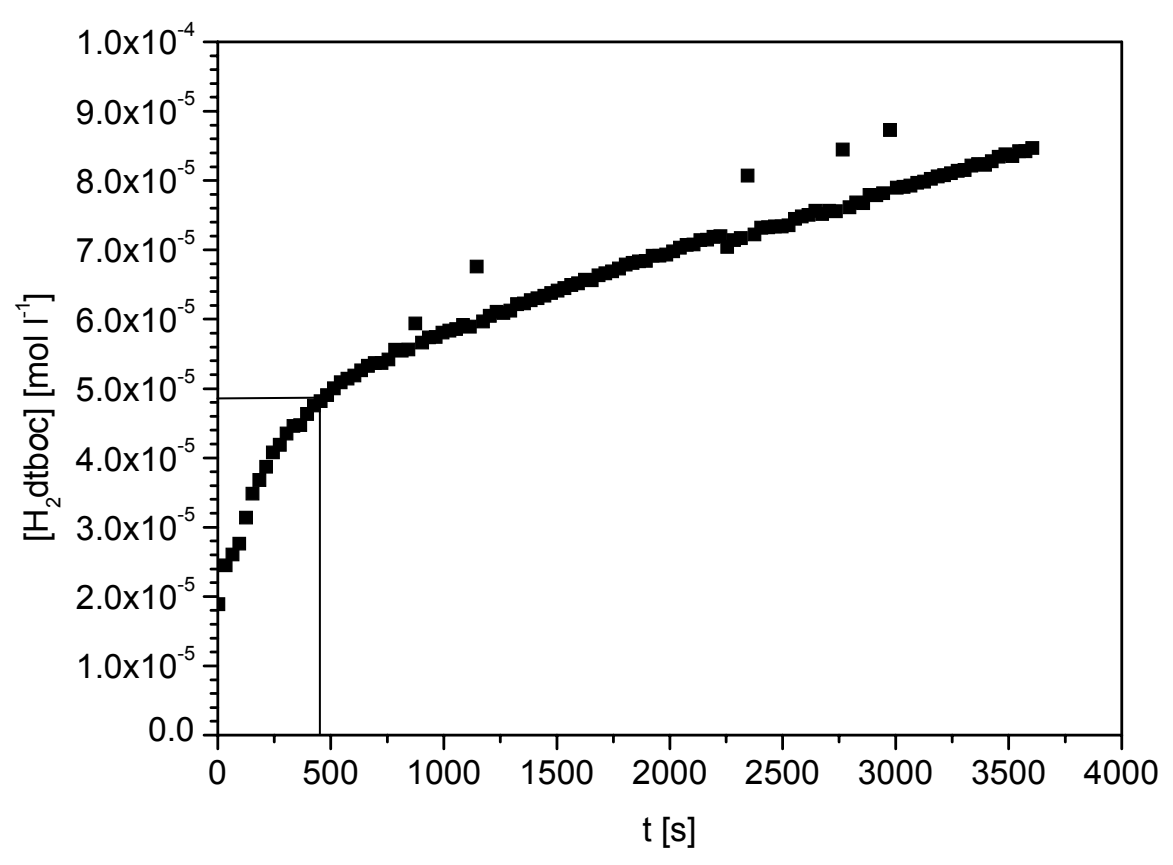

Abbildung 50: Konzentrations-Zeit-Verlauf der Chinonentwicklung mit dem Katalysator 15, [15] $]_{0}=$ 4.8·10 $0^{-5} \mathrm{M}$ mit Essigsäure/Acetat-Puffer.

Eindeutigere Ergebnisse lassen sich mit dem TRIS-Puffer erzielen. Da zudem TRISPuffer auch in den bisherigen Untersuchungen an den Komplexen 7a, 8 und 13 Verwendung fand, ist eine bessere Vergleichbarkeit gegeben. Gewählt wurde pH 8.0, da bei $\mathrm{pH} 9.0$ bereits verstärkte Autoxidation des Substrates beobachtet wurde.

In der ersten Meßreihe wurde [15] $]_{0}$ von $4.8 \cdot 10^{-5} \mathrm{M}$ bis $2.4 \cdot 10^{-4} \mathrm{M}$ variiert, $\left[\mathrm{H}_{2} \mathrm{dtbc}\right]_{0}$ lag konstant bei $2 \cdot 10^{-3} \mathrm{M}$. Hier ergibt sich eine lineare Abhängigkeit der Anfangsgeschwindigkeit von der Katalysatorkonzentration [15], durch lineare Regression erhält man $k_{\exp }=2.8 \mathrm{~h}^{-1}$ (Abbildung 51, Tabelle 17). Die Aktivität von 15 ist damit unter Berücksichtigung der erhöhten Temperatur während der Messung deutlich kleiner als bei LV. Dies entspricht der Erwartung, daß aufgrund des negativeren $E_{p}{ }^{R e d}$ von 15 die Reaktivität niedriger sein muß - ohne Puffer ist wie bereits erwähnt keine Aktivität zu beobachten. Der Abstand der Kupferzentren bei 15 
dürfte wegen der größeren Länge eines der Ligandseitenarme kleiner sein als bei LV, die Aktivität des Komplexes wird dadurch jedoch offensichtlich nicht begünstigt.

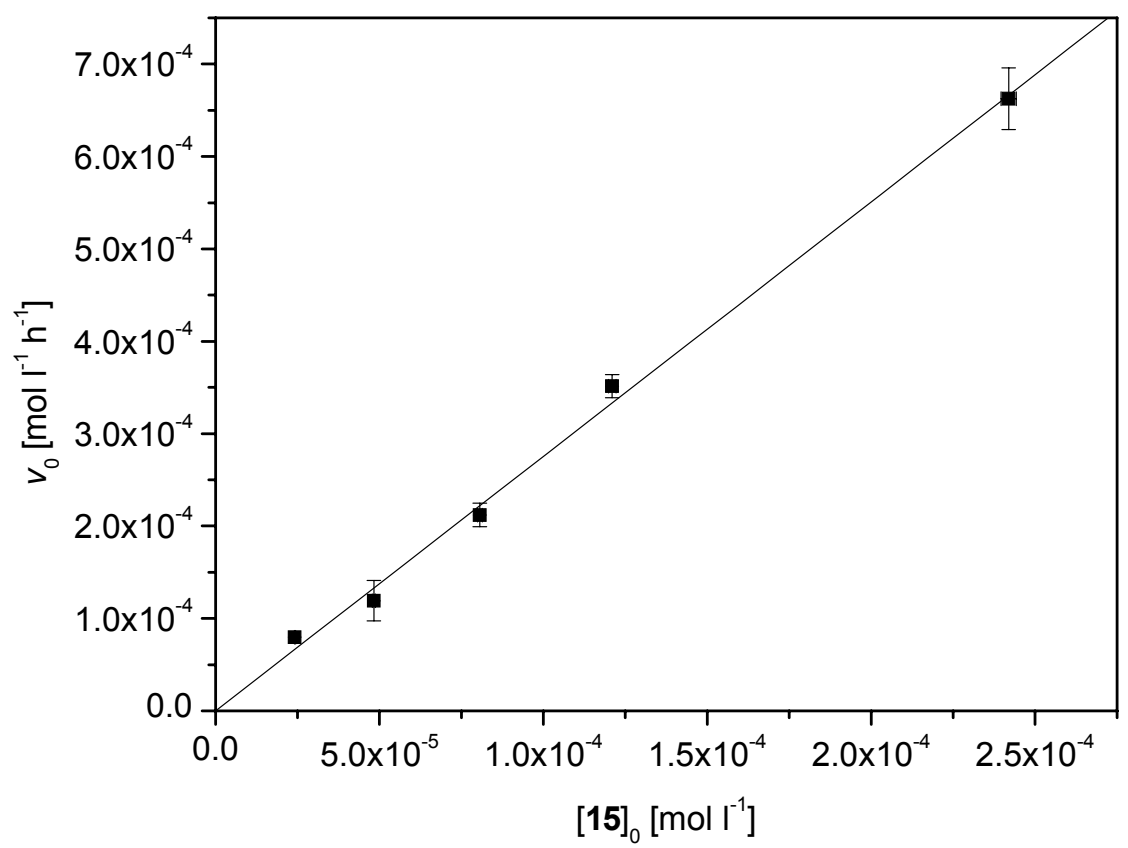

Abbildung 51: Abhängigkeit von $v_{0}$ von der gewählten Katalysator-Anfangskonzentration [Kat] $]_{0}$ von 15 bei $\mathrm{pH} 8.0$ bei konstanter Substrat-Anfangskonzentration von $2 \cdot 10^{-3} \mathrm{M}$ in Methanol mit TRIS-Puffer $(0.047 \mathrm{M})$.

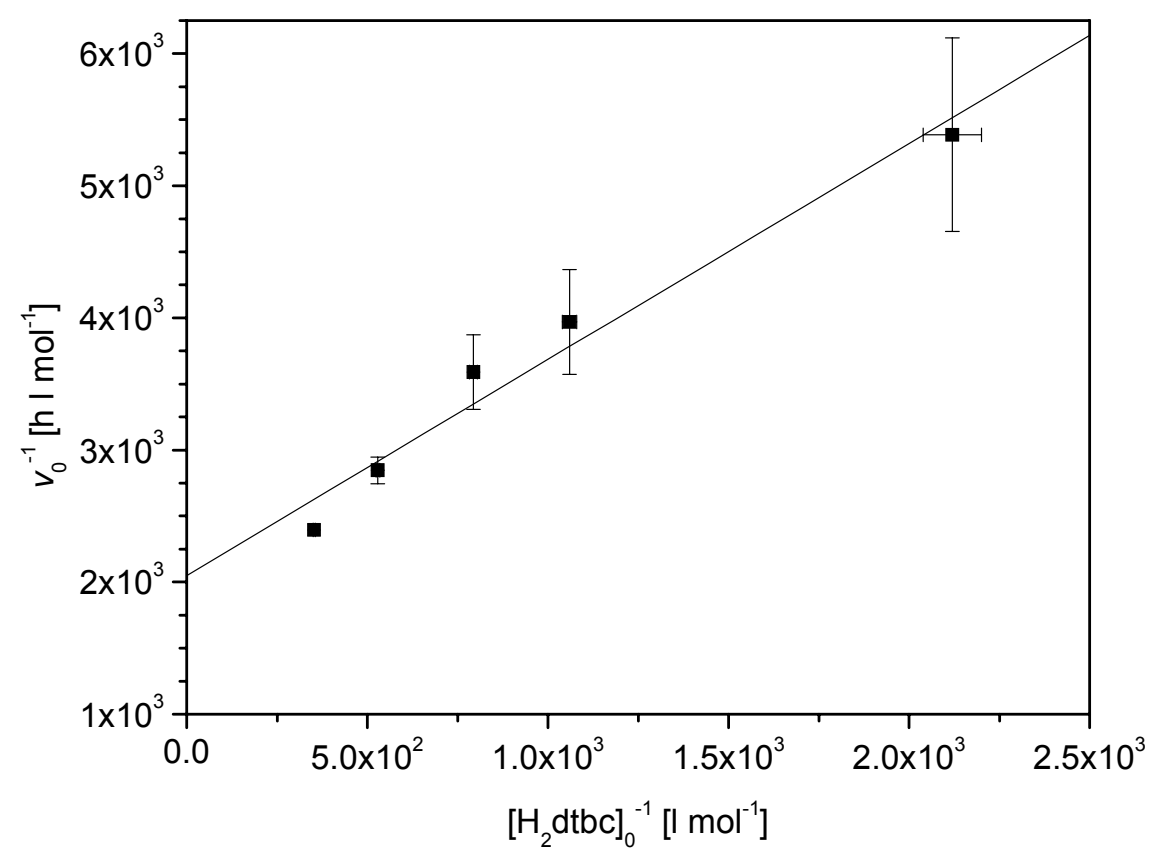

Abbildung 52: Lineweaver-Burke Plot für den Katalysator 15 bei $\mathrm{pH} 8,[15]_{0}=1.25 \cdot 10^{-4} \mathrm{M}$. 
Die in der zweiten Meßreihe erhaltene Abhängigkeit der Anfangsgeschwindigkeit von der Substratkonzentration kann auch hier wieder durch Anwendung einer MichaelisMenten-Kinetik ausgewertet werden (Abbildung 52). Der Lineweaver-Burke Plot bestätigt die in Meßreihe 1 erhaltenen Ergebnisse, $k_{c a t}$ liegt mit $3.9 \mathrm{~h}^{-1}$ höher als $k_{\exp }$ (Tabelle 17).

\begin{tabular}{cccccccc}
\hline & $\begin{array}{c}k_{\exp } \\
{\left[\mathrm{h}^{-1}\right]}\end{array}$ & $\begin{array}{c}T \\
{\left[{ }^{\circ} \mathrm{C}\right]}\end{array}$ & $\begin{array}{c}d_{\mathrm{Cu} \ldots \mathrm{Cu}} \\
{[\AA]}\end{array}$ & $\begin{array}{c}k_{\text {cat }} \\
{\left[\mathrm{h}^{-1}\right]}\end{array}$ & $\begin{array}{c}K_{\mathrm{M}} / 10^{-4} \\
{\left[\mathrm{~mol} \mathrm{I}^{-1}\right]}\end{array}$ & $\begin{array}{c}v_{\max } / 10^{-6} \\
{\left[\mathrm{~mol} \mathrm{I}^{-1} \mathrm{~min}^{-1}\right]}\end{array}$ & $\begin{array}{c}E_{\mathrm{p}}^{\text {Red }} \\
{[\mathrm{V}]}\end{array}$ \\
\hline $\mathbf{1 5}$ & $2.8 \pm 0.1$ & 40 & ca. 3.5 & $3.9 \pm 0.4$ & $18.0 \pm 1.1$ & $8.1 \pm 0.8$ & -1.38 \\
LV & $2.2 \pm 0.2$ & 20 & 4.41 & - & - & - & -1.05 \\
\hline
\end{tabular}

Tabelle 17: Kinetische Parameter der Oxidation von $\mathrm{H}_{2} \mathrm{dtbc}$ in Methanol der Katalysatoren 15 (mit 0.047 M TRIS) und LV, $v_{\max }$ wurde durch einen Lineweaver-Burke Plot erhalten, $E_{P}{ }^{R e d}$ wurde gegen Ferrocen bestimmt, [a] im analogen Dinickel(II)komplex LVI. ${ }^{[107]}$

Komplexe der Liganden $\mathrm{HL}^{9}$ und $\mathrm{HL}^{10}$ mit makrozyklischen Seitenarmen stellen offenbar nur schlechte Katalysatoren für die Catecholase-Reaktion dar. Kupfer(II) paßt hervorragend in die durch den Makrozyklus angebotene Kavität und wird somit gegenüber Kupfer(I) stabilisiert, was sich im sehr negativen $E_{\mathrm{p}}{ }^{\text {Red }}$ äußert. Zudem bildet der Makrozyklus mit dem Kupferzentrum stabile Chelatfünfringe, wodurch die Ausbildung freier Koordinationsstellen für die Substratanbindung erschwert wird.

Dagegen wurde bereits gezeigt, daß sich solche makrozyklischen Systeme wegen ihrer hohen Stabilitätskonstanten gut eignen, um Sauerstoffaddukte der Kupferkomplexe auch bei vergleichsweise hohen Temperaturen untersuchen zu können (siehe Kapitel 6). ${ }^{[107]}$

\subsection{Aktivität eines einkernigen Kupferkomplexes}

Zur Ergänzung der kinetischen Untersuchungen zweikerniger Kupferpyrazolatkomplexe wurde die Catecholase-Aktivität eines verwandten einkernigen Kupferpyrazolkomplexes untersucht (Abbildung 53). Dieser Komplex 16 weist einen Pyrazolliganden mit nur einem koordinierenden Seitenarm auf, das Kupferzentrum ist durch die drei N-Donoren des Pyrazolseitenarmes, das Pyrazol-NAtom und das N-Atom des Solvens Acetonitril fünffach koordiniert. 


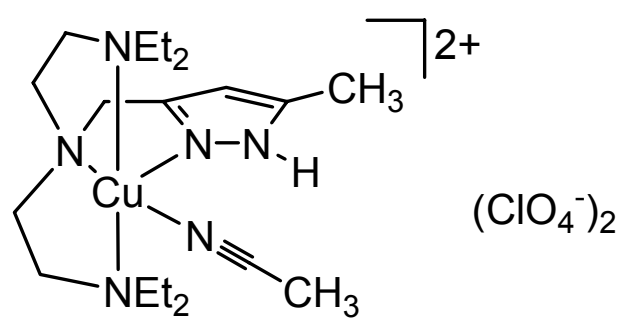

Abbildung 53: Struktur des einkernigen Kupferpyrazolkomplexes 16.

Da für die Zweielektronenoxidation des Catechols zwei Kupfer(II)zentren zu Kupfer(I) reduziert werden müssen, sollten im Falle eines einkernigen Komplexes im ersten Schritt der Reaktion zwei Katalysatormoleküle zur Oxidation eines Substrates notwendig sein, und somit eine Abhängigkeit zweiter Ordnung von $v_{0}$ von der Katalysatorkonzentration gefunden werden.

Der Komplex 16 ist im ungepufferten Medium recht aktiv, so daß auf einen Puffer verzichtet wurde. [Kat $]_{0}$ wurde zwischen $10^{-5} \mathrm{M}$ und $10^{-4} \mathrm{M}$ variiert, $\left[\mathrm{H}_{2} \mathrm{dtbc}\right]_{0}$ betrug $10^{-3} \mathrm{M}$. Die Messungen fanden in Methanol bei $20^{\circ} \mathrm{C}$ statt. Aus der Kurve ergibt sich zunächst, daß die Abhängigkeit der Anfangsgeschwindigkeit $v_{0}$ von [Kat] nicht linear ist (Abbildung 54).

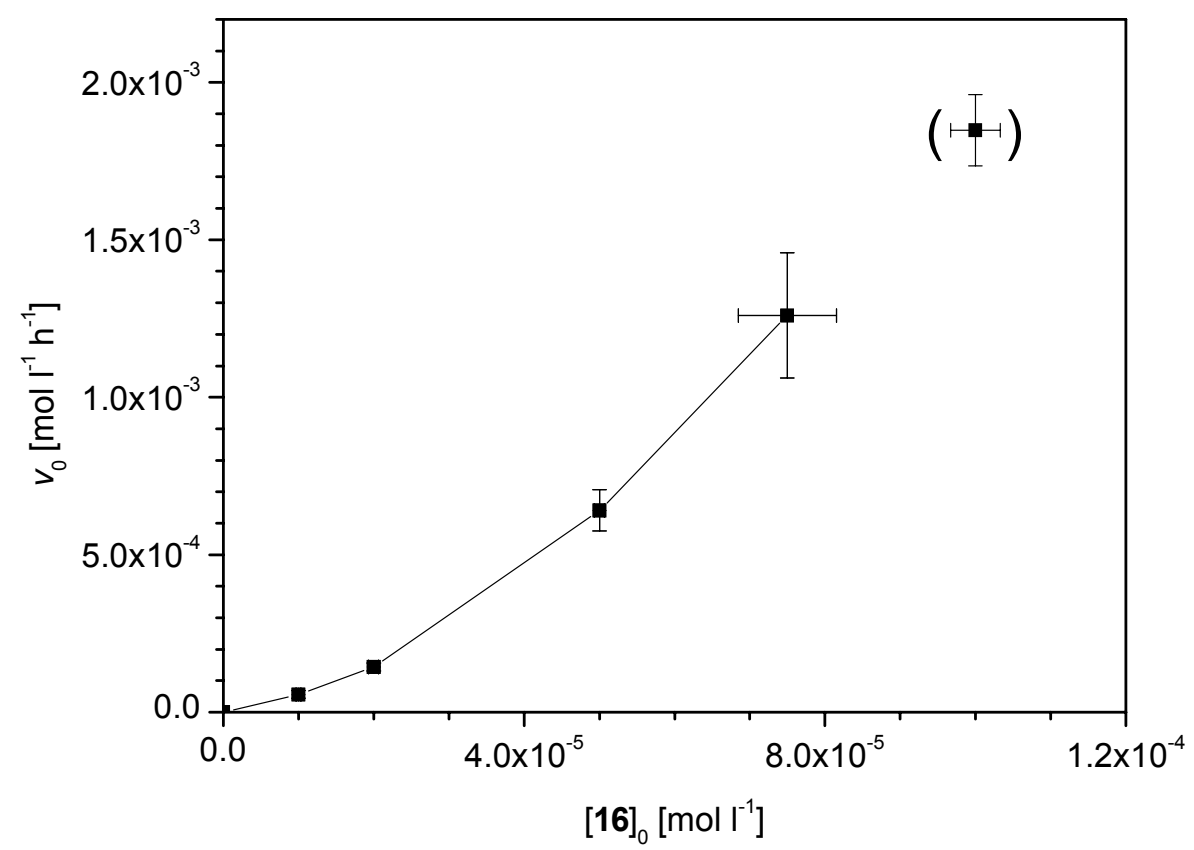

Abbildung 54: Abhängigkeit von $v_{0}$ von der gewählten Katalysator-Anfangskonzentration [Kat $]_{0}$ für Komplex 16 bei konstanter Substrat-Anfangskonzentration von $10^{-3} \mathrm{M}$ in Methanol. 
Unter der Annahme einer Abhängigkeit 2. Ordnung von der Katalysatorkonzentration ergibt sich das Geschwindigkeitsgesetz:

(16) $v_{0}=\left(\frac{\mathrm{d}([\mathrm{dtboc}])}{\mathrm{dt}}\right)_{0}=k_{\exp } \cdot[\mathrm{Kat}]_{0}^{2}$.

Trägt man also die experimentell erhaltenen $v_{0}$-Werte gegen das Quadrat der Katalysatorkonzentration auf, so sollte man eine Gerade erhalten, aus deren Steigung $k_{\exp }$ ermittelt werden kann. In der Tat zeigt $v_{0}$ über einen weiten Konzentrationsbereich eine lineare Abhängigkeit von der Katalysatorkonzentration, nur bei hoher Katalysatoranfangskonzentration von $10^{-4} \mathrm{M}$ und dementsprechend geringem Substratüberschuß von nur 10 Äquivalenten sinkt die Aktivität. Führt man für die restlichen Meßpunkte eine lineare Regression durch, so erhält man einen guten R-Wert von 0.99861, mit $k_{\exp }=2.30 \cdot 10^{5} \mathrm{I} \mathrm{mol}^{-1} \mathrm{~h}^{-1}$ (Abbildung 55). Somit ist in der Tat für einen mononuklearen Katalysator bei hohem Substratüberschuß wie erwartet eine Abhängigkeit 2. Ordnung gegeben.

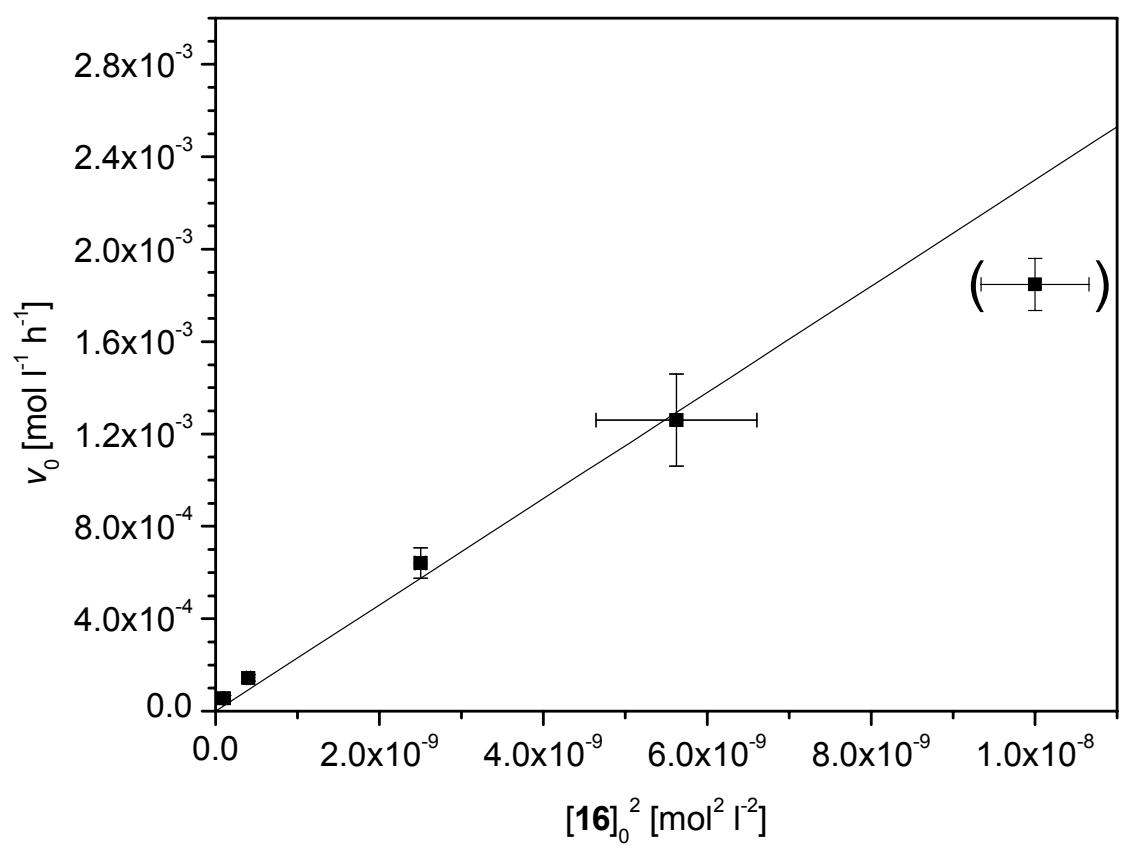

Abbildung 55: Abhängigkeit von vo vom Quadrat der gewählten Katalysator-Anfangskonzentration [Kat] von 16. 


\subsection{Nachweis der Bildung von $\mathrm{H}_{2} \mathrm{O}_{2}$}

Prinzipiell kann die Oxidation eines Catechols zum o-Chinon durch $\mathrm{O}_{2}$ auf verschiedenen Wegen mit zwei unterschiedlichen Stöchiometrien vonstatten gehen: Wird zur Oxidation des Substrats ein Äquivalent $\mathrm{O}_{2}$ benötigt (Weg I), so entstehen als Produkte der Reaktion Chinon und $\mathrm{H}_{2} \mathrm{O}_{2}$. Wird jedoch nur ein halbes Äquivalent gebraucht (Weg II), so erhält man als Endprodukte der Reaktion das Chinon und $\mathrm{H}_{2} \mathrm{O}$ (Schema 16). Die Identifizierung des Reduktionsproduktes von $\mathrm{O}_{2} \rightarrow \mathrm{H}_{2} \mathrm{O}$ oder $\rightarrow$ $\mathrm{H}_{2} \mathrm{O}_{2}$ liefert daher Hinweise auf den Reaktionsmechanismus.
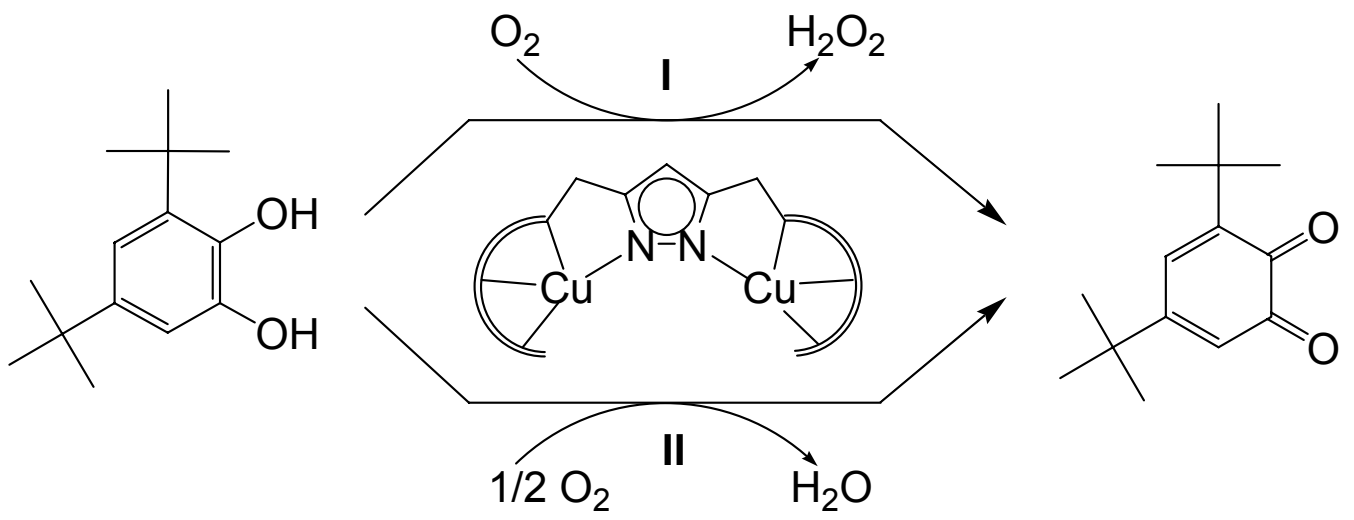

Schema 16: Catecholasereaktion, mögliche Nebenprodukte sind $\mathrm{H}_{2} \mathrm{O}_{2}$ oder $\mathrm{H}_{2} \mathrm{O}$.

Der Nachweis von $\mathrm{H}_{2} \mathrm{O}_{2}$ als Nebenprodukt der Reaktion erfolgte indirekt durch UV/Vis-Spektroskopie, indem zunächst die Catecholase-Reaktion durch Zugabe von $\mathrm{H}_{2} \mathrm{SO}_{4}$ gequencht wurde und nach Entfernen des o-Chinons (welches den $\mathrm{H}_{2} \mathrm{O}_{2}$ Nachweis stört) durch Ausschütteln mit Dichlormethan mit einer KI-Lösung versetzt wurde. $\mathrm{H}_{2} \mathrm{O}_{2}$ oxidiert das $\mathrm{I}^{-}$zum $\mathrm{I}_{3}^{-}$, welches eine starke Absorptionsbande bei 353 $\mathrm{nm} \quad\left(\varepsilon=26000 \mathrm{Imol}^{-1} \mathrm{~cm}^{-1}\right)$ aufweist. Diese Reaktion kann spezifisch durch das Enzym Lactoperoxidase beschleunigt werden. ${ }^{[62,63,147]}$ Zusätzlich wurde zur Bestätigung der erhaltenen Ergebnisse eine Kontrolle mit einem Standard $-\mathrm{H}_{2} \mathrm{O}_{2}$ Nachweisstäbchen der Firma Merck durchgeführt. In allen untersuchten Fällen stimmten die Ergebnisse beider Nachweismethoden überein.

Im Falle der Komplexe 1 - 3 wurde ein Anstieg der Absorption bei 353 nm beobachtet, der bei $171 \%$, bei 2 63\% und bei 3 58\% der theoretisch erwarteten maximalen Absorption bei vollständigem Substratumsatz entspricht, was das Auftreten von $\mathrm{H}_{2} \mathrm{O}_{2}$ als Nebenprodukt belegt. 4 liefert ein nicht so eindeutiges Bild, die Absorption steigt nur auf $17 \%$ der erwarteten Absorption. In jedem Falle ist ein 
gewisser Verlust an $\mathrm{H}_{2} \mathrm{O}_{2}$ während der Aufarbeitung zu erwarten, insbesondere durch das Schütteln mit Dichlormethan, da hier ein gewisser Prozentsatz Wasser und in diesem gelöste Substanzen in der organischen Phase verbleiben. Zudem dürfte sich das $\mathrm{H}_{2} \mathrm{O}_{2}$ schon während der Reaktion allmählich zersetzen, was insbesondere bei 4, dem mit Abstand langsamsten der Komplexe, dazu führen kann, daß letztlich nur ein geringer Anteil des ursprünglich gebildeten $\mathrm{H}_{2} \mathrm{O}_{2}$ noch detektiert wird (Abbildung 56).

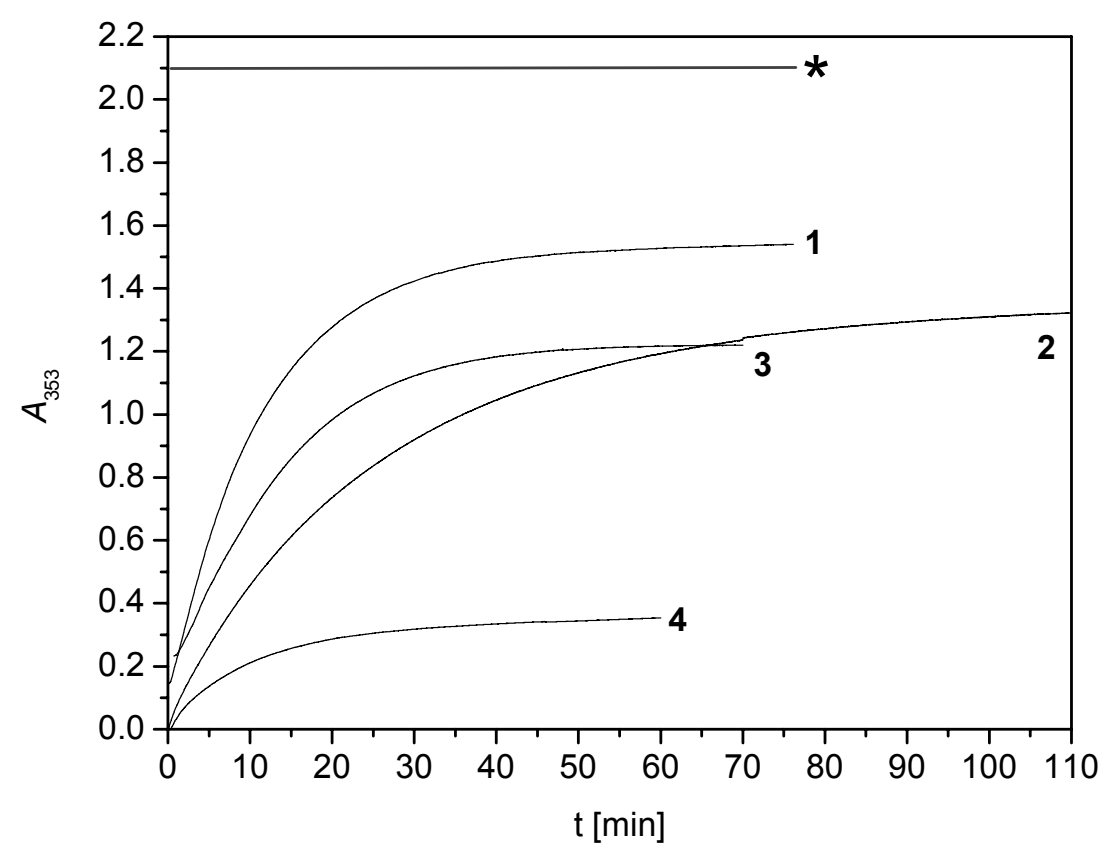

Abbildung 56: Entwicklung der $I_{3}^{-}$-Bande bei 353 nm für die Komplexe 1 - 4, * erwartete Absorption bei einer Stöchiometrie gemäß Weg I (Schema 16).

Für die aktiven Komplexe 1 - $\mathbf{3}$ wurde noch ein weiteres Experiment durchgeführt, um die Art des Nebenproduktes bei deren Catecholase-Reaktion zu ermitteln. Die Löslichkeit von $\mathrm{O}_{2}$ in Methanol beträgt unter Raumbedingungen $2.5 \cdot 10^{-3} \mathrm{M}^{[148]}$ Setzt man eine $5 \cdot 10^{-3}$ molare Lösung des Substrates zusammen mit dem Katalysator in luftgesättigtem Methanol in einer luftdicht abgeschlossenen Küvette um, so sollte das im Methanol befindliche $\mathrm{O}_{2}$ ausreichen, um entweder nach Weg I $2.5 \cdot 10^{-3} \mathrm{~mol} \mathrm{I}^{-1}$ Substrat unter $\mathrm{H}_{2} \mathrm{O}_{2}$-Entwicklung, oder im Weg II bei gleichzeitiger Entstehung von $\mathrm{H}_{2} \mathrm{O} 5 \cdot 10^{-3} \mathrm{~mol} \mathrm{I}^{-1}$ des Substrats zu oxidieren. Die Aktivität von 4 war für dieses Experiment zu gering, so daß selbst bei längerer Meßdauer von $15 \mathrm{~h}$ nur Bruchteile eines Äquivalentes des Substrates umgesetzt wurden. 
Setzt man eine $10^{-4}$ molare Katalysatorlösung mit $5 \cdot 10^{-3} \mathrm{~mol} \mathrm{I}^{-1}$ Substrat unter diesen Bedingungen um, so beobachtet man in der Tat, daß die Reaktion nach einigen Stunden nach Entstehung von $2.1 \cdot 10^{-3} \mathrm{~mol}^{-1} \mathrm{dtboc}$ stoppt. Erst nach einer Injektion von Luft in die Küvette startet die Reaktion von neuem und stoppt auch hier erst wieder, wenn weitere $2.4 \cdot 10^{-3} \mathrm{~mol} \mathrm{I}^{-1}$ Substrat umgesetzt sind (Abbildung 57). Dies bestätigt das Ergebnis des Lactoperoxidase-beschleunigten $\mathrm{I}_{3}{ }^{-}$-Nachweises: Zunächst wird das Substrat vom gelösten $\mathrm{O}_{2}$ zum o-Chinon oxidiert, wobei als Nebenprodukt $\mathrm{H}_{2} \mathrm{O}_{2}$ entsteht. Nachdem auf diese Weise der gesamte Sauerstoff aufgebraucht ist, kann erst nach erneuter Luftzugabe der Rest des $\mathrm{H}_{2} \mathrm{dtbc}$ zum Chinon oxidiert werden.

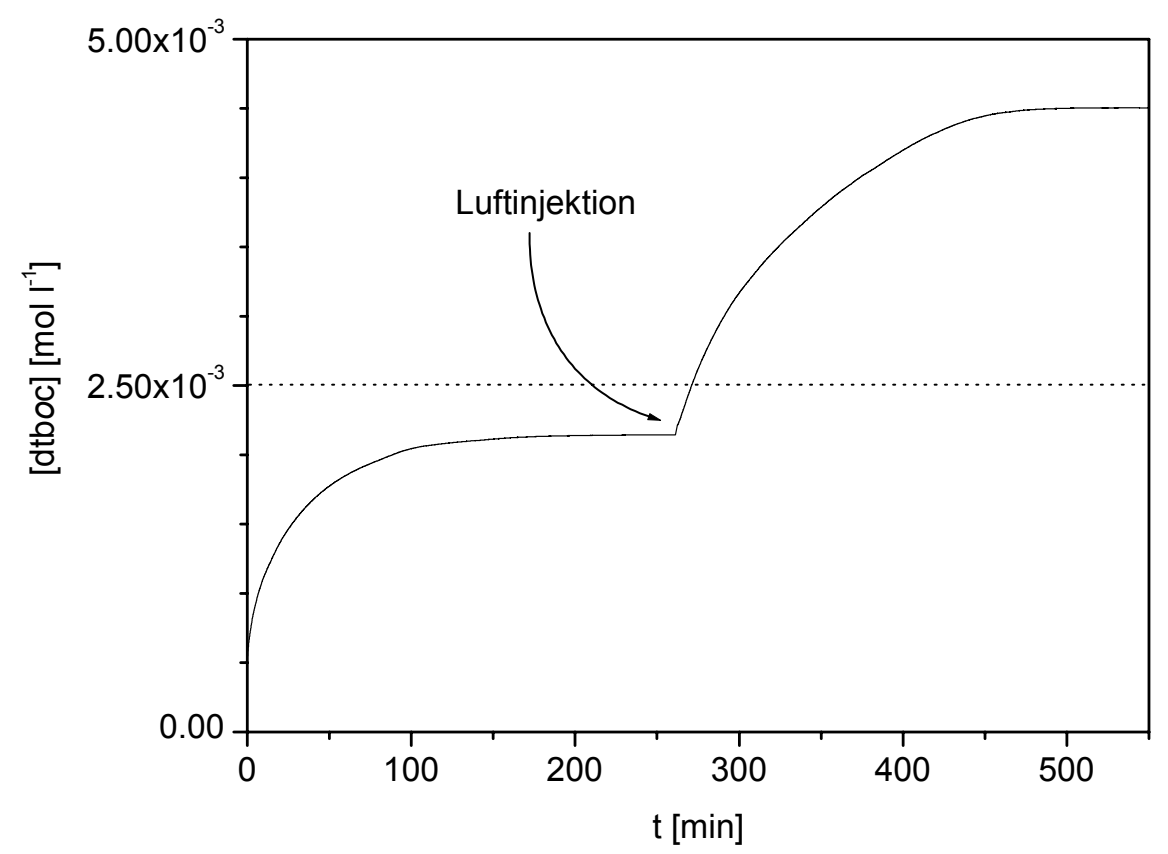

Abbildung 57: Zeitverlauf der Oxidation von $\mathrm{H}_{2}$ dtbc durch Komplex 1 in Methanol mit zusätzlicher Luftinjektion, $[1]_{0}=10^{-4} \mathrm{M},\left[\mathrm{H}_{2} \mathrm{dtbc}\right]_{0}=5 \cdot 10^{-3} \mathrm{M}$.

Die Komplexe 7a, 8 und 13 wurden ebenfalls auf eine mögliche $\mathrm{H}_{2} \mathrm{O}_{2}$-Entstehung hin untersucht. Der Lactoperoxidase-beschleunigte $\mathrm{I}_{3}{ }^{-}$-Nachweis fiel hier aber eindeutig negativ aus, ebenso wie der Nachweis mit dem Teststäbchen. Setzt man dagegen die Komplexe mit $\mathrm{O}_{2}$-gesättigtem Methanol in einer geschlossenen Küvette um, so erhält man das gleiche Ergebnis wie in Abbildung 57, d.h. es wird immer nur ein Äquivalent des Substrates umgesetzt. Daher wurde eine Blindprobe des Lactoperoxidase-Tests durchgeführt, indem zum Komplex in gepuffertem Methanol 
$\mathrm{H}_{2} \mathrm{O}_{2}$ zugegeben wurde. Mit dieser Probe wurde nach 15 min der $\mathrm{I}_{3}^{-}$-Nachweis durchgeführt, welcher eindeutig positiv war. Somit kann ausgeschlossen werden, daß während der Reaktion $\mathrm{H}_{2} \mathrm{O}_{2}$ entsteht, aber aufgrund der Reaktionsbedingungen oder durch den Katalysator selbst so schnell wieder zersetzt wird, daß es nicht mehr nachgewiesen werden kann. Möglicherweise kommen in der geschlossenen Küvette andere Effekte zum tragen. Zu beachten ist, daß anders als in der offenen Küvette die $\mathrm{O}_{2}$-Konzentration hier stetig abnimmt und dadurch die Reaktion stark verlangsamt werden bzw. ganz zum Stillstand kommen kann.

\subsection{Diskussion der katalytischen Aktivität der Dikupferpyrazolatkomplexe}

In Tabelle 19 sind die erhaltenen kinetischen Parameter der untersuchten

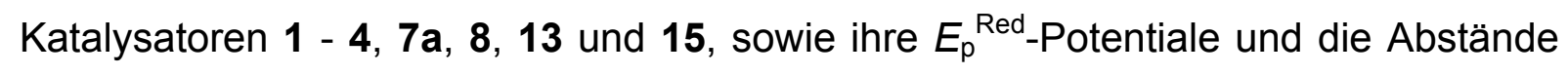
zwischen den Kupferzentren in der Festkörperstruktur angegeben. Zum Vergleich sind ebenfalls einige Systeme aufgelistet, die von anderen Arbeitsgruppen untersucht wurden. Die in dieser Arbeit untersuchten Dikupferpyrazolatkomplexe decken praktisch die ganze Bandbreite an katalytischer Aktivität biomimetischer Modellkomplexe der Catechol-Oxidase ab. Die Katalysatoren 2 und 13 bewegen sich hinsichtlich ihrer katalytischen Aktivität im Spitzenbereich, der Komplex 15 mit den makrozyklischen Ligand-Seitenarmen dagegen weist keine nennenswerte Aktivität auf.

Zum Vergleich sind in Tabelle 18 die Geschwindigkeits- und Michaelis-Konstanten für die Catecholase-Aktivität des Enzyms Tyrosinase für verschiedene natürliche Catecholamine aufgeführt. ${ }^{[149]}$

\begin{tabular}{ccc}
\hline Substrat & $k_{\text {cat }}\left[\mathrm{h}^{-1}\right]$ & $K_{M}\left[\mathrm{~mol}^{-1}\right]$ \\
\hline L-DOPA & 69480 & $4.55 \cdot 10^{-4}$ \\
D-DOPA & 109440 & $1.16 \cdot 10^{-3}$ \\
L-Adrenalin & 108 & $2.64 \cdot 10^{-4}$ \\
L-Noradrenalin & 7 & $1.01 \cdot 10^{-4}$ \\
\hline
\end{tabular}

Tabelle 18: Kinetische Parameter der Oxidation verschiedener Substrate durch das Enzym Tyrosinase. 


\begin{tabular}{|c|c|c|c|c|c|c|c|c|}
\hline & $\begin{array}{l}T \\
{\left[{ }^{\circ} \mathrm{C}\right]}\end{array}$ & $\begin{array}{l}k_{\exp } \\
{\left[\mathrm{h}^{-1}\right]}\end{array}$ & $\begin{array}{l}d_{\mathrm{Cu} \ldots \mathrm{Cu}} \\
[\AA]]\end{array}$ & $\begin{array}{l}k_{\text {cat }} \\
{\left[\mathrm{h}^{-1}\right]}\end{array}$ & $\begin{array}{l}K_{\mathrm{M}} \\
{\left[\mathrm{mol} \mathrm{I}^{-1}\right]}\end{array}$ & $\begin{array}{l}V_{\max } \\
{\left[\mathrm{mol} \mathrm{I}^{-1} \mathrm{~min}^{-1}\right]}\end{array}$ & $E_{\mathrm{p}}^{\text {Red, },}[\mathrm{V}]^{* \star * *}$ & Ref. \\
\hline 1 & 20 & $1400 \pm 200$ & 3.54 & $--^{* *}$ & $-* *$ & $-* *$ & -0.75 & [100] \\
\hline 2 & 20 & $2430 \pm 202$ & 3.45 & $2804 \pm 388$ & $(8.9 \pm 3.5) \cdot 10^{-5}$ & $(4.7 \pm 0.6) \cdot 10^{-4}$ & -0.68 & [100] \\
\hline 3 & 20 & $514 \pm 70$ & 4.09 & $--^{* *}$ & $-^{* *}$ & $-{ }^{* *}$ & -0.76 & [100] \\
\hline 4 & 20 & $22.8 \pm 1.2$ & 4.53 & $31.6 \pm 11.3$ & $(7.9 \pm 4.0) \cdot 10^{-4}$ & $(5.3 \pm 1.9) \cdot 10^{-6}$ & -0.90 & [100] \\
\hline 8 & 20 & $593 \pm 4$ & 4.36 & $1125 \pm 102$ & $(1.0 \pm 0.1) \cdot 10^{-3}$ & $(3.8 \pm 0.3) \cdot 10^{-4}$ & -0.67 & - \\
\hline $7 a$ & 20 & $629 \pm 12$ & 4.29 & $1432 \pm 93$ & $(1.4 \pm 0.1) \cdot 10^{-3}$ & $(4.8 \pm 0.3) \cdot 10^{-4}$ & $--^{* * *}$ & - \\
\hline 13 & 20 & $5178 \pm 62$ & $\begin{array}{l}3.51 / \\
3.52\end{array}$ & $6502 \pm 239$ & $(7.5 \pm 0.5) \cdot 10^{-4}$ & $(10.8 \pm 0.3) \cdot 10^{-4}$ & -0.53 & - \\
\hline 15 & 40 & $2.8 \pm 0.1$ & ca. $3.5^{*}$ & $3.9 \pm 0.4$ & $(18.0 \pm 1.1) \cdot 10^{-4}$ & $(8.1 \pm 0.8) \cdot 10^{-6}$ & -1.38 & - \\
\hline LV & 20 & $2.2 \pm 0.2$ & 4.41 & $-* * *$ & $-* * *$ & $-_{-* * *}$ & -1.05 & [107] \\
\hline$\left[\mathrm{Cu}_{2}(\mathbf{X X X V I})(\mathrm{OH})\right]^{2+}$ & 25 & 13827 & $\sim 3.7$ & $--_{* *}^{* *}$ & $-_{* *}^{* *}$ & & k.A. & [67] \\
\hline$\left[\mathrm{Cu}_{2}(\mathbf{X X X V})_{2}\right]^{2}$ & 25 & 279 & $\sim 3.7$ & $--^{* *}$ & $--^{* *}$ & & k.A. & [67] \\
\hline "Cu $\mathrm{Cu}_{3} \mathrm{XXXI1"}$ & k.A. & $102 \pm 4$ & $\begin{array}{l}3.21 \\
5.74\end{array}$ & $138 \pm 8$ & $(8.9 \pm 0.5) \cdot 10^{-4}$ & & k.A. & [66] \\
\hline "CuXXXXIII" & k.A. & $9927 \pm 392$ & k.A. & $9471 \pm 255$ & $(5.9 \pm 0.4) \cdot 10^{-3}$ & & k.A. & [66] \\
\hline $\begin{array}{l}{\left[\mathrm{Cu}_{2} \text { (XXXIV) }(\mathrm{OH})\right.} \\
\left.\left(\mathrm{H}_{2} \mathrm{O}\right)(\mathrm{EtOH})\right]^{2+}\end{array}$ & k.A. & k.A. & 2.90 & $214 \pm 5$ & $(2.4 \pm 0.2) \cdot 10^{-4}$ & & -0.80 & [68] \\
\hline $\begin{array}{l}{\left[\mathrm{Cu}_{2}(\mathbf{X X X V I I I )}\right.} \\
\left.(\mathrm{OAc})\left(\mathrm{H}_{2} \mathrm{O}\right)_{2}\right]^{2+}\end{array}$ & 25 & k.A. & $\sim 3.4$ & 28 & $7.9 \cdot 10^{-4}$ & & -0.93 & [69] \\
\hline
\end{tabular}

Tabelle 19: kinetische Daten verschiedener bei Catecholase-Reaktion aktiver Katalysatoren, ${ }^{*}$ im analogen Dinickel(II)komplex LVI, ${ }^{[90] \quad * *}$ Abhängigkeit 0. Ordnung bzgl. Substrat, ${ }^{* * *}$ unklare Ergebnisse, ${ }^{* * * *}$ gegen Ferrocen. 
Ein direkter Vergleich der Aktivität von z.T. sehr verschiedenen Modellsystemen macht aufgrund der Vielfalt der Parameter, welche die katalytische Aktivität bestimmen, keinen Sinn. Tendenzen innerhalb einer Modellkomplexklasse wie den hier untersuchten Dikupferpyrazolatkomplexen mit strukturell eng verwandten Katalysatoren können aber sehr wohl aussagekräftig sein. Die Komplexe 1 - 4, welche in reinem Methanol untersucht wurden, werden im Folgenden prinzipiell getrennt betrachtet von jenen im gepufferten Medium .

1. Abstand der Kupferzentren: Ein Abstand von ca. $3.5 \AA$ scheint ideal zu sein, mit zunehmendem Abstand nimmt die Aktivität deutlich ab (Abbildung 58).

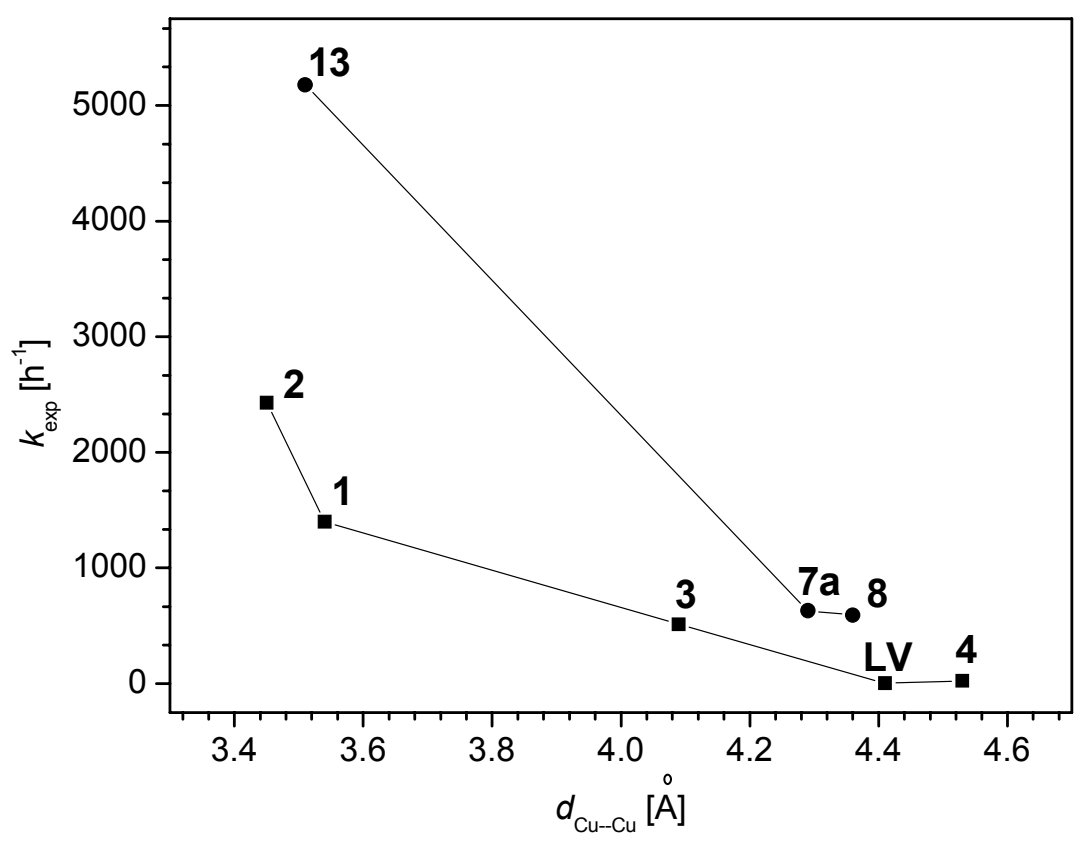

Abbildung 58: Kupfer-Kupfer-Abstand der Katalysatoren und ihre katalytische Aktivität.

Dies spricht für eine Korrelation des Kupfer-Kupfer-Abstandes mit der Catecholase-Aktivität der Komplexe. Für eine erfolgreiche Zweielektronenoxidation des Substrates muß also in einem Schritt der Katalyse eine Wechselwirkung zwischen dem Substrat und beiden Kupferzentren stattfinden, z.B. durch Ausbildung einer verbrückenden Koordination zwischen den Metallen. Es sollte jedoch immer berücksichtigt werden, daß die im Festkörper gefundenen Abstände nicht unbedingt jenen in Lösung entsprechen. Die verwendeten Liganden haben ausreichende konformationelle Freiheit, um den Einbau von Zweitbrücken unterschiedlicher 
Größe zwischen den Kupferzentren zu erlauben. Diese Zweitbrücke kann durchaus den Abstand der Kupferzentren in erheblichem Maße beeinflussen (Tabelle 20). Daraus folgt, daß der Kupfer-Kupfer-Abstand nach Abdissoziation der Zweitbrücke in Lösung sich durchaus von dem in der Kristallstruktur gefundenen unterscheiden kann. Wichtig ist jedoch festzustellen, daß z.B. der Ligand $\left[\mathrm{L}^{6}\right]^{-}$einen für die Substratanbindung günstigen kleinen Kupfer-Kupfer-Abstand, wie bei 10 und 13 gesehen, realisieren kann, was bei $\left[L^{5}\right]^{-}$nicht möglich zu sein scheint, da hier ausschließlich große Zweitbrücken eingebaut werden und Kupfer-KupferAbstände $>4 \AA$ beobachtet werden.

\begin{tabular}{lllll}
\hline Zweitbrücke & {$\left[L^{5} J\right.$} & $d_{\mathrm{Cu} \cdots \mathrm{Cu}}[\AA]$ & {$\left[L^{6} T\right.$} & $d_{\mathrm{Cu} \cdots \mathrm{Cu}}[\AA]$ \\
\hline Acetat & $\mathbf{6}$ & $4.045(1)$ & $\mathbf{9}$ & $4.268(1)$ \\
$\mathrm{MeOH} \cdots \mathrm{F}$ & $\mathbf{7 a}$ & $4.290(1)$ & $\mathbf{1 1}$ & $4.430(1)$ \\
$\mathrm{H}_{2} \mathrm{O} \cdots \mathrm{F}$ & $\mathbf{7 b}$ & $4.287(1)$ & &.$/$ \\
$\left(\mathrm{O}_{2} \mathrm{~N}\right) \mathrm{O} \cdots \mathrm{HOMe}$ & $\mathbf{8}$ & $4.357(1)$ & &.$/$. \\
$\mathrm{OH}+\mathrm{ClO}_{4}-$-Gegenion & &.$/$ & $\mathbf{1 0}$ & $3.441(1)$ \\
$\mathrm{OH}+\mathrm{CF}_{3} \mathrm{SO}_{3}-$-Gegenion & &.$/$ & $\mathbf{1 3}$ & $3.515(5)[3.518(5)]$ \\
$\mathrm{Cl}$ & &.$/$ & $\mathbf{1 2}$ & $3.829(2)$
\end{tabular}

Tabelle 20: Abstände der Kupferzentren von Komplexen der Liganden $\left[L^{5} J\right.$ und $\left[L^{6} J\right.$ bedingt durch unterschiedliche Zweitbrücken.

2. Koordination der Kupferzentren der untersuchten Komplexe: Die UV/VisSpektroskopie bestätigt, daß sich die Koordinationsgeometrie in Lösung im Vergleich zum Festkörper nicht dramatisch ändert. Die Komplexe 3, 4 und 15 bilden mit den Zentralionen stabile Chelatfünfringe, die ein Abdissoziieren der terminalen N-Donoren der Ligandmatrix erschweren. Dies gilt insbesondere für 15 aufgrund der hohen Stabilitätskonstanten des Kupferkomplexes mit dem Triazacyclononan/Pyrazolat-Hybridliganden. Komplex 3 hat allerdings noch apicale Bindungsstellen, die nur schwach durch Solvensmoleküle koordiniert sind, während bei 15, 4 und seinem makrocyclischen Äquivalent LV alle Koordinationsstellen durch den Chelatliganden belegt sind. Ist für die Anbindung des Substrates das Vorhandensein einer freien Koordinationsstelle erforderlich, so muß dies Auswirkungen auf die 
katalytische Aktivität des entsprechenden Komplexes haben. Die Unterschiede in der katalytischen Aktivität der strukturell eng verwandten Komplexe $\mathbf{4}$ und $\mathbf{L V}$ deuten darauf hin, daß eine solche Abdissoziation eines Seitenarmes bei der Katalyse eine Rolle spielen könnte. Die anderen Komplexe mit weniger stabilen Chelatsechsringen sind aktiver, dies kann aber auch daran liegen, daß die längeren Seitenarme kleinere Kupfer-KupferAbstände zulassen. Bei Katalysator 13 sind z.B. auch alle Koordinationsstellen durch den Liganden und die $\mathrm{OH}$-Brücke belegt, er weist aber einen kleinen Kupfer-Kupfer-Abstand und lange Ligandseitenarme auf, deren N-Donoren leicht abdissoziieren können.

3. Elektrochemische Eigenschaften: Eine Quantifizierung der Ergebnisse ist aufgrund der Irreversibilität der Reduktion nicht möglich. Eine Tendenz ist aber sehr wohl ersichtlich. So ergeben Katalysatoren mit niedrigem Potential, also einer stärkeren Stabilisierung der Kupfer(II)spezies, eine deutlich niedrigere Aktivität als Katalysatoren mit höherem Potential (Abbildung 59).

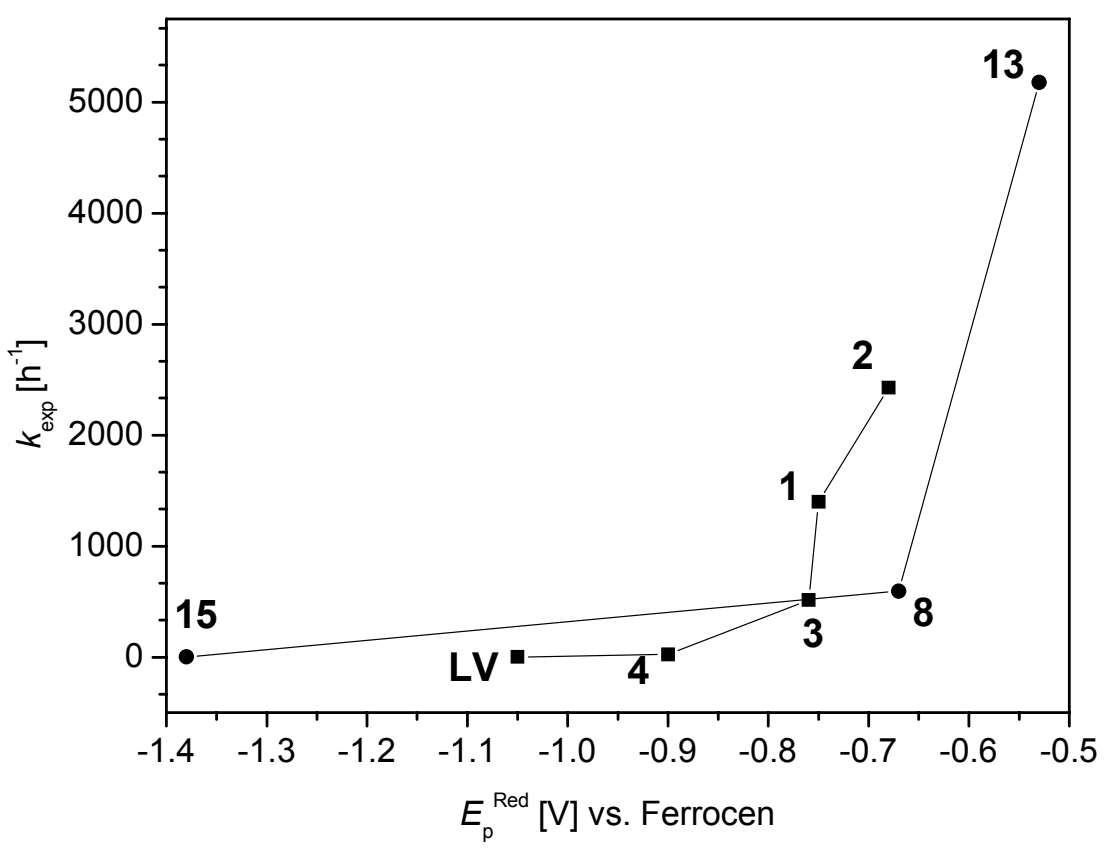

Abbildung 59: $E_{p}^{\text {Red }}$ der Katalysatoren und ihre katalytische Aktivität (vs. Ferrocen).

Dies bedeutet, daß Kupferverbindungen, bei welchen die Reduktion von Kupfer(II) zu Kupfer(I) erschwert ist, ungeeignete Katalysatoren für die Catecholase-Reaktion sind. Interessant wäre die Untersuchung eines Dikupferpyrazolatkomplexes mit sehr hohem $E_{\mathrm{p}}^{\text {Red }}$. Hier könnte dann die 
Kupfer(I)spezies so stabilisiert sein, daß der nächste Schritt, die Reoxidation mit $\mathrm{O}_{2}$, erschwert ist. Dies müßte dann zu einem schnellen stöchiometrischen Substratumsatz führen, gefolgt von einer langsamen katalytischen Phase.

4. pH-Wert und Puffer: Sowohl der pH-Wert als auch die Art des Puffers haben entscheidenden Einfluß auf die Aktivität. Bei niedrigem $\mathrm{pH}$ sind vermutlich einige N-Funktionen der Seitenarme vom Metallion losgelöst und protoniert, womit im Fall der offenkettigen Liganden Koordinationsstellen für eine Substratanbindung frei werden. Andererseits kann dies z.B. im Falle des Liganden $\left[\mathrm{L}^{6}\right]^{-} \mathrm{zu}$ einer Aufweitung des Kupfer-Kupfer-Abstandes führen, wenn die axial gebundenen Pyridin-N-Donoren abdissoziieren. Weiterhin dürfte für eine erfolgreiche Anbindung des Substrates dessen zumindest teilweise Deprotonierung erforderlich sein, was nur bei höheren $\mathrm{pH}-$ Werten möglich ist. Für eine Quantifizierung dieser Aussagen sollte allerdings noch eine Analyse der Speziesverteilung für den Katalysator bei verschiedenen pHs durchgeführt werden, was im Rahmen dieser Arbeit nicht möglich war. Ein weiteres Ergebnis der $\mathrm{pH}$-abhängigen Untersuchungen ist, daß über $\mathrm{pH}$ Effekte hinaus große Unterschiede der Aktivität je nach verwendetem Puffersystem beobachtet wurden. MES und MOPS koordinieren nur schlecht und gehen nur schwache Wechselwirkungen mit dem Katalysator ein. Dagegen ist beim Acetat von einer - wahrscheinlich verbrückenden Koordination an die Kupferzentren auszugehen. Diese Annahme wird unterstützt von den Festkörperstrukturen von $\mathbf{6}$ und 9, bei denen ein Acetat verbrückend zwischen die Kupferzentren tritt. Die Beobachtungen beim Umsetzen des Liganden $\mathrm{HL}^{4}$ mit Nickelacetat zeigen zudem, daß noch weitergehende Veränderungen der Kupferpyrazolatkomplexe im Essigsäure/Acetat-gepufferten Milieu möglich sein könnten. In diesem Fall findet man nämlich in der Festkörperstruktur des entstandenen dinuklearen Nickelkomplexes zum einen erwartungsgemäß eine Acetatbrücke zwischen den Nickelzentren, zum anderen aber auch ein weiteres Acetat, welches unter Verdrängung eines Seitenarmes an eines der Nickelzentren koordiniert. ${ }^{[150]}$ Genauso kann der TRIS-Puffer als Chelatligand verbrückend zwischen die Kupferzentren treten. Ein Austausch der ursprünglichen Zweitbrücke gegen ein Puffermolekül erklärt auch die gleichen Eigenschaften von $7 \mathrm{a}$ und $\mathbf{8}$ im gepufferten Medium. 


\section{Charakterisierung von Disauerstoffaddukten}

Um weitere Einblicke in den Mechanismus der Catecholase-Reaktion zu gewinnen, wurden Sauerstoffaddukte der reduzierten Dikupferkomplexe gezielt hergestellt und UV/Vis-spektroskopisch mit Hilfe der Stopped-Flow-Technik sowie der Ramanspektroskopie charakterisiert. Dies gibt Aufschluß über die mögliche Art der Anbindung von molekularem Sauerstoff während der Katalyse.

\subsection{Spektroskopische Eigenschaften von Dikupfersauerstoffaddukten}

Die UV/Vis- und Raman-spektroskopischen Eigenschaften der literaturbekannten, röntgenographisch charakterisierten Kupfer-Peroxokomplexe unterscheiden sich deutlich. Dies erlaubt auch die Charakterisierung von Peroxokomplexen, von denen noch keine Festkörperstruktur untersucht werden konnte. So wurden bereits mehrere Peroxid- und Bis( $\mu$-oxo)-Verbindungen auf diese Weise identifiziert. ${ }^{[21,151,152,153,154,155,156,157,158]}$ Nachfolgende Tabelle gibt einen Überblick über spektroskopische und strukturelle Daten ausgewählter Komplexe.

\begin{tabular}{|c|c|c|c|c|c|}
\hline Bindungsmodus & $\begin{array}{l}\mathrm{Cu} \cdots \mathrm{Cu} \\
[\AA]]\end{array}$ & $\begin{array}{l}O-O \\
{[\AA]}\end{array}$ & $\begin{array}{l}\text { UV/Vis }[\mathrm{nm}] \\
\left(\varepsilon\left[\mathrm{mol} \Gamma^{-1} \mathrm{~cm}^{-1}\right]\right)\end{array}$ & $\begin{array}{l}\text { Raman }\left[\mathrm{cm}^{-1}\right] \\
\text { Werte für }{ }^{18} \mathrm{O} \text { in } \\
\text { Klammern }\end{array}$ & Ref. \\
\hline$\mu-\eta^{2}: \eta^{2}$-Peroxo & & & & $0-0$ & \\
\hline Oxyhämocyanin & 3.62 & 1.36 & $350(20000)$ & 750 & {$[10],[159]$} \\
\hline V & 3.56 & 1.41 & $\begin{array}{l}349(21000), \\
551(800)\end{array}$ & 741 (698) & [14] \\
\hline VI & 3.48 & 1.49 & $\begin{array}{l}360(24700), \\
532(1530)\end{array}$ & 760 (719) & [15] \\
\hline $\operatorname{Bis}(\mu-0 \times 0)$ & & & & Cu-O & \\
\hline Tolman et al. & 2.79 & 2.29 & $\begin{array}{l}318(12000), \\
430(14000)\end{array}$ & $602,612(583)$ & [19] \\
\hline Tolman et al. & 2.35 & 2.78 & $\begin{array}{l}316(13000), \\
414(14000)\end{array}$ & $585(564)$ & [22] \\
\hline $\begin{array}{l}\text { trans- } \mu-\eta^{1}: \eta^{1}- \\
\text { Peroxo }\end{array}$ & & & & $0-0$ & \\
\hline $\mathbf{X I}$ & 4.36 & 1.43 & $\begin{array}{l}440(2000) \\
525(11500) \\
590(\text { sh, } 7600)\end{array}$ & $832(788)$ & [30] \\
\hline
\end{tabular}




\begin{tabular}{|c|c|c|c|c|c|}
\hline$\mu_{4}$-Peroxo & & & & $0-0$ & \\
\hline Krebs et al. (cis) & $2.99 / 3.03$ & 1.45 & $\begin{array}{l}384(9700), \\
587(610)\end{array}$ & $878(841)$ & [33] \\
\hline XIII (trans) & $2.99 / 3.90$ & 1.50 & $\begin{array}{l}360(3100), \\
631(260)\end{array}$ & $817(770)$ & [34] \\
\hline
\end{tabular}

Tabelle 21: Spektroskopische und strukturelle Eigenschaften von röntgenographisch charakterisierten Kupfer-Disauerstoffkomplexen.

\subsection{Synthese der $\mathrm{O}_{2}$-Addukte}

Setzt man die Liganden $\mathrm{HL}^{1}-\mathrm{HL}^{8}$, sowie $\mathrm{HL}^{9}$ mit einem Äquivalent Base und $\left[\mathrm{Cu}(\mathrm{MeCN})_{4}\right] \mathrm{X}\left(\mathrm{X}=\mathrm{CF}_{3} \mathrm{SO}_{3}^{-}, \mathrm{PF}_{6}^{-}, \mathrm{ClO}_{4}^{-}, \mathrm{BF}_{4}^{-}\right)$in Lösung bei tiefer Temperatur um, so bilden sich zunächst gelbe Lösungen der entsprechenden Dikupfer(I)komplexe. Die Kupfer(I)salze wurden nach Literaturvorschrift hergestellt. ${ }^{[160]}$ Diese Verbindungen sind bei Temperaturen unter $-20^{\circ} \mathrm{C}$ recht stabil. Als Lösungsmittel bieten sich insbesondere Nitrile wie Aceto- oder Propionitril an, deren Schmelzpunkt ausreichend tief liegt und welche die $\mathrm{Cu}(\mathrm{l})$ spezies im Gegensatz zu protischen Lösungsmitteln wie z.B. Methanol stabilisieren. In protischem Solvens kann zudem eine Protonierung der basischen Peroxoeinheit stattfinden. ${ }^{[161]}$

Es wurden zahlreiche Versuche durchgeführt, in Propionitril bei $-78^{\circ} \mathrm{C}$ aus den Dikupfer(I)komplexen durch Luft- oder $\mathrm{O}_{2}$-Zugabe die jeweiligen $\mathrm{O}_{2}$-Addukte zu isolieren und kristallin zur röntgenographischen Charakterisierung zu erhalten. Gibt man zu den Komplexen der Liganden $\mathrm{HL}^{1}-\mathrm{HL}^{2}$ sowie $\mathrm{HL}^{5}-\mathrm{HL}^{8}$ Sauerstoff, so wird entweder sofort eine grüne Lösung erhalten, oder aber zunächst eine Braunfärbung, welche spätestens nach einigen Tagen auch bei tiefer Temperatur in eine Grünfärbung umschlägt. Da Peroxoaddukte im allgemeinen violett gefärbt sind, ist eine Grünfärbung ein deutliches Zeichen für eine Zersetzung des Dikupfer(I)komplexes unter Bildung einer unerwünschten Kupfer(II)spezies. Es ist aber - wie bereits in Kapitel 2 erwähnt - auch eine grüne Dikupferperoxoverbindung XIII des Liganden $\mathrm{HL}^{3}$ bekannt. ${ }^{[34]}$ Das braune Zwischenprodukt ist entweder eine Mischung aus dem erwarteten violetten $\mathrm{O}_{2}$-Addukt und noch nicht umgesetztem Dikupfer(I)komplex oder es handelt sich hier um eine andere Art der Anbindung des Sauerstoffes an die Kupferzentren. Eine Charakterisierung dieser Produkte gelang jedoch nicht, da keine Kristalle für die Röntgenstrukturanalyse gewonnen werden 
konnten und die Verbindungen zu instabil für UV/Vis- und Ramanmessungen waren und sich beim Transfer vom Kolben in den Meßraum zersetzten. Bei der Umsetzung von $\mathrm{HL}^{6}$ mit $\left[\mathrm{Cu}(\mathrm{MeCN})_{4}\right] \mathrm{CF}_{3} \mathrm{SO}_{3}$ bei $-78^{\circ} \mathrm{C}$ in Dichlormethan mit einer Hilfsbase und Luftsauerstoff konnte durch anschließende Etherdiffusion ein grünes Zersetzungsprodukt $\left[\mathrm{L}^{6} \mathrm{Cu}_{2}(\mathrm{OH})\right]\left(\mathrm{CF}_{3} \mathrm{SO}_{3}\right)_{2}$ kristallin erhalten werden, dessen Festkörperstruktur jener von 13 entspricht (siehe Kapitel 4).

Erfolgreicher war die Synthese von Peroxoaddukten der Kupfer(I)komplexe der Liganden $\mathrm{HL}^{4}$ und $\mathrm{HL}^{9}$. Zwar konnten auch hier keine röntgenographisch verwertbaren Kristalle gewonnen werden, jedoch erhält man durch Umsetzen dieser Liganden mit $\left[\mathrm{Cu}(\mathrm{MeCN})_{4}\right] \mathrm{PF}_{6}$ in Propionitril bei $-78^{\circ} \mathrm{C}$ und anschließende Luftinjektion violette bis violettbraune Lösungen, welche auch bei höheren Temperaturen ausreichende Stabilität zeigen. Beim Übertragen in den Meßraum des Ramanspektrometers fand aber eine schnelle Zersetzung statt. Hier erwies sich Dichlormethan als geeigneteres Solvens. Die violette Spezies ist im Falle des Liganden $\mathrm{HL}^{4}$ unterhalb von $-78{ }^{\circ} \mathrm{C}$ über einige Stunden stabil und konnte auch ohne Zersetzung Raman- und UV/Vis-spektroskopisch vermessen werden. Das Sauerstoffaddukt der Kupfer(I)verbindung von $\mathrm{HL}^{9}$ ist dank des makrozyklischen Seitenarms noch wesentlich stabiler und konnte sogar für einige Sekunden in Raumatmosphäre gehandhabt werden. Die anderen Komplexe erwiesen sich auch in Dichlormethan als zu instabil für eine weitere Untersuchung.

Ausgehend von der Vierzähnigkeit beider Liganden ist eine $\mu-\eta^{1}: \eta^{1}$-peroxidische Koordination des Sauerstoffes zu erwarten (Abbildung 60). Die Informationen aus UV/Vis- und Ramanspektren der entsprechenden Sauerstoffaddukte sollten durch Vergleich mit den Spektren der bereits bekannten Sauerstoffaddukte eine Zuordnung der Art des Adduktes ermöglichen. 


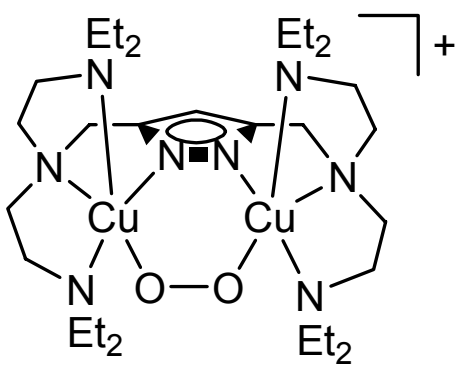

"L ${ }^{4} \mathrm{Cu}_{2}\left(\mathrm{O}_{2}\right)^{\prime \prime}$

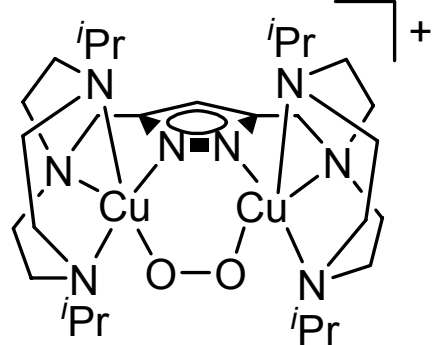

${ }^{\prime L}{ }^{9} \mathrm{Cu}_{2}\left(\mathrm{O}_{2}\right)^{\prime \prime}$

Abbildung 60: erwartete Struktur der Sauerstoffaddukte der Dikupfer(I)verbindungen von $L^{4}$ und $L^{9}$.

\subsection{UV/Vis-Spektroskopie und Stopped-Flow-Messungen}

Eine Tieftemperatur-UV/Vis-Messung des violetten Sauerstoffadduktes von $\mathrm{HL}^{4}$ gelang in Propionitril über die Darstellung des Dikupfer(I)komplexes " ${ }^{4} \mathrm{Cu}_{2}{ }^{\prime}$ und Transfer über eine Kanüle in eine $\mathrm{N}_{2(1)}$-gekühlte Küvette bei ca. $-80^{\circ} \mathrm{C}$ unter Luft- und Feuchtigkeitsauschluß. Anschließend wurde ebenfalls über eine Kanüle Luft injiziert und die Probe vermessen. Das Spektrum zeigt eine recht intensive Bande bei 544 $\mathrm{nm}$, sowie eine weitere Bande in Form einer Schulter bei $633 \mathrm{~nm}$ (Abbildung 61). Molare Extinktionskoeffizienten sind nicht angegeben, da es durch Kondensation von Wasser an der Küvette zu einer leichten Trübung an der Außenwand während der Messung kam, wodurch die beobachtete Absorption verschoben wurde. Dennoch läßt die Lage der Banden Schlüsse auf die Art und Weise der Anbindung des Sauerstoffes an den Komplex zu. Man kann beide Banden $\mathrm{O}_{2}{ }^{2-} \rightarrow \mathrm{Cu}(\mathrm{II})$ LMCTÜbergängen zuordnen, ${ }^{[162]}$ da die von Karlin et al. synthetisierte und röntgenographisch charakterisierte $\mu-\eta^{1}: \eta^{1}$-Peroxoverbindung $\mathbf{X I}$ typische Übergänge bei ähnlichen Wellenlängen aufweist (siehe Tabelle 21). Eine sichere Aussage über die Art der Anbindung des $\mathrm{O}_{2}^{2-}$ kann anhand dieser Daten jedoch noch nicht getroffen werden. 


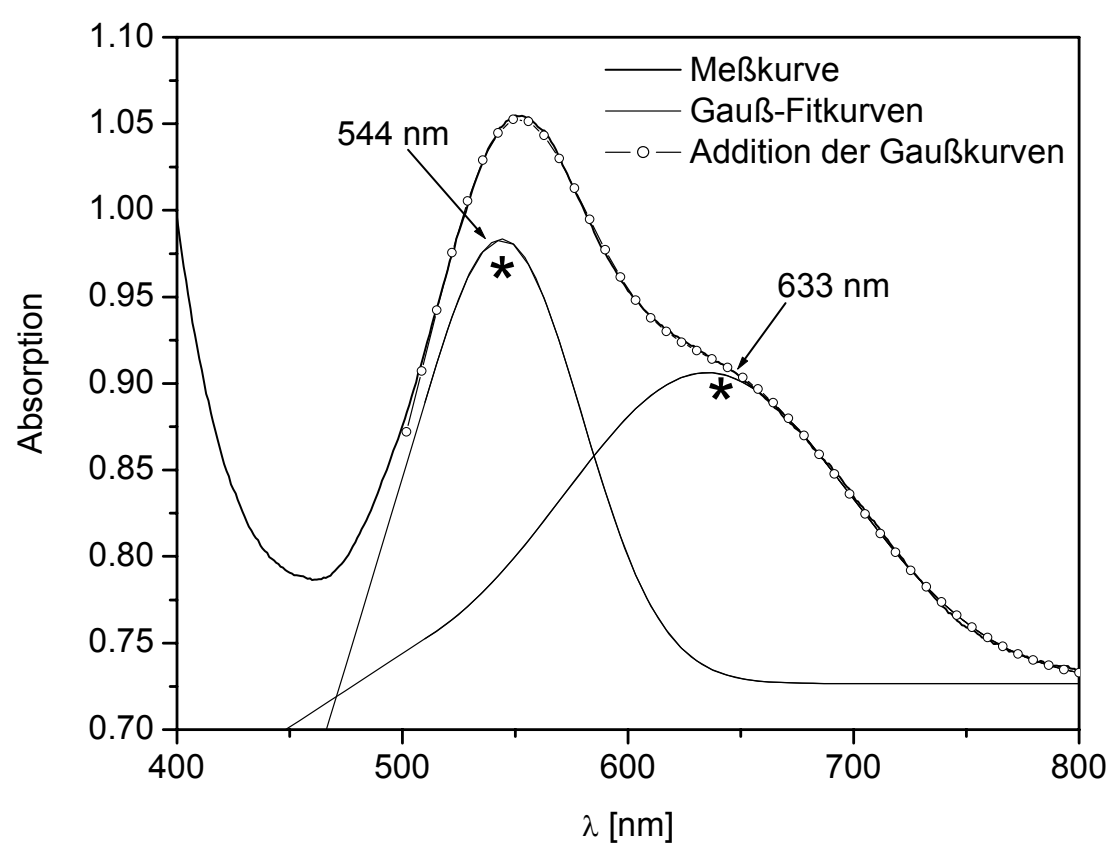

Abbildung 61: UV/Vis-Spektrum von ${ }^{4}{ }^{4} \mathrm{Cu}_{2} \mathrm{O}_{2}{ }^{\prime \prime}$, Konz.: $1.6 \cdot 10^{-3} \mathrm{M},{ }^{*}$ die genaue Lage und Intensität der Banden wurde mit Hilfe von zwei Gauß-Fitfunktionen ermittelt.

Die Peroxoverbindung " $\mathrm{L}^{4} \mathrm{Cu}_{2}\left(\mathrm{O}_{2}\right)^{\text {" }}$ wurde sodann mit Hilfe der Stopped-FlowTechnik in Dichlormethan untersucht. Mit der verwendeten Apparatur waren Meßtemperaturen von weniger als $-10^{\circ} \mathrm{C}$ nicht möglich, so daß der Komplex zu instabil für eine gute Detektion war. Jedoch war nach $2.7 \cdot 10^{-3} \mathrm{~s}$ noch eine leichte Bande bei ca. $565 \mathrm{~nm}$ zu erkennen, so daß auch in diesem Lösungsmittel die Bildung eines Peroxoadduktes wahrscheinlich ist. Innerhalb von einer Sekunde konnte eine Zersetzung unter Bildung einer grünen Spezies mit einer Bande bei ca. $675 \mathrm{~nm}$ im für Kupfer(II)verbindungen typischen Bereich festgestellt werden (Abbildung 62). Die Lage dieser Bande ist deutlich verschieden von der entsprechenden Bande des $\mathrm{H}_{3} \mathrm{O}_{2}$-verbrückten Komplexes 4, die bei $855 \mathrm{~nm}$ liegt. Möglicherweise entsteht aus der Peroxoverbindung direkt durch Chloridabstraktion die entsprechende Chlorverbindung " $\mathrm{L}^{4} \mathrm{Cu}_{2} \mathrm{Cl}$ " des Komplexes. 


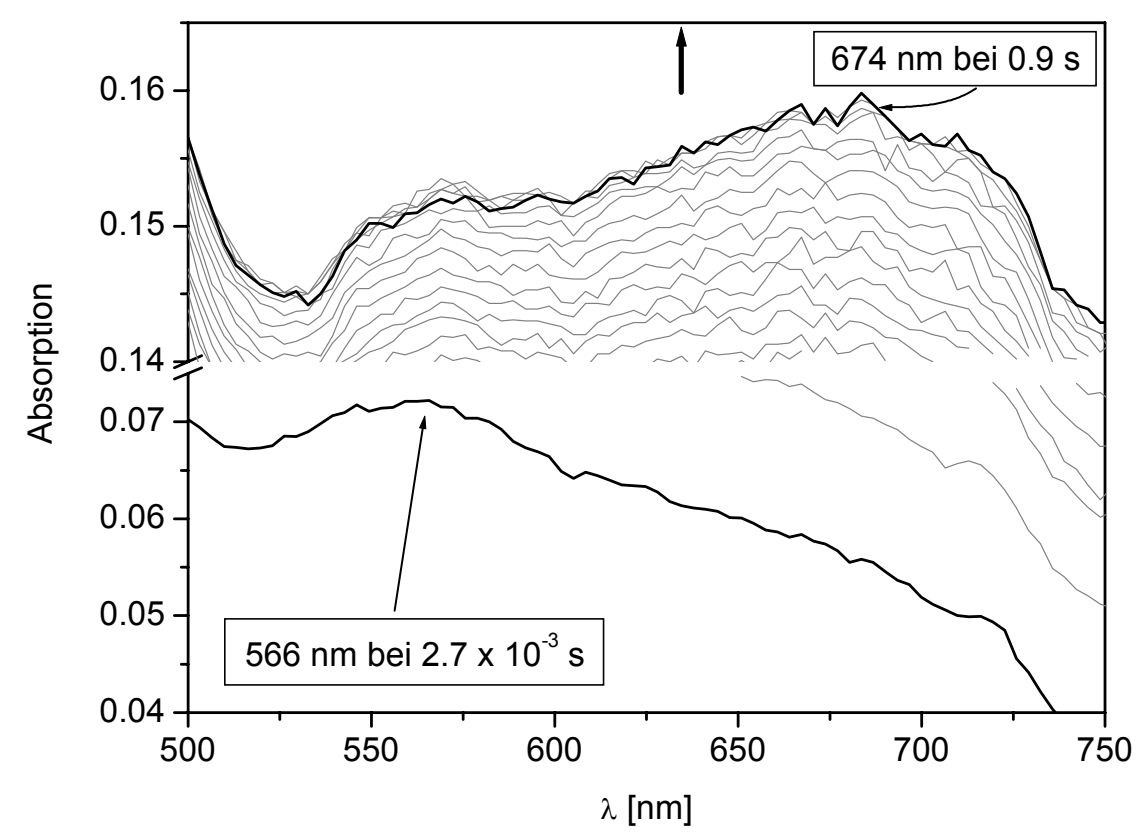

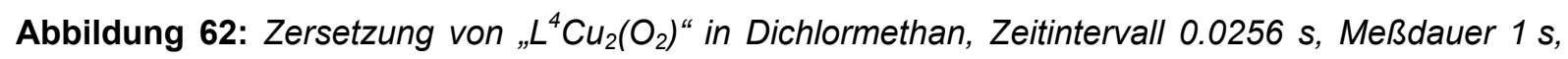
Temp. $-10^{\circ} \mathrm{C}$.

Das violette Peroxoaddukt " $\mathrm{L}^{9} \mathrm{Cu}_{2}\left(\mathrm{O}_{2}\right)^{\text {“ }}$ konnte bereits in vorhergehenden Arbeiten UV/Vis-spektroskopisch in Acetonitril untersucht werden, aufgrund der Lage seiner Banden bei $356 \mathrm{~nm}$ (Schulter), $527 \mathrm{~nm}\left(\varepsilon=3400 \mathrm{I} \mathrm{mol}^{-1} \mathrm{~cm}^{-1}\right)$ und $612 \mathrm{~nm}$ $\left(\varepsilon=2900 \mathrm{I} \mathrm{mol}^{-1} \mathrm{~cm}^{-1}\right)$ wurde es ebenfalls als Peroxoverbindung identifiziert. ${ }^{[107]}$

Die Stopped-Flow-Technik erlaubt nun einen besseren Einblick in die Kinetik der Zersetzung von " $\mathrm{L}^{9} \mathrm{Cu}_{2}\left(\mathrm{O}_{2}\right)^{\text {“. }}$. Hierbei wurde bei $-10{ }^{\circ} \mathrm{C}$ im Meßraum eine $1.7 \cdot 10^{-2} \mathrm{M}$ Lösung von " $\mathrm{L}^{9} \mathrm{Cu}_{2}$ " in Dichlormethan mit dem gleichen Volumen $\mathrm{O}_{2}$-gesättigten Dichlormethans gemischt und über einen Zeitraum von $1124 \mathrm{~s}$ das UV/Vis-Spektrum im Bereich von 300 - $1100 \mathrm{~nm}$ aufgenommen. Die Bildung der Peroxospezies erfolgte so schnell, daß sie mit der Stopped Flow Technik nicht mehr erfaßt werden konnte, jedoch ließ sich inr Zerfall gut beobachten. ", ${ }^{9} \mathrm{Cu}_{2}\left(\mathrm{O}_{2}\right)^{\text {“ }}$ hat in Dichlormethan eine Bande bei $490 \mathrm{~nm}\left(\varepsilon=2320 \mathrm{~mol} \mathrm{l}^{-1} \mathrm{~cm}^{-1}\right)$ und eine Bande bei $612 \mathrm{~nm}$ (Schulter, $\left.\varepsilon=1040 \mathrm{~mol} \mathrm{I}^{-1} \mathrm{~cm}^{-1}\right)$. Innerhalb der Meßzeit verringerte sich die Intensität der Bande bei $490 \mathrm{~nm}$ stark, die Bande bei $612 \mathrm{~nm}$ verschwand (Abbildung 63). Gleichzeitig bildete sich die Bande des Zersetzungsproduktes bei ca. $720 \mathrm{~nm}$, welches nicht näher charakterisiert wurde, jedoch unterscheidet sich dessen Spektrum klar von 
jenem des Zersetzungsproduktes in Propionitril, ${ }^{[107]}$ so daß auch hier die direkte Bildung der Chlorverbindung " $\mathrm{L}^{9} \mathrm{Cu}_{2} \mathrm{Cl}$ “ möglich ist.

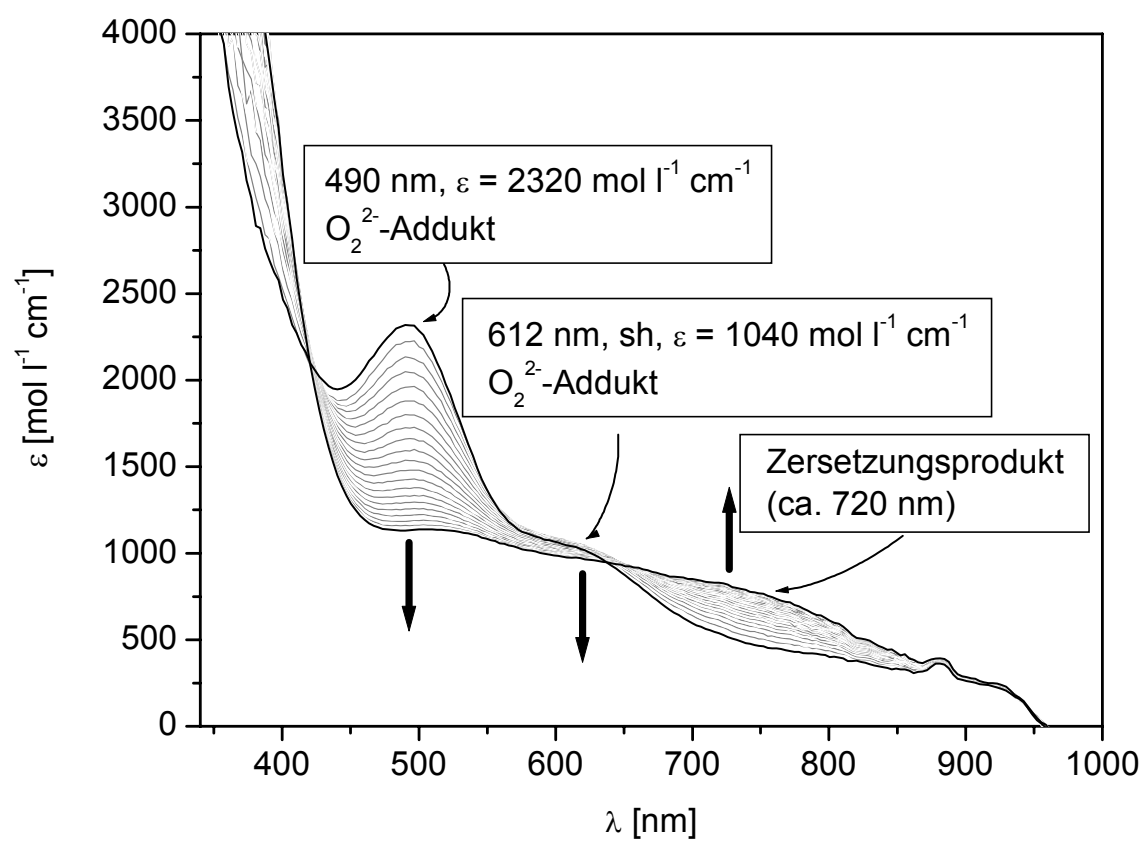

Abbildung 63: Zersetzung von " $\mathrm{L}^{9} \mathrm{Cu}_{2} \mathrm{O}_{2}$ " in Dichlormethan, Zeitintervall $13 \mathrm{~s}$, Meßdauer $1124 \mathrm{~s}$, Temp. $-10^{\circ} \mathrm{C}$, dargestellt ist jede vierte Meßkurve.

Trägt man den reziproken Wert der Konzentration des Peroxoadduktes gegen die Zeit auf, so erhält man einen linearen Abfall gemäß dem integrierten Geschwindigkeitsgesetz (Abbildung 64):

$$
\frac{1}{[A]}-\frac{1}{[A]_{0}}=2 k \cdot t
$$

Unter der Annahme, daß es sich beim Zerfall von " $\mathrm{L}^{9} \mathrm{Cu}_{2} \mathrm{O}_{2}$ " um eine Reaktion 2. Ordnung des Typs

(18) $2 \mathrm{~A} \rightarrow \mathrm{C}+\mathrm{D}+\ldots$

handelt, ergibt sich die Geschwindigkeitskonstante $k=(985 \pm 3) I \mathrm{~mol} \mathrm{~h}^{-1}$. Die Halbwertszeit $t_{1 / 2}$ beträgt $0.13 \mathrm{~h}$ bei $-10^{\circ} \mathrm{C}$. 


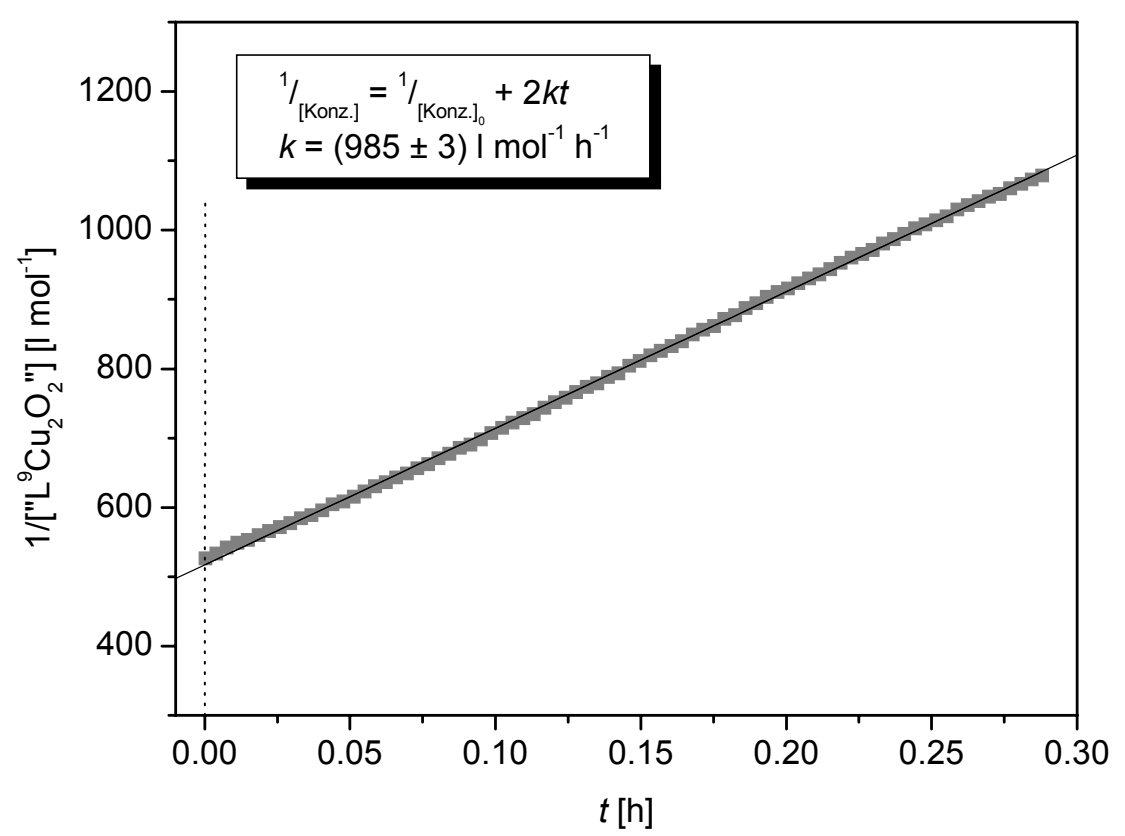

Abbildung 64: Reziproke Konzentration von " $L^{9} \mathrm{CuO}_{2}$ "vs. $t$, lineare Regression ergibt die Zerfallskonstante $k$ als Steigung der Geraden.

Für den Zerfall bzw. die Weiterreaktion von Dikupferperoxoaddukten wurden von anderen Forschungsgruppen Kinetiken 1. Ordnung gefunden. ${ }^{[155,163]}$ Zur Interpretation der gefundenen Abhängigkeit kann man spekulieren, daß zunächst im geschwindigkeitsbestimmenden Schritt die Bildung eines Dimers aus zwei dinuklearen Komplexen erfolgt, welche dann weiter zerfallen. So könnte die in Kapitel 2 erwähnte Verbindung $\left[\mathrm{L}_{2}^{3} \mathrm{Cu}_{4}\left(\mathrm{O}_{2}\right)(\mathrm{OH})_{2}\right]^{2+}$ (XIII) ebenfalls einer solchen dimeren Zwischenstufe für den Zerfall der Dikupfersauerstoffverbindung " $\mathrm{L}^{3} \mathrm{Cu}_{2}\left(\mathrm{O}_{2}\right)^{4}$ entsprechen. ${ }^{[34]}$

\subsection{Raman-Spektroskopie}

Die Ramanspektren der Peroxoverbindungen " $\mathrm{L}^{4} \mathrm{Cu}_{2}\left(\mathrm{O}_{2}\right)^{\text {“ }}$ und " ${ }^{9} \mathrm{Cu}_{2}\left(\mathrm{O}_{2}\right)^{\text {“ }}$ wurden in Dichlormethan bei Temperaturen von $-170^{\circ} \mathrm{C}$ bis $-80^{\circ} \mathrm{C}$ aufgenommen. Die Anregungswellenlänge des HeNe-Lasers betrug $623 \mathrm{~nm}$. Da die Energie der Anregungswellenlänge im Bereich der elektronischen Anregung der Peroxogruppe liegt, werden aufgrund des Resonanzeffektes deren Schwingungsbanden spezifisch verstärkt. ${ }^{[164]}$ 


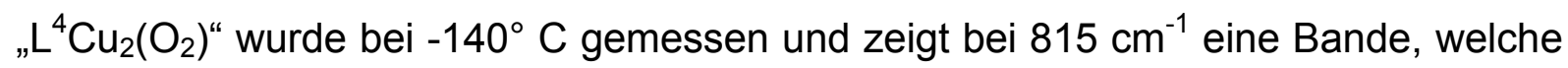
einer O-O-Streckschwingung eines $\mu-\eta^{1}: \eta^{1}$-Dikupfer(II)peroxokomplexes zugeordnet werden kann (siehe Tabelle 21). Hinzu kommt eine Bande bei $537 \mathrm{~cm}^{-1}$ die zu der entsprechenden Cu-O-Schwingung gehören könnte. Zum Nachweis, daß es sich bei diesen Banden tatsächlich um Schwingungen der Peroxogruppierung handelt, wurde diese in einem weiteren Experiment mit ${ }^{18} \mathrm{O}_{2}$ hergestellt. $\tilde{v}(\mathrm{O}-\mathrm{O})$ und $\tilde{v}(\mathrm{Cu}-\mathrm{O})$ sollten aufgrund der höheren Masse des ${ }^{18} \mathrm{O}$ nach der Gleichung

(19) $\tilde{v}_{0}=\frac{1}{2 \pi c} \sqrt{\frac{k}{\mu}}$ ( $\mu$ : reduzierte Masse, $k$ : Kraftkonstante, $c$ : Lichtgeschwindigkeit)

zu kleineren Werten verschoben, d.h. energieärmer werden. Alle weiteren Banden im Spektrum bleiben unverändert. Die zu erwartenden Wellenzahlen $\tilde{v}\left({ }^{18} \mathrm{O}-{ }^{18} \mathrm{O}\right)$ und $\tilde{v}\left(\mathrm{Cu}^{18}{ }^{18} \mathrm{O}\right)$ können berechnet werden anhand der Formel:

(20) $\tilde{v^{18} \mathrm{O}}=\sqrt{\frac{\mu_{16} \mathrm{O}}{\mu_{18} \mathrm{O}}} \cdot \tilde{v^{16} \mathrm{O}}$

Tatsächlich ist dieses Verhalten zu beobachten. Im Spektrum finden sich zwei neue Banden, die mit den berechneten Werten gut übereinstimmen (Tabelle 22):

\begin{tabular}{llll} 
& exp. ${ }^{16} \mathrm{O}$ & ber. ${ }^{18} \mathrm{O}$ & exp. ${ }^{18} \mathrm{O}$ \\
\hline$\tilde{v}(\mathrm{O}-\mathrm{O})$ & $815 \mathrm{~cm}^{-1}$ & $768 \mathrm{~cm}^{-1}$ & $769 \mathrm{~cm}^{-1}$ \\
$\tilde{v}$ (Cu-O) & $537 \mathrm{~cm}^{-1}$ & $513 \mathrm{~cm}^{-1}$ & $518 \mathrm{~cm}^{-1}$
\end{tabular}

Tabelle 22: Berechnete und experimentell ermittelte Werte der ${ }^{18} \mathrm{O}-{ }^{18} \mathrm{O}$ - und $\mathrm{Cu}^{18}{ }^{18} \mathrm{O}-\mathrm{Sch} w i n g u n g e n$ von ${ }^{4}{ }^{4} \mathrm{Cu}_{2}\left({ }^{18} \mathrm{O}_{2}\right)$.

Zugleich bleiben die Banden der entsprechenden ${ }^{16} \mathrm{O}-\mathrm{Schwingungen} \mathrm{erhalten,} \mathrm{d.h.}$ vor der Messung ist noch Luftsauerstoff aufgenommen worden, entweder schon während der Synthese der Peroxoverbindung oder bei dem Transfer der Lösung auf den Objektträger des Raman-Spektrometers (Abbildung 65). 


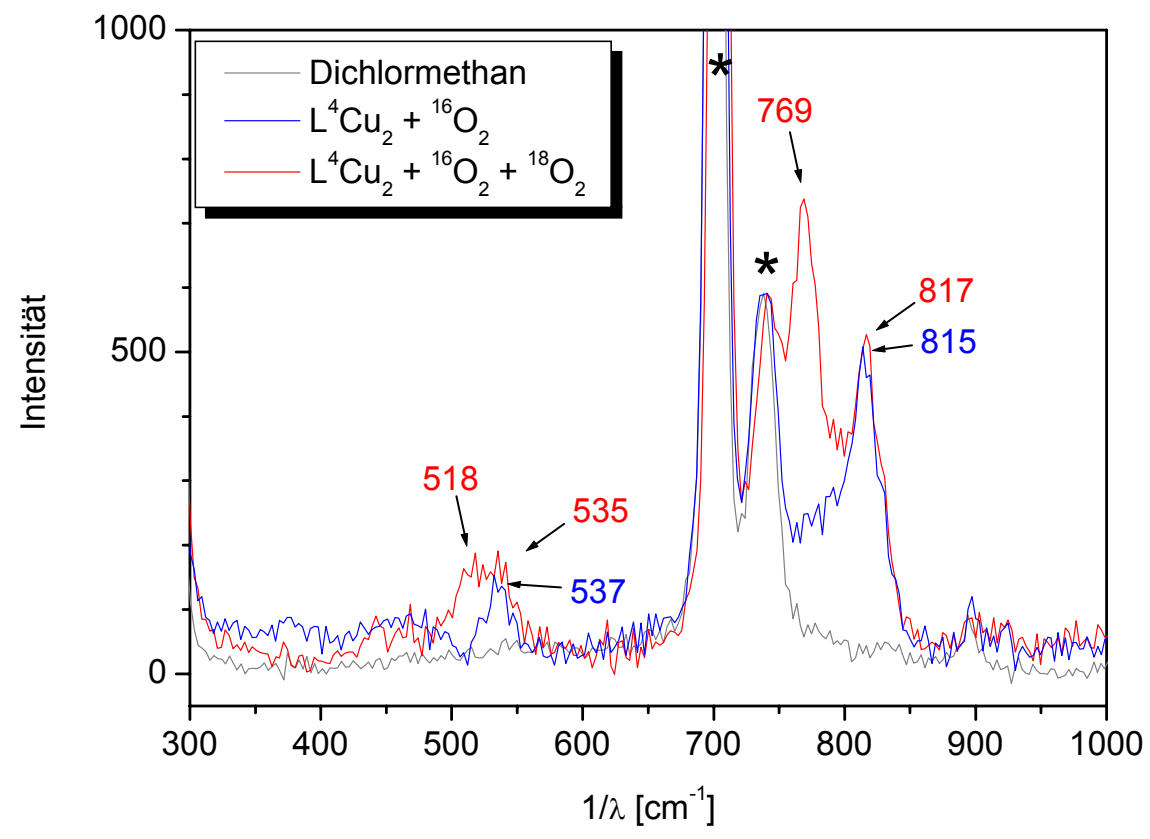

Abbildung 65: Raman-Spektrum der Peroxoverbindung von " $\mathrm{L}^{4} \mathrm{Cu}_{2}\left(\mathrm{O}_{2}\right)$ “ in Dichlormethan mit ${ }^{16} \mathrm{O}_{2}$ und ${ }^{18} \mathrm{O}_{2}$, *: Lösungsmittelpeaks.

Die Peroxoverbindung erwies sich bei Temperaturen zwischen $-170^{\circ} \mathrm{C}$ und $-140^{\circ} \mathrm{C}$ als stabil. Wie man an Abbildung 66 sehen kann, nimmt die Intensität $\tilde{v}(\mathrm{O}-\mathrm{O})$ bei einer Erhöhung der Temperatur von $-170^{\circ} \mathrm{C}$ auf $-140^{\circ} \mathrm{C}$ noch zu.

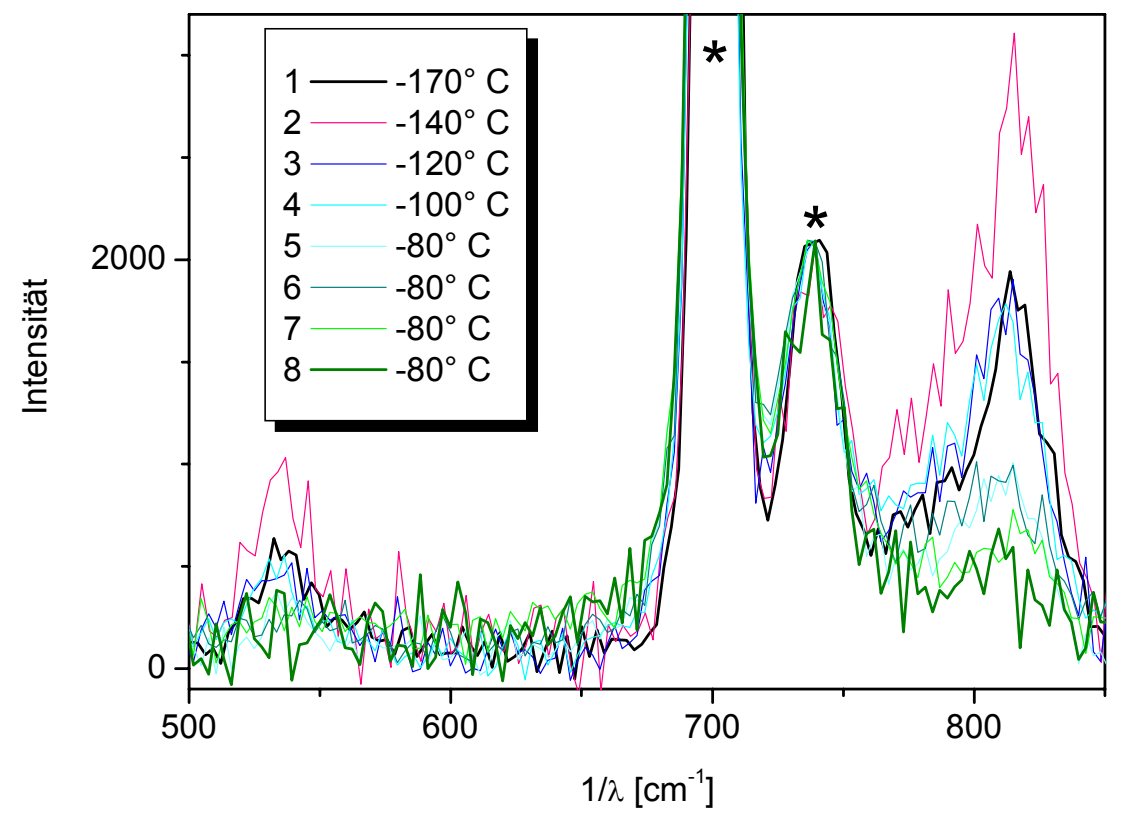

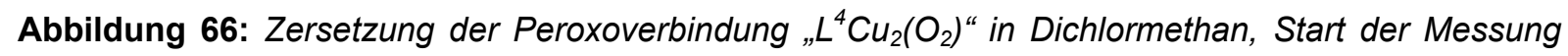
bei $-170^{\circ} \mathrm{C}$, bei $-80^{\circ} \mathrm{C}$ wurden 4 Messungen durchgeführt, *: Lösungsmittelpeaks. 
Es findet also noch weitere Aufnahme von $\mathrm{O}_{2}$ statt. $\mathrm{Ab}$ Temperaturen von $-120^{\circ} \mathrm{C}$ findet langsame Zersetzung statt, bei $-80^{\circ} \mathrm{C}$ beschleunigt sich die Zersetzung und die Bande verschwindet innerhalb einer Minute schließlich ganz.

Das Ramanspektrum der Spezies nach Umsetzen von " $\mathrm{L}^{9} \mathrm{Cu}_{2}$ " mit ${ }^{16} \mathrm{O}_{2}$ bei $-140{ }^{\circ} \mathrm{C}$ in Dichlormethan ist ähnlich: Man erhält eine Bande bei $808 \mathrm{~cm}^{-1}$, entsprechend einer O-O-Streckschwingung, eine weitere Bande bei $537 \mathrm{~cm}^{-1}$ kann auch hier wieder der Cu-O-Schwingung zugeordnet werden (Abbildung 67). Damit entspricht auch das Spektrum dieser Verbindung einem $\mu-\eta^{1}: \eta^{1}$-peroxidisch verbrückten Dikupfer(II)komplex. Ob es sich bei dem Maximum bei $786 \mathrm{~cm}^{-1}$ um eine Bande einer weiteren Spezies handelt, konnte nicht abschließend geklärt werden. Auch hier wurde wieder ein Kontrollexperiment mit ${ }^{18} \mathrm{O}_{2}$ durchgeführt. Die nach Formel (20) berechneten Werte für die der $\left({ }^{18} \mathrm{O}-{ }^{18} \mathrm{O}\right)$ - und $\left(\mathrm{Cu}^{-18} \mathrm{O}\right)$-Schwingungen ergeben sich zu $761 \mathrm{~cm}^{-1}$ bzw. $509 \mathrm{~cm}^{-1}$. Im Spektrum findet man weiterhin die Banden der entsprechenden Schwingungen mit ${ }^{16} \mathrm{O}$. Die Bande für die (Cu-O)-Schwingung ist deutlich verbreitert und liegt zwischen 505 und $528 \mathrm{~cm}^{-1}$. Wahrscheinlich entspricht dies in Wirklichkeit zwei Schwingungen, und zwar jener für ( $\left.\mathrm{Cu}-{ }^{16} \mathrm{O}\right)$ bei ca. $528 \mathrm{~cm}^{-1}$ und jener für $\left(\mathrm{Cu}^{-18} \mathrm{O}\right)$ bei ca. $505 \mathrm{~cm}^{-1}$, was im Bereich der berechneten Verschiebung liegt. Eine weitere Bande erscheint bei $779 \mathrm{~cm}^{-1}$, allerdings ist ihre Lage sehr nahe zum ungeklärten Maximum bei $786 \mathrm{~cm}^{-1}$, welches bei der Messung $\operatorname{der}\left({ }^{16} \mathrm{O}\right)_{2}{ }^{2-}$-Verbindung aufgetreten war. Zum anderen ist die Bande im Vergleich zur berechneten Bande der $\left({ }^{18} \mathrm{O}-{ }^{18} \mathrm{O}\right)$-Schwingung deutlich zu größeren Wellenzahlen verschoben, während der Lösungsmittelpeak bei $741 \mathrm{~cm}^{-1}$ verbreitert erscheint. Wahrscheinlich überlagern sich hier die Bande von Dichlormethan und die Bande der $\left({ }^{18} \mathrm{O}-{ }^{18} \mathrm{O}\right)$-Schwingung. Aufschlüsse darauf könnten Messungen in einem anderen Lösungsmittel wie Acetonitril geben, welches im fraglichen Bereich keine Banden aufweist. Bei allen im Rahmen dieser Arbeit durchgeführten Versuchen mit Acetonitril zersetzten sich aber die Peroxoverbindungen sehr schnell, so daß keine Messungen in diesem Lösungsmittel möglich waren. 


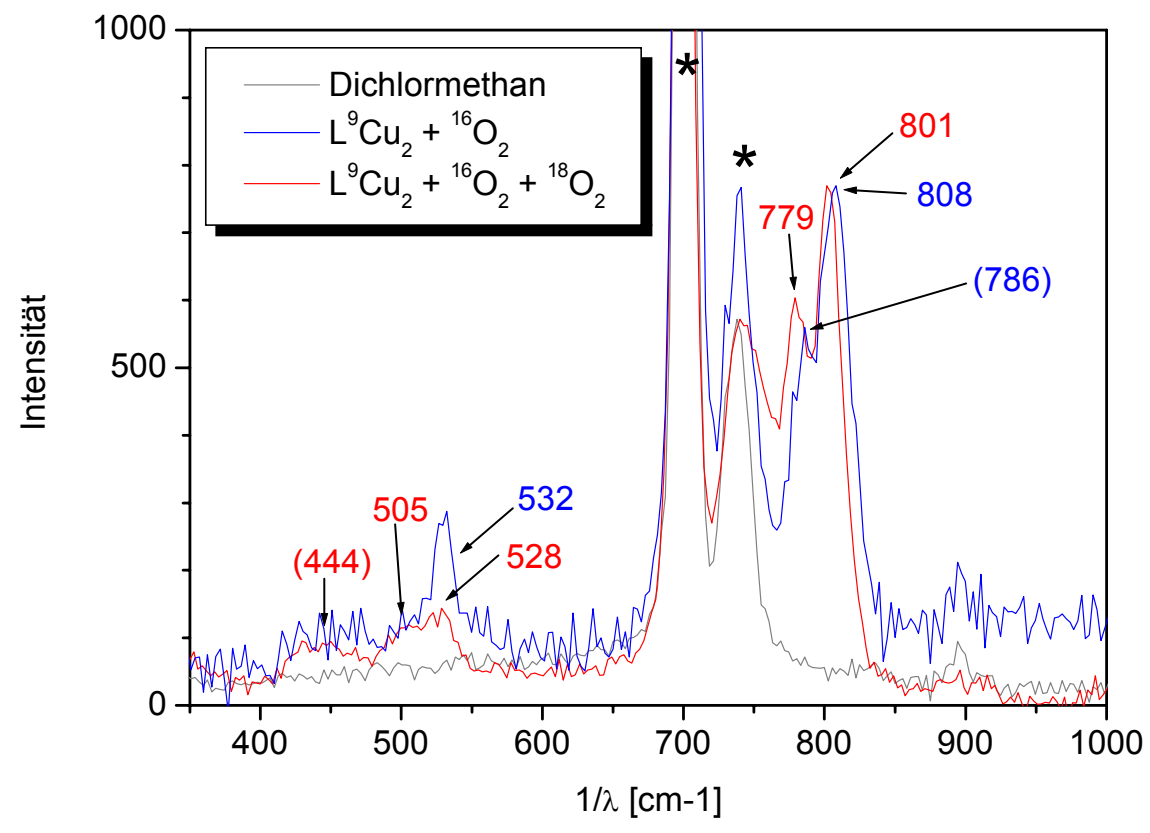

Abbildung 67: Raman-Spektrum der Peroxoverbindung " $L^{9} \mathrm{Cu}_{2}\left(\mathrm{O}_{2}\right)$ “ in Dichlormethan mit ${ }^{16} \mathrm{O}_{2}$ und ${ }^{18} \mathrm{O}_{2}$, *: Lösungsmittelpeaks.

Setzt man " ${ }^{9} \mathrm{Cu}_{2}\left(\mathrm{O}_{2}\right)^{\prime \prime}$ bei $-78^{\circ} \mathrm{C}$ ca. 30 min der Raumatmosphäre aus, so ist im Ramanspektrum die langsame Zersetzung der Verbindung zu beobachten (Abbildung 68).

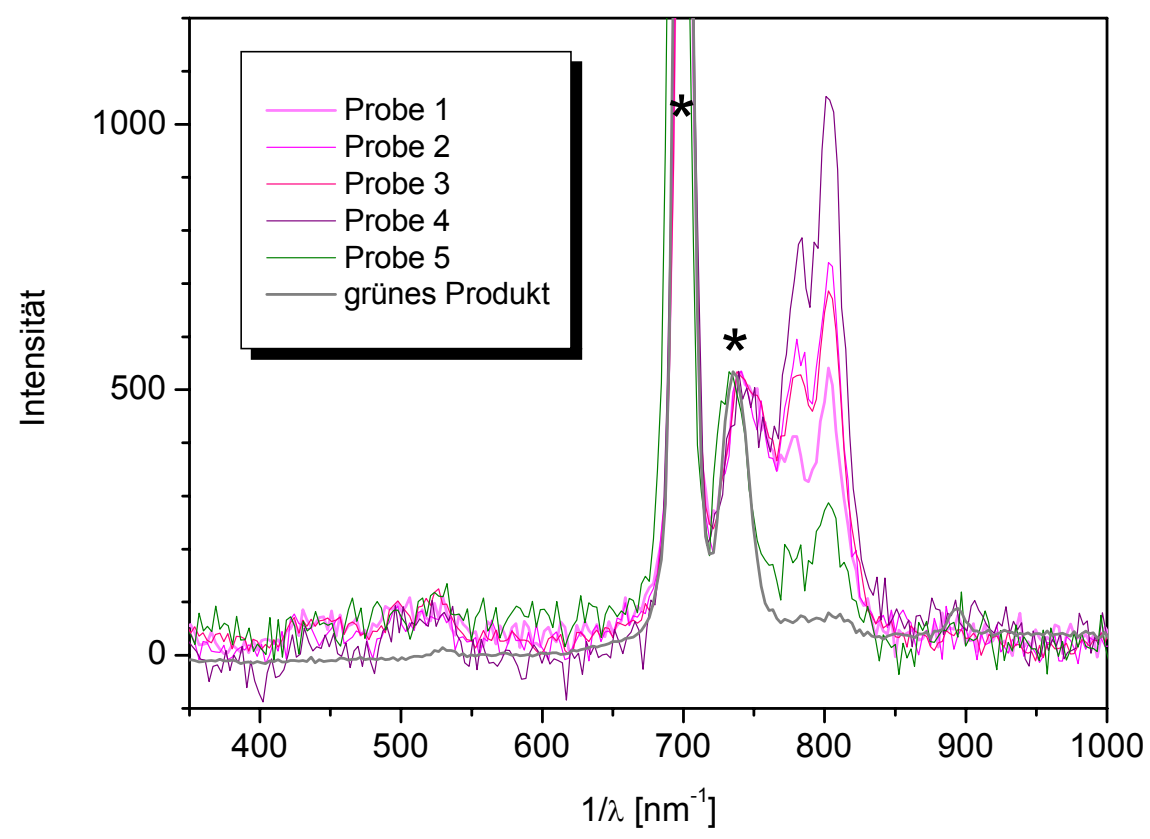

Abbildung 68: Zersetzung der Peroxoverbindung,${ }^{\circ}{ }^{9} \mathrm{Cu}_{2} \mathrm{O}_{2}{ }^{\prime \prime}$ in Dichlormethan bei $-78^{\circ} \mathrm{C}$ innerhalb von 30 min, * Lösungsmittelpeaks. 
Die Messung selbst wurde bei $-130^{\circ} \mathrm{C}$ mit einer Mischung von ${ }^{16} \mathrm{O}_{2}$ und ${ }^{18} \mathrm{O}_{2}$ durchgeführt. Die Stabilität des Peroxoadduktes erlaubte auch Untersuchungen zur Reversibilität der Sauerstoffaufnahme in den Komplex. Durch Anlegen von Vakuum bei $-78^{\circ} \mathrm{C}$ änderten sich Farbe und Ramanspektrum der Verbindung nicht, der Sauerstoff bleibt als Peroxid fest in den Komplex eingebunden.

\subsection{Schlußfolgerungen}

Anhand der erhaltenen spektroskopischen Ergebnisse, sowie struktureller Überlegungen ergeben sich Hinweise auf die Art der Anbindung des Sauerstoffes in den Dikupfersauerstoffverbindungen " $\mathrm{L}^{4} \mathrm{Cu}_{2}\left(\mathrm{O}_{2}\right)$ “ und " $\mathrm{L}^{9} \mathrm{Cu}_{2}\left(\mathrm{O}_{2}\right)$ “:

1. Zähnigkeit der Liganden: Wie bereits erwähnt, wird immer eine Fünffachkoordination der Kupferzentren angestrebt. Während dies bei dreizähnigen Liganden zu side on anbindenden $\eta^{2}$-Superoxoverbindungen, verbrückenden $\mu-\eta^{2}: \eta^{2}$-Peroxo-, bzw. nach Bruch der O-O-Bindung zu Bis $(\mu-$ oxo)-Spezies führt, oder auch zur Ausbildung von $\mu_{4}$-peroxidischen Verbrückungen von vier Kupferzentren, so wurden bei vierzähnigen Liganden $\mu-\eta^{1}: \eta^{1}$-verbrückende Peroxo- bzw. end on gebundene Hydroperoxoverbindungen gefunden. Bei den Liganden $\mathrm{HL}^{4}$ und $\mathrm{HL}^{9}$ wird somit für jede der beiden Kavitäten eine end-on-Anbindung zu erwarten sein, sofern alle Liganddonoratome koordinieren. Davon ist allerdings auszugehen, insbesondere beim makrozyklischen Liganden $\mathrm{HL}^{9}$ mit hoher Bindungsaffinität.

2. Anzahl der Kupferzentren: Bei Liganden, welche zwei Kupferzentren koordinieren können, besteht die Möglichkeit der Bildung intermolekularer $\left\{\mathrm{Cu}_{2}(\mathrm{~L}) \mathrm{O}_{2}\right\}_{\mathrm{n}}$-Oligomere. ${ }^{[155]}$ Allerdings wurde selbst bei solchen Liganden mit flexibler Ethylenbrücke zwischen zwei tripodalen $\mathrm{N}_{4}$-Einheiten eine Bevorzugung intramolekularer Addukte gefunden. ${ }^{[165]}$ Die bei "L ${ }^{9} \mathrm{Cu}_{2}\left(\mathrm{O}_{2}\right)^{\text {“- }}$ beobachtete Zerfallskinetik 2. Ordnung deutet dagegen auf die Existenz einer dimeren Verbindung hin. Möglich ist, daß zunächst die monomere Dikupfersauerstoffverbindung entsteht, die anschließend auf einem Reaktionsweg weiterreagiert, welcher ein dimeres Zwischenprodukt aufweist.

3. Spektroskopische Befunde: Die im UV/Vis-Spektren der Sauerstoffaddukte von $\mathrm{HL}^{4}$ und $\mathrm{HL}^{9}$ weisen aufgrund der Banden bei 633 bzw. $612 \mathrm{~nm}$ die größte 
Ähnlichkeit mit dem Spektrum der $\mu-\eta^{1}: \eta^{1}$-Peroxoverbindung $\mathbf{X I}$ auf. Eine dritte Bande im Bereich von $440 \mathrm{~nm}$ wurde zwar in beiden Fällen nicht gefunden, wird aber möglicherweise von den starken Banden der $\pi-\pi^{*}$-Übergänge des Liganden überdeckt. Die Spektren von $\operatorname{Bis}(\mu-0 \times 0)-$ und $\mu_{4^{-}}$ Peroxoverbindungen unterscheiden sich dagegen deutlich (vgl. Tabelle 21). Durch die Ramanspektren lassen sich die Möglichkeiten weiter eingrenzen: $\mu-\eta^{2}: \eta^{2}$-Peroxo- bzw. Bis( $\mu$-oxo)-Verbindungen zeigen nur jeweils eine charakteristische Bande bei deutlich kleineren Wellenzahlen zwischen $725-760 \mathrm{~cm}^{-1}$ für die O-O- bzw. $585-612 \mathrm{~cm}^{-1}$ für die Cu-O-Schwingung.

\begin{tabular}{|l|l|ll|}
\hline & \multicolumn{1}{|c|}{ UV/Vis [nm] } & \multicolumn{2}{|c|}{ Raman $\left[\mathrm{cm}^{-1}\right]$} \\
& \multicolumn{1}{|c}{$\left(\varepsilon\left[\mathrm{mol} \mathrm{I}{ }^{-1} \mathrm{~cm}^{-1}\right]\right)$} & $\mathbf{O}-\mathrm{O}$ & $\mathrm{Cu}-\mathrm{O}$ \\
\hline "L $\mathrm{L}^{4} \mathrm{Cu}_{2}\left(\mathbf{O}_{2}\right) “$ & $544,633(\mathrm{sh})$ & $815\left({ }^{16} \mathrm{O}\right)$ & $537\left({ }^{16} \mathrm{O}\right)$ \\
& & $769\left({ }^{18} \mathrm{O}\right)$ & $518\left({ }^{18} \mathrm{O}\right)$ \\
\hline "L ${ }^{9} \mathrm{Cu}_{2}\left(\mathrm{O}_{2}\right) “$ & $490(2320), 612(\mathrm{sh}, 1040)$ & $808\left({ }^{16} \mathrm{O}\right)$ & $532\left({ }^{16} \mathrm{O}\right)$ \\
& & $779\left({ }^{18} \mathrm{O}\right) ?$ & $505\left({ }^{18} \mathrm{O}\right)$ \\
\hline
\end{tabular}

Tabelle 23: Spektroskopische Daten von $L^{4} \mathrm{Cu}_{2}\left(\mathrm{O}_{2}\right)^{\text {“ }}$ und " ${ }^{9} \mathrm{Cu}_{2}\left(\mathrm{O}_{2}\right)^{\text {“. }}$.

Somit ist von einer $\mu-\eta^{1}: \eta^{1}$-peroxidischen Anbindung des Sauerstoffes bei den untersuchten Liganden auszugehen, jedoch ist es unwahrscheinlich, daß die sterischen Ansprüche des Chelatliganden eine trans-Verbrückung zulassen. Wahrscheinlicher ist eine $c i s-\mu-\eta^{1}: \eta^{1}$-Verbrückung des Sauerstoffes (Abbildung 69).

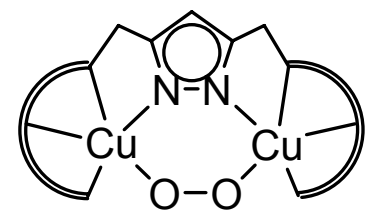

Abbildung 69: Postulierte Struktur des Sauerstoffadduktes von " $L^{4} \mathrm{Cu}_{2} \mathrm{O}_{2}$ “ und " $L^{9} \mathrm{Cu}_{2} \mathrm{O}_{2}$.

Für diese Art der Verbrückung sind bislang noch keine Vertreter strukturell charakterisiert worden. Endgültigen Aufschluß kann hier nur eine röntgenographische Untersuchung der entsprechenden Spezies liefern. DFTUntersuchungen untermauern allerdings die postulierte Konstitution, wobei die berechneten Energien für die (O-O)-Streckschwingung gut mit den experimentellen 
Werten übereinstimmen. ${ }^{[166]}$

Zusätzlich sollten in zukünftigen Arbeiten weitere Ramanmessungen von " $\mathrm{L}^{9} \mathrm{Cu}_{2} \mathrm{O}_{2}$ “ in einem anderen Lösungsmittel durchgeführt werden. So hat z.B. Acetonitril keine störenden Banden im Meßbereich. Möglicherweise könnte auch die Verwendung eines Lasers mit anderer Einstrahlwellenlänge Aufschluß über die Art des Peaks bei $779 \mathrm{~cm}^{-1}$ geben. Weitere UV/Vis-Messungen mit der Stopped-Flow-Technik für " $\mathrm{L}^{4} \mathrm{Cu}_{2}\left(\mathrm{O}_{2}\right)^{\text {“" und } ~} \mathrm{~L}^{9} \mathrm{Cu}_{2}\left(\mathrm{O}_{2}\right)^{\text {“ }}$ bei tieferer Temperatur $\left(-50^{\circ} \mathrm{C}\right)$ werden an der Universität Göttingen gemessen, was im Rahmen dieser Arbeit nicht mehr möglich war. Sie sollen zur Identifizierung von Zwischenprodukten führen - z.B. wäre im ersten Schritt eine $\eta^{1}$-superoxidische Anbindung des Sauerstoffes an ein Kupferzentrum denkbar, bevor es zur Ausbildung der Peroxidbrücke kommt. Die Identifizierung weiterer Zwischenprodukte kann auch Aufschluß über die Kinetik 2. Ordnung beim Zerfall des Komplexes " $\mathrm{L}^{9} \mathrm{Cu}_{2}\left(\mathrm{O}_{2}\right)$ “ liefern. Kinetische Daten über die Bildung des Adduktes könnten dann ebenfalls erhalten werden. Des weiteren sollte bei verschiedenen Temperaturen gemessen werden, um die thermodynamischen Daten von Bildung und Zerfall des Sauerstoffadduktes zu erhalten. 


\section{Addukte mit substratanalogen Molekülen}

\subsection{Allgemeines}

Wie könnte man sich die Anbindung des Substratmoleküls im Lauf der CatecholaseReaktion vorstellen? Klar ist, daß das Vorhandensein zweier Kupferzentren in enger Nachbarschaft (Abstand $<5 \AA$ ) eine Verstärkung der katalytischen Aktivität der betreffenden Komplexe im Vergleich zu einkernigen Kupferkomplexen mit sich bringt. ${ }^{[68,77]}$ Aber bedeutet das auch zwangsläufig, daß bei zweikernigen Komplexen eine gleichzeitige bidentate Anbindung des Substrats an beide Zentren erfolgt? In der Tat postulieren Solomon et al. für die Tyrosinase eine entsprechende bidentat verbrückende Koordination des Catechols im katalytischen Zyklus (siehe Schema 6). ${ }^{[31]}$ Bestes Gegenbeispiel ist aber die Catechol-Oxidase selbst: nach Krebs et al. erfolgt der Angriff in der Tat nur monodentat über eines der beiden Kupferzentren, während das andere Kupferion an diesem katalytischen Schritt unbeteiligt bleibt, ${ }^{[39]}$ weil es durch einen Phenylalaninrest für den Angriff großer Substratmoleküle abgeschirmt ist, während kleinere Moleküle wie $\mathrm{O}_{2}$ durchaus noch in die koordinative "Tasche" zwischen die beiden Kupferzentren eintreten können.

Um eine Vorstellung von der Art der Anbindung von Substratmolekülen an Enzyme und kleine biomimetische Koordinationsverbindungen zu erlangen, setzt man den Katalysator mit einer dem Substrat ähnlichen Verbindung um, die nach Bildung des Adduktes aber nicht weiterreagiert. Im Falle der Oxidation von Catecholen muß ein Catechol gewählt werden, dessen Normalpotential zu hoch für eine Oxidation zum oChinon mit $\mathrm{O}_{2}$ ist. Hier bieten sich verschiedene deaktivierte Catecholderivate wie Tetrachlorcatechol $\left(\mathrm{H}_{2} \mathrm{tcc}\right)$ oder Tetrabromcatechol $\left(\mathrm{H}_{2} \mathrm{tbc}\right)$ an, bei denen die Elektronendichte im aromatischen Kern durch den -I-Effekt der funktionellen Gruppen verringert ist, was eine Elektronenabgabe erschwert. So erhält man durch cyclovoltammetrische Untersuchungen für den Zweielektronenredoxprozess des Paares $\mathrm{H}_{2} \mathrm{dtbc} / \mathrm{dtboc}$ ein $E_{1 / 2}=-0.222 \mathrm{~V}$. Aufgrund des +l-Effektes der tert-butylGruppen liegt das Redoxpotential damit noch tiefer als bei unsubstituiertem Catechol (für $\mathrm{H}_{2}$ cat/o-Chinon ist $E_{1 / 2}=-0.046 \mathrm{~V}$ ). Dagegen hat das Paar $\mathrm{H}_{2} \mathrm{tcc} / \mathrm{tcoc}$ (tcoc $=$ Tetrachlor-o-chinon) ein positives Redoxpotential: $E_{1 / 2}=+0.312 \vee$ (in Methanol gegen $\mathrm{Ag} / \mathrm{AgCl}) .{ }^{[135]}$ 
Literaturbekannt sind bereits einige Tetrachlorcatecholataddukte. Die erste röntgenongraphisch charakterisierte Verbindung ist ein von Karlin et al. durch Reaktion eines phenoxoverbrückten zweikernigen Kupfer(I)komplexes mit Tetrachloro-benzochinon synthetisierter Dikupfer(II)komplex LVII, in dem tcc ${ }^{2-}$ als bidentater Ligand die Kupferzentren bei einem Cu-Cu-Abstand von $3.248 \AA$ verbrückt (Abbildung 70). ${ }^{[167]}$

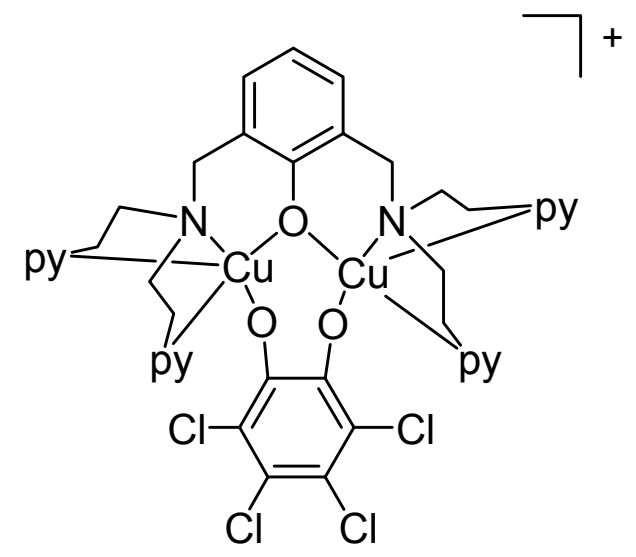

Abbildung 70: Dikupfer(II)komplex LVII von Karlin et al.

Eine weitere Dikupfer(II)verbindung mit verbrückender tcc--Einheit wurde von Comba et al. hergestellt. ${ }^{[72]}$ In derselben Arbeit wurde auch ein zweikerniger Kupferkomplex, vorgestellt, bei dem aufgrund größerer Kupfer-Kupfer-Abstände von $7.381 \AA$ das tcc $^{2-}$ nicht verbrückend zwischen die Kupferzentren tritt, sondern in bidentater Weise an nur ein Kupferzentrum bindet. Drei Bindungsweisen wurden für tcc ${ }^{2-}$ bisher gefunden: $\eta^{1}, \eta^{2}$ und $\mu-\eta^{1}: \eta^{1}$ (Abbildung 71). ${ }^{[107,57,72,168]}$

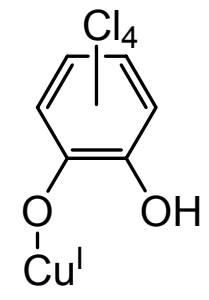

$\eta^{1}$

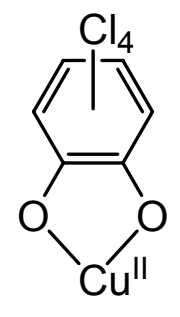

$\eta^{2}$

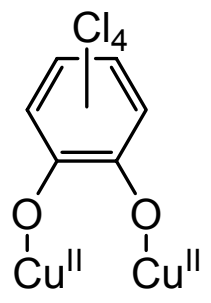

$\mu-\eta^{1}: \eta^{1}$

Abbildung 71: Bindungsmoden von tcc ${ }^{2-}$ bei Koordination an Kupfer. 


\subsection{Charakterisierung von $\operatorname{tbc}^{2-}-$ und tcc ${ }^{2-}$-Addukten an Dikupferpyrazolat- komplexe}

Von Kupferkomplexen des eigenen Ligandensystems ist ebenfalls in vorhergehenden Arbeiten die Synthese eines tcc ${ }^{2-}$-Adduktes 17 gelungen, ${ }^{[100]}$ bei dem das Catechol bidentat in der basalen Koordinationssphäre eines Metallzentrums unter Verdrängung eines chelatisierenden Seitenarmes anbindet. Bei größeren Kupfer-Kupfer-Abständen, die durch die kurzen chelatisierenden Seitenarme des Pyrazolatliganden erzwungen werden (4.202(1) $\AA$ bei 17 und 4.088(1) $\AA$ im entsprechenden substratfreien Komplex 3 ), bevorzugt das Catecholat offenbar eine nicht verbrückende Bindungsweise (Abbildung 72). ${ }^{[100]}$ Der gleiche Bindungsmodus wurde auch in der eigenen Arbeitsgruppe für das tcc ${ }^{2-}$-Addukt des Dikupferkomplexes des Liganden $\mathrm{HL}^{9}$ gefunden. ${ }^{[107]}$

Die Untersuchungen der Catecholase-Reaktion mit $\mathrm{H}_{2} \mathrm{dtbc}$ fanden unter neutralen Bedingungen in reinem Methanol sowie bei neutralen bis leicht basischen $\mathrm{pH}-$ Werten zwischen 7.3 und $8.0 \mathrm{im}$ gepufferten Milieu statt (siehe Kapitel 5). Durch Umsetzen von $\mathrm{H}_{2}$ tcc mit Dikupfer(II)pyrazolatkomplexen in reinem Methanol bildeten sich keine tcc $^{2-}$-Addukte, so daß mit einer Hilfsbase gearbeitet werden mußte, um das Tetrachlorcatechol zu deprotonieren. Um Aufschlüsse über das Aussehen eines Katalysator-Substrat-Adduktes während der Katalyse erhalten zu können, sollte das tcc ${ }^{2-}$-Addukt aber unter vergleichbaren - d.h. nicht zu basischen Reaktionsbedingungen wie bei den kinetischen Messungen hergestellt werden. Daher wurden bei der Herstellung des tcc ${ }^{2-}$-Adduktes des Dikupfer(II)komplexes von $\mathrm{HL}_{3}$ ein Äquivalent $\mathrm{H}_{2} \mathrm{tcc}$ und nur wenig $\mathrm{KO}^{t} \mathrm{Bu}$ als Hilfsbase (2.7 Äquivalente) verwendet. Dies führte zur Protonierung des freien Seitenarmes bei 17. Offen blieb zunächst die Frage, ob bei der Deprotonierung des Seitenarmes bei Verwendung einer größeren Basenmenge sich die Art der Anbindung des tcc $^{2-}$ noch ändern könnte - möglicherweise in Form einer "Verschiebung“ des tcc $^{2-}$ zwischen die Kupferzentren als Tetrachlorocatecholat-Brücke. 


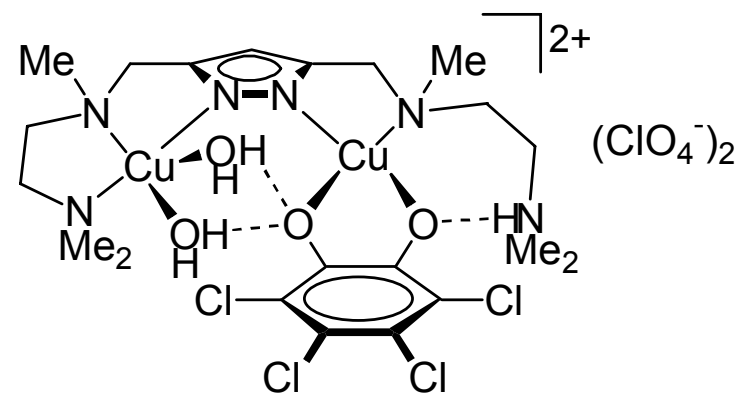

Abbildung 72: Struktur von 17.

Eine andere Methode zur Herstellung von tcc ${ }^{2-}$-Addukten, bei der weniger Hilfsbase benötigt wird, wurde schon von Karlin et al. vorgestellt. ${ }^{[167]}$ Man setzt dabei die Dikupfer(I)verbindung mit Tetrachlor-o-chinon (o-Chloranil) und mit nur einem Äquivalent Base um, welches zur Abstraktion des Pyrazol-H-Atoms dient. Die Synthese selbst muß aufgrund der Instabilität der Dikupfer(I)verbindungen bei tiefer Temperatur sowie unter Schutzgasatmosphäre stattfinden. Ist die Reaktion abgeschlossen, so kann zur Gewinnung der Substanz in kristalliner Form unter Raumtemperaturbedingungen weitergearbeitet werden.

Auf diese Weise gelang die Darstellung eines tcc ${ }^{2-}$-Adduktes an den Dikupferkomplex von $\mathrm{HL}^{3}$ unter Verwendung von $\left[\mathrm{Cu}^{\prime}(\mathrm{MeCN})_{4}\right] \mathrm{PF}_{6}$ in Propionitril. Anschließend konnte das Produkt $\left[\mathrm{L}^{3} \mathrm{Cu}_{2}\left(\mathrm{C}_{6} \mathrm{Cl}_{4} \mathrm{O}_{2}\right)\left(\mathrm{H}_{2} \mathrm{O}\right)(\mathrm{MeCN})\right] \mathrm{PF}_{6}$ (18) durch Diffusion von Petrolether kristallin erhalten werden. Das Produkt liegt in kristalliner Form in der Raumgruppe $P 1$ vor. In der triklinen Zelle befinden sich zwei Moleküle. In der Tat hat sich auch hier ein Addukt mit dem substratähnlichen Tetrachlorcatecholat gebildet, bei welchem das tcc $^{2-}$ nur an eines der beiden Kupferzentren in der basalen Ebene einer stark verzerrt quadratisch pyramidalen Koordinationsumgebung mit $\tau=0.31$ in bidentater Weise koordiniert und über das O2-Atom eine $\mathrm{H}$-Brücke zu einem $\mathrm{H}_{2} \mathrm{O}$-Molekül ausbildet, welches wiederum an das andere Kupferzentrum basal koordiniert ist $\left(d_{02 \cdots 03}=2.586(3) \AA\right.$, Abbildung 73). 


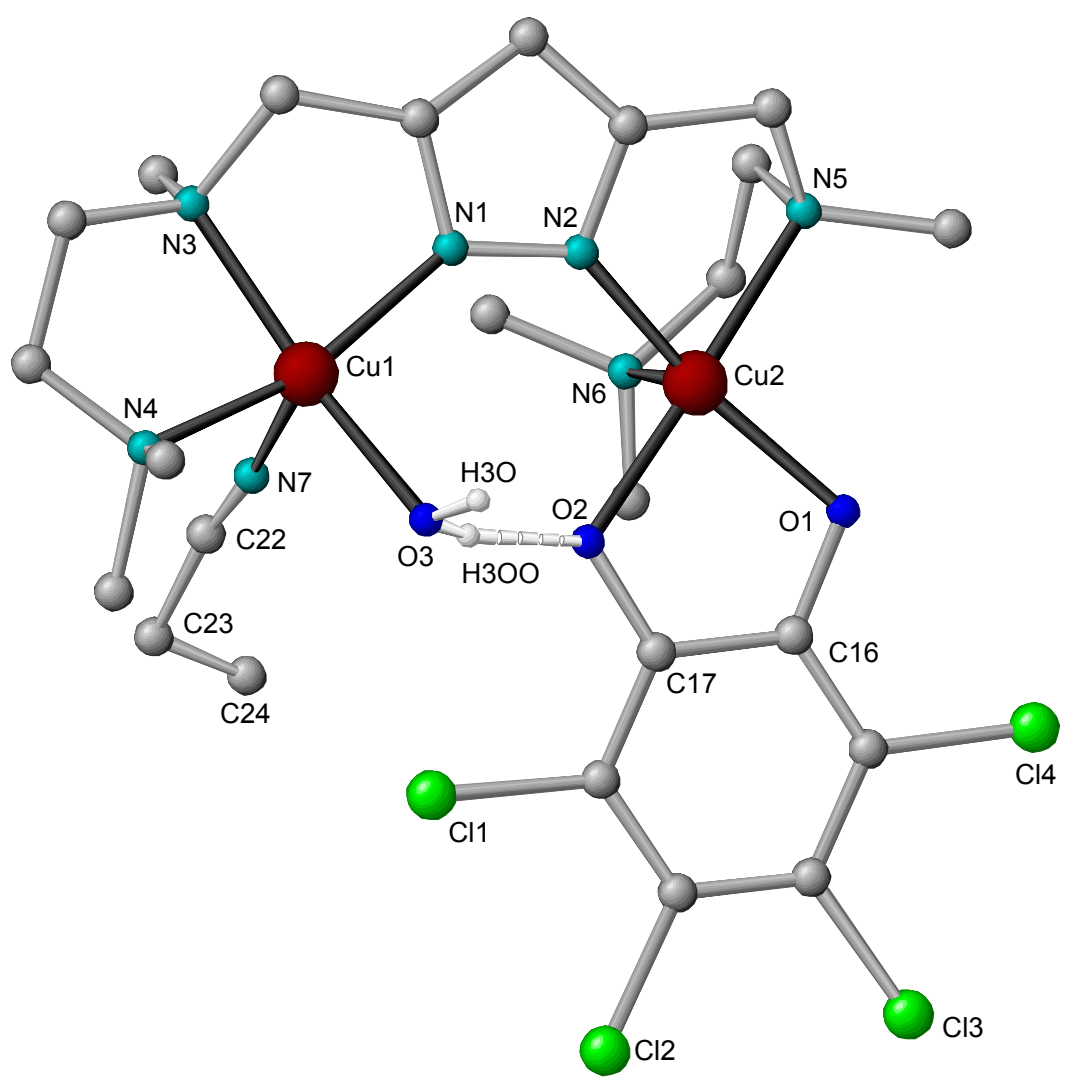

Abbildung 73: Festkörperstruktur des Kations von 18.

An Cu1 findet man ebenfalls eine verzerrt quadratisch pyramidale Koordinationsumgebung mit $\tau=0.18$. In der apicalen Position befindet sich ein MeCN-Solvensmolekül. Vergleicht man die Komplexe 17 und 18, stellt man fest, daß sich beide sehr ähnlich sind bis auf die Tatsache, daß im Falle von 18 alle Ligandseitenarme an die Kupferzentren koordiniert sind. Man könnte somit 18 als deprotonierte Form von $\mathbf{1 7}$ betrachten. Die Frage, ob sich bei weiterer Deprotonierung die Art der Anbindung von tcc ${ }^{2-}$ ändert, läßt sich nun anhand der Struktur von 18 verneinen - offensichtlich bevorzugt das tcc-Dianion bei größeren Kupfer-Kupfer-Abständen die bidentate Anbindung an nur eines der Kupferzentren unabhängig vom Protonierungsgrad des Liganden. Bedingt durch die geometrischen Vorgaben des Pyrazolatliganden liegt der Abstand der Metallzentren mit 4.288(1) $\AA$ auch beträchtlich höher als z.B. bei dem oben genannten Komplex von Karlin et al.

Bei der Reduktion von Chinon durch Kupfer(I) kann es anstelle einer Zweielektronenreduktion zum Catechol auch zu einer einfachen Reduktion zum Semichinon kommen. ${ }^{[169,170,171]}$ Dieses kann durch seine charakteristischen UV/Vis- 
und ESR-spektroskopischen Eigenschaften identifiziert werden. ${ }^{[57]}$ Ob bei der Reaktion der $\mathrm{Cu}^{\prime}$-Verbindung mit dem o-Chinon ein Semichinon oder tatsächlich durch vollständige Reduktion ein Catecholat einstanden ist, kann aber auch durch Vergleich der charakteristischen Bindungslängen von $\mathrm{C} 16-\mathrm{C} 17, \mathrm{O} 2-\mathrm{C} 17$ und $\mathrm{O} 1-$ C16 bestimmt werden. ${ }^{[135,167,172]}$ Während ein o-Semichinon durch stärker lokalisierte Doppelbindungen unterschiedliche $\mathrm{C}-\mathrm{C}-\mathrm{Bindungslängen} \mathrm{aufweist}$ und die C16-C17Bindung dann eher Einfachbindungscharakter hätte, sind in einem Catechol alle $\pi$ Bindungen delokalisiert und die Bindungslängen daher fast identisch. Zugleich weisen die C-O-Bindungslängen im Semichinon einen partiellen Doppelbindungscharakter auf und sind damit kürzer als die entsprechenden C-OEinfachbindungen im Catecholat. Im Falle von 18 liegen der C16-C17-Abstand bei 1.421(3) $\AA$ und die C-O-Abstände bei 1.333(3) bzw. 1.328(3) $\AA$ und sind somit im für bidentat koordinierte Catechole typischen Bereich. ${ }^{[135,167,172]}$

\begin{tabular}{|c|c|c|c|}
\hline \multicolumn{4}{|l|}{ Abstände } \\
\hline Cu1-O3 & $1.963(2)$ & Cu2-O2 & $1.9465(17)$ \\
\hline Cu1-N1 & $1.973(2)$ & Cu2-O1 & $1.9684(17)$ \\
\hline Cu1-N3 & $2.054(2)$ & Cu2-N2 & $1.969(2)$ \\
\hline Cu1-N4 & $2.057(2)$ & Cu2-N5 & $2.091(2)$ \\
\hline Cu1-N7 & $2.265(2)$ & Cu2-N6 & $2.331(2)$ \\
\hline \multirow[t]{4}{*}{ 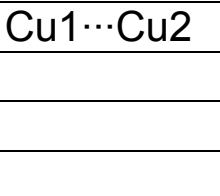 } & $4.288(1)$ & $\mathrm{O} 2 \cdots \mathrm{O} 3$ & $2.586(3)$ \\
\hline & & O1-C16 & $1.333(3)$ \\
\hline & & O2-C17 & $1.328(3)$ \\
\hline & & C16-C17 & $1.421(3)$ \\
\hline \multicolumn{4}{|l|}{ Winkel } \\
\hline O3-Cu1-N1 & $95.76(8)$ & O2-Cu2-O1 & $84.90(7)$ \\
\hline O3-Cu1-N3 & $168.12(8)$ & O2-Cu2-N2 & 97.34(7) \\
\hline N1- & $81.33(8)$ & O1-Cu2-N2 & $158.38(8)$ \\
\hline O3-Cu1-N4 & $93.41(8)$ & O2-Cu2-N5 & $176.87(8)$ \\
\hline N1-Cu1-N4 & 157.42(9) & O1-Cu2-N5 & $96.56(8)$ \\
\hline N3-Cu1-N4 & $85.47(9)$ & N2-Cu2-N5 & $82.33(8)$ \\
\hline O3-Cu1-N7 & $93.44(9)$ & O2-Cu2-N6 & $93.82(8)$ \\
\hline N1-Cu1-N7 & $104.26(9)$ & O1-Cu2-N6 & $97.10(8)$ \\
\hline N3-Cu1-N7 & $98.44(9)$ & N2-Cu2-N6 & 104.18(9) \\
\hline N4-Cu1-N7 & $95.72(9)$ & N5-Cu2-N6 & $83.27(8)$ \\
\hline
\end{tabular}

Tabelle 24: Ausgewählte Bindungslängen $[\AA]$ und -winkel $\left[^{\circ}\right]$ von 18.

Im ESI-Massenspektrum von 18 in Aceton sieht man neben dem Peak für $\left[\mathrm{L}^{3} \mathrm{Cu}_{2}\right]^{+}$ bei $\mathrm{m} / \mathrm{z}=421$ mit einer Intensität von $100 \%$ den Peak des Adduktes mit tcc ${ }^{2-}$ 
$\left[\mathrm{L}^{3} \mathrm{Cu}_{2}\left(\mathrm{C}_{6} \mathrm{Cl}_{4} \mathrm{O}_{2}\right)\right]^{+}$bei $\mathrm{m} / \mathrm{z}=667$ mit der Intensität $9 \%$. Das UV/Vis-Spektrum der Methanollösung von 18 zeigt neben den typischen Intraliganden- bzw. CTÜbergängen bei ca. $200<\lambda<300 \mathrm{~nm}$ den d-d-Übergang von quadratisch pyramidal koordiniertem Kupfer(II) bei $618 \mathrm{~nm}\left(\varepsilon=200 \mathrm{~mol} \mathrm{I}^{-1} \mathrm{~cm}^{-1}\right)$, aber auch eine schwache Bande bei $476 \mathrm{~nm}\left(\varepsilon=170 \mathrm{~mol} \mathrm{I}^{-1} \mathrm{~cm}^{-1}\right)$ (Abbildung 74). Für einen d-d-Übergang eines quadratisch pyramidal koordinierten Kupfer(II) ist die letztgenannte Wellenlänge zu niedrig, für einen CT-Übergang wäre eine stärkere Intensität zu erwarten. Ein Kupfer(II)semichinonatokomplex, hergestellt aus $\mathrm{H}_{2}$ tcc und einem Bis $(\mu$-oxo)komplex weist in diesem Bereich starke CT-Übergänge bei $434 \mathrm{~nm}$ (sh, $\varepsilon=$ $\left.3300 \mathrm{~mol} \mathrm{I}^{-1} \mathrm{~cm}^{-1}\right)$ und $444 \mathrm{~nm}\left(\varepsilon=3400 \mathrm{~mol} \mathrm{I}^{-1} \mathrm{~cm}^{-1}\right)$ auf, ein deutlicher Unterschied zur in der selben Arbeit vorgestellten analogen Verbindung mit tcc ${ }^{2-} .{ }^{[57]}$ Vielleicht liegt auch im hier vorgestellten Fall ein gewisser Anteil an Semichinonat in der Lösung vor, allerdings nur in kleinen Mengen. ESR-Messungen könnten darüber Aufschluß geben. So ergeben mononukleare Kupfer(II)-semichinonatokomplexe im ESR aufgrund antiferromagnetischer Wechselwirkung in der Regel kein Signal. ${ }^{[173]}$

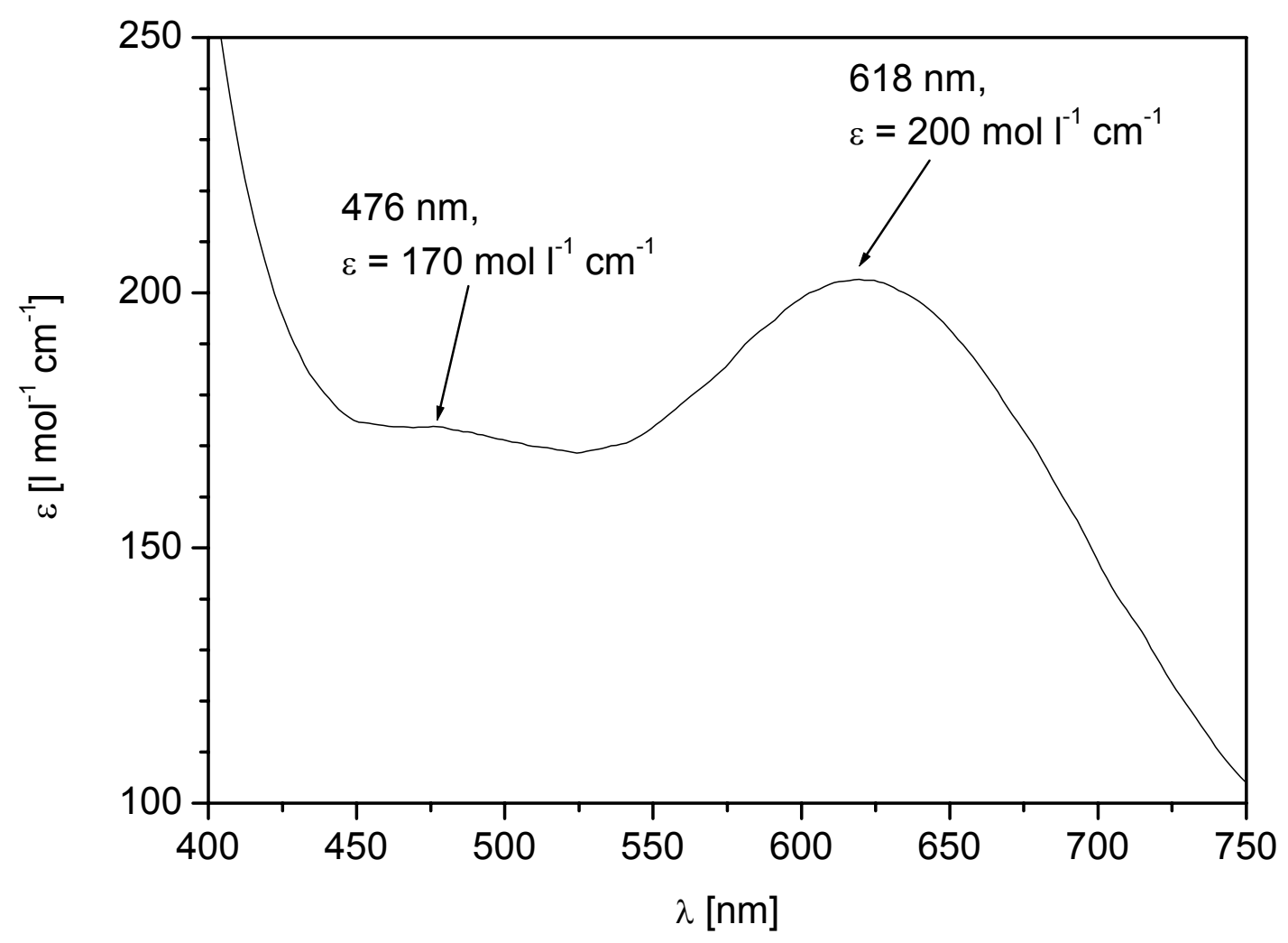

Abbildung 74: UV/Vis-Spektrum von 18 in Methanol. 
Im Rahmen dieser Arbeit konnten ESR-Messungen von 18 noch nicht durchgeführt werden, sind aber in Folgearbeiten geplant.

Röntgenographisch bestimmte Molekülstrukturen von Addukten des Tetrabromcatechols an Kupferzentren sind bisher nicht bekannt. Im Rahmen dieser Arbeit konnte das $\mathrm{tbc}^{2}$-Addukt eines Dikupfer(II)komplexes des Liganden $\mathrm{HL}^{3}$ hergestellt und röntgenographisch charakterisiert werden. Verwendet wurde hierzu $\mathrm{Cu}\left(\mathrm{NO}_{3}\right)_{2} \cdot 3 \mathrm{H}_{2} \mathrm{O}$, welches in Methanol mit dem deprotonierten $\left[\mathrm{L}^{3}\right]^{-}-$Liganden und tbc ${ }^{2-}$ umgesetzt wurde. Anschließend konnte nach Entfernen des Methanols im Vakuum der Komplex $\left[\mathrm{L}^{3} \mathrm{Cu}_{2}\left(\mathrm{C}_{6} \mathrm{Br}_{4} \mathrm{O}_{2}\right)\left(\mathrm{C}_{2} \mathrm{H}_{5} \mathrm{OH}\right) \mathrm{NO}_{3}\right]$ (19) aus Ethanol durch Etherdiffusion kristallin gewonnen werden. Der Dikupferkomplex 19 liegt im Festkörper in der P2 $1 / n-$ Raumgruppe vor. In der monoklinen Zelle befinden sich vier Moleküle. Die Art der Anbindung des Substrats entspricht im Prinzip jener des tcc- bei 17 und 18. Auch $\mathrm{tbc}^{2-}$ koordiniert als bidentater Ligand an nur eines der beiden Kupferzentren in der basalen Ebene einer verzerrt quadratisch pyramidalen Koordinationsumgebung mit $\tau_{\mathrm{Cu} 2}=0.10$. Weitere basale Donoren sind die Atome N5 und N6 des Seitenarmes, während in der apicalen Position aufgrund der Jahn-Teller-Verzerrung in deutlich erhöhtem Abstand von 2.295(6) $\AA$ das N6-Atom anbindet. Cu1 ist ebenfalls verzerrt quadratisch pyramidal koordiniert $\left(\tau_{\mathrm{Cu} 1}=0.29\right)$. Basal liegen dabei die Stickstoffatome N3 und N1 des Pyrazolatliganden, sowie die Sauerstoffatome O3 welches Teil eines Ethanolmoleküles ist - und $\mathrm{O} 4$ von einem koordinierenden Nitratanion. Apical koordiniert das N4-Atom des Pyrazolatliganden. Der Abstand der Kupferzentren ist mit 4.312(2) $\AA$ erwartungsgemäß groß (Abbildung 75). Ein H-Atom an $\mathrm{O} 3$ konnte nicht lokalisiert werden, muß aber zur Herstellung des

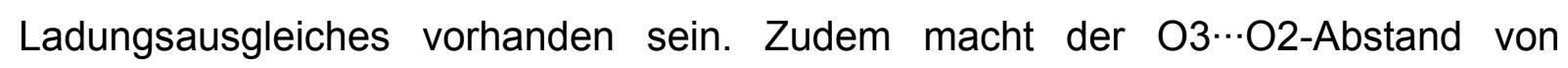
2.581(7) $\AA$ das Vorhandensein einer H-Brücke zwischen beiden Sauerstoffatomen wahrscheinlich, in Analogie zu der entsprechenden H-Brücke in 17. Die Verwendung des vollständig deprotonierten tbc-Dianions führt hier zu einer mit $\mathbf{1 8}$ fast analogen Struktur, so daß die Gleichwertigkeit beider Synthesemethoden für Catecholaddukte hiermit belegt wird. 


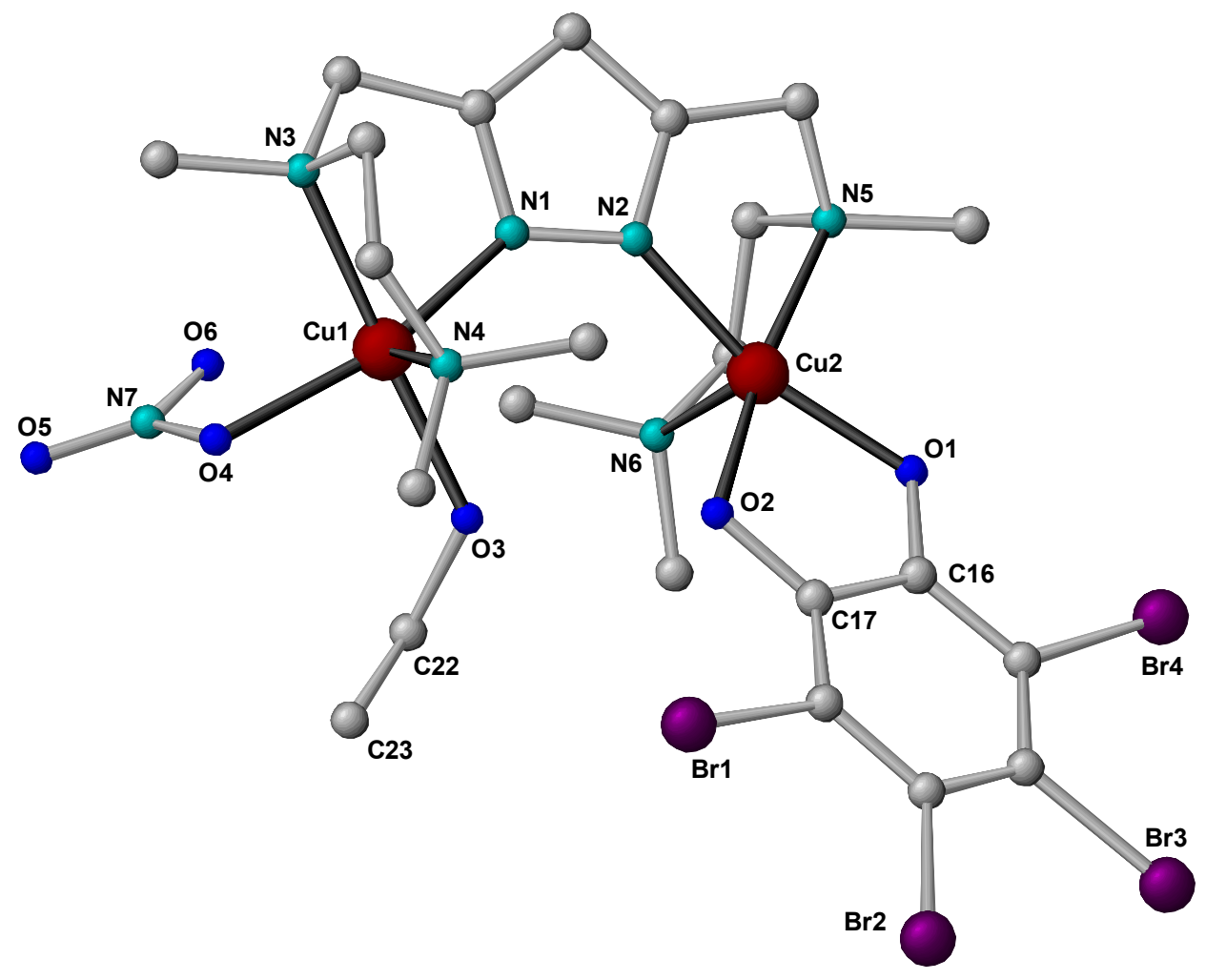

Abbildung 75: Festkörperstruktur des Kations von 19.

\begin{tabular}{|c|c|c|c|}
\hline \multicolumn{4}{|l|}{ Abstände } \\
\hline Cu1-N1 & $1.963(6)$ & Cu2-N2 & $1.970(6)$ \\
\hline Cu1-O4 & $2.003(5)$ & Cu2-N5 & $2.093(6)$ \\
\hline Cu1-O3 & $2.009(5)$ & Cu2-N6 & $2.295(6)$ \\
\hline Cu1-N3 & $2.131(6)$ & Cu2-O1 & $1.911(5)$ \\
\hline Cu1-N4 & $2.231(6)$ & Cu2-O2 & $1.982(5)$ \\
\hline \multirow[t]{4}{*}{ Cu1-Cu2 } & $4.3120(15)$ & O2-O3 & $2.581(7)$ \\
\hline & & O1-C16 & $1.322(8)$ \\
\hline & & O2-C17 & $1.338(8)$ \\
\hline & & C16-C17 & $1.408(11)$ \\
\hline \multicolumn{4}{|l|}{ Winkel } \\
\hline N1-Cu1-O4 & $159.7(2)$ & O1-Cu2-N2 & $163.9(2)$ \\
\hline N1-Cu1-O3 & $98.4(2)$ & O1-Cu2-O2 & $84.9(2)$ \\
\hline O4-Cu1-O3 & $90.3(2)$ & N2-Cu2-O2 & $100.7(2)$ \\
\hline N1-Cu1-N3 & $81.4(2)$ & O1-Cu2-N5 & $90.5(2)$ \\
\hline O4-Cu1-N3 & $90.8(2)$ & N2-Cu2-N5 & $81.5(2)$ \\
\hline O3-Cu1-N3 & $176.8(2)$ & O2-Cu2-N5 & $170.1(2)$ \\
\hline N1-Cu1-N4 & \begin{tabular}{|l|}
$104.2(2)$ \\
\end{tabular} & O1-Cu2-N6 6 & $94.1(2)$ \\
\hline O4-Cu1-N4 & $93.6(2)$ & N2-Cu2-N6 & $99.0(2)$ \\
\hline O3-Cu1-N4 & $92.8(2)$ & O2-Cu2-N6 & $104.6(2)$ \\
\hline N3-Cu1-N4 & $84.2(2)$ & N5-Cu2-N6 & $84.4(2)$ \\
\hline
\end{tabular}

Tabelle 25: Ausgewählte Bindungslängen $[\AA]$ und -winkel $\left[^{\circ}\right]$ von 19. 
Im FAB-Massenspektrum von 19 dominiert das Fragment $\left[\mathrm{L}^{3} \mathrm{Cu}_{2}\right]^{+}$mit einer Intensität von $100 \%$ bei $m / z=421$, allerdings findet man auch mit einer relativen Intensität von $12 \%$ die Spezies $\left[{ }^{3} \mathrm{Cu}_{2}\left(\mathrm{C}_{6} \mathrm{Br}_{4} \mathrm{O}_{2}\right)\right]^{+}$bei $\mathrm{m} / \mathrm{z}=841$. Das UV/Vis-Spektrum der Methanollösung von 19 zeigt auch hier neben der d-d-Bande bei $617 \mathrm{~nm}(\varepsilon=240$ $\left.\mathrm{mol} \mathrm{I}{ }^{-1} \mathrm{~cm}^{-1}\right)$ eine Bande bei $493 \mathrm{~nm}\left(\varepsilon=230 \mathrm{~mol} \mathrm{l}^{-1} \mathrm{~cm}^{-1}\right)$, die ebenfalls auf einen Semichinonatkomplex hindeuten könnte. Auch hier sind weitere Untersuchungen insbesondere ESR-spektroskopische Studien - von Interesse.

Ein tcc ${ }^{2-}$-Addukt $\left[\mathrm{L}^{4} \mathrm{HCu}_{2}\left(\mathrm{C}_{6} \mathrm{Cl}_{4} \mathrm{O}_{2}\right)\left(\mathrm{H}_{2} \mathrm{O}\right)\right]\left(\mathrm{ClO}_{4}\right)_{2}$ (20) des Dikupfer(II)komplexes mit dem Liganden $\mathrm{HL}^{4}$, welches durch Reaktion von $\left[\mathrm{L}^{4}\right]^{-}$mit zwei Äquivalenten $\mathrm{Cu}\left(\mathrm{ClO}_{4}\right)_{2} \cdot 6 \mathrm{H}_{2} \mathrm{O}$, einem Äquivalent $\mathrm{H}_{2} \mathrm{tcc}$ sowie 2.7 Äquivalenten $\mathrm{KO}^{\mathrm{t}} \mathrm{Bu}$ synthetisiert werden konnte, verhält sich analog zu 17. Der Komplex kristallisiert in der Raumgruppe $P 2_{1}$ mit vier Molekülen in der monoklinen Zelle. Diese bestehen aus zwei Paaren fast identischer Moleküle. Die Koordinationsgeometrie der beiden Kupferzentren des Komplexes ist recht unterschiedlich: Während Cu1 mit den basal koordinierenden 01- und O2-Atomen des $\mathrm{tcc}^{2-}$, sowie den N3- und N4-Atomen des Pyrazolatliganden einerseits und dem apical in großem Abstand von 2.423(4) [2.358(4)] anbindenden N4-Atom eindeutig quadratisch planar koordiniert ist $\left(\tau_{\mathrm{Cu} 1}=0.13[0.05]\right)$, so findet man für Cu2 eine verzerrt trigonal pyramidale Geometrie mit $\tau_{\mathrm{Cu} 2}=0.81[0.80]$. Hierbei koordinieren das N6-Atom des Pyrazolats und das O3-Atom eines Wassermoleküls in den axialen Positionen, und basal liegen mit den Atomen N2, N7 und N8 die restlichen N-Donoren des Pyrazolatliganden (Abbildung 76).

Somit greift das tcc $^{2-}$ auch in diesem Falle nur eines der beiden Kupferzentren an. Die Ausbildung einer quadratisch pyramidalen Geometrie um das Kupferzentrum könnte durch das Catecholderivat verursacht sein, da nur Cu2 die vom substratfreien Komplex 4 bekannte trigonal bipyramidale Koordination beibehält. Eine verbrückende Koordination durch das $\mathrm{tcc}^{2-}$ wird auch hier wieder durch den großen Abstand der beiden Kupferzentren von 4.439(1) [4.478(1)] $\AA$ (4.533(1) $\AA$ im substratfreien Komplex) verhindert. Das tcc ${ }^{2-}$ bildet des weiteren über das O2-Atom eine H-Brücke zum $\mathrm{H}$-Atom $\mathrm{H} 3 \mathrm{O}$ des an das andere Kupferzentrum koordinierenden $\mathrm{H}_{2} \mathrm{O}-$ Moleküls. Das andere $\mathrm{H}$-Atom des Wassers bildet eine $\mathrm{H}$-Brücke zum O-Atom eines 
Perchlorat-Gegenions aus $d_{03 \cdots 017}=3.050$ [2.815] $\AA$ ). Analog zu 17 wird auch hier ein Proton des $\mathrm{H}_{2}$ tcc durch ein N-Atom des Pyrazolatliganden abgefangen, so daß $\mathrm{N} 5$ protoniert vorliegt. Die entsprechenden $\mathrm{H}$-Atome befinden sich in $\mathrm{H}$-Brücken zu einem Perchloration in der einen Moleküleinheit bzw. zu einem O-Atom des tcc ${ }^{2-}$ in der anderen Einheit.

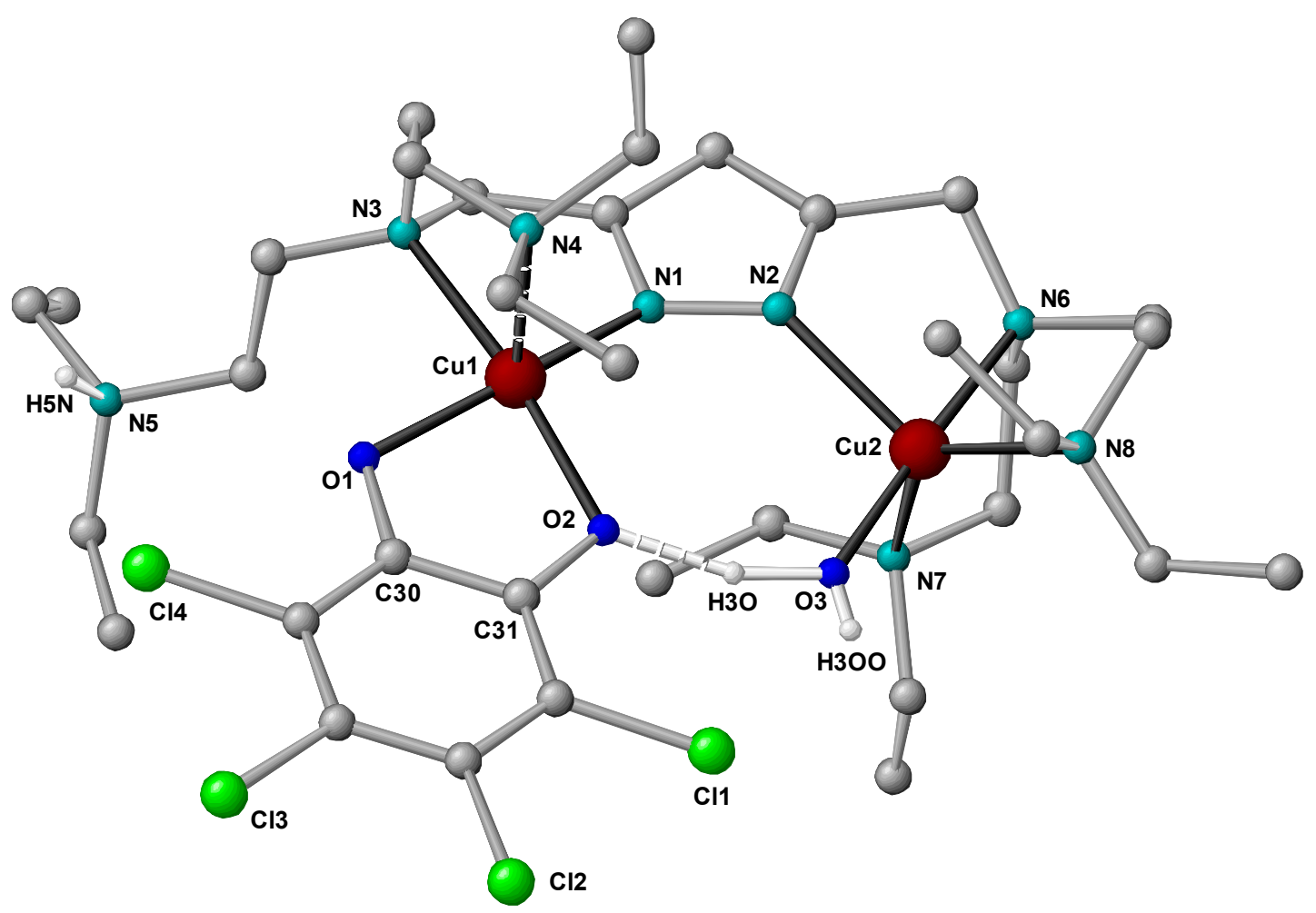

Abbildung 76: Festkörperstruktur eines Kations von 20, über H-Brücken verbundene Anionen sind aus Gründen der Übersichtlichkeit nicht aufgeführt.

Auch in diesem Komplex führt eine Analyse der entscheidenden Bindungslängen $d_{\mathrm{C} 30-\mathrm{C} 31}=1.401(7)[1.407(7)] \AA$ zum einen und $d_{01-\mathrm{C} 30}=1.322(6)[1.336(6)] \AA$ sowie $d_{\mathrm{O} 2-\mathrm{C} 31}=1.337(6)[1.333(5)] \AA$ zum anderen - zum Ergebnis, daß keine teilweise Oxidation des Catechols zum Semichinon durch Kupfer(II) stattgefunden hat.

\begin{tabular}{|c|c|c|c|c|}
\hline \multicolumn{5}{|l|}{ Abstände } \\
\hline Cu1-01 & 1.918(3) & {$[1.939(3)]$} & Cu2-O3 & $1.949(4)[1.951(4)]$ \\
\hline Cu1-N1 & $1.972(4)$ & {$[1.974(4)]$} & Cu2-N6 & $2.032(4)[2.049(4)]$ \\
\hline Cu1-O2 & $1.979(3)$ & [1.984(3)] & Cu2-N2 & $2.095(4)[2.078(4)]$ \\
\hline Cu1-N3 & $2.127(4)$ & {$[2.110(4)]$} & Cu2-N8 & $2.095(4)[2.111(4)]$ \\
\hline Cu1-N4 & $2.423(4)$ & {$[2.358(4)]$} & Cu2-N7 & $2.170(4)[2.232(5)]$ \\
\hline \multirow[t]{4}{*}{ 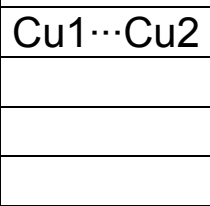 } & $4.439(1)$ & {$[4.478(1)]$} & O1-C30 & $1.322(6)[1.336(6)]$ \\
\hline & & & O2-C31 & $1.337(6)[1.333(5)]$ \\
\hline & & & C30-C31 & $1.401(7)[1.407(7)]$ \\
\hline & & & $\mathrm{O} 2 \cdots \mathrm{O} 3$ & $2.546(5)[2.604(6)]$ \\
\hline
\end{tabular}




\begin{tabular}{|l|ll|l|ll|}
\hline Winkel & \multicolumn{1}{l|}{} \\
\hline O1-Cu1-N1 & $164.1(2)$ & {$[166.1(2)]$} & O3-Cu2-N6 & $177.0(2)$ & {$[178.3(2)]$} \\
\hline O1-Cu1-O2 & $84.7(2)$ & {$[85.0(2)]$} & O3-Cu2-N2 & $99.5(2)$ & {$[98.1(2)]$} \\
\hline N1-Cu1-O2 & $100.9(2)$ & {$[100.3(2)]$} & N6-Cu2-N2 & $83.1(2)$ & {$[83.6(2)]$} \\
\hline O1-Cu1-N3 & $90.2(2)$ & {$[89.8(2)]$} & O3-Cu2-N8 & $91.5(2)$ & {$[93.6(2)]$} \\
\hline N1-Cu1-N3 & $82.4(2)$ & {$[82.8(2)]$} & N6-Cu2-N8 & $85.7(2)$ & {$[85.2(2)]$} \\
\hline O2-Cu1-N3 & $172.1(2)$ & {$[169.2(2)]$} & N2-Cu2-N8 & $125.2(2)$ & {$[130.1(2)]$} \\
\hline O1-Cu1-N4 & $92.9(2)$ & {$[90.7(2)]$} & O3-Cu2-N7 & $94.8(2)$ & {$[95.2(2)]$} \\
\hline N1-Cu1-N4 & $100.2(2)$ & {$[100.0(2)]$} & N6-Cu2-N7 & $86.1(2)$ & {$[84.5(2)]$} \\
\hline O2-Cu1-N4 & $102.8(2)$ & {$[106.3(2)]$} & N2-Cu2-N7 & $104.1(2)$ & {$[101.6(2)]$} \\
\hline N3-Cu1-N4 & $83.5(2)$ & {$[83.2(2)]$} & N8-Cu2-N7 & $128.4(2)$ & {$[125.5(2)]$} \\
\hline
\end{tabular}

Tabelle 26: Ausgewählte Bindungslängen $[\AA]$ und -winkel $\left[^{\circ}\right]$ von 20, Werte für das zweite unabhängige Molekül sind in Klammern angegeben.

Das ESI-Massenspektrum der Acetonlösung von 20 zeigt bei $\mathrm{m} / \mathrm{z}=648$ den Peak des einfach protonierten Liganden mit zwei koordinierten Kupferzentren $\left[\mathrm{L}^{4} \mathrm{Cu}_{2} \mathrm{H}\right]^{+}$mit der Intensität 100\%, in geringer Menge taucht jedoch auch das Fragment $\left[\mathrm{L}^{4} \mathrm{Cu}_{2}\left(\mathrm{C}_{6} \mathrm{Cl}_{4} \mathrm{O}_{2}\right)\right]^{+}$mit $1 \%$ bei $\mathrm{m} / \mathrm{z}=893$ auf. Im UV/Vis-Spektrum der Methanollösung von 20 sind hier nur Banden für Intraliganden- und CT-Übergange zwischen $\lambda=227 \mathrm{~nm}$ und $303 \mathrm{~nm}$, sowie eine $\mathrm{d}$-d-Bande bei $629 \mathrm{~nm}$ $\left(\varepsilon=240 \mathrm{~mol} \mathrm{I}^{-1} \mathrm{~cm}^{-1}\right)$ zu sehen.

Die Synthese des $\mathrm{tbc}^{2-}$-Adduktes eines Dikupferkomplexes mit einem Pyrazolatliganden mit langen Seitenarmen gelang nur im Falle des Liganden $\mathrm{HL}^{5}$, indem $\mathrm{H}_{2}$ tbc mit zwei Äquivalenten $\mathrm{KO}^{t} \mathrm{Bu}$ in Methanol mit dem deprotonierten Liganden und $\mathrm{Cu}\left(\mathrm{ClO}_{4}\right)_{2} \cdot 6 \mathrm{H}_{2} \mathrm{O}$ zur Reaktion gebracht wurde. Durch Kristallisation aus Aceton/Pentan konnten Kristalle des Komplexes $\left[\mathrm{L}^{5} \mathrm{Cu}_{2}\left(\mathrm{C}_{6} \mathrm{Br}_{4} \mathrm{O}_{2}\right)\left(\mathrm{H}_{2} \mathrm{O}\right) \mathrm{ClO}_{4}\right]$ (21) gewonnen werden. 21 kristallisiert in der Raumgruppe $P 2_{1} / c$ mit vier Molekülen in der monoklinen Zelle. Wie in allen übrigen Dikupferkomplexen des Liganden $\mathrm{HL}^{5}$ findet man auch hier trotz der langen Chelatseitenarme einen großen Abstand zwischen den beiden Kupferzentren: $d_{\mathrm{Cu} \text {...Cu }}=4.369 \AA$. Dementsprechend bindet auch hier das substratähnliche tbc-Dianion in der bekannten bidentaten Weise nur an eines der beiden Metallzentren (Cu1) an, während beim zweiten Kupferzentrum (Cu2) die nicht durch den Pyrazolatliganden belegten Koordinationsstellen durch ein $\mathrm{H}_{2} \mathrm{O}$ - und ein Perchlorat-Gegenion abgesättigt werden. 


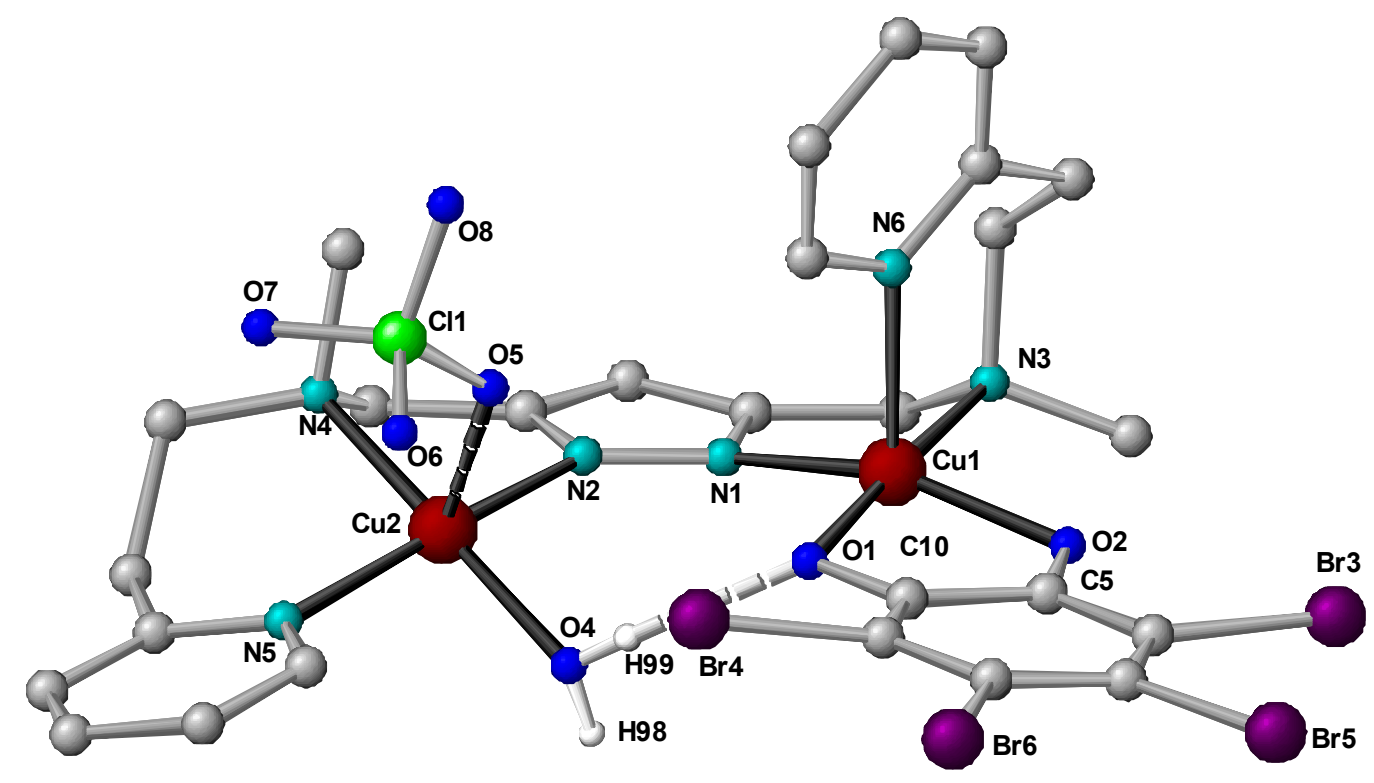

Abbildung 77: Festkörperstruktur von 21.

Im Falle von $\mathrm{Cu} 2$ handelt es sich dabei um eine fast ideal quadratisch pyramidale Koordination $\left(\tau_{\mathrm{Cu} 2}=0.08\right)$ mit einem apical befindlichen Perchlorat und den basalen Donoren N2, N4 und N5, welche vom Pyrazolatliganden stammen, sowie dem O4 des $\mathrm{H}_{2} \mathrm{O}$-Moleküls. $\tau_{\mathrm{Cu} 1}$ beträgt 0.30 und zeigt damit eine stark verzerrte quadratisch pyramidale Koordination mit dem basal anbindenden $\mathrm{tbc}^{2-}$, sowie den Donoren N1 und N3. Apical koordiniert das Pyridin-N6-Atom (Abbildung 77).

Eine H-Brücke zwischen dem O4-Atom des Wassermoleküls und dem O1-Atom des Catechols wird über das H99-Atom verwirklicht, während über die H98-Atome zweier Moleküleinheiten zwischen dem O4-Atom des einen Moleküls und dem $\mathrm{O} 2$ des anderen durch $\mathrm{H}$-Brücken im Festkörper eine dimere Anordnung entsteht (Abbildung 78). 


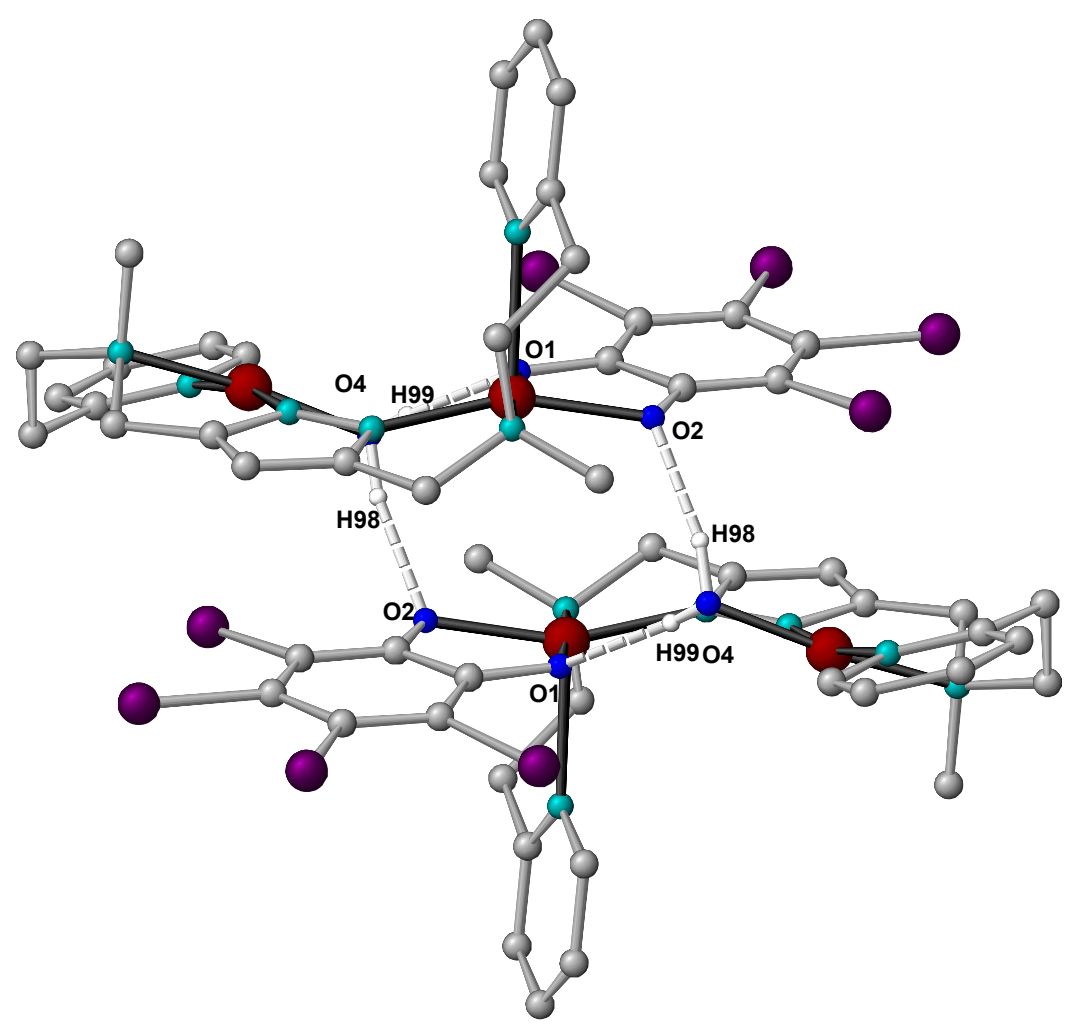

Abbildung 78: Durch H-Brücken gebildetes Dimer aus zwei Moleküleinheiten von 21, die koordinierenden Perchlorationen sind aus Gründen der Übersichtlichkeit nicht aufgeführt.

Auch im Falle von 21 sprechen die Bindungslängen $d_{\mathrm{O} 1-\mathrm{C} 10}=1.319(4)$, $d_{\mathrm{O} 2-\mathrm{C} 5}=1.328(4)$ und $d_{\mathrm{C} 5-\mathrm{C} 10}=1.429(5)$ für das Vorliegen des Adduktes in der nichtoxidierten aromatischen Catecholatform.

\begin{tabular}{|c|c|c|c|}
\hline \multicolumn{4}{|l|}{ Abstände } \\
\hline Cu1-O1 & 1.952(2) & Cu2-O4 & $1.954(3)$ \\
\hline Cu1-O2 & $1.964(2)$ & Cu2-N2 & $1.972(3)$ \\
\hline Cu1-N1 & $1.984(3)$ & Cu2-N5 & $2.007(3)$ \\
\hline Cu1-N3 & $2.087(3)$ & Cu2-N4 & $2.063(3)$ \\
\hline Cu1-N6 & $2.305(3)$ & Cu2-O5 & $2.475(3)$ \\
\hline Cu1...Cu2 & $4.3693(12)$ & O1-C10 & $1.319(4)$ \\
\hline $\mathrm{O} 1 \ldots \mathrm{O} 4$ & $2.568(4)$ & O2-C5 & $1.328(4)$ \\
\hline $\mathrm{O} 2 \ldots \mathrm{O} 4$ & $2.697(4)$ & C5-C10 & $1.429(5)$ \\
\hline \multicolumn{4}{|l|}{ Winkel } \\
\hline O1-Cu1-O2 & $84.36(10)$ & O4-Cu2-N2 & $94.43(12)$ \\
\hline O1-Cu1-N1 & 98.64(11) & O4-Cu2-N5 & $89.26(13)$ \\
\hline O2-Cu1-N1 & $159.15(12)$ & N2-Cu2-N5 & $168.74(14)$ \\
\hline O1-Cu1-N3 & 177.12(11) & O4-Cu2-N4 & $173.25(13)$ \\
\hline O2-Cu1-N3 & 92.92(11) & N2-Cu2-N4 & $81.01(13)$ \\
\hline N1-Cu1-N3 & $83.57(12)$ & N5-Cu2-N4 & $94.32(14)$ \\
\hline O1-Cu1-N6 & $87.42(11)$ & O4-Cu2-O5 & $85.59(12)$ \\
\hline O2-Cu1-N6 & 96.84(11) & N2-Cu2-O5 & 101.15(11) \\
\hline
\end{tabular}




\begin{tabular}{|l|l|l|l|}
\hline N1-Cu1-N6 & $103.89(12)$ & N5-Cu2-O5 & $89.73(12)$ \\
\hline N3-Cu1-N6 & $93.85(11)$ & N4-Cu2-O5 & $100.14(11)$ \\
\hline
\end{tabular}

Tabelle 27: Ausgewählte Bindungslängen $[\AA]$ und -winkel $\left[^{\circ}\right]$ von 21.

Die elementaranalytischen Befunde für $\mathbf{2 1}$ sind unbefriedigend, die Substanz ist trotz der Kristallinität des Produktes nicht rein. Möglicherweise liegt ein Konglomerat von Kristallen verschiedener Produkte vor. Dies wird durch FAB-massenspekrometrische Befunde unterstützt, in denen Fragmente einer wesentlich größeren Verbindung detektiert werden, welche mehr als einen Pyrazolatliganden enthalten muß. U.a. wurde bei $m / z=568$ das Fragment $\left[\mathrm{L}^{5} \mathrm{Cu}_{2} \mathrm{Br}\right]^{+}$mit einer Intensität von $14 \%$ gefunden, so daß z.T. Halogenidabstraktion eingetreten sein muß. Zwischen den Peaks bei $m / z=1393$ (Intensität $100 \%$ ) und $\mathrm{m} / \mathrm{z}=973$ (Intensität $22 \%$ ) wurde die Massendifferenz für ein $\mathrm{C}_{6} \mathrm{Br}_{4} \mathrm{O}_{2}$-Fragment gefunden. Leider konnten die Peaks nicht eindeutig zugeordnet werden, so daß die Frage nach der Art des Zweitproduktes unbeantwortet bleiben muß.

Setzt man Dikupferkomplexe von $\mathrm{HL}^{6}$ mit tcc ${ }^{2-}$ oder tbc $\mathrm{tam}^{2-}$ um, so erhält man generell keine Catecholaddukte. Anstattdessen ist eine Abspaltung des Halogensubstituenten $\left(\mathrm{Cl}\right.$ oder $\mathrm{Br}$ ) vom Catechol $\mathrm{zu}$ beobachten. Setzt man $\mathrm{HL}^{6}$ in Acetonitril mit $\mathrm{Cu}\left(\mathrm{ClO}_{4}\right)_{2} \cdot 6 \mathrm{H}_{2} \mathrm{O}$ und tcc ${ }^{2-}$ um, so findet man im ESI-Massenspektrum der Methanollösung keinen Hinweis auf ein koordiniertes Catecholat. Dagegen gehört der bestimmende Peak bei $\mathrm{m} / \mathrm{z}=805$ zum Fragment $\left[{ }^{6} \mathrm{Cu}_{2} \mathrm{Cl}\left(\mathrm{ClO}_{4}\right)\right]^{+}$. Die gleiche Beobachtung macht man im ESI-Spektrum der Aceton-Lösung nach dem Umsetzen von $\mathrm{HL}^{6}$ mit $\left[\mathrm{Cu}(\mathrm{MeCN})_{4}\right] \mathrm{PF}_{6}$ in Propionitril mit $o$-Chloranil und einer Base: Stärkster Peak ist hier die Spezies $\left[\mathrm{L}^{6} \mathrm{Cu}_{2} \mathrm{Cl}\left(\mathrm{PF}_{6}\right)\right]^{+}$, des weiteren findet man das Fragment $\left[{ }^{6} \mathrm{Cu}_{2} \mathrm{Cl}\right]^{+}$bei $\mathrm{m} / \mathrm{z}=706 \mathrm{zu} 21 \%$ und die Spezies $\left[\mathrm{L}^{6} \mathrm{Cu}_{2} \mathrm{ClF}\right]^{+}$bei $\mathrm{m} / \mathrm{z}=725$ - letztere entsteht offenbar durch Abspaltung eines Fluorids vom Gegenion. Kristalle, welche aus diesen Lösungen erhalten wurden, enthielten ausschließlich den chlorverbrückten Dikupferkomplex (vgl. 12). ${ }^{[174]}$

Zum gleichen Ergebnis gelangt man, wenn man auf analoge Weise den Dikupfer(II)komplex mit tbc ${ }^{2-}$ umsetzt. Mit einer Intensität von $100 \%$ gehört der Peak im ESI-Massenspektrum der Methanollösung bei $\mathrm{m} / \mathrm{z}=672$ zum Fragment $\left[{ }^{6} \mathrm{Cu}_{2}+\mathrm{H}\right]^{+}$. Einziger weiterer starker Peak im Spektrum ist bei $\mathrm{m} / \mathrm{z}=751$ jener des 
Fragmentes $\left[\mathrm{L}^{6} \mathrm{Cu}_{2} \mathrm{Br}+\mathrm{H}\right]^{+}(64 \%)$. Die Fähigkeit von Kupfer(I) zur radikalischen Halogenidabstraktion, z.B aus Dichlormethan, ist bereits bekannt. ${ }^{[161]}$

Natürlich sagen Massenspektren nur wenig darüber aus, in welchen Mengen eine Spezies in der untersuchten Probe tatsächlich vorhanden ist. Dennoch läßt sich anhand der erhaltenen Festkörperstruktur sagen, daß zumindest ein Teil des Catechols durch den Dikupferkomplex zerstört worden sein muß und sich davon abgespaltene Halogenide an die Kupferzentren angelagert haben. Zudem ließ sich das Catecholataddukt in allen Fällen bei Komplexen anderer Liganden, in denen es im Festkörper vorgefunden wurde, auch im ESI-Spektrum nachweisen, während entsprechende Signale hier völlig fehlen.

Mit Ligand $\mathrm{HL}^{7}$ wurden keine sauberen Produkte erhalten, sondern ausschließlich Pulver und Öle. Dennoch konnte ein tcc ${ }^{2-}$-Addukt im ESI-Massenspektrum der Methanollösung nachgewiesen werden. Die entsprechende Verbindung wurde durch Umsetzen von $\left[\mathrm{L}^{7}\right]^{-}$mit $\mathrm{Cu}\left(\mathrm{NO}_{3}\right)_{2} \cdot 3 \mathrm{H}_{2} \mathrm{O}$ und tcc ${ }^{2-}$ in Methanol gewonnen und nach Entfernen des Solvens im Vakuum in Acetonitril durch Etherdiffusion ausgefällt. Der stärkste Peak im Spektrum gehört mit $100 \%$ bei $\mathrm{m} / \mathrm{z}=643 \mathrm{zu}\left[\mathrm{L}^{7} \mathrm{Cu}_{2}\right]^{+}$, desweiteren ist bei $m / z=705$ mit $27 \%$ das Fragment $\left[\mathrm{L}^{7} \mathrm{Cu}_{2}\left(\mathrm{NO}_{3}\right)\right]^{+} \mathrm{zu}$ finden. Interessant ist der Peak bei $\mathrm{m} / \mathrm{z}=950$. Er entspricht einer Spezies $\left[\mathrm{L}^{7} \mathrm{Cu}_{2}\left(\mathrm{C}_{6} \mathrm{Cl}_{4} \mathrm{O}_{2}\right)\left(\mathrm{NO}_{3}\right)+\mathrm{H}\right]^{+}$und hat eine Intensität von $61 \%$. Theoretische und gefundene Isotopenverteilung stimmen gut überein. Allerdings ist auch in diesem Spektrum bei $\mathrm{m} / \mathrm{z}=768$ mit $25 \%$ ein Peak $\mathrm{zu}$ finden, der zu einer Spezies $\left[\mathrm{L}^{7} \mathrm{Cu}_{2} \mathrm{Cl}\right]^{+}$gehört, so daß auch hier von einer zumindest teilweisen Abspaltung von Chlor vom Substratimitat ausgegangen werden muß. 

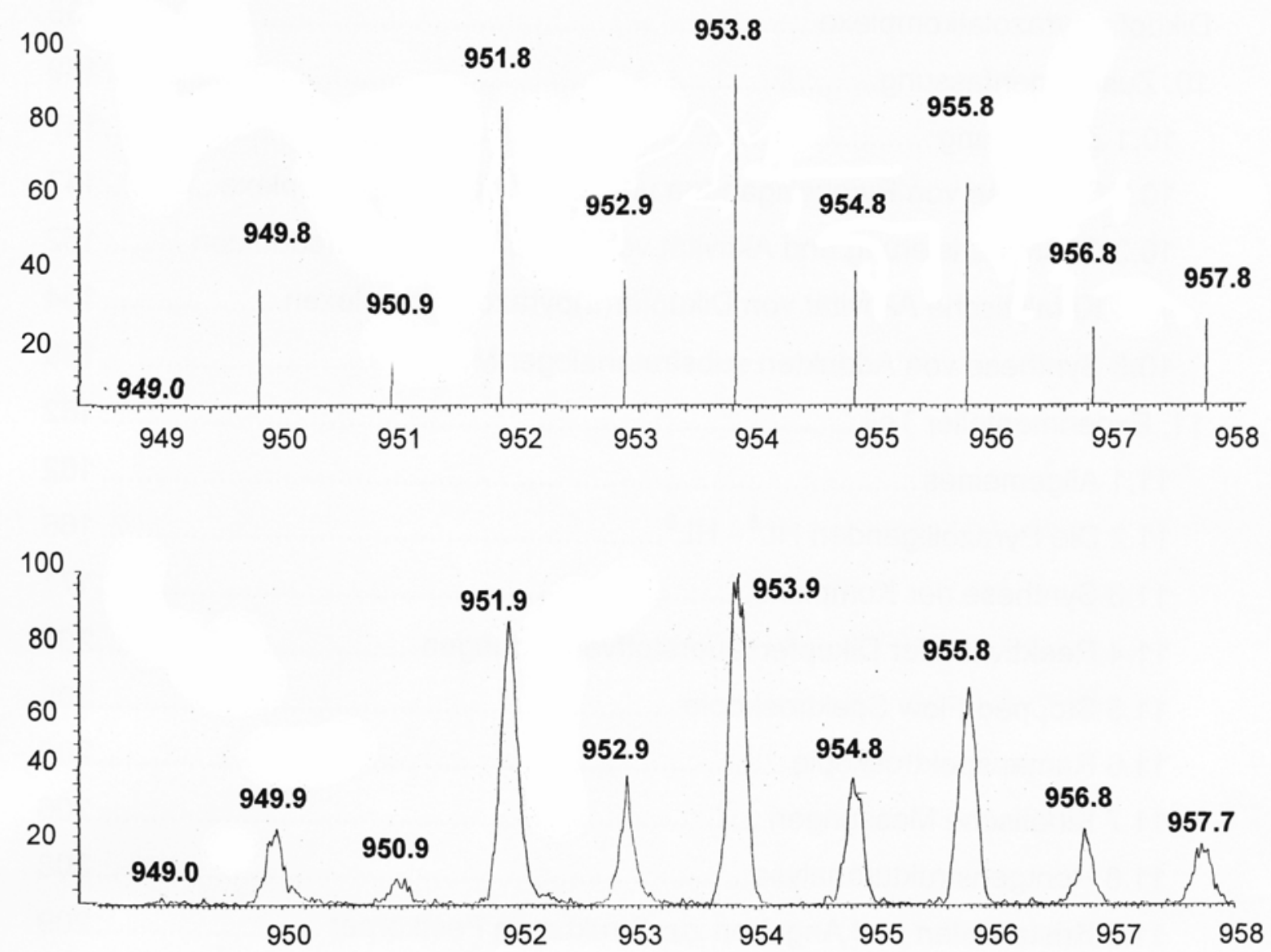

Abbildung 79: theoretisches (oben) und experimentell bestimmtes (unten) Isotopenmuster im ESIMassenspektrum von $\left[\mathrm{L}^{7} \mathrm{Cu}_{2}\left(\mathrm{C}_{6} \mathrm{Cl}_{4} \mathrm{O}_{2}\right)\left(\mathrm{NO}_{3}\right)+\mathrm{H}\right]^{+}$.

Aus dem Rahmen fällt die Festkörperstruktur des tcc ${ }^{2-}$-Adduktes $\left[\mathrm{L}^{8} \mathrm{Cu}_{2} \mathrm{TCC}_{2}\left(\mathrm{PF}_{6}\right)_{2}\right.$ (22) des Dikupferkomplexes von $\mathrm{HL}^{8}$. Man gewinnt die entsprechenden Kristalle nach Reaktion des deprotonierten Liganden mit $\left[\mathrm{Cu}(\mathrm{MeCN})_{4}\right] \mathrm{PF}_{6}$ und o-Chloranil bei tiefer Temperatur in Acetonitril sowie anschließender Etherdiffusion. Der Komplex liegt in der Raumgruppe C2/c mit vier Molekülen in der monoklinen Zelle vor. Auch dieser Komplex vermeidet eine verbrückende bidentate Anbindung des Catecholats an die Kupferzentren. Hier wird dies aber erreicht, indem zwei Kupferzentren nicht nur durch eine, sondern durch zwei Ligandeinheiten verbrückt werden, und damit von drei N-Atomen eines Ligandseitenarmes, einem Pyrazol-N-Atom derselben Ligandeinheit, sowie einem Pyrazol-N-Atom der anderen Ligandeinheit in verzerrt trigonal bipyramidaler Weise - analog zu der Koordinationsgeomtrie von 14 mit dem gleichen Liganden - koordiniert werden $\left(\tau_{\mathrm{Cu} 1 / \mathrm{Cu} 1}=0.74\right)$. Zugleich koordinieren die beiden restlichen Seitenarme je ein weiteres Kupferion durch ihre jeweils drei $\mathrm{N}$ Donoren, während jeweils ein tcc-Dianion die leicht verzerrt quadratisch pyramidale 
Koordination dieser Kupferzentren vervollständigt $\left(\tau_{\mathrm{Cu} / \mathrm{Cu} 2^{\prime}}=0.05\right)$. Hierbei bindet das Catechol wieder in der basalen Koordinationssphäre an, das aliphatische N6-Atom sitzt aufgrund der Jahn-Teller-Verzerrung in großem Abstand von 2.312(7) $\AA$ in der apicalen Position (Abbildung 80).

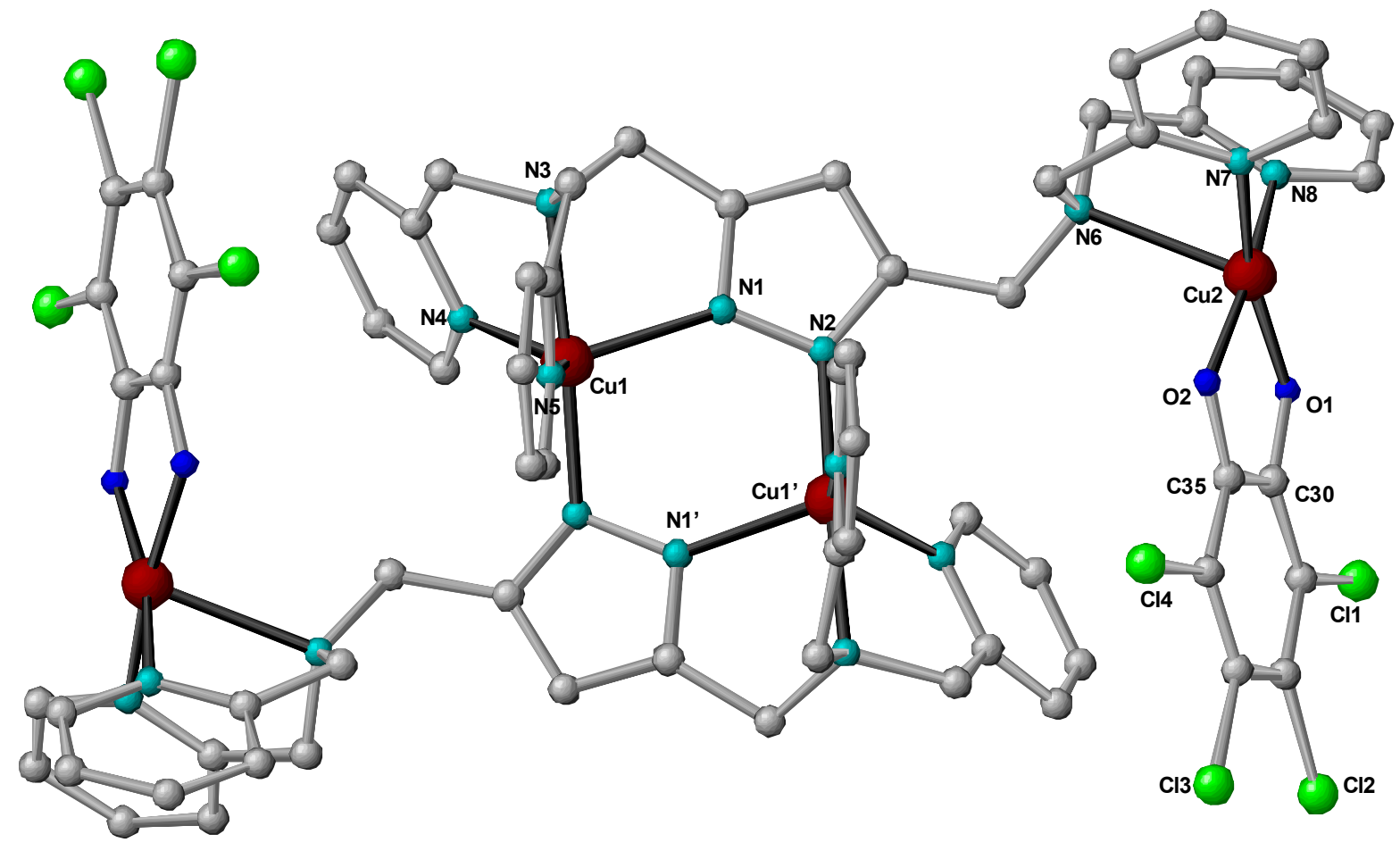

Abbildung 80: Festkörperstruktur eines Kations von 22.

Das Molekül hat eine kristallographische $\mathrm{C}_{2}$-Achse, welche u.a. die Mittelpunkte der

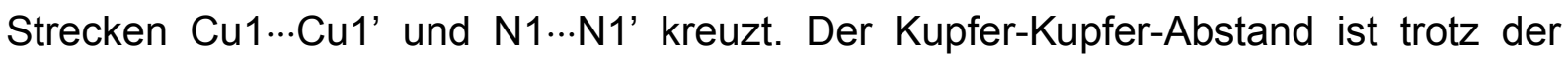
kurzen Ligandseitenarme mit $d_{\mathrm{Cu} 1 \ldots \mathrm{Cu}{ }^{\prime}}=3.757$ (3) $\AA$ vergleichsweise klein, liegt damit aber im Bereich einer Reihe literaturbekannter Bis(pyrazolato)-verbrückter Dikupfer(II)komplexe. ${ }^{[175,176,177,178,179]}$

Man erhält das gleiche Dimer, unabhängig davon, ob man unter völligem Luft- und Wasserausschluß in Acetonitril/Ether oder an Luft in Methanol/Ether auskristallisieren läßt. Sofern in Lösung ein Gleichgewicht zwischen monomeren und dimeren tcc ${ }^{2-}$ Komplex-Addukten vorliegt (Schema 17), ist die Bevorzugung der bidentat chelatisierenden Anbindung offenbar so groß, daß die dimere Verbindung 22 durch Ausfällen isoliert werden konnte. 


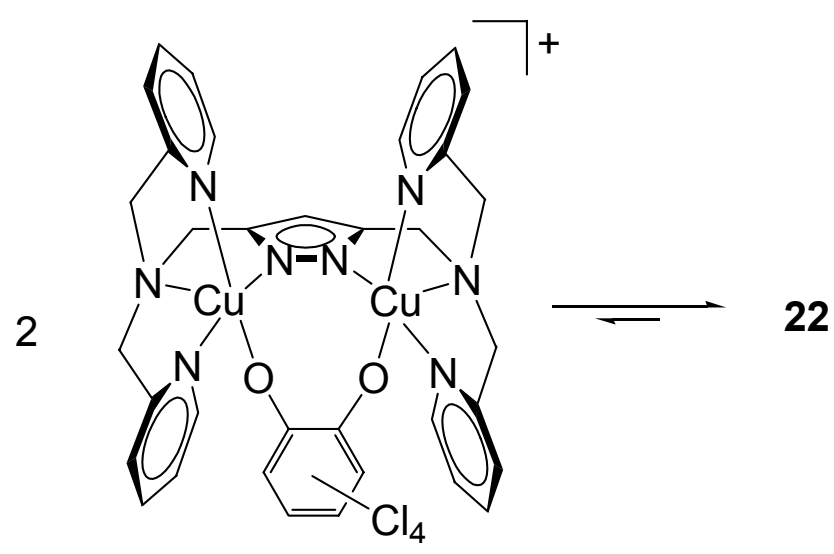

Schema 17: mögliches Gleichgewicht in Lösung zwischen einem Komplex mit bidentat verbrückendem tcc ${ }^{2-}$ und Komplex 22.

\begin{tabular}{|c|c|c|c|}
\hline \multicolumn{4}{|l|}{ Abstände } \\
\hline Cu1-N2' & 1.958(7) & Cu2-O1 & 1.921(6) \\
\hline Cu1-N3 & $2.047(7)$ & Cu2-O2 & $1.922(5)$ \\
\hline Cu1-N4 & $2.055(8)$ & Cu2-N8 & $1.998(6)$ \\
\hline Cu1-N5 & $2.055(8)$ & Cu2-N7 & $2.028(7)$ \\
\hline Cu1-N1 & $2.076(7)$ & Cu2-N6 & $2.312(7)$ \\
\hline \multirow{3}{*}{ Cu1 Cu1' } & $3.757(3)$ & O1-C30 & $1.324(10)$ \\
\hline & & O2-C35 & $1.330(10)$ \\
\hline & & C30-C35 & $1.410(13)$ \\
\hline \multicolumn{4}{|l|}{ Winkel } \\
\hline N2'-Cu1-N3 & $176.4(3)$ & O2-Cu2-O1 & $86.7(2)$ \\
\hline N2'-Cu1-N4 & $100.4(3)$ & O2-Cu2-N8 & $170.3(3)$ \\
\hline N3-Cu1-N4 & $80.0(3)$ & O1-Cu2-N8 & $88.4(3)$ \\
\hline N2'-Cu1-N5 & $94.8(3)$ & O2-Cu2-N7 & $90.6(3)$ \\
\hline N3-Cu1-N5 & $82.4(3)$ & O1-Cu2-N7 & 167.3(3) \\
\hline N4-Cu1-N5 & 132.3(3) & N8-Cu2-N7 & $96.0(3)$ \\
\hline N2'-Cu1-N1 & $102.7(3)$ & O2-Cu2-N6 & $94.7(2)$ \\
\hline N3-Cu1-N1 & $80.3(3)$ & O1-Cu2-N6 & 114.6(3) \\
\hline N4-Cu1-N1 & 113.4(3) & N8-Cu2-N6 & 79.8(3) \\
\hline N5-Cu1-N1 & $106.7(3)$ & N7-Cu2-N6 & 77.9(3) \\
\hline
\end{tabular}

Tabelle 28: Ausgewählte Bindungslängen $[\AA]$ und -winkel $\left[^{\circ}\right]$ von 22.

Das größte Fragment, das im ESI-Massenspektrum der Methanollösung von $22 \mathrm{zu}$ sehen ist, ist $\left[\mathrm{L}^{8} \mathrm{Cu}_{2}\left(\mathrm{C}_{4} \mathrm{Cl}_{4} \mathrm{O}_{2}\right)\right]^{+}$bei $\mathrm{m} / \mathrm{z}=859(20 \%)$. Es ist auch hier wieder eine teilweise Halogenidabspaltung vom Substrat festzustellen, da auch eine Spezies $\left[\mathrm{L}^{8} \mathrm{Cu}_{2} \mathrm{Cl}\right]^{+}$bei $\mathrm{m} / \mathrm{z}=650(28 \%)$ detektiert wurde. Der Basispeak bei $\mathrm{m} / \mathrm{z}=615$ gehört zu $\left[\mathrm{L}^{8} \mathrm{Cu}_{2}\right]^{+}$. Im UV/Vis-Spektrum in Acetonitril sieht man neben den Ligandenund CT-Übergängen bei ca. $245<\lambda<300 \mathrm{~nm}$ nur den $\mathrm{d}$-d-Übergang bei $732 \mathrm{~nm}$ 
$\left(\varepsilon=650 \mathrm{~mol} \mathrm{I}^{-1} \mathrm{~cm}^{-1}\right)$, was in Übereinstimmung mit der Festkörperstruktur auch in Lösung auf eine trigonal bipyramidale Koordinationsgeometrie an den Kupferzentren hinweist. 


\section{8. Überlegungen zum Mechanismus der Catecholase- Reaktion der Dikupferpyrazolatkomplexe}

Die Vorschläge für mögliche Mechanismen der bei den untersuchten Katalysatoren 1, 2, 3, 4, 7a, 8 und 13 gefundenen Catecholase-Aktivität basieren auf folgenden Beobachtungen:

1. Natur des Sauerstoffadduktes: Anhand der UV/Vis- und ramanspektroskopischen Messungen wurden die für die Liganden $\mathrm{HL}^{4}$ und $\mathrm{HL}^{9}$ detektierten Sauerstoffaddukte als $\mu-\eta^{1}: \eta^{1}$-Peroxoverbindung identifiziert. Aufgrund der sterischen Ansprüche des " $\mathrm{L}^{\mathrm{n}} \mathrm{Cu}_{2}{ }{ }-\mathrm{Fragmentes}$ wird von einer cis- $\mu-\eta^{1}: \eta^{1}-$ Peroxoverbindung ausgegangen. Die Annahme einer ähnlichen Art der Anbindung von molekularem $\mathrm{O}_{2}$ an die anderen Pyrazolatkomplexe ist aufgrund der Ähnlichkeit der räumlichen Umgebung der Kupferzentren, sowie des Kupfer-Kupfer-Abstandes von mindestens $3.45 \AA$ - was ungünstig für eine $\mu-\eta^{2}: \eta^{2}$-Verbrückung sein sollte - plausibel.

2. Addukte mit $\mathrm{tbc}^{2-}$ und $\mathrm{tcc}^{2-}:$ In allen beobachteten Fällen wurde eine verbrückende Anbindung des substratanalogen Moleküls an beide Kupferzentren vermieden. Anstattdessen wurde der bidentate Angriff an nur ein Kupferzentrum in dessen basaler Koordinationsspäre bevorzugt, unabhängig vom Abstand der Kupferzentren. Inwieweit dieses Ergebnis Relevanz für die katalytische Oxidation von $\mathrm{H}_{2} \mathrm{dtbc}$ hat, ist schwer abzuschätzen. So muß die von $\mathrm{tbc}^{2-}$ und $\mathrm{tcc}^{2-}$ bevorzugte Art der Anbindung nicht unbedingt mit der Art der Anbindung von $\mathrm{dtbc}^{2-}$ übereinstimmen bzw. zu einer erfolgreichen Oxidation im Falle des Substratmoleküls führen. So könnte für die $\eta^{2}$-Koordination ein Gleichgewicht vorliegen, während in einer Parallelreaktion ein $\mu-\eta^{1}: \eta^{1}$ - oder $\eta^{1}$-angreifendes Molekül zum Chinon oxidiert wird. Ebenso könnte das $\eta^{2}$-anbindende Substrat in einem nächsten Reaktionsschritt zwischen die Kupferzentren unter Ausbildung der $\mu-\eta^{1}: \eta^{1}$ Brücke wandern - dafür spräche die beobachtete Abhängigkeit der katalytischen Aktivität vom Abstand der Kupferzentren (zu große Abstände wirken sich, wenn ein verbrückender Angriff des Substrates erforderlich ist, 
negativ auf die Aktivität aus.) Aufgrund der eigenen Ergebnisse und ähnlich zu Vorschlägen von Belle et al. ${ }^{[180]}$ wird - zumindest im ersten Schritt - ein monodentater Angriff des Substrates vorgeschlagen. Desweiteren wird angenommen, daß vor oder bei dem Angriff an das Metallzentrum eine Abstraktion eines Protons stattfindet, da nicht deprotoniertes $\mathrm{H}_{2} \mathrm{tcc}$ keine Addukte mit den Kupferpyrazolatkomplexen bildet.

3. Abhängigkeit der Reaktionsgeschwindigkeit von Katalysator- und Substratkonzentration: Die lineare Abhängigkeit von der Katalysatorkonzentration, sowie die beobachtete Michaelis-Menten-Kinetik bei 2, 4, 7a, 8, 13 und 15 sind ein Hinweis darauf, daß es sich bei dem Intermediat der Katalyse um ein 1:1-Addukt aus Katalysator und Substrat handelt, die Reaktion also enzymanalog verläuft.

4. Bildung von $\mathrm{H}_{2} \mathrm{O}$ bzw. $\mathrm{H}_{2} \underline{O}_{2}: \mathrm{H}_{2} \mathrm{O}_{2}$ wurde als Nebenprodukt bei der Katalyse für 1, 2 und 3 eindeutig nachgewiesen. Für 7a, 8 und 11 war der Nachweis negativ, für 4 wurde eine geringe $\mathrm{H}_{2} \mathrm{O}_{2}$-Entwicklung beobachtet. Anhand dieser Ergebnisse werden für die unterschiedlichen Katalysatoren zwei verschiedene Reaktionswege vorgeschlagen, bei Weg I entsteht $\mathrm{H}_{2} \mathrm{O}_{2}$ als Nebenprodukt, bei Weg II $\mathrm{H}_{2} \mathrm{O}$. Im Falle von 4 ist es möglich, daß beide Reaktionswege beschritten werden. Eine weitere Möglichkeit im Falle von 4, 7a, 8 und 13 - die Zersetzung des $\mathrm{H}_{2} \mathrm{O}_{2}$ schon während der Reaktion oder der Aufarbeitung - wurde durch eine Blindprobe, in der nur der Katalysator und $\mathrm{H}_{2} \mathrm{O}_{2}$ vorlagen, ausgeschlossen.

Bei Weg I (Schema 18) tritt zunächst im ersten Schritt unter Verlust eines Protons das Substrat monodentat in die basale Kooridinationsumgebung eines Kupfer(II)ions. Möglicherweise erfolgt dann in einem nächsten Schritt die $\mu-\eta^{1}: \eta^{1}-$ Verbrückung des Substrates unter Verlust eines weiteren Protons. Diese Möglichkeit wird durch die besonders hohe Aktivität der Katalysatoren mit $d_{\mathrm{Cu} \cdots \mathrm{Cu}} \sim 3.5 \AA$ nahegelegt. Anschließend wird unter Reduktion beider Kupferzentren zu Kupfer $(I)$ das Substrat zum Chinon oxidiert. Die Dikupfer(I)spezies wird dann durch $\mathrm{O}_{2}$ unter Bildung von $\mathrm{H}_{2} \mathrm{O}_{2}$ wieder zu Kupfer(II) reoxidiert. Im Falle von $\mathbf{2}$ und $\mathbf{4}$ ist die Chinonbildung der geschwindigkeitsbestimmende Schritt. Da für 1 und 3 keine Abhängigkeit der Reaktionsgeschwindigkeit von $\left[\mathrm{H}_{2} \mathrm{dtbc}\right]_{0}$ beobachtet wurde, könnte hier ein anderer Schritt, wie z.B. die Reoxidation mit $\mathrm{O}_{2}$, geschwindigkeitsbestimmend sein. 


\section{Weg I}

Katalysatoren 1, 2, 3, (4)

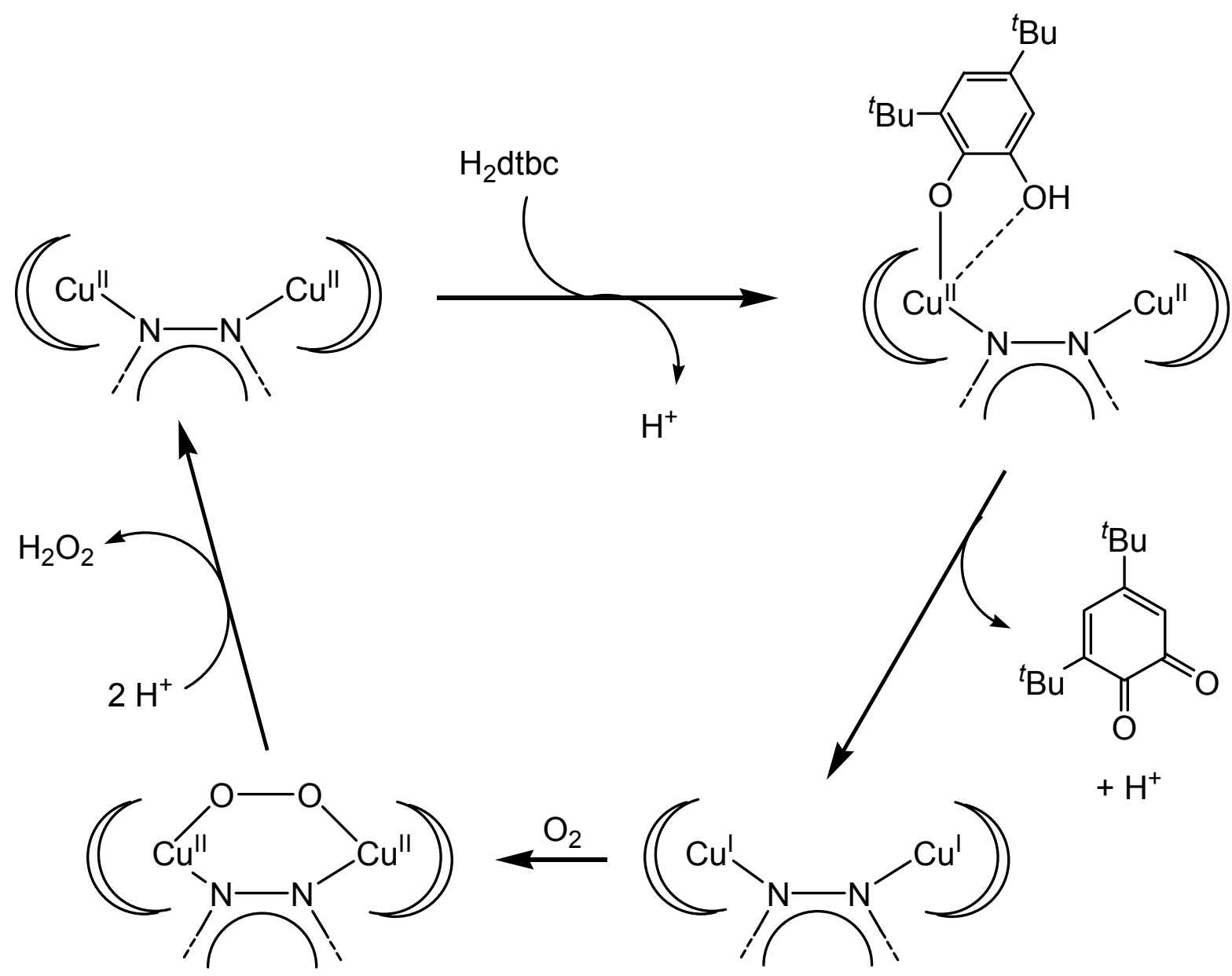

Schema 18: postulierter Mechanismus der Catecholase-Reaktion der Katalysatoren 1, 2 und 3.

Weg II (Schema 19) unterscheidet sich von Weg I, indem das Peroxoaddukt des Katalysators in der Lage ist, ein weiteres Substratmolekül unter Bildung von $\mathrm{H}_{2} \mathrm{O}$ zum Chinon zu oxidieren, wobei wieder die Kupfer(II)spezies entsteht. Der geschwindigkeitsbestimmende Schritt ist bei $\mathbf{7 a / 8}$ und 13 die Produktbildung. 


\section{Weg II}

Katalysatoren $7 \mathrm{a}, \mathbf{8}, 13$

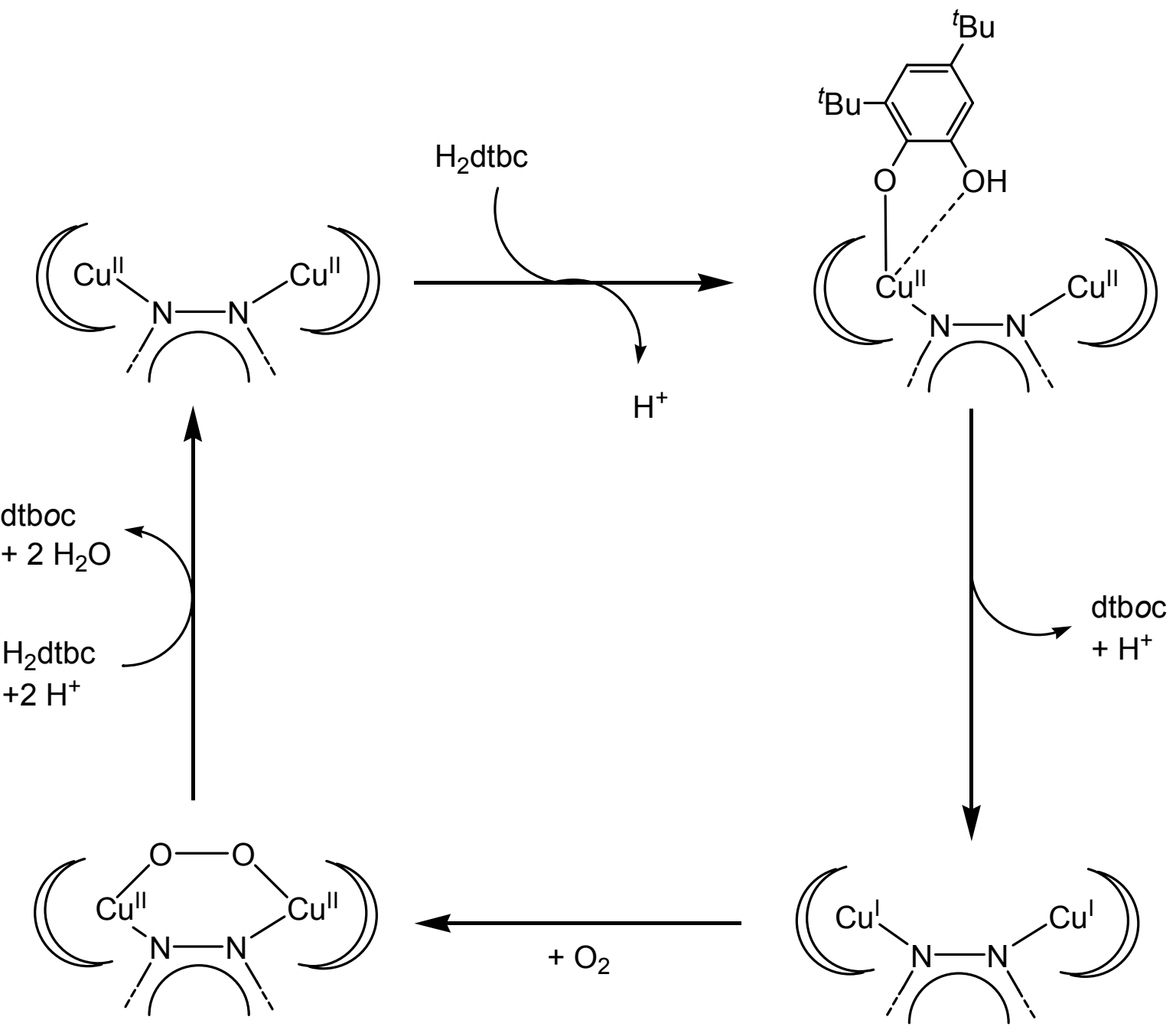

Schema 19: postulierter Mechanismus der Catecholase-Reaktion der Katalysatoren 7a, 8 und 13. 


\section{Reaktionen der Dikupferpyrazolatkomplexe mit Phenolen}

Dikupferkomplexe der Liganden $\mathrm{HL}^{1}-\mathrm{HL}^{8}$ wurden mit verschiedenen Phenolderivaten umgesetzt. Zum einen sollte dies dem Test auf ihre Fähigkeit zur oHydroxylierung von Phenolen dienen („Cresolase-Aktivität“), zum anderen sollten sie auf ihre Eignung als Katalysatoren der oxidativen Kupplung von 2,6-Dimethylphenol (DMP) zum Kunststoff Poly(1,4)phenylether (PPE) geprüft werden.

\subsection{Versuche zur o-Hydroxylierung von Phenolen}

Zum Test auf die Cresolase-Aktivität wurden die Dikupferperoxoaddukte der Pyrazolatliganden verwendet. Hierzu wurde der Ligand zunächst in Propionitril gelöst. Anschließend wurde bei $-78^{\circ} \mathrm{C}$ ein Äquivalent Kalium-tert-butylat als Base zugegeben und mit zwei Äquivalenten $\left[\mathrm{Cu}(\mathrm{MeCN})_{4}\right] \mathrm{PF}_{6}$ umgesetzt, wobei gelbe Dikupfer(I)komplexe entstanden. Aufgrund der Instabilität der meisten Sauerstoffaddukte wurde zunächst das Phenol zugegeben, bevor in die Lösung Luft eingeleitet wurde. Die Lösungen färbten sich daraufhin innerhalb kurzer Zeit grün und wurden unter Rühren aufgetaut. Nach dem Entfernen des Lösungsmittels im Vakuum wurde der Katalysator säulenchromatographisch abgetrennt und die Zusammensetzung der organischen Reaktionsprodukte mit Hilfe der GC/MS-Technik untersucht.

Als Testsubstrat wurden zwei verschiedene Phenole verwendet. 2,4-Di-tertbutylphenol sollte durch ortho-Hydroxylierung zum 3,5-Di-tert-butylcatechol umgesetzt werden, welches anschließend zum o-Chinon weiteroxidiert würde. Auf diese Weise sollte die Cresolase-Aktivität der eigenen Systeme getestet werden. Während dieses Phenol vor unerwünschten Nebenreaktionen durch die sterisch anspruchsvollen tert-Butylgruppen weitgehend geschützt ist, so sind bei dem zweiten verwendeten Phenol, dem 2,3,5-Trimethylphenol, auch weitere Oxidations- oder Oxygenierungsreaktionen denkbar, z.B. die Bildung von Oligomeren oder auch die Oxygenierung in para-Stellung unter Bildung von $p$-Chinon. Ein Grund für die Verwendung dieses Phenols ist das Interesse an seiner para-Oxygenierung zum 2,3,5-Trimethyl-p-hydrochinon und anschließender Oxidation zum 2,3,5-Trimethyl- $p$ - 
chinon. Das 2,3,5-Trimethyl-p-hydrochinon ist ein wichtiges Edukt bei der Synthese von Vitamin E, und Derivate davon weisen als Antioxidantien starke antimutagene Aktivität auf. ${ }^{[181,182]}$ Ein Metabolit des Vitamin E, das $\alpha$-Tocopherylchinon, ist ein potentes Antikoagulans. ${ }^{[181]}$ Derivate des 2,3,5-Trimethyl-p-hydrochinons und des 2,3,5-Trimethyl-p-chinons finden auch in der Bekämpfung der Alzheimerkrankheit und anderer neurodegenerativer Erkrankungen Verwendung. ${ }^{[183,184]}$

Der Test auf die Cresolase-Aktivität mit 2,4-Di-tert-butylphenol war in allen Fällen negativ, im GC/MS konnte ausschließlich Edukt nachgewiesen werden. Hinsichtlich der o-Hydroxylierung von 2,3,5-Trimethylphenol wurden im Rahmen dieser Arbeit erste Versuche mit Dikupferkomplexen der Liganden $\mathrm{HL}^{2}, \mathrm{HL}^{5}, \mathrm{HL}^{6}$ und $\mathrm{HL}^{8}$ unternommen. Als Reaktionsprodukt bei Verwendung des Komplexes " $\mathrm{L}^{1} \mathrm{Cu}_{2}\left(\mathrm{O}_{2}\right)^{\text {" }}$ wurde El-massenspektrometrisch neben dem Edukt bei $\mathrm{m} / \mathrm{z}=136$ (Intensität $100 \%$ ) nur der Molpeak eines Dimers $\left[\mathrm{C}_{6} \mathrm{H}_{2} \mathrm{O}\left(\mathrm{CH}_{3}\right)_{3}\right]_{2}$ bei $\mathrm{m} / \mathrm{z}=270(36 \%)$ gefunden. Die Konstitution des Dimers kann hierbei nicht bestimmt werden. Denkbar ist eine direkte Kupplung der Aromaten oder auch die Bildung eines Diphenylethers. Damit sind fünf verschiedene Produkte möglich (Abbildung 81).

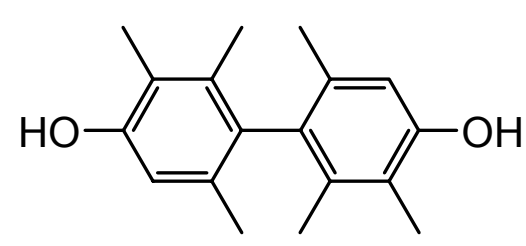

a

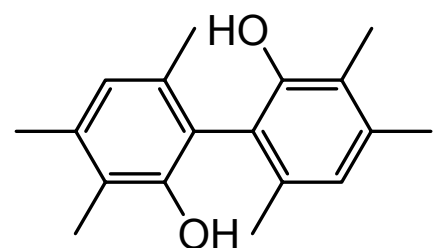

b

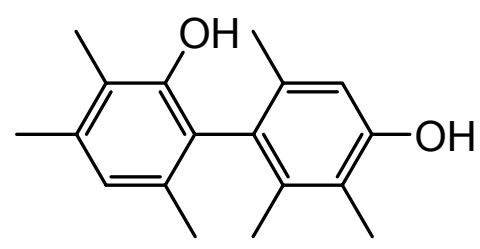

C

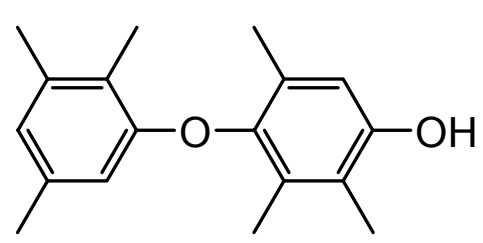

d

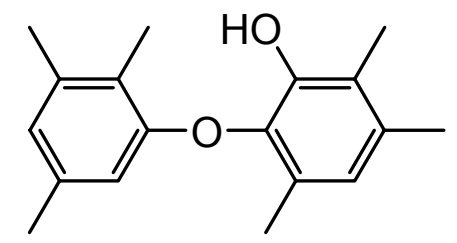

e

Abbildung 81: Mögliche Dimerisierungsprodukte von 2,3,5-Trimethylphenol mit der Molmasse 270 $\mathrm{g} / \mathrm{mol}$.

Bei der Reaktion mit "L ${ }^{5} \mathrm{Cu}_{2}\left(\mathrm{O}_{2}\right)^{\text {“ }}$ konnten ebenfalls nur Dimerisierungsprodukte nachgewiesen werden. Im GC/MS ist allerdings fast ausschließlich das Edukt mit einer Retentionszeit von 10.64 min zu sehen. In Spuren werden bei Retentionszeiten 
von 19.61, 20.69 und 20.82 min drei verschiedene Dimerisierungsprodukte mit einem Molpeak von jeweils $\mathrm{m} / \mathrm{z}=270$ detektiert, jedoch mit deutlich verschiedenen Zerfallsreihen. So ist bei dem Produkt mit der Retentionszeit 20.69 min neben dem Peak bei $\mathrm{m} / \mathrm{z}=135$ mit $30 \%$, welcher einem Fragment $\left[\mathrm{C}_{6} \mathrm{H}_{2}\left(\mathrm{CH}_{3}\right)_{3} \mathrm{O}\right]^{+}$entspricht, ein Peak mit $m / z=150$ (Intensität $100 \%$ ) zu sehen, welcher bei den beiden anderen Produkten völlig fehlt. Dieser Peak paßt zum Fragment:

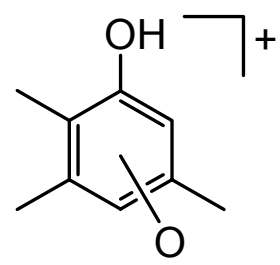

Abbildung 82: Dimerfragment bei $\mathrm{m} / \mathrm{z}=150$.

Das entsprechende Dimer ist damit entweder Diphenylether $\mathbf{d}$ oder $\mathbf{e}$. Dementsprechend liegt die Annahme nahe, daß die anderen Produkte bei " $\mathrm{L}^{5} \mathrm{Cu}_{2}\left(\mathrm{O}_{2}\right)$ ", aber auch das Produkt bei " $\mathrm{L}^{2} \mathrm{Cu}_{2}\left(\mathrm{O}_{2}\right)$ " Kupplungsprodukte zwischen den beiden aromatischen Kernen sein könnten, also eines der Dimere a - c. Hier findet man nur Peaks bei $m / z=255\left(\mathrm{M}^{+}-1 \times \mathrm{CH}_{3}\right), 240\left(\mathrm{M}^{+}-2 \mathrm{x} \mathrm{CH}_{3}\right), 237\left(\mathrm{M}^{+}-\right.$ 1x $\left.\mathrm{CH}_{3}-1 \mathrm{x}_{2} \mathrm{O}\right)$ und 135 (2,3,5-Trimethylphenoxylradikal). Somit erhält man für "L ${ }^{5} \mathrm{Cu}_{2}\left(\mathrm{O}_{2}\right)^{\prime \prime}$ ein Gemisch dreier verschiedener Dimere, von denen zwei Kupplungsprodukte der aromatischen Kerne darstellen und eines einen Diarylether.

Bei Verwendung von " $\mathrm{L}^{6} \mathrm{Cu}_{2}\left(\mathrm{O}_{2}\right)^{\text {" }}$ werden bei denselben Retentionszeiten ebenfalls Dimere gefunden, zusätzlich noch bei 19.7 und 21.8 zwei weitere Dimere. Ein

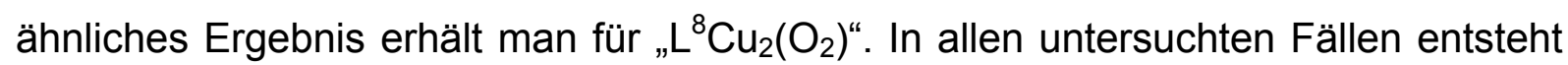
somit in Spuren in unspezifischer Weise - was auf eine radikalische Reaktion hindeutet - ein Gemisch verschiedener Dimere. Für " ${ }^{6} \mathrm{Cu}_{2}\left(\mathrm{O}_{2}\right)$ " und " $\mathrm{L}^{8} \mathrm{Cu}_{2}\left(\mathrm{O}_{2}\right)^{\text {" }}$ findet man jedoch zusätzlich bei kürzeren Retentionszeiten von 9.9 - 11.9 min verschiedene weitere Produkte mit der Molmasse $150 \mathrm{~g} / \mathrm{mol}$. Das Produkt mit der Retentionszeit $9.93 \mathrm{~min}$ konnte durch Vergleich seiner Fragmente mit den Literaturwerten als 2,3,5-Trimethyl-p-chinon identifiziert werden (Abbildung 84, Abbildung 85). Auch bei den übrigen Produkten handelt es sich wohl um Chinone oder andere Oxgenierungsprodukte. Die Dikupferkomplexe des Typs LVIII 
(Abbildung 83) sind hinsichtlich der Oxygenierung von 2,3,5-Trimethylphenol zu 2,3,5-Trimethyl-p-hydrochinon also am erfolgreichsten.

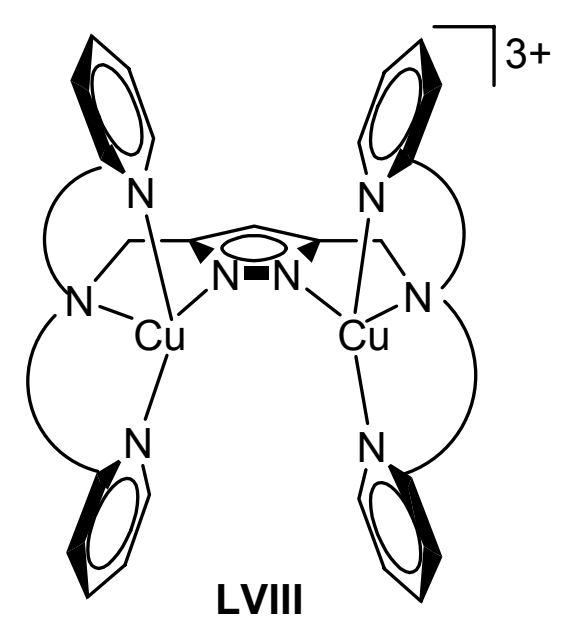

Abbildung 83: allgemeine Struktur $L$ VIII der Komplexe " $L^{6} C u_{2}$ “ und " $L^{8} C u_{2}$ “.
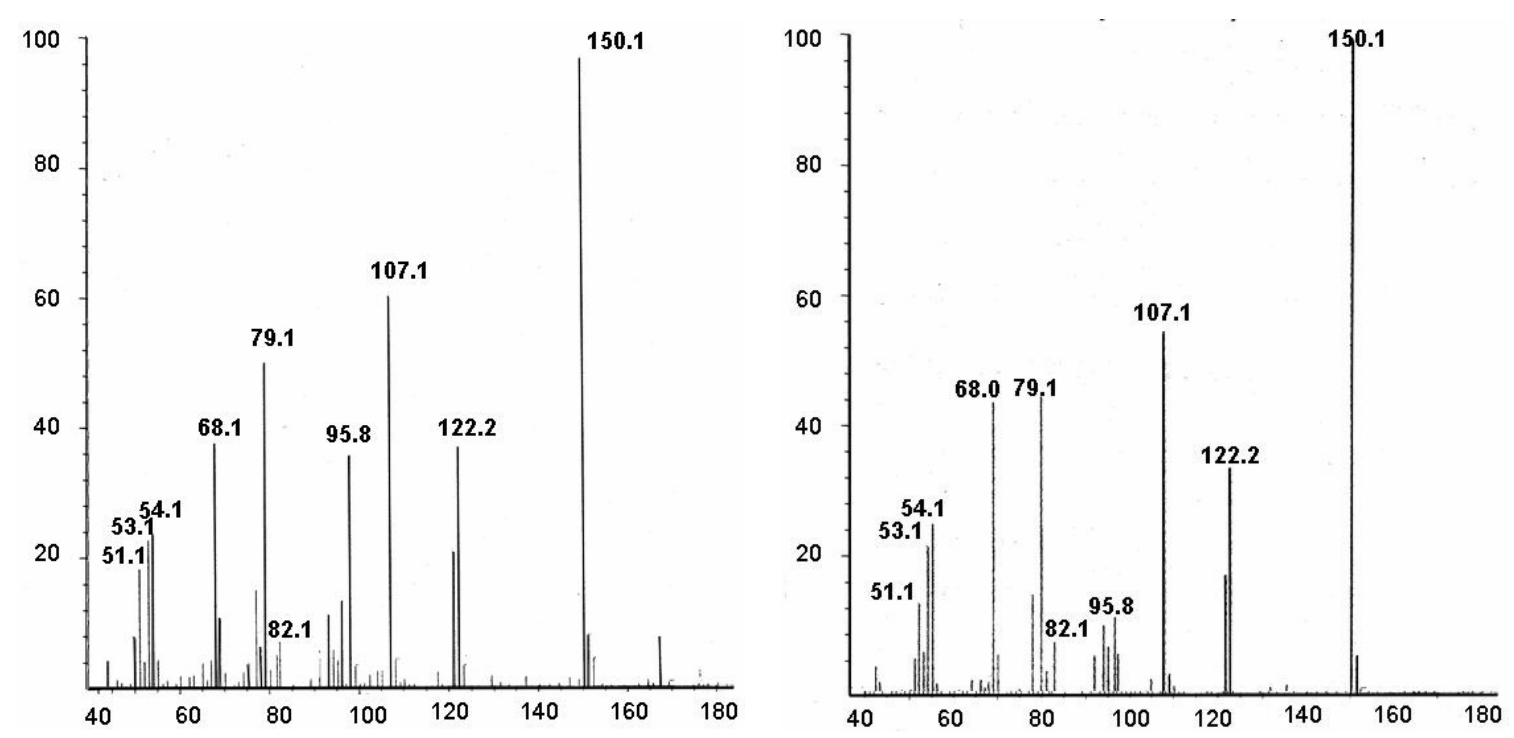

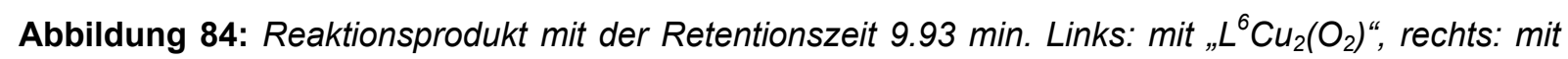
"L ${ }^{8} \mathrm{Cu}_{2}\left(\mathrm{O}_{2}\right)^{\prime \prime}$. 


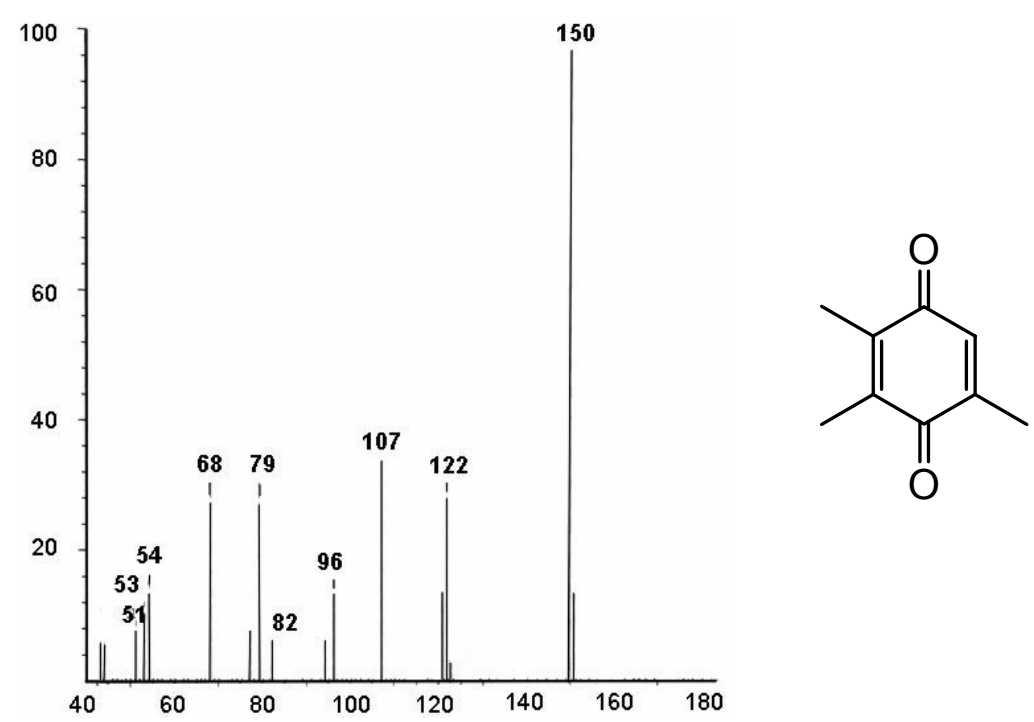

Abbildung 85: Referenzspektrum von 2,3,5-Trimethyl-p-chinon.

Unter den bislang gewählten Reaktionsbedingungen liefern die Dikupfersauerstoffaddukte jedoch noch ein Gemisch praktisch aller möglichen monomeren und dimeren Produkte in nur geringer Ausbeute. Allerdings zeigen diese ersten Versuche, daß die Dikupferpyrazolatkomplexe in der Lage sind, im Prinzip eine Vielzahl von Oxidations- und Oxygenierungsreaktionen zu vermitteln. Eine Variation der Versuchsbedingungen könnte die Effizienz beträchtlich steigern und die Spezifität erhöhen. So wurde z.B. bei Komplex XXIII (mit R = H, vgl. Schema 7) die intramolekulare Hydroxylierung des Liganden in Nitrilen unterdrückt, während in Lösungsmitteln wie Dichlormethan oder Aceton, die $\mathrm{Cu}(\mathrm{I})$ schlechter koordinieren, die Hydroxylierung beobachtet werden konnte. ${ }^{[185,186]}$ Bei höherer Effizienz der Reaktion könnte diese auch mit sterisch anspruchsvolleren Substraten wie 2,4-Di-tertbutylphenol zum Erfolg führen. Eine weitergehende Optimierung dieser Reaktivität unter Verwendung des Sortiments verfügbarer Dikupferpyrazolatkomplexe erscheint lohnend.

\subsection{Dinukleare Kupferkomplexe als Katalysatoren bei der oxidativen Kupplung von 2,6-Dimethylphenol}

Poly(1,4)phenylether (PPE) ist ein bedeutender Kunststoff aufgrund seiner chemischen Stabilität und seiner guten mechanischen Eigenschaften. ${ }^{[187,188]}$ Die oxidative Kupplung von 2,6-Dimethylphenol (DMP) zu PPE durch einen Kupfer(I)pyridinkomplex wurde im Jahre 1959 von Hay et al. gefunden (Schema 
20). ${ }^{[189]}$ Seitdem wurden viele Kupfer(II)komplexe mit stickstoffhaltigen Liganden entwickelt, welche mit Hilfe molekularen Sauerstoffs die Polymerisation von DMP katalysieren. ${ }^{[190,191,192,193,194]}$ Unter Verwendung von 4-Phenoxyphenol als Monomerem anstelle des DMP gelang auch die regioselektive oxidative Polymerisierung $\mathrm{zu}$ unsubstituiertem PPE mit Hilfe von Tyrosinasemodellkomplexen. ${ }^{[195]}$ Unerwünschtes Nebenprodukt bei der Polymerisation ist das 3,3',5,5'-Tetra-methyl-4,4'-diphenochinon (DPQ), welches aus einer C-C-Kupplung und anschließender Oxidation zweier DMP-Moleküle herrührt.<smiles>CC1=CC(=C2C=C(C)C(O[C@H](C)c3cc(C)c(O[C@H](C)CO)c(C)c3)C(C)=C2)C=C(C)C1=O</smiles>

Schema 20: Polymerisation von DMP zu PPE.

Der Mechanismus der Polymerisation ist noch immer nicht gut verstanden. Diskutiert wird ein radikalischer Reaktionsweg über Phenoxyradikale, sowie ein Mechanismus über einen zweikernigen phenolatverbrückten Kupfer(II)komplex. ${ }^{[196,197,198,199]}$

Von der Gruppe Reedijk et al. werden zur Zeit mononukleare Kupferkomplexe mit den Liganden 1-Methylimidazol (Meim), 1,2-Dimethylimidazol (Dmeim) und 4,4'Biimidazolen (bi1 und bi2) hinsichtlich ihrer Aktivität bei der Katalyse dieser Reaktion untersucht (Abbildung 86). ${ }^{[200,201,202]}$<smiles>Cn1ccnc1</smiles>

Meim<smiles>Cc1nccn1C</smiles>

Dmeim<smiles></smiles>

$\mathrm{R}=\mathrm{H}(\mathrm{bi} 1), \mathrm{Me}(\mathrm{bi})$

Abbildung 86: Liganden von Reedijk et al.

Während für die Kupferkomplexe von bi1 und bi2 im Festkörper dimere Strukturen mit zwei Kupferzentren gefunden werden, wird aus spektroskopischen Befunden geschlossen, daß sie in Lösung zu mononuklearen Spezies dissoziieren. In allen 
Fällen wird gefunden, daß die zusätzliche Methylgruppe bei Dmeim und bi2 zu einer Steigerung der katalytischen Aktivität im Vergleich zum einfach methylierten Liganden führt. Dies wird auf eine Verzerrung der mononuklearen Komplexe in Lösung zurückgeführt, was deren katalytische Aktivität beeinflußt. Zum anderen spielt die Basizität des Liganden eine Rolle, da dieser während der Katalyse als Brønstedbase wirken könnte.

Im Rahmen einer Zusammenarbeit mit der Arbeitsgruppe von Reedijk et al. wurden die Komplexe 1 - 4 auf ihre katalytische Aktivität in der DMP-Polymerisation geprüft, da bisher Daten über den Zusammenhang zwischen dem Abstand der Kupferzentren in dinuklearen Komplexen und der katalytischen Aktivität fehlen. Die katalytische Aktivität der Komplexe - bestimmt anhand der Anfangsgeschwindigkeit $v_{0}$ der Aufnahme von $\mathrm{O}_{2}$ beim Schütteln einer Mischung aus Katalysator und Substrat in Acetonitril mit dem basischen Cokatalysator $\mathrm{NaOMe}$ unter Sauerstoffatmosphäre bei Normaldruck - lag nur wenig unter jener der aktivsten der von Reedijk verwendeten Katalysatoren (Tabelle 29). Die katalytische Aktivität der Komplexe 1 - 4 ist in mehrerlei Hinsicht von Bedeutung:

1. Kupferpyrazolkomplexe galten bislang als nicht katalytisch aktiv bei der DMPPolymerisation. So sind Mischungen von 3,5-Dimethylpyrazol mit Kupfer(II)nitrat nicht katalytisch aktiv. ${ }^{[203]}$ Dagegen weisen die Komplexe 1 - 4 eine durchaus signifikante Aktivität auf.

2. Der Abstand der Kupferzentren spielt offensichtlich eine große Rolle für die katalytische Aktivität der Komplexe. Dies ist im Einklang mit dem mechanistischen Vorschlag von Reedijk et al., daß für den Fortschritt der Katalyse eine dinukleare Spezies benötigt wird.

Natürlich müssen noch weitere Faktoren bei der Betrachtung der katalytischen Aktivität berücksichtigt werden: So könnte die stärker koordinierende Methylcarbonatbrücke bei $\mathbf{3}$ dessen schwächere Aktivität im Vergleich mit $\mathbf{4}$ erklären. Anhand der Kupfer-Kupfer-Abstände wäre für $\mathbf{3}$ eigentlich die größere Aktivität zu erwarten. 2 verbraucht durch seine protonierten Seitenarme einen Teil des basischen Cokatalysators, so daß auch hier eine geringere Aktivität als erwartet resultiert. 


\begin{tabular}{|ccccc|}
\hline Katalysator & $d_{C u \cdots c u}[\AA]$ & $D M P$-Umsatz $[\%]$ & $v_{0}\left[\mathrm{~mol}^{-1} \mathrm{~s}^{-1}\right]$ & Reaktionszeit $[\mathrm{h}]$ \\
\hline bi1 & - & 68 & $3 \cdot 10^{-5}$ & 1.5 \\
bi2 & - & 43 & $31 \cdot 10^{-5}$ & 0.3 \\
Meim & - & 68 & $142 \cdot 10^{-5}$ & 0.08 \\
Dmeim & - & 76 & $200 \cdot 10^{-5}$ & 0.08 \\
$\mathbf{1}$ & 3.540 & 85 & $27.0 \cdot 10^{-5}$ & 1 \\
$\mathbf{2}$ & 3.448 & 61 & $9.4 \cdot 10^{-5}$ & 1 \\
3 & 4.088 & 54 & $1.5 \cdot 10^{-5}$ & 1 \\
4 & 4.533 & 37 & $3.0 \cdot 10^{-5}$ & 1 \\
\hline
\end{tabular}

Tabelle 29: Katalytische Aktivität bei der Polymerisation von DMP der eigenen dinuklearen Komplexe 1 - 4 und der mononuklearen Komplexe von Reedijk et al. in Acetonitril. 


\section{Zusammenfassung}

\subsection{Zielsetzung}

Ziel der Arbeit war es, neue Modellkomplexe für Enzyme mit einem Typ 3 Kupferzentrum zu entwickeln, zu charakterisieren und auf ihre katalytische Aktitvität zu überprüfen. Auch Modellkomplexe möglicher Intermediate bei der Umsetzung von Substratmolekülen sollten synthetisiert und charakterisiert werden. Hierzu sollten Pyrazolatkompartimentliganden mit zwei Koordinationstaschen zur Herstelllung dinuklearer Kupferkomplexe verwendet werden. Die Synthese einiger dieser Liganden wird in dieser Arbeit erstmals vorgestellt. Die Kupfer(I)komplexe dieser Liganden sollten zur Anbindung molekularen Sauerstoffs verwandt werden. Die erhaltenenen Sauerstoffaddukte sollten charakterisiert und zur Oxygenierung verschiedener Substrate eingesetzt werden. Neue Kupfer(II)pyrazolatkomplexe sollten synthetisiert und hinsichtlich ihrer Fähigkeit, Oxidationsreaktionen zu vermitteln, untersucht werden, insbesondere in Bezug auf die Oxidation von 1,2Diphenolen (Catecholase-Aktivität). Die Untersuchungen sollten dazu führen, kooperative Wechselwirkungen zweier Metallzentren bei der Vermittlung von Mehrelektronenredoxreaktionen mit Hilfe molekularen Sauerstoffs besser zu verstehen.

\subsection{Synthese von Pyrazolliganden und ihrer Dikupfer(II)komplexe}

Zur Herstellung neuer Kupfer(I)- und Kupfer(II)pyrazolatkomplexe wurden acht verschiedene Pyrazolliganden mit unterschiedlichen Seitenarmen in 3- und 5Stellung am Pyrazolkern hergestellt (Abbildung 87). All diese Liganden müssen über mehrstufige Synthesen hergestellt werden (in der Regel 6 - 7 Stufen). Die Synthese der Liganden $\mathrm{HL}^{5}-\mathrm{HL}^{8}$ wird in dieser Arbeit erstmals beschrieben. Die Dikupferkomplexe 1 - 4 konnten bereits in eigenen, vorhergehenden Arbeiten synthetisiert werden (Abbildung 88 ) $^{[100]}$ und wurden im Rahmen dieser Arbeit nun weitergehend untersucht. Wesentlich ist, daß sich durch die Wahl der Seitenarme des Liganden molekulare Parameter wie der Metall-Metall-Abstand und die individuelle Koordinationsumgebung gezielt einstellen lassen. 
<smiles>[R12]NCc1cc(CN[R10])[nH]n1</smiles>

$$
\text { ( }
$$

Abbildung 87: Synthetisierte Pyrazolliganden.

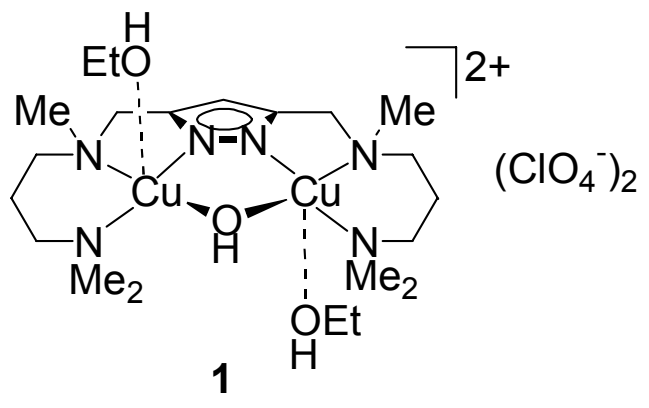

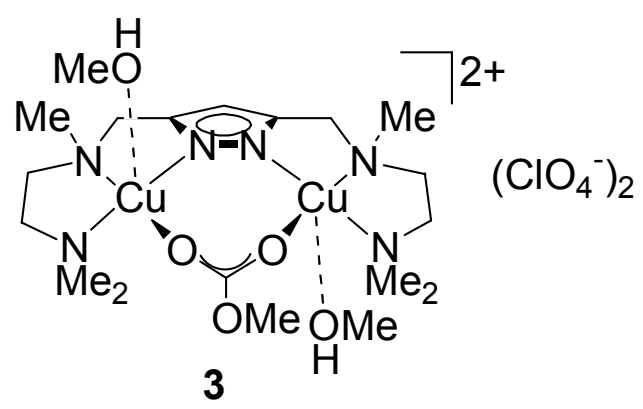

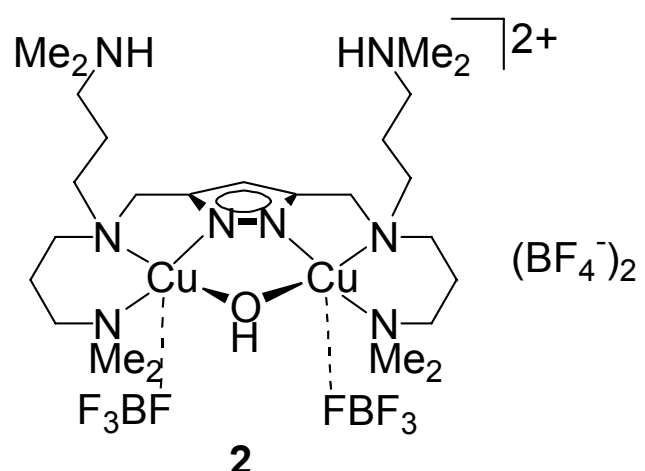

2

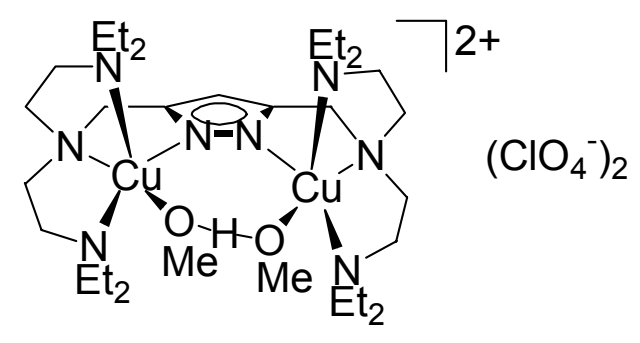

4

Abbildung 88: Die Komplexe 1 - 4 mit den Liganden $\left[L^{1} T-\left[L^{4} J\right.\right.$.

Im Rahmen der hier vorgestellten Arbeit konnte von $\mathrm{HL}^{3}$ aus Aceton ein neuer tetranuklearer Kupferkomplex gewonnen werden, welcher $\mathrm{CO}_{2}$ aus der Luft als $\mu_{3}-$ Carbonatbrücke in den Komplex eingebunden hat (Abbildung 89). In Methanol entsteht hingegen Komplex 3. 


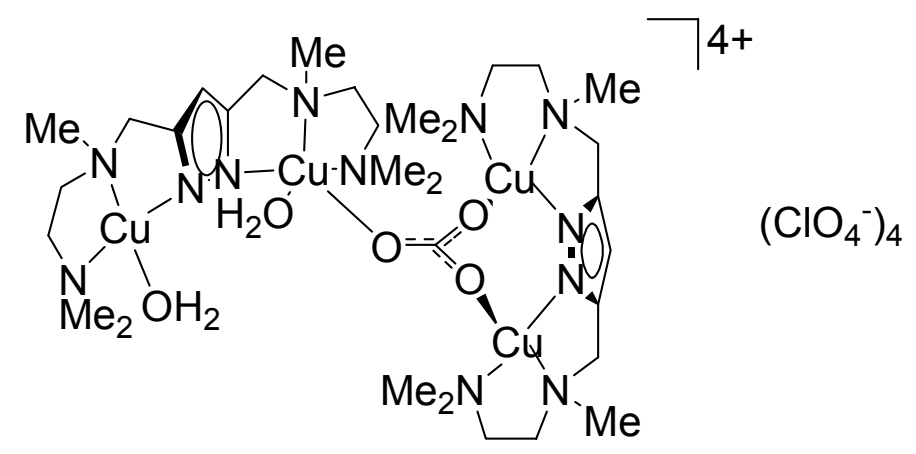

Abbildung 89: Der tetranukleare Kupferkomplex 5 mit einer $\mu^{3}$-Carbonat-Brücke.

Vom Liganden $\mathrm{HL}^{5}$ konnten vier verschiedene Dikupfer(II)komplexe strukturell charakterisiert werden (Abbildung 90).

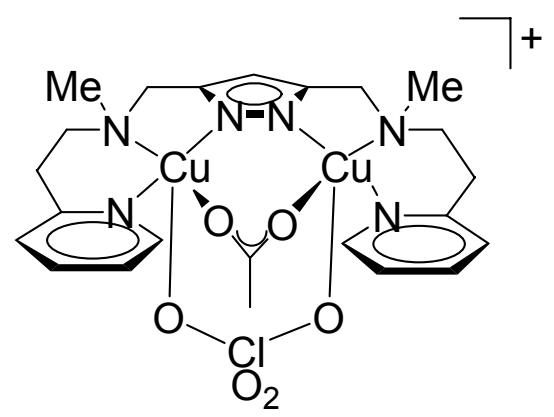

6

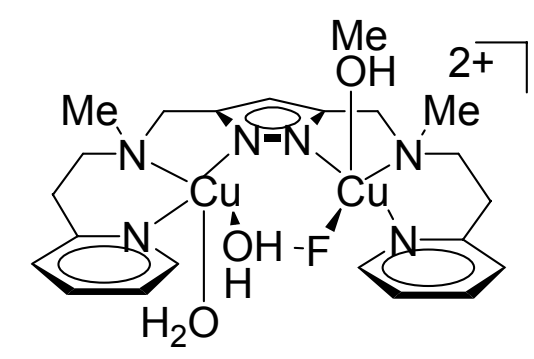

$7 b$

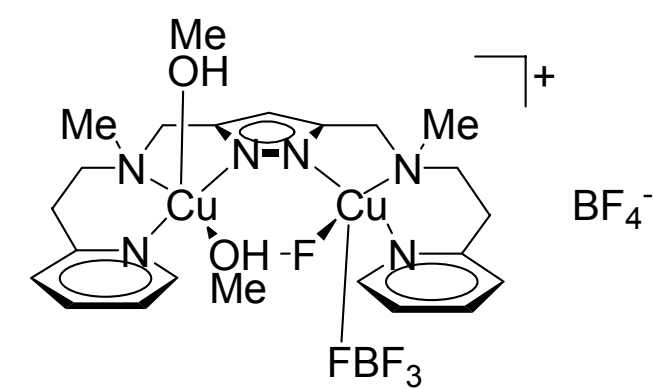

$7 a$

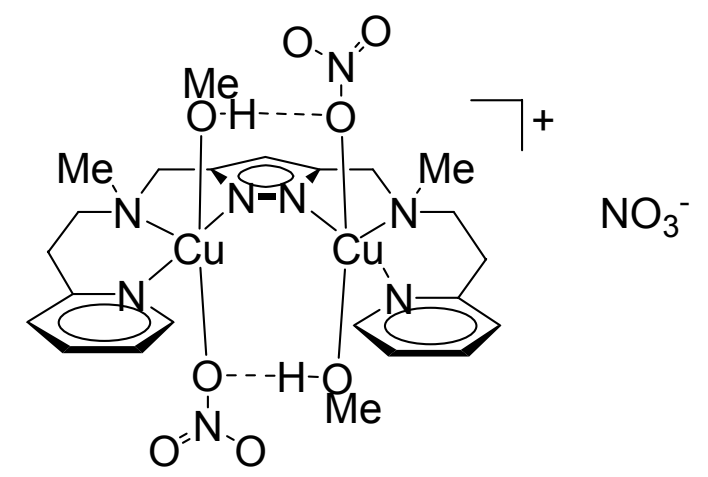

8

Abbildung 90: Dikupfer(II)komplexe des Liganden $H L^{5}$.

Ein Acetat-verbrückter Dikupfer(II)komplex 6 mit Perchlorat-Gegenion entstand zum einen durch Synthese in Acetonitril, wobei hydrolysiertes Solvens als Acetat eingebaut wurde und zum anderen durch direkte Synthese mit Kupfer(II)acetat in Aceton. Durch Vergleich der kristallographischen Daten der Bindungslängen 
zwischen Zweitbrücke und Kupferzentren, sowie durch ESI-Massenspekroskopie konnte ausgeschlossen werden, daß es sich bei 6 um ein Acetamid infolge einer nur teilweisen Hydrolysierung des Solvens handelt.

Komplex 7a wurde durch Verwendung von $\mathrm{Cu}\left(\mathrm{BF}_{4}\right)_{2} \cdot 6 \mathrm{H}_{2} \mathrm{O}$ hergestellt. Dabei kam es zu einer Abspaltung von Fluorid vom Gegenion. Der Komplex weist als Zweitbrücke zwischen den Kupferzentren eine MeO-H $\cdots$ F-Brücke auf. 7b entsteht als Neben- oder Folgeprodukt von $7 \mathrm{a}$ nach längerem Stehen unter Raumatmosphäre unter Verdrängung von koordinierendem Methanol. Setzt man $\mathrm{HL}^{5}$ mit Kupfer(II)nitratTrihydrat um, erhält man unabhängig von der Stöchiometrie der eingesetzten Edukte einen dinuklearen Komplex 8, in welchem die Kupferzentren durch eine zweifache $\left(\mathrm{O}_{2}\right) \mathrm{NO} \cdots \mathrm{H}-\mathrm{OMe}$-Brücke koordiniert sind. $\mathrm{HL}^{5}$ bevorzugt offensichtlich die Ausbildung von Komplexen mit enspannter Konformation des Liganden. Obwohl $\mathrm{HL}^{5}$ aufgrund der konformationellen Freiheit der Seitenarme in der Lage sein müßte, auch kurze Kupfer-Kupfer-Abstände zu ermöglichen, haben alle Komplexe relativ große Abstände (6: $d_{\mathrm{Cu} \cdots \mathrm{Cu}}=4.045(1) \AA, 7 \mathrm{a}: d_{\mathrm{Cu} \cdots \mathrm{Cu}}=4.290(1) \AA, 7 \mathbf{b}: d_{\mathrm{Cu} \cdots \mathrm{Cu}}=4.287(1) \AA, 8$ : $d_{\mathrm{Cu} \cdots \mathrm{Cu}}=4.357(1) \AA$ ), was zum Einbau großer Zweitbrücken führt.

Fünf dinukleare Kupfer(II)komplexe des Liganden $\mathrm{HL}^{6}$ konnten röntgenographisch charakterisiert werden (Abbildung 91). Auch hier führte die Synthese und Kristallisation des Perchloratsalzes in Acetonitril zu einer Verbindung 9, in die ein Hydrolyseprodukt von Acetonitril eingebaut war. Durch gezielte Synthese des Acetatverbrückten Komplexes 9 und Vergleich der massen- und IR-spektroskopischen Daten konnte das Hydrolyseprodukt auch hier zweifelsfrei als Acetat identifiziert werden. Der Einbau der Acetatbrücke führt zu einer starken Verzerrung des Komplexes. Der Einbau von Acetat ließ sich verhindern, indem die Kristallisation in Dichlormethan durchgeführt wurde, wobei der Hydroxid-verbrückte Komplex 10 erhalten wurde. Aufgrund der schlechten Löslichkeit des Perchloratsalzes in Methanol und anderen Lösungsmitteln wurde der Komplex 11 mit einem Tetrafluorborat-Gegenion synthetisiert. Die Löslichkeit konnte damit erhöht werden, zugleich wurde aber wiederum ein Fluor vom $\mathrm{BF}_{4}{ }^{-}$abgespalten unter Ausbildung

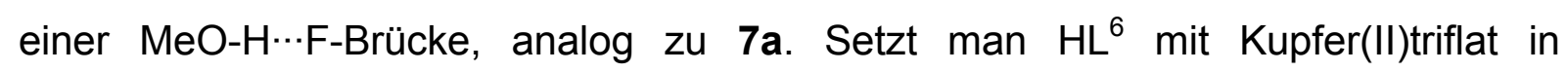
Dichlormethan durch und läßt aus diesem Solvens das Produkt auch auskristallisieren, so wird in der Festkörperstruktur des erhaltenen Produktes 12 ein 
$\mu$-koordiniertes Chlorid zwischen den Kupferzentren gefunden. Dies läßt sich entweder auf eine Verunreinigung des Solvens mit $\mathrm{HCl}$ zurückführen oder auf die Fähigkeit dieses Komplexes, eine Halogenabspaltung von Substraten zu bewirken, wie das auch bei späteren Umsetzungen mit chlor- und bromhaltigen Catecholderivaten beobachtet wurde (siehe 10.5). Die Struktur dieses Komplexes ist ebenfalls verzerrt, dies wurde auf das große Chloridion zurückgeführt.

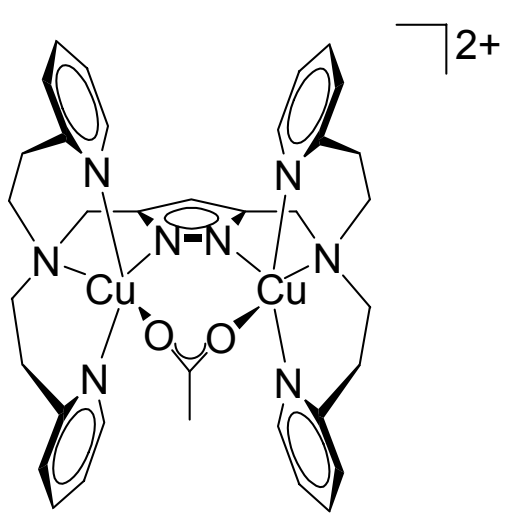

9

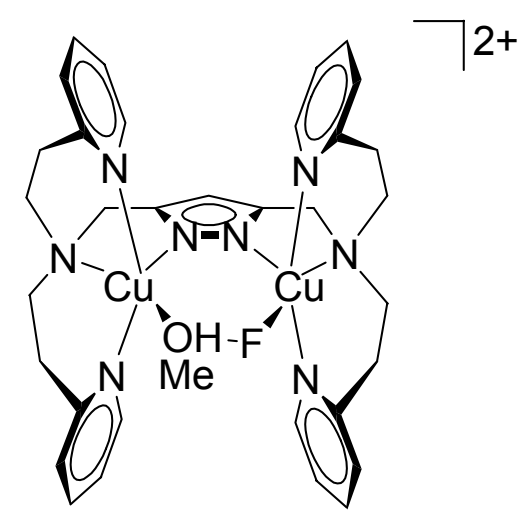

11

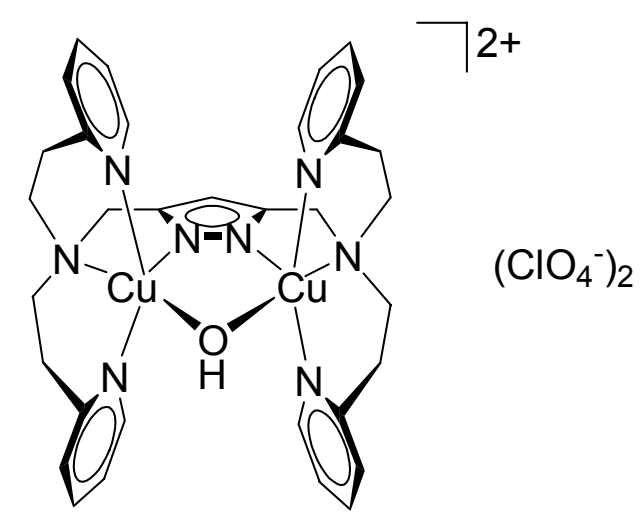

10

$\left(\mathrm{BF}_{4}{ }^{-}\right)_{2}$

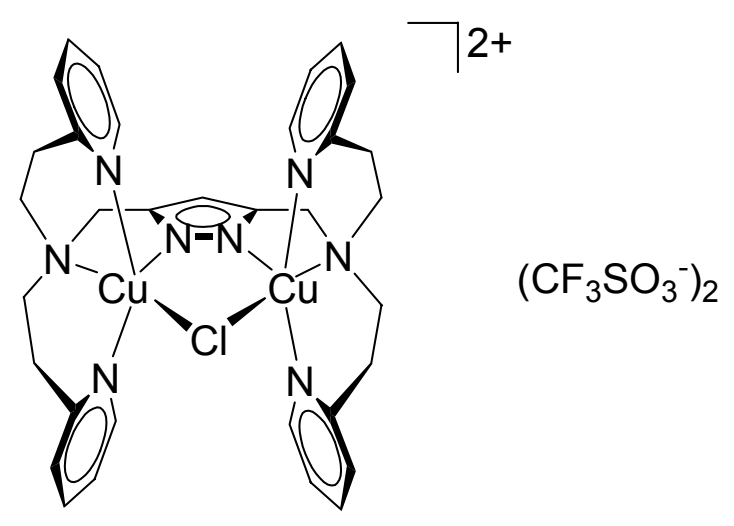

12

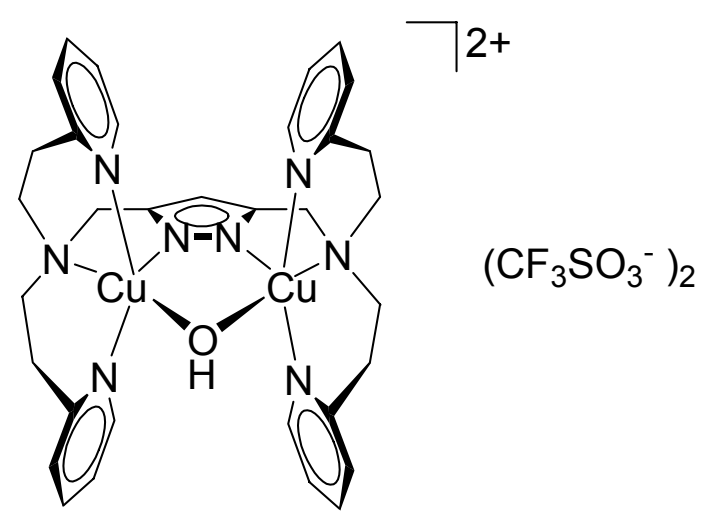

13

Abbildung 91: Dikupfer(II)komplexe des Liganden $\mathrm{HL}^{6}$. 
Ein Hydroxid-verbrückter Dikupfer(II)komplex 13 von $\mathrm{HL}^{6}$ mit Triflat-Gegenion konnte zum einen durch Umsetzen eines Kupfer(I)komplexes von $\mathrm{HL}^{6}$ mit Kupfer(I)triflat in Dichlormethan bei tiefer Temperatur nach Erwärmen auf Raumtemperatur oder durch direkte Umsetzung von $\mathrm{HL}^{6}$ mit Kupfer(II)triflat in Methanol gewonnen werden. Je nach Zweitbrücke variieren die Kupfer-Kupfer-Abstände in Komplexen von $\mathrm{HL}^{6}$ wesentlich mehr als bei Komplexen von $\mathrm{HL}^{5}\left(9: d_{\mathrm{Cu} \cdots \mathrm{Cu}}=4.268(1) \AA, 10: d_{\mathrm{Cu} \cdots \mathrm{Cu}}=\right.$ 3.441(1) $\AA$, 11: $d_{\mathrm{Cu} \cdots \mathrm{Cu}}=4.430(1) \AA$, 12: $d_{\mathrm{Cu} \cdots \mathrm{Cu}}=3.829(2) \AA$, 13: $d_{\mathrm{Cu} \cdots \mathrm{Cu}}$ $=3.515(5) / 3.518(5) \AA$ ). Wahrscheinlich bevorzugt das Fragment " $\mathrm{L}^{6} \mathrm{Cu}^{\prime \prime}{ }_{2}$ " aufgrund der konformativen Ansprüche des Liganden unter Wahrung einer Fünffachkoordination am Kupferzentrum einen kleinen Kupfer-Kupfer-Abstand, kann aber durch größere Liganden wie das Acetation auseinandergedrückt werden.

Dikupfer(II)komplexe des Liganden $\mathrm{HL}^{7}$ konnten zwar massenspektrometrisch nachgewiesen, aber nicht in kristalliner Form erhalten werden. Von $\mathrm{HL}^{8}$ gelang die röntgenographische Charakterisierung eines Dikupfer(II)komplexes 14 mit einem $\mathrm{BF}_{4}{ }^{-}-$Gegenion. Wie bei den oben genannten Komplexen mit diesem Gegenion fand auch hier Fluoridabstraktion statt, im Komplex wird eine MeO-H $\cdots$ F-Brücke gefunden (Abbildung 92).

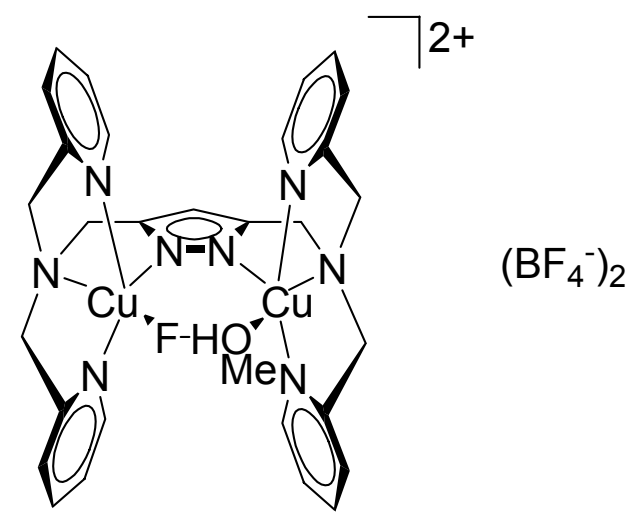

Abbildung 92: Der Komplex 14 mit dem $\left[L^{8} J\right.$-Liganden.

Die kurzen Seitenarme des Liganden verursachen einen großen Abstand der Kupferzentren von 4.291(1) $\AA$. Die Koordinationsgeometrie der Komplexe von Liganden mit langen Seitenarmen ist verzerrt quadratisch pyramidal, dieser Ligand mit kurzen Seitenarmen ergibt dagegen eine verzerrt trigonal bipyramidale Koordination von Kupfer(II). 


\subsection{Katalytische Aktivität von Dikupfer(II)pyrazolatkomplexen}

Die Komplexe 1 - 4, 7a, 8, 13 und 15 (Abbildung 93) wurden in Methanol auf ihre Catecholase-Aktivität mit dem Testsubstrat 3,5-Di-tert-butylcatechol $\left(\mathrm{H}_{2} \mathrm{dtbc}\right)$ getestet. Die Struktur von 15 ist nicht röntgenographisch charakterisiert, jedoch sprechen die erhaltenen massenspektrometrischen und elementaranalytischen Daten sowie der Vergleich mit dem entsprechenden Dinickel(II)komplex, dessen Struktur bekannt ist, ${ }^{[107]}$ für die hier vorgeschlagene Struktur. Da 7a, 8, 13 und 15 in reinem Methanol kaum katalytische Aktivität aufwiesen, wurden die Messungen im gepufferten Milieu durchgeführt, wobei insbesondere bei Verwendung des TRISPuffers große Aktivitätssteigerungen festgestellt wurden. Der makrozyklische Komplex 15 war allerdings selbst unter diesen Bedingungen kaum aktiv, so daß bei den Messungen seiner Aktivität die Temperatur auf $40^{\circ} \mathrm{C}$ erhöht wurde, während die anderen Katalysatoren bei $20^{\circ} \mathrm{C}$ untersucht wurden.

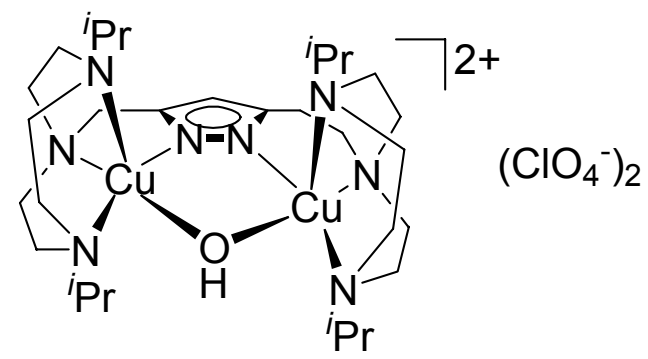

15

Abbildung 93: Struktur von 15.

Die Katalysatoren $7 \mathrm{a}$ und $\mathbf{8}$ wurden bei pH 7.3, sowie 13 und 15 bei pH 8.0 im TRISgepufferten Milieu eingesetzt - bei diesen pH-Werten liegt jeweils das Aktivitätsmaximum. Das Produkt der Catecholase-Reaktion ist das 3,5-Di-tert-butyl-o-chinon (dtboc). Dessen Entwicklung wurde UV/Vis-spektroskopisch untersucht und mit Hilfe der Methode der Anfangsgeschwindigkeiten kinetisch ausgewertet. Die Abhängigkeit der Catecholase-Aktivität der Komplexe von der Katalysatorkonzentration war im untersuchten Bereich linear, für die Abhängigkeit von der Substratkonzentration wurde meist eine Sättigungskinetik beobachtet, welche mit Hilfe einer MichaelisMenten-Kinetik beschrieben werden konnte. Ausnahmen ergaben sich nur für die Katalysatoren $\mathbf{1}$ und $\mathbf{3}$, bei denen keine Substratabhängigkeit gefunden wurde. Dies 
kann man zum einen mit einer besonders niedrigen Michaelis-Konstante, d.h. einer starken thermodynamischen Begünstigung der Anbindung des Substrats an den Katalysator erklären. Zum anderen kann in diesen Fällen auch ein anderer Schritt der Katalyse geschwindigkeitsbestimmend sein, z.B. die Reoxidation der Kupfer(I)spezies durch Sauerstoff. Die erhaltenen kinetischen Daten der Katalysatoren sind in Tabelle 30 zusammengefaßt, zusammen mit den röntgenographisch bestimmten Kupfer-Kupfer-Abstände im Festkörper.

\begin{tabular}{|c|c|c|c|c|c|c|c|}
\hline & $\begin{array}{l}T \\
{\left[{ }^{\circ} \mathrm{C}\right]}\end{array}$ & $\begin{array}{l}k_{\exp } \\
{\left[h^{-1}\right]}\end{array}$ & $\begin{array}{l}d_{\mathrm{Cu} \ldots \mathrm{Cu}} \\
[\AA]]\end{array}$ & $\begin{array}{l}k_{\text {cat }} \\
{\left[h^{-1}\right]}\end{array}$ & $\begin{array}{l}K_{\mathrm{M}} \\
{\left[\mathrm{mol} \mathrm{I}^{-1}\right]}\end{array}$ & $\begin{array}{l}V_{\max } \\
{\left[\mathrm{mol} \mathrm{I}^{-1} \mathrm{~min}^{-1}\right]}\end{array}$ & $\begin{array}{l}E_{\mathrm{p}}^{\text {Red,1 }} \\
{[\mathrm{V}]}\end{array}$ \\
\hline 1 & 20 & $1400 \pm 200$ & $3.540(1)$ & - & - & - & -0.75 \\
\hline 2 & 20 & $2430 \pm 202$ & $3.447(2)$ & $2804 \pm 388$ & $(8.9 \pm 3.5) \cdot 10^{-5}$ & $(4.7 \pm 0.6) \cdot 10^{-4}$ & -0.68 \\
\hline 3 & 20 & $514 \pm 70$ & $4.088(1)$ & - & - & - & -0.76 \\
\hline 4 & 20 & $22.8 \pm 1.2$ & $4.533(1)$ & $31.6 \pm 11.3$ & $(7.9 \pm 4.0) \cdot 10^{-4}$ & $(5.3 \pm 1.9) \cdot 10^{-6}$ & -0.90 \\
\hline $7 a^{*}$ & 20 & $629 \pm 12$ & $4.290(1)$ & $1432 \pm 93$ & $(1.4 \pm 0.1) \cdot 10^{-3}$ & $(4.8 \pm 0.3) \cdot 10^{-4}$ & $* * * *$ \\
\hline $8^{*}$ & 20 & $593 \pm 4$ & $4.357(1)$ & $1125 \pm 102$ & $(1.0 \pm 0.1) \cdot 10^{-3}$ & $(3.8 \pm 0.3) \cdot 10^{-4}$ & -0.67 \\
\hline $13^{*}$ & 20 & $5178 \pm 62$ & $\begin{array}{l}3.515(5) / \\
3.518(5)\end{array}$ & $6502 \pm 239$ & $(7.5 \pm 0.5) \cdot 10^{-4}$ & $(10.8 \pm 0.3) \cdot 10^{-4}$ & -0.53 \\
\hline $15^{*}$ & 40 & $2.8 \pm 0.1$ & ca. $3 \cdot 5^{\star * *}$ & $3.9 \pm 0.4$ & $(18.0 \pm 1.1) \cdot 10^{-4}$ & $(8.1 \pm 0.8) \cdot 10^{-6}$ & -1.38 \\
\hline $\mathbf{L V}^{\star * *}$ & 20 & $2.2 \pm 0.2$ & 4.41 & - & - & - & -1.05 \\
\hline
\end{tabular}

Tabelle 30: Geschwindigkeitskoeffizienten $k$, Michaelis-Konstante $K_{M}$, maximale Geschwindigkeit $v_{\max }$ und Reduktionspotential $E_{p}^{\text {Red, } 1}$ und Abstand der Kupferzentren $d_{C u \cdots C u}$ der Katalysatoren, * Messung im gepufferten Milieu, ${ }^{* *}$ aus vorhergehenden Arbeiten, ${ }^{[90]}{ }^{* * *}$ im entsprechenden Dinickel(II)komplex, ${ }^{* * * *} 7$ a lieferte keine verwertbaren Daten.

Zudem wurden cyclovoltammetrische Messungen der Katalysatoren durchgeführt, wobei sich die Reduktion in allen Fällen als elektrochemisch irreversibel erwies wahrscheinlich aufgrund der Abdissoziation der Zweitbrücke und den grundlegend verschiedenen Koordinationsgeometrien von Kupfer(I) und Kupfer(II). Die erhaltenen $E_{\mathrm{p}}{ }^{\text {Red }}$-Werte sind ebenfalls in der Tabelle aufgeführt. Aufgrund der ähnlichen Aktivitäten von $\mathbf{7 a}$ und 8 im gepufferten Milieu, sowie identischer UV/Vis- und Massenspektren ihrer Spezies in gepufferter Lösung liegt wahrscheinlich in beiden Fällen während der Katalyse dieselbe aktive Spezies vor. 
Die katalytische Aktivität kann mit dem Abstand der Kupferzentren korreliert werden. Die Katalysatoren 1, 2 und $\mathbf{1 3}$ mit einem Kupfer-Kupfer-Abstand von jeweils ca. $3.5 \AA$ weisen eine besonders hohe katalytische Aktivität auf, während bei großen Abständen von $>4 \AA$ deutlich geringere Aktivitäten gemessen werden. Dies bestätigt die Annahme, daß bei der Oxidation der Catechole ein Zusammenwirken beider Kupferzentren von Vorteil ist. Zudem ist es wahrscheinlich, daß das Substrat mit beiden Kupferzentren zugleich wechselwirkt, z.B. durch Ausbildung einer verbrückenden Koordination. Ebenso wirkt sich das Reduktionspotential auf die Aktivität der entsprechenden Komplexe aus. So haben die Komplexe mit hoher

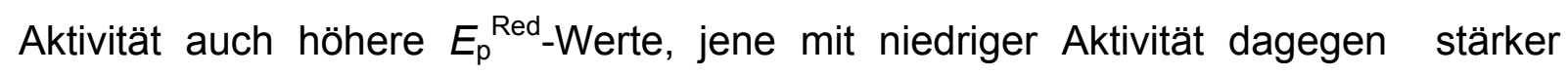
negative $E_{\mathrm{p}}^{\text {Red }}$-Werte, d.h. Komplexe, in denen die Kupfer(II)spezies zu stark stabilisiert wird, oxidieren Catechole unter Ausbildung der reduzierten Kupfer(I)verbindung nur schwer. Ein weiterer Grund für die geringere Aktivität der Komplexe mit kurzen Ligandseitenarmen ist die Ausbildung von Chelatfünfringen mit den Kupferzentren, die zu höheren Stabilitätskonstanten führen sollten als Chelatsechsringe bei Liganden mit längeren Seitenarmen. Dies sollte eine Abdissoziation von Liganddonoren und damit den Angriff des Substrates erschweren. Insbesondere beim makrocyclischen Komplex 15 mit besonders hohen Stabilitätskonstanten spielt dies eine Rolle. Die Zusammenhänge sind in Abbildung 94 und Abbildung 95 graphisch dargestellt.

Niedrige pH-Werte sind i.allg. ungünstig für die Katalyse, da hier die Deprotonierung des Substrates erschwert ist. Tatsächlich wird für $\mathbf{7 a}, \mathbf{8}$ und 13 eine besonders hohe Aktivität bei neutralem bis leicht basischen Milieu beobachtet. Erschwert wird die Analyse der pH-Abhängigkeit durch die Tatsache, daß gut koordinierende Puffer wie Essigsäure/Acetat und TRIS mit den Katalysatoren wechselwirken und deren Aktivität beeinflussen.

Als Reduktionsprodukt von $\mathrm{O}_{2}$ bei der Catecholase-Reaktion wurde bei 1, 2 und 3 $\mathrm{H}_{2} \mathrm{O}_{2}$ nachgewiesen, bei 4 wurde zwar die Entstehung von $\mathrm{H}_{2} \mathrm{O}_{2}$ detektiert, aber nur in geringen Mengen. Für 7a, 8 und 13 konnte keine $\mathrm{H}_{2} \mathrm{O}_{2}$-Entstehung beobachtet werden. Aufgrund dieses Befundes werden zwei verschiedene Reaktionswege vorgeschlagen (siehe 10.5) 


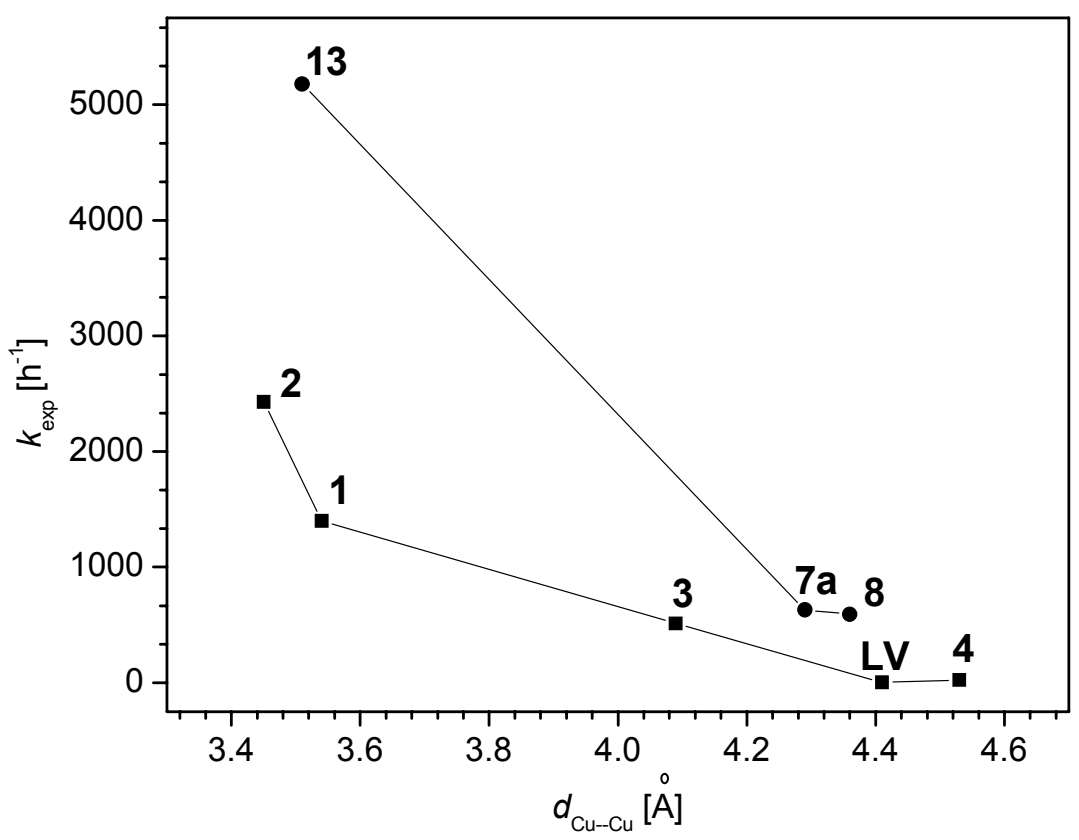

Abbildung 94: Kupfer-Kupfer-Abstand der Katalysatoren und ihre katalytische Aktivität.

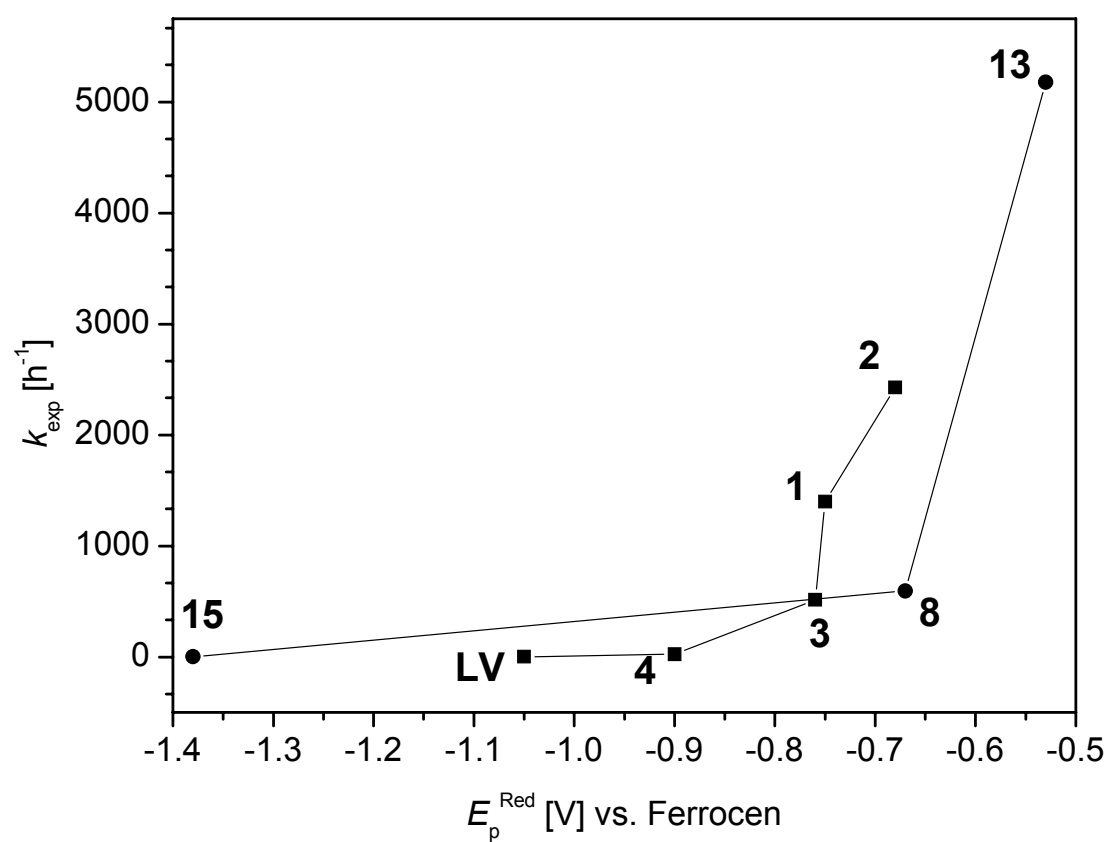

Abbildung 95: $E_{p}^{R e d}$ der Katalysatoren und ihre katalytische Aktivität (vs. Ferrocen). 
Die Untersuchung des mononuklearen Komplexes 16 (Abbildung 96) ergab wie erwartet eine Abhängigkeit 2. Ordnung vom Katalysator, d.h. zwei Katalysatormoleküle werden benötigt, um die Zweielektronenoxidation des Catechols zu bewirken.

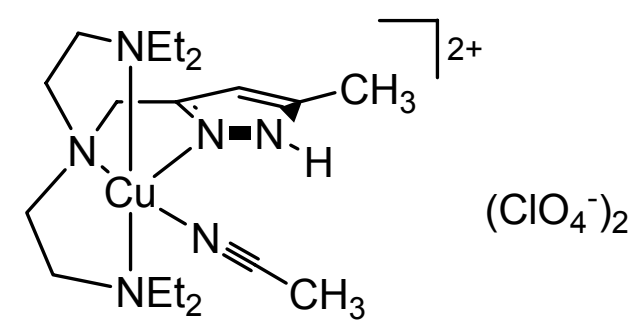

Abbildung 96: Der mononukleare Komplex 16.

\subsection{Charakterisierung und Aktivität von Dikupfersauerstoffaddukten}

Die violetten bis braunvioletten Dikupferperoxoverbindungen " ${ }^{4} \mathrm{Cu}_{2}\left(\mathrm{O}_{2}\right)^{\text {“ }}$ und " $\mathrm{L}^{9} \mathrm{Cu}_{2}\left(\mathrm{O}_{2}\right)$ " konnten bei tiefer Temperatur in Dichlormethan und Acetonitril durch Einleiten von Luft in Lösungen der entsprechenden Kupfer(I)verbindungen hergestellt und UV/Vis- und ramanspektroskopisch untersucht werden. Der Ligand $\mathrm{HL}^{9}$ ist ein Ligand mit einem Triazacyclononanseitenarm, der durch den makrocyclischen Effekt Dikupfer(II)sauerstoffverbindungen stabilisiert (Abbildung 97). Die Zersetzung von ${ } \mathrm{L}^{9} \mathrm{Cu}_{2}\left(\mathrm{O}_{2}\right)^{\prime}$ wurde UV/Vis-spektroskopisch mittels der Stopped-Flow-Technik verfolgt.

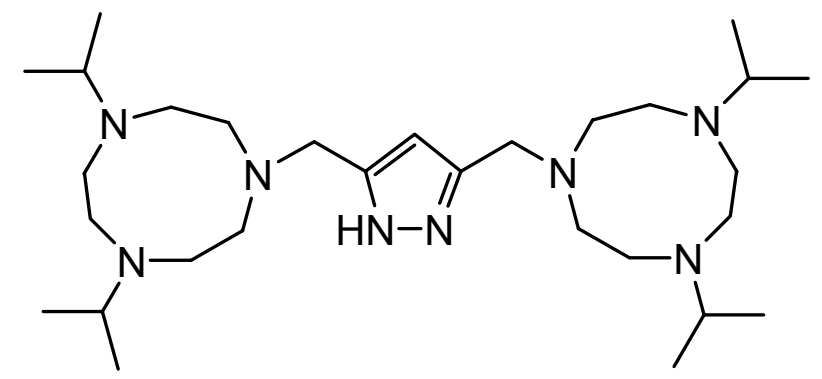

Abbildung 97: Der Ligand $\mathrm{HL}^{9}$ mit dem makrocyclischen Triazacyclononanseitenarm.

" $\mathrm{L}^{4} \mathrm{Cu}_{2}\left(\mathrm{O}_{2}\right)^{\text {“ }}$ ist bis $-80^{\circ} \mathrm{C}$ in Dichlormethan stabil und zersetzt sich bei höheren Temperaturen schnell zu einem grünen Produkt. " $\mathrm{L}^{9} \mathrm{Cu}_{2}\left(\mathrm{O}_{2}\right)^{\text {“ }}$ ist stabiler und kann 
kurze Zeit gekühlt unter Raumatmosphäre gehandhabt werden. Die erhaltenen spektroskopischen Befunde sind in Tabelle 31 zusammengefaßt. Die aus dem Ramanspektrum erhaltenen Schwingungsbanden für $\tilde{v}(\mathrm{O}-\mathrm{O})$ und $\tilde{v}(\mathrm{Cu}-\mathrm{O})$ wurden durch ${ }^{18} \mathrm{O}$-Isotopenmarkierung identifiziert.

\begin{tabular}{l|lll}
\hline & UV/Nis [nm] & Raman $\left[\mathrm{cm}^{-1}\right]$ \\
& $\left(\varepsilon\left[\mathrm{mol} \mathrm{l}^{-1} \mathrm{~cm}^{-1}\right]\right)$ & O-O & Cu-O \\
\hline "L $\mathrm{L}^{4} \mathrm{Cu}_{2}\left(\mathbf{O}_{2}\right) “$ & $544^{*}, 633(\mathrm{sh})^{*}$ & $815\left({ }^{16} \mathrm{O}\right)$ & $537\left({ }^{16} \mathrm{O}\right)$ \\
& & $769\left({ }^{18} \mathrm{O}\right)$ & $518\left({ }^{18} \mathrm{O}\right)$ \\
"L ${ }^{9} \mathrm{Cu}_{2}\left(\mathrm{O}_{2}\right) “$ & $490(2318), 612(\mathrm{sh}, 1041)$ & $808\left({ }^{16} \mathrm{O}\right)$ & $532\left({ }^{16} \mathrm{O}\right)$ \\
& & $* *$ & $505\left({ }^{18} \mathrm{O}\right)$ \\
\hline
\end{tabular}

Tabelle 31: UV/Vis- und ramanspektroskopisch detektierte Banden der Dikupferperoxoverbindungen " $\mathrm{L}^{4} \mathrm{Cu} \mathrm{Cu}_{2}\left(\mathrm{O}_{2}\right)$ " und " $\mathrm{L}^{9} \mathrm{Cu}_{2}\left(\mathrm{O}_{2}\right)$ “, * in Acetonitril, sonst: Dichlormethan, ${ }^{* *} \tilde{v}\left({ }^{18} \mathrm{O}-{ }^{18} \mathrm{O}\right)$ liegt wahrscheinlich im Bereich einer Lösungsmittelbande.

Die $\mathrm{O}_{2}{ }^{2-} \rightarrow \mathrm{Cu}(\mathrm{II})$ LMCT-Übergänge im UV/Vis-Spektrum, sowie die O-O- und Cu-OStreckschwingungen im Ramanspektrum wurden mit jenen bereits bekannter Kupfersauerstoffaddukte verglichen, wobei sich große Ähnlichkeiten mit den Spektren von trans- $\mu-\eta^{1}: \eta^{1}$-Dikupferperoxoverbindungen ergaben. Aufgrund des Aufbaus des Dikupferpyrazolatkomplexes wird eine cis- $\mu-\eta^{1}: \eta^{1}-$ Verbrückung anstelle einer trans-Verbrückung favorisiert (Abbildung 98).
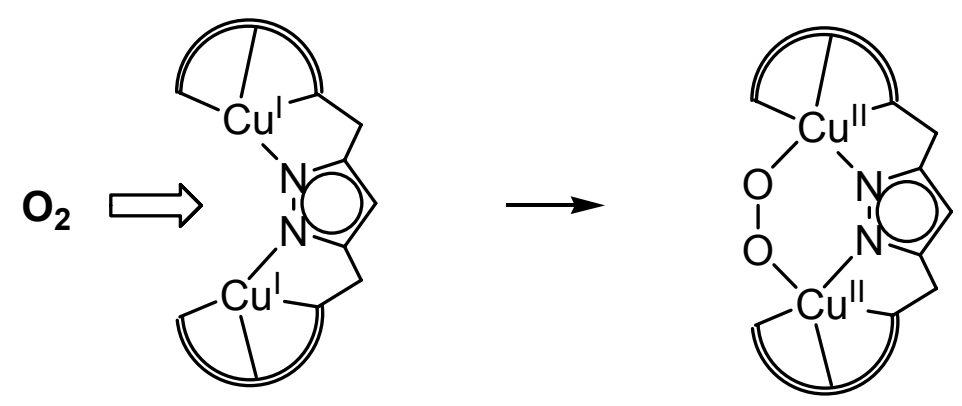

Abbildung 98: Angriff von molekularem $\mathrm{O}_{2}$ an einen Dikupfer(I)komplex unter Bildung des cis- $\mu-\eta^{1}: \eta^{1}-$ Adduktes.

Der Zerfall von " $\mathrm{L}^{9} \mathrm{Cu}_{2}\left(\mathrm{O}_{2}\right)^{\text {“ }}$ folgt einer Kinetik 2. Ordnung. Möglicherweise bildet sich zunächst ein Dimer, welches anschließend weiter zersetzt wird. Dies steht im 
Einklang mit der literaturbekannten Festkörperstruktur von Komplex XIII, bei welchem es sich um ein Dimer aus zwei $\left[\mathrm{L}^{3}\right]^{-}$-Ligandeinheiten mit vier trans- $\mu_{4}$-peroxidisch verbrückten Kupferzentren handelt (Abbildung 99). ${ }^{[34]}$

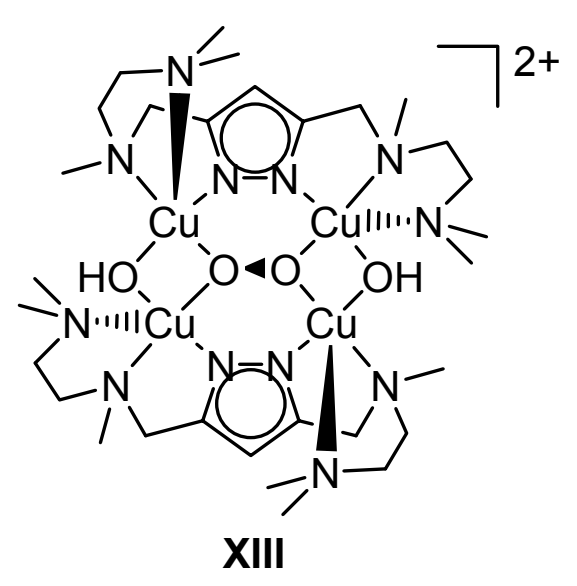

Abbildung 99: Struktur des trans- $\mu_{4}$-peroxidisch verbrückten Komplexes XIII.

\subsection{Synthese von Addukten substratanaloger Moleküle}

Um Hinweise auf die Art der Anbindung eines Catecholsubstrates während des Catecholase-Katalysezyklus zu gewinnen, wurden Modellkomplexe mit einem gebundenen substratähnlichen Molekül untersucht. Dazu wurden fünf verschiedene Addukte mit Tetrabromcatechol $\left(\mathrm{H}_{2} \mathrm{tbc}\right)$ und Tetrachlorcatechol $\left(\mathrm{H}_{2} \mathrm{tcc}\right)$ hergestellt (Abbildung 100). Mit nicht deprotoniertem Catechol findet keine Adduktbildung statt. Daher wurde im Falle von 19, 20 und 21 der Dikupfer(II)komplex mit dem Catechol in Gegenwart von Base umgesetzt, bei 18 und 22 wurde das Addukt durch Reaktion des Kupfer(I)komplexes mit o-Chloranil bei tiefer Temperatur hergestellt. In den erhaltenen Produkten koordinieren die Catecholate immer bidentat an eines der beiden Kupferzentren, wobei sie bei 18 - 21 in die basale Koordinationssphäre der verzerrt quadratisch pyramidal koordinierten Kupferzentren eintreten. 22 bildet eine dimere Struktur aus zwei Ligandeinheiten, welche durch zwei Kupferzentren verbrückt sind. 


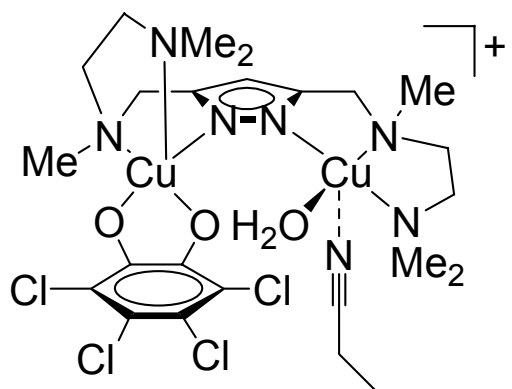

18

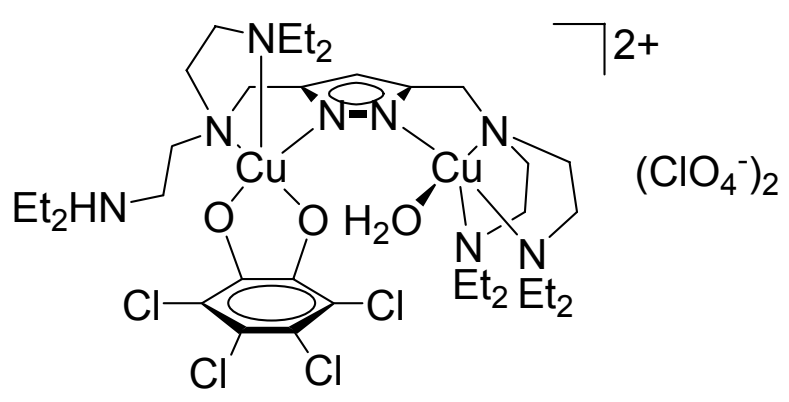

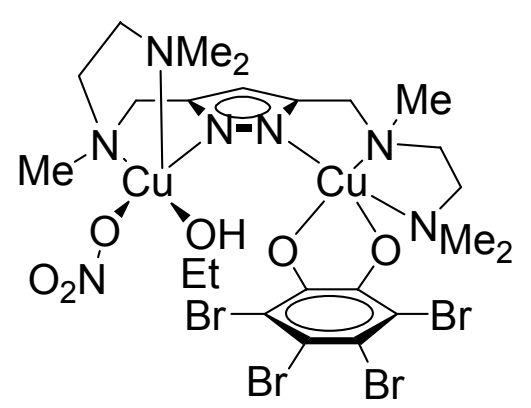

19

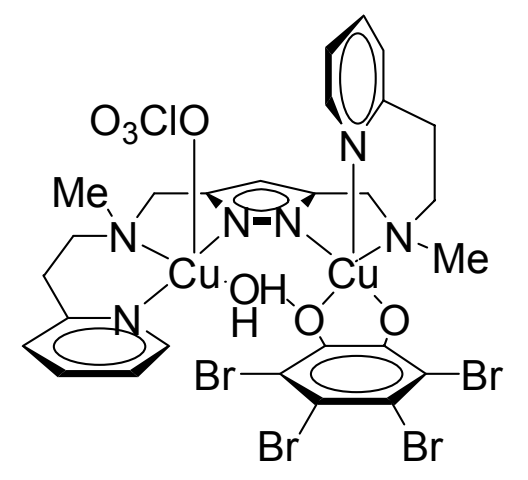

21

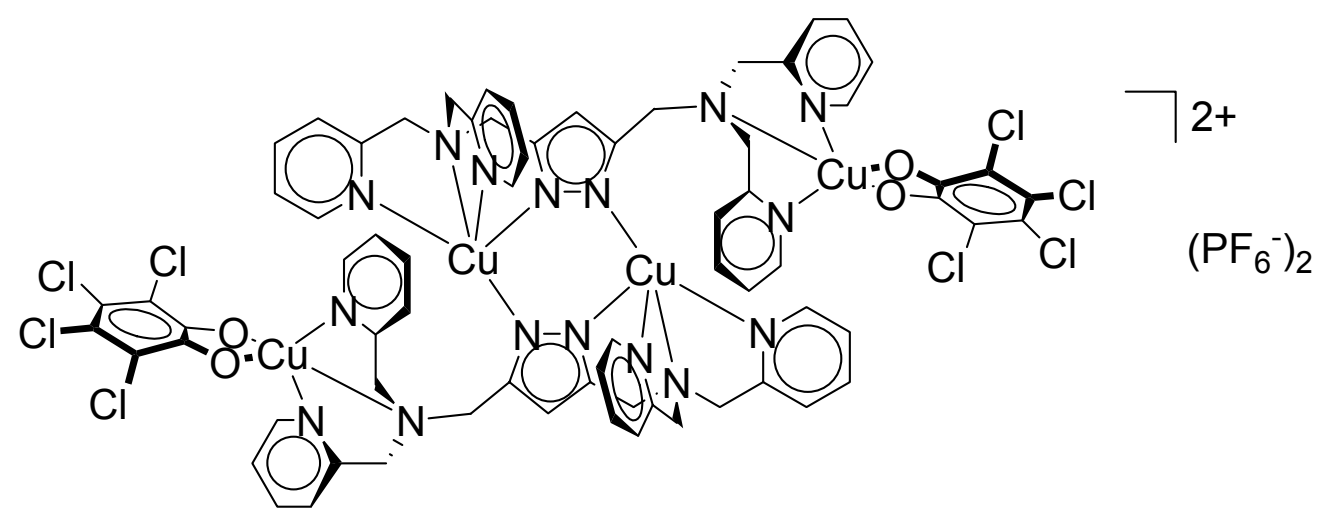

22

Abbildung 100: Modellkomplexe mit den substratähnlichen Catecholationen tbc $c^{2-}$ und $t c c^{2-}$.

Anhand der Strukturen der Modellkomplexe wird ein Mechanismus für die Catecholase-Reaktion der hier vorgestellten Dikupfer(II)komplexe 1 - 4, 7a, 8, und 13 vorgeschlagen, in welchem das Substrat zunächst nur an ein Kupfer(II)zentrum koordiniert, wonach dann - möglicherweise nach Ausbildung einer verbrückenden Koordination - das Substrat unter Reduktion beider Kupfer(II)zentren zu Kupfer(I) zum Chinon oxidiert wird. Aufgrund der spektroskopischen Befunde für die Dikupfer(II)sauerstoffverbindungen wird für den nächsten Schritt die Reoxidation mit $\mathrm{O}_{2}$ unter Ausbildung einer $\mu-\eta^{1}: \eta^{1}$-Peroxoverbindung postuliert. Die Katalysatoren 1, 
2 und 3 reagieren danach direkt unter $\mathrm{H}_{2} \mathrm{O}_{2}$-Abspaltung zur Ausgangsverbindung zurück, während $\mathbf{7 a}, \mathbf{8}$ und $\mathbf{1 3}$ unter Oxidation eines weiteren Substratmoleküls und $\mathrm{H}_{2} \mathrm{O}$-Abspaltung den katalytischen Zyklus vollenden (Abbildung 101). Der wenig aktive Komplex 4 reagiert entweder auf einem anderen Weg bzw. auf mehreren verschiedenen Reaktionswegen, oder es hat sich aufgrund der längeren Meßzeit bereits zu viel $\mathrm{H}_{2} \mathrm{O}_{2}$ zersetzt, so daß dieses nicht mehr vollständig detektiert werden kann (siehe Kapitel 10.3).

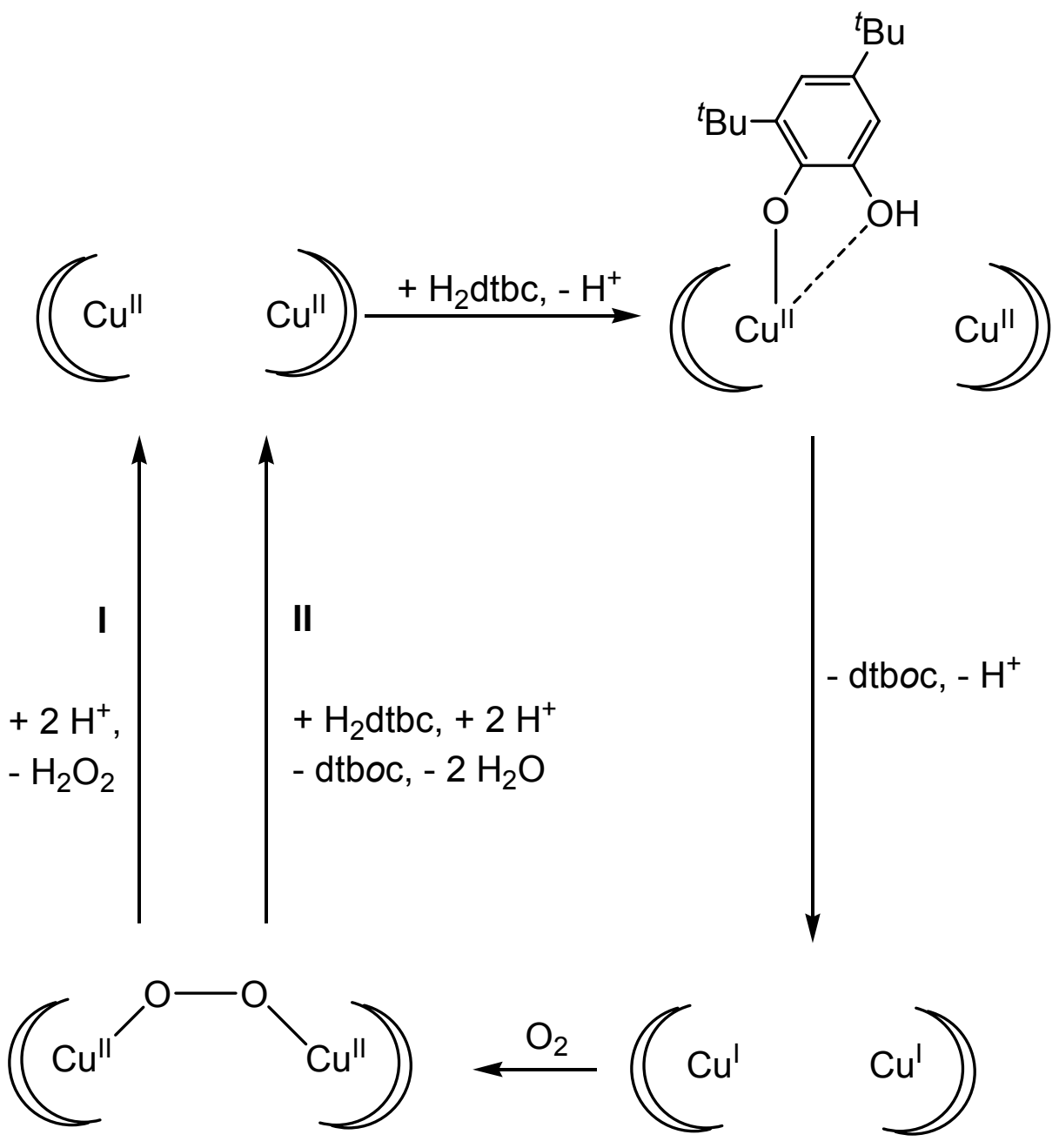

Abbildung 101: Postulierter Mechanismus der Catecholase-Reaktion der untersuchten Katalysatoren.

\subsection{Versuche zur Umsetzung von Phenolen}

Bei der Umsetzung von Kupfer(I)komplexen der Liganden $\mathrm{HL}^{2}, \mathrm{HL}^{5}, \mathrm{HL}^{6}$ und $\mathrm{HL}^{8}$ mit Luftsauerstoff und 2,4-Di-tert-butylphenol bei tiefer Temperatur in Acetonitril konnte in keinem Falle eine Cresolase-Aktivität, d.h. eine ortho-Hydroxylierung des Phenols 
festgestellt werden, es wurde El-massenspektroskopisch nur das Edukt nachgewiesen. Setzt man die Liganden mit 2,3,5-Trimethylphenol um, welches in para- und ortho-Stellung schlechter geschützt ist, so sieht man im GC/MS-Spektrum neben dem Edukt in kleinen Mengen eine Reihe verschiedener Kupplungsprodukte, darunter direkte Kupplungsprodukte der aromatischen Kerne und verschiedene Diphenylether (Abbildung 102).

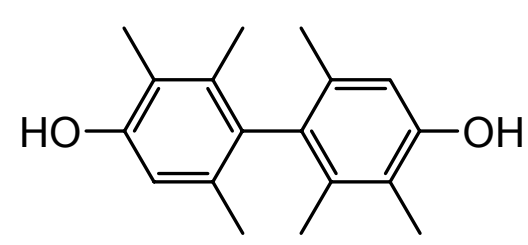

a

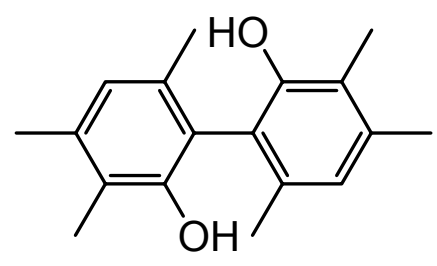

b

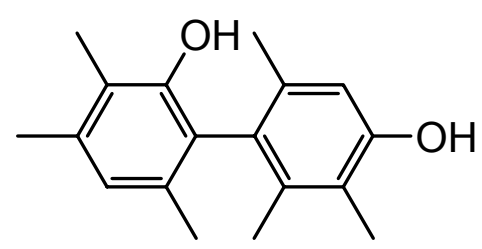

C

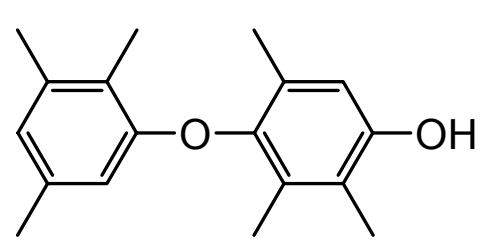

d

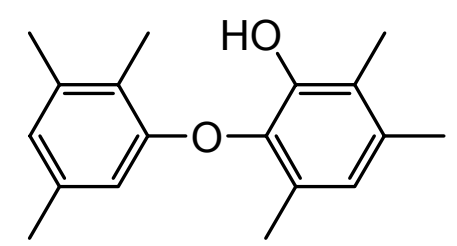

e

Abbildung 102: Mögliche Dimerisierungsprodukte a - e bei der Umsetzung von 2,3,5-Trimethylphenol mit verschiedenen Dikupfersauerstoffverbindungen der Liganden $H L^{2}, H L^{5}, H L^{6}$ und $H L^{8}$.

Daneben ist bei den Komplexen von $\mathrm{HL}^{6}$ und $\mathrm{HL}^{8}$ auch die Bildung verschiedener Oxygenierungsprodukte zu beobachten. So konnte in diesen Fällen die Bildung von 2,3,5-Trimethyl-p-chinon durch Vergleich mit dem bekannten Massenspektrum dieser Verbindung nachgewiesen werden.

Die Komplexe 1 - 4 wurden in Zusammenarbeit mit der Arbeitsgruppe Reedijk et al. (Leiden) auf ihre Eignung als Katalysatoren der oxidativen Kupplung von 2,5Dimethyphenol (DMP) zu Poly(1,4)phenylether (PPE) untersucht (Abbildung 103). 
$\mathrm{m}$

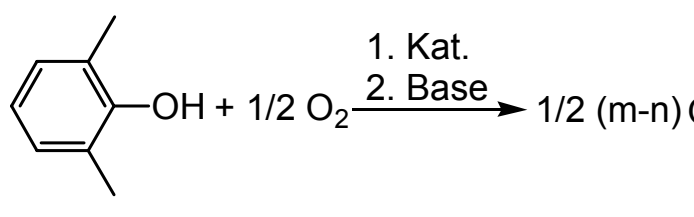

DMP<smiles>CC1=CC(=C2C=C(C)C(=O)C(C)=C2)C=C(C)C1=O</smiles>

$\mathrm{DPQ}$<smiles>Cc1cc(C)c(O[C@H](C)CO)c(C)c1</smiles>

PPE

Abbildung 103: Polymerisation von DMP zu PPE.

Hierbei wurden z.T. gute katalytische Eigenschaften festgestellt, obwohl einfachere Kupferpyrazolkomplexe bislang als nicht katalytisch aktiv galten. Desweiteren wurde beobachtet, daß das Zusammenwirken zweier Kupferzentren bei der Polymerisation von Bedeutung ist - auch hier werden mit kleinen Kupfer-Kupfer-Abständen die höchsten Aktivitäten erzielt. 


\section{Experimenteller Teil}

\subsection{Allgemeines}

Apparaturen: Es wurden konventionelle Glasapparaturen oder Schlenkkolben verwendet. Sofern nötig, wurden die Apparaturen unter Verwendung eines Heißluftföns ausgeheizt, mehrmals im Ölpumpenvakuum evakuiert und mit trockenem und sauerstoffreiem Stickstoff oder Argon (Trocknung durch Molsieb $3 \AA$ der Firma Merck und Desoxygenierung durch einen CuO-Katalysator BTS der Firma BASF) gespült.

Absolute Lösungsmittel: Alle Reaktionen wurden in frisch absolutierten, desoxygenierten und destillierten Lösungsmitteln durchgeführt. Folgende Trocknungsmittel fanden Verwendung:

$\begin{array}{llll}\text { Aceton: } & \text { Calciumchlorid } & \text { Pentan: } & \text { Natrium/Kalium } \\ \text { Acetonitril: } & \text { Phosphorpentoxid } & \text { Petrolether 40/60: } & \text { Calciumhydrid } \\ \text { Dichlormethan: } & \text { Calciumhydrid } & \text { Propionitril: } & \text { Phosphorpentoxid } \\ \text { Diethylether: } & \text { Natrium/Kalium } & \text { Tetrahydrofuran: } & \text { Natrium/Kalium } \\ \text { Ethanol: } & \text { Natrium } & & \\ \text { Methanol: } & \text { Magnesiumspäne } & & \end{array}$

Für die Ramanspektren und Stopped-Flow-Messungen wurde absolutes Dichlormethan über Molsieb der Firma Fluka verwendet.

NMR-Spektren: Die NMR-Spektren wurden in Heidelberg auf einem NMRSpektrometer AC 200 der Firma Bruker $\left({ }^{1} \mathrm{H}: 200.13 \mathrm{MHz},{ }^{13} \mathrm{C}: 50.32 \mathrm{MHz}\right)$ oder einem Bruker Avance DRX $300\left({ }^{1} \mathrm{H}: 300.13 \mathrm{MHz},{ }^{13} \mathrm{C}: 75.47 \mathrm{MHz}\right.$ ) aufgenommen. Die NMR-Spektren in Göttingen wurden mit Geräten des Typs Bruker Avance 500 $\left({ }^{1} \mathrm{H}: 500.13 \mathrm{MHz},{ }^{13} \mathrm{C}: 125.77 \mathrm{MHz}\right), 300\left({ }^{1} \mathrm{H}: 300.13 \mathrm{MHz},{ }^{13} \mathrm{C}: 75.47 \mathrm{MHz}\right)$ und 200 $\left({ }^{1} \mathrm{H}: 200.13 \mathrm{MHz},{ }^{13} \mathrm{C}: 50.32 \mathrm{MHz}\right.$ ) aufgenommen. Alle Spektren wurden bei $298 \mathrm{~K}$ aufgenommen. Die angegebenen Werte der Verschiebung $\delta$ beziehen sich auf den internen Standard durch die Lösungsmittel $\mathrm{CDCl}_{3}\left(\delta=7.27\right.$ für ${ }^{1} \mathrm{H}$-NMR bzw. $\delta=77.0$ für ${ }^{13} \mathrm{C}-\mathrm{NMR}$ ) und $\mathrm{D}_{6}$-Aceton ( $\delta=2.04$ für ${ }^{1} \mathrm{H}$-NMR bzw. $\delta=29.8$ für $\left.{ }^{13} \mathrm{C}-\mathrm{NMR}\right)$ relativ zu externem Tetramethylsilan (jeweils $\delta=0$ ). 
Die ${ }^{13} \mathrm{C}$-Spektren wurden ${ }^{1} \mathrm{H}$-entkoppelt aufgenommen.

Abkürzungen: $\mathrm{s}=$ Singulett, $\mathrm{d}=$ Dublett, $\mathrm{dt}=$ Dublett von Tripletts, $\mathrm{t}=$ Triplett, $\mathrm{m}=$ Multiplett.

Massenspektren: In Heidelberg wurden die Massenspektren auf einem Massenspektrometer Finnigan MAT 8230 mit integriertem Spectro-System 300 aufgenommen. Die untersuchten Ionen wurden entweder durch Elektronenstoßionisation ( $\mathrm{El}, 70 \mathrm{eV}$ ) oder durch Fast Atom Bombardment (FAB, Xenon als Stoßgas) der Substanz in einer 4-Nitrobenzylalkohol-Matrix (Nibeol) erzeugt. ESI-Spektren wurden auf dem Massenspektrometer Finnigan TSQ 700 aufgenommen. Die Massenspektren in Göttingen wurden auf den Geräten Finnigan MAT 8200 für El-Spektren, Finnigan MAT 95 für FAB-Spektren und Finnigan MAT LCQ für ESI-Spektren aufgenommen.

Elementaranalysen: Die Elementaranalyse erfolgte in Heidelberg durch das Mikroanalytische Labor des Chemischen Institutes der Universität Heidelberg mit einem CHN-Analysator Vario EL der Firma Elementar und in Göttingen durch das Mikroanalytische Labor des Instituts für Anorganische Chemie der Universität Göttingen mit einem CHN-O-Rapid der Firma Elementar. Sofern nicht anders vermerkt, sind die Werte für die angegebene Summenformel berechnet.

Infrarotspektren: Die Infrarotspektren wurden in Heidelberg auf einem Bruker IFS-66 FT-IR-Spektrometer und in Göttingen auf einem Digilab Excalibur Spektrometer aufgenommen. Die Feststoffe wurden als KBr-Preßling gemessen. Die Lage der Banden ist in Wellenzahlen angegeben $\left(\tilde{v}\right.$ in $\left.\mathrm{cm}^{-1}\right)$. Die Intensitäten sind wie folgt abgekürzt:

$\mathrm{m}=$ mittel

$\mathrm{s}=$ stark

vs $=$ sehr stark

$\mathrm{w}=$ schwach

UV/Vis-Spektren: In Heidelberg wurden die Tieftemperaturspektren auf einem PerkinElmer Lambda 19 Spektrometer aufgenommen. Die Spektren wurden in Lösung mit einer Quarzküvette der Dicke $1.0 \mathrm{~cm}$ gemessen. Die kinetischen Messungen wurden 
auf einem Varian Cary 1E Spektrometer aufgenommen mit einer Quarzküvette der Dicke $1.0 \mathrm{~cm}$. Die Wellenlängen $\lambda$ sind in $\mathrm{nm}$, die Extinktionskoeffizienten $\varepsilon$ sind in mol $\mathrm{I}^{-1} \mathrm{~cm}^{-1}$ angegeben. In Göttingen erfolgten die Messungen auf einem Analytik Jena Specord S 100 Spektrometer.

Stopped-Flow-Untersuchungen: Die Messung der UV/Vis-Spektren mittels der Stopped-Flow-Technik erfolgte an der Universität Heidelberg auf einem SX.18MV-R Stopped Flow Reaction Analyzer der Firma Applied Photophysics. Die Messung erfolgte in einem Meßraum von $20 \mu$ l Volumen mit einer Durchstrahllänge von $0.2 \mathrm{~cm}$ bei $-10^{\circ} \mathrm{C}$. Die Messungen wurden unter Argonatmosphäre durchgeführt.

Raman-Spektren: Die ramanspektroskopischen Untersuchungen wurden an der Universität Heidelberg auf einem Labram-Spektrometer der Firma Jobin Yvon Horiba mit einem HeNe-Laser mit der Einstrahlwellenlänge $632 \mathrm{~nm}$ bei Temperaturen zwischen -170 und $-80^{\circ} \mathrm{C}$ unter Stickstoff durchgeführt.

GC/MS-Spektren: Die GC/MS-Spektren wurden an der Universität Göttingen auf einem Finnigan INCOS 50 mit dem angeschlossenem Gaschromatographen Varian 3400 aufgenommen.

Cyclovoltammetrie: Die cyclovoltammetrischen Messungen wurden an einem Potentiostat/Galvanostat Model 263A der Firma Perkin-Elmer / Princeton Applied Research mit einem Model K0264 Micro-Cell Kit durchgeführt. Als Solvens wurde Acetonitril mit Tetrabutylammonium-hexafluorophosphat bzw. -tetrafluoroborat $(0.1 \mathrm{M})$ als Leitsalz verwendet, als Arbeitselektrode eine Glassy Carbon Elektrode, als Gegenelektrode eine Platinelektrode und als Referenzelektrode eine AgElektrode. Die Spektren wurden gegen einen internen Ferrocen-Standard gemessen $\left(E^{0}\left(\mathrm{FeCp}_{2} / \mathrm{FeCp}_{2}{ }^{+}=0.40 \mathrm{~V}\right.\right.$ gegen Normalwasserstoffhalbzelle in $\left.\mathrm{H}_{2} \mathrm{O}\right) .{ }^{[204]}$

Kristallisationsversuche: Die Kristallisationsversuche wurden durch Überschichten einer filtrierten Lösung der zu kristallisierenden Substanz mit einem 2- bis 3-fachen Überschuß an Fällungsmittel durchgeführt.

Reagentien: Die nicht aufgeführten Verbindungen waren durch die 
Chemikalienausgabe des Chemischen Institutes erhältlich. Folgende Reagentien wurden über die angegebenen Firmen bezogen:

3,5-Di-tert-butylcatechol

Kalium-tert-butylat

Kupfer(II)nitrat-Trihydrat

Kupfer(II)perchlorat-Hexahydrat

Kupfer(II)tetrafluorborat-Hexahydrat

Kupfer(II)trifluormethylsulfonat

MES

MOPS

$\mathrm{H}_{2} \mathrm{O}_{2}$-Teststäbchen

Lactoperoxidase

Methanol

Methyl-[2-(2-pyridyl)ethyl]amin (MePEA)

o-Chloranil

Propionitril

Tetrakis(acetonitril)kupfer(I)-

hexafluorphosphat

Tetrabromcatechol

Tetrachlorcatechol

2,3,5-Trimethylphenol

TRIS
Aldrich

Aldrich

Lancaster

Aldrich

Avocado

Aldrich

Aldrich

Aldrich

Merck

Aldrich

Grüssing

Aldrich

Merck

Fluka

Aldrich

Aldrich

Lancaster

Aldrich

Aldrich

Die folgenden Reagentien wurden nach literaturbekannten Vorschriften dargestellt:

BPEMA, BPEA, BPMA ${ }^{[97,98]}$

3,5-Bis(chlormethyl)-1-(tetrahydropyran-2-yl)pyrazol ${ }^{[94,95,96]}$

$\left[\mathrm{Cu}(\mathrm{MeCN})_{4}\right]^{+} \mathrm{X}^{-}\left(\mathrm{X}^{-}=\mathrm{PF}_{6}^{-}, \mathrm{ClO}_{4}^{-}, \mathrm{CF}_{3} \mathrm{SO}_{3}^{-}, \mathrm{BF}_{4}^{-}\right)^{[160]}$ 
11.2 Die Pyrazolliganden $\mathrm{HL}^{5}-\mathrm{HL}^{8}$
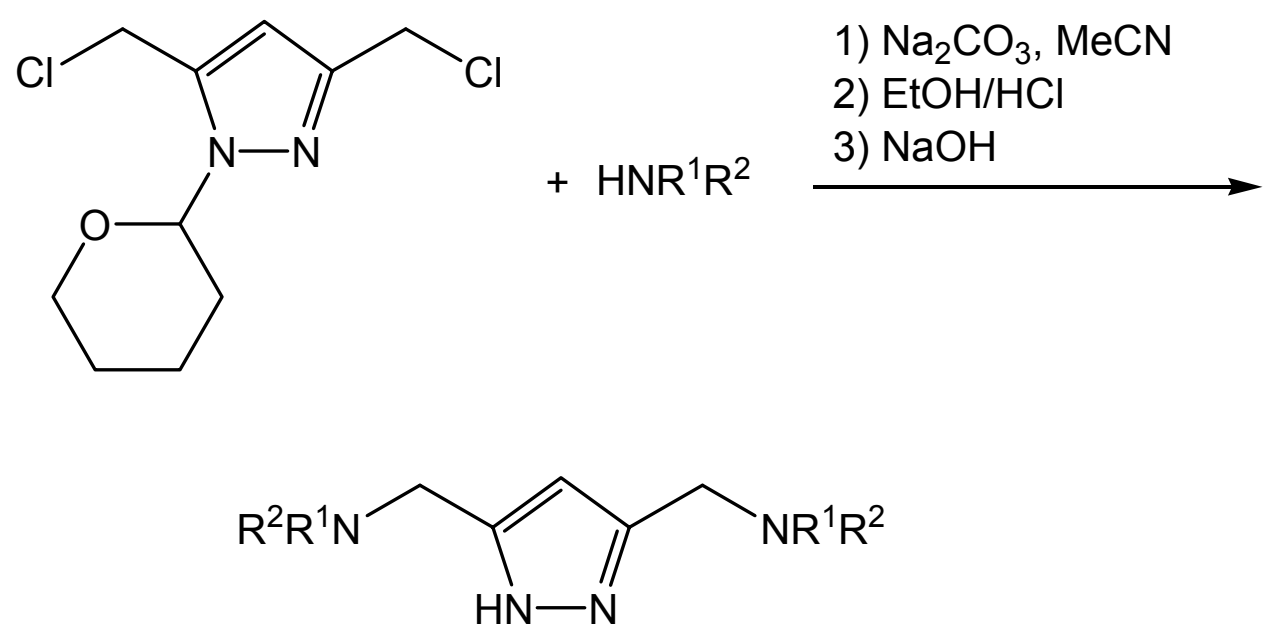

$\mathrm{N}_{\mathrm{R}^{2}}^{-\mathrm{R}^{1}}=\mathrm{HN}_{-}^{-\mathrm{Me}}$

$\left(\mathrm{HL} \mathrm{L}^{5}\right)$<smiles>CCCc1ccccn1</smiles>

$\left(\mathrm{HL}^{6}\right)$<smiles>c1ccc(CCNCc2ccccn2)nc1</smiles>

$\left(H L^{7}\right)$<smiles>CNCc1ccccn1</smiles>

$\left(\mathrm{HL}^{8}\right)$

\section{Ansatz:}

$2.3 \mathrm{mmol} \mathrm{HL} \mathrm{L}^{\mathrm{n}}$ : $313 \mathrm{mg}$ MePEA

$523 \mathrm{mg}$ BPEA

$490 \mathrm{mg}$ BPEMA

$458 \mathrm{mg}$ BPMA

$273 \mathrm{~g}$ (1.1 mmol) 3,5-Bis(chlormethyl)-1-(tetrahydropyran-2-yl)pyrazol

$1.2 \mathrm{~g}(11.4 \mathrm{mmol}) \mathrm{Na}_{2} \mathrm{CO}_{3}$

$50 \mathrm{ml}$ Acetonitril 
Durchführung:

Zunächst wird $\mathrm{Na}_{2} \mathrm{CO}_{3}$ eine Stunde lang im Vakuum auf $100^{\circ} \mathrm{C}$ erhitzt. Anschließend werden das Amin (MePEA, BPEMA, BPEA oder BPMA) und das 3,5Bis(chlormethyl)-1-(tetrahydropyran-2-yl)pyrazol in Acetonitril gelöst und zugegeben. Man läßt nun 24 Stunden unter Rückfluß kochen, filtriert dann und entfernt das Solvens im Vakuum. Der THP-geschützte Ligand bleibt als gelbes Öl zurück. Die Schutzgruppe wird mit ethanolischer $\mathrm{HCl}$ abgespalten, indem man das Öl eine Stunde lang mit $\mathrm{EtOH} / \mathrm{HCl}$ rühren läßt. Dabei fällt das Hydrochlorid des Liganden in Form eines weißen Pulvers aus, welches abfiltriert und im Vakuum getrocknet wird. Das Pulver wird in wäßriger $\mathrm{NaOH}(\mathrm{pH}=12)$ aufgenommen und mit zweimal $50 \mathrm{ml}$ Dichlormethan extrahiert. Nach Trocknen mit Magnesiumsulfat wird das Solvens im Vakuum entfernt. Man erhält den Liganden als gelb-braunes, viskoses Öl.

$\mathrm{HL}^{5}$ :

Summenformel: $\mathrm{C}_{21} \mathrm{H}_{28} \mathrm{~N}_{6}(364.49 \mathrm{~g} / \mathrm{mol})$

${ }^{1} \mathrm{H}-\mathrm{NMR}\left(\mathrm{CDCl}_{3}\right)$ : 2.23 (s, $\left.6 \mathrm{H}, \mathrm{NCH}_{3}\right), 2.80,2.97$ (t, $\left.7 \mathrm{~Hz}, 8 \mathrm{H}, \mathrm{NCH}_{2} \mathrm{CH}_{2}\right), 3.60$ (s, $\left.4 \mathrm{H}, \mathrm{CH}_{2}\right), 6.00\left(\mathrm{~s}, 1 \mathrm{H}, \mathrm{H}^{4, \mathrm{pz}}\right), 7.06-7.13\left(\mathrm{~m}, 4 \mathrm{H}, \mathrm{H}^{3, \mathrm{Py}}, \mathrm{H}^{5, \mathrm{py}}\right)$, $7.55\left(\mathrm{dt},{ }^{3} \mathrm{JHH}_{\mathrm{HH}}=2 \mathrm{~Hz}, 8 \mathrm{~Hz}, 2 \mathrm{H}, \mathrm{H}^{4, \mathrm{Py}}\right), 8.50\left(\mathrm{~m}, 2 \mathrm{H}, \mathrm{H}^{6, \mathrm{Py}}\right)$

${ }^{13} \mathrm{C}-\mathrm{NMR}\left(\mathrm{CDCl}_{3}\right): 35.7\left(\mathrm{NCH}_{2} \mathrm{CH}_{2}\right), 42.3\left(\mathrm{NCH}_{3}\right), 53.4\left(\mathrm{CH}_{2}\right), 56.6\left(\mathrm{NCH}_{2} \mathrm{CH}_{2}\right)$, $104.3\left(C^{4, p z}\right), 121.2\left(C^{3, p y}\right), 123.3\left(C^{5, p y}\right), 136.4\left(C^{4, p y}\right), 149.1$ $\left(C^{6, p y}\right), 149.1\left(C^{3, p z}, C^{5, p z}\right), 160.3\left(C^{2, p y}\right)$

IR (Film): $\tilde{v}=3355$ (s), 3195 (s), 3102 (s), 3011 (s), 2945 (s), 2844 (s), $2797(\mathrm{~s}), 1659(\mathrm{w}), 1594(\mathrm{~s}), 1570(\mathrm{~m}), 1477(\mathrm{~s}), 1437(\mathrm{~s}), 1357$ $(m), 1310(w), 1251(w), 1227(w), 1183(w), 1150(m), 1122(m)$, $1094(w), 1050(m), 1000(m), 874(w), 765(m), 633(w), 608$ $(w), 522(w), 404(w)$

MS (EI): m/z (\%): $65\left(19,\left[\mathrm{C}_{3} \mathrm{~N}_{2} \mathrm{H}\right]^{+}\right), 106\left(45,\left[\mathrm{CH}_{2} \mathrm{CH}_{2} \mathrm{Py}\right]^{+}\right), 135$ (38, $\left.\left[\mathrm{H}_{3} \mathrm{CNCH}_{2} \mathrm{CH}_{2} \mathrm{Py}\right]^{+}\right), 136\left(80, \quad\left[\left(\mathrm{C}_{3} \mathrm{~N}_{2} \mathrm{H}\right)\left(\mathrm{CH}_{2} \mathrm{~N}\right)_{2} \mathrm{CH}_{3}\right]^{+}\right), 165(9$, $\left.\left[\left(\mathrm{C}_{3} \mathrm{~N}_{2} \mathrm{H}\right)\left(\mathrm{CH}_{2} \mathrm{~N}\right)_{2}\left(\mathrm{CH}_{3}\right)_{2}\left(\mathrm{CH}_{2}\right)\right]^{+}\right), \quad 179 \quad\left(19, \quad\left[\left(\mathrm{C}_{3} \mathrm{~N}_{2} \mathrm{H}\right)\right.\right.$ $\left.\left.\left.\left(\mathrm{CH}_{2} \mathrm{~N}\right)_{2}\left(\mathrm{CH}_{3}\right)_{2}\left(\mathrm{CH}_{2}\right)\right]^{+}\right), 228\left(83, \mathrm{HL}^{5}-\mathrm{CH}_{2} \mathrm{CH}_{2} \mathrm{Py}-2 \mathrm{CH}_{3}\right]^{+}\right), 229$ (78, [HL $\left.\left.{ }^{5}-\mathrm{H}_{3} \mathrm{CNCH}_{2} \mathrm{CH}_{2} \mathrm{Py}\right]^{+}\right), 230\left(100,\left[\mathrm{HL}^{5}-\mathrm{H}_{3} \mathrm{CNCH}_{2} \mathrm{CH}_{2} \mathrm{Py}\right.\right.$ $\left.+1]^{+}\right), 258\left(18,\left[\mathrm{HL}^{5}-\mathrm{CH}_{2} \mathrm{CH}_{2} \mathrm{Py}\right]^{+}\right), 272\left(19,\left[\mathrm{HL}^{5}-\mathrm{CH}_{2} \mathrm{Py}\right]^{+}\right)$, $364\left(23,\left[\mathrm{HL}^{5}\right]^{+}\right)$ 
EA: ber. C 46.13; $\mathrm{H} 6.08 ; \mathrm{N} 15.37$ für $\mathrm{HL}^{5} \cdot 5 \mathrm{HCl}$ exp. C 45.28; H 6.98; N 13.80

Ausbeute: $292 \mathrm{mg}(0.8 \mathrm{mmol}, 73 \%)$

$H^{6}$ :

Summenformel: $\mathrm{C}_{33} \mathrm{H}_{38} \mathrm{~N}_{8}(546.72 \mathrm{~g} / \mathrm{mol})$

${ }^{1} \mathrm{H}-\mathrm{NMR}\left(\mathrm{CDCl}_{3}\right): 2.89\left(\mathrm{~m}, 16 \mathrm{H}, \mathrm{NCH}_{2} \mathrm{CH}_{2}\right), 3.74\left(\mathrm{~s}, 4 \mathrm{H}\right.$, PyrazolCH $\left.\mathrm{CH}_{2}\right), 5.85$ (s, 1 $\left.\mathrm{H}, \mathrm{H}^{4, \mathrm{pz}}\right), 6.98-7.01\left(\mathrm{~m}, 8 \mathrm{H}, \mathrm{H}^{3, \mathrm{py}}, \mathrm{H}^{5, \mathrm{py}}\right), 7.43(\mathrm{dt}, 2 \mathrm{~Hz}, 8 \mathrm{~Hz}, 4$ $\left.\mathrm{H}, \mathrm{H}^{4, \mathrm{py}}\right), 8.44\left(\mathrm{~m}, 4 \mathrm{H}, \mathrm{H}^{6, \mathrm{py}}\right)$

${ }^{13} \mathrm{C}-\mathrm{NMR}\left(\mathrm{CDCl}_{3}\right): 36.1\left(\mathrm{NCH}_{2} \mathrm{CH}_{2}\right), 53.7\left(\mathrm{NCH}_{2} \mathrm{CH}_{2}\right), 54,0\left(\mathrm{CH}_{2}\right), 103.0\left(\mathrm{C}^{4, \mathrm{pz}}\right)$, $120.9\left(C^{5, p y}\right), 123.3\left(C^{3, p y}\right), 136.1\left(C^{4, p y}\right), 149.0\left(C^{3, p z}, C^{5, p z}\right)$, $149.0\left(C^{6, p y}\right), 160.5\left(C^{2, p y}\right)$

IR (Film): $\tilde{v}=3186$ (s), 3132 (s), 3084 (s), 3067 (s), 3008 (s), 2931 (s), 2822 (s), 1669 (w), 1632 (m), 1595 (vs), 1569 (s), 1477 (vs), 1436 (vs), 1359 (m), 1309 (w), 1249 (w), 1223 (w), 1191 (w), $1149(\mathrm{~m}), 1120(\mathrm{~s}), 1093(\mathrm{~m}), 1052(\mathrm{~m}), 1025(\mathrm{w}), 994(\mathrm{~s}), 964$ (w), $879(w), 845(w), 751(\mathrm{~s}), 630(\mathrm{w}), 612(\mathrm{w}), 520(\mathrm{w}), 404(\mathrm{~m})$ MS (EI): $\left.m / z(\%): 65\left(8, \mathrm{C}_{3} \mathrm{~N}_{2} \mathrm{H}\right]^{+}\right), 106\left(100,\left[\mathrm{CH}_{2} \mathrm{CH}_{2} \mathrm{Py}\right]^{+}\right), 135(80$, $\left.\left[\mathrm{H}_{2} \mathrm{CNCH}_{2} \mathrm{CH}_{2} \mathrm{Py}+1\right]^{+}\right), 319\left(40,\left[\mathrm{H}_{2} \mathrm{C}\left(\mathrm{C}_{3} \mathrm{~N}_{2} \mathrm{H}\right) \mathrm{CH}_{2} \mathrm{~N}\left(\mathrm{CH}_{2} \mathrm{CH}_{2-}\right.\right.\right.$ $\left.\left.\mathrm{Py})_{2}\right]^{+}\right), 321\left(76,\left[\mathrm{HL}^{6}-\mathrm{N}\left(\mathrm{CH}_{2} \mathrm{CH}_{2} \mathrm{Py}\right)_{2}+1\right]^{+}\right), 440\left(12,\left[\mathrm{HL}^{6}-\right.\right.$ $\left.\left.\mathrm{CH}_{2} \mathrm{CH}_{2} \mathrm{Py}\right]^{+}\right), 454\left(13,\left[\mathrm{HL}^{6}-\mathrm{CH}_{2} \mathrm{Py}\right]^{+}\right), 546\left(16,\left[\mathrm{HL}^{6}\right]^{+}\right)$

EA: ber. C 49.43; $\mathrm{H} 5.66 ; \mathrm{N} 13.97$ für $\mathrm{HL}^{6} \cdot 7 \mathrm{HCl}$ exp. C 49.70; H $6.22 ; \mathrm{N} 13.55$

Ausbeute: $492 \mathrm{mg}$ (0.9 mmol, $82 \%)$

$\mathrm{HL}^{7}$ :

Summenformel: $\mathrm{C}_{31} \mathrm{H}_{34} \mathrm{~N}_{8}(518.66 \mathrm{~g} / \mathrm{mol})$

${ }^{1} \mathrm{H}-\mathrm{NMR}\left(\mathrm{CDCl}_{3}\right): 2.9\left(\mathrm{~m}, 4 \mathrm{H}, \mathrm{NCH}_{2} \mathrm{CH}_{2}\right), 2.99\left(\mathrm{~m}, 4 \mathrm{H}, \mathrm{NCH}_{2} \mathrm{CH}_{2}\right), 3.75(\mathrm{~s}, 8 \mathrm{H}$, $\left.\mathrm{CH}_{2}\right), 5.99\left(\mathrm{~s}, 1 \mathrm{H}, \mathrm{H}^{4, \mathrm{pz}}\right), 7.03-7.08\left(\mathrm{~m}, 6 \mathrm{H}, \mathrm{H}^{5, \mathrm{py}}, \mathrm{H}^{5, \mathrm{py}}, \mathrm{H}^{6, \mathrm{py}}\right)$, $7.14\left(\mathrm{~m}, 2 \mathrm{H}, \mathrm{H}^{6, \mathrm{py} y}\right), 7.45-7.52\left(\mathrm{~m}, 4 \mathrm{H}, \mathrm{H}^{3, \mathrm{py}}, \mathrm{H}^{3, \mathrm{py}}\right)$ ), $8.43-8.50$ $\left(\mathrm{m}, 4 \mathrm{H}, \mathrm{H}^{4, \mathrm{py}}, \mathrm{H}^{4, \mathrm{py}}\right)$ 
${ }^{13} \mathrm{C}-\mathrm{NMR}\left(\mathrm{CDCl}_{3}\right): 30.9$ (Pyrazol- $\left.\mathrm{CH}_{2}\right), 36.1\left(\left(\mathrm{NCH}_{2} \mathrm{CH}_{2}\right), 54.0\left(\mathrm{NCH}_{2} \mathrm{CH}_{2}\right), 59.8\right.$ (Pyridin- $\left.\mathrm{CH}_{2}\right), 104.0\left(\mathrm{C}^{4, \mathrm{pz}}\right), 121.1,121.9,123.1,123.5\left(\mathrm{C}^{3, \mathrm{py}}\right.$, $\left.C^{3, p y}, C^{5, p y}, C^{5, p y}\right), 136.2,136.4\left(C^{4, p y}, C^{4, p y \prime}\right), 148.8,149.0,149.0$ $\left(C^{6, p y}, C^{6, p y}, C^{3, p z}, C^{5, p z}\right), 159.6,160.5\left(C^{2, p y}, C^{2, p y}\right)$

IR (Film): $\tilde{v}=3188$ (s), 3086 (s), 3009 (s), 2931 (s), 2828 (s), 2485 (w), $2302(w), 2210(w), 1984(w), 1955(w), 1879(w), 1857(w)$, $1657(\mathrm{~m}), 1593(\mathrm{~s}), 1570(\mathrm{~s}), 1476(\mathrm{~s}), 1435(\mathrm{~s}), 1363(\mathrm{~m}), 1310$ (m), $1251(\mathrm{~m}), 1202(\mathrm{w}), 1149(\mathrm{~s}), 1122(\mathrm{~s}), 1092(\mathrm{~m}), 1050(\mathrm{~m})$, $1025(\mathrm{~m}), 996(\mathrm{~s}), 761(\mathrm{~s}), 632(\mathrm{~m}), 617(\mathrm{~m}), 510(\mathrm{w}), 404(\mathrm{~m})$

MS (EI): $m / z(\%): 65\left(8,\left[\mathrm{C}_{3} \mathrm{~N}_{2} \mathrm{H}\right]^{+}\right), 93\left(52,\left[\mathrm{CH}_{2} \mathrm{Py}+1\right]^{+}\right), 106(30$, $\left.\left[\mathrm{CH}_{2} \mathrm{CH}_{2} \mathrm{Py}\right]^{+}\right), 121\left(39, \quad\left[\mathrm{NCH}_{2} \mathrm{CH}_{2} \mathrm{Py}+1\right]^{+}\right), 213$ (56, $\left.\left[\mathrm{PyCH}_{2} \mathrm{NCH}_{2} \mathrm{CH}_{2} \mathrm{Py}+1\right]^{+}\right), 307\left(30,\left[\mathrm{HL}^{7}-\mathrm{PyCH}_{2} \mathrm{~N}-\mathrm{CH}_{2} \mathrm{CH}_{2} \mathrm{Py}\right.\right.$ $\left.+1]^{+}\right), 321\left(28,\left[\mathrm{HL}^{7}-\mathrm{CH}_{2} \mathrm{Py}-\mathrm{CH}_{2} \mathrm{CH}_{2} \mathrm{Py}+1\right]^{+}\right), 426\left(100,\left[\mathrm{HL}^{7}-\right.\right.$ $\left.\left.\mathrm{CH}_{2} \mathrm{Py}\right]^{+}\right), 518\left(2,\left[\mathrm{HL}^{7}\right]^{+}\right)$

EA: ber. C 48.11; $\mathrm{H} 5.34 ; \mathrm{N} 14.48$ für $\mathrm{HL}^{7} \cdot 7 \mathrm{HCl}$ exp. C 48.89; H 6.00; N 13.95

Ausbeute: $519 \mathrm{mg}(1.0 \mathrm{mmol}, 91 \%)$

$H L^{8}$ :

Summenformel: $\mathrm{C}_{29} \mathrm{H}_{30} \mathrm{~N}_{8}(490.61 \mathrm{~g} / \mathrm{mol})$

${ }^{1} \mathrm{H}-\mathrm{NMR}\left(\mathrm{CDCl}_{3}\right): 3.76\left(\mathrm{~s}, 4 \mathrm{H}, \mathrm{CH}_{2}\right), 3.91\left(\mathrm{~s}, 8 \mathrm{H}, \mathrm{CH}_{2}\right), 6.12\left(\mathrm{~s}, 1 \mathrm{H}, \mathrm{H}^{4, \mathrm{pz}}\right), 7.07$ $\left(\mathrm{m}, 4 \mathrm{H}, \mathrm{H}^{5, \mathrm{py}}\right), 7.28\left(\mathrm{~d},{ }^{3} \mathrm{~J}_{\mathrm{HH}}=1 \mathrm{~Hz}, 8 \mathrm{~Hz}, 4 \mathrm{H}, \mathrm{H}^{3, \mathrm{py}}\right), 7.54(\mathrm{dt}$, $\left.{ }^{3} J_{H H}=1 \mathrm{~Hz}, 8 \mathrm{~Hz}, 4 \mathrm{H}, \mathrm{H}^{4, \mathrm{py}}\right), 8.47\left(\mathrm{~m},{ }^{3} \mathrm{~J}_{\mathrm{HH}}=4 \mathrm{H}, \mathrm{H}^{6, \mathrm{py}}\right)$

${ }^{13} \mathrm{C}-\mathrm{NMR}\left(\mathrm{CDCl}_{3}\right)$ : 54.6 (Pyrazol- $\left.\mathrm{CH}_{2}\right), 59.5\left(\right.$ Pyridin- $\left.\mathrm{CH}_{2}\right), 104.5\left(\mathrm{C}^{4, \mathrm{pz}}\right), 121.9$, $122.1\left(C^{3, p y}, C^{5, p y}\right), 136.3\left(C^{4, p y}\right), 149.2\left(C^{6, p y}\right), 148.9\left(C^{3, p z}, C^{5, p z}\right)$, $159.5\left(\mathrm{C}^{2, \mathrm{py}}\right)$

IR (Film): $\tilde{v}=3186$ (s), 3140 (s), 3116 (s), 3008 (s), 2982 (s), 2922 (s), 2881 (s), 2821 (s), $2703(\mathrm{~m}), 2606(\mathrm{w}), 2477$ (w), 2297 (w), 2003 $(\mathrm{w}), 1905(\mathrm{w}), 1674(\mathrm{~m}), 1645(\mathrm{~m}), 1594(\mathrm{~s}), 1570(\mathrm{~s}), 1519(\mathrm{~m})$, 1475 (s), 1435 (s), 1385 (s), $1362(\mathrm{~s}), 1313(\mathrm{~m}), 1257(\mathrm{~m}), 1238$ (s), $1220(\mathrm{~m}), 1147(\mathrm{~s}), 1123(\mathrm{~s}), 1094(\mathrm{~m}), 1047(\mathrm{~s}), 996(\mathrm{~s}), 973$ (s), $959(\mathrm{~m}), 898(\mathrm{~m}), 884(\mathrm{~s}), 847(\mathrm{~s}), 802(\mathrm{~s}), 767(\mathrm{~s}), 707(\mathrm{w})$, $667(\mathrm{w}), 643(\mathrm{w}), 629(\mathrm{~m}), 620(\mathrm{~m}), 613(\mathrm{~s}), 521(\mathrm{w}), 512(\mathrm{w}), 500$ (w), $458(w), 404(m)$ 
MS (EI): $\left.m / z(\%): 65\left(8, \mathrm{C}_{3} \mathrm{~N}_{2} \mathrm{H}\right]^{+}\right), 93\left(45,\left[\mathrm{CH}_{2} \mathrm{Py}+1\right]^{+}\right), 200\left(38,\left[\mathrm{HL}^{8}-\right.\right.$ $\left.\left.\mathrm{N}\left(\mathrm{CH}_{2} \mathrm{Py}\right)_{2}-\mathrm{CH}_{2} \mathrm{Py}\right]^{+}\right), 293\left(28,\left[\mathrm{HL}^{8}-\mathrm{N}\left(\mathrm{CH}_{2} \mathrm{Py}\right)_{2}{ }^{+1}\right]^{+}\right), 398(100$, $\left.\left[\mathrm{HL}^{8}-\mathrm{CH}_{2} \mathrm{Py}\right]^{+}\right), 490\left(2,\left[\mathrm{HL}^{8}\right]^{+}\right)$

EA: ber. C 46.70; $\mathrm{H} 5.00 ; \mathrm{N} 15.02$ für $\mathrm{HL}^{8} \cdot 7 \mathrm{HCl}$

exp. C 46.82; H 5.48; N 14.70

Ausbeute: $393 \mathrm{mg}$ (0.8 mmol, $73 \%)$ 


\subsection{Synthese der Komplexe}

\subsection{1 $\left[\left(\mathrm{L}^{3} \mathrm{Cu}_{2}\right)\left(\mathrm{CO}_{3}\right)\left(\mathrm{H}_{2} \mathrm{O}\right)_{2}\left(\mathrm{ClO}_{4}\right)\right]\left(\mathrm{ClO}_{4}\right)_{3} \quad(5)$}

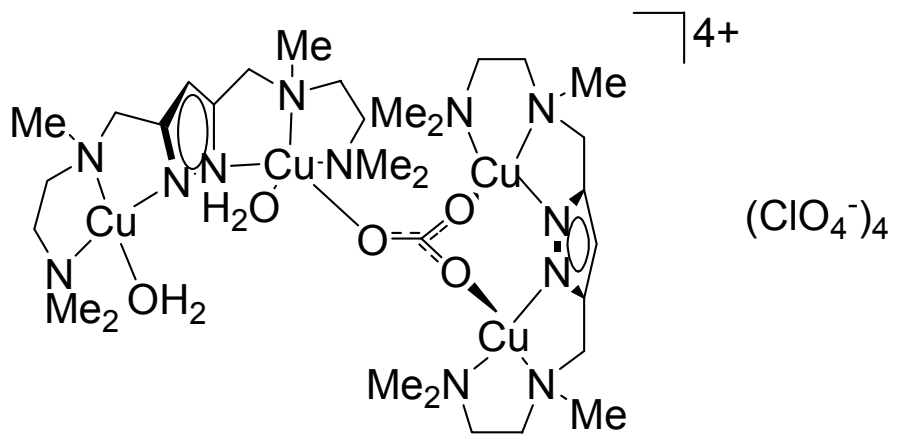

Ansatz:

$370 \mathrm{mg}(1.25 \mathrm{mmol}) \mathrm{HL}^{3}$

$281 \mathrm{mg}(2.50 \mathrm{mmol})$ Kalium-tert-butylat

$926 \mathrm{mg}(2.50 \mathrm{mmol}) \mathrm{Cu}\left(\mathrm{ClO}_{4}\right)_{2} \cdot 6 \mathrm{H}_{2} \mathrm{O}$

$150 \mathrm{ml}$ Methanol

\section{Durchführung:}

$\mathrm{HL}^{3}$ und wird mit zwei Äquivalenten der Base in $150 \mathrm{ml}$ Methanol gelöst und anschließend das Kupferperchlorat zugegeben. Anschließend leitet man eine halbe Stunde $\mathrm{CO}_{2}$ in die Lösung, wobei diese blau wird. Das Lösungsmittel wird im Vakuum entfernt und der Rückstand in wenig Aceton aufgenommen. Anschließend wird die Lösung filtriert und mit Pentan überschichtet. Die erhaltenen blauen Kristalle sind analysenrein und für die Röntgenstrukturanalyse geeignet.

Summenformel: $\mathrm{C}_{31} \mathrm{H}_{66} \mathrm{Cu}_{4} \mathrm{Cl}_{4} \mathrm{~N}_{12} \mathrm{O}_{21}+2$ Aceton $(1455,08 \mathrm{~g} / \mathrm{mol})$

IR $(\mathrm{KBr}): \tilde{v}=3589(\mathrm{~m}), 3504(\mathrm{~m}), 3392(\mathrm{~m}), 3118(\mathrm{w}), 2984(\mathrm{~m}), 2964(\mathrm{~m})$, 2908(m), 2820(m), 2015(w), 1701(s), 1637(w), 1517(s), $1471(\mathrm{~s}), \quad 1440(\mathrm{~m}), \quad 1422(\mathrm{~m}), \quad 1367(\mathrm{~s}), \quad 1318(\mathrm{~m}), \quad 1285(\mathrm{w})$, 1263(w), 1230(w), 1090(s), 1029(s), 987(s), 942(m), 847(m), 833(w), 806(w), 793(w), 776(m), 623(s), 533(w), 504(w), 473(w), 436(w), 397(w), 352(w),321(w)

MS (FAB, nibeol): $m / z$ (\%): 257 (80, [L $\left.\left.{ }^{3} \mathrm{Cu}-\mathrm{Me}_{2} \mathrm{NCH}_{2} \mathrm{CH}_{2} \mathrm{NMe}\right]^{+}\right), 421$ (100,

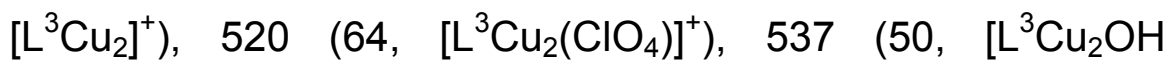
$\left.\left.\left(\mathrm{ClO}_{4}\right)\right]^{+}\right), \quad 619 \quad\left(11, \quad\left[\mathrm{~L}^{3} \mathrm{Cu}_{2}\left(\mathrm{ClO}_{4}\right)_{2}\right]^{+}\right), \quad 1100 \quad\left(8, \quad\left[\left(\mathrm{~L}^{3} \mathrm{Cu}_{2}\right)_{2}\right.\right.$ $\left.\left.\left(\mathrm{CO}_{3}\right)\left(\mathrm{ClO}_{4}\right)_{2}\right]^{+}\right), 1199\left(12,\left[\left(\mathrm{~L}^{3} \mathrm{Cu}_{2}\right)_{2}\left(\mathrm{CO}_{3}\right)\left(\mathrm{ClO}_{4}\right)_{3}\right]^{+}\right)$ 
EXPERIMENTELLER TEIL

EA: ber. C 30.54; H 5.40; N 11.55; O 25.29; Cl 9.75; Cu 17.47 exp. C 29.93; H 5.25; N 11.53

UV/Vis (Aceton) $\lambda(\varepsilon) 226$ (18090), 286 (sh, 6790), 614 (450)

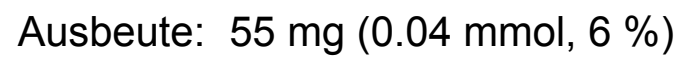




\subsection{2 $\left[\mathrm{L}^{5} \mathrm{Cu}_{2}(\mathrm{OAC})\left(\mathrm{ClO}_{4}\right)\right] \mathrm{ClO}_{4}(6)$}

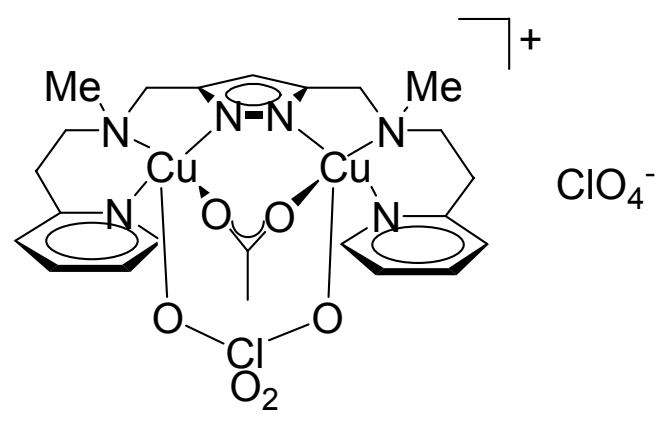

Ansatz 1:

$110 \mathrm{mg}(0.30 \mathrm{mmol}) \mathrm{HL}^{5}$

$68 \mathrm{mg}(0.60 \mathrm{mmol})$ Kalium-tert-butylat

$224 \mathrm{mg}(0.60 \mathrm{mmol}) \mathrm{Cu}\left(\mathrm{ClO}_{4}\right)_{2} \cdot 6 \mathrm{H}_{2} \mathrm{O}$

$75 \mathrm{ml}$ Acetonitril

Durchführung:

In einem $250 \mathrm{ml}$ Kolben wird der Ligand $\mathrm{HL}^{5}$ zunächst in $75 \mathrm{ml}$ Acetonitril mit zwei Äquivalenten Kalium-tert-butylat umgesetzt. Anschließend werden zwei Äquivalente Kupfer(II)perchlorat zugegeben. Die blaue Lösung wird sechs Stunden gerührt. Nach Entfernen des Lösungsmittels im Vakuum wird der Rückstand wieder in wenig Dichlormethan aufgenommen, filtriert und in einem Schlenckkolben mit Diethylether überschichtet. Die erhaltenen blauen Kristalle sind analysenrein und für die Röntgenstrukturanalyse geeignet.

Summenformel: $\mathrm{C}_{23} \mathrm{H}_{30} \mathrm{Cu}_{2} \mathrm{Cl}_{2} \mathrm{~N}_{6} \mathrm{O}_{10}+\mathrm{CH}_{2} \mathrm{Cl}_{2}(833,45 \mathrm{~g} / \mathrm{mol})$

IR $(\mathrm{KBr}): \tilde{v}=3443(\mathrm{~m}), 3275(\mathrm{~m}), 3127(\mathrm{~m}), 3088(\mathrm{~m}), 2928(\mathrm{~m}), 2880(\mathrm{~m})$, 2175(w), 2021(w), 1612(s), 1571(s), 1528(w), 1489(m), 1449(s), 1373(w), 1317(m), 1249(w), 1096(s), 1011(s), 959(m), 929(w), 913(w), 844(w), 824(w), 786(m), 772(m), 732(w), 623(s), 590(w), 537(w), 429(w) 
MS (FAB, nibeol): $m / z(\%): 291\left(60,\left[\mathrm{~L}^{5} \mathrm{Cu}-\mathrm{MeN}\left(\mathrm{CH}_{2} \mathrm{CH}_{2} \mathrm{Py}\right]^{+}\right), 355\left(23,\left[\mathrm{~L}^{5} \mathrm{Cu}_{2}\right.\right.\right.$ - $\left.\mathrm{MeN}\left(\mathrm{CH}_{2} \mathrm{CH}_{2} \mathrm{Py}+1\right]^{+}\right), 489\left(100,\left[\mathrm{~L}^{5} \mathrm{Cu}_{2}\right]^{+}\right), 588$ (42, $\left.\left[\mathrm{L}^{5} \mathrm{Cu}_{2}\left(\mathrm{ClO}_{4}\right)\right]^{+}\right), 687\left(22,\left[\mathrm{~L}^{5} \mathrm{Cu}_{2}\left(\mathrm{ClO}_{4}\right)_{2}\right]^{+}\right)$

EA: ber. C 34.59; H 3.87; N 10.08; O 19.20; Cl 17.02; Cu 15.25 exp. C 34.18; H 4.24; N 10.96

UV/Vis (MeCN) $\lambda(\varepsilon) 233$ (15580), 258 (17570), 290 (sh, 6860), 631 (440)

Ausbeute: $76 \mathrm{mg}(0.09 \mathrm{mmol}, 30 \%)$

Ansatz 2:

$184 \mathrm{mg}(0.50 \mathrm{mmol}) \mathrm{HL}^{5}$

$56 \mathrm{mg}(0.50 \mathrm{mmol})$ Kalium-tert-butylat

$278 \mathrm{mg}(0.75 \mathrm{mmol}) \mathrm{Cu}\left(\mathrm{ClO}_{4}\right)_{2} \cdot 6 \mathrm{H}_{2} \mathrm{O}$

$50 \mathrm{mg}(0.25 \mathrm{mmol}) \mathrm{Cu}\left(\mathrm{CH}_{3} \mathrm{COO}^{-}\right)_{2} \cdot \mathrm{H}_{2} \mathrm{O}$

$125 \mathrm{ml}$ Aceton

Durchführung:

In einem $250 \mathrm{ml}$ Kolben wird in $100 \mathrm{ml}$ Aceton der Ligand $\mathrm{HL}^{5}$ zunächst mit einem Äquivalent Kalium-tert-butylat umgesetzt. Anschließend werden 1,5 Äquivalente Kupfer(II)perchlorat zugegeben. Hierzu lässt man eine Lösung von $50 \mathrm{mg}$ Kupfer(II)acetat in $25 \mathrm{ml}$ Aceton zutropfen. Die blaue Lösung wird eine Stunde gerührt. Nach Entfernen des Lösungsmittels im Vakuum wird der Rückstand wieder in wenig Dichlormethan aufgenommen, filtriert und in einem Schlenckkolben mit Diethylether überschichtet. Die erhaltenen blauen Kristalle sind analysenrein und für die Röntgenstrukturanalyse geeignet.

Summenformel: $\mathrm{C}_{23} \mathrm{H}_{30} \mathrm{Cu}_{2} \mathrm{Cl}_{2} \mathrm{~N}_{6} \mathrm{O}_{10}+\mathrm{CH}_{2} \mathrm{Cl}_{2}(833.45 \mathrm{~g} / \mathrm{mol})$

IR $(\mathrm{KBr}): \tilde{v}=3474(\mathrm{~m}), 3120(\mathrm{w}), 3062(\mathrm{w}), 3006(\mathrm{~m}), 2966(\mathrm{~m}), 2932(\mathrm{~m})$, 2914(m), 2879(m), 2820(w), 2023(w), 1612(s), 1566(s), 1488(s), 1447(s), 1415(s), 1374(w), 1333(w), 1312(m), 1273(w), 1244(w), 1223(w), 1162(m), 1095(s), 1050(s), 1006(s), 979(s), 952(m), 930(m), 913(m), 848(m), 822(m), 775(s), 768(s), 734(s), 700(w), 654(w), 623(s), 594(w), 535(w), 481(w), 437(w), 385(w), 359(w), 333(w) 
MS (ESI, MeOH): m/z (\%): $489\left(11,\left[{ }^{5} \mathrm{Cu}_{2}\right]^{+}\right), 548\left(10,\left[\mathrm{~L}^{5} \mathrm{Cu}_{2}(\mathrm{OAc})\right]^{+}\right), 607$ (100, $\left.\left[\mathrm{L}^{5} \mathrm{Cu}_{2}(\mathrm{OAc})_{2}\right]^{+}\right), 647\left(37,\left[\mathrm{~L}^{5} \mathrm{Cu}_{2}(\mathrm{OAc})\left(\mathrm{ClO}_{4}\right)\right]^{+}\right)$

EA: ber. C $34,59 \mathrm{H} 3,87 \mathrm{~N} 10,08 \mathrm{O} 19.20 \mathrm{Cl} 17.02 \mathrm{Cu} 15.25$ exp. C $34,28 \mathrm{H} 3,91 \mathrm{~N} 9,96$

UV/Vis (MeCN) $\lambda(\varepsilon) 235$ (13300), 259 (15550), 287 (sh, 6470), 628 (300)

Ausbeute: $48 \mathrm{mg}(0.06 \mathrm{mmol}, 12 \%)$ 


\subsection{3 $\left[\mathrm{L}^{5} \mathrm{Cu}_{2} \mathrm{~F}(\mathrm{MeOH})_{2}\left(\mathrm{BF}_{4}\right)\right] \mathrm{BF}_{4}(7 \mathrm{a})$}

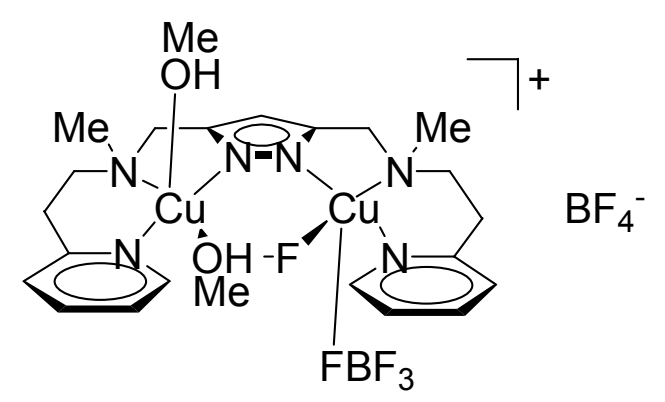

Ansatz:

$175 \mathrm{mg}(0.48 \mathrm{mmol}) \mathrm{HL}^{5}$

$108 \mathrm{mg}$ (0.96 mmol) Kalium-tert-butylat

$331 \mathrm{mg}(0.96 \mathrm{mmol}) \mathrm{Cu}\left(\mathrm{BF}_{4}\right)_{2} \cdot 6 \mathrm{H}_{2} \mathrm{O}$

$150 \mathrm{ml}$ Methanol

Durchführung:

In einem $250 \mathrm{ml}$ Kolben wird der Ligand $\mathrm{HL}^{5}$ zunächst in $150 \mathrm{ml}$ Methanol mit zwei Äquivalenten Kalium-tert-butylat umgesetzt. Anschließend werden zwei Äquivalente Kupfer(II)tetrafluorborat zugegeben. Die blaue Lösung wird sechs Stunden gerührt. Nach Entfernen des Lösungsmittels im Vakuum wird der Rückstand wieder in wenig Methanol aufgenommen, filtriert und in einem Schlenckkolben mit Diethylether überschichtet. Die erhaltenen blauen Kristalle sind analysenrein und für die Röntgenstrukturanalyse geeignet.

Summenformel: $\mathrm{C}_{23} \mathrm{H}_{35} \mathrm{Cu}_{2} \mathrm{~B}_{2} \mathrm{~F}_{9} \mathrm{~N}_{6} \mathrm{O}_{2}(747,26 \mathrm{~g} / \mathrm{mol})$

IR (KBr): $\tilde{v}=3551(\mathrm{~m}), 3420(\mathrm{~m}), 3128(\mathrm{~m}), 3090(\mathrm{~m}), 2985(\mathrm{~m}), 2950(\mathrm{~s})$, 2886(m), 2710(m), 2609(m), 2542(m), 1613(s), 1573(m), 1524(w), 1490(m), 1466(m), 1449(s), 1375(w), 1321(m), 1289(w), 1249(w), 1183(w), 1069(s), 1029(s), 913(w), 848(w), 826(w), 789(w), 765(m), 653(w), 591(w), 522(w), 454(w), 427(w), 329(w)

MS (FAB, nibeol): $m / z$ (\%): 291 (28, $\left[\mathrm{L}^{5} \mathrm{Cu}-\mathrm{MeN}\left(\mathrm{CH}_{2} \mathrm{CH}_{2} \mathrm{Py}\right]^{+}\right), \quad 489$ (100, $\left.\left[\mathrm{L}^{5} \mathrm{Cu}_{2}\right]^{+}\right), 508\left(79,\left[\mathrm{LCu}_{2} \mathrm{~F}\right]^{+}\right), 595\left(32,\left[\mathrm{LCu}_{2} \mathrm{~F}\left(\mathrm{BF}_{4}\right)\right]^{+}\right)$ 
EXPERIMENTELLER TEIL

EA: ber. C 36.97; H 4.72; N 11.25; B 2.89; O 4.28; F 22.88;

Cu 17.01

exp. C 36.71; H 4.66; N 11.19

UV/Vis (MeOH) $\lambda(\varepsilon) 206$ (14800), 224 (14540), 259 (11820), 284 (sh, 4710), 644 (200)

Ausbeute: $92 \mathrm{mg}(0.12 \mathrm{mmol}, 25 \%)$ 


\subsection{4 $\left[\mathrm{L}^{5} \mathrm{Cu}_{2}(\mathrm{MeOH})_{2}\left(\mathrm{NO}_{3}\right)_{2}\right] \mathrm{NO}_{3}(8)$}

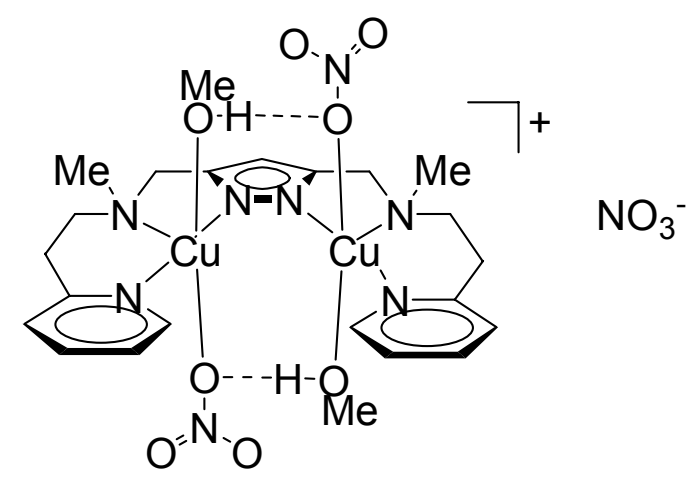

Ansatz:

$295 \mathrm{mg}(0.81 \mathrm{mmol}) \mathrm{HL}^{5}$

$91 \mathrm{mg}(0.81 \mathrm{mmol})$ Kalium-tert-butylat

$391 \mathrm{mg}(1.62 \mathrm{mmol}) \mathrm{Cu}\left(\mathrm{NO}_{3}\right)_{2} \cdot 3 \mathrm{H}_{2} \mathrm{O}$

$150 \mathrm{ml}$ Methanol

Durchführung:

In einem $250 \mathrm{ml}$ Kolben wird der Ligand $\mathrm{HL}^{5}$ zunächst in $150 \mathrm{ml}$ Methanol mit einem Äquivalent Kalium-tert-butylat umgesetzt. Anschließend werden zwei Äquivalente Kupfer(II)nitrat zugegeben. Die blaugrüne Lösung wird zwei Stunden gerührt. Nach Entfernen des Lösungsmittels im Vakuum wird der Rückstand wieder in wenig Methanol aufgenommen, filtriert und in einem Schlenckkolben mit Diethylether überschichtet. Die erhaltenen blauen Kristalle sind analysenrein und für die Röntgenstrukturanalyse geeignet.

Summenformel: $\mathrm{C}_{23} \mathrm{H}_{35} \mathrm{Cu}_{2} \mathrm{~N}_{9} \mathrm{O}_{11}(740,67 \mathrm{~g} / \mathrm{mol})$

IR (KBr): $\tilde{v}=3365(\mathrm{~m}), 2931(\mathrm{~m}), 2876(\mathrm{~m}), 2486(\mathrm{w}), 2397(\mathrm{w})$, 1764(w), 1611(s), $1570(\mathrm{~m}), \quad 1484(\mathrm{~s}), \quad 1437(\mathrm{~s}), \quad 1384(\mathrm{~s}), \quad 1357(\mathrm{~s})$, 1286(s), $1160(\mathrm{~m}), \quad 1105(\mathrm{~m}), \quad 1062(\mathrm{w}), \quad 1012(\mathrm{~m}), \quad 979(\mathrm{w})$, 959(w), 911(w), 841(w), 825(w), 812(w), 769(m),709(w), 649(w), 590(w), 537(w), 472(w), 429(w), 317(w) 
MS (FAB, nibeol): $m / z$ (\%): 291 (39, $\left[\mathrm{L}^{5} \mathrm{Cu}-\mathrm{MeN}\left(\mathrm{CH}_{2} \mathrm{CH}_{2} \mathrm{Py}\right]^{+}\right), 355$ (70, $\left[\mathrm{L}^{5} \mathrm{Cu}_{2}-\mathrm{MeN}\left(\mathrm{CH}_{2} \mathrm{CH}_{2} \mathrm{Py}+1\right]^{+}\right), 489\left(32,\left[\mathrm{~L}^{5} \mathrm{Cu}_{2}\right]^{+}\right), 551(66$, $\left.\left[\mathrm{L}^{5} \mathrm{Cu}_{2} \mathrm{NO}_{3}\right]^{+}\right), 613\left(100,\left[\mathrm{~L}^{5} \mathrm{Cu}_{2}\left(\mathrm{NO}_{3}\right)_{2}\right]^{+}\right)$

EA: ber. C 37.30; H 4.76; N 17.02; O 23.76; Cu 17.16 exp. C 37.06; H 4.98; N 17.05

UV/Vis (MeOH) $\lambda(\varepsilon) 216$ (26600), 259 (16010), 284 (sh, 7190), 635 (290) Ausbeute: $330 \mathrm{mg}(0.45 \mathrm{mmol}, 56 \%)$ 


\subsection{5 $\left[\mathrm{L}^{6} \mathrm{Cu}_{2}(\mathrm{OAC})\right]\left(\mathrm{ClO}_{4}\right)_{2}$ (9)}

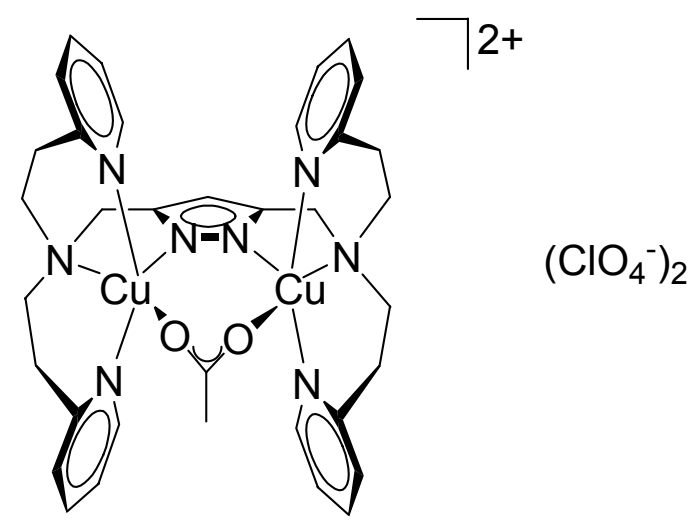

Ansatz 1:

$350 \mathrm{mg}(0.64 \mathrm{mmol}) \mathrm{HL}^{6}$

$144 \mathrm{mg}$ (1.28 mmol) Kalium-tert-butylat

$474 \mathrm{mg}(1.28 \mathrm{mmol}) \mathrm{Cu}\left(\mathrm{ClO}_{4}\right)_{2} \cdot 6 \mathrm{H}_{2} \mathrm{O}$

$150 \mathrm{ml}$ Acetonitril

Durchführung:

In einem $250 \mathrm{ml}$ Kolben wird der Ligand $\mathrm{HL}^{6}$ zunächst in $150 \mathrm{ml}$ Acetonitril mit zwei Äquivalenten Kalium-tert-butylat umgesetzt. Anschließend werden zwei Äquivalente Kupfer(II)perchlorat zugegeben. Die dunkelgrüne Lösung wird zwei Stunden gerührt. Nach Entfernen des Lösungsmittels im Vakuum wird der Rückstand wieder in wenig Acetonitril aufgenommen, filtriert und in einem Schlenckkolben mit Diethylether überschichtet. Die erhaltenen blauen Kristalle sind analysenrein und für die Röntgenstrukturanalyse geeignet.

Summenformel: $\mathrm{C}_{35} \mathrm{H}_{40} \mathrm{Cu}_{2} \mathrm{Cl}_{2} \mathrm{~N}_{8} \mathrm{O}_{10}(930,74 \mathrm{~g} / \mathrm{mol})$

IR $(\mathrm{KBr}): \tilde{v}=3440(\mathrm{~m}), 3118(\mathrm{w}), 3082(\mathrm{w}), 3042(\mathrm{w}), 2948(\mathrm{~m}), 2884(\mathrm{~m})$, 2244(w), 2171(w), 2112(w), 2015(w), 1731(w), 1607(s), 1570(s), 1550(m), 1487(s), 1444(s), 1383(m), 1324(m), 1309(m), 1255(w), 1160(w), 1093(s), 1009(m), 934(w), 836(w), 787(m), 768(s), 623(s), 589(w), 532(w), 472(w), 429(w), 414(w), $380(w)$ 
MS (ESI, MeOH): $m / z(\%): 382\left(24,\left[\mathrm{~L}^{6} \mathrm{Cu}-\mathrm{N}\left(\mathrm{CH}_{2} \mathrm{CH}_{2} \mathrm{Py}\right)_{2}\right]^{+}\right), 671\left(15,\left[\mathrm{~L}^{6} \mathrm{Cu}_{2}\right]^{+}\right)$, $735\left(46,\left[\mathrm{~L}^{6} \mathrm{Cu}_{2}(\mathrm{MeOH})_{2}\right]^{+}\right), 767\left(44,\left[\mathrm{~L}^{6} \mathrm{Cu}_{2}(\mathrm{MeOH})_{3}\right]^{+}\right), 789(24$, $\left.\left[\mathrm{L}^{6} \mathrm{Cu}_{2}(\mathrm{OAc})_{2}\right]^{+}\right), 829\left(27,\left[\mathrm{~L}^{6} \mathrm{Cu}_{2}(\mathrm{OAc})\left(\mathrm{ClO}_{4}\right)\right]^{+}\right)$

EA: ber. C 45.17; H 4.33; N 12.04; O 17.19; $\mathrm{Cl} 7.62$; $\mathrm{Cu} 13.66$ exp. C 45.13; H 4.33; N 12.85

UV/Nis (MeCN) $\lambda(\varepsilon) 225$ (18430), 257 (20650), 267 (sh, 16080), 646 (280)

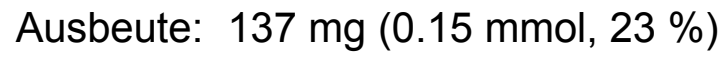

Ansatz 2:

$188 \mathrm{mg}(0.34 \mathrm{mmol}) \mathrm{HL}^{6}$

$38 \mathrm{mg}(0.34 \mathrm{mmol})$ Kalium-tert-butylat

$189 \mathrm{mg}(0.51 \mathrm{mmol}) \mathrm{Cu}\left(\mathrm{ClO}_{4}\right)_{2} \cdot 6 \mathrm{H}_{2} \mathrm{O}$

$34 \mathrm{mg}(0.17 \mathrm{mmol}) \mathrm{Cu}\left(\mathrm{CH}_{3} \mathrm{COO}\right)_{2} \cdot \mathrm{H}_{2} \mathrm{O}$

$125 \mathrm{ml}$ Aceton

Durchführung:

In einem $250 \mathrm{ml}$ Kolben wird der Ligand $\mathrm{HL}^{6}$ zunächst in $100 \mathrm{ml}$ Aceton mit einem Äquivalent Kalium-tert-butylat umgesetzt. Anschließend werden 1.5 Äquivalente Kupfer(II)perchlorat zugegeben, sowie 0.5 Äquivalente Kupfer(II)acetat. Die grüne Lösung wird eine Stunde gerührt. Nach Entfernen des Lösungsmittels im Vakuum wird der Rückstand wieder in wenig Dichlormethan aufgenommen, filtriert und in einem Schlenckkolben mit Diethylether überschichtet. Man erhält ein grünes Pulver.

Summenformel: $\mathrm{C}_{35} \mathrm{H}_{40} \mathrm{Cu}_{2} \mathrm{Cl}_{2} \mathrm{~N}_{8} \mathrm{O}_{10}+1 \mathrm{CH}_{2} \mathrm{Cl}_{2}(1015,67 \mathrm{~g} / \mathrm{mol})$

IR (KBr): $\tilde{v}=3609(\mathrm{~m}), 3526(\mathrm{~m}), 3449(\mathrm{~m}), 3076(\mathrm{w}), 2970(\mathrm{w}), 2921$ $(w), 2871(w), 1610(s), 1569(m), 1488(m), 1442(s), 1384$ (w), $1329(w), 1309(w), 1278(w), 1256(w), 1212(w), 1162$ (w), $1088(v s), 1019(m), 959(m), 933(m), 840(w), 786(m)$, $769(s), 731(w), 697(w), 653(w), 623(s), 587(w), 532(w)$, $470(w), 427(w), 357(w)$ 
MS (ESI, MeOH): $m / z$ (\%): $382\left(74,\left[{ }^{6} \mathrm{Cu}-\mathrm{N}\left(\mathrm{CH}_{2} \mathrm{CH}_{2} \mathrm{Py}\right)_{2}\right]^{+}\right), 671\left(47,\left[\mathrm{~L}^{6} \mathrm{Cu}_{2}\right]^{+}\right)$, $706\left(60,\left[\mathrm{~L}^{6} \mathrm{Cu}_{2} \mathrm{Cl}\right]^{+}\right), 730\left(71,\left[\mathrm{~L}^{6} \mathrm{Cu}_{2}(\mathrm{OAc})\right]^{+}\right), 767\left(80,\left[\mathrm{~L}^{6} \mathrm{Cu}_{2}\right.\right.$ $\left.\left.(\mathrm{MeOH})_{3}\right]^{+}\right), \quad 787 \quad\left(100, \quad\left[\mathrm{~L}^{6} \mathrm{Cu}_{2}(\mathrm{OH})\left(\mathrm{ClO}_{4}\right)\right]^{+}\right), \quad 829 \quad(71$, $\left.\left[\mathrm{L}^{6} \mathrm{Cu}_{2}(\mathrm{OAc})\left(\mathrm{ClO}_{4}\right)\right]^{+}\right)$

EA: ber. C 42.57; H 4.17; N 11.03; O 15.75; Cl 13.96; Cu 12.51 exp. C 42.70; H 4.25; N 11.75

UV/Nis (MeCN) $\lambda(\varepsilon) 226$ (15320), 258 (18910), 262 (sh, 18740), 653 (250) Ausbeute: $217 \mathrm{mg}(0.23 \mathrm{mmol}, 68 \%)$ 


\subsection{6 $\left[\mathrm{L}^{6} \mathrm{Cu}_{2}(\mathrm{OH})\right]\left(\mathrm{ClO}_{4}\right)_{2}(10)$}

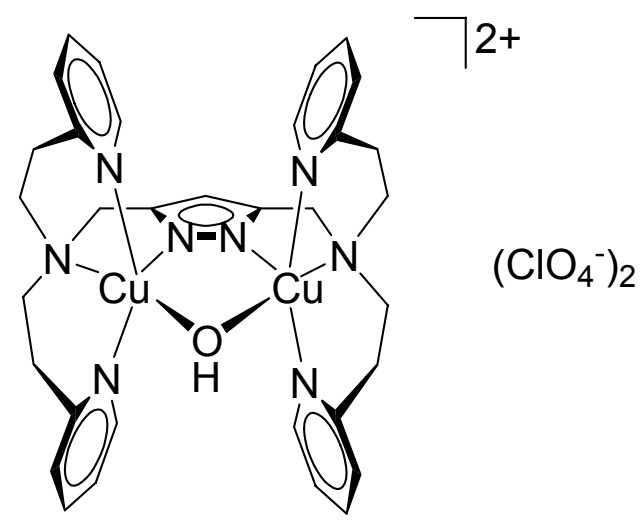

Ansatz:

$120 \mathrm{mg}(0.22 \mathrm{mmol}) \mathrm{HL}^{6}$

$49 \mathrm{mg}(0.44 \mathrm{mmol})$ Kalium-tert-butylat

$163 \mathrm{mg}(0.44 \mathrm{mmol}) \mathrm{Cu}\left(\mathrm{ClO}_{4}\right)_{2} \cdot 6 \mathrm{H}_{2} \mathrm{O}$

$150 \mathrm{ml}$ Acetonitril

Durchführung:

In einem $250 \mathrm{ml}$ Kolben wird der Ligand $\mathrm{HL}^{6}$ zunächst in $150 \mathrm{ml}$ Acetonitril mit zwei Äquivalenten Kalium-tert-butylat umgesetzt. Anschließend werden zwei Äquivalente Kupfer(II)perchlorat zugegeben. Die dunkelgrüne Lösung wird sechs Stunden gerührt. Nach Entfernen des Lösungsmittels im Vakuum wird der Rückstand wieder in wenig Dichlormethan aufgenommen, filtriert und in einem Schlenckkolben mit Diethylether überschichtet. Die nach Kristallisation bei $4^{\circ} \mathrm{C}$ erhaltenen blauen Kristalle sind analysenrein und für die Röntgenstrukturanalyse geeignet.

Summenformel: $\mathrm{C}_{33} \mathrm{H}_{38} \mathrm{Cu}_{2} \mathrm{Cl}_{2} \mathrm{~N}_{8} \mathrm{O}_{9}(888,70 \mathrm{~g} / \mathrm{mol})$

IR (KBr): $\tilde{v}=3524(\mathrm{~m}), 3443(\mathrm{~m}), 3121(\mathrm{w}), 3076(\mathrm{w}), 2959(\mathrm{~m}), 2923(\mathrm{~m})$, $2867(\mathrm{~m}), \quad 2184(\mathrm{w}), \quad 2107(\mathrm{w}), \quad 2011(\mathrm{w}), \quad 1606(\mathrm{~s}), \quad 1569(\mathrm{~m})$, 1487(s), 1443(s), 1381(w), 1329(m), 1309(m), 1263(w), 1160(m), 1091(s), 1017(m), 957(w), 932(w), 835(w), 786(m), 768(s), 731(w), 697(w), 638(w), 622(s), 589(w), 531(w), 471(w), 428(w), 413(w) 
MS (FAB, nibeol): $m / z(\%): 382\left(7,\left[\mathrm{~L}^{6} \mathrm{Cu}-\mathrm{N}\left(\mathrm{CH}_{2} \mathrm{CH}_{2} \mathrm{Py}\right)\right]^{+}\right), 566\left(18,\left[\mathrm{~L}^{6} \mathrm{Cu}_{2}-\right.\right.$ $\left.\left.\left.\mathrm{CH}_{2} \mathrm{CH}_{2} \mathrm{Py}+1\right]^{+}\right), 671\left(100,\left[\mathrm{~L}^{6} \mathrm{Cu}_{2}\right]^{+}\right]\right), 770\left(27,\left[\mathrm{~L}^{6} \mathrm{Cu}_{2}\left(\mathrm{ClO}_{4}\right)\right]^{+}\right)$

EA: ber. C 44.60; H 4.31; N 12.61; O 16.20; Cl 7.98; Cu 14.30 exp. C 46.05; H 4.59; N 13.02

UV/Vis $\left(\mathrm{CH}_{2} \mathrm{Cl}_{2}\right) \lambda(\varepsilon) 234$ (18200), 257 (21820), 644 (270)

Ausbeute: $67 \mathrm{mg}(0.08 \mathrm{mmol}, 36 \%)$ 


\subsection{7 $\left[\mathrm{L}^{6} \mathrm{Cu}_{2} \mathrm{~F}(\mathrm{MeOH})\right]\left(\mathrm{BF}_{4}\right)_{2} \quad(11)$}

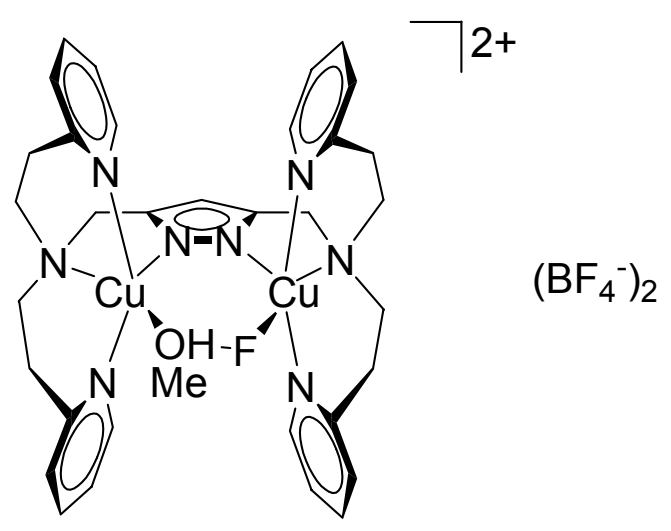

Ansatz:

$152 \mathrm{mg}(0.28 \mathrm{mmol}) \mathrm{HL}^{6}$

$63 \mathrm{mg}(0.56 \mathrm{mmol})$ Kalium-tert-butylat

193mg (0.56 mmol) $\mathrm{Cu}\left(\mathrm{BF}_{4}\right)_{2} \cdot 6 \mathrm{H}_{2} \mathrm{O}$

$150 \mathrm{ml}$ Methanol

Durchführung:

In einem $250 \mathrm{ml}$ Kolben wird der Ligand $\mathrm{HL}^{6}$ zunächst in $150 \mathrm{ml}$ Methanol mit zwei Äquivalenten Kalium-tert-butylat umgesetzt. Anschließend werden zwei Äquivalente Kupfer(II)tetrafluorborat zugegeben. Die blaugrüne Lösung wird zwei Stunden gerührt. Nach Entfernen des Lösungsmittels im Vakuum wird der Rückstand wieder in wenig Methanol aufgenommen, filtriert und in einem Schlenckkolben mit Diethylether überschichtet. Die erhaltenen blauen Kristalle sind analysenrein und für die Röntgenstrukturanalyse geeignet.

Summenformel: $\mathrm{C}_{34} \mathrm{H}_{41} \mathrm{~B}_{2} \mathrm{Cu}_{2} \mathrm{~F}_{9} \mathrm{~N}_{8} \mathrm{O}(897,44 \mathrm{~g} / \mathrm{mol})$

$\operatorname{IR}(\mathrm{KBr}): \tilde{v}=3460(\mathrm{~m}), 3210(\mathrm{w}), 3147(\mathrm{w}), 3115(\mathrm{w}), 3086(\mathrm{w}), 3044(\mathrm{w})$, 2980(w), 2952(w), 2933(w), 2858(w), 2698(w), 1635(w), 1606(s), 1573(w), 1487(m), 1462(w), 1446(s), 1384(w), 1356(w), 1327(w), 1307(w), 1284(w), 1259(w), 1159(m), 1070(s), 1033(s), 830(w), 806(w), 788(m), 772(s), 765(s), $640(w), 590(w), 520(w), 470(w), 430(w), 414(w), 313(w)$ 
MS (FAB, nibeol): $m / z(\%): 382\left(34,\left[\mathrm{~L}^{6} \mathrm{Cu}-\mathrm{N}\left(\mathrm{CH}_{2} \mathrm{CH}_{2} \mathrm{Py}\right)_{2}\right]^{+}\right), 671\left(52,\left[\mathrm{~L}^{6} \mathrm{Cu}_{2}\right]^{+}\right)$, $\left.690\left(100,\left[\mathrm{~L}^{6} \mathrm{Cu}_{2} \mathrm{~F}\right]\right]^{+}\right), 777\left(46,\left[\mathrm{~L}^{6} \mathrm{Cu}_{2} \mathrm{~F}\left(\mathrm{BF}_{4}\right)\right]^{+}\right)$

EA: ber. C 45.50; H 4.60; N 12.49; B 2.41; O 1.78; F 19.05;

Cu 14.16

exp. C 44.83; H 4.50; N 12.30

UV/Vis (MeOH) $\lambda(\varepsilon) 209$ (18340), 223 (18220), 257 (21030), 669 (210), 946 (sh, 100)

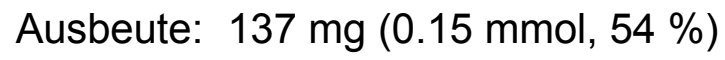




\subsection{8 $\left[\mathrm{L}^{6} \mathrm{Cu}_{2} \mathrm{Cl}\right]\left(\mathrm{CF}_{3} \mathrm{SO}_{3}\right)_{2}(12)$}

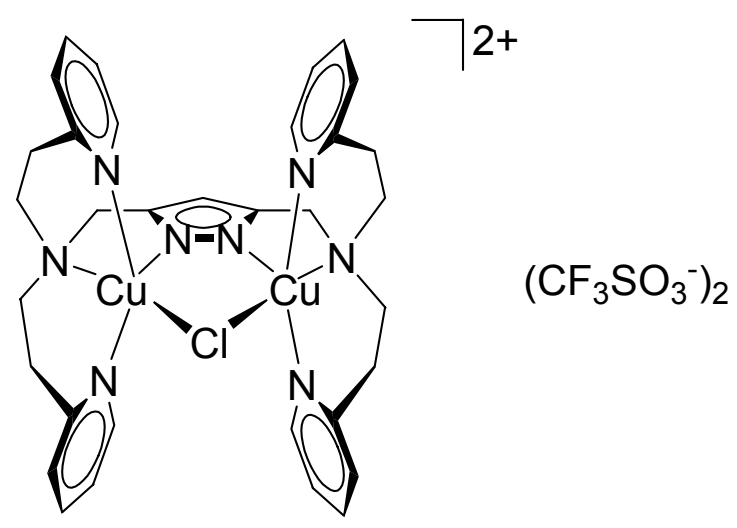

Ansatz:

$399 \mathrm{mg}(0.73 \mathrm{mmol}) \mathrm{HL}^{6}$

$164 \mathrm{mg}(1.46 \mathrm{mmol})$ Kalium-tert-butylat

$528 \mathrm{mg}(1.46 \mathrm{mmol}) \mathrm{Cu}\left(\mathrm{CF}_{3} \mathrm{SO}_{3}\right)_{2}$

$150 \mathrm{ml}$ Dichlormethan

Durchführung:

In einem $250 \mathrm{ml}$ Kolben wird der Ligand $\mathrm{HL}^{6}$ zunächst in $150 \mathrm{ml}$ Dichlormethan mit zwei Äquivalenten Kalium-tert-butylat umgesetzt. Anschließend werden zwei Äquivalente Kupfer(II)trifluormethansulfonat zugegeben. Die dunkelgrüne Lösung wird sechs Stunden gerührt. Nach Entfernen des Lösungsmittels im Vakuum wird der Rückstand wieder in wenig Dichlormethan aufgenommen, filtriert und in einem Schlenckkolben mit Diethylether überschichtet. Die nach Kristallisation erhaltenen grünen Kristalle sind analysenrein und für die Röntgenstrukturanalyse geeignet.

Summenformel: $\mathrm{C}_{35} \mathrm{H}_{37} \mathrm{Cu}_{2} \mathrm{ClF}_{6} \mathrm{~N}_{8} \mathrm{O}_{6} \mathrm{~S}_{2}+\mathrm{CH}_{2} \mathrm{Cl}_{2}(1091,32 \mathrm{~g} / \mathrm{mol})$

$\operatorname{IR}(\mathrm{KBr}): \tilde{v}=3454(\mathrm{~m}), \quad 3084(\mathrm{w}), 2953(\mathrm{w}), 2920(\mathrm{w}), 2871(\mathrm{w})$, 2293(w), 1610(s), 1571(m), 1488(m), 1447(s), 1384(w), 1276(s), 1224(s), 1155(s), 1109(m), 1058(w), 1030(s), 957(w), 932(w), 837(w), 786(m), 770(s), 731(w), 699(w), 637(s), 588(w), 573(m), 516(s), 473(w), 427(w), 414(w) 
MS (ESI, MeOH): $m / z \quad \%): 382 \quad\left(28, \quad\left[\mathrm{~L}^{6} \mathrm{Cu}-\mathrm{N}\left(\mathrm{CH}_{2} \mathrm{CH}_{2} \mathrm{Py}\right)\right]^{+}\right), \quad 671(9$, $\left.\left[\begin{array}{llll}\mathrm{L}^{6} \mathrm{Cu}_{2} & \left.\left.]^{+}\right]\right), \quad 767 \quad\left(15, \quad\left[\mathrm{~L}^{6} \mathrm{Cu}_{2}\right.\right. & (\mathrm{MeOH})_{3}\end{array}\right]^{+}\right), \quad 855 \quad(35$, $\left.\left[{ }^{6} \mathrm{Cu}_{2} \mathrm{Cl}\left(\mathrm{CF}_{3} \mathrm{SO}_{3}\right)\right]^{+}\right)$

EA: ber. C 39.62; H 3.60; N 10.27; O 8.80; F 10.45; S 5.89; Cl 9.75; Cu 11.65 exp. C 39.14; H 3.71; N 10.27

UV/Vis (MeOH) $\lambda(\varepsilon) 210$ (17790), 221 (17330), 259 (19780), 652 (270) Ausbeute: $205 \mathrm{mg}(0.19 \mathrm{mmol}, 26 \%)$ 
11.3.9 $\left[\mathrm{L}^{6} \mathrm{Cu}_{2}(\mathrm{OH})\right]\left(\mathrm{CF}_{3} \mathrm{SO}_{3}\right)_{2}(13)$

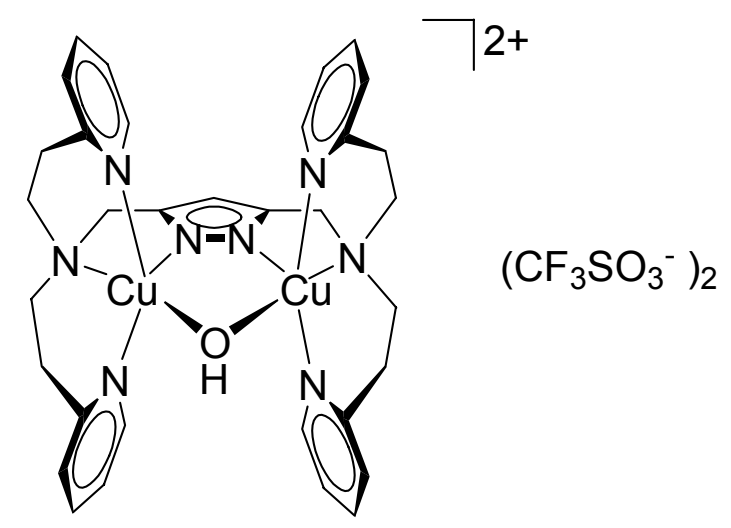

Ansatz 1:

$186 \mathrm{mg}(0.34 \mathrm{mmol}) \mathrm{HL}^{6}$

$38 \mathrm{mg}$ (0.34 mmol) Kalium-tert-butylat

$214 \mathrm{mg}(0.68 \mathrm{mmol})\left[\mathrm{Cu}(\mathrm{MeCN})_{4}\right] \mathrm{CF}_{3} \mathrm{SO}_{3}$

$50 \mathrm{ml}$ Dichlormethan

Durchführung:

In einem $100 \mathrm{ml}$ Schlenckkolben wird der Ligand $\mathrm{HL}^{6}$ unter Stickstoffatmosphäre bei $-78^{\circ} \mathrm{C}$ zunächst in $50 \mathrm{ml}$ Dichlormethan mit einem Äquivalent Kalium-tert-butylat umgesetzt. Anschließend werden zwei Äquivalente Kupfer(I)trifluormethansulfonat zugegeben. Die gelbe Lösung wird 10 Minuten gerührt und anschließend über eine Spritze Luftsauerstoff zugegeben, worauf sich die Lösung zunächst braun, dann grün färbt. Nach dem Auftauen wird mit Diethylether überschichtet. Die erhaltenen grünen Kristalle sind für die Röntgenstruktur-analyse geeignet.

Summenformel: $\mathrm{C}_{35} \mathrm{H}_{38} \mathrm{Cu}_{2} \mathrm{~F}_{6} \mathrm{~N}_{8} \mathrm{O}_{7} \mathrm{~S}_{2}(987,94 \mathrm{~g} / \mathrm{mol})$

IR $(\mathrm{KBr}): \tilde{v}=3546(\mathrm{~m}), 3452(\mathrm{~m}), 3075(\mathrm{w}), 2964(\mathrm{~s}), 2907(\mathrm{~m}), 2861(\mathrm{w})$, 1662(w), 1645(w), 1571(w), 1488(w), 1445(w), 1417(w), 1262(s), 1224(s), 1098(s), 1030(s), 864(m), 801(s), 699(w), 660(w), 637(s), 572(w), 516(w), 394(m)

MS (FAB, nibeol): $m / z(\%): 382\left(8,\left[\mathrm{~L}^{6} \mathrm{Cu}-\mathrm{N}\left(\mathrm{CH}_{2} \mathrm{CH}_{2} \mathrm{Py}\right)\right]^{+}\right), 566\left(3,\left[\mathrm{~L}^{6} \mathrm{Cu}_{2}-\right.\right.$ $\left.\left.\left.\mathrm{CH}_{2} \mathrm{CH}_{2} \mathrm{Py}+1\right]^{+}\right), 671\left(5,\left[\mathrm{~L}^{6} \mathrm{Cu}_{2}\right]^{+}\right]\right)$ 
EA: ber. C 42.55; H 3.88; N 11.34; O 11.34; F 11.54; S 6.49;

Cu 12.86

exp. C $37.69 ; \mathrm{H} 4.45 ; \mathrm{N} 8.26$

UV/Vis (MeOH) $\lambda(\varepsilon) 208$ (15540), 222 (sh, 13530), 260 (15100), 638 (290)

Ausbeute: $225 \mathrm{mg}(0.23 \mathrm{mmol}, 68 \%)$

\section{Ansatz 2:}

$285 \mathrm{mg}(0.58 \mathrm{mmol}) \mathrm{HL}^{6}$

$117 \mathrm{mg}(1.04 \mathrm{mmol})$ Kalium-tert-butylat

$377 \mathrm{mg}(1.04 \mathrm{mmol}) \mathrm{Cu}\left(\mathrm{CF}_{3} \mathrm{SO}_{3}\right)_{2}$

$150 \mathrm{ml}$ Methanol

\section{Durchführung:}

In einem $250 \mathrm{ml}$ Kolben wird der Ligand $\mathrm{HL}^{6}$ zunächst in $150 \mathrm{ml}$ Methanol mit zwei Äquivalenten Kalium-tert-butylat umgesetzt. Anschließend werden zwei Äquivalente Kupfer(II)trifluormethansulfonat zugegeben. Die grüne Lösung wird zwei Stunden gerührt. Nach Entfernen des Lösungsmittels im Vakuum wird der Rückstand wieder in wenig Aceton aufgenommen, filtriert und in einem Schlenckkolben mit Pentan überschichtet. Die nach Kristallisation erhaltenen grünen Kristalle sind analysenrein und für die Röntgenstruktur-analyse geeignet.

Summenformel: $\mathrm{C}_{35} \mathrm{H}_{38} \mathrm{Cu}_{2} \mathrm{~F}_{6} \mathrm{~N}_{8} \mathrm{O}_{7} \mathrm{~S}_{2}(987,94 \mathrm{~g} / \mathrm{mol})$

IR (KBr): $\tilde{v}=3566(\mathrm{~m}), 3076(\mathrm{w}), 2961(\mathrm{w}), 2922(\mathrm{w}), 1708(\mathrm{~m}), 1607$ (s), $1571(\mathrm{~m}), 1487(\mathrm{~m}), 1445$ (s), $1366(\mathrm{~m}), 1281$ (vs), 1260 (vs), $1225(\mathrm{~s}), 1152(\mathrm{~s}), 1107(\mathrm{~m}), 1030(\mathrm{vs}), 809(\mathrm{w}), 789(\mathrm{~m})$, $768(\mathrm{~s}), 637$ (vs), $573(\mathrm{w}), 517(\mathrm{~m})$

MS (FAB, nibeol): $m / z(\%): 382\left(32,\left[\mathrm{~L}^{6} \mathrm{Cu}-\mathrm{N}\left(\mathrm{CH}_{2} \mathrm{CH}_{2} \mathrm{Py}\right)\right]^{+}\right), 566\left(20,\left[\mathrm{~L}^{6} \mathrm{Cu}_{2}-\right.\right.$ $\left.\left.\mathrm{CH}_{2} \mathrm{CH}_{2} \mathrm{Py}+1\right]^{+}\right), 671\left(100, \quad\left[{ }^{6} \mathrm{Cu}_{2}\right]^{+}\right], \quad 820 \quad\left(59, \quad\left[\mathrm{~L}^{6} \mathrm{Cu}_{2}\left(\mathrm{CF}_{3}\right.\right.\right.$ $\left.\left.\left.\mathrm{SO}_{3}\right)\right]^{+}\right), \quad 969 \quad\left(7, \quad\left[\mathrm{~L}^{6} \mathrm{Cu}_{2}\left(\mathrm{CF}_{3} \mathrm{SO}_{3}\right)_{2}\right]^{+}\right), \quad 986 \quad\left(2, \quad\left[\mathrm{~L}^{6} \mathrm{Cu}_{2}\right.\right.$ $\left.\left.(\mathrm{OH})\left(\mathrm{CF}_{3} \mathrm{SO}_{3}\right)_{2}\right]^{+}\right)$

EA: ber. C 42.55; H 3.88; N 11.34; O 11.34; F 11.54; S 6.49;

$\mathrm{Cu} 12.86$

exp. C 43.30; H 4.22; N 10,92 
EXPERIMENTELLER TEIL

UV/Vis (MeOH) $\lambda(\varepsilon) 206$ (19050), 221 (sh, 15940), 258 (16870), 648 (250)

Ausbeute: $83 \mathrm{mg}(0.08 \mathrm{mmol}, 14 \%)$ 


\subsubsection{0 $\left[\mathrm{L}^{8} \mathrm{Cu}_{2} \mathrm{~F}(\mathrm{MeOH})\right]\left(\mathrm{BF}_{4}\right)_{2}(14)$}

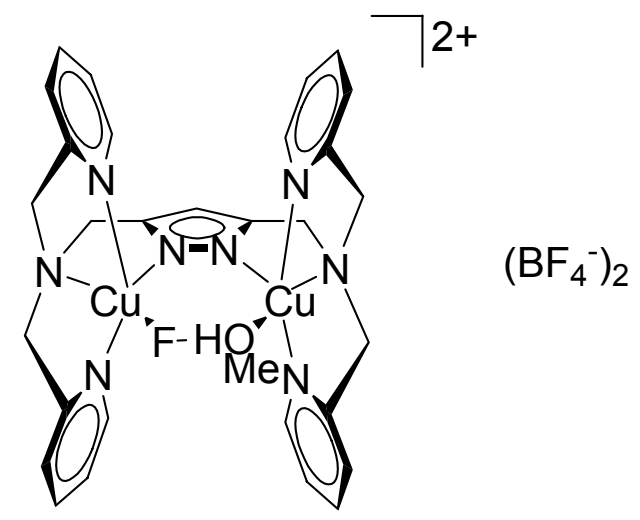

Ansatz:

$185 \mathrm{mg}(0.38 \mathrm{mmol}) \mathrm{HL}^{8}$

$85 \mathrm{mg}(0.76 \mathrm{mmol})$ Kalium-tert-butylat

$262 \mathrm{mg}(0.76 \mathrm{mmol}) \mathrm{Cu}\left(\mathrm{BF}_{4}\right)_{2} \cdot 6 \mathrm{H}_{2} \mathrm{O}$

$150 \mathrm{ml}$ Methanol

Durchführung:

In einem $250 \mathrm{ml}$ Kolben wird der Ligand $\mathrm{HL}^{8}$ zunächst in $150 \mathrm{ml}$ Methanol mit zwei Äquivalenten Kalium-tert-butylat umgesetzt. Anschließend werden zwei Äquivalente Kupfer(II)tetrafluorborat zugegeben. Die blaugrüne Lösung wird zwei Stunden gerührt. Nach Entfernen des Lösungsmittels im Vakuum wird der Rückstand wieder in wenig Methanol aufgenommen, filtriert und in einem Schlenckkolben mit Diethylether überschichtet. Die erhaltenen blauen Kristalle sind analysenrein und für die Röntgenstrukturanalyse geeignet.

Summenformel: $\mathrm{C}_{30} \mathrm{H}_{33} \mathrm{~B}_{2} \mathrm{Cu}_{2} \mathrm{~F}_{9} \mathrm{~N}_{8} \mathrm{O}(841,34 \mathrm{~g} / \mathrm{mol})$

IR $(\mathrm{KBr}): \tilde{v}=3576(\mathrm{~m}), 3476(\mathrm{~m}), 3146(\mathrm{w}), 3105(\mathrm{w}), 3080(\mathrm{w}), 3038(\mathrm{w})$, 2928(w), 2870(w), 1609(s), 1573(m), 1483(m), 1446(m), 1369(w), 1330(w), 1309(w), 1285(w), 1261(w), 1159(m), 1060(s), 1034(s), 948(m), 902(w), 879(w), 840(w), 822(w), 773(s), 726(w), 649(w), 556(w), 533(w), 521(w), 491(w), 437(w), 412(w) 
MS (FAB, nibeol): $m / z$ (\%): $262\left(100,\left[\mathrm{~L}^{8} \mathrm{Cu}-\mathrm{N}\left(\mathrm{CH}_{2} \mathrm{Py}\right)_{2}-\mathrm{CH}_{2} \mathrm{Py}\right]^{+}\right), 354$ (18, $\left.\left[\mathrm{L}^{8} \mathrm{Cu}-\mathrm{N}\left(\mathrm{CH}_{2} \mathrm{Py}\right)_{2}\right]^{+}\right), 615\left(35,\left[\mathrm{~L}^{8} \mathrm{Cu}_{2}\right]^{+}\right), 634\left(24,\left[\mathrm{~L}^{8} \mathrm{Cu}_{2} \mathrm{~F}\right]^{+}\right)$, $721\left(7,\left[\mathrm{~L}^{8} \mathrm{Cu}_{2} \mathrm{~F}\left(\mathrm{BF}_{4}\right)\right]^{+}\right)$

EA: ber. C 42.83; H 3.95; N 13.32; B 2.57; O 1.90; F 20.32;

Cu 15.11

exp. C 42.02; H 4.18; N 12.53

UV/Vis (MeOH) $\lambda(\varepsilon) 212$ (19540), 221 (19590), 254 (17550), 297 (5290), 691 (sh, 190), 900 (280)

Ausbeute: $67 \mathrm{mg}(0.08 \mathrm{mmol}, 21 \%)$ 


\subsubsection{1 $\left[\mathrm{L}^{10} \mathrm{Cu}_{2}(\mathrm{OH})\right]\left(\mathrm{ClO}_{4}\right)_{2}(15)$}

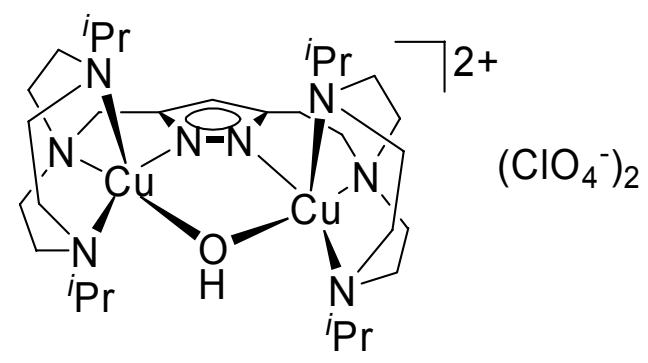

Ansatz:

$205 \mathrm{mg}(0.38 \mathrm{mmol}) \mathrm{HL}^{10}$

$85 \mathrm{mg}(0.76 \mathrm{mmol})$ Kalium-tert-butylat

$282 \mathrm{mg}(0.76 \mathrm{mmol}) \mathrm{Cu}\left(\mathrm{ClO}_{4}\right)_{2} \cdot 6 \mathrm{H}_{2} \mathrm{O}$

$150 \mathrm{ml}$ Methanol

Durchführung:

In einem $250 \mathrm{ml}$ Kolben wird der Ligand $\mathrm{HL}^{10}$ zunächst in $150 \mathrm{ml}$ Methanol mit zwei Äquivalenten Kalium-tert-butylat umgesetzt. Anschließend werden zwei Äquivalente Kupfer(II)perchlorat zugegeben. Die grüne Lösung wird über Nacht gerührt. Nach Entfernen des Lösungsmittels im Vakuum wird der Rückstand wieder in wenig Aceton aufgenommen, filtriert und in einem Schlenckkolben mit Pentan überschichtet. Es fällt ein grüner Feststoff aus.

Summenformel: $\mathrm{C}_{30} \mathrm{H}_{60} \mathrm{Cl}_{2} \mathrm{Cu}_{2} \mathrm{~N}_{8} \mathrm{O}_{9}(847,83 \mathrm{~g} / \mathrm{mol})^{*}$

IR (KBr): $\tilde{v}=3600$ (s), 3451 (m), 2970 (vs), 2942 (s), 2862 (s), 2017 (w), $1710(\mathrm{~m}), 1636(\mathrm{w}), 1516(\mathrm{~m}), 1495(\mathrm{~s}), 1464(\mathrm{~s}), 1440(\mathrm{~m})$, 1389 (s), 1372 (s), $1355(\mathrm{~m}), 1330$ (s), $1293(\mathrm{~m}), 1268(\mathrm{~m})$, $1226(\mathrm{~m}), 1174$ (s), 1093 (vs), 1024 (s), 990 (s), 967 (s), 958 (s), $909(w), 874(w), 844(w), 806(m), 786(m), 769(m), 734$ $(\mathrm{m}), 715(\mathrm{~m}), 623(\mathrm{~s}), 593(\mathrm{w}), 458(\mathrm{~m})$ 
MS (FAB, nibeol): $m / z(\%): 657\left(20,\left[\mathrm{~L}^{10} \mathrm{Cu}_{2}\right]^{+}\right), 674\left(44,\left[\mathrm{~L}^{10} \mathrm{Cu}_{2} \mathrm{OH}\right]^{+}\right), 773(100$, $\left.\left[\mathrm{L}^{10} \mathrm{Cu}_{2} \mathrm{OHClO}_{4}\right]^{+}\right)$

EA: ber. C 41.19; H 6.91; N 12.81; Cl 8.10; O 16.46; Cu 14.53 exp. C 41.26; H 6.97; N 12.62

UV/Vis (MeOH) $\lambda(\varepsilon) 210$ (7920), 267 (6900), 325 (sh, 3180), 375 (3150), 626 (230) Ausbeute: $110 \mathrm{mg}(0.13 \mathrm{mmol}, 34 \%)^{*}$ 


\subsubsection{2 $\left[\mathrm{L}^{3} \mathrm{Cu}_{2}\left(\mathrm{C}_{6} \mathrm{Cl}_{4} \mathrm{O}_{2}\right)\left(\mathrm{H}_{2} \mathrm{O}\right)(\mathrm{MeCN})\right] P F_{6}$ (18)}

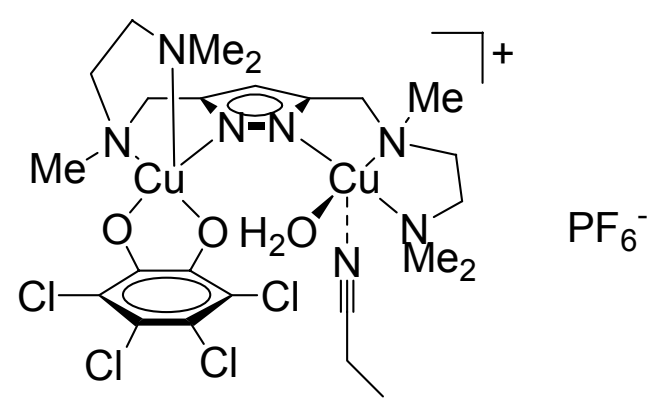

Ansatz:

$115 \mathrm{mg}(0.39 \mathrm{mmol}) \mathrm{HL}^{3}$

$44 \mathrm{mg}(0.39 \mathrm{mmol})$ Kalium-tert-butylat

$291 \mathrm{mg}(0.78 \mathrm{mmol})\left[\mathrm{Cu}(\mathrm{MeCN})_{4}\right] \mathrm{PF}_{6}$

$96 \mathrm{mg}(0.39 \mathrm{mmol})$ o-Chloranil

$50 \mathrm{ml}$ Acetonitril

Durchführung:

In einem $100 \mathrm{ml}$ Schlenckkolben wird der Ligand $\mathrm{HL}^{3}$ unter Stickstoffatmosphäre bei $-70^{\circ} \mathrm{C}$ zunächst in $50 \mathrm{ml}$ Propionitril mit einem Äquivalent Kalium-tert-butylat umgesetzt. Anschließend werden zwei Äquivalente Kupfer(I)hexafluorophosphat zugegeben. $\mathrm{Zu}$ der gelben Lösung wird 1 Äquivalent o-Chloranil gegeben, anschließend lässt man die grüne Lösung unter Rühren auftauen. Nach dem Auftauen wird filtriert und mit Petrolether überschichtet. Die erhaltenen grünen Kristalle sind für die Röntgenstrukturanalyse geeignet.

Summenformel: $\mathrm{C}_{21} \mathrm{H}_{33} \mathrm{Cl}_{4} \mathrm{Cu}_{2} \mathrm{~F}_{6} \mathrm{~N}_{6} \mathrm{O}_{3} \mathrm{P}(831.39 \mathrm{~g} / \mathrm{mol})$

$\operatorname{IR~(KBr):~} \tilde{v}=3636(\mathrm{~m}), 3570(\mathrm{~m}), 3417(\mathrm{~m}), 2985(\mathrm{~s}), 2926(\mathrm{~s}), 1631(\mathrm{w})$, $1587(w), 1529(m), 1510(w), 1438(s), 1371(s), 1327(w), 1312$ $(\mathrm{m}), 1291(\mathrm{w}), 1248(\mathrm{~s}), 1175(\mathrm{w}), 1160(\mathrm{w}), 1091(\mathrm{w}), 1068(\mathrm{w})$, $1036(\mathrm{~m}), 1024(\mathrm{~m}), 997(\mathrm{~s}), 981(\mathrm{~s}), 958(\mathrm{~m}), 944(\mathrm{~m}), 840(\mathrm{~s})$, $802(\mathrm{~s}), 785(\mathrm{~s}), 737(\mathrm{w}), 681(\mathrm{w}), 591(\mathrm{w}), 555(\mathrm{~s}) \mathrm{cm}^{-1}$ 
MS (ESI, Aceton): $m / z(\%): 667\left(9,\left[\mathrm{~L}^{3} \mathrm{Cu}_{2} \mathrm{C}_{6} \mathrm{Cl}_{4} \mathrm{O}_{2}\right]^{+}\right), 421\left(100,\left[\mathrm{~L}^{3} \mathrm{Cu}_{2}\right]^{+}\right)$

EA: ber. C 30.34; H 4.00; N 10.11; O 5.77; F 13.71; P 3.73; Cl 17.06;

Cu 15.29

exp. C 28.86; H 4.21; N 9.84

UV/Vis (MeOH) $\lambda(\varepsilon) 200$ (14370, sh), 223 (37870), 257 (14600), 299 (sh, 7600), 476 (170), $618(200)$

Ausbeute: $13 \mathrm{mg}(0.02 \mathrm{mmol}, 4 \%)$ 


\subsubsection{3 $\left[\mathrm{L}^{3} \mathrm{Cu}_{2}\left(\mathrm{C}_{6} \mathrm{Br}_{4} \mathrm{O}_{2}\right)\left(\mathrm{C}_{2} \mathrm{H}_{5} \mathrm{OH}\right) \mathrm{NO}_{3}\right]$ (19)}

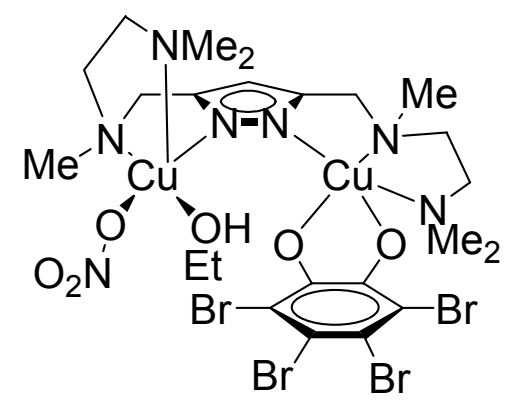

Ansatz:

$94 \mathrm{mg}(0.32 \mathrm{mmol}) \mathrm{HL}^{3}$

$107 \mathrm{mg}(0.96 \mathrm{mmol})$ Kalium-tert-butylat

$153 \mathrm{mg}(0.64 \mathrm{mmol}) \mathrm{Cu}\left(\mathrm{NO}_{3}\right)_{2} \cdot 3 \mathrm{H}_{2} \mathrm{O}$

$135 \mathrm{mg}(0.32 \mathrm{mmol})$ Tetrabromcatechol

$100 \mathrm{ml}$ Methanol

Durchführung:

In einem $250 \mathrm{ml}$ Kolben wird der Ligand $\mathrm{HL}^{3}$ zunächst in $150 \mathrm{ml}$ Methanol mit drei Äquivalenten Kalium-tert-butylat umgesetzt. Anschließend werden zwei Äquivalente Kupfer(II)-nitrat zugegeben. Zu der dunkelgrünen Lösung wird ein Äquivalent Tetrabromcatechol zugegeben und zwei Stunden gerührt. Nach Entfernen des Lösungsmittels im Vakuum wird der Rückstand wieder in wenig Ethanol aufgenommen, filtriert und in einem Schlenckkolben mit Diethylether überschichtet. Die nach Kristallisation erhaltenen dunkelgrünen Kristalle sind analysenrein und für die Röntgenstrukturanalyse geeignet.

Summenformel: $\quad \mathrm{C}_{23} \mathrm{H}_{37} \mathrm{Br}_{4} \mathrm{Cu}_{2} \mathrm{~N}_{7} \mathrm{O}_{6}(954.29 \mathrm{~g} / \mathrm{mol})$

IR (KBr): $\tilde{v}=3416(w), 3134(w), 3016(w), 2978(w), 2888(m), 2829(m)$, $2799(\mathrm{~m}), 2720(\mathrm{w}), 2563(\mathrm{w}), 1490(\mathrm{~s}), 1451$ (vs), $1383(\mathrm{~m}), 1344$ $(\mathrm{m}), 1321(\mathrm{~m}), 1276(\mathrm{~s}), 1259(\mathrm{~s}), 1238(\mathrm{~s}), 1211(\mathrm{~m}), 1177(\mathrm{w})$, $1143(w), 1096(w), 1062(w), 1036(s), 1003(m), 985(w), 962$ $(m), 928(w), 875(w), 833(w), 806(m), 771(w), 737(m), 619(w)$, $598(w), 570(w), 550(w), 483(w), 438(w), 417(w) \mathrm{cm}^{-1}$ 
MS (FAB, Nibeol): $m / z(\%): 421\left(100,\left[\mathrm{~L}^{3} \mathrm{Cu}_{2}\right]^{+}\right), 483\left(24,\left[\mathrm{~L}^{3} \mathrm{Cu}_{2} \mathrm{NO}_{3}\right]^{+}\right), 762(12$, $\left.\left[\mathrm{L}^{3} \mathrm{Cu}_{2}\left(\mathrm{C}_{6} \mathrm{Br}_{3} \mathrm{O}_{2}\right)\right]^{+}\right), 841\left(12,\left[\mathrm{~L}^{3} \mathrm{Cu}_{2}\left(\mathrm{C}_{6} \mathrm{Br}_{4} \mathrm{O}_{2}\right)\right]^{+}\right)$

EA: ber. C 28.95; H 3.91; N 10.27; O 10.06; Cu 13.32; Br 33.49 exp. C 29.02; H 3.98; N 10.33

UV/Vis (MeOH) $\lambda(\varepsilon) 233$ (18490), 262 (14490), 292 (10170), 493 (230), 617 (240) Ausbeute: $68 \mathrm{mg}(0.07 \mathrm{mmol}, 22 \%)$ 


\subsubsection{4 $\left[\mathrm{L}^{4} \mathrm{HCu}_{2}\left(\mathrm{C}_{6} \mathrm{Cl}_{4} \mathrm{O}_{2}\right)\left(\mathrm{H}_{2} \mathrm{O}\right)\right]\left(\mathrm{ClO}_{4}\right)_{2}(20)$}

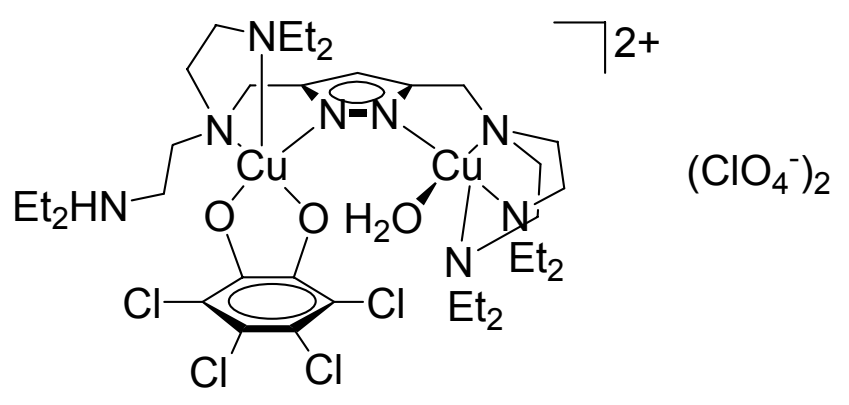

Ansatz:

$262 \mathrm{mg}(0.50 \mathrm{mmol}) \mathrm{HL}^{4}$

$150 \mathrm{mg}(1.34 \mathrm{mmol})$ Kalium-tert-butylat

$371 \mathrm{mg}(1.00 \mathrm{mmol}) \mathrm{Cu}\left(\mathrm{ClO}_{4}\right)_{2} \cdot 6 \mathrm{H}_{2} \mathrm{O}$

$124 \mathrm{mg}(0.50 \mathrm{mmol})$ Tetrachlorcatechol

$150 \mathrm{ml}$ Methanol

Durchführung:

In einem $250 \mathrm{ml}$ Kolben wird der Ligand $\mathrm{HL}^{4}$ zunächst in $150 \mathrm{ml}$ Methanol mit 1.34 mmol Kalium-tert-butylat umgesetzt. Anschließend werden zwei Äquivalente Kupfer(II)-perchlorat zugegeben. Zu der grünen Lösung wird ein Äquivalent Tetrachlorcatechol zugegeben und eine Stunde gerührt. Nach Entfernen des Lösungsmittels im Vakuum wird der Rückstand wieder in wenig Aceton aufgenommen, filtriert und in einem Schlenckkolben mit Petrolether überschichtet. Die nach Kristallisation erhaltenen grünen Kristalle sind analysenrein und für die Röntgenstrukturanalyse geeignet.

Summenformel: $\mathrm{C}_{35} \mathrm{H}_{64} \mathrm{Cl}_{6} \mathrm{Cu}_{2} \mathrm{~N}_{8} \mathrm{O}_{11}(1112.74 \mathrm{~g} / \mathrm{mol})$

IR (KBr): $\tilde{v}=3441$ (s), 2970 (s), 2929 (s), $2874(\mathrm{~s}), 1701(\mathrm{w}), 1635(\mathrm{w})$, $1571(w), 1521(w), 1444(s), 1371(s), 1328(m), 1255(m)$, $1218(w), 1182(m), 1088(s), 968(s), 927(w), 826(w), 806$ (s), $787(\mathrm{~s}), 733(\mathrm{~m}), 682(\mathrm{w}), 622(\mathrm{~s}) \mathrm{cm}^{-1}$ 
MS (ESI, Aceton): $m / z(\%): 893\left(1,\left[{ }^{4} \mathrm{Cu}_{2} \mathrm{C}_{6} \mathrm{Cl}_{4} \mathrm{O}_{2}\right]^{+}\right), 648\left(100,\left[\mathrm{~L}^{4} \mathrm{Cu}_{2} \mathrm{H}\right]^{+}\right)$

EA: ber. C 37.78; H 5.80; N 10.07; O 15.82; Cl 19.12; Cu 11.42

exp. C 36.95; H 5.63; N 9.66

UV/Vis (MeOH) $\lambda(\varepsilon) 227$ (13960), 247 (13790), 303 (sh, 7670), 629 (240)

Ausbeute: $43 \mathrm{mg}(0.04 \mathrm{mmol} ; 8 \%)$ 


\subsubsection{5 $\left[{ }^{5} \mathrm{Cu}_{2}\left(\mathrm{C}_{6} \mathrm{Br}_{4} \mathrm{O}_{2}\right)\left(\mathrm{H}_{2} \mathrm{O}\right) \mathrm{ClO}_{4}\right](21)$}

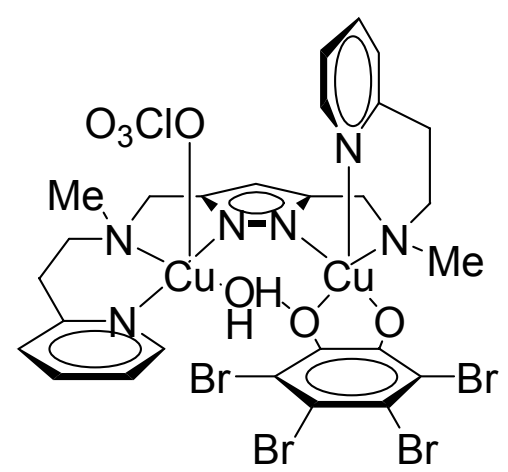

Ansatz:

$86 \mathrm{mg}(0.24 \mathrm{mmol}) \mathrm{HL}^{5}$

$81 \mathrm{mg}$ (0.72 mmol) Kalium-tert-butylat

$178 \mathrm{mg}(0.48 \mathrm{mmol}) \mathrm{Cu}\left(\mathrm{ClO}_{4}\right)_{2} \cdot 6 \mathrm{H}_{2} \mathrm{O}$

$102 \mathrm{mg}$ (0.24 mmol) Tetrabromcatechol

$150 \mathrm{ml}$ Methanol

Durchführung:

In einem $250 \mathrm{ml}$ Kolben wird der Ligand $\mathrm{HL}^{5}$ zunächst in $150 \mathrm{ml}$ Methanol mit drei Äquivalenten Kalium-tert-butylat umgesetzt. Anschließend werden zwei Äquivalente Kupfer(II)-perchlorat zugegeben. Zu der braunen Lösung wird ein Äquivalent Tetrabromcatechol zugegeben und zwei Stunden gerührt. Nach Entfernen des Lösungsmittels im Vakuum wird der Rückstand wieder in wenig Aceton aufgenommen, filtriert und in einem Schlenckkolben mit Pentan überschichtet. Die nach Kristallisation erhaltenen grünbraunen Kristalle sind analysenrein und für die Röntgenstrukturanalyse geeignet. 
Summenformel: $\mathrm{C}_{27} \mathrm{H}_{29} \mathrm{Br}_{4} \mathrm{Cl}_{1} \mathrm{Cu}_{2} \mathrm{~N}_{6} \mathrm{O}_{7}(1031.72 \mathrm{~g} / \mathrm{mol})$

IR (KBr): $\tilde{v}=3449(\mathrm{~m}), 2962(\mathrm{w}), 2923(\mathrm{w}), 2854(\mathrm{w}), 1611(\mathrm{~m}), 1440(\mathrm{~s})$, $1350(\mathrm{~m}), 1312(\mathrm{~m}), 1238(\mathrm{~s}), 983(\mathrm{~m}), 1103(\mathrm{vs}), 924(\mathrm{w}), 837$ $(w), 804(w), 766(w), 739(w), 625(s)$

MS (FAB, Nibeol): $m / z \quad(\%): 489\left(82, \quad\left[L^{5} \mathrm{Cu}_{2}\right]^{+}\right), 568 \quad\left(14, \quad\left[\mathrm{~L}^{5} \mathrm{Cu}_{2} \mathrm{Br}\right]^{+}\right), 588 \quad(4$, $\left.\left[\mathrm{L}^{5} \mathrm{Cu}_{2}\left(\mathrm{ClO}_{4}\right)\right]^{+}\right)$

EA: ber. C $31.43 \mathrm{H} 2.83 \mathrm{~N} 8.15 \mathrm{O} 10.86 \mathrm{Cl} 3.44 \mathrm{Cu} 12.32 \mathrm{Br} 30.98$ exp. C $27.25 \mathrm{H} 2.36 \mathrm{~N} 6.35$

UV/Vis (Aceton) $\lambda(\varepsilon) 329$ (4910), 485 (1000), 671 (sh, 580)

Ausbeute: $79 \mathrm{mg}(0.07 \mathrm{mmol}, 29 \%)$ 
11.3.16 $\left[\mathrm{L}^{8} \mathrm{Cu}_{2} \mathrm{TCC}_{2}\left(\mathrm{PF}_{6}\right)_{2}(22)\right.$

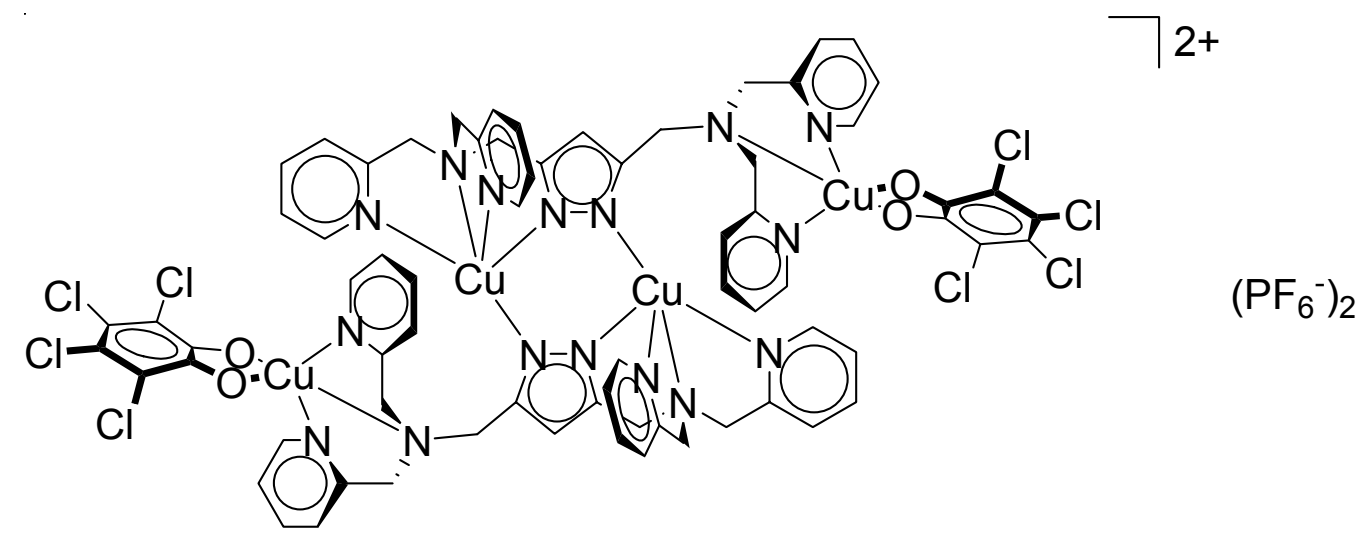

Ansatz:

$160 \mathrm{mg}(0.33 \mathrm{mmol}) \mathrm{HL}^{8}$

$37 \mathrm{mg}(0.33 \mathrm{mmol})$ Kalium-tert-butylat

$246 \mathrm{mg}(0.66 \mathrm{mmol})\left[\mathrm{Cu}(\mathrm{MeCN})_{4}\right] \mathrm{PF}_{6}$

$81 \mathrm{mg}(0.33 \mathrm{mmol})$ o-Chloranil

$50 \mathrm{ml}$ Acetonitril

Durchführung:

In einem $100 \mathrm{ml}$ Schlenckkolben wird der Ligand $\mathrm{HL}^{8}$ bei $-40^{\circ} \mathrm{C}$ unter Stickstoffatmosphäre zunächst in $50 \mathrm{ml}$ Acetonitril mit einem Äquivalent Kalium-tertbutylat umgesetzt. Anschließend werden zwei Äquivalente Kupfer(I)hexafluorophosphat zugegeben. Zu der gelben Lösung wird 1 Äquivalent oChloranil gegeben, anschließend lässt man die braune Lösung unter Rühren auftauen. Nach dem Auftauen wird filtriert und mit Diethylether überschichtet. Die erhaltenen grünen Kristalle sind für die Röntgenstrukturanalyse geeignet.

Summenformel: $\mathrm{C}_{70} \mathrm{H}_{58} \mathrm{Cl}_{8} \mathrm{Cu}_{4} \mathrm{~F}_{12} \mathrm{~N}_{16} \mathrm{O}_{4} \mathrm{P}_{2}(2015.05 \mathrm{~g} / \mathrm{mol})$

$\mathrm{IR}(\mathrm{KBr}): \tilde{v}=3643(\mathrm{~m}), 3410(\mathrm{~m}), 3110(\mathrm{w}), 3071(\mathrm{w}), 3037(\mathrm{w}), 2965(\mathrm{w})$, 2924(w), 2858(w),1608(s), 1574(m), 1527(w), 1483(m), 1453(s), 1372(m), 1347(w), 1308(w), 1291(m), 1256(s), 1159(m), $1100(\mathrm{~m}), 1055(\mathrm{~m}), 1025(\mathrm{~m}), 971(\mathrm{~m}), 840(\mathrm{~s}), 803(\mathrm{~s})$, 786(s), 763(s), 682(w), 650(w), 558(s) 
MS (ESI, MeOH): m/z (\%): $615\left(100,\left[\mathrm{~L}^{8} \mathrm{Cu}_{2}\right]^{+}\right), 650\left(28,\left[\mathrm{~L}^{8} \mathrm{Cu}_{2} \mathrm{Cl}\right]^{+}\right), 859(20$, $\left.\left[\mathrm{L}^{8} \mathrm{Cu}_{2}\left(\mathrm{C}_{6} \mathrm{Cl}_{4} \mathrm{O}_{2}\right)\right]^{+}\right)$

EA: ber. $\mathrm{C}$ 41.72; H 2.90; N 11.12; O 3.18; F 11.31; P 3.07; Cl 14.08; Cu 12.61 exp. C 40.89; H 3.32; N 10.88

UV/Nis (MeCN) $\lambda(\varepsilon) 246$ (25810), 264 (23120), 299 (14620), 732 (sh, 650) Ausbeute: $88 \mathrm{mg}(0.04 \mathrm{mmol}, 24 \%)$ 


\subsection{Kinetische Messungen}

Messung der pH-Abhängigkeit:

Eine Messung der pH-Abhängigkeit der Catecholase-Aktivität wurde für die Katalysatoren 2, 7a, 8, 13 und 15 durchgeführt. Hierzu wurden bei $20^{\circ} \mathrm{C}$ in einer offenen Quarzglasküvette mit Rührer $(\mathrm{d}=1 \mathrm{~cm})$ jeweils $1.45 \mathrm{ml}$ Katalysatorlösung mit $1.45 \mathrm{ml}$ Substratlösung und $0.1 \mathrm{ml}$ Pufferlösung gemischt und die Entwicklung der Bande des entstehenden $o$-Chinons bei $400 \mathrm{~nm}\left(\varepsilon=1810 \mathrm{~mol} \mathrm{I}^{-1} \mathrm{~cm}^{-1}\right)$ UV/Visspektroskopisch gemessen. Die katalytische Aktivität wurde mit Hilfe der Methode der Anfangsgeschwindigkeiten bestimmt. Die Konzentration des Katalysators in der Küvette betrug bei $210^{-5} \mathrm{M}$, bei $7 \mathrm{a}$ und $82 \cdot 10^{-5} \mathrm{M}$, bei $1310^{-5} \mathrm{M}$ und bei $1510^{-4} \mathrm{M}$. Die Anfangskonzentration an $\mathrm{H}_{2} \mathrm{dtbc}$ betrug konstant $2 \cdot 10^{-3} \mathrm{M}$. Als Puffer wurden wäßrige Lösungen von $0.02 \mathrm{M}$ Essigsäure/Natriumacetat ( $\mathrm{pH} 4.05$ - 5.6), MES (5.6 6.6), MOPS (6.6 - 7.6), TRIS mit $\mathrm{Cl}^{-}-$Anion und TRIS mit $\mathrm{NO}_{3}{ }^{-}$-Anion ( $\mathrm{pH} 7.3$ - 9.0) verwendet. Die Meßdauer betrug jeweils $15 \mathrm{~min}$, bei 15 eine Stunde. Die jeweiligen Aktivitätsmaxima lagen bei pH 5.6 (2) 7.3 (7a), 7.6 (8), 8.0 (13) und 5.6 (15). Es wurde eine starke Abhängigkeit der Aktivität von der Art des verwendeten Puffers beobachtet.

\section{Bestimmung der Catecholase-Aktivität:}

Die Catecholase-Aktivität der Komplexe 1 - 4, 7a, 8, 13, 15 und 16 bei $20^{\circ} \mathrm{C}$ wurde UV/Vis-spektroskopisch anhand der Entwicklung der Bande des Produktes $\mathrm{H}_{2} \mathrm{dtboc}$ bei $20^{\circ} \mathrm{C}$ in einer offenen Quarzglasküvette mit Rüher $(\mathrm{d}=1 \mathrm{~cm})$ bei $400 \mathrm{~nm}$ verfolgt. Die Bestimmung der katalytischen Aktivität ergab sich aus der Methode der Anfangsgeschwindigkeiten. Jede Messung wurde mindestens dreimal durchgeführt. Die Meßfehler ergeben sich aus der resultierenden Standardabweichung des Mittelwertes der Messungen, sowie aus den Volumen- und Massefehlern der verwendeten Gefäße und der Feinwaage. Im Falle der Komplexe 1 - 4 und 16 wurden $1.5 \mathrm{ml}$ der Katalysatorlösung mit $1.5 \mathrm{ml}$ Substratlösung umgesetzt. Die Konzentration von 1 - 3 wurde zwischen $2 \cdot 10^{-6} \mathrm{M}$ und $10^{-5} \mathrm{M}$ variiert, diejenige von 4 zwischen $5 \cdot 10^{-6} \mathrm{M}$ und $2 \cdot 10^{-5} \mathrm{M}$. Die Konzentration von 16 betrug zwischen $10^{-5}$ und $10^{-4} \mathrm{M}$. Die Anfangskonzentration von $\mathrm{H}_{2} \mathrm{dtbc}$ betrug dabei $10^{-3} \mathrm{M}$. In einer zweiten

Meßreihe wurde bei konstanter Katalysatorkonzentration von $10^{-5} \mathrm{M}$ die Substratkonzentration zwischen $10^{-4} \mathrm{M}$ und $10^{-3} \mathrm{M}$ im Falle von 1 - 3 bzw. zwischen 
$2 \cdot 10^{-4} \mathrm{M}$ und $2 \cdot 10^{-3} \mathrm{M}$ im Falle von 4 variiert.

Die Aktivität der Katalysatoren 7a, 8, 13 und 15 wurde im gepufferten Milieu gemessen. Der Ablauf der Messung erfolgt wie bereits beschrieben. Die Messungen für 15 erfolgten bei $40^{\circ} \mathrm{C}$ in einer geschlossenen Küvette. Die Pufferkonzentration betrug immer $0.047 \mathrm{M}$, in der ersten Meßreihe betrug die Katalysatorkonzentration

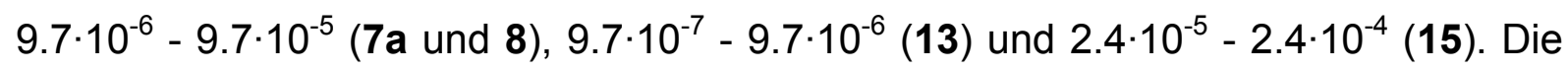
Substratkonzentration betrug dabei konstant $1.9 \cdot 10^{-3} \mathrm{M}$. In der zweiten Meßreihe wurde die Substratkonzentration zwischen $3.9 \cdot 10^{-4}$ und $2.7 \cdot 10^{-3} \mathrm{M}$ (für $7 \mathrm{a}, 8$ und 13) und $4.7 \cdot 10^{-4}$ und $2.8 \cdot 10^{-3} \mathrm{M}(15)$ variiert. Die Katalysatorkonzentration betrug dabei $1.9 \cdot 10^{-5} \mathrm{M}(7 \mathrm{a}$ und 8$), 10^{-5} \mathrm{M}(\mathbf{1 3})$ und $1.2 \cdot 10^{-4} \mathrm{M}$.

Bei allen Katalysatoren außer dem einkernigen 16 wurde bei diesen Messungen eine Abhängigkeit 1. Ordnung vom Katalysator beobachtet, bei 16 ergab sich eine Abhängigkeit 2. Ordnung. Für die Substratabhängigkeit wurde bei 2, 4, 7a, 8, 13 und 15 eine Sättigungskinetik beobachtet, die mit einem Michaelis-Menten-Ansatz ausgewertet wurde. Für $\mathbf{1}$ und $\mathbf{3}$ wurde keine Abhängigkeit von der Substratkonzentration beobachtet.

Als Blindproben wurden Messungen mit einfachen Kupfer(II)salzen wie Kupfer(II)perchlorat-Hexahydrat, Kupfer(II)tetrafluorborat-Hexahydrat oder Kupfer(II)nitrat-Trihydrat durchgeführt, die alle eine deutlich niedrigere Aktivität als die Komplexe mit dem entsprechenden Anion aufwiesen.

Test auf $\mathrm{H}_{2} \mathrm{O}_{2}$ :

Für die Komplexe 1 - 4, 7a, 8 und 13 wurde geprüft, ob bei der Reaktion als Nebenprodukt $\mathrm{H}_{2} \mathrm{O}_{2}$ entsteht. Zunächst wurde dazu das $\mathrm{H}_{2}$ dtbc mit dem Katalysator in Gegenwart von Luftsauerstoff soweit oxidiert, bis eine Absorption $A_{400}$ von 0.4 erreicht war. Anschließend wurde die Reaktion mit dem gleichen Volumen $0.005 \mathrm{M}$ $\mathrm{H}_{2} \mathrm{SO}_{4}$ gequencht und das störende $\mathrm{H}_{2} \mathrm{dtboc}$ sowie nicht umgesetztes $\mathrm{H}_{2} \mathrm{dtbc}$ mit Dichlormethan extrahiert. Zu $2 \mathrm{ml}$ der Reaktionslösung wurde anschließend in die Referenzküvette $1 \mathrm{ml} \mathrm{H} \mathrm{H}_{2} \mathrm{O}$ und in die Meßküvette $1 \mathrm{ml} 0.30 \mathrm{M} \mathrm{KI}$-Lösung zugegeben. In die Meßküvette wurden weiterhin katalytische Mengen Lactoperoxidase zur spezifischen Beschleunigung der Oxidation von $\mathrm{I}^{-} \mathrm{zu} \mathrm{I}_{3}^{-}$durch $\mathrm{H}_{2} \mathrm{O}_{2}$ gegeben. Die 
Bande $A_{353}\left(\varepsilon=26000 \mathrm{~mol} \mathrm{I}^{-1} \mathrm{~cm}^{-1}\right)$ des entstehenden $\mathrm{I}_{3}^{-}$wurde UV/Visspektroskopisch verfolgt. Als Blindprobe wurde dieselbe Messung mit Katalysator, aber ohne Substrat durchgeführt. Weiterhin wurde jede Messung zusätzlich durch $\mathrm{H}_{2} \mathrm{O}_{2}$-Teststäbchen verifiziert. Die Ergebnisse der $\mathrm{I}^{-}$-Oxidation und der $\mathrm{H}_{2} \mathrm{O}_{2}-$ Teststäbchen stimmten in allen Fällen überein. Eine eindeutige $\mathrm{H}_{2} \mathrm{O}_{2}$-Entwicklung konnte für die Katalysatoren 1, 2 und 3 nachgewiesen werden, die Entwicklung der $\mathrm{I}_{3}{ }^{-}$-Bande bei 4 war schwach, eine klare Aussage läßt sich hier nicht treffen. In allen anderen Fällen verlief der Test auf $\mathrm{H}_{2} \mathrm{O}_{2}$ negativ.

\subsection{Synthese und Charakterisierung von Dikupferperoxoverbindungen}

\section{Versuche zur Kristallisierung von Dikupferperoxoverbindungen}

In einem $100 \mathrm{ml}$ Schlenckkolben wurden ca. $0.3 \mathrm{mmol}$ des Liganden $\mathrm{HL}^{1}-\mathrm{HL}^{8}$ unter Stickstoffatmosphäre bei $-78^{\circ} \mathrm{C}$ zunächst in $50 \mathrm{ml}$ des Lösungsmittels mit einem Äquivalent Kalium-tert-butylat umgesetzt. Als Lösungsmittel dienten Propionitril, Dichlormethan oder Aceton. Anschließend wurden zwei Äquivalente $\left[\mathrm{Cu}(\mathrm{MeCN})_{4}\right]^{+} \mathrm{X}^{-}$ $\left(\mathrm{X}^{-}=\mathrm{PF}_{6}^{-}, \mathrm{ClO}_{4}^{-}, \mathrm{CF}_{3} \mathrm{SO}_{3}^{-}, \mathrm{BF}_{4}^{-}\right)$zugegeben. Die gelbe Lösung wurde 10 Minuten gerührt und anschließend über eine Spritze Luftsauerstoff zugegeben, worauf sich die Lösung braun färbte. Über eine Kanüle wurde die braune Lösung langsam mit Diethylether überschichtet. Anschließend wurde der Kolben bis zur Kristallisation des Produktes bei $-80^{\circ} \mathrm{C}$ aufbewahrt. Bei allen Kristallisationsversuchen konnten nur grüne Zersetzungsprodukte erhalten werden. Ein solches Zersetzungsprodukt mit dem Triflat-Anion wurde kristallographisch charakterisiert und auf diese Weise als OH-verbrückter Dikupfer(II)komplex 13 identifiziert.

\section{Stopped Flow Spektroskopie:}

Zunächst wurden in einem Kolben 0.17 mmol des Liganden $\mathrm{HL}^{9}$ in $10 \mathrm{ml}$ entgastem Dichlormethan gelöst und bei $-70^{\circ} \mathrm{C}$ unter Inertgasatmosphäre mit einem Äquivalent Kalium-tert-butylat deprotoniert und dann zwei Äquivalente $\left[\mathrm{Cu}(\mathrm{MeCN})_{4}\right] \mathrm{PF}_{6}$ zugegeben, worauf eine gelbe Lösung entstand. In eine vorgekühlte Spritze wurden

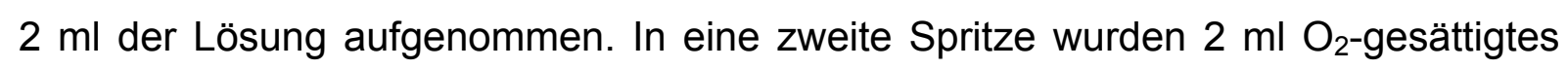
Dichlormethan aufgenommen (die Sättigungskonzentration von $\mathrm{O}_{2}$ in Dichlormethan bei $25^{\circ} \mathrm{C}$ beträgt $\left.0.38 \mathrm{mmol}^{-1}\right){ }^{[163,205]}$ Beide Lösungen wurden unter Inertgasatmosphäre in die Stopped Flow Anlage injiziert und im Meßraum bei $-10^{\circ} \mathrm{C}$ 
zur Reaktion gebracht. Die Entstehung des Peroxoadduktes erfolgte zu schnell, so daß seine Entwicklung nicht verfolgt werden konnte. Jedoch konnte der Zerfall von "L $\mathrm{L}^{9} \mathrm{Cu}_{2} \mathrm{O}_{2}$ " UV/Vis-spektroskopisch beobachtet werden.

Ramanspektroskopie:

Zur Messung der Raman-Spektren von " $\mathrm{L}^{4} \mathrm{Cu}_{2}\left(\mathrm{O}_{2}\right)$ " und " $\mathrm{L}^{9} \mathrm{Cu}_{2}\left(\mathrm{O}_{2}\right)$ " wurde zunächst in einem Kolben mit Hahn unter Schutzgas bei $-70^{\circ} \mathrm{C} 0.2 \mathrm{mmol}$ des Liganden in 10 $\mathrm{ml}$ entgastem Dichlormethan unter Inertgasatmosphäre gelöst, mit einem Äquivalent Kalium-tert-butylat deprotoniert und dann zwei Äquivalente $\left[\mathrm{Cu}(\mathrm{MeCN})_{4}\right] \mathrm{PF}_{6}$ zugegeben. Anschließend wurde ein leichtes Vakuum angelegt und durch Öffnen des Hahns Luft in den Kolben gezogen, woraufhin die Lösung violett bzw. braunviolett wurde. Bei Messungen mit ${ }^{18} \mathrm{O}_{2}$ wurde nach Anlegen des Vakuums eine kleine Menge ${ }^{18} \mathrm{O}_{2}$ in einen evakuierten Glaskolben mit drei Hähnen gezogen, aus welchem wiederum das Gas in den Reaktionskolben gezogen wurde. Mit einer vorgekühlten Spritze wurde die Lösung auf den gekühlten Objektträger des Raman-Spektrometers gebracht. Die Messungen erfolgten bei $-170^{\circ}$ bzw. $-140^{\circ} \mathrm{C}$.

\subsection{Reaktivität der Dikupfersauerstoffverbindungen}

$0.5 \mathrm{mmol}$ des Liganden wurden bei $-70^{\circ} \mathrm{C}$ unter Inertgasatmosphäre in $50 \mathrm{ml}$ Propionitril gelöst und mit einem Äquivalent Kalium-tert-butylat, sowie zwei Äquivalenten $\left[\mathrm{Cu}(\mathrm{MeCN})_{4}\right] \mathrm{PF}_{6}$ umgesetzt, worauf sich eine gelbe bis gelb-braune Lösung bildete. Anschließend wurde ein Äquivalent des Substrats 2,4-Di-tertbutylphenol oder 2,3,5-Trimethylphenol zugegeben und Luft in die Lösung eingeleitet. Die Lösung wurde langsam unter Rühren auf Raumtemperatur erwärmt, wobei sie sich grün färbte. Nach Entfernen des Lösungsmittels im Vakuum wurde der Katalysator säulenchromatographisch weitestgehend abgetrennt (statonäre Phase: $\mathrm{SiO}_{2}$, mobile Phase: zunächst Methanol/Essigester 1:2, dann Methanol, zuletzt Dichlormethan). Die Lösung der Reaktionsprodukte in Dichlormethan wurde GC/MSspektroskopisch untersucht.

\subsection{Röntgenstrukturanalyse}

Die Bestimmung der Elementarzelle und die Sammlung der Meßdaten erfolgte in 
Heidelberg auf einem Siemens (Nicolet Syntex) R3m/V-Diffraktometer und einem Nonius Kappa CCD Diffraktometer mit graphitmonochromatisierter Mo-K $\mathrm{K}_{\alpha}$-Strahlung $(\lambda=0.711 \AA$ ). An der Universität Göttingen erfolgten die Messungen an einem STOEIPDS-2 Gerät, einem STOEAED-2 Gerät und einem Vierkreisdiffraktometer mit Offset-Eulerwiege der Firma Huber mit einem Bruker SMART 4K CCD-Zähler mit graphitmonochromatisierter Mo- $K_{\alpha}-$ Strahlung $(\lambda=0.711 \AA)$, sowie an einem SMART 6000 CCD Diffraktometer mit Cu- $K_{\alpha}$-Strahlung $(\lambda=1.542 \AA)$. Die Meßwerte wurden bezüglich Lorentz- und Polarisationseffekten korrigiert. Zusätzlich erfolgten Absorptionskorrekturen. Die Lösung und Verfeinerung wurde mit den Programmen SHELXS-97 $7^{[206]}$ und SHELXL-97 ${ }^{[207]}$ durchgeführt. Bei den Kristalldaten in Kapitel 3.2 und den folgenden Gitterkonstanten sind in Klammern hinter den jeweiligen Werten die Standardabweichungen in Einheiten der letzten Dezimale angegeben.

Kristall- oder Röntgenstrukturanalysen wurden durchgeführt von Fabio Dall'Antonia, Thomas Labahn, Dr. Mathias Noltemeyer, Dr. Peter Pritzkow, José Antonio Cuesta Seijo und Denis Vidovic. 


\subsection{Kristalldaten und Angaben zur Struktur im Festkörper}

\begin{tabular}{|c|c|c|}
\hline & 5 & 6 \\
\hline \multirow[t]{2}{*}{ Formel } & $\mathrm{C}_{31} \mathrm{H}_{66} \mathrm{Cu}_{4} \mathrm{Cl}_{4} \mathrm{~N}_{12} \mathrm{O}_{21}$ & $\mathrm{C}_{23} \mathrm{H}_{30} \mathrm{Cu}_{2} \mathrm{Cl}_{2} \mathrm{~N}_{6} \mathrm{O}_{10}$ \\
\hline & + 1.5 Aceton & $+\mathrm{CH}_{2} \mathrm{Cl}_{2}$ \\
\hline $\mathrm{M}\left[\mathrm{g} \mathrm{mol}^{-1}\right]$ & 1426.04 & 833.44 \\
\hline Kristallgröße [mm] & $0.26 \times 0.24 \times 0.24$ & $0.35 \times 0.17 \times 0.09$ \\
\hline Kristallsystem & Monoklin & Triklin \\
\hline Raumgruppe & $C 2 / c$ & $P_{1}^{\overline{1}}$ \\
\hline$a[\AA]$ & $25.4187(12)$ & $10.6954(5)$ \\
\hline $\mathrm{b}[\AA]$ & $12.2144(6)$ & $10.9317(5)$ \\
\hline$c[\AA]$ & $36.9585(17)$ & $14.3903(7)$ \\
\hline$\alpha\left[^{\circ}\right]$ & 90 & $75.299(1)$ \\
\hline$\beta\left[^{\circ}\right]$ & $96.057(1)$ & $85.568(1)$ \\
\hline$\gamma\left[{ }^{\circ}\right]$ & 90 & $78.999(1)$ \\
\hline$V\left[\AA^{3}\right]$ & $11410.6(9)$ & $1596.78(13)$ \\
\hline$Z$ & 8 & 2 \\
\hline$\rho_{\text {ber. }}\left[\mathrm{g} \mathrm{cm}^{-3}\right]$ & 1.659 & 1.733 \\
\hline$T[\mathrm{~K}]$ & $190(2)$ & $190(2)$ \\
\hline$\mu\left(\mathrm{Mo}-\mathrm{K}_{\alpha}\right)\left[\mathrm{mm}^{-1}\right]$ & 1.742 & 1.730 \\
\hline$h k l$ Bereich & $\pm 37,0-18,0-54$ & $\pm 14,-13-14,0-19$ \\
\hline$\theta$ Bereich $\left[{ }^{\circ}\right]$ & $1.61-32.04$ & $1.94-28.34$ \\
\hline Gemessene Reflexe & 62350 & 23129 \\
\hline Unabhängige Refl. (Rint) & $19249(0.0416)$ & $7959(0.0408)$ \\
\hline Verfeinerte Parameter & 986 & 532 \\
\hline Maximale Restelektronendichte $\left[\mathrm{e} \AA^{-3}\right]$ & 1.410 & 0.761 \\
\hline$R 1(I>2 \sigma(I))$ & 0.0563 & 0.0380 \\
\hline wR2 (alle Daten) & 0.1526 & 0.1072 \\
\hline Goodness-of-fit & 1.042 & 1.032 \\
\hline
\end{tabular}




\begin{tabular}{|c|c|c|}
\hline & $7 a$ & $7 b$ \\
\hline Formel & $\mathrm{C}_{23} \mathrm{H}_{35} \mathrm{Cu}_{2} \mathrm{~B}_{2} \mathrm{~F}_{9} \mathrm{~N}_{6} \mathrm{O}_{2}$ & $\mathrm{C}_{22} \mathrm{H}_{35} \mathrm{~B}_{2} \mathrm{Cu}_{2} \mathrm{~F}_{9} \mathrm{~N}_{6} \mathrm{O}_{3}$ \\
\hline $\mathrm{M}\left[\mathrm{g} \mathrm{mol}^{-1}\right]$ & 747.27 & 751.26 \\
\hline Kristallgröße [mm] & $0.30 \times 0.28 \times 0.25$ & $0.25 \times 0.12 \times 0.09$ \\
\hline Kristallsystem & Orthorhombisch & Monoklin \\
\hline Raumgruppe & Pbca & $P 2_{1} / c$ \\
\hline $\mathrm{a}[\AA]$ & $14.878(3)$ & $13.762(3)$ \\
\hline $\mathrm{b}[\AA]]$ & $14.853(3)$ & $15.346(4)$ \\
\hline$c[\AA]$ & $27.554(6)$ & $14.810(3)$ \\
\hline$\alpha\left[^{\circ}\right]$ & 90 & 90 \\
\hline$\beta\left[^{\circ}\right]$ & 90 & $109.955(5)$ \\
\hline$\gamma\left[^{\circ}\right]$ & 90 & 90 \\
\hline$V\left[\AA^{3}\right]$ & $6089(2)$ & $2940.0(12)$ \\
\hline$Z$ & 8 & 4 \\
\hline$\rho_{\text {ber. }}\left[\mathrm{g} \mathrm{cm}^{-3}\right]$ & 1.630 & 1.697 \\
\hline$T[\mathrm{~K}]$ & $106(2)$ & $106(2)$ \\
\hline$\mu\left(\mathrm{Mo}-\mathrm{K}_{\alpha}\right)\left[\mathrm{mm}^{-1}\right]$ & 1.485 & 1.541 \\
\hline$h k l$ Bereich & $0-22,0-22,0-41$ & $-19-17,0-21,0-20$ \\
\hline$\theta$ Bereich $\left[^{\circ}\right]$ & $2.01-32.02$ & $1.98-30.01$ \\
\hline Gemessene Reflexe & 56268 & 24698 \\
\hline Unabhängige Refl. (Rint) & $10498(0.0500)$ & $8543(0.0480)$ \\
\hline Verfeinerte Parameter & 537 & 523 \\
\hline Maximale Restelektronendichte $\left[\mathrm{e} \AA^{-3}\right]$ & 1.547 & 0.950 \\
\hline$R 1(I>2 \sigma(I))$ & 0.0486 & 0.0425 \\
\hline$w R 2$ (alle Daten) & 0.1251 & 0.1117 \\
\hline Goodness-of-fit & 1.106 & 1.032 \\
\hline
\end{tabular}




\begin{tabular}{|c|c|c|}
\hline & 8 & 9 \\
\hline \multirow[t]{2}{*}{ Formel } & $\mathrm{C}_{23} \mathrm{H}_{35} \mathrm{Cu}_{2} \mathrm{~N}_{9} \mathrm{O}_{11}$ & $\mathrm{C}_{35} \mathrm{H}_{40} \mathrm{Cu}_{2} \mathrm{Cl}_{2} \mathrm{~N}_{8} \mathrm{O}_{10}$ \\
\hline & & +1 Diethyether \\
\hline $\mathrm{M}\left[\mathrm{g} \mathrm{mol}^{-1}\right]$ & 740.68 & 1006.85 \\
\hline Kristallgröße [mm] & $0.35 \times 0.24 \times 0.22$ & $0.36 \times 0.35 \times 0.33$ \\
\hline Kristallsystem & Triklin & Monoklin \\
\hline Raumgruppe & $P_{1}^{-}$ & $C 2 / c$ \\
\hline$a[\AA]$ & $8.3820(17)$ & $34.901(7)$ \\
\hline $\mathrm{b}[\AA]$ & $13.924(3)$ & $11.491(2)$ \\
\hline$c[\AA]$ & $14.606(3)$ & $25.963(5)$ \\
\hline$\alpha\left[^{\circ}\right]$ & $62.94(3)$ & 90 \\
\hline$\beta\left[^{\circ}\right]$ & $83.62(3)$ & $124.24(3)$ \\
\hline$\gamma\left[\left[^{\circ}\right]\right.$ & $85.64(3)$ & 90 \\
\hline$V\left[\AA^{3}\right]$ & $1508.1(5)$ & $8608(3)$ \\
\hline$z$ & 2 & 8 \\
\hline$\rho_{\text {ber. }}\left[\mathrm{g} \mathrm{cm}^{-3}\right]$ & 1.631 & 1.554 \\
\hline$T[\mathrm{~K}]$ & $133(2)$ & $133(2)$ \\
\hline$\mu\left(\mathrm{Mo}-\mathrm{K}_{\alpha}\right)\left[\mathrm{mm}^{-1}\right]$ & 1.483 & 1.182 \\
\hline$h k l$ Bereich & $\pm 9, \pm 16,-14-17$ & $-40-33,0-13,0-30$ \\
\hline$\theta$ Bereich $\left[^{\circ}\right]$ & $2.45-24.63$ & $1.61-24.70$ \\
\hline Gemessene Reflexe & 12347 & 23497 \\
\hline Unabhängige Refl. (Rint) & $5049(0.0952)$ & $7297(0.1001)$ \\
\hline Verfeinerte Parameter & 409 & 637 \\
\hline Maximale Restelektronendichte $\left[\mathrm{e} \AA^{-3}\right]$ & 1.446 & 1.014 \\
\hline$R 1(I>2 \sigma(I))$ & 0.0597 & 0.0621 \\
\hline$w R 2$ (alle Daten) & 0.1507 & 0.1685 \\
\hline Goodness-of-fit & 0.983 & 1.002 \\
\hline
\end{tabular}




\begin{tabular}{|c|c|c|}
\hline & 10 & 11 \\
\hline \multirow[t]{2}{*}{ Formel } & $\mathrm{C}_{33} \mathrm{H}_{38} \mathrm{Cu}_{2} \mathrm{Cl}_{2} \mathrm{~N}_{8} \mathrm{O}_{9}$ & $\mathrm{C}_{34} \mathrm{H}_{41} \mathrm{~B}_{2} \mathrm{Cu}_{2} \mathrm{~F}_{9} \mathrm{~N}_{8} \mathrm{O}$ \\
\hline & $+2 \mathrm{CH}_{2} \mathrm{Cl}_{2}$ & + 1.5 Methanol \\
\hline $\mathrm{M}\left[\mathrm{g} \mathrm{mol}^{-1}\right]$ & 1058.55 & 945.50 \\
\hline Kristallgröße [mm] & $0.43 \times 0.39 \times 0.11$ & $0.30 \times 0.27 \times 0.11$ \\
\hline Kristallsystem & Monoklin & Triklin \\
\hline Raumgruppe & $P 2_{1} / n$ & $P_{1}^{-}$ \\
\hline$a[\AA]]$ & $9.0111(8)$ & $9.167(3)$ \\
\hline $\mathrm{b}[\AA]]$ & $39.929(3)$ & $11.544(3)$ \\
\hline$c[\AA]]$ & $12.1896(10)$ & $20.436(6)$ \\
\hline$\alpha\left[^{\circ}\right]$ & 90 & $101.798(5)$ \\
\hline$\beta\left[^{\circ}\right]$ & $106.223(2)$ & $95.253(5)$ \\
\hline$\gamma\left[^{\circ}\right]$ & 90 & $107.189(6)$ \\
\hline$V\left[\AA^{3}\right]$ & $4211.2(6)$ & $1995.5(10)$ \\
\hline Z & 4 & 2 \\
\hline$\rho_{\text {ber. }}\left[\mathrm{g} \mathrm{cm}^{-3}\right]$ & 1.670 & 1.572 \\
\hline$T[\mathrm{~K}]$ & $190(2)$ & $106(2)$ \\
\hline$\mu\left(\mathrm{Mo}-\mathrm{K}_{\alpha}\right)\left[\mathrm{mm}^{-1}\right]$ & 1.454 & 1.154 \\
\hline$h k l$ Bereich & $-12-11,0-53,0-16$ & $\pm 13,-17-16,0-30$ \\
\hline$\theta$ Bereich $\left[^{\circ}\right]$ & $2.81-28.70$ & $1.90-32.04$ \\
\hline Gemessene Reflexe & 29282 & 34727 \\
\hline Unabhängige Refl. (Rint) & $10802(0.0430)$ & $13460(0.0397)$ \\
\hline Verfeinerte Parameter & 701 & 789 \\
\hline Maximale Restelektronendichte $\left[\mathrm{e} \AA^{-3}\right]$ & 1.162 & 0.941 \\
\hline$R 1(I>2 \sigma(I))$ & 0.0501 & 0.0445 \\
\hline$w R 2$ (alle Daten) & 0.1193 & 0.1176 \\
\hline Goodness-of-fit & 1.067 & 1.046 \\
\hline
\end{tabular}




\begin{tabular}{|c|c|c|}
\hline & 12 & 13 \\
\hline \multirow[t]{2}{*}{ Formel } & $\mathrm{C}_{35} \mathrm{H}_{37} \mathrm{Cu}_{2} \mathrm{ClF}_{6} \mathrm{~N}_{8} \mathrm{O}_{6} \mathrm{~S}_{2}$ & $\mathrm{C}_{35} \mathrm{H}_{38} \mathrm{Cu}_{2} \mathrm{~F}_{6} \mathrm{~N}_{8} \mathrm{O}_{7} \mathrm{~S}_{2}$ \\
\hline & $+\mathrm{CH}_{2} \mathrm{Cl}_{2}$ & +1 Aceton \\
\hline $\mathrm{M}\left[\mathrm{g} \mathrm{mol}^{-1}\right]$ & 1091.32 & 1040.79 \\
\hline Kristallgröße [mm] & $0.60 \times 0.45 \times 0.34$ & $0.43 \times 0.29 \times 0.28$ \\
\hline Kristallsystem & Monoklin & Orthorhombisch \\
\hline Raumgruppe & $P 2_{1} / c$ & $P_{n a 2}$ \\
\hline$a[\AA]$ & $21.609(4)$ & $22.552(14)$ \\
\hline $\mathrm{b}[\AA]$ & $13.260(3)$ & $13.21(2)$ \\
\hline$c[\AA]$ & $16.663(3)$ & $28.35(5)$ \\
\hline$\alpha\left[^{\circ}\right]$ & 90 & 90 \\
\hline$\beta\left[^{\circ}\right]$ & $107.21(3)$ & 90 \\
\hline$\gamma\left[^{\circ}\right]$ & 90 & 90 \\
\hline$V\left[\AA^{3}\right]$ & $4560.9(16)$ & $8449(21)$ \\
\hline$z$ & 4 & 8 \\
\hline$\rho_{\text {ber. }}\left[\mathrm{g} \mathrm{cm}^{-3}\right]$ & 1.586 & 1.636 \\
\hline$T[\mathrm{~K}]$ & $277(2)$ & $133(2)$ \\
\hline$\mu\left[\mathrm{mm}^{-1}\right]$ & $4.302\left(\mathrm{Cu}-\mathrm{K}_{\alpha}\right)$ & $1.193\left(\mathrm{Mo}^{\left.-\mathrm{K}_{\alpha}\right)}\right.$ \\
\hline$h k l$ Bereich & $-23-24, \pm 14, \pm 18$ & $-29-18, \pm 17, \pm 36$ \\
\hline$\theta$ Bereich $\left[^{\circ}\right]$ & 2.14 to 59.97 & $1.79-27.59$ \\
\hline Gemessene Reflexe & 28340 & 125703 \\
\hline Unabhängige Refl. (Rint) & $6582(0.0231)$ & $19490(0.0705)$ \\
\hline Verfeinerte Parameter & 579 & 1166 \\
\hline Maximale Restelektronendichte $\left[\mathrm{e} \AA^{-3}\right]$ & 1.272 & 0.688 \\
\hline$R 1(I>2 \sigma(I))$ & 0.0606 & 0.0459 \\
\hline$w R 2$ (alle Daten) & 0.1792 & 0.0997 \\
\hline Goodness-of-fit & 1.060 & 1.071 \\
\hline
\end{tabular}




\begin{tabular}{|c|c|c|}
\hline & 14 & 18 \\
\hline \multirow[t]{2}{*}{ Formel } & $\mathrm{C}_{30} \mathrm{H}_{33} \mathrm{~B}_{2} \mathrm{Cu}_{2} \mathrm{~F}_{9} \mathrm{~N}_{8} \mathrm{O}$ & $\mathrm{C}_{21} \mathrm{H}_{33} \mathrm{Cl}_{4} \mathrm{Cu}_{2} \mathrm{~F}_{6} \mathrm{~N}_{6} \mathrm{O}_{3} \mathrm{P}$ \\
\hline & & $+1 \mathrm{EtCN}$ \\
\hline $\mathrm{M}\left[\mathrm{g} \mathrm{mol}^{-1}\right]$ & 841.34 & 904.48 \\
\hline Kristallgröße [mm] & $0.23 \times 0.19 \times 0.07$ & $0.30 \times 0.22 \times 0.22$ \\
\hline Kristallsystem & Monoklin & Triklin \\
\hline Raumgruppe & $P 2_{1} / c$ & $P_{1}^{-}$ \\
\hline $\mathrm{a}[\AA]$ & $17.178(3)$ & $12.4327(6)$ \\
\hline $\mathrm{b}[\AA]$ & $14.858(3)$ & $13.1551(7)$ \\
\hline$c[\AA]$ & $15.109(3)$ & $13.7200(7)$ \\
\hline$\alpha\left[^{\circ}\right]$ & 90 & $62.154(1)$ \\
\hline$\beta\left[^{\circ}\right]$ & $104.583(4)$ & $87.539(1)$ \\
\hline$\gamma\left[{ }^{\circ}\right]$ & 90 & $73.926(1)$ \\
\hline$V\left[\AA^{3}\right]$ & $3731.9(12)$ & $1896.7(2)$ \\
\hline$Z$ & 4 & 2 \\
\hline$\rho_{\text {ber. }}\left[\mathrm{g} \mathrm{cm}^{-3}\right]$ & 1.497 & 1.584 \\
\hline$T[\mathrm{~K}]$ & $106(2)$ & $190(2)$ \\
\hline$\mu\left(\mathrm{Mo}-\mathrm{K}_{\alpha}\right)\left[\mathrm{mm}^{-1}\right]$ & 1.221 & 1.512 \\
\hline$h k l$ Bereich & $-18-17,0-15,0-15$ & $\pm 18,-16-19,0-20$ \\
\hline$\theta / 2 \theta$ Bereich $\left[{ }^{\circ}\right]$ & $1.84-21.97$ & $4.2-64.1$ \\
\hline Gemessene Reflexe & 16068 & 33357 \\
\hline Unabhängige Refl. (Rint) & $4550(0.0668)$ & $12555(0.0356)$ \\
\hline Verfeinerte Parameter & 440 & 570 \\
\hline Maximale Restelektronendichte $\left[\mathrm{e} \AA^{-3}\right]$ & 0.772 & 1.32 \\
\hline$R 1(I>2 \sigma(I))$ & 0.0484 & 0.043 \\
\hline$w R 2$ (alle Daten) & 0.1321 & 0.130 \\
\hline Goodness-of-fit & 1.007 & 1.017 \\
\hline
\end{tabular}




\begin{tabular}{|c|c|c|}
\hline & 19 & 20 \\
\hline \multirow[t]{2}{*}{ Formel } & $\mathrm{C}_{23} \mathrm{H}_{37} \mathrm{Br}_{4} \mathrm{Cu}_{2} \mathrm{~N}_{7} \mathrm{O}_{6}$ & $\mathrm{C}_{35} \mathrm{H}_{64} \mathrm{Cl}_{6} \mathrm{Cu}_{2} \mathrm{~N}_{8} \mathrm{O}_{11}$ \\
\hline & & + 1 Aceton \\
\hline $\mathrm{M}\left[\mathrm{g} \mathrm{mol}^{-1}\right]$ & 954.29 & 1170.80 \\
\hline Kristallgröße [mm] & $1.00 \times 0.60 \times 0.20$ & $0.38 \times 0.26 \times 0.17$ \\
\hline Kristallsystem & Monoklin & Monoklin \\
\hline Raumgruppe & $P 2_{1} / n$ & $P 2_{1}$ \\
\hline$a[\AA]]$ & $15.386(3)$ & $14.1449(3)$ \\
\hline $\mathrm{b}[\AA]]$ & $12.7015(15)$ & $13.8461(3)$ \\
\hline$c[\AA]$ & $17.045(4)$ & $26.6750(7)$ \\
\hline$\alpha\left[^{\circ}\right]$ & 90 & 90.00 \\
\hline$\beta\left[^{\circ}\right]$ & $94.97(2)$ & $100.64(1)$ \\
\hline$\gamma\left[^{\circ}\right]$ & 90 & 90.00 \\
\hline$V\left[\AA^{3}\right]$ & $3318.6(11)$ & $5134.5(2)$ \\
\hline$z$ & 4 & 4 \\
\hline$\rho_{\text {ber. }}\left[\mathrm{g} \mathrm{cm}^{-3}\right]$ & 1.908 & 1.515 \\
\hline$T[\mathrm{~K}]$ & $200(2)$ & $173(2)$ \\
\hline$\mu\left(\mathrm{Mo}-\mathrm{K}_{\alpha}\right)\left[\mathrm{mm}^{-1}\right]$ & 6.145 & 1.204 \\
\hline$h k l$ Bereich & $\pm 18, \pm 15, \pm 20$ & $\pm 17, \pm 17,0-33$ \\
\hline$\theta / 2 \theta$ Bereich $\left[^{\circ}\right]$ & $3.64-25.00$ & $3.0-52.8$ \\
\hline Gemessene Reflexe & 11648 & 40021 \\
\hline Unabhängige Refl. (Rint) & $5824(0.0977)$ & $20430(0.0726)$ \\
\hline Verfeinerte Parameter & 386 & 1237 \\
\hline Maximale Restelektronendichte $\left[\mathrm{e} \AA^{-3}\right]$ & 2.749 & 1.11 \\
\hline$R 1(I>2 \sigma(I))$ & 0.0760 & 0.047 \\
\hline$w R 2$ (alle Daten) & 0.1978 & 0.121 \\
\hline Goodness-of-fit & 1.077 & 0.984 \\
\hline
\end{tabular}




\begin{tabular}{|c|c|c|}
\hline & 21 & 22 \\
\hline \multirow[t]{2}{*}{ Formel } & $\mathrm{C}_{27} \mathrm{H}_{29} \mathrm{Br}_{4} \mathrm{Cl}_{1} \mathrm{Cu}_{2} \mathrm{~N}_{6} \mathrm{O}$ & $\mathrm{C}_{70} \mathrm{H}_{58} \mathrm{Cl}_{8} \mathrm{Cu}_{4} \mathrm{~F}_{12} \mathrm{~N}_{16} \mathrm{O}_{4} \mathrm{P}_{2}$ \\
\hline & + 0.5 Aceton & $+6 \mathrm{MeCN}$ \\
\hline $\mathrm{M}\left[\mathrm{g} \mathrm{mol}^{-1}\right]$ & 1060.76 & 2261.35 \\
\hline Kristallgröße [mm] & $0.60 \times 0.50 \times 0.40$ & $1.00 \times 1.00 \times 0.50$ \\
\hline Kristallsystem & Monoklin & Monoklin \\
\hline Raumgruppe & $P 2_{1} / c$ & $C 2 / c$ \\
\hline$a[\AA]]$ & $13.636(3)$ & $2917.5(6)$ \\
\hline $\mathrm{b}[\AA]]$ & $12.363(3)$ & $1633.9(3)$ \\
\hline$c[\AA]$ & $22.274(4)$ & $2372.7(5)$ \\
\hline$\alpha\left[^{\circ}\right]$ & 90 & 90 \\
\hline$\beta\left[^{\circ}\right]$ & $103.67(3)$ & $113.25(3)$ \\
\hline$\gamma\left[{ }^{\circ}\right]$ & 90 & 90 \\
\hline$V\left[\AA^{3}\right]$ & $3648.7(13)$ & $10390(10)$ \\
\hline$z$ & 4 & 4 \\
\hline$\rho_{\text {ber. }}\left[\mathrm{g} \mathrm{cm}^{-3}\right]$ & 1.922 & 1.445 \\
\hline$T[\mathrm{~K}]$ & $133(2)$ & $346(2)$ \\
\hline$\mu\left(\mathrm{Mo}-\mathrm{K}_{\alpha}\right)\left[\mathrm{mm}^{-1}\right]$ & 5.672 & 1.121 \\
\hline$h k l$ Bereich & $-16-17, \pm 15, \pm 27$ & $-31-28, \pm 17,0-25$ \\
\hline$\theta$ Bereich $\left[^{\circ}\right]$ & $1.88-26.37$ & $3.61-22.53$ \\
\hline Gemessene Reflexe & 50672 & 6813 \\
\hline Unabhängige Refl. (Rint) & $7440(0.0523)$ & $6765(0.1657)$ \\
\hline Verfeinerte Parameter & 475 & 607 \\
\hline Maximale Restelektronendichte $\left[\mathrm{e} \AA^{-3}\right]$ & 1.000 & 1.198 \\
\hline$R 1(I>2 \sigma(I))$ & 0.0316 & 0.0783 \\
\hline$w R 2$ (alle Daten) & 0.0758 & 0.2503 \\
\hline Goodness-of-fit & 1.039 & 1.032 \\
\hline
\end{tabular}




\section{LITERATURVERZEICHNIS}

\section{Literaturverzeichnis}

${ }^{1}$ Römpp Chemie Lexikon, 9. Aufl., Georg Thieme Verlag, Stuttgart, 1990.

${ }^{2}$ N.N. Greenwood, A. Earnshaw, Chemie der Elemente, 1. Aufl., VCH, Weinheim, 1988.

${ }^{3}$ F.A. Carey, R.J. Sundberg, Organische Chemie, 1. Aufl., VCH, Weinheim, 1995.

${ }^{4}$ A. Streitwieser, C.H. Heathcock, E.M. Kosower, Organische Chemie, 2. Aufl., VCH, Weinheim, 1994.

${ }^{5}$ W. Kaim, B. Schwederski, Bioanorganische Chemie, Teubner Studienbücher, Stuttgart, 1991.

${ }^{6}$ D. Voet, J.G. Voet, C.W. Pratt, Lehrbuch der Biochemie, Wiley-VCH, Weinheim, 2002.

${ }^{7}$ C. Gerdemann, C. Eicken, B. Krebs, Acc. Chem. Res. 2002, 35, 183-191.

${ }^{8}$ S.J. Lippard, J.M. Berg, Principles of Bioinorganic Chemistry, University Science Books, Mill Valley, CA, 1994.

${ }^{9}$ W.P.J. Gaykema, W.G.J. Hol, J.M. Vereijken, N.M. Soeter, H.J. Bak, J.J. Beintema, Nature, 1984, 309, 23-29.

${ }^{10}$ K.A. Magnus, H. Thon-Tat, J.E Carpenter, Chem. Rev. 1994, 94, 727-735.

${ }^{11}$ M.E. Cuff, K.I. Miller, K.E. van Holde, W.A. Hendrickson, J. Mol. Biol. 1998, 278, 855-870.

12 K.A. Magnus, B. Hazes, H. Thon-Tat, C. Bonaventura, J. Bonaventura, W.G.J. Hol, Proteins : Structure, Function and Genetics 1994, 19, 302-309.

${ }^{13}$ N. Kitajima, T. Koda, S. Hashimoto, T. Kitagawa, Y. Moro-oka, J. Chem. Soc., Chem. Commun. 1988, 151-152.

${ }^{14}$ N. Kitajima, K. Fujisawa, Y. Moro-oka, J. Am. Chem. Soc. 1989, 111, 8975-8976.

${ }^{15}$ M. Kodera, K. Katayama, Y. Tachi, K. Kano, S. Hirota, S. Fujinami, M. Suzuki, J. Am. Chem. Soc. 1999, 121, 11006-11007.

${ }^{16}$ A.G. Blackman, W.B. Tolman, Structure and Bonding 2000, 97, 179-211.

${ }^{17}$ S. Mahapatra, J.A. Halfen, E.C. Wilkinson, L. Que, W.B. Tolman, J. Am. Chem. Soc. 1994, 116, 9785-9786.

${ }^{18}$ S. Mahapatra, J.A. Halfen, E.C. Wilkinson, G. Pan, C.J. Cramer, L. Que, W.B. Tolman, J. Am. Chem. Soc. 1995, 117, 8865-8866.

${ }^{19}$ S. Mahapatra, J.A. Halfen, E.C. Wilkinson, G. Pan, X. Wang, V.G. Young, C.J. Cramer, L. Que, W.B. Tolman, J. Am. Chem. Soc. 1996, 118, 11555-11574.

${ }^{20}$ W.B. Tolman, Acc. Chem. Res. 1997, 30, 227-237.

${ }^{21}$ S. Mahapatra, S. Kaderli, A. Llobet, Y. Neuhold, T. Palanché, J.A. Halfen, V.G. Young, T.A. Kaden, L. Que, A.D. Zuberbühler, W.B. Tolman, Inorg. Chem. 1997, 36, 6343-6356.

${ }^{22}$ S. Mahapatra, V.G. Young, S. Kaderli, A.D. Zuberbühler, W.B. Tolman, Angew. Chem. 1997, 109, 125-127; Angew. Chem., Int. Ed. 1997, 36, 130-133.

${ }^{23}$ J.A. Halfen, S. Mahapatra, E.C. Wilkinson, S. Kaderli, V.G. Young, L. Que, A.D. Zuberbühler, W.B. Tolman, Science 1996, 271, 1397-1400.

${ }^{24}$ S. Itoh, M. Taki, H. Nakao, P.L. Holland, W.B. Tolman, L. Que, S. Fukuzumi, Angew. Chem. 2000, 112, 409-411; Angew. Chem., Int. Ed. 2000, 39, 398-400.

${ }^{25}$ L. Que, W.B. Tolman, Angew. Chem. 2002, 114, 1160-1185; Angew. Chem, Int. Ed. 2002, 41, 1114-1137. 
${ }^{26}$ H.V. Obias, Y. Lin, N.N. Murthy, E. Pidcock, E.I. Solomon, M. Ralle, N.J. Blackburn, Y. Neuhold, A.D. Zuberbühler, K.D. Karlin, J. Am. Chem. Soc. 1998, 120, 12960-12961.

${ }^{27}$ E. Pidcock, S. DeBeer, H.V. Obias, B. Hedman, K.O. Hodgson, K.D. Karlin, E.I. Solomon, J. Am. Chem. Soc. 1999, 121, 1870-1878.

${ }^{28}$ D.J.E. Spencer, N.W. Aboellela, A.M. Reynolds, P.L. Holland, W.B. Tolman, J. Am. Chem. Soc. 2002, 124, 2108-2109.

${ }^{29}$ D.J.E. Spencer, A.M. Reynolds, P.L. Holland, B.A. Jazdzewski, C. Duboc-Toia, L. Le Pape, S. Yokota, Y. Tachi, S. Itoh, W.B. Tolman, Inorg. Chem. 2002, 41, 6307-6321.

${ }^{30}$ R.R. Jacobson, Z. Tyeklar, A. Farooq, K.D. Karlin, S. Liu, J. Zubieta, J. Am. Chem. Soc. 1988, 110, 3690-3692.

${ }^{31}$ E.I. Solomon, U.M. Sundaram, T.E. Machonkin, Chem. Rev. 1996, 96, 2563-2605.

${ }^{32}$ A.P. Cole, D.E. Root, P. Mukherjee, E.I. Solomon, T.D.P. Stack, Science 1996, 273, 1848-1850.

33 J. Reim, B. Krebs, Angew. Chem. 1994, 106, 2040-2041; Angew. Chem., Int. Ed. 1994, 33, $1969-$ 1971.

${ }^{34}$ F. Meyer, H. Pritzkow, Angew. Chem. 2000, 112, 2199-2200; Angew. Chem., Int. Ed. 2000, 39, 2112-2115.

${ }^{35}$ K. Fujisawa, M. Tanaka, Y. Moro-oka, N. Kitajima, J. Am. Chem. Soc. 1994, 116, 12079-12080.

${ }^{36}$ A. Wada, M. Harata, K. Hasegawa, K. Jitsukawa, H. Masuda, M. Mukai, T. Kitagawa, H. Einaga, Angew. Chem. 1998, 110, 874-875; Angew. Chem., Int. Ed. 1998, 37, 798-799.

${ }^{37}$ C. Gerdemann, C. Eicken, B. Krebs, Acc. Chem. Res. 2002, 35, 183-191.

${ }^{38}$ H.-J. Krüger, Angew. Chem. 1999, 111, 659-663; Angew. Chem., Int. Ed. 1999, 38, 627-631.

${ }^{39}$ T. Klabunde, C. Eicken, J.C. Sacchettini, B. Krebs, Nat. Struct. Biol. 1998, 5, 1084-1090.

${ }^{40}$ H. Decker, R. Dillinger, F. Tuczek, Angew. Chem. 2000, 112, 1656-1660.

${ }^{41}$ P.L. Holland, W.B. Tolman, Coord. Chem. Rev. 1999, 192, 855-869.

${ }^{42}$ P.Kroneck in Handbook of Metalloproteins, Vol. 2 (Hrsg. A. Messerschmidt, R. Huber, T. Poulos, K. Wieghardt), 1. Aufl., Wiley-VCH, Weinheim, 2001, 1331 - 1341.

43 T. Rasmussen, B.C. Berks, J. Sanders-Loehr, D.M. Dooley, W.G. Zumft, A.J. Thomson, Biochemistry 2000, 39, 12753-12756.

${ }^{44}$ K. Brown, K. Djinovic-Carugo, T. Haltia, I. Cabrito, M. Saraste, J.J.G. Moura, I. Moura, M. Tegoni, C. Cambillau, J. Biol. Chem. 2000, 275, 41133-41136.

${ }^{45}$ P. Chen, S. George, I. Cabrito, W.E. Antholine, J.J.G. Moura, I. Moura, B. Hedman, K. Hodgson, E.I. Solomon, J. Am. Chem. Soc. 2002, 124, 744-745.

${ }^{46}$ P. Chen, I. Cabrito, J.J.G. Moura, I. Moura, E.I. Solomon, J. Am. Chem. Soc. 2002, 124, 1049710507.

${ }^{47}$ K.D. Karlin, J.C. Hayes, Y. Gultneh, R.W. Cruse, J.W. McKown, J.P. Hutchinson, J. Zubieta, J. Am. Chem. Soc. 1984, 106, 2121-2128.

${ }^{48}$ L. Casella, M. Gullotti, G. Pallanza, L. Rigoni, J. Am. Chem. Soc. 1988, 110, 4221-4227.

${ }^{49}$ R. Menif, A.E. Martell, P.J. Squattrito,A. Clearfield, Inorg. Chem. 1990, 29, 4723-4729.

${ }^{50}$ T.N. Sorrell, M.L. Garrity, Inorg. Chem. 1991, 30, 210-215. 
${ }^{51}$ E. Pidcock, H.V. Obias, C.X. Zhang, K.D. Karlin, E.I. Solomon, J. Am. Chem. Soc 1998, 120, 78417847.

${ }^{52}$ L. Santagostini, M. Gullotti, E. Monzani, L. Casella, R. Dillinger, F. Tuczek, Chem. Eur. J. 2000, 6, 519-522.

${ }^{53}$ N.N. Murthy, M. Mahroof-Tahir, K.D. Karlin, Inorg. Chem. 2001, 40, 628-635.

${ }^{54}$ D. Ghosh, R. Mukherjee, Inorg. CHem. 1998, 37, 6597-6605.

${ }^{55}$ H. Arii, Y. Saito, S. Nagatomo, T. Kitagawa, Y. Funahashi,K. Jitskawa, H. Masuda, Chem. Lett. 2003, 32, 156-157.

${ }^{56}$ I. Blain, M. Giorgi, I De Riggi, M. Réglier, Eur. J. Inorg. Chem. 2000, 393-398.

${ }^{57}$ L.M. Berreau, S. Mahapatra, J.A. Halfen, R.P. Houser, V.G. Young, W.B. Tolman, Angew. Chem. 1999, 111, 180-183; Angew. Chem., Int. Ed. 1999, 38, 207-210.

${ }^{58}$ P.L. Holland, K.R. Rodgers, W.B. Tolman, Angew. Chem. 1999, 111, 1210-1213; Angew. Chem., Int. Ed. 1999, 38, 1139-1142.

${ }^{59}$ S. Itoh, M. Taki, H. Nakao, P. L. Holland, W.B. Tolman, L. Que,S. Fukuzumi, Angew. Chem. 2000, 112, 409-411; Angew. Chem., Int. Ed. 2000, 39, 398-400.

${ }^{60}$ K.D. Karlin, S. Kaderli, A.D. Zuberbühler, Acc. Chem. Res. 1997, 30, 139-147.

${ }^{61}$ M. Réglier, C. Jorand, B Waegell, J. Chem. Soc., Chem. Commun. 1990, 1752-1755.

${ }^{62}$ E. Monzani, L. Quinti, A. Perotti, L. Casella,M. Gullotti, L. Randaccio, S. Geremia, G. Nardin, P. Faleschini, G. Tabbi, Inorg. Chem. 1998, 37, 553-562.

${ }^{63}$ E. Monzani, G. Battaini, A. Perotti, L. Casella,M. Gullotti, L. Santagostini, L. Randaccio, S. Geremia, G. Nardin, P. Zanello, G. Opromolla, Inorg. Chem. 1999, 38, 5359-5369.

${ }^{64}$ L. Casella, E. Monzani, M. Gullotti, D. Cavagnino, G. Cerina, L. Santagostini, R. Ugo, Inorg. Chem. 1996, 35, 7516-7525.

${ }^{65}$ S. Itoh, H. Kumei, M. Taki, S. Nagatomo, T. Kitagawa, S. Fukuzumi, J. Am. Chem. Soc. 2001, 67086709.

${ }^{66}$ R. Wegner, M. Gottschaldt, H. Görls, E. Jäger, D. Klemm, Chem. Eur. J. 2001, 7, 2143-2155.

67 J. Chyn, F.L. Urbach, Inorg. Chim. Acta, 1991, 189, 157-163.

${ }^{68}$ J. Reim. B. Krebs, J. Chem. Soc., Dalton Trans. 1997, 3793-3804.

${ }^{69}$ A. Neves, L.M. Rossi, A.J. Bortoluzzi, B. Szpoganicz, C. Wiezbicki, E. Schwingel, Inorg. Chem. 2002, 41, 1788-1794.

${ }^{70}$ F. Meyer, S. Beyreuther, K. Heinze, L. Zsolnai, Chem. Ber./ Receuil 1997, 130, 605-613.

${ }^{71}$ G. Battaini, E. Monzani, L. Casella, L. Santagostini, R. Pagliarin, J. Bioinorg. Chem., 2000, 5, 262268.

${ }^{72}$ H. Börzel, P. Comba, H. Pritzkow, Chem. Commun. 2001, 97-98.

${ }^{73}$ P. Gentschev, N. Möller, B. Krebs, Inorg. Chim. Acta 2000, 442-452.

${ }^{74}$ F. Zippel, F. Ahlers, R. Werner, W. Haase, H. Nolting, B. Krebs, Inorg. Chem. 1996, 35, 3409-3419.

${ }^{75}$ M.R. Malachowski, L.J. Tomlinson, M.G. Davidson, M.J. Hall, J. Coord. Chem. 1992, 25, 171-174.

${ }^{76}$ M.R. Malachowski, H.B. Huynh, L.J. Tomlinson, R.S. Kelly, J.W. Furbee, J. Chem. Soc., Dalton Trans. 1995, 31-36.

${ }^{77}$ M.R. Malachowski, M.G. Davidson, Inorg. Chim. Acta 1989, 162, 199-204. 


\section{LITERATURVERZEICHNIS}

${ }^{78}$ J. Manzur, A.M. Garcia, V. Rivas, A.M. Atria, J. Valenzuela, E. Spodine, Polyhedron 1997, 16, 22992305.

${ }^{79}$ D.A. Rockcliffe; E. Martell, J. Mol. Cat. A 1995, 99, 101-114.

${ }^{80}$ M. Gupta, P. Mathur, R.J. Butcher, Inorg. Chem. 2001, 40, 878-885.

${ }^{81}$ S. Torelli, C. Belle, I. Gautier-Luneau, J.L. Pierre, E. Saint-Aman, J.M. Latour, L. Le Pape, D. Luneau, Inorg. Chem. 2000, 39, 3526-3536.

${ }^{82}$ D. Bolus, G.S. Vigee, Inorg. Chim. Acta 1982, 67, 19-25.

${ }^{83}$ C. Belle, C. Beguin, I. Gautier-Luneau, S. Hamman, C. Philouze, J.L. Pierre, F. Thomas, S. Torelli, E. Saint-Aman, M. Bonin, Inorg. Chem. 2002, 41, 479-491.

${ }^{84}$ J. Mukherjee, R. Mukherjee, Inorg. Chim. Acta 2002, 337, 429-438.

${ }^{85}$ S. Cheng, H. Wei, Inorg. Chim. Acta 2002, 340, 105-113.

${ }^{86}$ J. Kaizer, J. Pap, G. Speier, L. Parkanyi, L. Korecz, A. Rockenbauer, J. Inorg. Biochem. 2002, 91, 190-198.

${ }^{87}$ G. Speier, J. Mol. Catal. 1986, 37, 259-267.

${ }^{88}$ J. Balla, T. Kiss, R.F. Jameson, Inorg. Chem. 1992, 31, 58-62.

${ }^{89}$ L.H. Gade, Koordinationschemie, Wiley-VCH, Weinheim, 1998.

${ }^{90}$ S. Buchler, F. Meyer, E. Kaifer, H. Pritzkow, Inorg. Chim. Acta 2002, 337, 371-386.

${ }^{91}$ F. Meyer, U. Ruschewitz, P. Schober, B. Antelmann, L. Zsolnai, J. Chem. Soc., Dalton Trans. 1998, 1181-1186.

${ }^{92}$ F. Meyer, S. Beyreuther, K. Heinze, L. Zsolnai, Chem. Ber./Recueil 1997, 130, 605-613.

${ }^{93}$ C. Acerete, Tetrahedron, 1994, 50, 4765-4774.

${ }^{94}$ T.G. Schenck, J.M. Downes, C.R.C. Milne, P.B. MacKenzie, H. Boucher, J. Whelan, B. Bosnich, Inorg. Chem. 1985, 24, 2334-2337.

95 J.S. Bradshaw, R.B. Nielsen, P. Tse, G. Arena, B.E. Wilson, N.K. Dalley, J.D. Lamb, J.C. Christensen, R.M. Izatt, J. Heterocyclic. Chem. 1986, 23, 361-368.

${ }^{96}$ J.C. Röder, F. Meyer, H. Pritzkow, Organomet. 2001, 20, 811-817.

${ }^{97}$ L.E. Brady, M. Freifelder, G.R. Stone, J. Org. Chem. 1961, 26, 4757-4758.

${ }^{98}$ H.J. Hoorn, P. de Joode, W.L. Driessen, J. Reedijk, Recl. Trav. Chim. Pays-Bas 1996, 115, $191-$ 197.

99 J. Ackermann, Diplomarbeit Universität Heidelberg 1999.

100 J. Ackermann, F. Meyer, E. Kaifer, H. Pritzkow, Chem. Eur. J. 2002, 8, 247-258.

${ }^{101}$ A.W. Addison, T.N. Rao, J. Reedijk, J. van Rijn, G.C. Verschoor, J. Chem. Soc., Dalton Trans. 1984, 1349-1356.

${ }^{102}$ G.A. von Albada, I. Mutikainen, O. Roubeau, U. Turpeinen, J. Reedijk, Inorg. Chim. Acta 2002, 331, 208-215.

${ }^{103}$ M. Zong-Wan, G. Liehr, R. van Eldik, J. Chem. Soc., Dalton Trans. 2001, 1593-1600.

${ }^{104}$ G.A. van Albada, I. Mutikainen, O.S. Roubeau, U. Turpeinen, J. Reedijk, Eur. J. Inorg. Chem. 2000, 10, 2179-2184.

${ }^{105}$ C. Bazzicalupi, A. Bencini, A. Bianchi, V. Fusi, P. Paoletti, B. Valtancoli, J. Chem. Soc., Chem. Commun. 1995, 15, 1555-1556. 
${ }^{106}$ A. Escuer, R. Vicente, E. Penalba, X. Solans, M. Font-Bardia, Inorg. Chem. 1996, 35, 248-251.

${ }^{107}$ S. Buchler, Dissertation Universität Heidelberg 2002.

${ }^{108}$ R. Alsfasser, M. Ruf, S. Trofimenko, H. Vahrenkamp, Chem. Ber. 1993, 126, 703-710.

${ }^{109}$ M. Ito, Y. Takita, Chem. Lett. 1996, 929-930.

${ }^{110}$ Y. Nishida, A. Yatani, Y. Nakao, J. Taka, S. Kashino, W. Mori, S. Suzuki, Chem. Lett., 1999, 135136.

${ }^{111}$ A. Escuer, F.A. Mautner, E. Penalba, R. Vicente, Inorg. Chem. 1998, 37, 4190-4196.

112 M. Kato, T. Ito, Inorg. Chem. 1985, 24, 509-514.

${ }^{113}$ P. Chakrabarti, V.G. Puranik, J.P. Haskar, S. Hati, D. Datta, Indian J. Chem., Sect. A 2000, 39, 571-578.

114 B.J. Hathaway in Comprehensive Coordination Chemistry Vol. 5 (Hrsg. Sir G. Wilkinson), Pergamon Press, Vol. 5, Oxford, 1987, 533 - 774.

${ }^{115}$ N.F. Curtis, R.W. Hay, Y.M. Curtis, J. Chem. Soc. (A) 1968, 182-187.

${ }^{116}$ A. Neves, L.M. Rossi, I. Vencato, V. Drago, W. Haase, R. Werner, Inorg. Chim. Acta 1998, 281, 111-115.

${ }^{117}$ R.W.M ten Hoedt, J. Reedijk, G.C. Verschoor, Rec. Trav. Chim. Pays-Bas 1981, 100, 400-405.

${ }^{118}$ F.J. Rietmeijer, R.A.G. de Graaff, J. Reedijk, Inorg. Chem. 1984, 23, 151-156.

${ }^{119}$ S.C. Lee, R.H. Holm, Inorg. Chem. 1993, 32, 4745-4753.

120 S.J. Barlow, S.J. Hill, J.E. Hocking, P. Hubberstey, W. Li, J. Chem. Soc., Dalton Trans. 1997, 4701-4703.

${ }^{121}$ D.A. Handley, P.B. Hitchcock, T.H. Lee, G.J. Leigh, Inorg. Chim. Acta 2001, 316, 59-64.

${ }^{122}$ B.F. Straub, F. Rominger, P. Hofmann, Inorg. Chem. 2000, 39, 2113-2119.

${ }^{123}$ J. Huheey, E. Keiter, R. Keiter, Anorganische Chemie, 2. Aufl., de Gruyter, Berlin, 1995.

${ }^{124}$ M.A. Rouet-Mayer, J. Philippon, F. Meudon, Bulletin de Liaison - Groupe Polyphenols 1986, 13, 499-501.

${ }^{125}$ M.A. Rouet-Mayer, J. Philippon, F. Meudon, Phytochemistry 1986, 25, 2717-2719.

${ }^{126}$ H. Nagao, N. Komeda, M. Mukaida, M. Suzuki, K. Tanaka, Inorg. Chem. 1996, 35, 6809-6815.

${ }^{127}$ H. Oshio, H. Ichida, J. Phys. Chem. 1995, 99, 3294-

${ }^{128}$ N. Komeda, H. Nagao, Y. Kushi, G. Adachi, M. Suzuki, A. Uehara, K. Tanaka, Bull. Chem. Soc. Jpn. 1995, 68, 581-

${ }^{129}$ C.S. Allen, Chang-Lin Chuang, M. Cornebise, J.W. Canary, Inorg. Chim. Acta 1995, 239, 29-

${ }^{130}$ R.J. Parker, L. Spiccia, B. Moubaraki, K.S. Murray, B.W. Skelton, A.H. White, Inorg. Chim. Acta 2000, 300, 922-

${ }^{131}$ N. Oishi, Y. Nishida, K. Ida, S. Kida, Bull. Chem. Soc. Jpn. 1980, 53, 2847-2850.

${ }^{132}$ R. Wegner, M. Gottschaldt, H. Görls, E.-G. Jäger, D. Klemm, Angew. Chem. 2000, 112, 608-612; Angew. Chem., Int. Ed. 2000, 39, 595-599.

${ }^{133}$ Y.-H. Chung, H.-H. Wei, Y.-H. Liu, G.-H. Lee, Y. Wang, J. Chem. Soc., Dalton Trans. 1997, 28252829.

${ }^{134}$ M.D. Stallings, M.M Morrison, D.T. Sawyer, Inorg. Chem. 1981, 20, 2655-2660. 


\section{LITERATURVERZEICHNIS}

${ }^{135}$ M. Pascaly, M. Duda, F. Schweppe, K. Zurlinden, F.K. Müller, B. Krebs, J. Chem. Soc., Dalton Trans. 2001, 828-837.

${ }^{136}$ R.G. Wilkins, Kinetics and Mechanism of Reactions of Transition Metal Complexes, 2. Aufl., VCH, Weinheim,1991.

${ }^{137}$ P.W. Atkins, Physikalische Chemie, 1. Aufl., VCH, Weinheim, 1990.

${ }^{138}$ Sigma Plot 2001 für Windows 7.0, SPSS Inc. 2001.

${ }^{139}$ Origin 6.2, Origin Lab Corporation 2000.

${ }^{140}$ W.J. Moore, D.O. Hummel, Physikalische Chemie, 3. Aufl., de Gruyter, Berlin, 1983.

${ }^{141}$ G. Wedler, Lehrbuch der physikalischen Chemie, 4. Aufl., Wiley-VCH, Weinheim, 1997.

${ }^{142}$ C.A. Tyson, A.E. Martell, J. Am. Chem. Soc. 1968, 90, 3379-3386.

${ }^{143}$ L. Casella, O. Carugo, M. Gullotti, S. Garofani, P. Zanello, Inorg. Chem. 1993, 32, 2056-2067.

${ }^{144}$ R. R. Gagné, R. P. Kreh, J. A. Dodge, J. Am. Chem. Soc. 1979, 101, 6917-6927.

145 J.A. Halfen, V.G. Young, W.B. Tolman, Inorg. Chem. 1998, 37, 2102-2103.

146 J.L. Schneider, V.G. Young, W.B. Tolman, Inorg. Chem. 1996, 35, 5410-5411.

${ }^{147}$ H. Jenzer, W. Jones, H. Kohler, J. Biol. Chem. 1986, 261, 15550-15556.

148 J. Tokunaga, J. Chem. Eng. Data 1975, 20, 41-46.

149 S.Z. Senior, L.L. Mans, H.D. VanGuilder, K.A. Kelly, M.P. Hendrich, T.E. Elgren, Biochem. 2003, 42, 4392-4397.

${ }^{150}$ F. Meyer, E. Kaifer, P. Kircher, K. Heinze, H. Pritzkow, Chem. Eur. J. 1999, 5, 1617-1630.

151 J.E. Pate, R.W. Cruse, K.D. Karlin, E.I. Solomon, J. Am. Chem. Soc. 1987, 109, 2624-2630.

${ }^{152}$ M. Hahroof-Tahir, N.N. Murthy, K.D. Karlin, N.J. Blackburn, S.N. Shaikh, J. Zubieta, Inorg. Chem. 1992, 31, 3001-3002.

${ }^{153}$ Z. Tyeklar, R.R. Jacobson, N. Wie, N.N. Murthy, J. Zubieta, K.D. Karlin, J. Am. Chem. Soc. 1993, 115, 2677-2689.

154 J.A. Halfen, V.G. Young, W.B. Tolman, J. Am. Chem. Soc 1996, 118, 10920-10921.

155 J.E. Bol, W.L. Driessen, R.Y.N. Ho, B. Maase, L Que, J. Reedijk, Angew. Chem. 1997, 109, $1022-$ 1025; Angew. Chem., Int. Ed. 1997, 36, 998-1000.

${ }^{156}$ S. Mahapatra, S. Kaderli, A. Llobet, Y. Neuhold, T. Palanche, J.A. Halfen, V.G. Young, T.A. Kaden, L. Que, A.D. Zuberbühler, W.B. Tolman, Inorg. Chem. 1997, 36, 6343-6356.

${ }^{157}$ H. Börzel, P. Comba, ,C. Katsichtes, W. Kiefer, A. Linke, V. Nagel, H. Pritzkow, Chem. Eur. J. 1999, 5, 1716-1721.

${ }^{158}$ C. He, H.L. DuBois, B. Hedman, K.O. Hodgson, S.J. Lippard, Angew. Chem. 2001, 113, $1532-$ 1535; Angew. Chem., Int. Ed. 2001, 40, 1484-1487.

159 E.I. Solomon, P. Chen, M. Metz, S. Lee, A.E. Palmer, Angew. Chem. 2001, 113, 4702-4724; Angew. Chem., Int. Ed. 2001, 40, 4570-4590.

${ }^{160}$ G.J. Kubas, Inorg. Synth. 1990, 28, 68-70.

${ }^{161}$ H. Börzel, Dissertation Universität Heidelberg 2001.

162 N. Kitajima, Y. Moro-oka, Chem. Rev. 1994, 94, 737-757.

${ }^{163}$ K.D. Karlin, M.S. Nasir, I. Cohen, R.W. Cruse, S. Kaderli, A.D. Zuberbühler, J. Am. Chem. Soc. 1994, 116, 1324-1336. 


\section{LITERATURVERZEICHNIS}

${ }^{164}$ E.I. Solomon, F. Tuczek, D.E. Root, C.A. Brown, Chem. Rev. 1994, 94, 827-856.

${ }^{165}$ DH. Lee, N. Wie, N.N. Murthy, Z. Tyeklar, K.D. Karlin, S. Kaderli, B. Jung, A.K. Zuberbühler, J. Am. Chem. Soc., 1995, 117, 12498-12513.

${ }^{166}$ I. Hyla-Kryspin, F. Meyer, unveröffentlichte Ergebnisse.

${ }^{167}$ K.D. Karlin, Y. Gultneh, T. Nicholson, J. Zubieta, Inorg. Chem. 1985, 24, 3725-3727.

${ }^{168}$ T.A. Annan, J.E. Kickham, D.G. Tuck, Can. J. Chem. 1991, 69, 251-256.

169 J.S. Thompson, J.C. Calabrese, Inorg. Chem. 1985, 24, 3167-3171.

${ }^{170}$ M. Ruf, B.C. Noll, M.D. Groner, G.T. Yee, C.G. Pierpont, Inorg. Chem. 1997, 36, 4860-4865.

${ }^{171}$ C. Benelli, A. Dei, D. Gatteschi, L. Pardi, Inorg. Chem. 1990, 29, 3409-3415.

172 W.O. Koch, V. Schünemann, M. Gerdan, A.X. Trautwein, H.-J. Krüger, Chem.-Eur. J. 1998, 4, 1255-1265.

${ }^{173}$ A. Die, D. Gatteschi, L. Pardi, A.L. Barra, L.C. Brunel, Chem. Phys. Lett. 1990, 175, 589-592.

${ }^{174}$ Persönliche Information von H. Pritzkow, Anorganisch-chemisches Institut, Universität Heidelberg.

175 G.A. Ardizzoia, S. Cenini, G. La Monica, N. Masciocchi, A. Maspero, M. Moret, Inorg. Chem. 1998, 37, 4284-4292.

${ }^{176}$ G.A. Ardizzoia, M.A. Angaroni, G. La Monica, N. Masciocchi, M. Moret, J. Chem. Soc., Dalton Trans. 1990, 7, 2277-2281.

${ }^{177}$ V.P. Hanot, T.D. Robert, J. Kolnaar, J.G. Haasnoot, J. Reedijk, H. Kooijman, A.L. Spek, J. Chem. Soc., Dalton Trans. 1996, 22, 4275-4281.

${ }^{178}$ F. Meyer, A. Jacobi, L. Zsolnai, Chem. Ber. 1997, 130, 1441-1447.

${ }^{179}$ B. Mernari, F. Abraham, M. Lagrenee, M. Drillon, P. Legoll, J. Chem. Soc, Dalton Trans. 1993, 11, 1707-1711.

${ }^{180}$ S. Torelli, C. Belle, S. Hamman, J. Pierre, Inorg. Chem. 2002, 41, 3983-3989.

${ }^{181}$ http://www.gettingwell.com/drug_info/nmdrugprofiles/nutsupdrugs/vit_0266.shtml.

${ }^{182}$ M. Hirose, S. Iwata, E. Ito, Y. Nihro, S. Takahashi, Y. Mizoguchi, Y. Miki, T. Satoh, N. Ito, T. Shirai, Carcinogenesis 1995, 16, 2227-2232.

${ }^{183}$ T. Nabeshima, Yakugaku Zasshi 1995, 115, 499-512.

${ }^{184}$ C. Atterwill, W.M. Purcell, F.M.D. Ismail (Roche Discovery), WO 98/34646 1998.

${ }^{185}$ M. Becker, S. Schindler, K.D. Karlin, T.K. Kaden, S. Kaderli, T. Palanché, A.D. Zuberbühler, Inorg. Chem. 1999, 1989-1995.

${ }^{186}$ S. Schindler, Eur. Inorg. Chem. 2000, 2311-2326.

187 J. Heijboer, J. Polym. Sci. C 1968, 16, 3755-3763.

${ }^{188}$ F.E. Karasz, J.M. O'Reilly, J. Polym. Sci., Pol. Lett. 1965, 3, 561-563.

${ }^{189}$ A.S. Hay, H.S. Stofford, G.F. Endres, J.W. Eustance, J. Am. Chem. Soc 1959, 81, 6335-6336.

${ }^{190}$ W. Chen, G. Challa, Eur. Polym. J. 1990, 26, 1211-1216.

191 S.S. Mahajan, B.B. Idage, B.D. Sarawade, N.N. Chavan, S. Sivaram, Macromol. Rep., A 1991, 28 (Suppl. 1), 7-13.

192 A.H.J. Tullemans, E. Bouwman, R.A.G. de Graaff, W.L. Driessen, J. Reedijk, Reel. Trav. Chim. Pays-Bas 1990, 109, 70-76.

${ }^{193}$ F.J. Viersen, G. Challa, J. Reedijk, Polymer 1990, 31, 1361-1367. 
194 J. Gao, J.H. Reibenspies, A.E. Martell, Inorg. Chim. Acta 2002, 338, 157-164.

195 H. Higashimura, K. Fujisawa, Y. Moro-oka, M. Kubota, A. Shiga, A. Terahara, H. Uyama, S. Kobayashi, J. Am. Chem. Soc. 1998, 120, 8529-8530.

${ }^{196}$ A.S. Hay, J. Polym. Sci. 1962, 58, 581-591.

197 P.J. Baesjou, W.L. Driessen, G. Challa, J. Reedijk, J. Mol. Catal. A 1998, 135, 273-283.

${ }^{198}$ P.J. Baesjou, W.L. Driessen, G. Challa, J. Reedijk, J. Mol. Catal. A 1999, 140, 241-253.

${ }^{199}$ F.J. Viersen, G. Challa, J. Reedijk, Polymer 1990, 31, 1368-1373.

${ }^{200}$ P. Gamez, J.A.P.P. van Dijk, W.L. Driessen, G. Challa, J. Reedijk, Adv. Synth. Catal. 2002, 344, 890-893.

201 P. Gamez, C. Simons, G. Aromi, W.L. Driessen, G. Challa, J. Reedijk, Appl. Catal. A 2001, 214, 187-192.

${ }^{202}$ G. Aromi, P. Gamez, H. Kooijman, A.L. Spek, W.L. Driessen, J. Reedijk, Eur. J. Inorg. Chem. 2003, 1394-1400.

203 persönliche Mitteilung P. Gamez.

${ }^{204}$ N.G. Connelly, W.E. Geiger, Chem. Rev. 1996F, 96, 877-910.

${ }^{205}$ M. Cvetkovic, S.R. Batten, B. Moubaraki, K.S. Murray, L. Spiccia, Inorg. Chim. Acta 2001, 324, 131-140.

${ }^{206}$ G.M. Sheldrick, SHELXS-97, „Program for Crystal Structure Solution“, Universität Göttingen, 1997.

207 G.M. Sheldrick, SHELXL-97, „Program for Crystal Structure Refinement“, Universität Göttingen, 1997. 


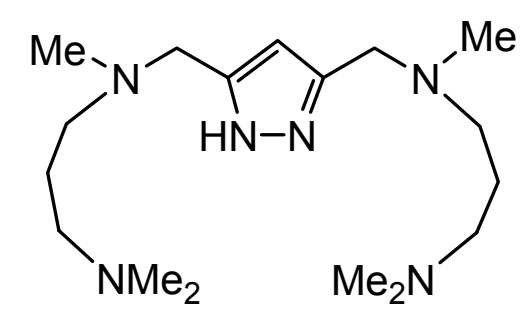

$H L^{1}$<smiles>CN(C)CCN(C)Cc1cc(CN(C)C)[nH]n1</smiles>

$\mathrm{HL}^{3}$<smiles>CN(C)Cc1cc(CN(C)C)[nH]n1</smiles>

$\mathrm{HL}^{5}$

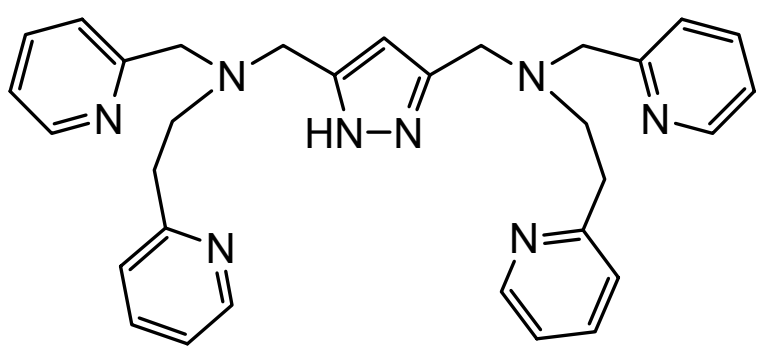

$\mathrm{HL}^{7}$

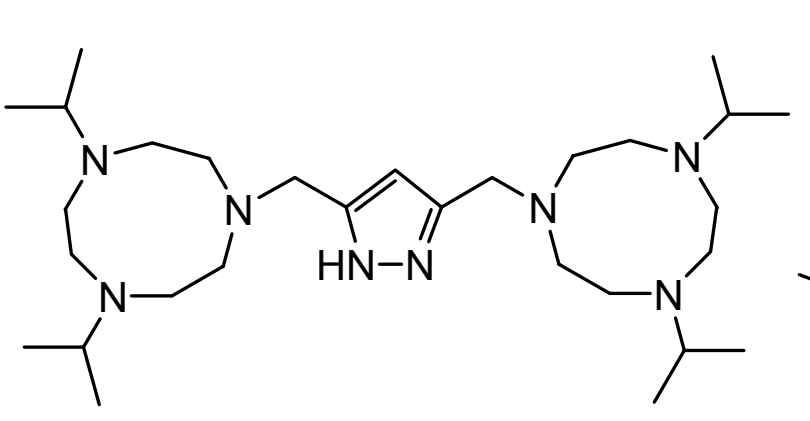

$\mathrm{HL}^{9}$

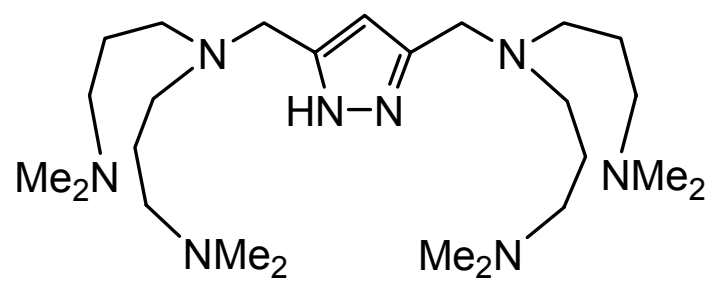

$\mathrm{HL}^{2}$

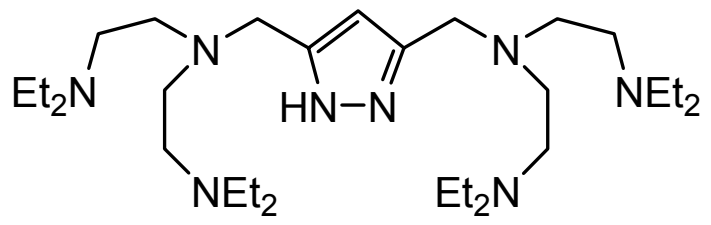

$\mathrm{HL}^{4}$

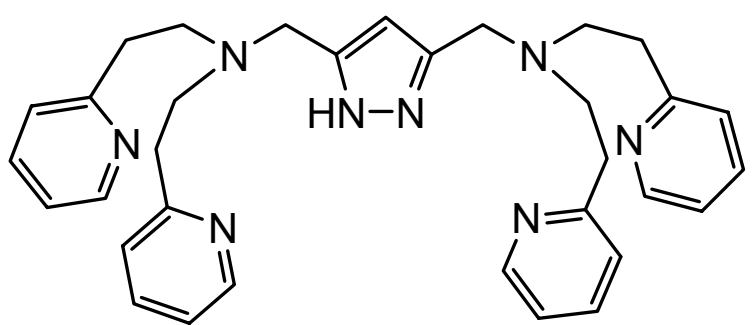

$\mathrm{HL}^{6}$

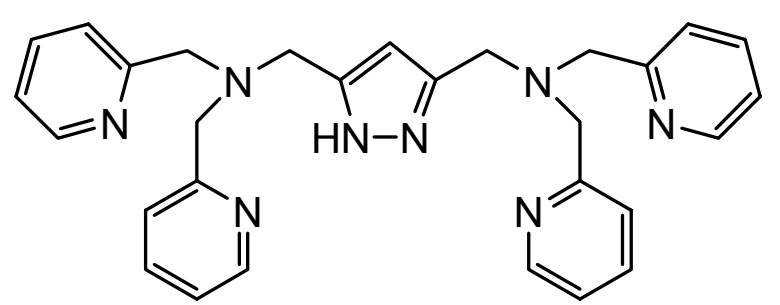

$\mathrm{HL}^{8}$

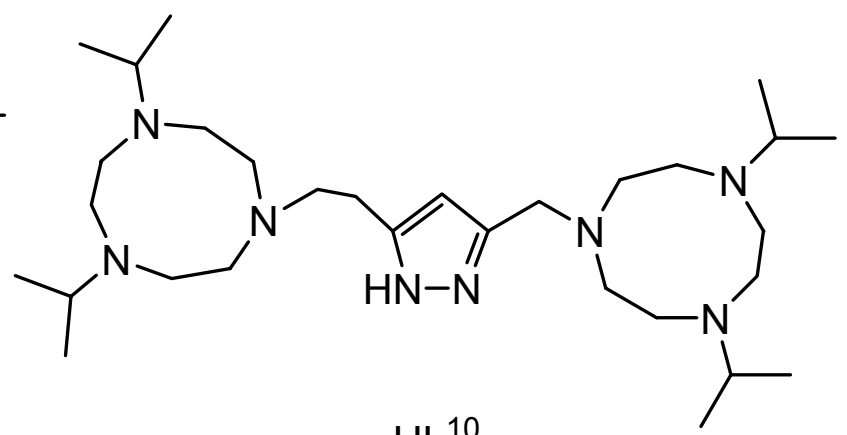

$\mathrm{HL}^{10}$ 
FORMELVERZEICHNIS
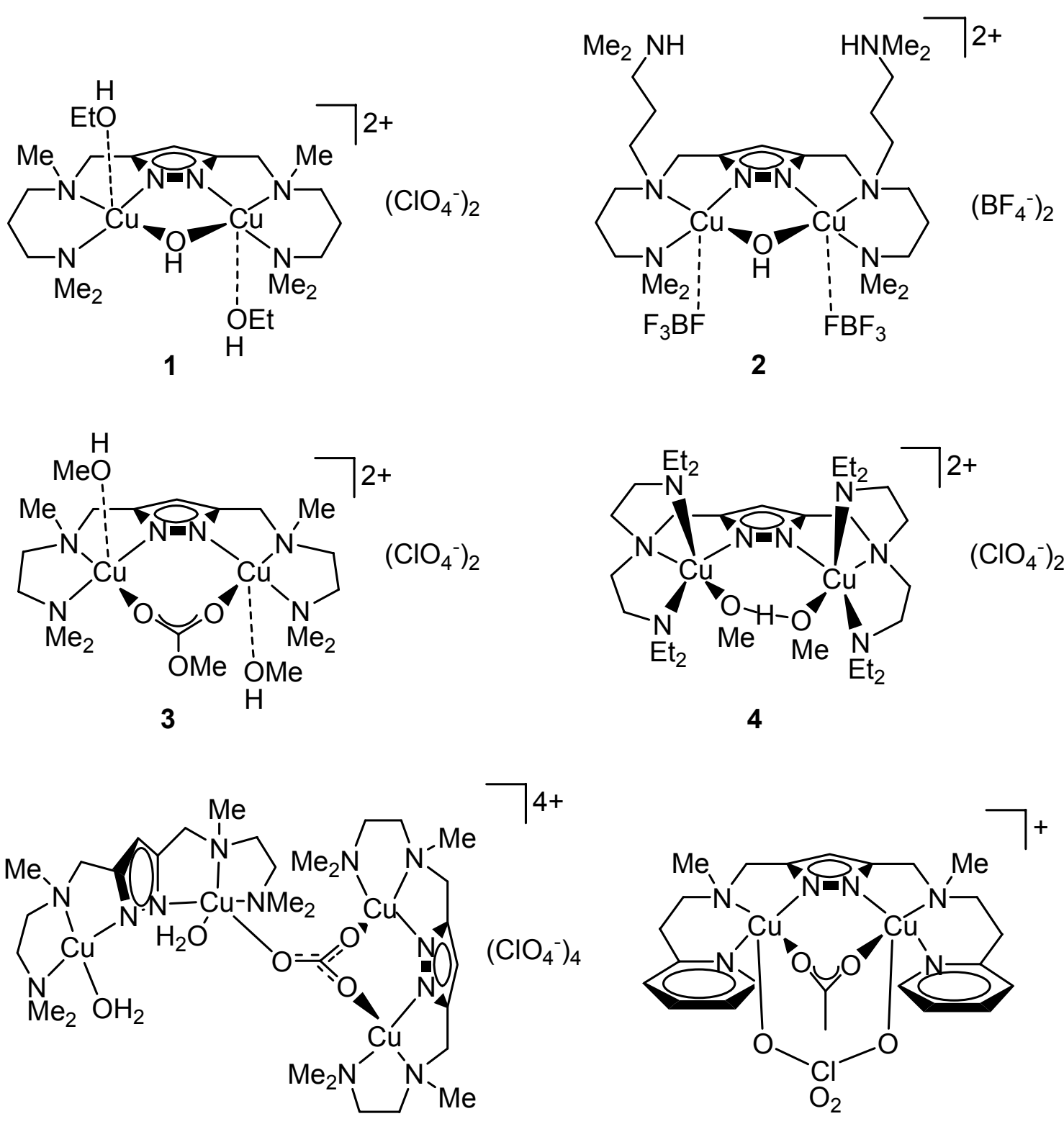

5
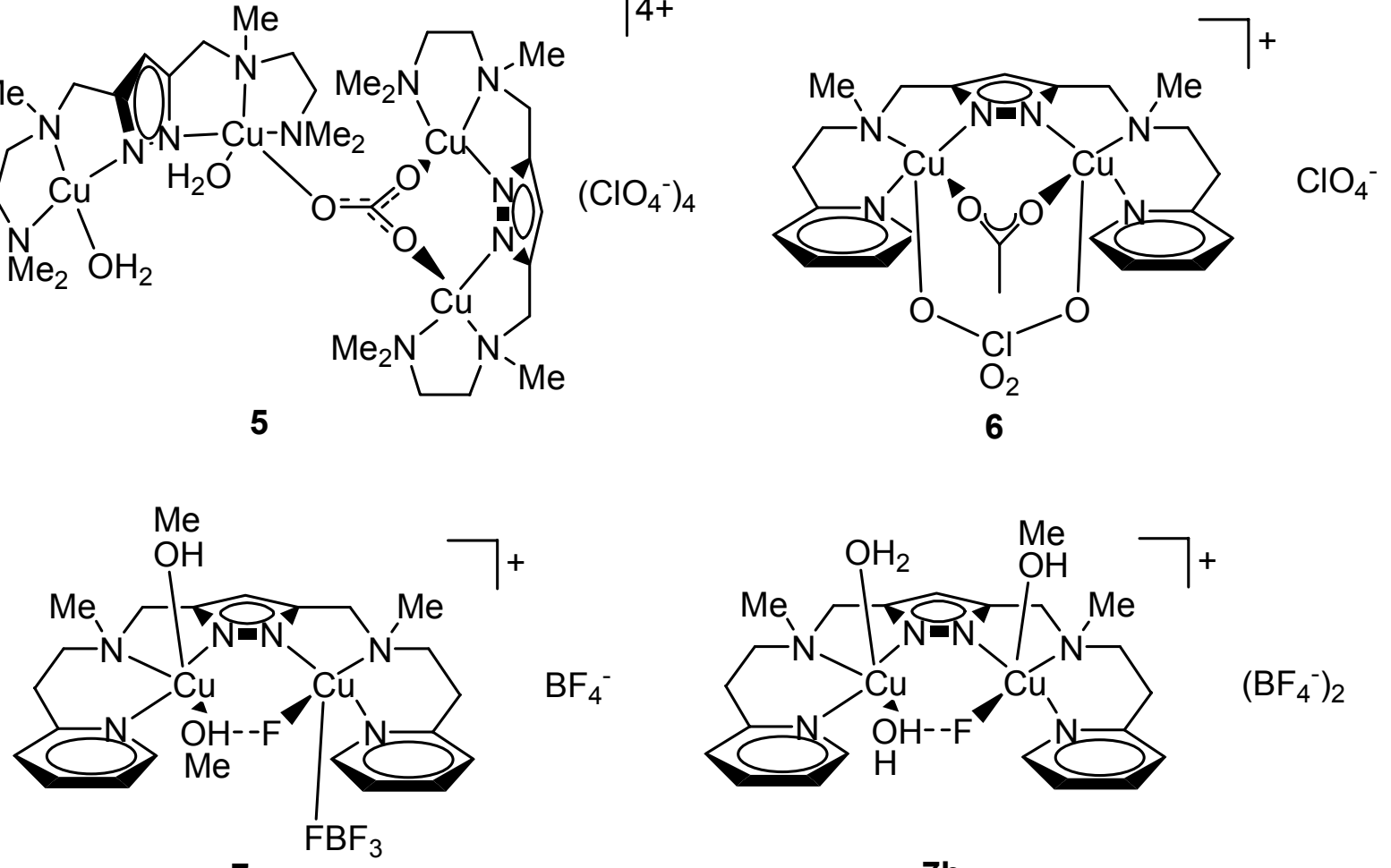

$\mathrm{BF}_{4}^{-}$

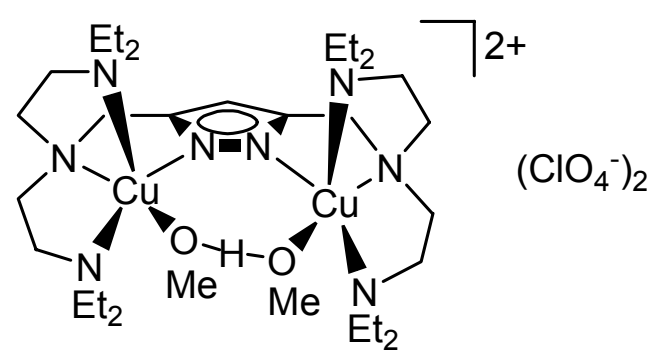

4

$7 a$

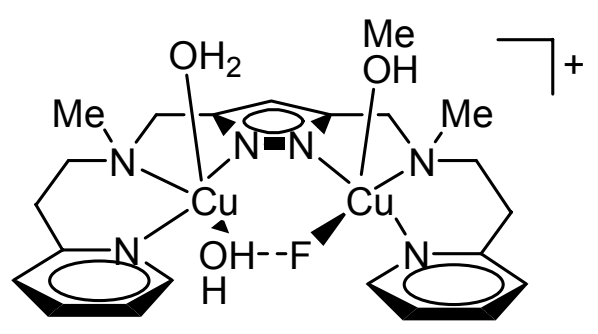

$\left(\mathrm{BF}_{4}{ }^{-}\right)_{2}$

$7 b$ 
FORMELVERZEICHNIS
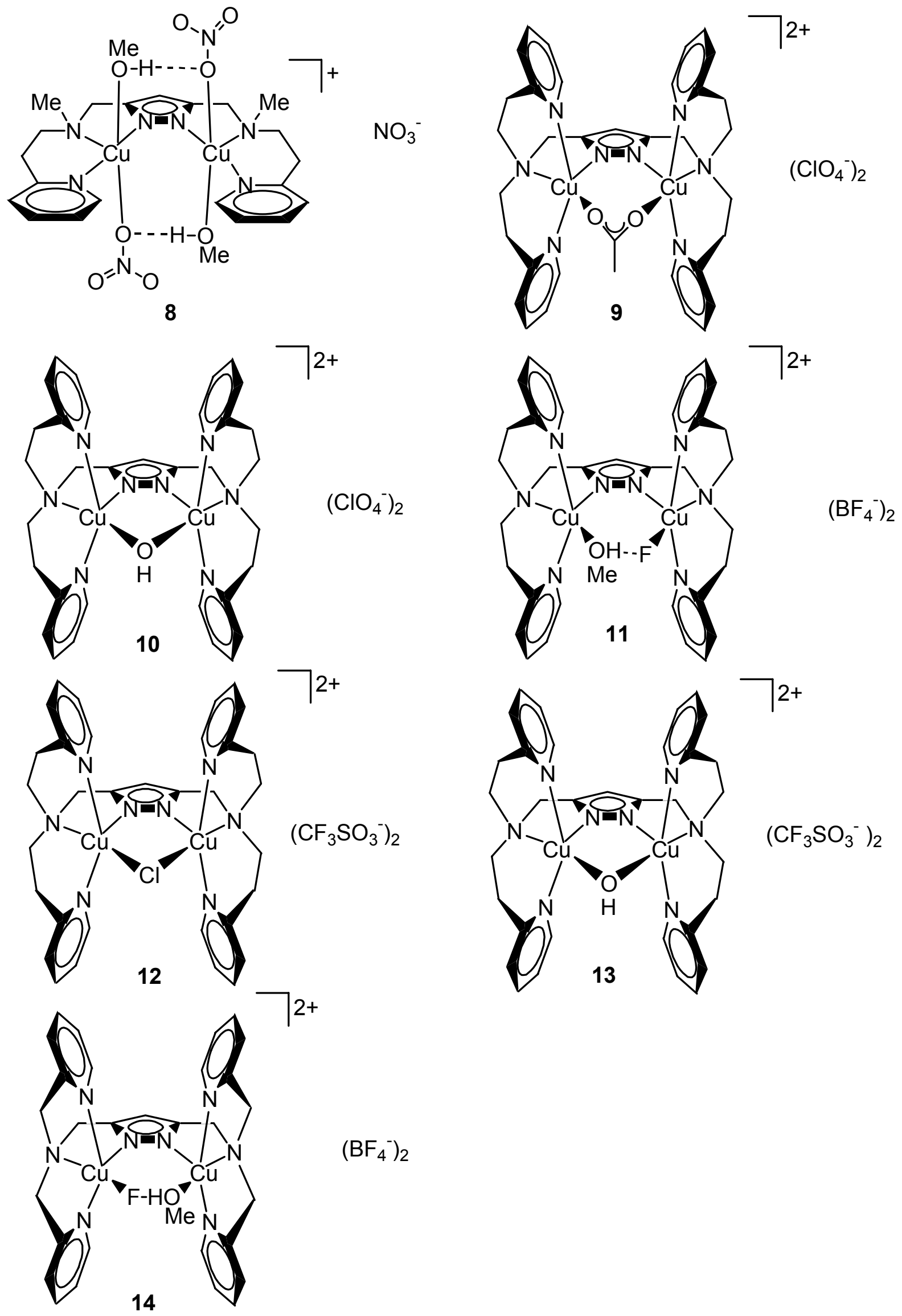

$\left(\mathrm{BF}_{4}^{-}\right)_{2}$ 
FORMELVERZEICHNIS

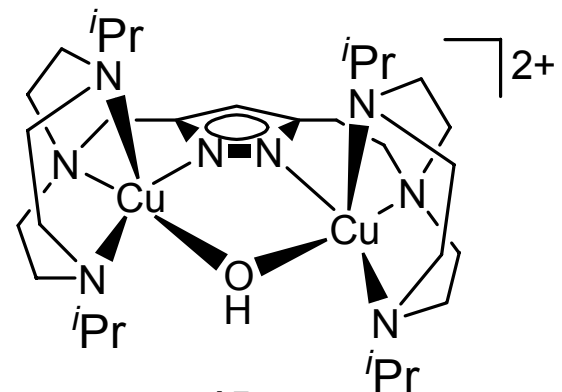

15

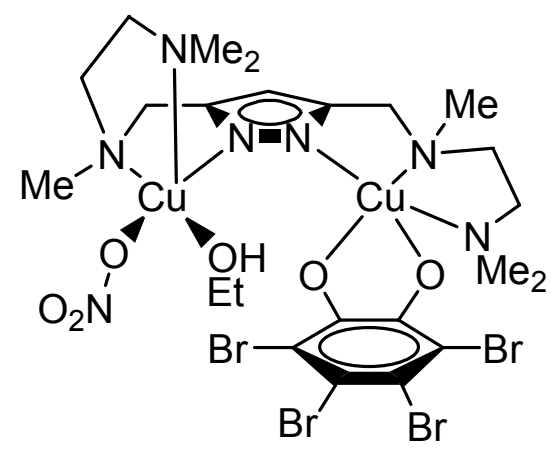

19

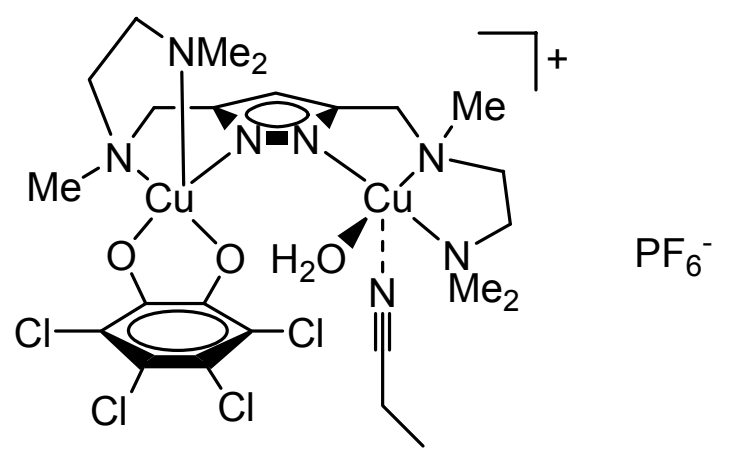

18

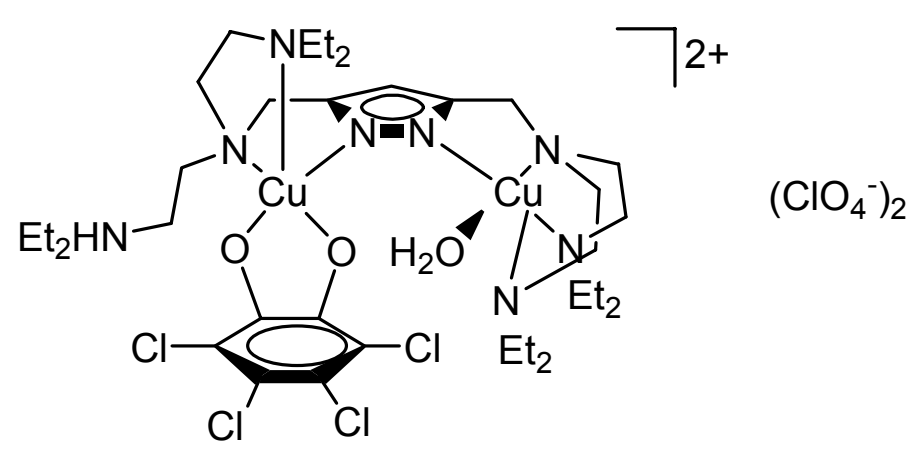

20

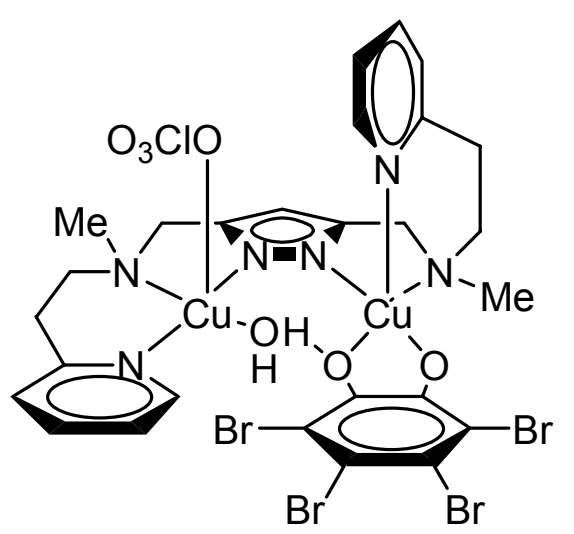

21

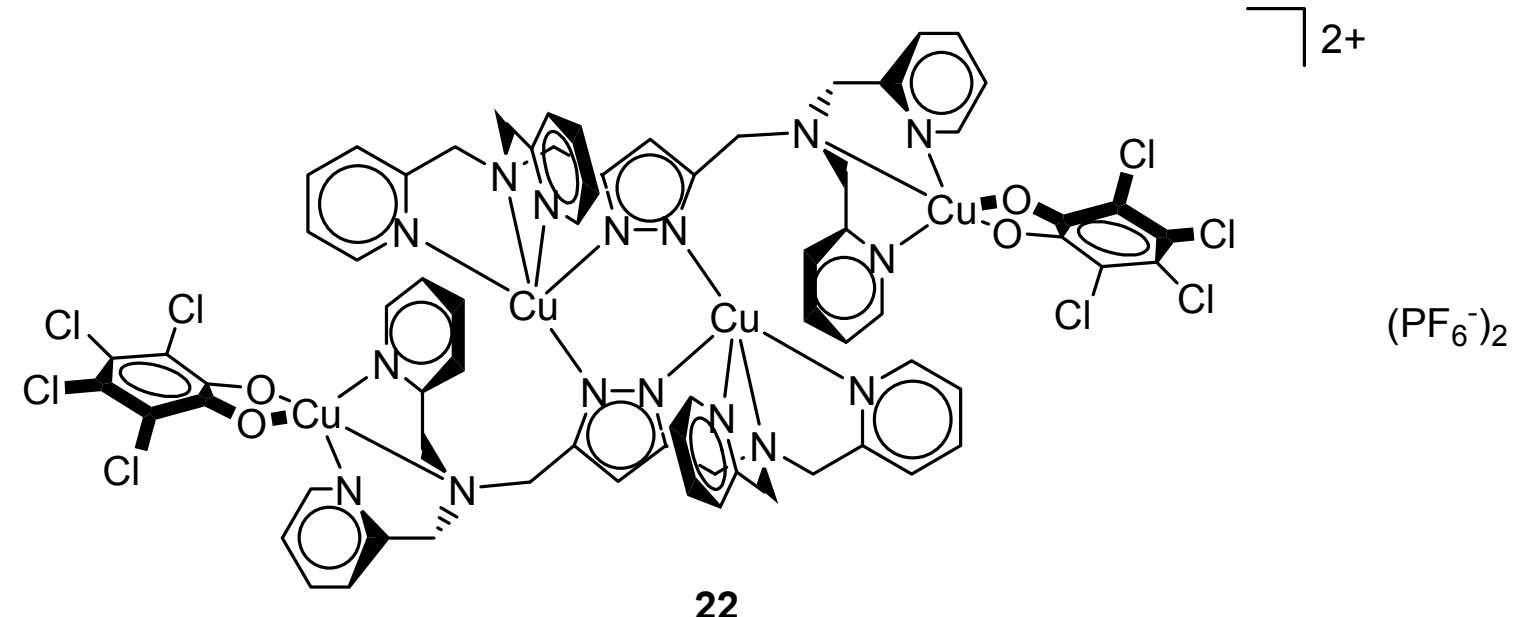




\section{Dank}

Ich danke allen Mitgliedern des AK Meyer in Heidelberg und Göttingen für das sehr gute und freundschaftliche Arbeitsklima und auch die schönen Abende in Göttingen und beim Grillen, ebenso wie die tollen (leider zu seltenen) Frühstücke auf der grünen Wiese vor Fünfnulldrei in Heidelberg.

Dem AK Comba danke ich für die gute Zusammenarbeit und das kollegiale Verhältnis sowie auch für die zur Verfügung gestellten Geräte und zahlreichen Hilfestellungen, auch bei Computerproblemen.

Den Mitgliedern des AK Sheldrick danke ich für das freundschaftliche Verhältnis während der Arbeit und beim Betreuen des AC0 Praktikums sowie auch außerhalb der Universität.

Den AKs Huttner und Limberg danke ich für die gute Zusammenarbeit und die zur Verfügung gestellten Geräte.

Silke Buchler danke ich für die vielen netten Unterhaltungen, die guten Tips und die sportliche Begeisterung für das AK-Joggen.

Jens Röder danke ich für die tiefenpsychologischen Gespräche und das - trotz mancher Probleme - gute kollegiale Arbeitsklima in Fünfnulldrei.

Michael Merz danke ich für die Unterstützung bei den Stopped-Flow- und RamanMessungen.

Jörg Teichgräber und Dr. Maringgele danke ich für die großartige Unterstützung beim Aufbau des neuen Labors in Göttingen.

Dr. Pritzkow in Heidelberg danke ich für die viele Arbeit, die er sich mit meinen nicht immer perfekten Kristallen gemacht hat, ebenso danke ich allen Kristallographen in Göttingen, die für mich Kristalle gemessen haben: Denis Vidovic, Thomas Labahn, José Antonio Cuesta Seijo, Dr. Noltemeyer und Fabio Dall'Antonia.

An Gilles Noël geht ein Merci für die NMR-Messungen und für seine Aufmunterungen bei meinem Französischkurs.

Rosana Ferrari, Serhiy Demeshko, Miguel Alvariño, Bernhard Bauer, Stamatia Katsiaouni, Gilles Noël, Guido Leibeling und Sebastian Dechert danke ich für die vielen interessanten (und lustigen) Gespräche nicht nur über Chemie. Ich danke Bernhard Bauer, Stamatia Katsiaouni, Sebastian Dechert und insbesondere Renate Schwiedernoch für das Korrekturlesen meiner Arbeit. 
Prof. Meyer danke ich dafür, daß er meine Arbeit ermöglicht und mich immer mit Rat und Tat unterstützt hat.

Axel Hoffmann, Carsten Schäfer und Renate Schwiedernoch danke ich für ihre Freundschaft, die mich in all diesen Jahren begleitet hat.

Ich danke meinen Eltern für ihre Unterstützung. Ihr wart immer da, wenn ich Euch gebraucht habe.

Meiner Freundin Rosana Ferrari danke ich für ihre Liebe und ihr Vertrauen. Du hast mein Leben vollständig gemacht. 


\section{Lebenslauf}

Name

Geburtsdatum

Geburtsort

Familienstand

Staatsangehörigkeit

Schulbildung

Wehrdienst

Hochschulstudium

Promotion:

Beschäftigungsverhältnisse

Stipendien:
Jens Ackermann

8. Mai 1973

Weinheim /Bergstr.

ledig

deutsch

1979-1983 Grundschule Birkenau

1983-1992 Werner-Heisenberg-Gymnasium Weinheim

Abitur: 19. Mai 1992

1992-1993 Philippsburg

Chemie (Diplom) seit dem Wintersemester 1993/1994

an der Ruprecht-Karls-Universität Heidelberg

Diplom-Vorprüfung: 2. Mai 1996

Diplom-Hauptprüfung: 17. Dezember 1998

Diplomarbeit: Januar 1999 bis Oktober 1999 mit dem Thema: „Catecholase-Aktivität neuer zweikerniger Kupfer-Pyrazolatkomplexe"

Beginn: 15. Oktober 1999 an der Ruprecht-Karls-Universität Heidelberg mit dem Thema: „Biomimetische Oxidationsreaktionen zweikerniger Kupferpyrazolatkomplexe“"

seit dem 01.11.01 an der Georg-August-Universität Göttingen Januar 1999 bis September 1999 als ungeprüfte wissenschaftliche Hilfskraft an der Ruprecht-Karls-Universität Heidelberg

Oktober 1999 bis September 2001 als wissenschaftlicher Angestellter an der Ruprecht-Karls-Universität Heidelberg seit November 2001 als wissenschaftlicher Angestellter an der Georg-August-Universität Göttingen

Oktober 2000 bis Oktober 2001 Stipendium nach dem Landesgraduiertenförderungsgesetz des Landes BadenWuerttemberg 